University of Nebraska - Lincoln

DigitalCommons@University of Nebraska - Lincoln

1980

\title{
A Statistical Analysis of the Quality of Surface Water in Nebraska
}

R. A. Engberg

Follow this and additional works at: https://digitalcommons.unl.edu/usgspubs

Part of the Geochemistry Commons, Geology Commons, Geomorphology Commons, Hydrology Commons, and the Other Earth Sciences Commons

This Article is brought to you for free and open access by the US Geological Survey at DigitalCommons@University of Nebraska - Lincoln. It has been accepted for inclusion in Publications of the US Geological Survey by an authorized administrator of DigitalCommons@University of Nebraska - Lincoln. 
A STATISTICAL ANALYSIS OF THE QUALITY OF SURFACE WATER IN NEBRASKA

\section{U.S. GEOLOGICAL SURVEY}

Water-Resources Investigations 80-43

Prepared in cooperation with

Nebraska Natural Resources Commission

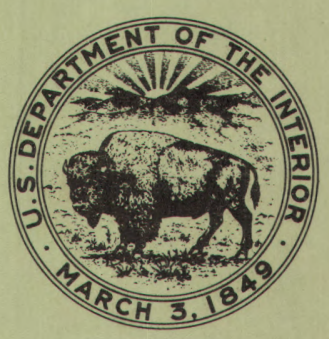


4. Title and Subtitle

A STATISTICAL ANALYSIS OF THE QUALITY OF SURFACE WATER IN NEBRASKA

\section{Author(s)}

R. A. Engberg

9. Performing Organization Name and Address

U.S. Geological Survey, Water Resources Division

Room 406 Federal Building

100 Centennial Mal1, North

Lincoln, Nebraska 68508

12. Sponsoring Organization Name and Address

U.S. Geological Survey, Water Resources Division

Room 406 Federal Building

100 Centennial Ma11, North

Lincoln, Nebraska 68508

15. Supplementary Notes

Prepared in cooperation with the Nebraska Natural Resources Commission
16. Abstract (Limit: 200 words) This report provides descriptive statistics for 29 chemical or
biological constituents for 109 stream sites in Nebraska sampled by the U.S. Geological Survey beginning in 1946. Also provided for each site are regression equations relating specific conductance to each of 12 chemical constituents and a regression equation relating specific conductance to stream discharge.

The descriptive statistics are presented by river basins. Water leaving these basins that has the lowest mean specific conductance (266 umho/cm) is from the Niobrara River at Verdel, and water that has the highest $(1,890$ umho/cm) is from the South P1atte River at Roscoe. Statewide, the principal cations in streamflow are calcium and sodium, and the principal anions are bicarbonate and sulfate. Calcium and bicarbonate usually predominate where mean specific conductance is less than $1,000 \mathrm{umho} / \mathrm{cm}$, and sodium and sulfate predominate where the mean exceeds 1,000 umho/ cm.

Based on regression equations, dissolved solids, hardness, calcium, magnesium and bicarbonate, with some exceptions, correlate well with specific conductance for all stream sites.

Illustrations of how regression equations may be used to estimate water quality of streamflow are given and are compared with values from actual analyses.

17. Document Analysis a. Descriptors

Streamflow, *water quality, surface waters, overland runoff, river basins

b. Identifiers/Open-Ended Terms

Specific conductance, regression equations, chemical constituents, *Nebraska, correlation coefficients

c. COSATI Field/Group

18. Availability Statement

№ restriction on distribution

19. Security Class (This Report)

21. No. of Pages 282

22. Price 
A STATISTICAL ANALYSIS OF THE QUALITY OF SURFACE WATER IN NEBRASKA By R. A. Engberg

U.S. GEOLOGICAL SURVEY

Water-Resources Investigations 80-43

Prepared in cooperation with Nebraska Natural Resources Commission

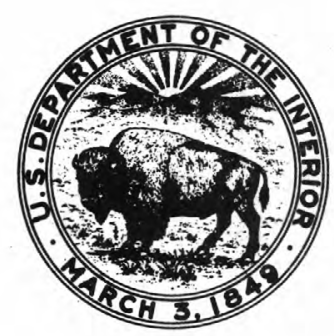

June 1980 
UNITED STATES DEPARTMENT OF THE INTERIOR

CECIL D. ANDRUS, Secretary

GEOLOGICAL SURVEY

H. William Menard, Director

For additional information write to:

U.S. Geological Survey

406 Federal Building

100 Centennial Ma11, North

Lincoln, Nebr. 68508 
Abstract......................................... 1

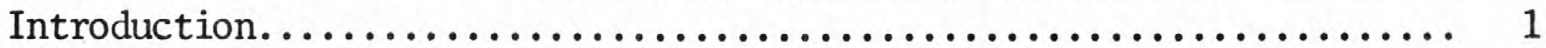

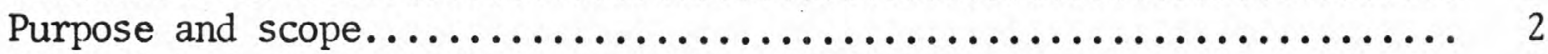

Natural hydrologic influences on water quality ............... 2

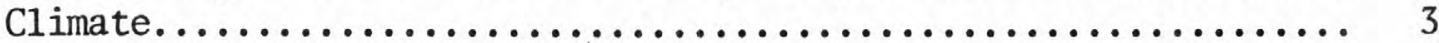

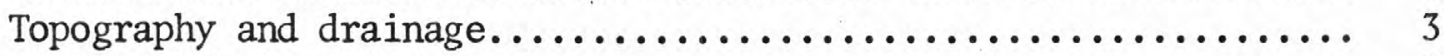

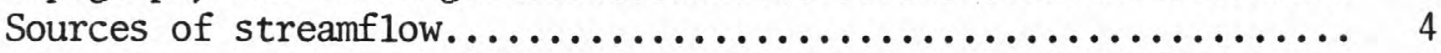

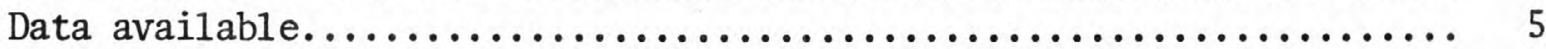

Programs through which data was obtained................ 6

Constituents selected for statistical analysis............. 6

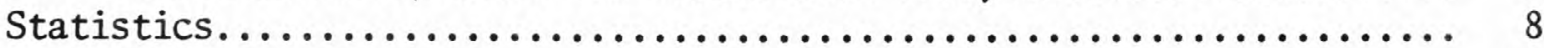

Explanation for blanks in the data tables................. 10

Analogous constituents........................... 11

Statistical data by basin........................... 12

White River - Hat Creek basin....................... 12

Niobrara River basin............................ 17

Missouri River Tributaries basin and Missouri River Main Stem

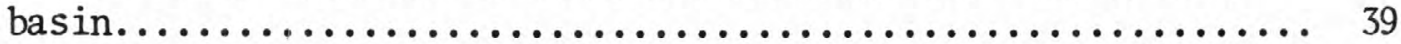

North Platte River basin......................... 49

South Platte River basin.......................... 82

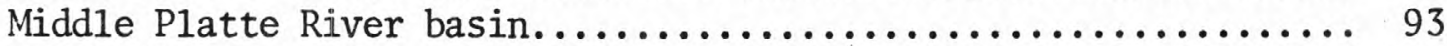

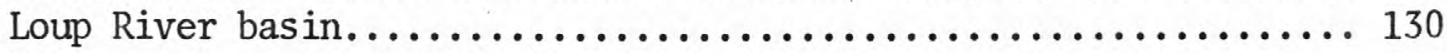

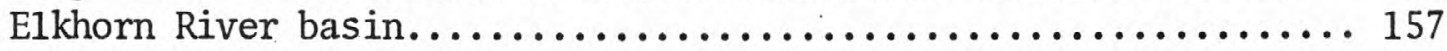

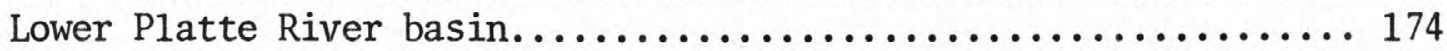

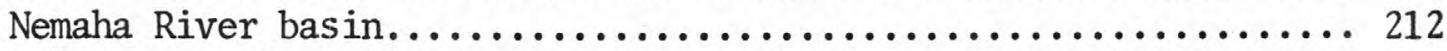

Republican River basin............................ 220

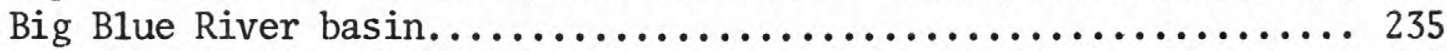

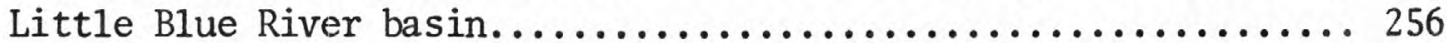

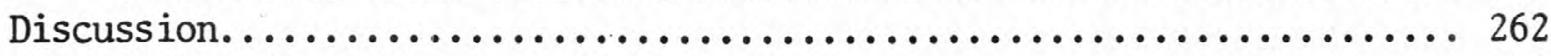

Application of descriptive statistics.................. 263

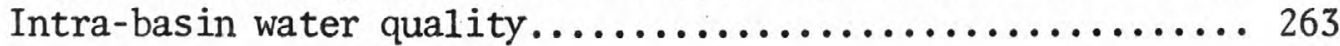

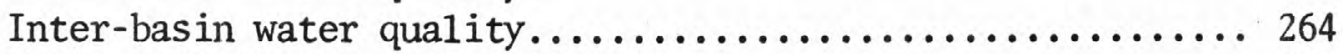

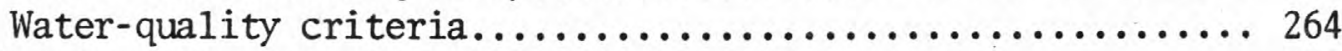

Application of regression equations.................... 267

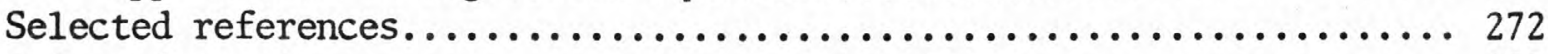

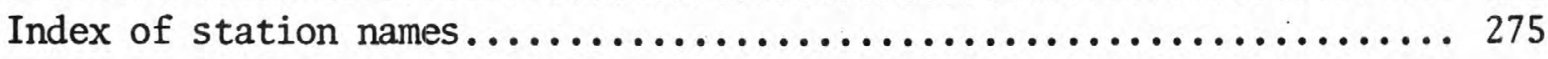


Figure 1. Map showing river basins of Nebrask ................ Page

Figure 1. Map showing river basins of Nebraska................ 13

Figures 2-14 - Maps showing location of water-quality sampling sites:

2. White River - Hat Creek basin..................... 14

3. Niobrara River basin.......................... 18

4. Missouri River tributaries basin and Missouri River

main stem basin.............................. 40

5. North Platte River basin....................... 50

6. South Platte River basin........................ 83

7. Middle Platte River basin......................... 94

8. Loup River basin............................. 131

9. Elkhorn River basin.......................... 158

10. Lower Platte River basin........................ 175

11. Nemaha River basin............................ 213

12. Republican River basin......................... 221

13. Big Blue River basin.......................... 236

14. Little Blue River basin........................ 257

TABLES

Table 1. Summary of principal Federal and cooperative surface-water quality programs in Nebraska, 1946 to present.......... 7

2. Comparison of concentrations of selected constituents to show the effect of sewage effluent on Spring Creek near Lexington................................. 95

3. Comparison of concentrations of selected constituents to show the effect of sewage effluent on Wood River near Grand Island................................ 95

4. Median fecal coliform and fecal streptococcus concentrations for stations on Salt Creek................ 176

5. Comparison of mean and median concentrations of several constituents to show assimilative capacity of the West Fork Big Blue River.

6. Comparison of mean concentrations of chemical constituents in outflow from each of 13 river basins in Nebraska..... 265

7. Comparison of nitrite + nitrate, total phosphorus, BOD, fecal coliform, and fecal streptococcus in outflow from 13 river basins in Nebraska...................... 266

8. Examples showing comparison of frequency statistics with irrigation criteria for two constituents............. 268

9. Comparison between concentrations of chemical constituents determined by chemical analysis with those concentrations computed from regression equations. 
The International System (SI) is a consistent system of metric units adopted by the Eleventh General Conference of Weights and Measures in 1960. Selected factors for converting inch-pound units used in this report to SI metric units are given below.

$\begin{array}{lcl}\text { Multiply inch-pound units } & \underline{\text { By }} & \text { To obtain SI units } \\ \text { acre } & 0.0040 & \text { square kilometer }\left(\mathrm{km}^{2}\right) \\ \text { acre-foot (acre-ft) } & 1,233 & \text { cubic meter }\left(\mathrm{m}^{3}\right) \\ \text { foot } & 0.3048 & \text { meter (m) } \\ \text { cubic foot per second }\left(\mathrm{ft}^{3} / \mathrm{s}\right) & 28.3162 & \text { liter per second }(\mathrm{L} / \mathrm{s}) \\ \text { inch (in) } & 25.4 & \text { millimeter (m) } \\ \text { mile (mi) } & 1.609 & \text { kilometer }(\mathrm{km}) \\ \text { square mile }\left(\mathrm{mi}^{2}\right) & 2.590 & \text { square kilometer }\left(\mathrm{km}^{2}\right) \\ \text { degree Fahrenheit }\left({ }^{\circ} \mathrm{F}\right) & 5 / 9(\mathrm{~F}-32) & \text { degree Celsius }\left({ }^{\circ} \mathrm{C}\right)\end{array}$



A STATISTICAL ANALYSIS OF THE QUALITY OF SURFACE WATER IN NEBRASKA

By

R. A. Engberg

ABSTRACT

This report provides descriptive statistics for 29 chemical or biological constituents for 109 stream sites in Nebraska sampled by the U.S. Geological Survey beginning in 1946. Also provided for each site are regression equations relating specific conductance to each of 12 chemical constituents and a regression equation relating specific conductance to stream discharge.

The descriptive statistics are presented by river basins. Water leaving these basins that has the lowest mean specific conductance (266 umho/cm) is from the Niobrara River at Verdel, and water that has the highest $(1,890$ umho/cm) is from the South Platte River at Roscoe. Statewide, the principal cations in streamflow are calcium and sodium, and the principal anions are bicarbonate and sulfate. Calcium and bicarbonate usually predominate where mean specific conductance is less than $1,000 u m h / c m$, and sodium and sulfate predominate where the mean exceeds 1,000 umho/cm.

Based on regression equations, dissolved solids, hardness, calcium, magnesium and bicarbonate, with some exceptions, correlate well with specific conductance for all stream sites.

Illustrations of how regression equations may be used to estimate water quality of streamflow are given and are compared with values from actual analyses.

\section{INTRODUCTION}

Systematic collection of water-quality data by the U.S. Geological Survey for streams in Nebraska began in 1946 when the Geological Survey office and laboratory were established in Lincoln. These data, together with a few scattered data collected prior to 1946, constitute a rich source of information on the quality of streamflow in Nebraska that can be important in a wide range of potential uses if they are reduced to a manageable and useful form. 
Section 208 of Public Law 92-500 is directed toward the investigation of and planning measures for the control of nonpoint-source pollution in streams. The Nebraska Natural Resources Commission--the agency having overall responsibility for 208 planning in Nebraska--recognized the need in the planning process to know what can be learned from existing records and approved a proposal to summarize and reduce the Survey's existing records to a manageable and useful form. The purpose of this report is to present Nebraska surface-water-quality data in such form. Although information in this report is of particular importance to those involved in 208 planning, it also should prove useful to planners at many levels, including Natural Resources District managers, officials of State and Federal agencies, and private individuals.

Data for 109 stream-sampling sites in Nebraska are summarized by river basin in this report. Maximums, minimums, means, medians, standard deviations, and percentiles have been determined for 29 different physical, chemical, and microbiological constituents. Such descriptive statistics provide a means for comparing constituent values within an analysis and for comparing individual constituents between analyses and between stations.

Functional relationships between specific conductance and each of 12 different constituents, as well as the functional relationship between specific conductance and water discharge, have been determined by the use of regression equations. These relationships allow users to estimate constituent concentrations when only specific conductance is known, or to estimate specific conductance when only water discharge is known.

Trend analyses or analyses of variance of data between stations have not been included in this report. These and other relationships might form the basis for future reports.

\section{NATURAL HYDROLOGIC FACTORS THAT INFLUENCE WATER QUALITY}

The quality of streamflow is influenced by many hydrologic factors of which several are discussed below. Climate affects stream-water quality through variations in temperature and precipitation. Topography and drainage affect stream quality by influencing the magnitude and rate of overland runoff. Sources of streamflow affect stream quality in that a high fraction of ground-water inflow tends to make streamflow constant in quality, whereas a high fraction of overland runoff tends to make it highly variable. 
Nebraska's climate is characterized by extremes. Hot summers and cold winters are normal, with wide and frequent temperature variations common at all times of the year. Statewide, January was the coldest month for the period 1941-70, according to Lawson and others (1977), with mean monthly average temperatures of $25^{\circ} \mathrm{F}\left(-4^{\circ} \mathrm{C}\right)$ or less for more than two-thirds of the State. July was the hottest month for the same period, with mean monthly average temperatures exceeding $75^{\circ} \mathrm{F}\left(24^{\circ} \mathrm{C}\right)$ for more than half the State.

Precipitation varies greatly across Nebraska. For the period 194170 , the mean annual precipitation ranged from 36 inches in extreme southeast Nebraska to 15 inches in western Nebraska (Lawson and others, 1977). Precipitation is unevenly distributed during the year, averaging five times greater in June, the wettest month, than in January, the dryest month.

\section{Topography and Drainage}

Land-surface elevations increase from east to west in Nebraska. The greatest relief is observed along the upper reaches of stream valleys, especially along the North Platte River in western Nebraska.

Topography in eastern Nebraska is related to glaciation during the Pleistocene Epoch. The eastern one-fourth of Nebraska was covered at least once by advancing glaciers during this period. Much of this area is composed of rolling hills made up of clayey till deposited by the glacier and covered by loess of aeolian origin. Southwest of the rolling hills are loess plains underlain by sands and gravels, some of which originate from the glaciers. Some of the plains in central and southwest Nebraska have been modified greatly by water and wind erosion to moderate to steeply sloping hills.

About one-fourth of Nebraska is covered by sandhills. These deposits are mostly stabilized by vegetation and are characterized by dunes, the axes of which lie generally in a northwesterly to southeasterly direction. The sandhills geologically are young. Keech and Bentall (1971) believe that they were formed during the last period of continental glaciation in late Pleistocene time.

Much of western Nebraska is high plains, characterized by bedrock covered in some areas by thin mantle rock. In southwest Nebraska, the bedrock is covered by loess. Topographic surfaces range from flatlands to rolling hills in the bedrock areas. 
Drainage follows the regional topography in an eastward or southeastward direction with the principal exception of the Platte River in the central part of the State. Here the channel describes a broad arc flowing to the northeast for approximately $100 \mathrm{mi}$ before returning to a southeasterly course. Increases of land-surface elevation as a result of deposition from glaciation in southeast Nebraska probably caused the river to change to its present course.

\section{Sources of Streamflow}

An annual average of approximately 1 million acre-ft of streamflow enters Nebraska from Colorado, Wyoming, Kansas, and South Dakota. However, approximately 7.1 million acre-ft leaves Nebraska each year (Nebraska Soil and Water Conservation Commission, 1971). The increase is derived from two sources within Nebraska--discharge from the groundwater reservoir and overland runoff. Of these two sources, the groundwater reservoir provides approximately 3.3 million acre- $\mathrm{ft}$ and overland runoff provides the remainder (Bentall and Shaffer, 1979).

Ground water is not evenly distributed throughout the State. Of the approximately 2 billion acre-ft of ground water estimated to be in storage in Nebraska, nearly half is in the Sandhills area where saturated thicknesses of 50 to $1,000 \mathrm{ft}$ of water-bearing material are common (University of Nebraska, 1966). In contrast, at many places in the extreme northwest corner of the State, in Republican River drainage, and in the glaciated part of eastern Nebraska there is less than $20 \mathrm{ft}$ of water-bearing material and in some places none at all.

In areas where ground water is abundant and soils are sandy, such as in the Sandhills, streamflow is maintained mostly by ground-water discharge. Such streams as the Niobrara, Middle Loup, and North Loup Rivers are characterized by relatively small ranges of water discharge owing to the constancy in amount of ground-water inflow and to the fact that only small fractions of their drainage areas contribute overland runoff. For example, the range in daily discharge for the Middle Loup River at Dunning from 1945 to 1978 was only 100 to $1,020 \mathrm{ft}^{3} / \mathrm{s}$. The 33year average discharge for this stream site was $398 \mathrm{ft}^{3} / \mathrm{s}$. The drainage area for this station is $1,850 \mathrm{mi}^{2}$ of which only $80 \mathrm{mi}^{2}$ contributes directly to surface runoff. Probably more than 75 percent of the flow at this station is derived from ground water.

On the other hand, in areas where ground water is not abundant and soils are clayey, streamflow is maintained mostly from overland runoff. This is true particularly for streams in the glaciated area of eastern Nebraska, such as the Big Blue River and the Big and Little Nemaha 
Rivers. The range in discharge for the Big Nemaha River at Falls City for the period 1944 to present, for example, was 3.0 to $71,600 \mathrm{ft}^{3} / \mathrm{s}$; the 32-year average discharge was $562 \mathrm{ft}^{3} / \mathrm{s}$. The drainage area of this station is $1,340 \mathrm{mi}^{2}$, all of which contributes directly to surface runoff. Probably 90 percent of the streamflow at this station is derived from overland runoff.

\section{DATA AVAILABLE}

The earliest sample for chemical analysis for a Nebraska stream in Geological Survey files was collected in 1912 from the Elkhorn River at Waterloo. Since then, samples have been collected at least once from approximately 425 surface-water sites in Nebraska. Analyses from scattered locations exist in the files through the early 1940's. Most were collected by Geological Survey headquarters personnel as part of specific projects or for general information as part of nationwide surveys. Sampling on a regular basis at specific stations did not begin until 1946 with the advent in Nebraska of the Missouri River Basin (MRB) program.

In recent years, the need for data on the quality of surface water in Nebraska has broadened considerably. Prior to the 1960 's, most sampling in the State was conducted to obtain information for project planning and development, particularly in relation to irrigation. In the mid-1960's attention began to focus on the need for data to be used in the control and abatement of pollution, and this need now has become the most important one. Even in regard to pollution, emphasis on data needs is changing from point sources to nonpoint sources.

One reason for the broadening of water-quality data is an increasing awareness of the importance of water quality as both a management and predictive tool. This is coupled with recent developments in analytical methodology that make possible the measurement of many constituents now that could not be measured routinely only a few years ago.

As a result of these broadened data needs, there is now a much wider variety of data available than there was even 10 years ago. In 1978 , water-quality data were collected on a monthly basis at 53 sites in Nebraska as compared to 22 sites in 1968, and during the same period the number of constituents analyzed increased from an average of about 12 per sample to nearly 30. Prior to 1968, the constituents determined commonly included only major cations and anions such as calcium, sodium, sulfate, chloride, and nitrate. Since then, although measurements of the foregoing constituents continue, considerable emphasis has been placed on measurements of trace constituents such as cadmium, arsenic, and selenium, on nutrients including phosphorus, ammonia, and organic nitrogen, on organic substances such as total organic carbon, lindane, 
and methoxychlor, and on biological or microbiological organisms including phytoplankton, periphyton, coliform bacteria and fecal streptococcal bacteria. Also during the last 10 years, the importance of on-site data collection has been recognized. Specific conductance, pH, water temperature, dissolved oxygen, and sometimes bicarbonate are now measured routinely at most sampling sites.

Sampling techniques and analytical methodologies have improved considerably over the period during which the samples discussed in this report have been collected. As a greater understanding of the hydrology of streamflow and of sediment movement developed, improved methods for securing truly representative water-quality samples followed. Also more precise and accurate instrumental analytical methods for determining constituent concentrations have been developed over the years.

Samples collected from 1946 to 1971 were analyzed in the district water-quality laboratory at Lincoln. Samples collected after 1971 were analyzed in the U.S. Geological Survey Central Laboratory system which presently has laboratories in Arvada, Colo., and Doraville, Ga.

\section{Programs through which Data was Obtained}

Studies funded through the Missouri River Basin (MRB) program provided the bulk of the data for nearly two decades and continue to be important. However, studies from other programs now also contribute substantially to the available data. The principal programs under which the U.S. Geological Survey has obtained or is now obtaining waterquality data for Nebraska are summarized in table 1.

\section{Constituents Selected for Statistical Analysis}

Of the nearly 425 sites at which samples were collected in Nebraska, data sufficient for statistical analyses are available for only 109. The criterion used to make this determination was that if 15 or more concurrent water-discharge and specific-conductance measurements were available for a specific site, data for that site were considered sufficient for statistical analyses.

Descriptive statistics for as many as 29 chemical and microbiological constituents are presented on the first page of data for each station. These constituents were selected mostly on the basis of availability of data. Included in the 29 constituents are specific conductance, $\mathrm{pH}$, dissolved oxygen, fecal coliform and fecal streptococcal bacteria, all of which are now measured streamside. Several nutrients, including five forms of nitrogen and two forms of phosphorus, were selected because of 
Table 1.--Summary of principal Federal and cooperative surface-water quality programs in Nebraska, 1946 to present Cooperating agencies: EPA, U.S. Environmental Protection Agency; LPSNRD, Lower Platte South Natural Resources District; NDWR, Nebraska Department of Water Resources; NNRC, Nebraska Natural Resources Commission; SVWD, Salt Valley Watershed District

\begin{tabular}{|c|c|c|c|c|c|}
\hline Program & Type & $\begin{array}{l}\text { Cooperating } \\
\text { agencies }\end{array}$ & $\begin{array}{l}\text { Year } \\
\text { begun }\end{array}$ & $\begin{array}{l}\text { Year } \\
\text { ended }\end{array}$ & Purpose and type of data \\
\hline Missouri River & Federa1 $1 /$ & $-\cdots-\cdots$ & 1946 & --- & $\begin{array}{l}\text { General assessment of quality of water for project planning, prin- } \\
\text { cipally irrigation projects; monitoring; chemical constituents. }\end{array}$ \\
\hline Irrigation Net & Federal & $-\cdots-1-1$ & 1952 & 1972 & $\begin{array}{l}\text { Quality of water for irrigation and the effects of irrigation } \\
\text { development on the quality of streamflow; chemical constituents. }\end{array}$ \\
\hline Benchmark & Federal & 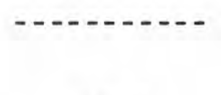 & 1967 & $-\cdots$ & $\begin{array}{l}\text { Analysis of pristine streams--common and trace chemical constitu- } \\
\text { ents; microbiological constituents. }\end{array}$ \\
\hline $\begin{array}{l}\text { Low-flow } \\
\text { quality and } \\
\text { quantity }\end{array}$ & $\begin{array}{l}\text { Cooper- } \\
\text { ative }\end{array}$ & NDWR & 1967 & 1970 & $\begin{array}{l}\text { Assessment of water quality at low flow in tributary streams in } \\
\text { Elkhorn and Blue River basins; common constituents. }\end{array}$ \\
\hline $\begin{array}{l}\text { Salt Creek } \\
\text { basin study }\end{array}$ & $\begin{array}{l}\text { Cooper- } \\
\text { ative }\end{array}$ & $\begin{array}{l}\text { SVWD } \\
\text { LPSNRD }\end{array}$ & 1968 & 1975 & $\begin{array}{l}\text { Assessment of water quality in Sa1t Creek basin--common constitu- } \\
\text { ents, nutrients, microbiology. }\end{array}$ \\
\hline $\begin{array}{l}\text { Dual-station } \\
\text { water pollution } \\
\text { assessment }\end{array}$ & Federal & EPA & 1969 & --- & $\begin{array}{l}\text { Assessment of water quality in streams above and below major } \\
\text { cities--nutrients, common constituents. }\end{array}$ \\
\hline $\begin{array}{l}\text { Statewide } \\
\text { monitoring } \\
\text { program }\end{array}$ & $\begin{array}{l}\text { Cooper- } \\
\text { ative }\end{array}$ & NNRC & 1971 & --- & $\begin{array}{l}\text { Long-term monitoring of water quality with emphasis on point- and } \\
\text { nompoint-source pollution--common and trace constituents, } \\
\text { nutrients, microbiology, sediment. }\end{array}$ \\
\hline NASQAN $2 /$ & Federal & 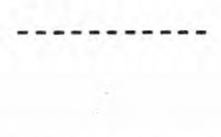 & 1974 & $-\cdots$ & $\begin{array}{l}\text { Assessment of quality of water leaving nationwide accounting } \\
\text { units--common and trace constituents, nutrients, biology and } \\
\text { microbiology, sediment. }\end{array}$ \\
\hline
\end{tabular}

\footnotetext{
1/ Work under this program requested by the U.S. Bureau of Reclamation and the U.S. Fish and Wildlife Service. 2/ National Stream Quality Accounting Network.
} 
their importance in eutrophication, their significance as indicators of possible contamination, or their potential effects on public health. Color and biochemical oxygen demand were selected because they are indicators of organic material in streamflow. The remainder of the constituents selected are those that occur most commonly in natural water and that have been measured most frequently in the past. Iron and manganese, generally considered trace constituents, have been measured routinely for many more years than most other trace constituents.

Trace constituents such as arsenic, lead, selenium, and cadmium are important because of their toxicity in small quantities to humans and animals. However, they are not included in the report because they have been measured on only a few samples from less than half the sites.

Results of regression analyses relating concentrations of each of 12 constituents to specific conductance and results of a regression analysis relating specific conductance to water discharge are presented on the second page of data for each station. These 12 constituents were chosen after first examining regression relationships between specific conductance and all 29 constituents for 7 stations that are representative of a variety of hydrologic settings in Nebraska. These stations are Salt Creek at Lincoln, Weeping Water.Creek near Union, Big Nemaha River at Falls City, Spring Creek at Lexington, Republican River at Guide Rock, North Loup River at Taylor, and North Platte River at Lisco. If specific conductance failed to explain 50 percent of the variance for any constituent at any of the 7 representative stations, that constituent was not included in the subsequent regression analyses for all 109 stations. Whereas the figure of 50 percent was somewhat arbitrary, the author concluded that if factors other than specific conductance explained more than 50 percent of the variance for a constituent for the 7 representative stations, a regression relationship for specific conductance and that constituent was not likely to be significant for any of the stations.

Discussions of the properties and significance of each of the 29 constituents described in this report may be found in Hem (1970), and McKee and Wolf (1963).

\section{STATISTICS}

Several available USGS Statpac computer programs and SAS (Statistical Analysis System) programs (Barr and others, 1976) were used in data reduction and table preparation.

Statistical terms used in this report are defined as follows:

Mean is the sum of the values of individual observations divided by the total number of observations in the group. 
Median is the value of the middle observation of an uneven number of ordered observations or the mean of the two middle observations when there are an even number of observations.

Maximum is the observation of largest value in a group of observations.

Minimum is the observation of smallest value in a group of observations.

Standard deviation is the square root of the sum of the squares of deviations from the mean of all observations in a group divided by the number of all observations. It is expressed mathematically as

$$
s=\sqrt{\frac{\sum\left(x_{i}-\bar{x}\right)^{2}}{n-1}}
$$

where $s$ is the standard deviation, $x_{i}$ is the $i$ th value of $x, \bar{x}$ is the mean value of $x$, and $n$ is the totaI number of observations.

Ninetieth percentile is that value in a group of observations below which 90 percent of the total number of observations occur.

Tenth percentile is that value in a group of observations below which 10 percent of the total number of observations occur.

Regression equation is an equation defining the relationship between a dependent and an independent variable. For a linear relationship, the equation takes the following form:

$$
\mathrm{Y}=\mathrm{b} \mathrm{X}+\mathrm{a}
$$

where $X$ and $Y$ are values of the independent and dependent variables, respectively. The coefficient $b$ is the slope of the line and the constant a is the Y-axis intercept. For a logarithmic relationship, the equation takes the following form:

$$
\mathrm{Y}=\mathrm{a} \mathrm{X}^{\mathrm{b}}
$$

Standard error of estimate, as used in this report, is a measurement of the variation around the line of regression. It is in actuality the standard deviation about the regression line.

Correlation coefficient is a term relating the standard error of estimate and the standard deviation of the values of the dependent variable. It takes the form of 


$$
r=\frac{b s_{x}}{s_{y}}
$$

where $\mathrm{r}$ is the correlation coefficient, $\mathrm{b}$ is the slope of the regression line, $s_{x}$ is the standard deviation of the independent variable, and $s_{y}$ is the standard deviation of the dependent variable. A perfect relationship has a value of \pm 1 . A completely imperfect relationship has a value of zero.

Percent explained variance is the coefficient of determination expressed as a percent. The coefficient of determination is the square of the correlation coefficient and is the proportion of variance in the dependent variable that is explained by variation in the independent variable.

\section{Explanation for Blanks in the Data Tables}

None of the tables of descriptive statistics (first page for each station) are complete. There are several reasons for this. If there is no value for number of measurements, then that particular constituent has never been measured at that station. When the number of measurements for a particular constituent is less than 10 , neither the median nor the percentile values are shown because they have limited significance. Samples for some stations consisted of composites of discharge-weighted increments of daily samples covering as many as 31 days each. These samples were not used in computing percentiles.

Blanks occur in the regression tables because of insufficient data. If less than 15 measurements for any of the 12 constituents or specific conductance were available, the regression equation for the relationship of that constituent to specific conductance was not determined.

Means and standard deviations are not shown for fecal coliform and fecal streptococcal bacteria enumerations. To explain why, the following paragraphs discuss continuous and discrete data and normal distribution.

With the exception of fecal coliform and fecal streptococcal bacteria, all constituent data in this report are continuous. This means that an infinite number of positive values for the constituent theoretically is possible between zero and its maximum concentration. Of course, this is limited in actuality by the sensitivity of the method by which the constituent is measured. Continuous data will of ten, but not always, tend to be distributed normally; that is, observed values of the constituent measured cluster around the mean and, if plotted, approximate a bell-shaped curve. 
Fecal coliform and fecal streptococcal bacteria data represent counts of individual colonies and can assume only positive interger values. Therefore, these data are not continuous but are limited only to certain discrete values. These data tend not to be distributed normally because they represent the association of organisms with each other and with their environment, and this is indeed the case with the fecal coliform and fecal streptococcal bacteria data in this report. Usually discrete data are highly skewed, that is, individual values do not cluster around the mean. As a result, measurements such as the mean and standard deviation are of limited use in analyzing fecal coliform and fecal streptococcal bacteria data. Frequency distributions represented by percentiles are much more useful tools than the mean and standard deviation for interpreting these fecal coliform and fecal streptococcal bacteria data.

\section{Analogous Constituents}

Statistical data are reported for certain constituents in more than one form. This is true for dissolved-solids residue and sum and for nitrate as $\mathrm{N}$, nitrate as $\mathrm{NO}_{3}$, and nitrite plus nitrate as $\mathrm{N}$.

Both dissolved-solids residue and dissolved-solids sum are shown in the tables because, in recent years, the residue of ten was not measured if a sufficient number of constituents were measured so that a sum could be computed. Conversely, the residue often was measured if a sufficient number of constituents were not measured. Although residue and sum represent nearly the same thing, they may represent different aspects of the same hydrologic condition; therefore, descriptive statistics for both are included in this report.

The three nitrogen constituents represent three different ways of reporting essentially the same data. Prior to the 1971 water year, USGS laboratories reported all values of nitrate as $\mathrm{NO}_{3}$. In 1971 the reporting convention was changed to report all values of nitrate as $\mathrm{N}$. Nitrate as $\mathrm{N}$ differs by a factor of 4.43 from nitrate as $\mathrm{NO}_{3}$. In 1972 the USGS Central Laboratory adopted an automated method for nitrate in which both nitrite and nitrate are measured together. Because nitrite is unstable and occurs generally in very small concentrations, this measurement of nitrite + nitrate as $\mathrm{N}$ is considered, for most conditions, to be equivalent to a measurement of nitrate only. Because of differences in laboratory methods over the years and because the different measurements are not precisely equivalent, these three constituents are included separately. in this report to make the nitrogen data more manageable and useful. 
For ease of examination, the statistical data presented herein are grouped by river basin (fig. 1). These are the same basin designations used by the Nebraska Natural Resources Commission, previously called the Nebraska Soil and Water Conservation Commission, in preparation of the Framework Study for the State Water Plan (NSWCC, 1971). Some of the basins are complete drainage units, some are parts of drainage units, and others are groupings of sma1l drainage units which are hydrologically similar.

Data are identified in the following sections both by station number and station name. Station numbers are official Geological Survey eight-digit downstream-order numbers. The first two digits are "06" for all stations, indicating that all stations in Nebraska are in the Missouri River basin. The other six digits indicate the downstream location within the basin.

\section{White River - Hat Creek basin}

The White River - Hat Creek basin is the smallest of the 13 basins in Nebraska. It is also one of the roughest areas of the State topographically, one of the areas least suited for cultivation, and one of the areas of lowest ground-water availability.

Sufficient data for statistical analyses are available for only one surface-water sampling site in the basin. That site, the White River near Whitney, is located in the central part of the basin (fig. 2) and was operated from 1969 to 1972 . The White River is fed mostly by overland runoff. Part of its drainage basin is composed of fine surficial clays, some of which are carried into the stream. Much of the time the river is extremely turbid and has a whitish appearance, which probably accounts for its name. Base flow is maintained by small amounts of ground water discharged into tributaries flowing from Pine Ridge which forms the southern boundary of part of the basin.

The mean specific conductance of the White River is 1,320 umho/cm which makes this one of the more highly mineralized streams in Nebraska. Sodium is the predominant cation (positively charged ion) with a mean concentration of $157 \mathrm{mg} / \mathrm{L}$. Sulfate is the predominate anion (negatively charged ion) with a mean concentration of $416 \mathrm{mg} / \mathrm{L}$.

Regression equations were developed for the relationships between specific conductance and seven constituents. Correlation coefficients were 0.91 or greater for each of these relationships. 


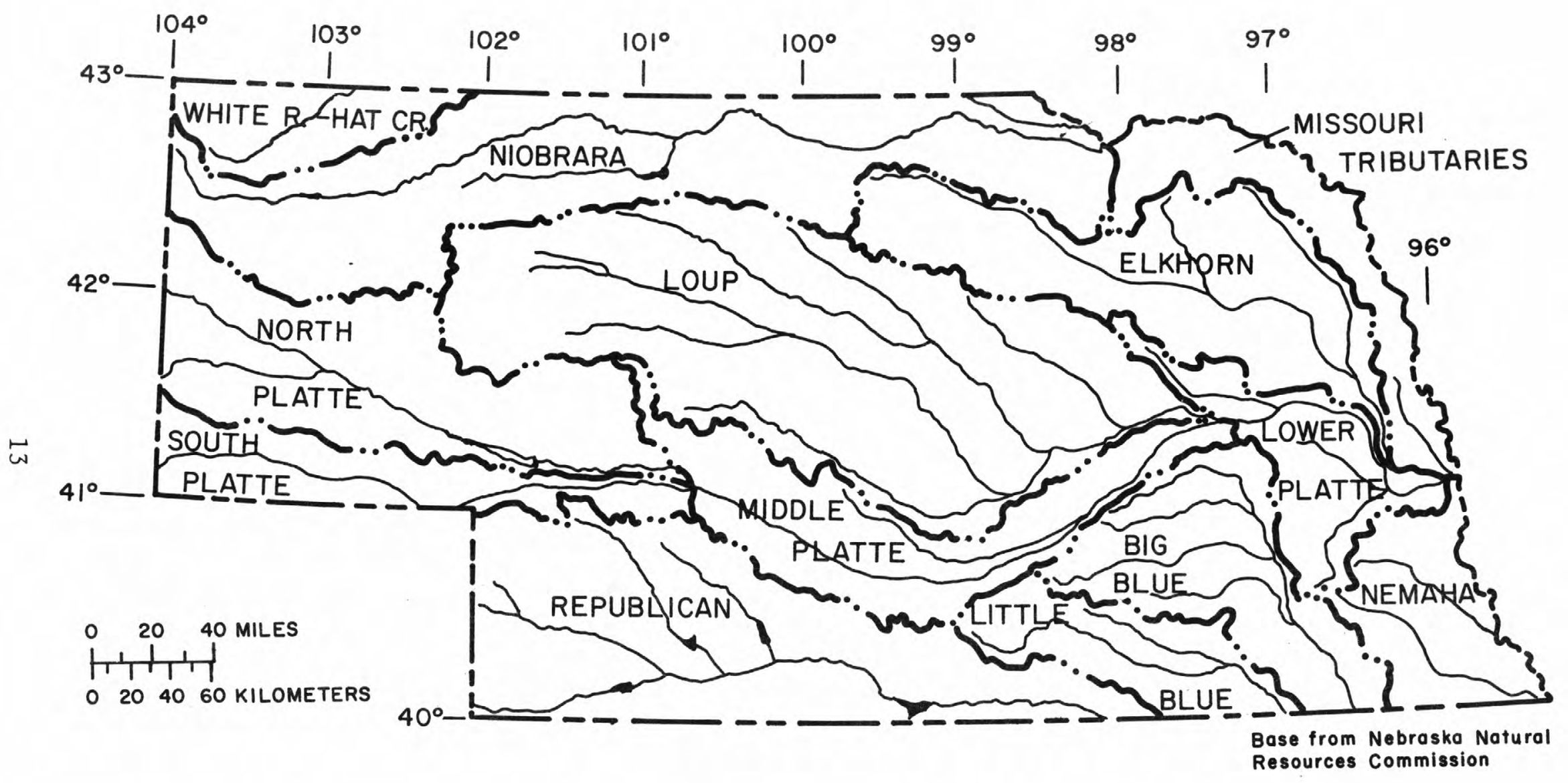

FIGURE 1.-River basins of Nebraska 


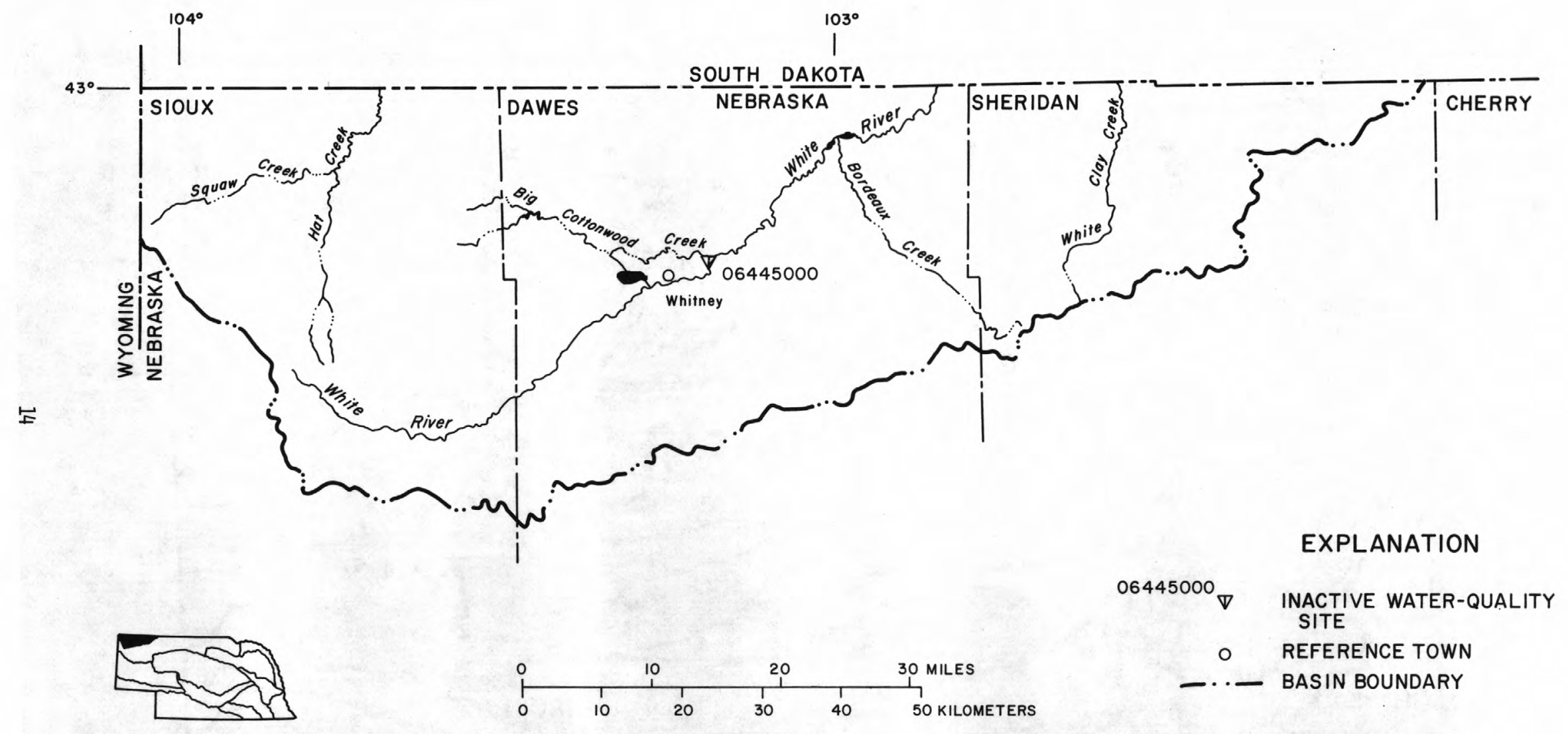

FIGURE 2.-Location of water-quality sampling site, White River - Hat Creek basin 
INHTE RIVER - IIAT CREEK BASIN

06445000 White River near Whitney, Nebr.

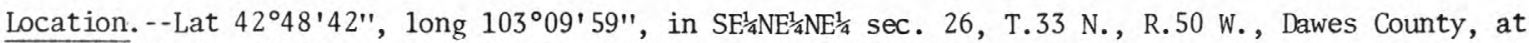
county road bridge $1.7 \mathrm{mi}$ north and $4.5 \mathrm{mi}$ east of Whitney.

Drainage area. $--676 \mathrm{mi}^{2}$.

Period of record.--August 1969 to June 1972.

Statistical data for selected chemical constituents

\begin{tabular}{|c|c|c|c|c|c|c|c|c|c|}
\hline Constituent & Units & $\begin{array}{l}\text { No. of } \\
\text { measure- } \\
\text { ments }\end{array}$ & $\begin{array}{l}\text { Max- } \\
\text { imum }\end{array}$ & $\begin{array}{l}\text { Min- } \\
\text { imum }\end{array}$ & Mean & Median & $\begin{array}{c}\text { Standard } \\
\text { devi- } \\
\text { ation }\end{array}$ & $\begin{array}{l}\text { Ninetieth } \\
\text { percent- } \\
\text { ile }\end{array}$ & $\begin{array}{l}\text { Tenth } \\
\text { percent- } \\
\text { ile }\end{array}$ \\
\hline Specific conductance & umho/cm & 73 & 3,500 & 495 & 1,320 & 1,190 & 616 & 2,200 & 680 \\
\hline Dissolved solids, residue & $\mathrm{mg} / \mathrm{L}$ & 36 & 2,290 & 298 & 961 & 813 & 466 & 1,700 & 470 \\
\hline Dissolved solids, sum & $\mathrm{mg} / \mathrm{L}$ & & & & & 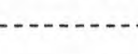 & 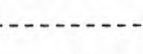 & & $-\cdots$ \\
\hline Hardness as $\mathrm{CaCO}_{3}$ & $\mathrm{mg} / \mathrm{L}$ & 36 & 736 & 170 & 329 & 288 & 132 & 550 & 212 \\
\hline Calcium, dissolved & $\mathrm{mg} / \mathrm{L}$ & 24 & 140 & 53 & 83 & 83 & 20 & 110 & 62 \\
\hline Magnesium, dissolved & $\mathrm{mg} / \mathrm{L}$ & 24 & 52 & 9.5 & 23 & 22 & 11 & 39 & 11 \\
\hline Sodium, dissolved & $\mathrm{mg} / \mathrm{L}$ & 23 & 424 & 52 & 157 & 140 & 96 & 250 & 60 \\
\hline Potassium, dissolved & $\mathrm{mg} / \mathrm{L}$ & 5 & 20 & 12 & 16 & $-\cdots-1$ & -1.6 & - & -- \\
\hline Bicarbonate ion & $\mathrm{mg} / \mathrm{L}$ & 12 & 502 & 198 & 313 & 300 & 92 & 450 & 206 \\
\hline Sulfate, dissolved & $\mathrm{mg} / \mathrm{L}$ & 36 & 1,300 & 67 & 416 & 332 & 296 & 850 & 104 \\
\hline Chloride, dissolved & $\mathrm{mg} / \mathrm{L}$ & 26 & 28 & 7.4 & 14 & 14 & 5.1 & 22 & 7.9 \\
\hline Fluoride, dissolved & $\mathrm{mg} / \mathrm{L}$ & 23 & 2.5 & 0.5 & 0.8 & 0.7 & 0.4 & 0.9 & 0.6 \\
\hline Silica, dissolved & $\mathrm{mg} / \mathrm{L}$ & 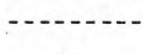 & . & 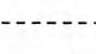 & 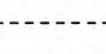 & 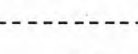 & 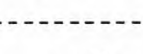 & & $\ldots$ \\
\hline Boron, dissolved & $\mathrm{mg} / \mathrm{L}$ & 23 & 740 & 60 & 253 & 207 & 165 & 420 & 87 \\
\hline Iron, dissolved & $\mathrm{ug} / \mathrm{L}$ & 5 & 49 & 6 & 28 & $\cdots$ & $21-$ & & -- \\
\hline Manganese, dissolved & $\mathrm{ug} / \mathrm{L}$ & 21 & 420 & 0 & 121 & 54 & 140 & 360 & 0 \\
\hline $\begin{array}{l}\text { Nitrite + nitrate as } \mathrm{N} \\
\text { dissolved }\end{array}$ & $\mathrm{mg} / \mathrm{L}$ & 12 & 1.5 & 0.03 & 0.59 & 0.58 & 0.49 & 1.1 & 0.06 \\
\hline Nitrate as $\mathrm{N}$, dissolved & $\mathrm{mg} / \mathrm{L}$ & 23 & 2.0 & 0.00 & 0.63 & 0.58 & 0.53 & 1.1 & 0.12 \\
\hline Nitrate as $\mathrm{NO}_{3}$, dissolved & $\mathrm{mg} / \mathrm{L}$ & & & & & & & & \\
\hline Ammonia $\mathrm{N}$, total & $\mathrm{mg} / \mathrm{L}$ & & & & & & & & \\
\hline Organic N, total & $\mathrm{mg} / \mathrm{L}$ & & & & & & & & \\
\hline Phosphorus, total & $\mathrm{mg} / \mathrm{L}$ & 36 & 1.1 & 0.04 & 0.27 & 0.20 & 0.22 & 0.55 & 0.10 \\
\hline Phosphorus, dissolved & $\mathrm{mg} / \mathrm{L}$ & 12 & 0.32 & 0.00 & 0.09 & 0.09 & 0.08 & 0.12 & 0.01 \\
\hline Fecal coliform & $\mathrm{col} / 100 \mathrm{ml}$ & 21 & 2,800 & 0 & 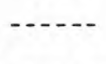 & 100 & 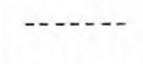 & 1,220 & 4 \\
\hline Fecal streptococci & $\mathrm{col} / 100 \mathrm{~m} 1$ & & & & 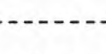 & & & & \\
\hline Oxygen, dissolved & $\mathrm{mg} / \mathrm{L}$ & 37 & 13.4 & 6.1 & 10.3 & 10.2 & 1.8 & 12.6 & 7.8 \\
\hline \multirow{2}{*}{$\begin{array}{l}\text { Biochemical } \\
\text { (5 day) }\end{array}$} & $\mathrm{mg} / \mathrm{L}$ & 36 & 6.7 & 0.6 & 2.5 & 2.2 & 1.4 & 5.2 & 1.2 \\
\hline & units & 52 & 9.3 & 7.3 & 8.1 & 8.2 & 0.3 & 8.4 & 7.9 \\
\hline $\mathrm{Co}-\mathrm{Pt}$ & units & 6 & 32 & 5 & 14 & 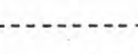 & -- & - & - \\
\hline
\end{tabular}


IMIITE RIVER - HAT CREEK BASIN

06445000 White River near Whitney, Nebr.--Continued

Results of regression analyses relating concentrations of selected chemical constituents to specific conductance $(\mathrm{SpC})$

\begin{tabular}{|c|c|c|c|c|c|}
\hline $\begin{array}{l}\text { Dissolved } \\
\text { constituents } \\
(\mathrm{mg} / \mathrm{L})\end{array}$ & $\begin{array}{l}\text { No. of } \\
\text { measure- } \\
\text { ments }\end{array}$ & Regression equation & $\begin{array}{l}\text { Corre- } \\
\text { lation } \\
\text { coeffi- } \\
\text { cient }\end{array}$ & $\begin{array}{l}\text { Percent } \\
\text { explained } \\
\text { variance }\end{array}$ & $\begin{array}{c}\text { Standard } \\
\text { error of } \\
\text { estimate } \\
(\mathrm{mg} / \mathrm{L}) \\
\end{array}$ \\
\hline Dissolved solids, residue (RDS) & 36 & $\mathrm{RDS}=0.838(\mathrm{SpC})-127.060$ & 0.99 & 98 & 61 \\
\hline \multicolumn{6}{|l|}{ Dissolved solids, sum (SDS) } \\
\hline Hardness as $\mathrm{CaCO}_{3}(\mathrm{TH})$ & 36 & $\mathrm{TH}=0.224(\mathrm{SpC})+38.351$ & .93 & 87 & 49 \\
\hline Calcium (Ca) & 24 & $\mathrm{Ca}=0.042(\mathrm{SpC})+35.143$ & .91 & 83 & 8.6 \\
\hline Magnesium (Mg) & 24 & $\mathrm{Mg}=0.023(\mathrm{SpC})-4.003$ & .96 & 92 & 3.0 \\
\hline Sodium (Na) & 23 & $\mathrm{Na}=0.165(\mathrm{SpC})-44.779$ & .99 & 99 & 11 \\
\hline Potassium (K) & $-\cdots-1$ & 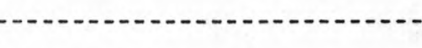 & -- & 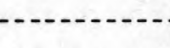 & \\
\hline Bicarbonate $\left(\mathrm{HCO}_{3}\right)$ & $-\cdots$ & 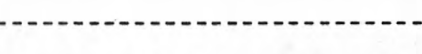 & & & \\
\hline Sulfate $\left(\mathrm{SO}_{4}\right)$ & 36 & $\mathrm{SO}_{4}=0.529(\mathrm{SpC})-271.345$ & .98 & 97 & 52 \\
\hline Chloride (C1) & 26 & $C l=0.010(\mathrm{SpC})+2.895$ & .92 & 84 & 2.1 \\
\hline Silica (Si) & $-\cdots--1$ & 年- & $-\cdots-1$ & (n) & \\
\hline Nitrite + Nitrate as $\mathrm{N}\left(\mathrm{NO}_{2}-\mathrm{NO}_{3}\right)$ & & & & & \\
\hline
\end{tabular}

Results of regression analyses relating specific conductance to water discharge (Q)

\begin{tabular}{ccccccc}
\hline $\begin{array}{c}\text { No. of } \\
\text { measure } \\
\text { ments }\end{array}$ & $\begin{array}{c}\text { Water discharge } \\
\left(\mathrm{ft}^{3} / \mathrm{s}\right)\end{array}$ & Maximum Minimum & Regression equation & $\begin{array}{c}\text { Correlation } \\
\text { coefficient }\end{array}$ & $\begin{array}{c}\text { Percent } \\
\text { explained } \\
\text { variance }\end{array}$ & $\begin{array}{c}\text { Standard error } \\
\text { of estimate }\end{array}$ \\
\hline 73 & $45 \quad 1.9$ & $\mathrm{SpC}=2356 \mathrm{Q}^{-0.36264}$ & -0.70 & 49 & 0.136 \\
\hline
\end{tabular}


The Niobrara River basin in Nebraska is long and narrow and lies mostly along the northern border of the State. The entire basin includes parts of Wyoming and South Dakota. In Nebraska, it extends for about $300 \mathrm{mi}$ from the Wyoming border to the confluence of the Niobrara and Missouri Rivers. For convenience, the small Ponca Creek basin is included in this discussion with the Niobrara, but water quality of Ponca Creek is not related to that of the Niobrara River and its tributaries.

Sufficient data for statistical analysis are available for eight surface-water sampling sites in the Niobrara River basin and for two on Ponca Creek. These sites are shown on figure 3.

During most of its passage across Nebraska, the Niobrara River flows through sandhills. Because the contributing drainage area is small compared to the total drainage, relatively small amounts of overland runoff reach the stream. For the 83 times that the Niobrara River at Norden was sampled for water quality, the maximum discharge was only 5.7 times the minimum, indicating relatively small overall fluctuations of stream discharge. This means that flow of the stream is derived mostly from ground-water seepage. On the other hand, the maximum water discharge of Ponca Creek at Anoka for the 22 times that it was sampled for water quality was more than 17,000 times the minimum discharge. Flow in Ponca Creek carries very little ground-water seepage, being maintained mostly by overland runoff.

Mean specific conductance averaged about $400 \mathrm{umho} / \mathrm{cm}$ for the four uppermost stations in the Niobrara River basin. By contrast, the mean near the mouth of the Niobrara River at Verdel was only $266 \mathrm{umho} / \mathrm{cm}$. The improvement in overall quality of water in a downstream direction is attributed to tributary inflow of water of excellent quality combined with seepage of ground water into the Niobrara during its passage through the sandhills. The principal cation throughout the entire reach of the Niobrara River is calcium, and the principal anion is bicarbonate.

Water quality of Long Pine Creek is probably representative of that of most tributaries of the Niobrara River having their origin in the sandhills, because geology and hydrology of most tributaries are very similar. Mean specific conductance for Long Pine Creek at Riverview is 179 umho/cm.

Concentrations of chloride, nitrate, BOD, fecal coliform, and fecal streptococcal bacteria are low in the Niobrara River and its tributaries, indicating that the stream is relatively unpolluted. 


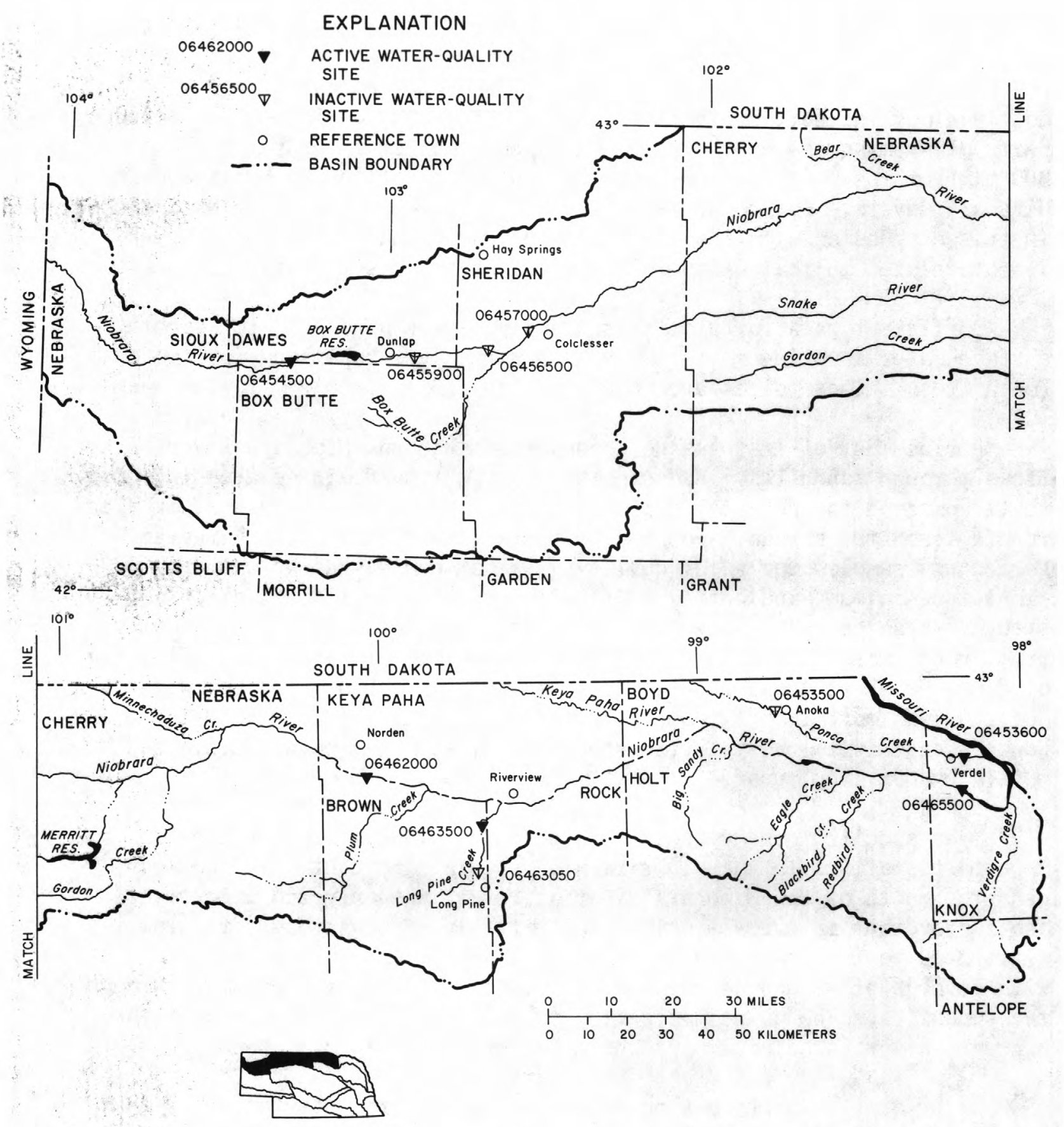

FIGURE 3.-Location of water-quality sampling sites, Niobrara River basin 
Regression equations were developed for the relationships between specific conductance and those constituents for which sufficient data has been collected. Correlation coefficients were low for several of the relationships and correlations were not significant at the 95 percent confidence level for several relationships. The only constituents for which the percent explained variance exceeded 50 for all Niobrara River and tributary stations, except those for which there was insufficient data, were dissolved-solids residue and bicarbonate.

Correlations are relatively poor for most constituents because concentrations are low and may vary over narrow ranges. A1so, because concentration ranges are low for most constituents, small natural variations in ion ratios between these constituents and variations resulting from analytical methodology in the laboratory tend to reduce correlation. According to Irwin and Lemons (1975), temporal variations, often seasonal, may be responsible for differences in chemical composition of water, causing correlation coefficients to be affected.

Mean specific conductance for samples from Ponca Creek at Anoka is $820 \mathrm{umho} / \mathrm{cm}$ and for samples from Ponca Creek at Verdel is $1,125 \mathrm{umho} / \mathrm{cm}$. Calcium is the predominate cation and bicarbonate and sulfate are the predominate anions in samples from Ponca Creek. 


\section{NIOBRARA RIVER BASIN}

06453500 Ponca Creek at Anoka, Nebr.

Location. --Lat $42^{\circ} 45^{\prime} 25^{\prime \prime}$, long $98^{\circ} 50^{\prime} 30^{\prime \prime}$, in NE $\frac{1}{4} \mathrm{sec} .9$, T. 34 N., R.13 W. , Boyd County, Hydrologic Unit 10150001, on downstream side of left pier of bridge on State Highway 11, 0.5 mi southwest of Anoka and $0.5 \mathrm{mi}$ upstream from Dry Creek.

Drainage area. $--505 \mathrm{mi}^{2}$.

Period of record.--December 1963 to September 1964, October 1966 to September 1967.

Statistical data for selected chemical constituents

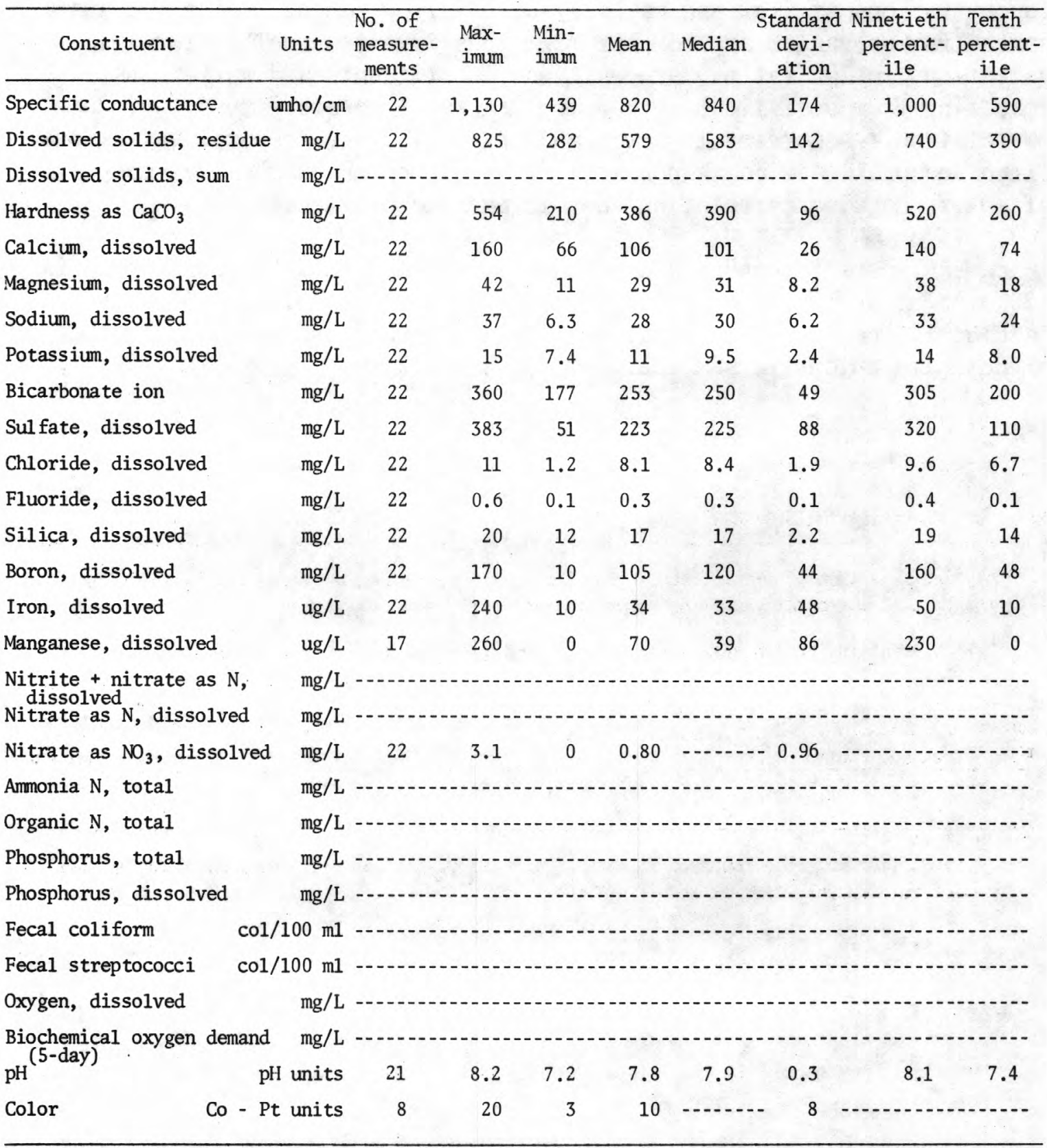


Results of regression analyses relating concentrations of selected chemical constituents to specific conductance $(\mathrm{SpC})$

\begin{tabular}{|c|c|c|c|c|c|}
\hline $\begin{array}{l}\text { Dissolved } \\
\text { constituents } \\
(\mathrm{mg} / \mathrm{L})\end{array}$ & $\begin{array}{l}\text { No. of } \\
\text { measure- } \\
\text { ments }\end{array}$ & Regression equation & $\begin{array}{l}\text { Corre- } \\
\text { lation } \\
\text { coeffi- } \\
\text { cient }\end{array}$ & $\begin{array}{l}\text { Percent } \\
\text { explained } \\
\text { variance }\end{array}$ & $\begin{array}{c}\text { Standard } \\
\text { error of } \\
\text { estimate } \\
(\mathrm{mg} / \mathrm{L})\end{array}$ \\
\hline Dissolved solids, residue (RDS) & 22 & $\mathrm{RDS}=0.814(\mathrm{SpC})-89.369$ & 1.0 & 99 & 13 \\
\hline Dissolved solids, sum (SDS) & $-\cdots$ & $-1-2-2-1$ & & & - \\
\hline Hardness as $\mathrm{CaCO}_{3}(\mathrm{TH})$ & 22 & $\mathrm{TH}=0.544(\mathrm{SpC})-60.696$ & .98 & 97 & 17 \\
\hline Calcium (Ca) & 22 & $\mathrm{Ca}=0.144(\mathrm{SpC})-11.876$ & .97 & 93 & 6.8 \\
\hline Magnesium (Mg) & 22 & $M g=0.044(S p C)-7.260$ & .94 & 89 & 2.8 \\
\hline Sodium (Na) & 22 & $\mathrm{Na}=0.027(\mathrm{SpC})+5.824$ & .76 & 58 & 4.1 \\
\hline Potassium (K) & 22 & $K=0.005(\mathrm{SpC})+6.733$ & $* .34$ & 12 & 2.3 \\
\hline Bicarbonate $\left(\mathrm{HCO}_{3}\right)$ & 22 & $\mathrm{HCO}_{3}=0.124(\mathrm{SpC})+151.537$ & .44 & 20 & 45 \\
\hline Sulfate $\left(\mathrm{SO}_{4}\right)$ & 22 & $\mathrm{SO}_{4}=0.473(\mathrm{SpC})-165.121$ & .93 & 87 & 33 \\
\hline Chloride (C1) & 22 & $\mathrm{Cl}=0.006(\mathrm{SpC})+3.361$ & .51 & 26 & 1.7 \\
\hline Silica (Si) & 22 & $\mathrm{Si}-0.003(\mathrm{SpC})+14.083$ & $* .26$ & 6.8 & 2.2 \\
\hline
\end{tabular}

* Not significant at 95 percent confidence level; the regression equation should not be used to predict the concentration of the dependent variable.

Results of regression analyses relating specific conductance to water discharge (Q)

\begin{tabular}{ccccccc}
\hline $\begin{array}{c}\text { No. of } \\
\text { measure- } \\
\text { ments }\end{array}$ & $\begin{array}{c}\text { Water discharge } \\
\left(\mathrm{ft}^{3} / \mathrm{s}\right)\end{array}$ & Maximum Minimum & Regression equation & $\begin{array}{c}\text { Correlation } \\
\text { coefficient }\end{array}$ & $\begin{array}{c}\text { Percent } \\
\text { explained } \\
\text { variance }\end{array}$ & $\begin{array}{c}\text { Standard error } \\
\text { of estimate }\end{array}$ \\
\hline 22 & $1,740 \quad 0.10$ & $\mathrm{SpC}=943 \mathrm{Q}^{-0.08756}$ & -0.82 & 67 & 0.059 & 14 \\
\hline
\end{tabular}

* Not significant at 95 percent confidence level; the regression equation should not be used to predict the concentration of the dependent variable. 


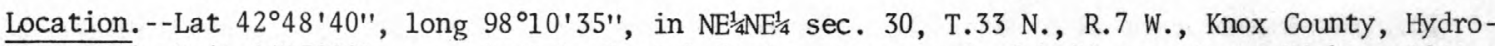
logic Unit 10150001, near left bank at left downstream end of bridge on State Highway 12 , $0.6 \mathrm{mi}$ east of Verdel and $3.1 \mathrm{mi}$ upstream from mouth.

Drainage area. $--812 \mathrm{mi}^{2}$.

Period of record.--July 1975 to current year.

Statistical data for selected chemical constituents

\begin{tabular}{|c|c|c|c|c|c|c|c|c|c|}
\hline Constituent & Units & $\begin{array}{l}\text { No. of } \\
\text { measure- } \\
\text { ments }\end{array}$ & $\begin{array}{l}\text { Max- } \\
\text { imum }\end{array}$ & $\begin{array}{l}\text { Min- } \\
\text { imum }\end{array}$ & Mean & Median & $\begin{array}{l}\text { Standard } \\
\text { devi- } \\
\text { ation } \\
\end{array}$ & $\begin{array}{l}\text { Ninetieth } \\
\text { percent- } \\
\text { ile }\end{array}$ & $\begin{array}{l}\text { Tenth } \\
\text { percent- } \\
\text { ile }\end{array}$ \\
\hline Specific conductance & umho/cm & 21 & 1,660 & 540 & 1,125 & 1,120 & 305 & 1,533 & 755 \\
\hline Dissolved solids, residue & $\mathrm{mg} / \mathrm{L}$ & 13 & 1,880 & 391 & 978 & 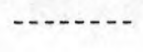 & $414-$ & & $-\cdots$ \\
\hline Dissolved solids, sum & $\mathrm{mg} / \mathrm{L}$ & 6 & 1,020 & 561 & 761 & -........ & $180-$ & & - \\
\hline Hardness as $\mathrm{CaCO}_{3}$ & $\mathrm{mg} / \mathrm{L}$ & 8 & 720 & 380 & 546 & & $131-$ & & - \\
\hline Calcium, dissolved & $\mathrm{mg} / \mathrm{L}$ & 7 & 190 & 110 & 148 & 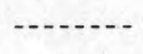 & $33-$ & & -- \\
\hline Magnesium, dissolved & $\mathrm{mg} / \mathrm{L}$ & 7 & 49 & 21 & 36 & 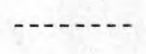 & $9.8-$ & & $\cdots$ \\
\hline Sodium, dissolved & $\mathrm{mg} / \mathrm{L}$. & 8 & 51 & 26 & 40 & 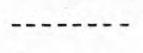 & $9.0-$ & & -1 \\
\hline Potassium, dissolved & $\mathrm{mg} / \mathrm{L}$ & 7 & 14 & 12 & 13 & $\ldots \ldots$ & $0.9-$ & & $\cdots$ \\
\hline Bicarbonate ion & $\mathrm{mg} / \mathrm{L}$ & 8 & 320 & 124 & 219 & 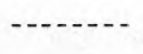 & $58-$ & & - n \\
\hline Sulfate, dissolved & $\mathrm{mg} / \mathrm{L}$ & 8 & 590 & 250 & 430 & $\ldots$ & $126-$ & & - \\
\hline Chloride, dissolved & $\mathrm{mg} / \mathrm{L}$ & 20 & 23 & 5.6 & 16 & 16 & 4.6 & 22 & 11 \\
\hline Fluoride, dissolved & $\mathrm{mg} / \mathrm{L}$ & 6 & 0.4 & 0.3 & 0.4 & ........... & $0.05-$ & & \\
\hline Silica, dissolved & $\mathrm{mg} / \mathrm{L}$ & 7 & 17 & 9.7 & 14 & $-\cdots$ & $2.6-$ & & -- \\
\hline Boron, dissolved & $\mathrm{mg} / \mathrm{L}$ & 7 & 340 & 130 & 187 & $-\cdots$ & $69-$ & & -- \\
\hline Iron, dissolved & $\mathrm{ug} / \mathrm{L}$ & 7 & 60 & 10 & 24 & & $24-$ & & \\
\hline Manganese, dissolved & ug/L & 7 & 350 & 0 & 163 & & 132 & & \\
\hline $\begin{array}{l}\text { Nitrite + nitrate as } \mathrm{N} \text {, } \\
\text { dissolved } \\
\text { Nitrate as N, dissolved }\end{array}$ & $\begin{array}{l}\mathrm{mg} / \mathrm{L} \\
\mathrm{mg} / \mathrm{L}\end{array}$ & 2 & 0.60 & 0.00 & 0.30 & & & & \\
\hline Nitrate as $\mathrm{NO}_{3}$, dissolved & $\mathrm{mg} / \mathrm{L}$ & - & 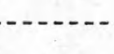 & & & & & & \\
\hline Ammonia $\mathrm{N}$, total & $\mathrm{mg} / \mathrm{L}$ & 19 & 0.41 & 0.00 & 0.07 & 0.05 & 0.09 & 0.13 & 0.00 \\
\hline Organic $N$, total & $\mathrm{mg} / \mathrm{L}$ & 19 & 1.6 & 0.12 & 0.55 & 0.44 & 0.38 & 1.4 & 0.15 \\
\hline Phosphorus, total & $\mathrm{mg} / \mathrm{L}$ & 19 & 0.52 & 0.01 & 0.10 & 0.06 & 0.12 & 0.26 & 0.02 \\
\hline Phosphorus, dissolved & $\mathrm{mg} / \mathrm{L}$ & 13 & 0.11 & 0.00 & 0.03 & 0.02 & 0.03 & 0.06 & 0.01 \\
\hline Fecal coliform & $\mathrm{col} / 100 \mathrm{ml}$ & 10 & 5,100 & 13 & $\ldots$ & 140 & $\ldots$ & 880 & 63 \\
\hline Fecal streptococci & $\mathrm{col} / 100 \mathrm{ml}$ & 15 & 10,000 & 68 & $-\ldots$ & 1,400 & - n & 7,100 & 140 \\
\hline Oxygen, dissolved & $\mathrm{mg} / \mathrm{L}$ & 19 & 13.3 & 6.7 & 9.7 & 9.4 & 2.0 & 12.5 & 7.2 \\
\hline \multirow{2}{*}{$\begin{array}{l}\text { Biochemical oxygen demar } \\
\text { (5-day) }\end{array}$} & $\mathrm{mg} / \mathrm{L}$ & 17 & 9.0 & 1.4 & 3.6 & 2.6 & 2.2 & 7.6 & 1.7 \\
\hline & $H$ units & 20 & 8.4 & 7.0 & 7.7 & 7.7 & 0.4 & 8.3 & 7.1 \\
\hline Co $-\mathrm{Pt}$ & $t$ units & 6 & 35 & 2 & 12 & - & $13-$ & & \\
\hline
\end{tabular}


Results of regression analyses relating concentrations of selected chemical constituents to specific conductance $(\mathrm{SpC})$

\begin{tabular}{|c|c|c|c|c|c|}
\hline $\begin{array}{l}\text { Dissolved } \\
\text { constituents } \\
(\mathrm{mg} / \mathrm{L})\end{array}$ & $\begin{array}{l}\text { No. of } \\
\text { measure- } \\
\text { ments }\end{array}$ & Regression equation & $\begin{array}{l}\text { Corre- } \\
\text { lation } \\
\text { coeffi- } \\
\text { cient }\end{array}$ & $\begin{array}{l}\text { Percent } \\
\text { explained } \\
\text { variance }\end{array}$ & $\begin{array}{c}\text { Standard } \\
\text { error of } \\
\text { estimate } \\
(\mathrm{mg} / \mathrm{L})\end{array}$ \\
\hline Dissolved·solids, residue (RDS) & & & & & \\
\hline Dissolved solids, sum (SDS) & $-\cdots-1$ & - & $\ldots-$ & - & -- \\
\hline Hardness as $\mathrm{CaCO}_{3}(\mathrm{TH})$ & 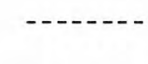 & 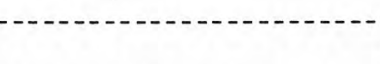 & $-\ldots-$. & & \\
\hline Calcium $(\mathrm{Ca})$ & $--1--1$ & 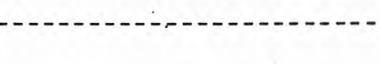 & -1 & & \\
\hline Magnesium (Mg) & 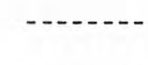 & 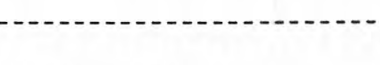 & $\ldots$ & $\ldots \ldots$ & \\
\hline Sodium (Na) & 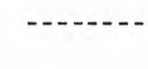 & 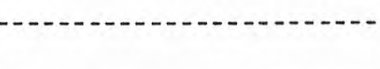 & ---1 & $\ldots \ldots$ & \\
\hline Potassium (K) & 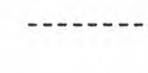 & - - & & $\ldots$ & -- \\
\hline Bicarbonate $\left(\mathrm{HCO}_{3}\right)$ & 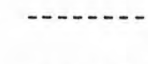 & 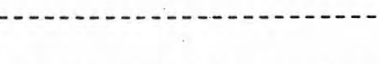 & & $--n-1$ & \\
\hline Sulfate $\left(\mathrm{SO}_{4}\right)$ & 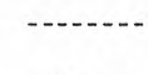 & - & - - - - & 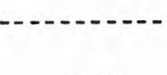 & \\
\hline Chloride (C1) & 20 & $\mathrm{C} 1=0.014(\mathrm{SpC})+0.443$ & $\cdot 0.90$ & 81 & 2.1 \\
\hline Silica (Si) & $-\cdots-1$ & 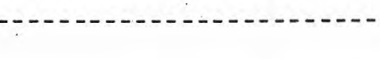 & ----1 & $-1-1$ & \\
\hline Nitrite + Nitrate as $\mathrm{N}\left(\mathrm{NO}_{2}-\mathrm{NO}_{3}\right)$ & $-\cdots-1$ & (n) & & & \\
\hline
\end{tabular}

Results of regression analyses relating specific conductance to water discharge (Q)

\begin{tabular}{ccccccc}
\hline $\begin{array}{c}\text { No. of } \\
\text { measure- } \\
\text { ments }\end{array}$ & $\begin{array}{c}\text { Water discharge } \\
\left(\mathrm{ft}^{3} / \mathrm{s}\right)\end{array}$ & Maximum Minimum & Regression equation & $\begin{array}{c}\text { Correlation } \\
\text { coefficient }\end{array}$ & $\begin{array}{c}\text { Percent } \\
\text { explained } \\
\text { variance }\end{array}$ & $\begin{array}{c}\text { Standard error } \\
\text { of estimate }\end{array}$ \\
\hline 20 & $170 \quad 0.08$ & $\mathrm{SpC}=1228 \mathrm{Q}^{-0.07273}$ & -0.52 & 27 & 0.113 \\
\hline
\end{tabular}




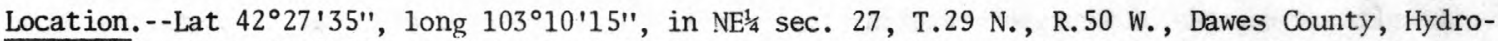
logic Unit 10150002 , on right bank $1 \mathrm{mi}$ upstream from high-water line of Box Butte Reservoir and $6 \mathrm{mi}$ east of Marsland.

Drainage area. $--1,400 \mathrm{mi}^{2}$, approximately.

Period of record.--July 1975 to current year.

Statistical data for selected chemical constituents

\begin{tabular}{|c|c|c|c|c|c|c|c|c|c|}
\hline Constituent & Units & $\begin{array}{l}\text { No. of } \\
\text { measure- } \\
\text { ments }\end{array}$ & $\begin{array}{l}\text { Max- } \\
\text { imum }\end{array}$ & $\begin{array}{l}\text { Min- } \\
\text { imum }\end{array}$ & Mean & Median & $\begin{array}{c}\text { Standard } \\
\text { devi- } \\
\text { ation }\end{array}$ & $\begin{array}{c}\text { Ninetieth } \\
\text { percent- } \\
\text { ile }\end{array}$ & $\begin{array}{l}\text { Tenth } \\
\text { percent- } \\
\text { ile }\end{array}$ \\
\hline Specific conductance & $\mathrm{mho} / \mathrm{cm}$ & 27 & 472 & 358 & 413 & 414 & 28 & 450 & 370 \\
\hline Dissolved solids, residue & $\mathrm{mg} / \mathrm{L}$ & 17 & 325 & 263 & 286 & 282 & 17 & 310 & 270 \\
\hline Dissolved solids, sum & $\mathrm{mg} / \mathrm{L}$ & 9 & 300 & 266 & 281 & $\ldots \ldots$ & 13 & $\ldots \ldots$ & $\cdots-$ \\
\hline Hardness as $\mathrm{CaCO}_{3}$ & $\mathrm{mg} / \mathrm{L}$ & 8 & 180 & 150 & 160 & 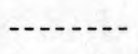 & 12 & & \\
\hline Calcium, dissolved & $\mathrm{mg} / \mathrm{L}$ & $8^{\circ}$ & 54 & 45 & 49 & $\ldots \ldots$ & 3.8 & & - - \\
\hline Magnesium, dissolved & $\mathrm{mg} / \mathrm{L}$ & 8 & 10 & 8.1 & 9.3 & 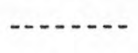 & 0.7 & & \\
\hline Sodium, dissolved & $\mathrm{mg} / \mathrm{L}$ & 8 & 37 & 22 & 26 & 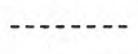 & 4.6 & & \\
\hline Potassium, dissolved & $\mathrm{mg} / \mathrm{L}$ & 8 & 8.7 & 6.3 & 7.4 & 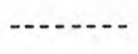 & 1.0 & & \\
\hline Bicarbonate ion & $\mathrm{mg} / \mathrm{L}$ & 8 & 270 & 210 & 243 & $\cdots$ & 21 & & \\
\hline Sulfate, dissolved & $\mathrm{mg} / \mathrm{L}$ & 8 & 33 & 11 & 16 & $\cdots-\cdots$ & 7.1 & & \\
\hline Chloride, dissolved & $\mathrm{mg} / \mathrm{L}$ & 25 & 8.3 & 3.5 & 5.3 & 4.9 & 1.2 & 7.1 & 3.9 \\
\hline Fluoride, dissolved & $\mathrm{mg} / \mathrm{L}$ & 9 & 0.8 & 0.6 & 0.7 & 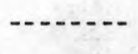 & 0.07 & & \\
\hline Silica, dissolved & $\mathrm{mg} / \mathrm{L}$ & 8 & 52 & 39 & 47 & 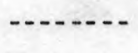 & 4.2 & & \\
\hline Boron, dissolved & $\mathrm{mg} / \mathrm{L}$ & 8 & 160 & 40 & 68 & 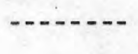 & 38 & & \\
\hline Iron, dissolved & ug/L & 8 & 180 & 0 & 46 & 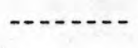 & 58 & & \\
\hline Manganese, dissolved & $u g / L$ & 8 & 200 & 5.0 & 46 & 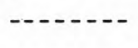 & 64 & & -- \\
\hline $\begin{array}{l}\text { Nitrite + nitrate as } \mathrm{N} \text {, } \\
\text { dissolved } \\
\text { Nitrate as } \mathrm{N} \text {, dissolved }\end{array}$ & $\mathrm{mg} / \mathrm{L}$ & -- & ---1 & $\ldots$ & $\ldots$ & & & & \\
\hline Nitrate as $\mathrm{NO}_{3}$, dissolved & $\mathrm{mg} / \mathrm{L}$ & --- & $--\cdots$ & --- & - & & & & -- \\
\hline Ammonia $\mathrm{N}$, total & $\mathrm{mg} / \mathrm{L}$ & 28 & 0.07 & 0.00 & 0.02 & 0.02 & 0.02 & 0.05 & 0.00 \\
\hline Organic $N$, total & $\mathrm{mg} / \mathrm{L}$ & 28 & 0.81 & 0.15 & 0.43 & 0.38 & 0.19 & 0.71 & 0.21 \\
\hline Phosphorus, total & $\mathrm{mg} / \mathrm{L}$ & 28 & 0.12 & 0.00 & 0.04 & 0.04 & 0.03 & 0.09 & 0.01 \\
\hline Phosphorus, dissolved & $\mathrm{mg} / \mathrm{L}$ & 20 & 0.08 & 0.00 & 0.02 & 0.02 & 0.02 & 0.05 & 0.00 \\
\hline Fecal coliform & $/ 100 \mathrm{ml}$ & 15 & 1,800 & 1 & $\cdots \cdots$ & $66-$ & -1 & 465 & 0 \\
\hline Fecal streptococci & $/ 100 \mathrm{ml}$ & 23 & 4,200 & 9 & $\cdots \cdots$ & $56 \cdots$ & & 660 & 16 \\
\hline Oxygen, dissolved & $\mathrm{mg} / \mathrm{L}$ & 28 & 16.5 & 8.2 & 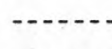 & $-10.0-$ & 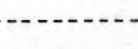 & 13.0 & 8.5 \\
\hline \multirow{2}{*}{$\begin{array}{l}\text { Biochemical oxygen demand } \\
\text { pH }(5-\text { day) }\end{array}$} & $\mathrm{mg} / \mathrm{L}$ & 26 & 9.4 & 0.6 & 2.9 & 2.5 & 1.9 & 5.2 & 1.1 \\
\hline & $H$ units & 28 & 7.8 & 7.1 & 7.4 & 7.4 & 0.2 & 7.7 & 7.2 \\
\hline Co $-\mathrm{Pt}$ & $t$ units & 9 & 25 & 3 & 14 & 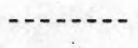 & 8 & & \\
\hline
\end{tabular}


06454500 Niobrara River above Box Butte Reservoir, Nebr.--Continued

Results of regression analyses relating concentrations of selected chemical constituents to specific conductance $(\mathrm{SpC})$

\begin{tabular}{|c|c|c|c|c|c|}
\hline $\begin{array}{l}\text { Dissolved } \\
\text { constituents } \\
(\mathrm{mg} / \mathrm{L})\end{array}$ & $\begin{array}{l}\text { No. of } \\
\text { measure- } \\
\text { ments }\end{array}$ & Regression equation & $\begin{array}{l}\text { Corre- } \\
\text { lation } \\
\text { coeffi- } \\
\text { cient }\end{array}$ & $\begin{array}{l}\text { Percent } \\
\text { explained } \\
\text { variance }\end{array}$ & $\begin{array}{c}\text { Standard } \\
\text { error of } \\
\text { estimate } \\
(\mathrm{mg} / \mathrm{L})\end{array}$ \\
\hline Dissolved solids, residue (RDS) & 17 & $\mathrm{RDS}=0.110(\mathrm{SpC})+240.947$ & $* 0.17$ & 3.0 & 17 \\
\hline Dissolved solids, sum (SDS) & $-\cdots$ & 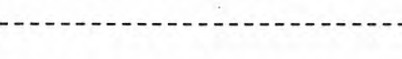 & 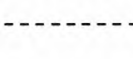 & 列 & - - - - - \\
\hline Hardness as $\mathrm{CaCO}_{3}(\mathrm{TH})$ & $-\cdots-1$ & - & - & & 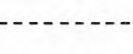 \\
\hline Calcium (Ca) & $\cdots-1$ & 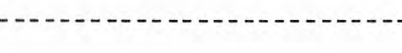 & - & ----- & $-\cdots$ \\
\hline Magnesium (Mg) & $-\cdots$ & 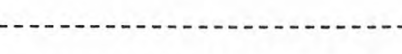 & & $-\cdots$ & 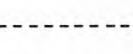 \\
\hline Sodium $(\mathrm{Na})$ & $-\cdots$ & & & & \\
\hline Potassium (K) & $-\cdots-1$ & - & ---- & 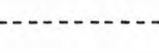 & 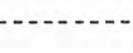 \\
\hline Bicarbonate $\left(\mathrm{HCO}_{3}\right)$ & $-\cdots$ & 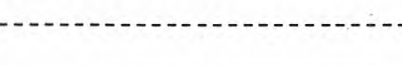 & & ---- & - \\
\hline Sulfate $\left(\mathrm{SO}_{4}\right)$ & $-\cdots-1$ & 等, & & & \\
\hline Chloride (C1) & 25 & $\mathrm{Cl}=0.003(\mathrm{SpC})+4.187$ & $* .06$ & 0.42 & 1.2 \\
\hline Silica (Si) & $-\cdots-1$ & (1) & & & \\
\hline Nitrite + Nitrate as $\mathrm{N}\left(\mathrm{NO}_{2}-\mathrm{NO}_{3}\right)$ & & & & & \\
\hline
\end{tabular}

* Not significant at 95 percent confidence level; the regression equation should not be used to predict the concentration of the dependent variable.

Results of regression analyses relating specific conductance to water discharge (Q)

\begin{tabular}{ccccccc}
\hline \multirow{2}{*}{$\begin{array}{l}\text { No. of } \\
\text { measure- } \\
\text { ments }\end{array}$} & $\begin{array}{c}\text { Water discharge } \\
\left(\mathrm{ft}^{3} / \mathrm{s}\right)\end{array}$ & Maximum Minimum & Regression equation & $\begin{array}{c}\text { Correlation } \\
\text { coefficient }\end{array}$ & $\begin{array}{c}\text { Percent } \\
\text { explained } \\
\text { variance }\end{array}$ & $\begin{array}{c}\text { Standard error } \\
\text { of estimate }\end{array}$ \\
\hline 27 & $86 \quad 6.6$ & $\mathrm{SpC}=406 \mathrm{Q}^{0.00508}$ & $* 0.04$ & 0.20 & 0.031 & 7.1 \\
\hline
\end{tabular}

*Not significant at 95 percent confidence level; the regression equation should not be used to predict the concentration of the dependent variable. 
Location.--Lat $42^{\circ} 27^{\prime} 48^{\prime \prime}$, long $102^{\circ} 55^{\prime} 47^{\prime \prime}$, in SE $\frac{1}{4} N W^{\prime}-4$ sec. 26, T.29 N., R.48 W., Dawes County, $0.5 \mathrm{mi}$ upstream from gaging station, at county road bridge $0.7 \mathrm{mi}$ upstream from Cottonwood Creek, and $2.0 \mathrm{mi}$ east of Dunlap.

Drainage area. $--1,580 \mathrm{mi}^{2}$, approximately (at gaging station).

Period of record. --August 1969 to September 1973.

Statistical data for selected chemical constituents

\begin{tabular}{|c|c|c|c|c|c|c|c|c|c|}
\hline Constituent & Units & $\begin{array}{l}\text { No. of } \\
\text { measure- } \\
\text { ments }\end{array}$ & $\begin{array}{l}\text { Max- } \\
\text { imum }\end{array}$ & $\begin{array}{l}\text { Min- } \\
\text { imum }\end{array}$ & Mean & Median & $\begin{array}{c}\text { Standard } \\
\text { devi- } \\
\text { ation }\end{array}$ & $\begin{array}{l}\text { Ninetieth } \\
\text { percent- } \\
\text { ile }\end{array}$ & $\begin{array}{c}\text { Tenth } \\
\text { percent- } \\
\text { ile }\end{array}$ \\
\hline Specific conductance & umho/cm & 79 & 510 & 290 & 382 & 389 & 46 & 433 & 316 \\
\hline Dissolved solids, residue & $\mathrm{mg} / \mathrm{L}$ & 2 & 279 & 221 & 250 & 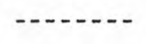 & 41 & & $-\cdots$ \\
\hline Dissolved solids, sum & $\mathrm{mg} / \mathrm{L}$ & 7 & 284 & 212 & 247 & 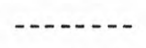 & 30 & & -- \\
\hline Hardness as $\mathrm{CaCO}_{3}$ & $\mathrm{mg} / \mathrm{L}$. & 7 & 150 & 99 & 124 & $-\cdots-1--$ & 21 & & -- \\
\hline Calcium, dissolved & $\mathrm{mg} / \mathrm{L}$ & 7 & 46 & 25 & 35 & 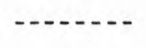 & 8.8 & & -- \\
\hline Magnesium, dissolved & $\mathrm{mg} / \mathrm{L}$ & 7 & 9.9 & 8.3 & 9.2 & $\cdots-\cdots$ & 0.5 & & $-\cdots$ \\
\hline Sodium, dissolved & $\mathrm{mg} / \mathrm{L}$ & 7 & 28 & 23 & 27 & $-\cdots-1$ & 1.9 & & -- \\
\hline Potassium, dissolved & $\mathrm{mg} / \mathrm{L}$ & 7 & 9.2 & 6.4 & 7.9 & $-\cdots-1$ & 0.9 & & -- \\
\hline Bicarbonate ion & $\mathrm{mg} / \mathrm{L}$ & 7 & 237 & 179 & 210 & 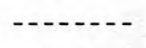 & 24 & & \\
\hline Sulfate, dissolved & $\mathrm{mg} / \mathrm{L}$ & 7 & 18 & 11 & 15 & $-\cdots---$ & 2.3 & & \\
\hline Chloride, dissolved & $\mathrm{mg} / \mathrm{L}$ & 7 & 6.3 & 2.0 & 4.4 & $-\cdots---$ & 1.4 & & \\
\hline Fluoride, dissolved & $\mathrm{mg} / \mathrm{L}$ & 7 & 0.9 & 0.7 & 0.7 & $-\cdots-1$ & 0.1 & & \\
\hline Silica, dissolved & $\mathrm{mg} / \mathrm{L}$ & 7 & 51 & 31 & 42 & - n & 8.9 & & \\
\hline Boron, dissolved & $\mathrm{mg} / \mathrm{L}$ & 7 & 60 & 20 & 44 & & 15 & & \\
\hline Iron, dissolved & $\mathrm{ug} / \mathrm{L}$ & & -1 & 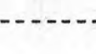 & --- & & & & \\
\hline Manganese, disso1ved & $\mathrm{ug} / \mathrm{L}$ & & - & 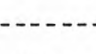 & - & & & & \\
\hline Nitrite + nitrate as $\mathrm{N}$, & $\mathrm{mg} / \mathrm{L}$ & 5 & 1.1 & 0.05 & 0.56 & $-\cdots---$ & 0.50 & & \\
\hline Nitrate as $\mathrm{N}$, dissolved & $\mathrm{mg} / \mathrm{L}$ & 2 & 0.86 & 0.03 & 0.45 & & & & \\
\hline Nitrate as $\mathrm{NO}_{3}$, dissolved & $\mathrm{mg} / \mathrm{L}$ & 2 & 3.8 & 0.20 & 2.0 & & & & \\
\hline Ammonia N, total & $\mathrm{mg} / \mathrm{L}$ & & & . & . & & & & \\
\hline Organic N, total & $\mathrm{mg} / \mathrm{L}$ & 2 & 0.57 & 0.20 & 0.39 & & & & \\
\hline Phosphorus, total & $\mathrm{mg} / \mathrm{L}$ & 2 & 0.21 & 0.18 & 0.20 & & & & \\
\hline Phosphorus, dissolved & $\mathrm{mg} / \mathrm{L}$ & 7 & 0.04 & 0.02 & 0.03 & & 0.01 & & \\
\hline $\begin{array}{ll}\text { Fecal coliform } & \mathrm{col} / \mathrm{l} \\
\text { Fecal streptococci } & \mathrm{col}\end{array}$ & $\operatorname{col} / 100 \mathrm{ml}$ & & 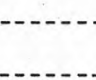 & 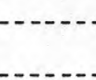 & -1 & & & & \\
\hline Fecal streptococci col/ & $\mathrm{col} / 100 \mathrm{ml}$ & 75 & & & & 06 & 20 & 120 & 67 \\
\hline $\begin{array}{l}\text { Oxygen, dissolved } \\
\text { Biochemical oxygen demand }\end{array}$ & $\begin{array}{l}\mathrm{mg} / \mathrm{L} \\
\mathrm{mg} / \mathrm{L}\end{array}$ & 75 & 13.8 & 5.3 & 9.7 & 9.0 & 2.0 & 12.0 & 0.1 \\
\hline $\mathrm{pH}^{(5-\text { day })}$ & $\mathrm{pH}$ units & 82 & 8.6 & 7.2 & 8.1 & 8.2 & 0.2 & 8.4 & 7.8 \\
\hline Co $-\mathrm{Pt}$ & co - Pt units & 7 & 18 & 0 & 5 & $-\cdots-1$ & 6 & $-\cdots$ & $-\ldots$ \\
\hline
\end{tabular}




\section{NIOBRARA RIVER BASIN}

06456500 Niobrara River near Hay Springs, Nebr.

Location. --Lat $42^{\circ} 28^{\prime} 30^{\prime \prime}$, long $102^{\circ} 41^{\prime} 50^{\prime \prime}$, in NE $\frac{1}{4} \mathrm{sec} .22$, T.29 N., R.46 W., on right bank about $500 \mathrm{ft}$ upstream from bridge on State Highway 87, $14 \mathrm{mi}$ south of Hay Springs, in Sheridan County, and about $4 \mathrm{mi}$ upstream from Box Butte Creek.

Period of record.-- October 1963 to September 1964.

Statistical data for selected chemical constituents

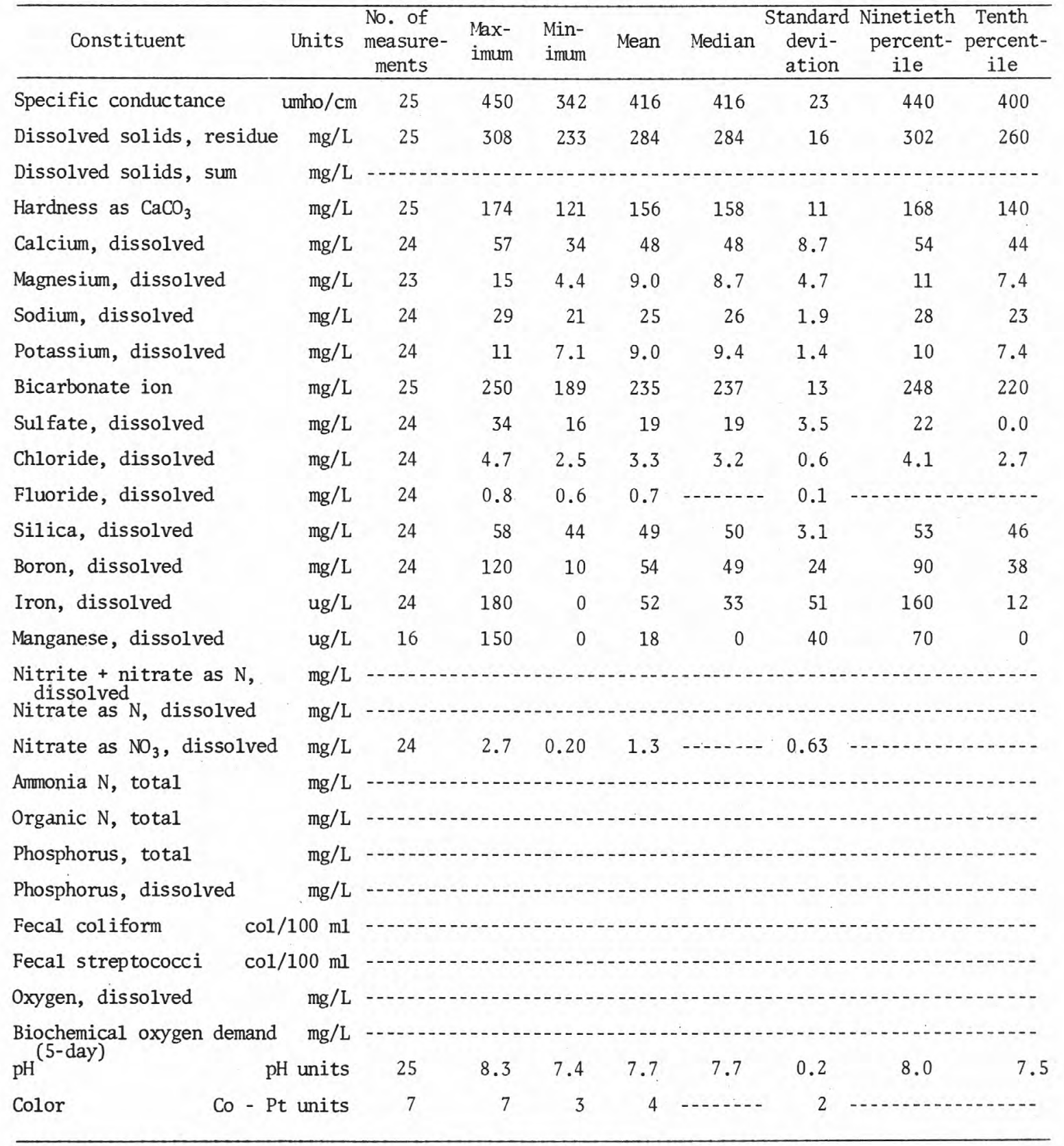


Results of regression analyses relating concentrations of selected chemical constituents to specific conductance $(\mathrm{SpC})$

\begin{tabular}{|c|c|c|c|c|c|}
\hline $\begin{array}{l}\text { Dissolved } \\
\text { constituents } \\
(\mathrm{mg} / \mathrm{L})\end{array}$ & $\begin{array}{l}\text { No. of } \\
\text { measure- } \\
\text { ments }\end{array}$ & Regression equation & $\begin{array}{l}\text { Corre- } \\
\text { lation } \\
\text { coeffi- } \\
\text { cient }\end{array}$ & $\begin{array}{l}\text { Percent } \\
\text { explained } \\
\text { variance }\end{array}$ & $\begin{array}{l}\text { Standard } \\
\text { error of } \\
\text { estimate } \\
(\mathrm{mg} / \mathrm{L})\end{array}$ \\
\hline Dissolved solids, residue (RDS) & 25 & $\mathrm{RDS}=0.659(\mathrm{SpC})+9.565$ & 0.94 & 89 & 5.4 \\
\hline Dissolved solids, sum (SDS) & & & & & \\
\hline Hardness as $\mathrm{CaCO}_{3}(\mathrm{TH})$ & 25 & $\mathrm{TH}=0.448(\mathrm{SpC})-30.677$ & .89 & 79 & 5.3 \\
\hline Calcium (Ca) & 24 & $\mathrm{Ca}=0.135(\mathrm{SpC})-8.214$ & .66 & 44 & $3.6 \mathrm{gge}$ \\
\hline Magnesium (Mg) & 23 & $\mathrm{Mg}=0.022(\mathrm{SpC})-0.215$ & *.25 & 6.4 & 1.9 it \\
\hline Sodium (Na) & 24 & $\mathrm{Na}=0.030(\mathrm{SpC})+12.819$ & $* .34$ & 11 & 1.8 \\
\hline Potassium (K) & 24 & $K=0.016(\mathrm{SpC})+2.350$ & $* .26$ & 6.5 & 1.3 \\
\hline Bicarbonate $\left(\mathrm{HCO}_{3}\right)$ & 25 & $\mathrm{HCO}_{3}=0.517(\mathrm{SpC})+20.026$ & .90 & 82 & 5.6 ? \\
\hline Sulfate $\left(\mathrm{SO}_{4}\right)$ & 24 & $\mathrm{SO}_{4}=0.075(\mathrm{SpC})-11.915$ & .46 & 22 & 3.2 \\
\hline Chloride (C1) & 24 & $\mathrm{Cl}=0.006(\mathrm{SpC})+0.597$ & $* .23$ & 5.3 & 0.6 \\
\hline Silica (Si) & 24 & $\mathrm{Si}=0.011(\mathrm{SpC})+44.764$ & *.08 & 0.60 & 3.2 \\
\hline
\end{tabular}

* Not significant at the 95 percent confidence level; the regression equation should not be used to predict the concentration of the dependent variable.

Results of regression analyses relating specific conductance to water discharge (Q) $\mathrm{IO}]$

\begin{tabular}{ccccccc}
\hline $\begin{array}{c}\text { No. of } \\
\text { measure- } \\
\text { ments }\end{array}$ & $\begin{array}{c}\text { Water discharge } \\
\left(\mathrm{ft}^{3} / \mathrm{s}\right)\end{array}$ & Maximum Minimum & Regression equation & $\begin{array}{c}\text { Correlation } \\
\text { coefficient }\end{array}$ & $\begin{array}{c}\text { Percent } \\
\text { explained } \\
\text { variance }\end{array}$ & $\begin{array}{c}\text { Standard error } \\
\text { of estimate }\end{array}$ \\
\hline 25 & $87 \quad 10$ & $\mathrm{SpC}=423 \mathrm{Q}^{-0.00580}$ & $* 0.04$ & 0.17 & 0.026 \\
\hline
\end{tabular}

* Not significant at the 95 percent confidence level; the regression equation should not be used to predict the concentration of the dependent variable. 


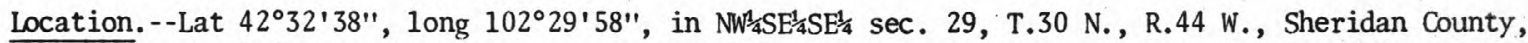
at wooden bridge $1 \mathrm{mi}$ west of Colclesser Mill School, about $2 \mathrm{mi}$ upstream from Pine Creek, and $2.2 \mathrm{mi}$ southwest of State Highway 250 bridge crossing.

Drainage area. $--2,220 \mathrm{mi}^{2}$, approximately.

Period of record.-- August 1909 to September 1973.

Statistical data for selected chemical constituents

\begin{tabular}{|c|c|c|c|c|c|c|c|c|c|}
\hline Constituent & Units & $\begin{array}{l}\text { No. of } \\
\text { measure- } \\
\text { ments }\end{array}$ & $\begin{array}{l}\text { Max- } \\
\text { imum }\end{array}$ & $\begin{array}{l}\text { Min- } \\
\text { imum }\end{array}$ & Mean & Median & $\begin{array}{c}\text { Standard } \\
\text { devi- } \\
\text { ation }\end{array}$ & $\begin{array}{c}\text { Ninetieth } \\
\text { percent- } \\
\text { ile }\end{array}$ & $\begin{array}{l}\text { Tenth } \\
\text { percent- } \\
\text { ile }\end{array}$ \\
\hline Specific conductance & umho/cm & 82 & 500 & 170 & 394 & 399 & 50 & 442 & 340 \\
\hline Dissolved solids, residue & $\mathrm{mg} / \mathrm{L}$ & 2 & 289 & 288 & 288 & $-\cdots-$ & - & & $\cdots$ \\
\hline Dissolved solids, sum & $\mathrm{mg} / \mathrm{L}$ & 7 & 298 & 254 & 272 & $-\cdots-1$ & 16 & & $\cdots$ \\
\hline Hardness as $\mathrm{CaCO}_{3}$ & $\mathrm{mg} / \mathrm{L}$ & 7 & 170 & 139 & 150 & -...... & 12 & & $\cdots$ \\
\hline Calcium, dissolved & $\mathrm{mg} / \mathrm{L}$ & 7 & 52 & 41 & 46 & $-\ldots$ & 4.3 & $\cdots+\cdots$ & $\cdots$ \\
\hline Magnesium, dissolved & $\mathrm{mg} / \mathrm{L}$ & 7 & 9.0 & 7.5 & 8.5 & 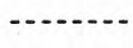 & 0.6 & $\ldots . .$. & $\cdots$ \\
\hline Sodium, dissolved & $\mathrm{mg} / \mathrm{L}$ & 7 & 29 & 18 & 23 & - n & 3.8 & &.- \\
\hline Potassium, dissolved & $\mathrm{mg} / \mathrm{L}$ & 7 & 14 & 7.7 & 10 & 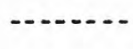 & 2.1 & & $\cdots$ \\
\hline Bicarbonate ion & $\mathrm{mg} / \mathrm{L}$ & 7 & 245 & 206 & 220 & 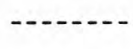 & 14 & & $-\cdots$ \\
\hline Sulfate, dissolved & $\mathrm{mg} / \mathrm{L}$ & 7 & 29 & 17 & 23 & - n & 4.6 & & $-\cdots$ \\
\hline Chloride, dissolved & $\mathrm{mg} / \mathrm{L}$ & 7 & 7.3 & 2.2 & 4.0 & $-\cdots$ & 1.6 & & $\cdots$ \\
\hline Fluoride, dissolved & $\mathrm{mg} / \mathrm{L}$ & 7 & 0.8 & 0.5 & 0.6 & 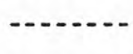 & 0.1 & & \\
\hline Silica, dissolved & $\mathrm{mg} / \mathrm{L}$ & 7 & 52 & 42 & 46 & $\cdots$ & 3.5 & & $\cdots$ \\
\hline Boron, dissolved & $\mathrm{mg} / \mathrm{L}$ & 7 & 71 & 35 & 51 & 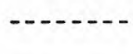 & 14 & & $-\cdots$ \\
\hline Iron, dissolved & $\mathrm{ug} / \mathrm{L}$ & 2 & 38 & 0 & 19 & & & & $\cdots$ \\
\hline Manganese, dissolved & $\mathrm{ug} / \mathrm{L}$ & 2 & 28 & 7 & 18 & & & & \\
\hline $\begin{array}{l}\text { Nitrite }+ \text { nitrate as } \mathrm{N} \text {, } \\
\text { dissolved }\end{array}$ & $\mathrm{mg} / \mathrm{L}$ & 5 & 0.96 & 0.05 & 0.43 & & 0.38 & & $\cdots$ \\
\hline Nitrate as $N$, dissolved & $\mathrm{mg} / \mathrm{L}$ & 2 & 0.97 & 0.13 & 0.55 & & & & \\
\hline $\begin{array}{l}\text { Nitrate as } \mathrm{NO}_{3} \text {, dissolved } \\
\text { Ammonia } \mathrm{N} \text {, total }\end{array}$ & $\begin{array}{l}\mathrm{mg} / \mathrm{L} \\
\mathrm{mg} / \mathrm{L}\end{array}$ & $\cdots-1$ & --- & -2 & $-\infty$ & & & & \\
\hline Organic $N$, total & $\mathrm{mg} / \mathrm{L}$ & 2 & 0.38 & 0.30 & 0.34 & $\cdots$ & & & \\
\hline Phosphorus, total & $\mathrm{mg} / \mathrm{L}$ & 1 & $-\cdots$ & $\cdots$ & 0.09 & & & & \\
\hline Phosphorus, dissolved & $\mathrm{mg} / \mathrm{L}$ & 7 & 0.08 & 0.02 & 0.05 & $\cdots+\cdots$ & 0.02 & & \\
\hline $\begin{array}{ll}\text { Fecal coliform } & \mathrm{col} / \\
\text { Fecal streptococci } & \mathrm{col} /\end{array}$ & $\mathrm{col} / 100 \mathrm{ml}$ & $-\cdots$ & 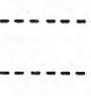 & & 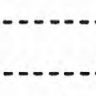 & & & & 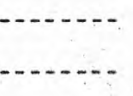 \\
\hline Oxygen, dissolved & $\mathrm{mg} / \mathrm{L}$ & 76 & 12.4 & 5.3 & 9.5 & 9.4 & 1.7 & 11.8 & 7.2 \\
\hline $\begin{array}{l}\text { Biochemical oxygen demand } \\
\mathrm{pH}^{(5-\text { day) }}\end{array}$ & $\begin{array}{r}\mathrm{mg} / \mathrm{L} \\
\mathrm{H} \text { units }\end{array}$ & 83 & 8.7 & 7.2 & 8.2 & 8.2 & 0.25 & 8.5 & 7.9 \\
\hline $\mathrm{Co}-\mathrm{Pt}$ & $t$ units & 7 & 20 & 3 & 9 & - n & 6.6 & 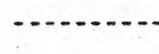 & \\
\hline
\end{tabular}


06457000 Niobrara River near Colclesser, Nebr.--Continued

Results of regression analyses relating concentrations of selected chemical constituents to specific conductance $(\mathrm{SpC})$

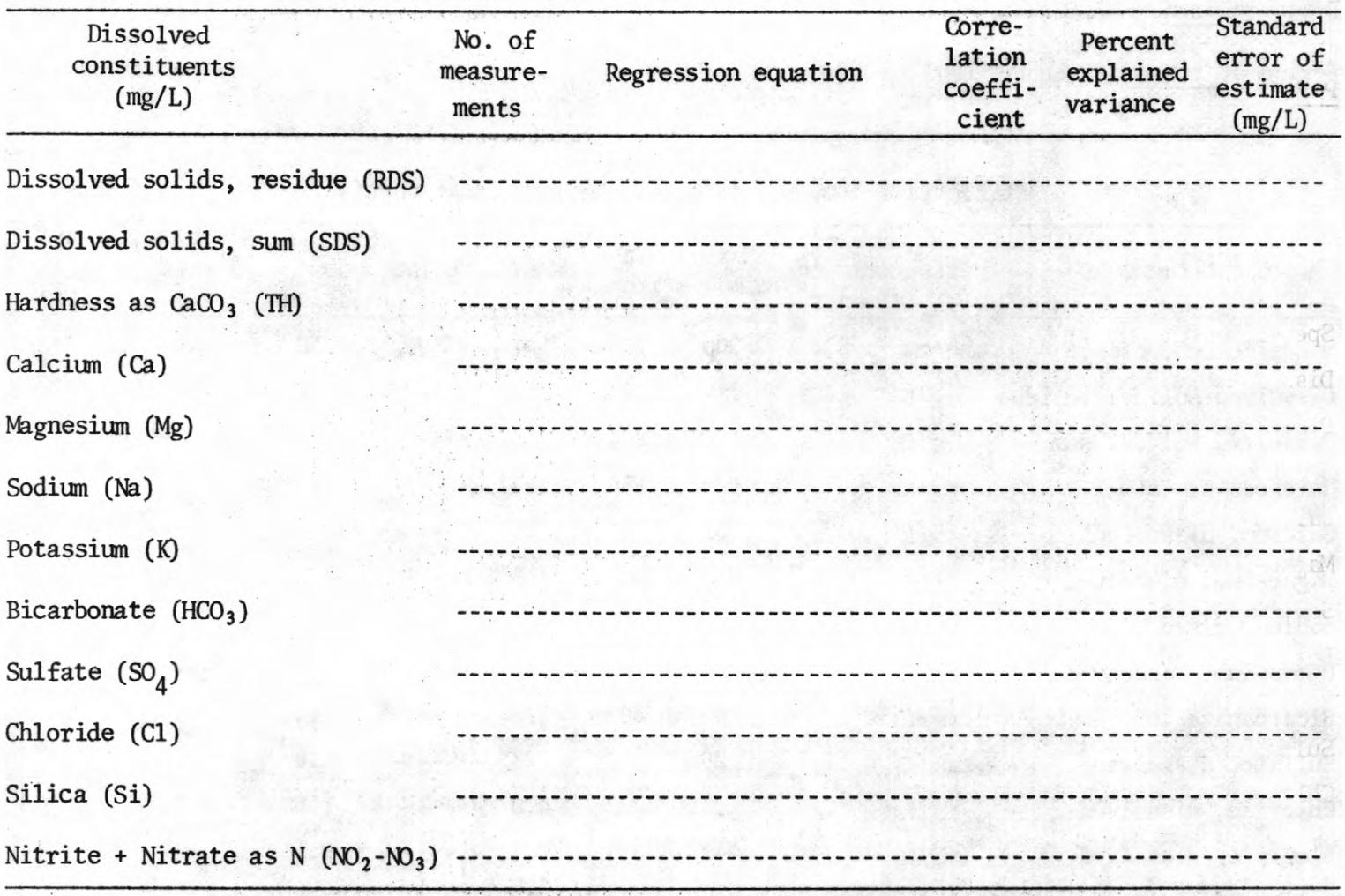

Results of regression analyses relating specific conductance to water discharge (Q)

\begin{tabular}{cccccccc}
\hline \multirow{2}{*}{$\begin{array}{c}\text { No. of } \\
\text { measure- }\end{array}$ Water discharge } & $\left(\mathrm{ft}^{3} / \mathrm{s}\right)$ & Rents & Maximum Minimum & Regression equation & $\begin{array}{c}\text { Correlation } \\
\text { coefficient }\end{array}$ & $\begin{array}{c}\text { Percent } \\
\text { explained } \\
\text { variance }\end{array}$ & \multicolumn{2}{c}{$\begin{array}{c}\text { Standard error } \\
\text { of estimate }\end{array}$} \\
\hline 82 & $75 \quad 16$ & $\mathrm{SpC}=263 \mathrm{Q}^{0.10724}$ & 0.26 & 6.9 & 0.062 & 14 \\
\hline
\end{tabular}


Location. --Lat $42^{\circ} 47^{\prime} 13^{\prime \prime}$, long $100^{\circ} 02^{\prime} 06^{\prime \prime}$, in $\mathrm{N}^{\frac{1}{2}} \mathrm{SW} \frac{\mathrm{f}}{4} \mathrm{sec} .33$, T.33 N., R.23 W., Keya Paha County, Hydrologic Unit 10150004, on left bank $60 \mathrm{ft}$ downstream from county road bridge, $1.5 \mathrm{mi}$ downstream from Fairfield Creek, and $6 \mathrm{mi}$ south of Norden.

Drainage area. $--8,390 \mathrm{mi}^{2}$, approximately.

Period of record.--Water years 1963-66, 1974 to current year.

Statistical data for selected chemical constituents

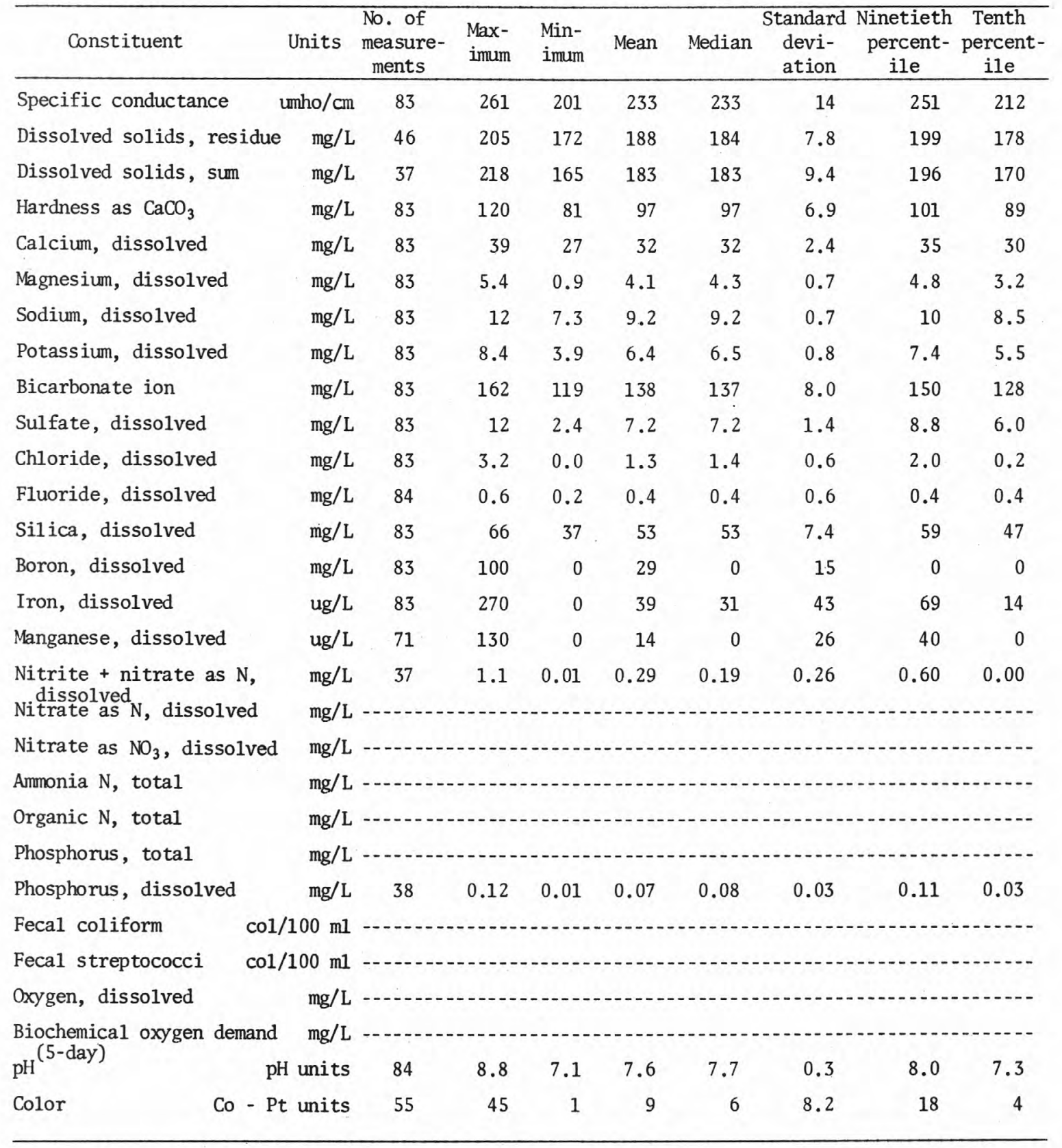




\section{NIOBRARA RIVER BASIN}

06462000 Niobrara River near Norden, Nebr.--Continued

Results of regression analyses relating concentrations of selected chemical constituents to specific conductance $(\mathrm{SpC})$

\begin{tabular}{|c|c|c|c|c|c|}
\hline $\begin{array}{c}\text { Dissolved } \\
\text { constituents } \\
(\mathrm{mg} / \mathrm{L})\end{array}$ & $\begin{array}{l}\text { No. of } \\
\text { measure- } \\
\text { ments }\end{array}$ & Regression equation & $\begin{array}{l}\text { Corre- } \\
\text { lation } \\
\text { coeffi- } \\
\text { cient }\end{array}$ & $\begin{array}{l}\text { Percent } \\
\text { explained } \\
\text { variance }\end{array}$ & $\begin{array}{l}\text { Standard } \\
\text { error of } \\
\text { estimate } \\
(\mathrm{mg} / \mathrm{L})\end{array}$ \\
\hline Dissolved solids, residue (RDS) & 46 & $\mathrm{RDS}=0.441(\mathrm{SpC})+83.587$ & 0.72 & 51 & 5.5 \\
\hline Dissolved solids, sum (SDS) & 36 & $\mathrm{SDS}=0.345(\mathrm{SpC})+103.812$ & .46 & 21 & 9.5 \\
\hline Hardness as $\mathrm{CaCO}_{3}$ (TH) & 82 & $\mathrm{TH}=0.326(\mathrm{SpC})+20.657$ & .67 & 44 & 5.2 \\
\hline Calcium (Ca). & 82 & $\mathrm{Ca}=0.117(\mathrm{SpC})+4.857$ & .68 & 46 & 1.8 \\
\hline Magnesium (Mg) & 82 & $M g=0.006(\mathrm{SpC})+2.549$ & $* .12$ & 1.5 & .7 \\
\hline Sodium (Na) & 82 & $\mathrm{Na}=0.022(\mathrm{SpC})+4.152$ & .43 & 18 & .6 \\
\hline Potassium (K) & 82 & $\mathrm{~K}=0.018(\mathrm{SpC})+2.112$ & .31 & 9.4 & .8 \\
\hline Bicarbonate $\left(\mathrm{HCO}_{3}\right)$ & 82 & $\mathrm{HCO}_{3}=0.432(\mathrm{SpC})+37.230$ & .76 & 57 & 5.3 \\
\hline Sulfate $\left(\mathrm{SO}_{4}\right)$ & 82 & $\mathrm{SO}_{4}=0.040(\mathrm{SpC})-2.038$ & .39 & 15 & 1.4 \\
\hline Chloride $(\mathrm{Cl})$ & 82 & CI $-0.011(\mathrm{SpC})-1.377$ & .26 & 7.0 & .6 \\
\hline Silica (Si) & 83 & $\mathrm{Si}=0.005(\mathrm{SpC})+52.071$ & $* .01$ & 0.02 & 5.2 \\
\hline Nitrite + Nitrate as $\mathrm{N}\left(\mathrm{NO}_{2}-\mathrm{NO}_{3}\right)$ & 36 & $\mathrm{NO}_{2}-\mathrm{NO}_{3}=0.002(\mathrm{SpC})-0.107$ & *.09 & 0.85 & 0.27 \\
\hline
\end{tabular}

* Not significant at the 95 percent confidence level; the regression equation should not be used to predict the concentration of the dependent variable.

Results of regression analyses relating specific conductance to water discharge (Q)

\begin{tabular}{ccccccc}
\hline $\begin{array}{c}\text { No. of } \\
\text { measure- } \\
\text { ments }\end{array}$ & $\begin{array}{c}\text { Mater discharge } \\
\left(\mathrm{ft}^{3} / \mathrm{s}\right)\end{array}$ & Maximum Minimum & Regression equation & $\begin{array}{c}\text { Correlation } \\
\text { coefficient }\end{array}$ & $\begin{array}{c}\text { Percent } \\
\text { explained } \\
\text { variance }\end{array}$ & $\begin{array}{c}\text { Standard error } I \\
\text { of estimate }\end{array}$ \\
\hline 83 & $2,230 \quad 390$ & SpC $-226 \Omega^{0.00407}$ & $* 0.02$ & 0.05 & 0.026 & 6.0 \\
\hline
\end{tabular}

* Not significant at the 95 percent confidence level; the regression equation should not be used to predict the concentration of the dependent variable. 


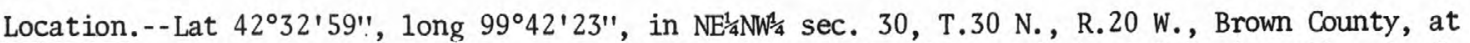
timber bridge $0.1 \mathrm{mi}$ downstream from U.S. Highway 20 bridge and about $0.9 \mathrm{mi}$ northwest of Long Pine.

Period of record.-- October 1973 to June 1975.

Statistical data for selected chemical constituents

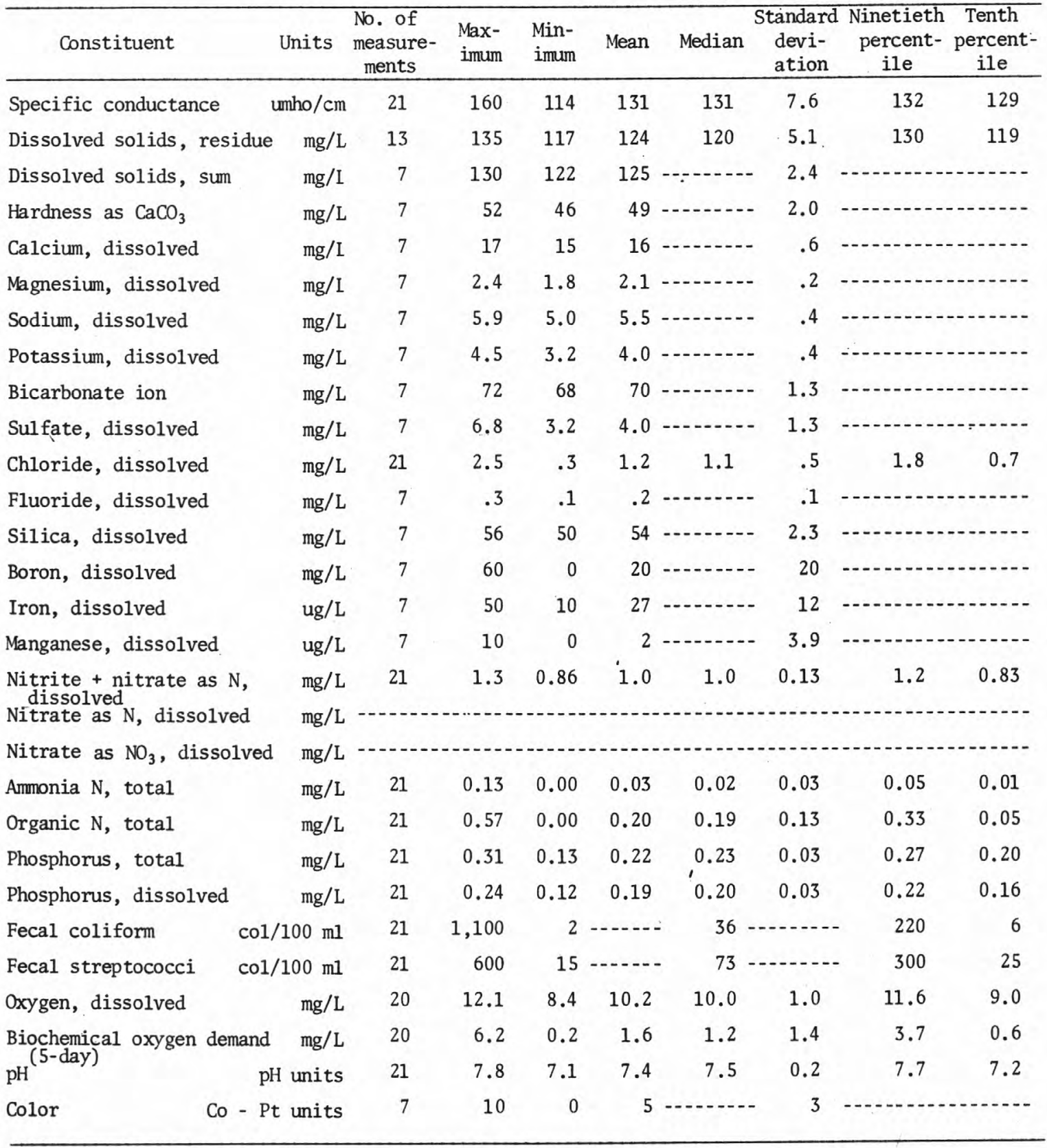


Results of regression analyses relating concentrations of selected chemical constituents to specific conductance (SpC)

\begin{tabular}{|c|c|c|c|c|c|}
\hline $\begin{array}{l}\text { Dissolved } \\
\text { constituents } \\
(\mathrm{mg} / \mathrm{L})\end{array}$ & $\begin{array}{l}\text { No. of } \\
\text { measure- } \\
\text { ments }\end{array}$ & Regression equation & $\begin{array}{l}\text { Corre- } \\
\text { lation } \\
\text { coeffi- } \\
\text { cient }\end{array}$ & $\begin{array}{l}\text { Percent } \\
\text { explained } \\
\text { variance }\end{array}$ & $\begin{array}{c}\text { Standard } \\
\text { error of } \\
\text { estimate } \\
(\mathrm{mg} / \mathrm{L})\end{array}$ \\
\hline Dissolved solids, residue (RDS) & ---- & 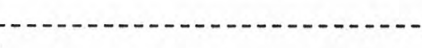 & $-\ldots$ & & 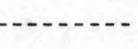 \\
\hline Dissolved solids, sum (SDS) & ---- & 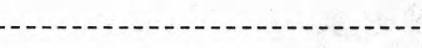 & & & \\
\hline Hardness as $\mathrm{CaCO}_{3}(\mathrm{TH})$ & --- & 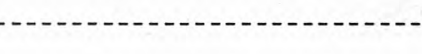 & & & $-\ldots$ \\
\hline Calcium (Ca) & $-\ldots$ & 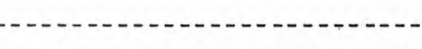 & & & 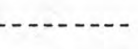 \\
\hline Magnesium (Mg) & $-\cdots-$ & $-8-1$ & & & \\
\hline Sodium (Na) & ----- & 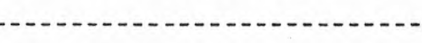 & - - - & $\ldots \ldots$ & -.. \\
\hline Potassium (K) & $---\cdots$ & 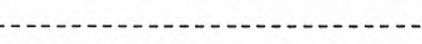 & & & \\
\hline Bicarbonate $\left(\mathrm{HCO}_{3}\right)$ & $-\ldots-\ldots$ & - & & & \\
\hline Sulfate $\left(\mathrm{SO}_{4}\right)$ & ----- & - - - & & & \\
\hline Chloride (Cl) & 21 & $\mathrm{Cl}=0.006(\mathrm{SpC})+0.436$ & $* 0.08$ & 0.73 & 0.5 \\
\hline Silica (Si) & $-\cdots-1$ & 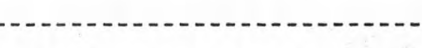 & & 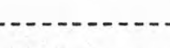 & 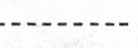 \\
\hline Nitrite + Nitrate as $\mathrm{N}\left(\mathrm{NO}_{2}-\mathrm{NO}_{3}\right)$ & 21 & $\mathrm{NO}_{2}-\mathrm{NO}_{3}=-0.004(\mathrm{SpC})+1.512$ & $* . .23$ & 5.2 & 0.13 \\
\hline
\end{tabular}

* Not significant at the 95 percent confidence level; the regression equation should not be used to predict the concentration of the dependent variable.

Results of regression analyses relating specific conductance to water discharge (Q)

\begin{tabular}{ccccccc}
\hline $\begin{array}{c}\text { No. of } \\
\text { measure } \\
\text { ments }\end{array}$ & $\begin{array}{c}\text { Water discharge } \\
\left(\mathrm{ft}^{3} / \mathrm{s}\right)\end{array}$ & Maximum Minimum & Regression equation & $\begin{array}{c}\text { Correlation } \\
\text { coefficient }\end{array}$ & $\begin{array}{c}\text { Percent } \\
\text { explained } \\
\text { variance }\end{array}$ & $\begin{array}{c}\text { Standard error } \\
\text { of estimate }\end{array}$ \\
\hline 21 & 58 & 50 & $\mathrm{SpC}=146 \mathrm{Q}^{-0.02806}$ & $*-0.03$ & 0.08 & 0.025 \\
\hline
\end{tabular}

* Not significant at the 95 percent confidence 1 evel; the regression equation should not be used to predict the concentration of the dependent variable. 


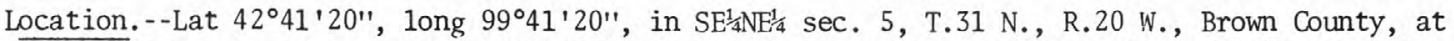
gaging station at county road bridge, $1 \mathrm{mi}$ downstream from Bone Creek, and $5.5 \mathrm{mi}$ southwest of Riverview.

Drainage area. $--390 \mathrm{mi}^{2}$, approximately.

Period of record.--Chemical analyses: October 1969 to July 1970, April 1973 to September 1973, October 1976 to current year.

Statistical data for selected chemical constituents

\begin{tabular}{|c|c|c|c|c|c|c|c|c|c|}
\hline Constituent & Units & $\begin{array}{l}\text { No. of } \\
\text { measure- } \\
\text { ments }\end{array}$ & $\begin{array}{l}\text { Max- } \\
\text { imum }\end{array}$ & $\begin{array}{l}\text { Min- } \\
\text { imum }\end{array}$ & Mean & Median & $\begin{array}{c}\text { Standard } \\
\text { devi- } \\
\text { ation }\end{array}$ & $\begin{array}{l}\text { Ninetieth } \\
\text { percent- } \\
\text { ile }\end{array}$ & $\begin{array}{l}\text { Tenth } \\
\text { percent- } \\
\text { ile }\end{array}$ \\
\hline Specific conductance & umho/cm & 49 & 297 & 151 & 179 & 172 & 28 & 210 & 153 \\
\hline Dissolved solids, residue & $\mathrm{mg} / \mathrm{L}$ & 16 & 267 & 143 & 165 & 152 & 32 & 195 & 146 \\
\hline Dissolved solids, sum & $\mathrm{mg} / \mathrm{L}$ & 24 & 229 & 120 & 152 & 150 & 19 & 159 & 137 \\
\hline Hardness as $\mathrm{CaCO}_{3}$ & $\mathrm{mg} / \mathrm{L}$ & 23 & 102 & 57 & 72 & 71 & 10 & 80 & 62 \\
\hline Calcium, dissolved & $\mathrm{mg} / \mathrm{L}$ & 23 & 34 & 20 & 24 & 23 & 3.4 & 28 & 21 \\
\hline Magnesium, dissolved & $\mathrm{mg} / \mathrm{L}$ & 23 & 5.5 & 0.5 & 3.0 & 3.1 & 1.0 & 3.8 & 1.9 \\
\hline Sodium, dissolved & $\mathrm{mg} / \mathrm{L}$ & 13 & 29 & 4.6 & 8.7 & 7.2 & 6.2 & 8.5 & 6.5 \\
\hline Potassium, dissolved & $\mathrm{mg} / \mathrm{L}$ & 13 & 17 & 4.6 & 6.5 & 5.6 & 3.2 & 7.5 & 0.0 \\
\hline Bicarbonate ion & $\mathrm{mg} / \mathrm{L}$ & 23 & 146 & 86 & 101 & 97 & 15 & 120 & 90 \\
\hline Sulfate, dissolved & $\mathrm{mg} / \mathrm{L}$ & 23 & 16 & 0.4 & 6.2 & 5.8 & 3.7 & 11 & 1.6 \\
\hline Chloride, dissolved & $\mathrm{mg} / \mathrm{L}$ & 27 & 21 & 0.0 & 2.5 & 1.9 & 3.8 & 3.2 & 1.0 \\
\hline Fluoride, dissolved & $\mathrm{mg} / \mathrm{L}$ & 24 & 0.6 & 0.2 & 0.3 & 0.3 & 0.1 & 0.5 & 0.2 \\
\hline Silica, dissolved & $\mathrm{mg} / \mathrm{L}$ & 22 & 58 & 27 & 50 & 53 & 9.5 & 58 & 42 \\
\hline Boron, dissolved & $\mathrm{mg} / \mathrm{L}$ & 20 & 60 & 0 & 27 & 21 & 17 & 53 & 10 \\
\hline Iron, dissolved & $\mathrm{ug} / \mathrm{L}$ & 24 & 130 & 0 & 38 & 32 & 25 & 60 & 20 \\
\hline Manganese, dissolved & $\mathrm{ug} / \mathrm{L}$ & 13 & 20 & 0 & 4.7 & 0 & 6 & 10 & 0 \\
\hline Nitrite + nitrate as $\mathrm{N}$, & & 15 & 1.4 & 0.47 & 1.0 & 1.1 & 0.28 & 1.3 & 0.50 \\
\hline $\begin{array}{l}\text { Nitrate as } \mathrm{N} \text {, dissolved } \\
\text { Nitrate as } \mathrm{NO}_{3} \text {, dissolved }\end{array}$ & $\begin{array}{l}\mathrm{mg} / \mathrm{L} \\
\mathrm{mg} / \mathrm{L}\end{array}$ & 2 & 0.62 & 0.31 & 0.47 & & & & \\
\hline Ammonia $\mathrm{N}$, total & $\mathrm{mg} / \mathrm{L}$ & 6 & 0.14 & 0.03 & 0.06 & $-\cdots-1$ & -0.04 & & \\
\hline Organic $N$, total & $\mathrm{mg} / \mathrm{L}$ & 8 & 3.5 & 0.16 & 0.83 & $-\cdots-1$ & 1.1 & & \\
\hline Phosphorus, total & $\mathrm{mg} / \mathrm{L}$ & 8 & 1.8 & 0.21 & 0.46 & $-1-1-1$ & -0.55 & & $\cdots$ \\
\hline Phosphorus, dissolved & $\mathrm{mg} / \mathrm{L}$ & 18 & 0.97 & 0.12 & 0.25 & 0.20 & 0.19 & 0.33 & 0.15 \\
\hline Fecal coliform & $\mathrm{col} / 100 \mathrm{ml}$ & 6 & 150 & 30 & $-\ldots$ & & & & \\
\hline Fecal streptococci & $\operatorname{col} / 100 \mathrm{ml}$ & 6 & 200 & 96 & & & & & -- \\
\hline Oxygen, dissolved & $\mathrm{mg} / \mathrm{L}$ & 27 . & 12.2 & 8.4 & 10.2 & 9.8 & 1.4 . & 12.0 & 8.5 \\
\hline \multirow{2}{*}{$\begin{array}{l}\text { Biochemical oxygen demand } \\
\text { (5-day) }\end{array}$} & $\mathrm{mg} / \mathrm{L}$ & 6 & 3.3 & 0.6 & 1.3 & - - - - & 1.0 & & \\
\hline & units & 53 & 8.4 & 7.0 & 7.8 & 8.0 & 0.3 & 8.2 & 7.4 \\
\hline $\mathrm{Co}-\mathrm{Pt}$ & $t$ units & 14 & 60 & 3 & 18 & 12 & 16 & 35 & 4 \\
\hline
\end{tabular}


NIOBRARA RIVER BASIN

06463500 Long Pine Creek near Riverview, Nebr.--Continued

Results of regression analyses relating concentrations of selected chemical constituents to specific conductance $(\mathrm{SpC})$

\begin{tabular}{|c|c|c|c|c|c|}
\hline $\begin{array}{c}\text { Dissolved } \\
\text { constituents } \\
(\mathrm{mg} / \mathrm{L})\end{array}$ & $\begin{array}{l}\text { No. of } \\
\text { measure- } \\
\text { ments }\end{array}$ & Regression equation & $\begin{array}{l}\text { Corre- } \\
\text { lation } \\
\text { coeffi- } \\
\text { cient }\end{array}$ & $\begin{array}{l}\text { Percent } \\
\text { explained } \\
\text { variance }\end{array}$ & $\begin{array}{c}\text { Standard } \\
\text { error of } \\
\text { estimate } \\
(\mathrm{mg} / \mathrm{L})\end{array}$ \\
\hline Dissolved solids, residue (RDS) & 16 & $\mathrm{RDS}=0.752(\mathrm{SpC})+25.424$ & 0.94 & 88 & 11 \\
\hline Dissolved solids, sum (SDS) & 23 & $S D S=0.470(S p C)+64.843$ & .81 & 65 & 12 \\
\hline Hardness as $\mathrm{CaCO}_{3}(\mathrm{TH})$ & 23 & $\mathrm{TH}=0.163(\mathrm{SpC})+42.492$ & .51 & 26 & 9.2 \\
\hline Calcium (Ca) & 23 & $\mathrm{Ca}=0.057(\mathrm{SpC})+13.520$ & .55 & 31 & 2.9 \\
\hline Magnesium (Mg) & 23 & $\mathrm{Mg}=0.005(\mathrm{SpC})+2.110$ & $* .15$ & 2.2 & 1.1 \\
\hline Sodium $(\mathrm{Na})$ & 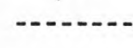 & 年- & & - & 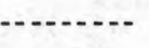 \\
\hline Potassium (K) & 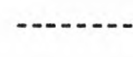 & - & & & \\
\hline Bicarbonate $\left(\mathrm{HCO}_{3}\right)$ & 23 & $\mathrm{HCO}_{3}=0.380(\mathrm{SpC})+30.863$ & .84 & 71 & 8.2 \\
\hline Sulfate $\left(\mathrm{SO}_{4}\right)$ & 23 & $\mathrm{SO}_{4}=0.031(\mathrm{SpC})+0.468$ & $* .28$ & 7.7 & 3.6 \\
\hline Chloride $(\mathrm{Cl})$ & 27 & $\mathrm{Cl}=0.092(\mathrm{SpC})-14.285$ & .74 & 55 & 2.6 \\
\hline Silica (Si) & 22 & $S i=-0.134(S p C)+74.598$ & -.47 & 22 & 8.6 \\
\hline Nitrite + Nitrate as $\mathrm{N}\left(\mathrm{NO}_{2}-\mathrm{NO}_{3}\right)$ & 15 & $\mathrm{NO}_{2}+\mathrm{NO}_{3}=-0.002(\mathrm{SpC})+1.283$ & $*-.04$ & 0.18 & 0.30 \\
\hline
\end{tabular}

* Not significant at the 95 percent confidence level; the regression equation should not be used to predict the concentration of the dependent variable.

Results of regression analyses relating specific conductance to water discharge (Q)

\begin{tabular}{ccccccc}
\hline $\begin{array}{c}\text { No. of } \\
\text { measure- } \\
\text { ments }\end{array}$ & $\begin{array}{c}\text { Water discharge } \\
\left(\mathrm{ft}^{3} / \mathrm{s}\right)\end{array}$ & Maximum Minimum & Regression equation & $\begin{array}{c}\text { Correlation } \\
\text { coefficient }\end{array}$ & $\begin{array}{c}\text { Percent } \\
\text { explained } \\
\text { variance }\end{array}$ & $\begin{array}{c}\text { Standard error } \\
\text { of estimate }\end{array}$ \\
\hline 49 & $762 \quad 89$ & $\mathrm{SpC}=54 \mathrm{Q}^{0.24302}$ & 0.58 & 34 & 0.049 \\
\hline
\end{tabular}


Location.--Lat $42^{\circ} 44^{\prime} 25^{\prime \prime}$, long $98^{\circ} 12^{\prime} 45^{\prime \prime}$, near center of $\mathrm{N}^{\frac{1}{2}} \mathrm{sec} .23$, T.32 N., R. 8 W., Knox County, Hydrologic Unit 10150007, on left bank 4 ft downstream from Pishelville Bridge, $6 \mathrm{mi}$ south of Verdel, and $7 \mathrm{mi}$ upstream from Verdigre Creek.

Drainage area.--12,600 $\mathrm{mi}^{2}$, approximately.

Period of record. --Water years 1958-65, 1967 to current year.

Statistical data for selected chemical constituents

\begin{tabular}{|c|c|c|c|c|c|c|c|c|c|}
\hline Constituent & Units & $\begin{array}{l}\text { No. of } \\
\text { measure- } \\
\text { ments }\end{array}$ & $\begin{array}{l}\text { Max- } \\
\text { imum }\end{array}$ & $\begin{array}{l}\text { Min- } \\
\text { imum }\end{array}$ & Mean & Median & $\begin{array}{l}\text { Standard } \\
\text { devi- } \\
\text { ation }\end{array}$ & $\begin{array}{l}\text { Ninetieth } \\
\text { percent- } \\
\text { ile }\end{array}$ & $\begin{array}{l}\text { Tenth } \\
\text { percent- } \\
\text { ile }\end{array}$ \\
\hline Specific conductance & umho/cin & 54 & 339 & 216 & 266 & 262 & 28 & 312 & 234 \\
\hline Dissolved solids, residue & $\mathrm{mg} / \mathrm{L}$ & 46 & 262 & 165 & 200 & 197 & 21 & 230 & 180 \\
\hline Dissolved solids, sum & $\mathrm{mg} / \mathrm{L}$ & 35 & 231 & 162 & 194 & 193 & 15 & 210 & 174 \\
\hline Hardness as $\mathrm{CaCO}_{3}$ & $\mathrm{mg} / \mathrm{L}$ & 35 & 140 & 93 & 110 & 113 & 12 & 130 & 96 \\
\hline Calcium, dissolved & $\mathrm{mg} / \mathrm{L}$ & 35 & 44 & 31 & 36 & 36 & 3.5 & 42 & 38 \\
\hline Magnesium, dissolved & $\mathrm{mg} / \mathrm{L}$ & 35 & 6.4 & 3.7 & 4.8 & 4.8 & 0.7 & 5.9 & 3.9 \\
\hline Sodium, dissolved & $\mathrm{mg} / \mathrm{L}$ & 35 & 13 & 7.1 & 9.9 & 9.8 & 1.3 & 12 & 8.6 \\
\hline Potassium, dissolved & $\mathrm{mg} / \mathrm{L}$ & 35 & 9.0 & 4.9 & 6.6 & 6.4 & 1.1 & 8.1 & 5.1 \\
\hline Bicarbonate ion & $\mathrm{mg} / \mathrm{L}$ & 35 & 164 & 109 & 142 & 144 & 12 & 162 & 122 \\
\hline Sulfate, dissolved & $\mathrm{mg} / \mathrm{L}$ & 35 & 34 & 9.2 & 16 & 15 & 5.8 & 26 & 11 \\
\hline Chloride, dissolved & $\mathrm{mg} / \mathrm{L}$ & 51 & 3.9 & 0.7 & 1.9 & 1.8 & 0.6 & 2.6 & 1.4 \\
\hline Fluoride, dissolved & $\mathrm{mg} / \mathrm{L}$ & 37 & 0.6 & 0.0 & 0.4 & 0.4 & 0.1 & 0.4 & 0.3 \\
\hline Silica, dissolved & $\mathrm{mg} / \mathrm{L}$ & 35 & 59 & 27 & 47 & 47 & 5.9 & 55 & 42 \\
\hline Boron, dissolved & $\mathrm{mg} / \mathrm{L}$ & 35 & 60 & 0 & 33 & 30 & 12 & 50 & 20 \\
\hline Iron, dissolved & $\mathrm{ug} / \mathrm{L}$ & 35 & 150 & 0 & 40 & 29 & 34 & 89 & 12 \\
\hline Manganese, dissolved & $\mathrm{ug} / \mathrm{L}$ & 35 & 210 & 0 & 22 & 10 & 44 & 40 & 0 \\
\hline $\begin{array}{l}\text { Nitrite + nitrate as } \mathrm{N} \text {, } \\
\text { dissolved } \\
\text { Nitrate as } \mathrm{N} \text {, dissolved }\end{array}$ & $\begin{array}{l}\mathrm{mg} / \mathrm{L} \\
\mathrm{mg} / \mathrm{L}\end{array}$ & 33 & 0.96 & 0.00 & 0.40 & 0.32 & 0.33 & 0.77 & 0.01 \\
\hline Nitrate as $\mathrm{NO}_{3}$, dissolved & $\mathrm{mg} / \mathrm{L}$ & & & & & & 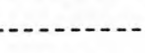 & 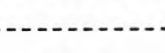 & -..... \\
\hline Ammonia $\mathrm{N}$, total & $\mathrm{mg} / \mathrm{L}$ & 53 & 0.64 & 0.00 & 0.07 & 0.04 & 0.11 & 0.15 & 0.00 \\
\hline Organic $\mathrm{N}$, total & $\mathrm{mg} / \mathrm{L}$ & 51 & 4.7 & 0.11 & 0.82 & 0.65 & 0.77 & 1.5 & 0.19 \\
\hline Phosphorus, tota1 & $\mathrm{mg} / \mathrm{L}$ & 53 & 1.4 & 0.05 & 0.27 & 0.23 & 0.22 & 0.44 & 0.13 \\
\hline Phosphorus, dissolved & $\mathrm{mg} / \mathrm{L}$ & 53 & 0.29 & 0.01 & 0.08 & 0.08 & 0.05 & 0.14 & 0.03 \\
\hline Fecal coliform & $\mathrm{col} / 100 \mathrm{ml}$ & 38 & 900 & 5 & 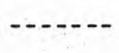 & 32 & -............ & 395 & 12 \\
\hline Fecal streptococci & $\mathrm{col} / 100 \mathrm{ml}$ & 46 & 2,600 & 12 & $-\cdots+-$ & 118 & $\cdots+\cdots$ & 510 & 40 \\
\hline Oxygen, dissolved & $\mathrm{mg} / \mathrm{L}$ & 51 & 13.4 & 7.0 & 9.8 & 9.4 & 1.6 & 12.2 & 7.9 \\
\hline \multirow{2}{*}{$\begin{array}{l}\text { Biochemical oxygen demand } \\
\mathrm{pH}^{(5-\text { day })}\end{array}$} & $\mathrm{mg} / \mathrm{L}$ & 45 & 9.4 & 0.4 & 3.2 & 2.7 & 2.0 & 5.8 & 0.8 \\
\hline & units & 55 & 8.6 & 6.5 & 7.7 & 7.7 & 0.4 & 8.2 & 7.4 \\
\hline Co $-\mathrm{Pt}$ & Co - Pt units & 35 & 130 & 2 & 21 & 10 & 24 & 45 & 5 \\
\hline
\end{tabular}


06465500. Niobrara River near Verdel, Nebr.--Continued

Results of regression analyses relating concentrations of selected chemical constituents to specific conductance $(\mathrm{SpC})$

\begin{tabular}{|c|c|c|c|c|c|}
\hline $\begin{array}{l}\text { Dissolved } \\
\text { constituents } \\
(\mathrm{mg} / \mathrm{L})\end{array}$ & $\begin{array}{l}\text { No. of } \\
\text { measure- } \\
\text { ments }\end{array}$ & Regression equation & $\begin{array}{l}\text { Corre- } \\
\text { lation } \\
\text { coeffi- } \\
\text { cient }\end{array}$ & $\begin{array}{l}\text { Percent } \\
\text { explained } \\
\text { variance }\end{array}$ & $\begin{array}{c}\text { Standard } \\
\text { error of } \\
\text { estimate } \\
(\mathrm{mg} / \mathrm{L})\end{array}$ \\
\hline Dissolved solids, residue (RDS) & 44 & $\mathrm{RDS}=0.670(\mathrm{SpC})+22.362$ & 0.91 & 84 & 8.7 \\
\hline Dissolved solids, sum (SDS) & 33 & $\mathrm{SDS}=0.510(\mathrm{SpC})+61.097$ & .89 & 79 & 7.2 \\
\hline Hardness as $\mathrm{CaCO}_{3}(\mathrm{TH})$ & 33 & $\mathrm{TH}=0.368(\mathrm{SpC})+13.852$ & .82 & 68 & 6.9 \\
\hline Calcium (Ca) & 33 & $\mathrm{Ca}=0.108(\mathrm{SpC})+7.662$ & .83 & 70 & 2.0 \\
\hline Magnesium (Mg) & 33 & $\mathrm{Mg}=0.020(\mathrm{SpC})-0.244$ & .72 & 52 & .5 \\
\hline Sodium (Na) & 33 & $\mathrm{Na}=0.042(\mathrm{SpC})-1.027$ & .85 & 73 & .7 \\
\hline Potassium $(\mathrm{K})$ & 33 & $K=0.023(\mathrm{SpC})+0.497$ & .56 & 31 & 1.0 \\
\hline Bicarbonate $\left(\mathrm{HCO}_{3}\right)$ & 33 & $\mathrm{HCO}_{3}=0.366(\mathrm{SpC})+46.842$ & .82 & 67 & 7.1 \\
\hline Sulfate $\left(\mathrm{SO}_{4}\right)$ & 33 & $\mathrm{SO}_{4}=0.114(\mathrm{SpC})-13.187$ & .53 & 28 & 5.0 \\
\hline Chloride (C1) & 49 & $\mathrm{Cl}=0.008(\mathrm{SpC})-0.332$ & .40 & 16 & .6 \\
\hline Silica (Si) & 33 & $\mathrm{Si}+0.004(\mathrm{SpC})+46.275$ & *.02 & 0.03 & 6.0 \\
\hline Nitrite + Nitrate as $\mathrm{N}\left(\mathrm{NO}_{2}-\mathrm{NO}_{3}\right)$ & 32 & $\mathrm{NO}_{2}-\mathrm{NO}_{3}=0.002(\mathrm{SpC})-0.267$ & $* .21$ & 4.4 & 0.33 \\
\hline
\end{tabular}

* Not significant at the 95 percent confidence level; the regression equation should not be used to predict the concentration of the dependent variable.

Results of regression analyses relating specific conductance to water discharge (Q)

\begin{tabular}{|c|c|c|c|c|c|c|c|}
\hline \multirow{2}{*}{$\begin{array}{l}\text { No. of } \\
\text { measure- } \\
\text { ments }\end{array}$} & \multicolumn{2}{|c|}{$\begin{array}{c}\text { Water discharge } \\
\left(\mathrm{ft}^{3} / \mathrm{s}\right) \\
\end{array}$} & \multirow{2}{*}{ Regression equation } & \multirow{2}{*}{$\begin{array}{l}\text { Correlation } \\
\text { coefficient }\end{array}$} & \multirow{2}{*}{$\begin{array}{c}\text { Percent } \\
\text { explained } \\
\text { variance }\end{array}$} & \multicolumn{2}{|c|}{$\begin{array}{c}\text { Standard error } \\
\text { of estimate }\end{array}$} \\
\hline & Maximum & Minimum & & & & Log units & Percent \\
\hline 52 & 4,240 & 508 & $\mathrm{SpC}=132 \mathrm{Q}^{0.09718}$ & 0.39 & 15 & 0.042 & 9.7 \\
\hline
\end{tabular}

* Not significant at the 95 percent confidence leve1; the regression equation should not be used to predict the concentration of the dependent variable. 
The series of basins collectively referred to as the Missouri River Tributaries basin consists of all direct drainage from Nebraska into the Missouri River between its confluence with the Niobrara River and its confluence with the Platte River. Data from sites in the Missouri River Tributaries basin were not sufficient for statistical analysis.

For convenience, stations on the Missouri River Main Stem are included in this part of the report. Data included for the Missouri River are limited to those obtained by the Nebraska District of the U.S. Geological Survey.

Data for five sites on the Missouri River Main Stem are sufficient for some statistical representation. Three of these sites are shown on figure 4 and the other two are shown on figure 11. Means for specific conductance at the five stations ranged from 676 to 723 umho/cm. Predominate cations at all stations are calcium and sodium; predominant anions are bicarbonate and sulfate.

Effluent from sewage plants of the major cities, Sioux City, Iowa, and Omaha, Nebr., is carried by the Missouri River. The median fecal coliform concentration at Decatur, which is about 35 river miles downstream from Sioux City, was 3,020 colonies per $100 \mathrm{ml}$. Near the Mormon Bridge at Omaha, which is at the northeast edge of the city and upstream from the Omaha sewage-treatment plant, it was 1,400 colonies per $100 \mathrm{ml}$. This more than two-fold improvement is related to several factors including bacterial die-off, tributary dilution, and settling of bacteria to the streambed. At Bellevue, just downstream from Omaha, the median fecal coliform concentration was 24,000 colonies per $100 \mathrm{~m} 1$. This improved to 17,000 colonies per $100 \mathrm{ml}$ at Plattsmouth. Some of this improvement may be the result of a dilution of 10 to 20 percent because of inflow of water from the Platte River.

Most nutrients increased in concentration as the water passed Omaha. Mean concentration of ammonia- $\mathrm{N}$ was $0.11 \mathrm{mg} / \mathrm{L}$ near the Mormon Bridge but increased to 0.22 at Plattsmouth. Mean total phosphorus was $0.23 \mathrm{mg} / \mathrm{L}$ at the Mormon Bridge and $0.39 \mathrm{mg} / \mathrm{L}$ at Plattsmouth.

Regression equations were developed for constituents at three stations. Data for the Missouri River at Nebraska City indicates reasonably good correlation with most constituents and specific conductance. Regulation of flows in the upper reaches of the Missouri River coupled with unregulated tributary inflow in the area of study may combine to decrease the percent explained variances for all constituents. 


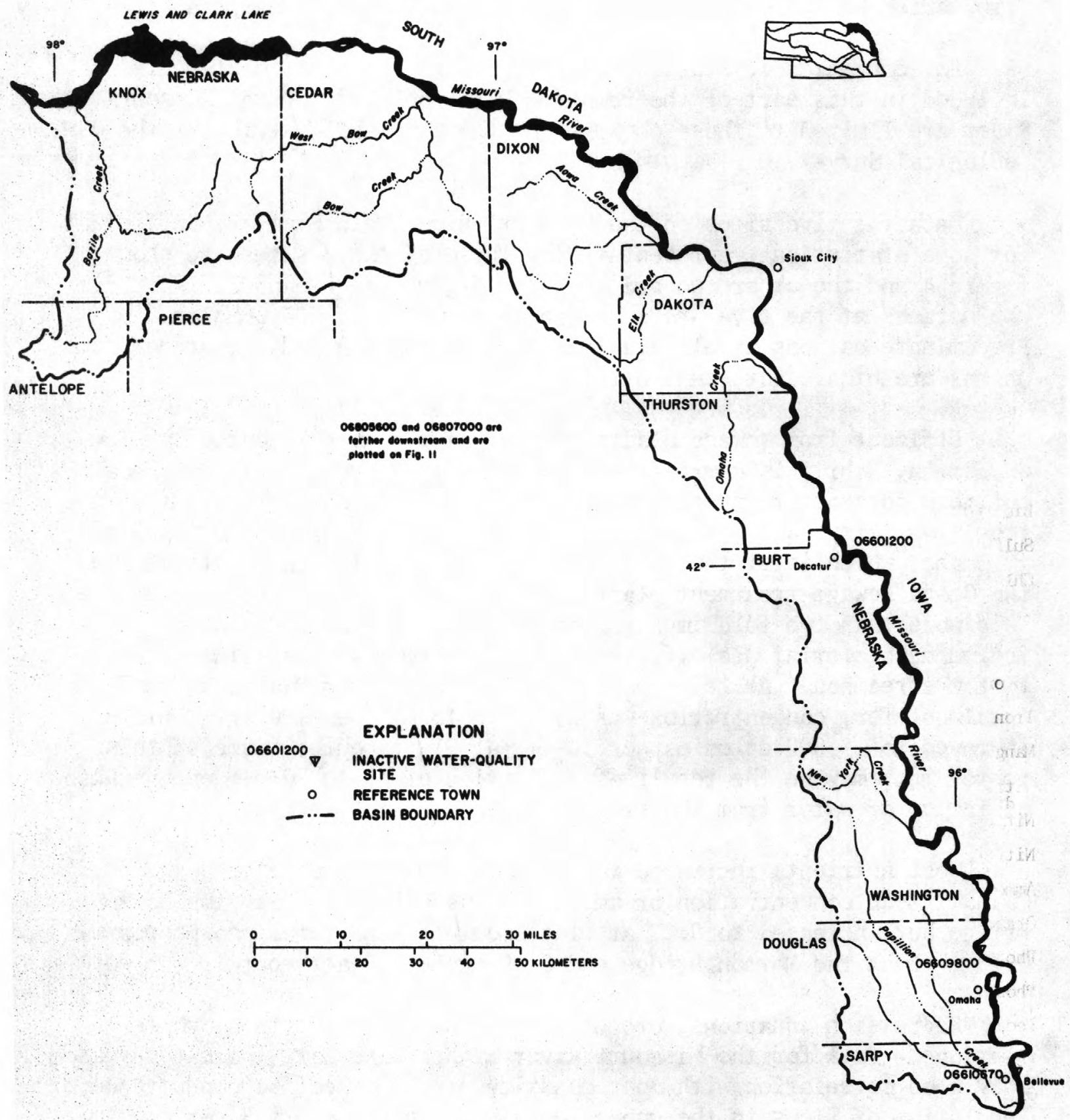

FICUnE 4.-Lecetion of woter-qualiny sompling sitos, Missouri River Tributaries basin and Missouri River mainstem 
MISSOURI RIVER MAIN STEM

06601200 Missouri River at Decatur, Nebr.

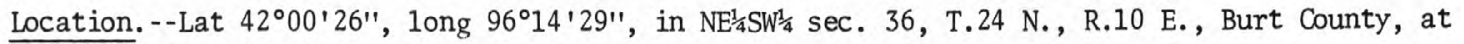
bridge at Decatur.

Drainage area. $--316,160 \mathrm{mi}^{2}$.

Period of record. - July 1959 to June 1970, August 1971 to September 1973.

Statistical data for selected chemical constituents

\begin{tabular}{|c|c|c|c|c|c|c|c|c|c|}
\hline Constituent & Units & $\begin{array}{l}\text { No. of } \\
\text { measure- } \\
\text { ments }\end{array}$ & $\begin{array}{l}\text { Max- } \\
\text { imum }\end{array}$ & $\begin{array}{l}\text { Min- } \\
\text { imum }\end{array}$ & Mean & Median & $\begin{array}{c}\text { Standard } \\
\text { devi- } \\
\text { ation }\end{array}$ & $\begin{array}{c}\text { Ninetieth } \\
\text { percent- } \\
\text { ile }\end{array}$ & $\begin{array}{c}\text { Tenth } \\
\text { percent- } \\
\text { ile } \\
\end{array}$ \\
\hline Specifiç conductance & umho/cm & 138 & 920 & 412 & 723 & 726 & 69 & 790 & 660 \\
\hline Dissolved solids, residue & $\mathrm{mg} / \mathrm{L}$ & 59 & 589 & 390 & 487 & 486 & 29 & 522 & 450 \\
\hline Dissolved solids, sum & $\mathrm{mg} / \mathrm{L}$ & 4 & 517 & 360 & 460 & 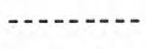 & --69 & $-1-n-1$ & $-1-2$ \\
\hline Hardness as $\mathrm{CaCO}_{3}$ & $\mathrm{mg} / \mathrm{L}$ & 36 & 285 & 210 & 242 & 246 & 55 & 262 & 220 \\
\hline Calcium, dissolved & $\mathrm{mg} / \mathrm{L}$ & 26 & 68 & 53 & 61 & 61 & 3.6 & 66 & 57 \\
\hline Magnesium, dissolved & $\mathrm{mg} / \mathrm{L}$ & 26 & 24 & 18 & 22 & 22 & 1.6 & 24 & 20 \\
\hline Sodium, dissolved & $\mathrm{mg} / \mathrm{L}$ & 7 & 72 & 33 & 62 & $\cdots-1-1$ & $14--$ & $--n-1$ & $-\cdots$ \\
\hline Potassium, dissolved & $\mathrm{mg} / \mathrm{L}$ & 4 & 8.1 & 5.3 & 6.1 & $\cdots+\cdots$ & $-1.4--$ & & 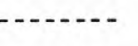 \\
\hline Bicarbonate ion & $\mathrm{mg} / \mathrm{L}$ & 79 & 238 & 150 & 190 & 193 & 21 & 208 & 170 \\
\hline Sulfate, dissọlved & $\mathrm{mg} / \mathrm{L}$ & 63 & 236 & 140 & 201 & 206 & 17 & 225 & 187 \\
\hline Chloride, dissolved & $\mathrm{mg} / \mathrm{L}$ & 52 & 22 & 8.5 & 11 & 11 & 1.9 & 13 & 9.6 \\
\hline Fluoride, dissolved & $\mathrm{mg} / \mathrm{L}$ & 7 & 0.6 & $0: 3$ & 0.5 & 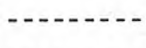 & $--0.1-$ & 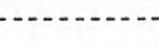 & \\
\hline Silica, dissolved & $\mathrm{mg} / \mathrm{L}$ & 4 & 12 & 7.4 & 8.8 & 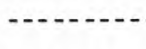 & --2.1 & & \\
\hline Boron, dissolved & $\mathrm{mg} / \mathrm{L}$ & 7 & 140 & 80 & 121 & $-\cdots-1$ & $21-$ & & \\
\hline Iron, dissolved & $\mathrm{ug} / \mathrm{L}$ & (5) & 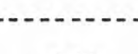 & - & 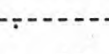 & & $-\cdots$ & & \\
\hline Manganese, dissolved & ug/L & 7 & 140 & 10 & 48 & 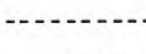 & $56-$ & & - \\
\hline $\begin{array}{l}\text { Nitrite + nitrate as } \mathrm{N} \text {, } \\
\text { dissolved }\end{array}$ & $\mathrm{mg} / \mathrm{L}$ & 26 & 1.0 & 0.04 & 0.25 & 0.23 & 0.19 & 0.39 & 0.07 \\
\hline Nitrate as $N$, dissolved & $\mathrm{mg} / \mathrm{L}$ & 12 & 1.0 & 0.10 & 0.40 & 0.36 & 0.26 & 0.62 & 0.13 \\
\hline Nitrate as $\mathrm{NO}_{3}$, dissolved & $\mathrm{mg} / \mathrm{L}$ & & - & & 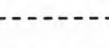 & & & & \\
\hline Ammonia $\mathrm{N}$, total & $\mathrm{mg} / \mathrm{L}$ & 37 & 1.1 & 0.00 & 0.10 & 0.06 & 0.19 & 0.23 & 0.00 \\
\hline Organic $\mathrm{N}$, tota1 & $\mathrm{mg} / \mathrm{L}$ & 31 & 1.9 & 0.13 & 0.39 & 0.34 & 0.30 & 0.44 & 0.23 \\
\hline Phosphorus, total & $\mathrm{mg} / \mathrm{L}$ & 65 & $1.3^{\circ}$ & 0.04 & 0.15 & 0.11 & 0.18 & 0.25 & 0.06 \\
\hline Phosphorus, dissolved & $\mathrm{mg} / \mathrm{L}$ & 28 & 0.14 & 0.00 & 0.05 & 0.05 & 0.04 & 0.10 & 0.01 \\
\hline Fecal coliform & $100 \mathrm{ml}$ & 76 & 16,000 & 360 & $-\cdots$ & 3,020 & 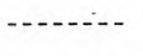 & 10,300 & 1,300 \\
\hline Fecal streptococci & $100 \mathrm{ml}$ & & 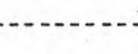 & $x^{2}$ & (2) & & & & \\
\hline Oxygen, dissolved & $\mathrm{mg} / \mathrm{L}$ & 100 & 17.4 & 6.6 & 10.4 & 10.0 & 2.2 & 13.5 & 7.8 \\
\hline \multirow{2}{*}{$\begin{array}{l}\text { Biochemical oxygen demand } \\
\mathrm{pH}^{(5-\text { day })}\end{array}$} & $\mathrm{mg} / \mathrm{L}$ & 38 & 9.2 & 0.5 & 2.2 & 1.8 & 1.7 & 4.1 & 1.0 \\
\hline & I units & 118 & 8.8 & 7.0 & 7.9 & 8.0 & 0.3 & 8.3 & 7.4 \\
\hline Co - Pt & units & 5 & 50 & 2 & 13 & - & $20-$ & & $-\cdots$ \\
\hline
\end{tabular}


Results of regression analyses relating concentrations of selected chemical constituents to specific conductance $(\mathrm{SpC})$

\begin{tabular}{|c|c|c|c|c|c|}
\hline $\begin{array}{l}\text { Dissolved } \\
\text { constituents } \\
(\mathrm{mg} / \mathrm{L})\end{array}$ & $\begin{array}{l}\text { No. of } \\
\text { measure- } \\
\text { ments }\end{array}$ & Regression equation & $\begin{array}{l}\text { Corre- } \\
\text { lation } \\
\text { coeffi- } \\
\text { cient }\end{array}$ & $\begin{array}{l}\text { Percent } \\
\text { explained } \\
\text { variance }\end{array}$ & $\begin{array}{l}\text { Standard } \\
\text { error of } \\
\text { estimate } \\
(\mathrm{mg} / \mathrm{L})\end{array}$ \\
\hline Dissolved solids, residue (RDS) & 58 & $\mathrm{RDS}=0.473(\mathrm{SpC})+143.051$ & 0.63 & 40 & 23 \\
\hline Dissolved solids, sum (SDS) & & & & & \\
\hline Hardness as $\mathrm{CaCO}_{3}(\mathrm{TH})$ & 36 & $\mathrm{TH}=0.240(\mathrm{SpC})+66.993$ & .77 & 59 & 9.8 \\
\hline Calcium (Ca) & 26 & $\mathrm{Ca}=0.056(\mathrm{SpC})+19.746$ & .72 & 52 & 2.5 \\
\hline Magnesium (Mg) & 26 & $\mathrm{Mg}=0.028(\mathrm{SpC})+0.685$ & .82 & 68 & .9 \\
\hline Sodium (Na) & $-\cdots--$ & - & $\ldots$ & -... & - \\
\hline Potassium (K) & $-\ldots$ & 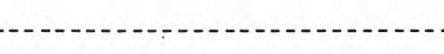 & & & \\
\hline Bicarbonate $\left(\mathrm{HCO}_{3}\right)$ & 79 & $\mathrm{HCO}_{3}=0.088(\mathrm{SpC})+125.754$ & .26 & 6.6 & 16 \\
\hline Sulfate $\left(\mathrm{SO}_{4}\right)$ & 62 & $\mathrm{SO}_{4}=0.302(\mathrm{SpC})-18.131$ & .76 & 57 & 11 \\
\hline Chloride (C1) & 52 & $\mathrm{C} 1=0.010(\mathrm{SpC})+3.749$ & $* .21$ & 4.6 & 1.9 \\
\hline Silica (Si) & $---\cdots$ & - & & & \\
\hline Nitrite + Nitrate as $\mathrm{N}\left(\mathrm{NO}_{2}-\mathrm{NO}_{3}\right)$ & 26 & $\mathrm{NO}_{2}+\mathrm{NO}_{3}=-0.003(\mathrm{SpC})+2.478$ & $3-.73$ & 54 & 0.13 \\
\hline
\end{tabular}

* Not significant at 95 percent confidence level; the regression equation should not be used to predict the concentration of the dependent variable.

Results of regression analyses relating specific conductance to water discharge (Q)

\begin{tabular}{cccccc}
\hline $\begin{array}{c}\text { No. of } \\
\text { measure } \\
\text { ments }\end{array}$ & $\begin{array}{c}\text { Water discharge } \\
\text { Maximum Minimum }\end{array}$ & Regression equation & $\begin{array}{c}\text { Correlation } \\
\text { coefficient }\end{array}$ & $\begin{array}{c}\text { Percent } \\
\text { explained } \\
\text { variance }\end{array}$ & $\begin{array}{c}\text { Standard error } \\
\text { of estimate }\end{array}$ \\
\hline 137 & $62,300 \quad 10,000$ & $\mathrm{SpC}=4030^{0.05578}$ & 0.18 & 3.2 & 0.048 \\
\hline
\end{tabular}




\section{MISSOURI RIVER MAIN STEM}

06609800 Missouri River near Mormon Bridge at Omaha, Nebr.

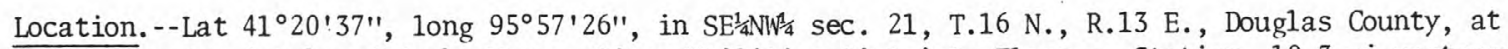
raw-water intake of Omaha Metropolitan Utilities District, Florence Station, 10.3 mi upstream from gaging station, $0.2 \mathrm{mi}$ downstream from Mormon Bridge, at river mile 626.2.

Period of record.-- February 1974 to June 1975.

Statistical data for selected chemical constituents

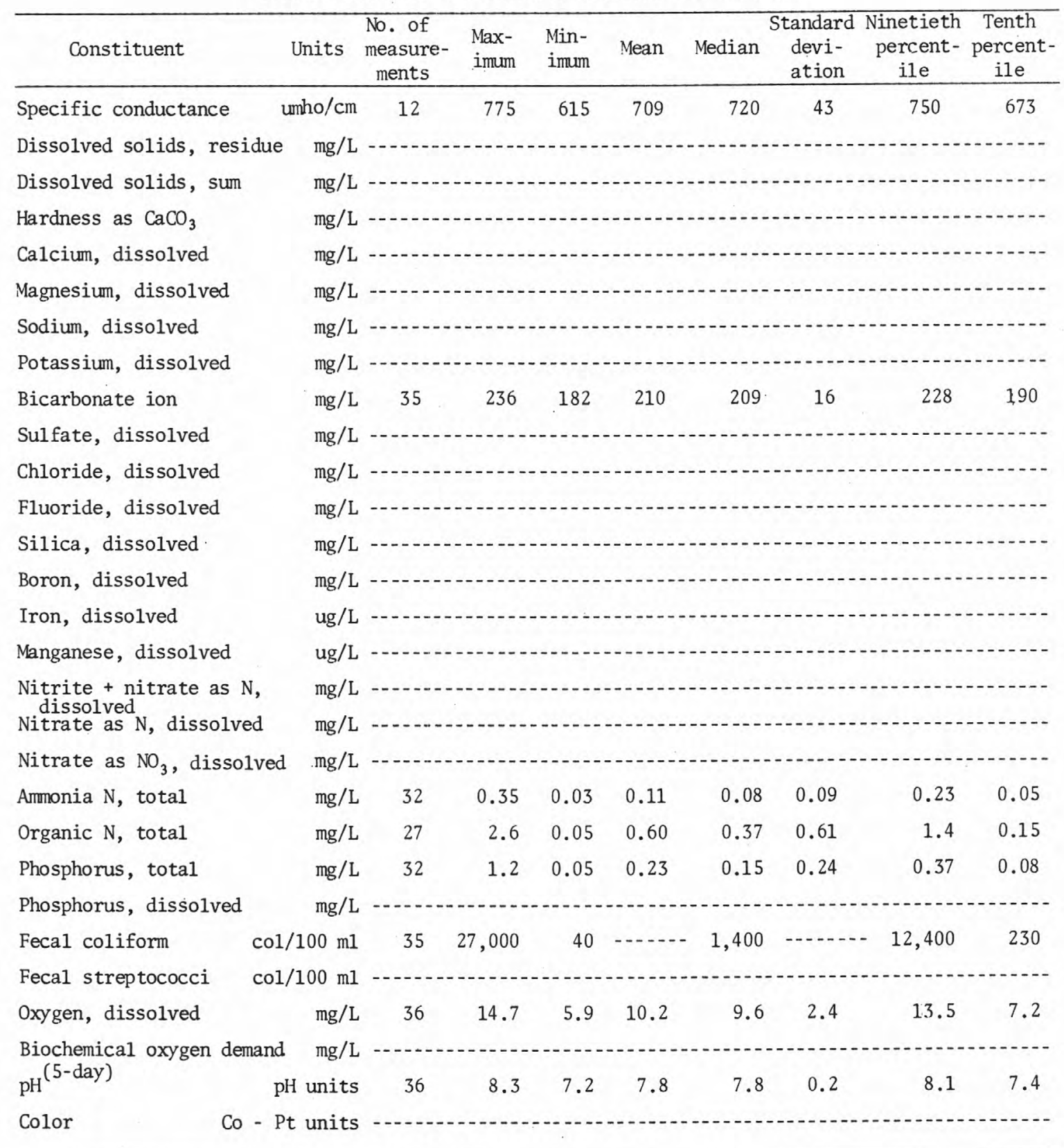




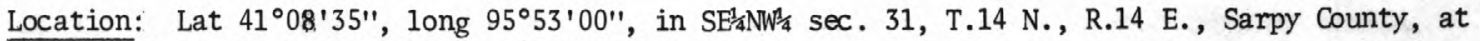
inlet to Kramer Power Plant in Bellevue.

Period of record.--July'1969 to June 1970, August 1971 to September 1973.

Statistical data for selected chemical constituents

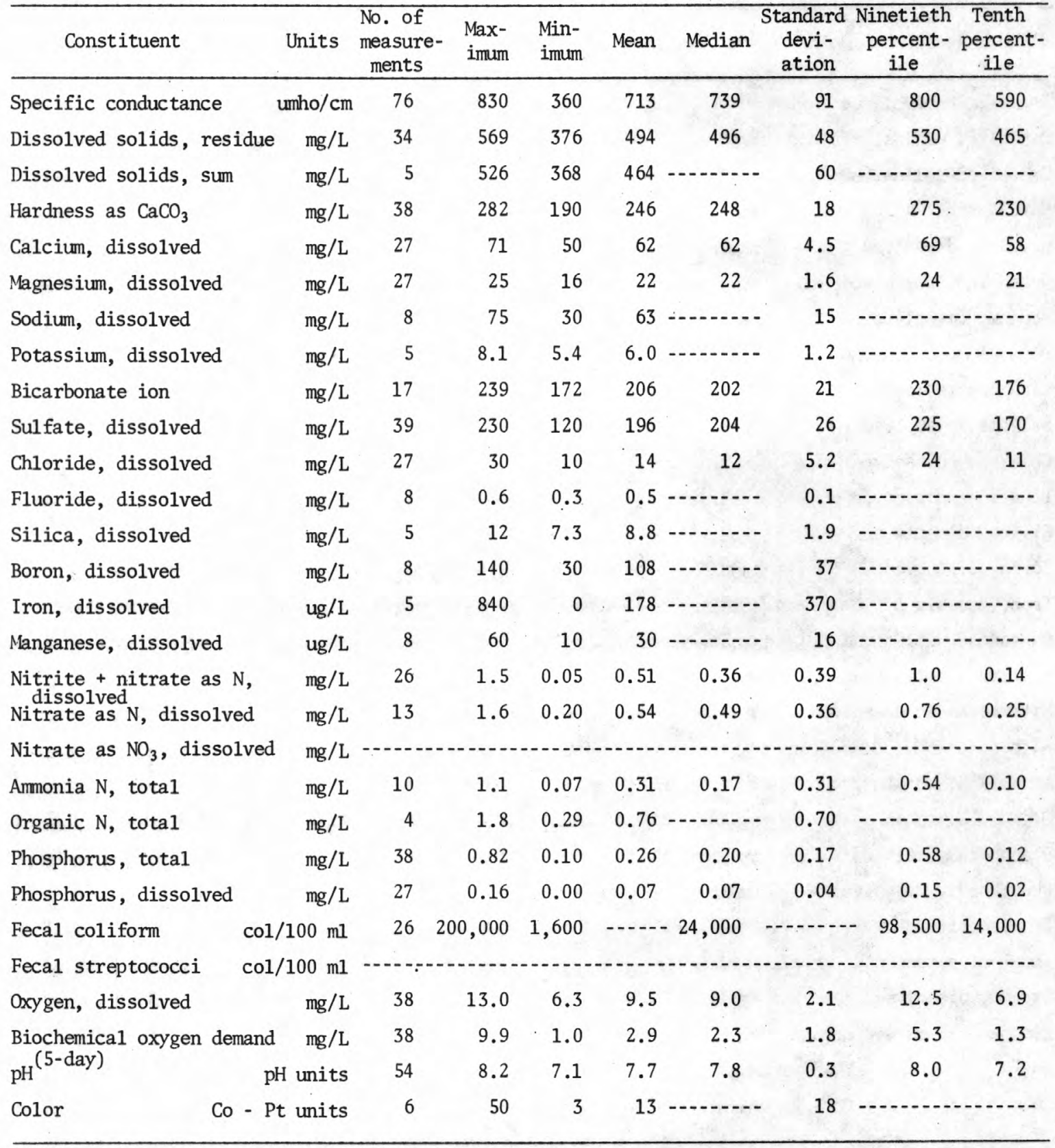


Results of regression analyses relating concentrations of selected chemical constituents to specific conductance $(\mathrm{SpC})$

\begin{tabular}{|c|c|c|c|c|c|}
\hline $\begin{array}{c}\text { Dissolved } \\
\text { constituents } \\
(\mathrm{mg} / \mathrm{L})\end{array}$ & $\begin{array}{l}\text { No. of } \\
\text { measure- } \\
\text { ments }\end{array}$ & Regression equation & $\begin{array}{l}\text { Corre- } \\
\text { lation } \\
\text { coeffi- } \\
\text { cient }\end{array}$ & $\begin{array}{l}\text { Percent } \\
\text { explained } \\
\text { variance }\end{array}$ & $\begin{array}{c}\text { Standard } \\
\text { error of } \\
\text { estimate } \\
(\mathrm{mg} / \mathrm{L})\end{array}$ \\
\hline Dissolved solids, residue (RDS) & 33 & $\mathrm{RDS}=0.688(\mathrm{SpC})-11.676$ & 0.94 & 89 & 13 \\
\hline Dissolved solids, sum (SDS) & & & & & \\
\hline Hardness as $\mathrm{CaCO}_{3}(\mathrm{TH})$ & 37 & $\mathrm{TH}=0.226(\mathrm{SpC})+80.187$ & .70 & 49 & 13 \\
\hline Calcium (Ca) & 27 & $\mathrm{Ca}=0.057(\mathrm{SpC})+19.715$ & .66 & 43 & 3.5 \\
\hline Magnesium (Mg) & 27 & $\mathrm{Mg}=0.019(\mathrm{SpC})+8.002$ & .60 & 35 & 1.3 \\
\hline Sodium (Na) & $\cdots \cdots$ & - n & - & $\ldots$ & . . . . . \\
\hline Potassium (K) & $-\ldots$ & 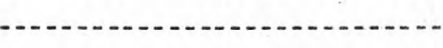 & & & \\
\hline Bicarbonate $\left(\mathrm{HCO}_{3}\right)$ & 16 & $\mathrm{HCO}_{3}=0.200(\mathrm{SpC})+61.838$ & .67 & 46 & 16 \\
\hline Sulfate $\left(\mathrm{SO}_{4}\right)$ & 38 & $\mathrm{SO}_{4}=0.411(\mathrm{SpC})-105.020$ & .90 & 80 & 12 \\
\hline Chloride (C1) & 27 & $\mathrm{Cl}=-0.004(\mathrm{SpC})+16.850$ & $*-.04$ & .13 & 5.3 \\
\hline Silica (Si) & 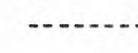 & - & - & $\cdots-\cdots$ & \\
\hline Nitrite + Nitrate as $\mathrm{N}\left(\mathrm{NO}_{2}-\mathrm{NO}_{3}\right)$ & 26 & $\mathrm{NO}_{2}-\mathrm{NO}_{3}=-0.004(\mathrm{SpC})+3.527$ & -.54 & 29 & 0.34 \\
\hline
\end{tabular}

* Not significant at the 95 percent confidence level; the regression equation should not be used to predict the concentration of the dependent variable.

Results of regression analyses relating specific conductance to water discharge (Q)

\begin{tabular}{ccccccc}
\hline $\begin{array}{c}\text { No. of } \\
\text { measure- } \\
\text { ments }\end{array}$ & $\begin{array}{c}\text { Mater discharge } \\
\left(\mathrm{ft}^{3} / \mathrm{s}\right)\end{array}$ & Maximum Minimum & Regression equation & $\begin{array}{c}\text { Correlation } \\
\text { coefficient }\end{array}$ & $\begin{array}{c}\text { Percent } \\
\text { explained } \\
\text { variance }\end{array}$ & $\begin{array}{c}\text { Standard error } \\
\text { of estimate }\end{array}$ \\
\hline 76 & $57,000 \quad 16,000$ & $\mathrm{SpC}=254 \mathrm{Q}^{0.09718}$ & & & & \\
\hline
\end{tabular}

* Not significant at the 95 percent confidence level; the regression equation should not be used to predict the concentration of the dependent variable. 


\section{MISSOURI RIVER MAIN STEM}

06805600 Missouri River at Plattsmouth, Nebr.

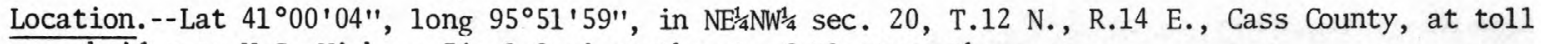
bridge on U.S. Highway $34,1.0 \mathrm{mi}$ southeast of Plattsmouth.

Period of record.-- February 1974 to June 1975.

Statistical data for selected chemical constituents

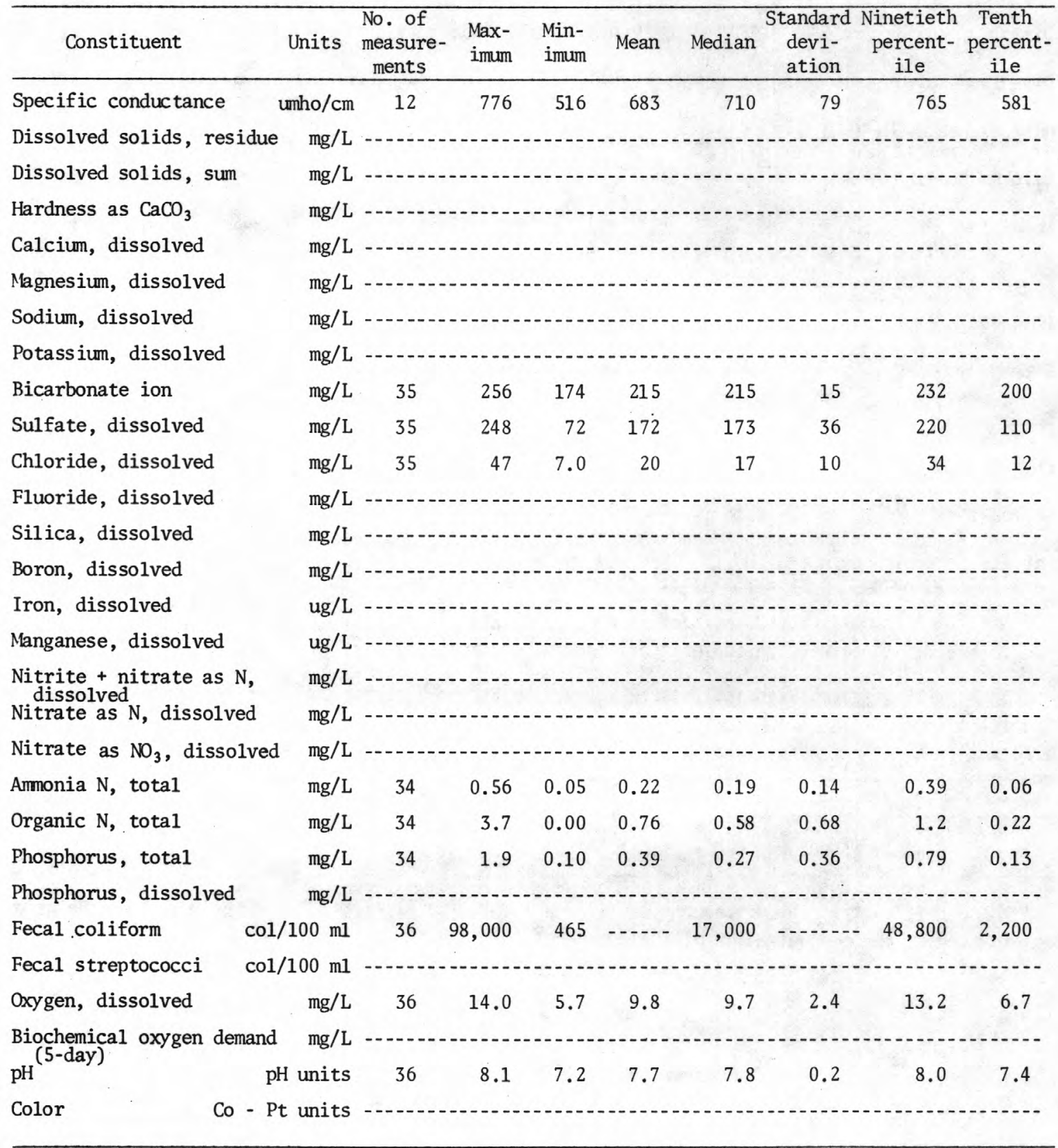




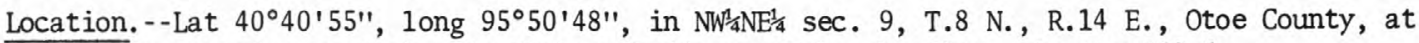
gaging station at Waubonsie Highway Bridge at Nebraska City, at mile 562.6.

Drainage area. $--414,400 \mathrm{mi}^{2}$, approximately.

Period of record. -- January 1951 to current year.

Statistical data for selected chemical constituents

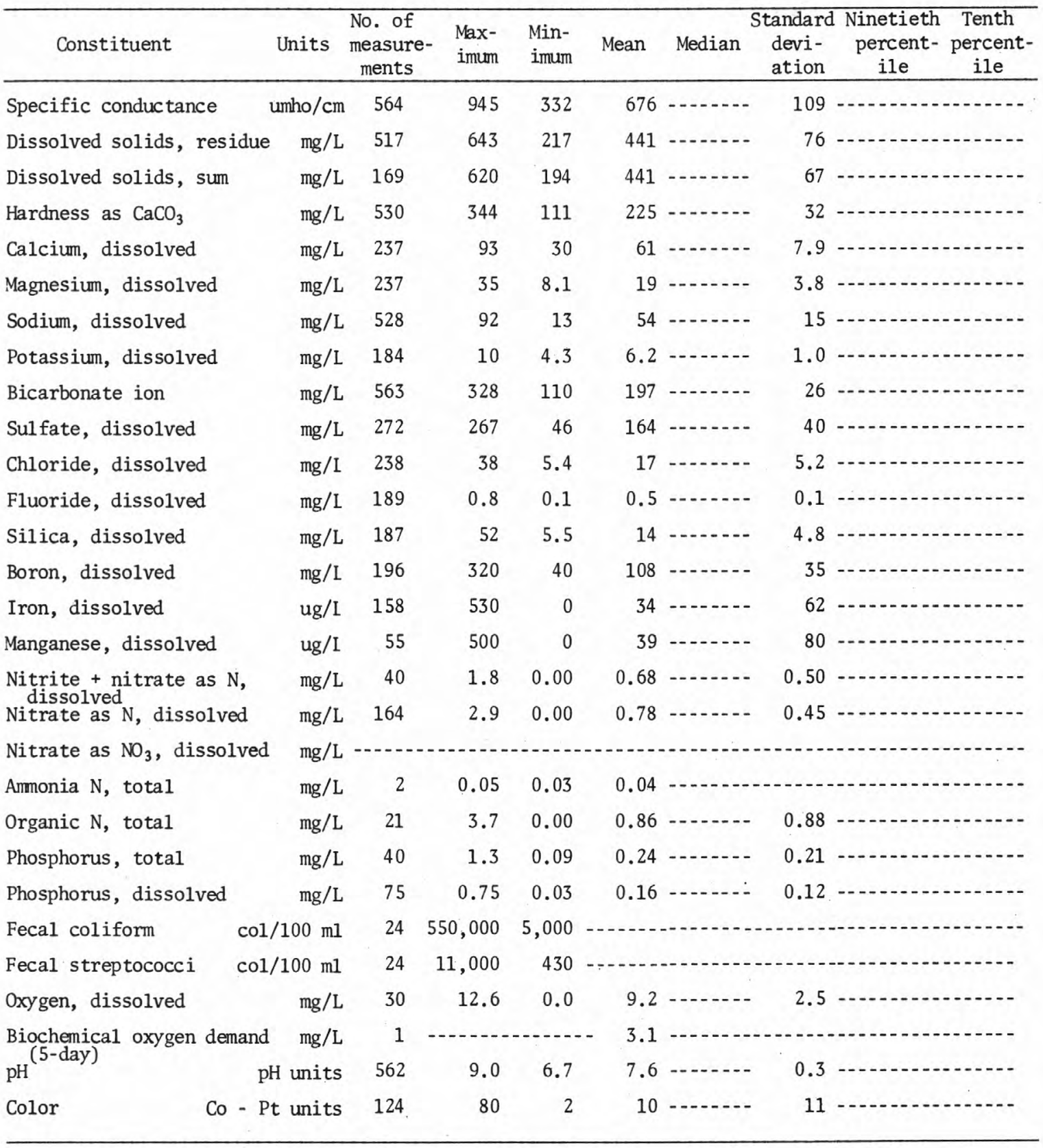


Results of regression analyses relating concentrations of selected chemical constituents to specific conductance $(\mathrm{SpC})$

\begin{tabular}{|c|c|c|c|c|c|}
\hline $\begin{array}{c}\text { Dissolved } \\
\text { constituents } \\
(\mathrm{mg} / \mathrm{L})\end{array}$ & $\begin{array}{l}\text { No. of } \\
\text { measure- } \\
\text { ments }\end{array}$ & Regression equation & $\begin{array}{l}\text { Corre- } \\
\text { lation } \\
\text { coeffi- } \\
\text { cient }\end{array}$ & $\begin{array}{l}\text { Percent } \\
\text { explained } \\
\text { variance }\end{array}$ & $\begin{array}{c}\text { Standard } \\
\text { error of } \\
\text { estimate } \\
(\mathrm{mg} / \mathrm{L})\end{array}$ \\
\hline Dissolved solids, residue (RDS) & 514 & $\mathrm{RDS}=0.675(\mathrm{SpC})-11.458$ & 0.98 & 96 & 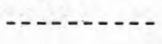 \\
\hline Dissolved solids, sum (SDS) & 167 & $\mathrm{SDS}=0.634(\mathrm{SpC})+4.833$ & .96 & 93 & $-\cdots-1-1$ \\
\hline Hardness as $\mathrm{CaCO}_{3}(\mathrm{TH})$ & 527 & $\mathrm{TH}=0.270(\mathrm{SpC})+43.302$ & .91 & 83 & -........ \\
\hline Calcium (Ca) & 236 & $\mathrm{Ca}=0.058(\mathrm{SpC})+21.592$ & .77 & 59 & 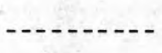 \\
\hline Magnesium (Mg) & 236 & $\mathrm{Mg}=0.031(\mathrm{SpC})-2.450$ & .86 & 73 & 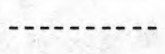 \\
\hline Sodium $(\mathrm{Na})$ & 527 & $\mathrm{Na}=0.125(\mathrm{SpC})-29.724$ & .93 & 87 & $-\ldots$ \\
\hline Potassium (K) & 183 & $K=-0.002(\mathrm{SpC})+7.835$ & -.26 & 6.5 & -....... \\
\hline Bicarbonate $\left(\mathrm{HCO}_{3}\right)$ & 562 & $\mathrm{HCO}_{3}=0.176(\mathrm{SpC})+78.683$ & .74 & 55 & \\
\hline Sulfate $\left(\mathrm{SO}_{4}\right)$ & 271 & $\mathrm{SO}_{4}=0.344(\mathrm{SpC})-71.379$ & .91 & 83 & - n \\
\hline Chloride (C1) & 236 & $\mathrm{Cl}=0.029(\mathrm{SpC})-3.525$ & .60 & 36 & - - - - \\
\hline Silica (Si) & 186 & $\mathrm{Si}=-0.008(\mathrm{SpC})+18.819$ & -.17 & 3.0 & $-\cdots+-.-1$ \\
\hline Nitrite + Nitrate as $\mathrm{N}\left(\mathrm{NO}_{2}-\mathrm{NO}_{3}\right)$ & 39 & $\mathrm{NO}_{2}-\mathrm{NO}_{3}=-0.002(\mathrm{SpC})+2.367$ & $7 *-.28$ & 7.6 & $-\cdots----$ \\
\hline
\end{tabular}

* Not significant at the 95 percent confidence interval; the regression equation should not be used to predict the concentration of the dependent variable.

Results of regression analyses relating specific conductance to water discharge (Q)

\begin{tabular}{|c|c|c|c|c|c|c|}
\hline \multirow{2}{*}{$\begin{array}{l}\text { No. of } \\
\text { measure- } \\
\text { ments }\end{array}$} & \multirow{2}{*}{$\begin{array}{l}\text { Water discharge } \\
\frac{\left(\mathrm{ft}^{3} / \mathrm{s}\right)}{\text { Maximum Minimum }}\end{array}$} & \multirow{2}{*}{ Regression equation } & \multirow{2}{*}{$\begin{array}{l}\text { Correlation } \\
\text { coefficient }\end{array}$} & \multirow{2}{*}{$\begin{array}{l}\text { Percent } \\
\text { explained } \\
\text { variance }\end{array}$} & \multicolumn{2}{|c|}{$\begin{array}{c}\text { Standard error } \\
\text { of estimate }\end{array}$} \\
\hline & & & & & Log̀ units & Percent \\
\hline 562 & 327,000 & $\mathrm{SpC}=2865 \mathrm{Q}^{-0.13994}$ & -0.48 & 23 & & $-\cdots$ \\
\hline
\end{tabular}


The North Platte River basin in Nebraska lies mostly in western Nebraska, extending from the Wyoming border to the confluence of the North and South P1atte Rivers in Lincoln County. Included in the basin is Lake McConaughy, the largest lake in Nebraska.

The North Platte River valley from the Wyoming border to Lake McConaughy is irrigated extensively with diverted surface water. Streamflow is maintained by controlled release of water from several reservoirs in Wyoming. Water is diverted both in Wyoming and in Nebraska into an extensive canal system which provides irrigation water for more than 170,000 acres in the North Platte Valley above Lake McConaughy (Nebraska Soil and Water Conservation Commission, 1971). Between Lake Mc Conaughy and the North Platte - South Platte River confluence, approximately 50,000 acres of the basin are irrigated by diversion of surface water. Irrigation water returns to the North Platte River in the form of groundwater seepage and direct runoff. Many drains, which prior to the development of the irrigation project were only intermittent streams, now flow the year around.

Data sufficient for statistical analysis are available for 15 sampling sites in the North Platte River basin (fig. 5). Of these sites, five are on the main stem and the rest on tributaries.

The mean specific conductance for the North Platte River at the Wyoming-Nebraska State line was 802 umho/cm and for 118 samples downstream at Bridgeport it was $931 \mathrm{umho} / \mathrm{cm}$. This 15 percent increase probably results mostly from irrigation return flow. Calcium and sodium are the principal cations and bicarbonate and sulfate are the principal anions in North Platte River water. An examination of data collected on a monthly basis indicates that sodium usually is higher in concentration near the end of the irrigation season than at other times.

Pumpkin Creek, a major tributary, joins the North Platte River below Bridgeport. Some Pumpkin Creek water and some ground water is used for irrigation in Pumpkin Creek basin. Calcium is the principal cation and bicarbonate is the principal anion in water from Pumpkin Creek near Bridgeport; the mean specific conductance is $592 \mathrm{umho} / \mathrm{cm}$.

The North Platte River near Keystone is just downstream from the outlet of Lake McConaughy. Specific conductance at this station averaged $741 \mathrm{umho} / \mathrm{cm}$. This is 26 percent less than the mean for the North Platte River at Bridgeport. Inflow of less highly mineralized water from tributaries draining sandhills areas and from seepage directly into the lake, and storage of relatively dilute storm rumoff are most likely 

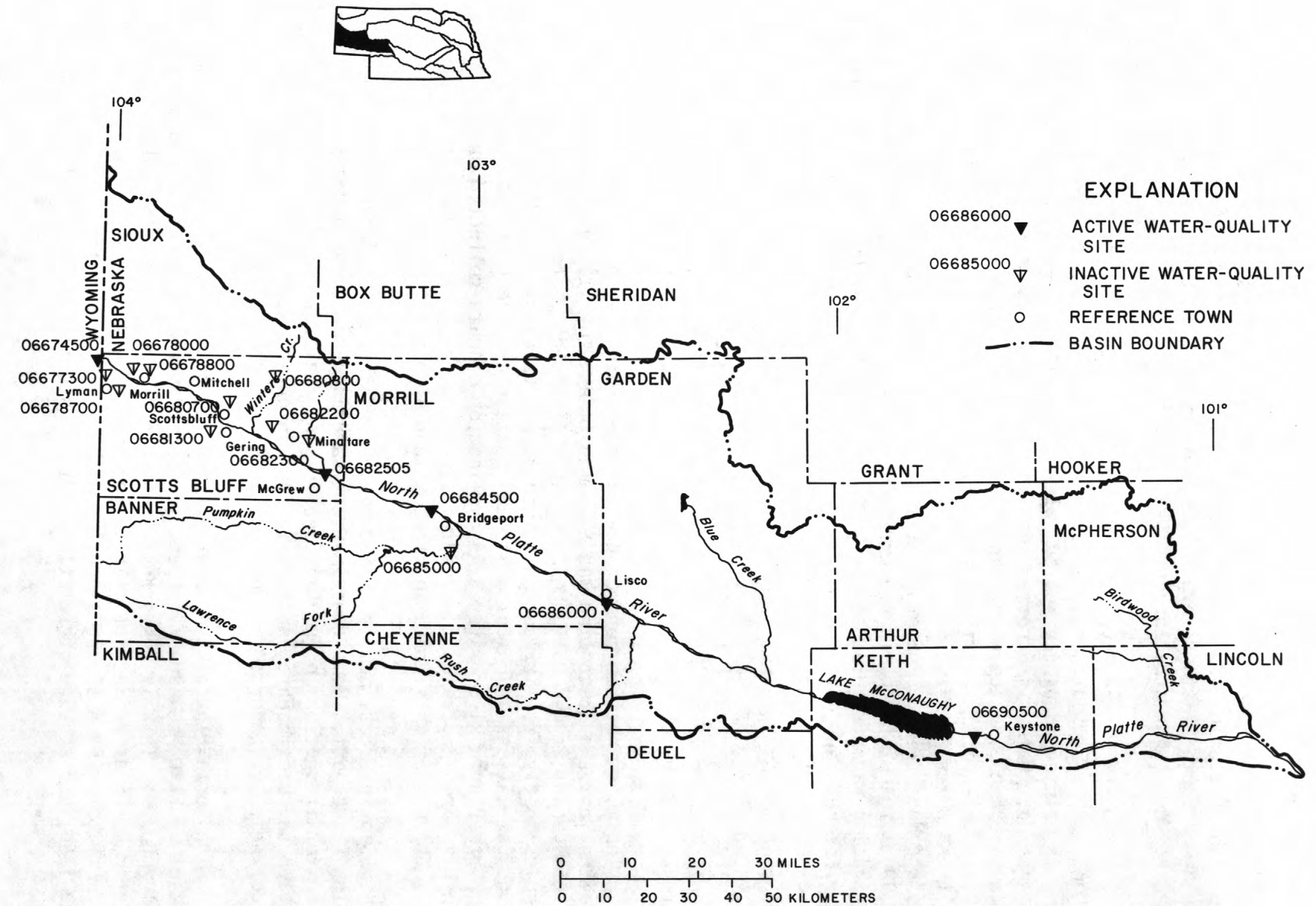

FIGURE 5.-Location of water-quality sampling sites, North Platte River basin 
the cause of this decrease. Large lakes often serve as "sinks" for some constituents, especially nutrients. Lake McConaughy seems to be a sink for nitrogen, because the mean value for nitrite + nitrate as $\mathrm{N}$ is 1.7 $\mathrm{mg} / \mathrm{L}$ at Lisco but only $0.41 \mathrm{mg} / \mathrm{L}$ at Keystone.

Results of regression analyses for the North Platte River at Wyoming-Nebraska State 1 ine indicate that percent explained variance for most constituents as related to specific conductance exceeds 50 and all relationships are significant at the 95 percent confidence.leve1. In contrast, none of the percent explained variances for the North Platte River near Keystone exceed 50 and most relationships are not significant at the 95 percent confidence level. This illustrates, dramatically, the effects that return flow from irrigation and impoundment have on regression relationships. Correlations for the Keystone station are poor; ion ratios of the constituents probably vary more considerably than does conductance. 


\section{NORTH PLATTE RIVER BASIN}

06674500 North Platte River at Wyoming-Nebraska State Line

Location.--Lat $41^{\circ} 59^{\prime} 25^{\prime \prime}$, long $104^{\circ} 02^{\prime} 57^{\prime \prime}$, in SW $\mathrm{NE}^{1} \mathrm{SE}^{\frac{1}{4}} \mathrm{sec} .4$, T.23 N., R. 58 W., Scotts Bluff County, Nebr., Hydrologic Unit 10180009, on right bank $650 \mathrm{ft}$ upstream from bridge on Nebraska State Highway 86, $700 \mathrm{ft}$ downstream from Wyoming-Nebraska State 1ine, and $0.5 \mathrm{mi}$ south of Henry, Nebr.

Drainage area (revised). $--22,218 \mathrm{mi}^{2}$, of which $1,929 \mathrm{mi}^{2}$ is probably noncontributing. The 3,959 $\mathrm{mi}^{2}$ in Great Divide basin is not included.

Period of record. -- Water year 1966 to current year.

Statistical data for selected chemical constituents

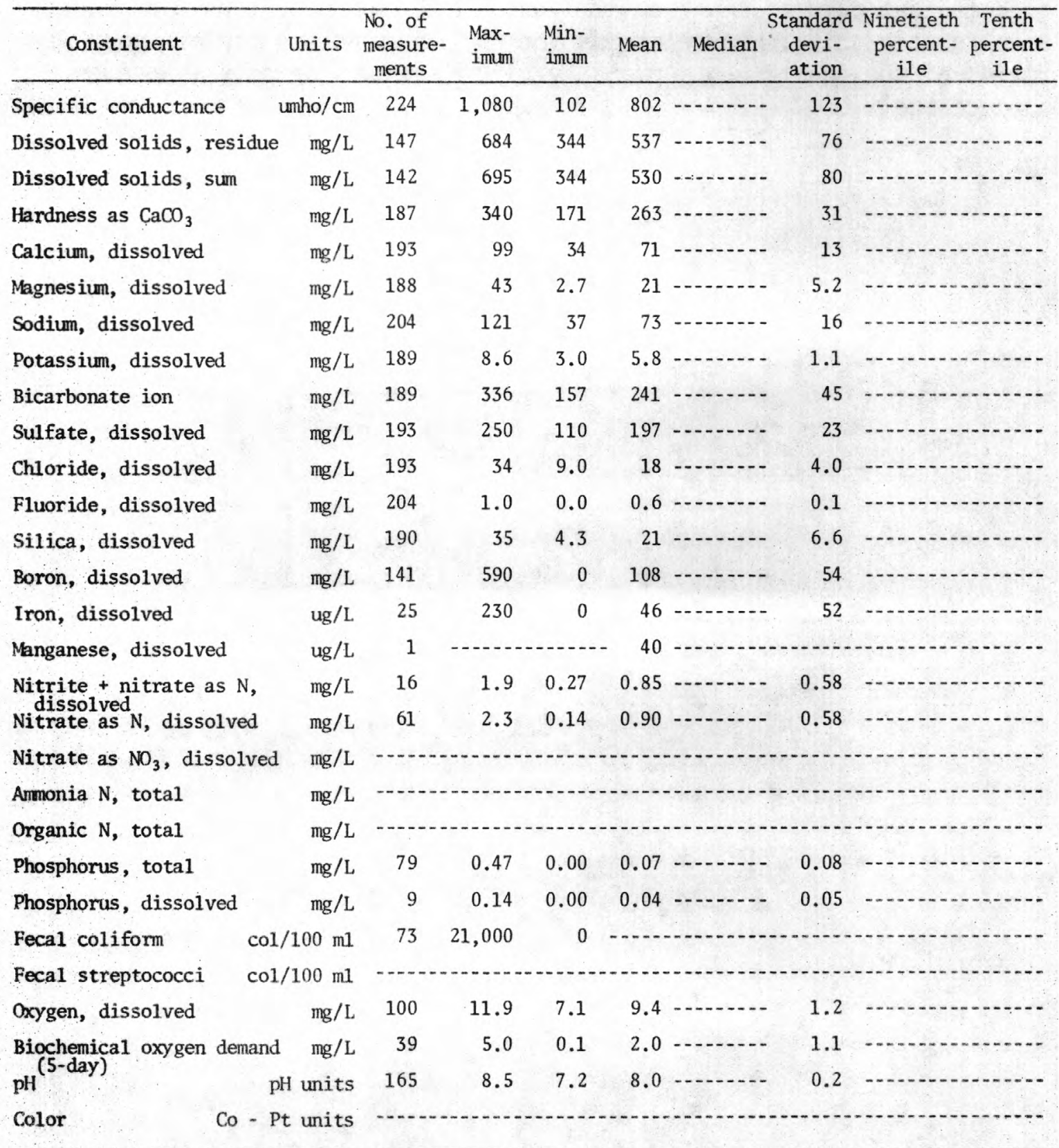


Results of regression analyses relating concentrations of selected chemical constituents to specific conductance $(\mathrm{SpC})$

\begin{tabular}{|c|c|c|c|c|c|}
\hline $\begin{array}{l}\text { Dissolved } \\
\text { constituents } \\
(\mathrm{mg} / \mathrm{L})\end{array}$ & $\begin{array}{l}\text { No. of } \\
\text { measure- } \\
\text { ments }\end{array}$ & Regression equation & $\begin{array}{l}\text { Corre- } \\
\text { lation } \\
\text { coeffi- } \\
\text { cient }\end{array}$ & $\begin{array}{l}\text { Percent } \\
\text { explained } \\
\text { variance }\end{array}$ & $\begin{array}{c}\text { Standard } \\
\text { error of } \\
\text { estimate } \\
(\mathrm{mg} / \mathrm{L})\end{array}$ \\
\hline Dissolved solids, residue (RDS) & 146 & $\operatorname{RDS}=0.723(\mathrm{SpC})-36.970$ & 0.98 & 96 & 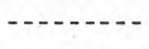 \\
\hline Dissolved solids, sum (SDS) & 131 & $\mathrm{SDS}=0.664(\mathrm{SpC})-7.347$ & .95 & 91 & 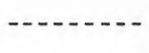 \\
\hline Hardness as $\mathrm{CaCO}_{3}(\mathrm{TH})$ & 176 & $\mathrm{TH}=0.232(\mathrm{SpC})+74.861$ & .79 & 63 & $-\cdots-1-1$ \\
\hline Calcium (Ca) & 182 & $\mathrm{Ca}=0.078(\mathrm{SpC})+8.244$ & .65 & 42 & $-1-n$ \\
\hline Magnesium (Mg) & 177 & $\mathrm{Mg}=0.010(\mathrm{SpC})+12.362$ & .21 & 4.4 & 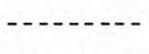 \\
\hline Sodium $(\mathrm{Na})$ & 185 & $\mathrm{Na}=0.129(\mathrm{SpC})-30.991$ & .86 & 75 & $\cdots+\cdots$ \\
\hline Potassium (K) & 178 & $\mathrm{~K}=0.007(\mathrm{SpC})-0.166$ & .73 & 53 & $-1-n$ \\
\hline Bicarbonate $\left(\mathrm{HCO}_{3}\right)$ & 178 & $\mathrm{HCO}_{3}=0.371(\mathrm{SpC})-58.898$ & .88 & 78 & (n) \\
\hline Sulfate $\left(\mathrm{SO}_{4}\right)$ & 182 & $\mathrm{SO}_{4}=0.182(\mathrm{SpC})+50.333$ & .83 & 70 & 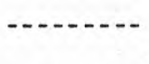 \\
\hline Chloride (Cl) & 182 & $\mathrm{Cl}=0.024(\mathrm{SpC})-1.221$ & .63 & 40 & - n \\
\hline Silica (Si) & 179 & $\mathrm{Si}=0.041(\mathrm{SpC})-12.362$ & .66 & 44 & $-\cdots-1$ \\
\hline Nitrite + Nitrate as $\mathrm{N}\left(\mathrm{NO}_{2}-\mathrm{NO}_{3}\right)$ & 15 & $\mathrm{NO}_{2}-\mathrm{NO}_{3}=0.003(\mathrm{SpC})-1.648$ & .79 & 62 & 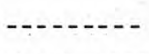 \\
\hline
\end{tabular}

Results of regression analyses relating specific conductance to water discharge (Q)

\begin{tabular}{|c|c|c|c|c|c|c|c|}
\hline \multirow{2}{*}{$\begin{array}{l}\text { No. of } \\
\text { measure- } \\
\text { ments }\end{array}$} & \multicolumn{2}{|c|}{$\begin{array}{l}\text { Water discharge } \\
\left(\mathrm{ft}^{3} / \mathrm{s}\right) \\
\end{array}$} & \multirow{2}{*}{ Regression equation } & \multirow{2}{*}{$\begin{array}{l}\text { Correlation } \\
\text { coefficient }\end{array}$} & \multirow{2}{*}{$\begin{array}{l}\text { Percent } \\
\text { explained } \\
\text { variance }\end{array}$} & \multicolumn{2}{|c|}{$\begin{array}{c}\text { Standard error } \\
\text { of estimate }\end{array}$} \\
\hline & Maximum & Minimum & & & & Log units & Percent \\
\hline 223 & 3,760 & 33 & $\mathrm{SpC}=1,740 \mathrm{Q}^{-0.12226}$ & -0.53 & 28 & & \\
\hline
\end{tabular}


Location.--Lat $41^{\circ} 55^{\prime}$, long $104^{\circ} 00^{\prime}$ in $\mathrm{NW}^{\frac{1}{4}} \mathrm{sec}$. 36, T.23 N., R. $58 \mathrm{~W}$., on right bank $65 \mathrm{ft}$ upstream from Union Pacific Railroad bridge, $0.8 \mathrm{mi}$ upstream from mouth, and 2.2 mi northeast of Lyman, Scotts Bluff County.

Period of record.-- September 1961 to September 1965.

Statistical data for selected chemical constituents

\begin{tabular}{|c|c|c|c|c|c|c|c|c|c|}
\hline Constituent & Units & $\begin{array}{l}\text { No. of } \\
\text { measure- } \\
\text { ments }\end{array}$ & $\begin{array}{l}\text { Max- } \\
\text { imum }\end{array}$ & $\begin{array}{l}\text { Min- } \\
\text { imum }\end{array}$ & Mean & Median & $\begin{array}{c}\text { Standard } \\
\text { devi- } \\
\text { ation }\end{array}$ & $\begin{array}{l}\text { Ninetieth } \\
\text { percent- } \\
\text { ile }\end{array}$ & $\begin{array}{l}\text { Tenth } \\
\text { percent- } \\
\text { ile }\end{array}$ \\
\hline Specific conductance & umho/cm & 43 & 1,560 & 751 & 1,280 & 1,380 & 229 & 1,500 & 860 \\
\hline Dissolved solids, residue & $\mathrm{mg} / \mathrm{L}$ & 43 & 1,060 & 503 & 874 & 940 & 153 & 998 & 568 \\
\hline Dissolved solids, sum & $\mathrm{mg} / \mathrm{L}$ & & & & & & & & \\
\hline Hardness as $\mathrm{CaCO}_{3}$ & $\mathrm{mg} / \mathrm{L}$ & 43 & 240 & 99 & 142 & 127 & 36 & 190 & 178 \\
\hline Calcium, dissolved & $\mathrm{mg} / \mathrm{L}$ & 43 & 63 & 20 & 38 & 35 & 10 & 52 & 28 \\
\hline Magnesium, dissolved & $\mathrm{mg} / \mathrm{L}$ & 43 & 20 & 7.3 & 11 & 9.8 & 3.3 & 17 & 8.6 \\
\hline Sodium, dissolved & $\mathrm{mg} / \mathrm{L}$ & 43 & 318 & 82 & 242 & 280 & 72 & 316 & 110 \\
\hline Potassium, dissolved & $\mathrm{mg} / \mathrm{L}$ & 43 & 15 & 5.8 & 11 & 12 & 2.0 & 14 & 9.3 \\
\hline Bicarbonate ion & $\mathrm{mg} / \mathrm{L}$ & 43 & 582 & 204 & 462 & 490 & 111 & 564 & 280 \\
\hline Sulfate, dissolved & $\mathrm{mg} / \mathrm{L}$ & 43 & 283 & 168 & 241 & 244 & 26 & 270 & 205 \\
\hline Chloride, dissolved & $\mathrm{mg} / \mathrm{L}$ & 43 & 39 & 15 & 28 & 30 & 5.5 & 34 & 18 \\
\hline Fluoride, dissolved & $\mathrm{mg} / \mathrm{L}$ & 43 & 1.0 & 0.4 & 0.8 & 0.8 & 0.1 & 0.9 & 0.6 \\
\hline Silica, dissolved & $\mathrm{mg} / \mathrm{L}$ & 43 & 63 & 14 & 48 & 53 & 13 & 61 & 29 \\
\hline Boron, dissolved & $\mathrm{mg} / \mathrm{L}$ & 43 & 370 & 10 & 269 & 305 & 84 & 357 & 140 \\
\hline Iron, dissolved & ug/L & 25 & 120 & 10 & 47 & 40 & 34 & 100 & 0 \\
\hline Manganese, dissolved & $u g / L$ & 1 & $-x_{1}$ & 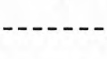 & 0 & & . & & \\
\hline $\begin{array}{l}\text { Nitrite + nitrate as } \mathrm{N}, \\
\text { dissolved } \\
\text { Nitrate as } N \text {, dissolved }\end{array}$ & $\begin{array}{l}\mathrm{mg} / \mathrm{L} \\
\mathrm{mg} / \mathrm{L}\end{array}$ & - & . & ( & . & & 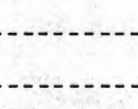 & & \\
\hline Nitrate as $\mathrm{NO}_{3}$, dissolved & $\mathrm{mg} / \mathrm{L}$ & 43 & 14 & 0.70 & 6.8 & & 3.4 & & \\
\hline Ammonia $\mathrm{N}$, total & $\mathrm{mg} / \mathrm{L}$ & & & & & & & & \\
\hline Organic N, total & $\mathrm{mg} / \mathrm{L}$ & & & & & & & & \\
\hline $\begin{array}{l}\text { Phosphorus, total } \\
\text { Phosphorus, dissol }\end{array}$ & $\begin{array}{l}\mathrm{mg} / \mathrm{L} \\
\mathrm{mg} / \mathrm{L}\end{array}$ & & & & & & & & \\
\hline Fecal coliform & $\mathrm{col} / 100 \mathrm{ml}$ & & & & & & & & \\
\hline Fecal streptococci & $\operatorname{col} / 100 \mathrm{ml}$ & & & & & & & & \\
\hline Oxygen, dissolved & $\mathrm{mg} / \mathrm{L}$ & & & & & & & & \\
\hline $\begin{array}{l}\text { Biochemical oxygen demand } \\
\text { (5-day) }\end{array}$ & $\begin{array}{l}\mathrm{mg} / \mathrm{L} \\
\text { units }\end{array}$ & 43 & 8.7 & 7.2 & 7.9 & 8.0 & 0.3 & 8.4 & 7.6 \\
\hline Co $-\mathrm{Pt}$ & units & 7 & 20 & 7 & 11 & 列- & 4.6 & & \\
\hline
\end{tabular}


Results of regression analyses relating concentrations of selected chemical constituents to specific conductance $(\mathrm{SpC})$

\begin{tabular}{|c|c|c|c|c|c|}
\hline $\begin{array}{l}\text { Dissolved } \\
\text { constituents } \\
\text { (mg/L) }\end{array}$ & $\begin{array}{l}\text { No. of } \\
\text { measure- } \\
\text { ments }\end{array}$ & Regression equation & $\begin{array}{l}\text { Corre- } \\
\text { lation } \\
\text { coeffi- } \\
\text { cient }\end{array}$ & $\begin{array}{l}\text { Percent } \\
\text { explained } \\
\text { variance }\end{array}$ & $\begin{array}{c}\text { Standard } \\
\text { error of } \\
\text { estimate } \\
(\mathrm{mg} / \mathrm{L})\end{array}$ \\
\hline Dissolved solids, residue (RDS) & 43 & $\operatorname{RDS}=0.662(\mathrm{SpC})+26.927$ & 0.99 & 98 & 23 \\
\hline Dissolved solids, sum (SDS) & $\cdots$ & & & & $\cdots$ \\
\hline Hardness as $\mathrm{CaCO}_{3}(\mathrm{TH})$ & 43 & $\mathrm{TD}=-0.137(\mathrm{SpC})+317.344$ & -.87 & 76 & 18 \\
\hline Calcium (Ca) & 43 & $\mathrm{Ca}=-0.036(\mathrm{SpC})+83.813$ & -.80 & 64 & 6.2 \\
\hline Magnesium (Mg) & 43 & $\mathrm{Mg}=-0.011(\mathrm{SpC})+26.024$ & -.79 & 63 & 2.0 \\
\hline Sodium $(\mathrm{Na})$ & 43 & $\mathrm{Na}=0.309(\mathrm{SpC})-153.763$ & .99 & 98 & 11 \\
\hline Potassium (K) & 43 & $K=0.005(\mathrm{SpC})+5.097$ & .55 & 30 & 1.6 \\
\hline Bicarbonate $\left(\mathrm{HCO}_{3}\right)$ & 43 & $\mathrm{HCO}_{3}=0.470(\mathrm{SpC})-140.012$ & .97 & 94 & 28 \\
\hline Sulfate $\left(\mathrm{SO}_{4}\right)$ & 43 & $\mathrm{SO}_{4}=0.093(\mathrm{SpC})+121.911$ & .83 & 69 & 14 \\
\hline Chloride (C1) & 43 & $\mathrm{Cl}=0.023(\mathrm{SpC})-1.067$ & .94 & 88 & 2.0 \\
\hline Silica (Si) & 43 & $\mathrm{Si}=0.052(\mathrm{SpC})-18.958$ & .90 & 81 & 5.8 \\
\hline
\end{tabular}

Results of regression analyses relating specific conductance to water discharge (Q)

\begin{tabular}{|c|c|c|c|c|c|c|c|}
\hline \multirow{2}{*}{$\begin{array}{l}\text { No. of } \\
\text { measure- } \\
\text { ments }\end{array}$} & \multicolumn{2}{|c|}{$\begin{array}{l}\text { Water discharge } \\
\left(\mathrm{ft}^{3} / \mathrm{s}\right)\end{array}$} & \multirow{2}{*}{ Regression equation } & \multirow{2}{*}{$\begin{array}{l}\text { Correlation } \\
\text { coefficient }\end{array}$} & \multirow{2}{*}{$\begin{array}{l}\text { Percent } \\
\text { explained } \\
\text { variance }\end{array}$} & \multicolumn{2}{|c|}{$\begin{array}{c}\text { Standard error } \\
\text { of estimate }\end{array}$} \\
\hline & Maximum & Minimum & & & & Log units & Percent \\
\hline 43 & 188 & 2.7 & $\mathrm{SpC}=2,178 \mathrm{Q}^{-0.17352}$ & 0.80 & 64 & 0.053 & 12 \\
\hline
\end{tabular}


NORTH PLATTE RIVER BASIN

06678000 Sheep Creek near Morrill, Nebr.

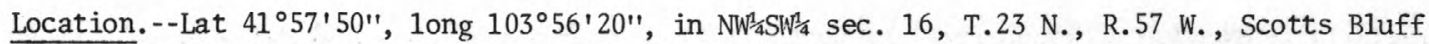
County, at gaging station at Burlington Northern Railroad bridge, $50 \mathrm{ft}$ downstream from bridge on U.S. Highway 26,1 mi west of Morrill, and $1.5 \mathrm{mi}$ upstream from mouth.

Drainage area. $--362 \mathrm{mi}^{2}$, of which about $25 \mathrm{mi}^{2}$ is noncontributing.

Period of record.--July 1970 to September 1973.

Statistical data for selected chemical constituents

\begin{tabular}{|c|c|c|c|c|c|c|c|c|}
\hline Constituent & Units & $\begin{array}{l}\text { No. of } \\
\text { measure- } \\
\text { ments }\end{array}$ & $\begin{array}{l}\text { Max- } \\
\text { imum }\end{array}$ & $\begin{array}{l}\text { Min- } \\
\text { imum }\end{array}$ & Mean & Median & $\begin{array}{l}\text { Standard } \\
\text { devi- } \\
\text { ation }\end{array}$ & $\begin{array}{l}\text { Ninetieth } \\
\text { percent- percent- } \\
\text { ile } \quad \text { ile } \\
\end{array}$ \\
\hline Specific conductance & umho/cm & 114 & 1,150 & 575 & 830 & 817 & 115 & 990 \\
\hline Dissolved solids, residue & $\mathrm{mg} / \mathrm{L}$ & 2 & 591 & 557 & 574 & $-\cdots$ & & \\
\hline Dissolved solids, sum & $\mathrm{mg} / \mathrm{L}$ & 6 & 594 & 534 & 560 & --- & 20 & \\
\hline Hardness as $\mathrm{CaCO}_{3}$ & $\mathrm{mg} / \mathrm{L}$ & 7 & 280 & 268 & 277 & $-\cdots-1$ & 5.4 & \\
\hline Calcium, dissolved & $\mathrm{mg} / \mathrm{I}$ & 7 & 80 & 76 & 78 & 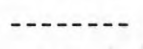 & 1.3 & \\
\hline Magnesium, dissolved & $\mathrm{mg} / \mathrm{L}$ & 7 & 21 & 18 & 19 & --- & 1.0 & \\
\hline Sodium, dissolved & $\mathrm{mg} / \mathrm{L}$ & 7 & 77 & 56 & 64 & $-1-$ & 6.8 & \\
\hline Potassium, dissolved & $\mathrm{mg} / \mathrm{L}$ & 7 & 12 & 10 & 11 & $-\cdots-1$ & 0.8 & \\
\hline Bicarbonate ion & $\mathrm{mg} / \mathrm{L}$ & 7 & 277 & 250 & 262 & --- & 8.4 & \\
\hline Sulfate, dissolved & $\mathrm{mg} / \mathrm{L}$ & 7 & 190 & 168 & 180 & --- & 8.6 & --- \\
\hline Chloride, dissolved & $\mathrm{mg} / \mathrm{L}$ & 7 & 16 & 14 & 15 & - n & 0.9 & \\
\hline Fluoride, dissolved & $\mathrm{mg} / \mathrm{L}$ & 7 & 1.0 & 0.6 & 0.7 & $\cdots$ & 0.2 & \\
\hline Silica, dissolved & $\mathrm{mg} / \mathrm{L}$ & 7 & 56 & 15 & 49 & - & 15 & \\
\hline Boron, dissolved & $\mathrm{mg} / \mathrm{L}$ & 7 & 120 & 90 & 97 & & 11 & \\
\hline Iron, dissolved & ug/L & & & & & & & \\
\hline Manganese, dissolved & ug/L & & & & & & & \\
\hline $\begin{array}{l}\text { Nitrite + nitrate as } \mathrm{N}, \\
\text { dissolved } \\
\text { Nitrate as N, dissolved }\end{array}$ & $\begin{array}{l}\mathrm{mg} / \mathrm{L} \\
\mathrm{mg} / \mathrm{L}\end{array}$ & 1 & 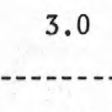 & 2.3 & 2.6 & $-\cdots-1$ & 0.30 & --- \\
\hline $\begin{array}{l}\text { Nitrate as } \mathrm{NO}_{3} \text {, dissolved } \\
\text { Anmonia } \mathrm{N} \text {, total }\end{array}$ & $\begin{array}{l}\mathrm{mg} / \mathrm{L} \\
\mathrm{mg} / \mathrm{L}\end{array}$ & 2 & 12 & 7.2 & 9.6 & & & \\
\hline Organic N, total & $\mathrm{mg} / \mathrm{L}$ & $-\cdots$ & & & & & & \\
\hline Phosphorus, total & $\mathrm{mg} / \mathrm{L}$ & & & & & & & \\
\hline Phosphorus, dissolved & $\mathrm{mg} / \mathrm{L}$ & 6 & 0.14 & 0.02 & 0.06 & $\cdots$ & 0.04 & \\
\hline $\begin{array}{ll}\text { Fecal coliform } & \mathrm{col} / \\
\text { Fecal streptococci } & \mathrm{col} /\end{array}$ & $\mathrm{col} / 100 \mathrm{ml}$ & $\cdots-$ & $-4=-1$ & - & -1 & & & 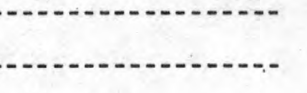 \\
\hline Oxygen, dissolved & $\mathrm{mg} / \mathrm{L}$ & 108 & 13.0 & 7.7 & 10.2 & 10.0 & 1.4 & 12.0 \\
\hline $\begin{array}{l}\text { Biochemical oxygen demand } \\
\text { (5-day) }\end{array}$ & $\begin{array}{l}\mathrm{mg} / \mathrm{L} \\
\text { units }\end{array}$ & 113 & 8.6 & 7.0 & 7.7 & 7.7 & 0.3 & 7.4 \\
\hline Co $-\mathrm{Pt}$ & units & 6 & 10 & 0 & 4 & 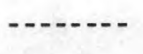 & 3.6 & - \\
\hline
\end{tabular}


Results of regression analyses relating concentrations of selected chemical constituents to specific conductance $(\mathrm{SpC})$

\begin{tabular}{|c|c|c|c|c|c|}
\hline $\begin{array}{c}\text { Dissolved } \\
\text { constituents } \\
(\mathrm{mg} / \mathrm{L})\end{array}$ & $\begin{array}{l}\text { No. of } \\
\text { measure- } \\
\text { ments }\end{array}$ & Regression equation & $\begin{array}{l}\text { Corre- } \\
\text { lation } \\
\text { coeffi- } \\
\text { cient }\end{array}$ & $\begin{array}{l}\text { Percent } \\
\text { explained } \\
\text { variance }\end{array}$ & $\begin{array}{c}\text { Standard } \\
\text { error of } \\
\text { estimate } \\
(\mathrm{mg} / \mathrm{L})\end{array}$ \\
\hline Dissolved solids, residue (RDS) & (n-n & $-1-2-1$ & -.... & & \\
\hline Dissolved solids, sum (SDS) & 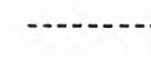 & & & & \\
\hline Hardness as $\mathrm{CaCO}_{3}(\mathrm{TH})$ & 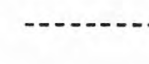 & - & $\ldots$ & 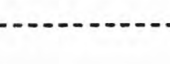 & $-\ldots$ \\
\hline Calcium (Ca) & $-1-2-1$ & - & 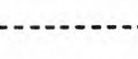 & $--1-2-1-2$ & 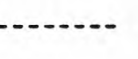 \\
\hline Magnesium (Mg) & $-1-n$ & 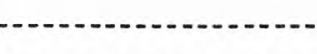 & $\ldots$ & (n) & \\
\hline Sodium (Na) & $-1-n-1$ & - & $-\cdots$ & (n) & $-\cdots$ \\
\hline Poțassium (K) & $-\cdots-1$ & 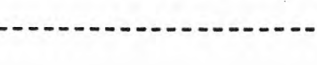 & $\ldots$ & 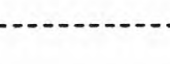 & 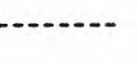 \\
\hline Bicarbonate $\left(\mathrm{HCO}_{3}\right)$ & $-\cdots+-1$ & - - - & -3 & $-1-n-1$ & \\
\hline Sulfate $\left(\mathrm{SO}_{4}\right)$ & $-\cdots-1$ & (1) & (5) & $--2---2$ & - \\
\hline Chloride (Cl) & 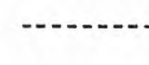 & - & & $-\ldots$ & \\
\hline Silica (Si) & $-1-1-1$ & 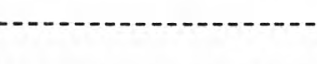 & & - n & $\cdots$ \\
\hline Nitrite + Nitrate as $\mathrm{N}\left(\mathrm{NO}_{2}-\mathrm{NO}_{3}\right)$ & $-\cdots-1$ & & & & \\
\hline
\end{tabular}

Results of regression analyses relating specific conductance to water discharge (Q)

\begin{tabular}{ccccccc}
\hline $\begin{array}{c}\text { No. of } \\
\text { measure- } \\
\text { ments }\end{array}$ & $\begin{array}{c}\text { Water discharge } \\
\left(\mathrm{ft}^{3} / \mathrm{s}\right)\end{array}$ & Maximum Minimum & Regression equation & $\begin{array}{c}\text { Correlation } \\
\text { coefficient }\end{array}$ & $\begin{array}{c}\text { Percent } \\
\text { explained } \\
\text { variance }\end{array}$ & $\begin{array}{c}\text { Standard error } \\
\text { of estimate }\end{array}$ \\
\hline 114 & 154 & 2.6 & $\mathrm{SpC}=771 \mathrm{Q}^{0.01905}$ & $* 0.18$ & 3.4 & 0.060 \\
\hline
\end{tabular}

* Not significant at the 95 percent confidence level; the regression equation should not be used to predict the concentration of the dependent variable. 
Location.--At Mitchel1-Gering Canal, $13 \mathrm{ft}$ upstream from culvert and 2.7 miles southwest of Mitche11, Scotts Bluff County.

Period of record.--September 1961 to September 1965.

Statistical data for selected chemical constituents

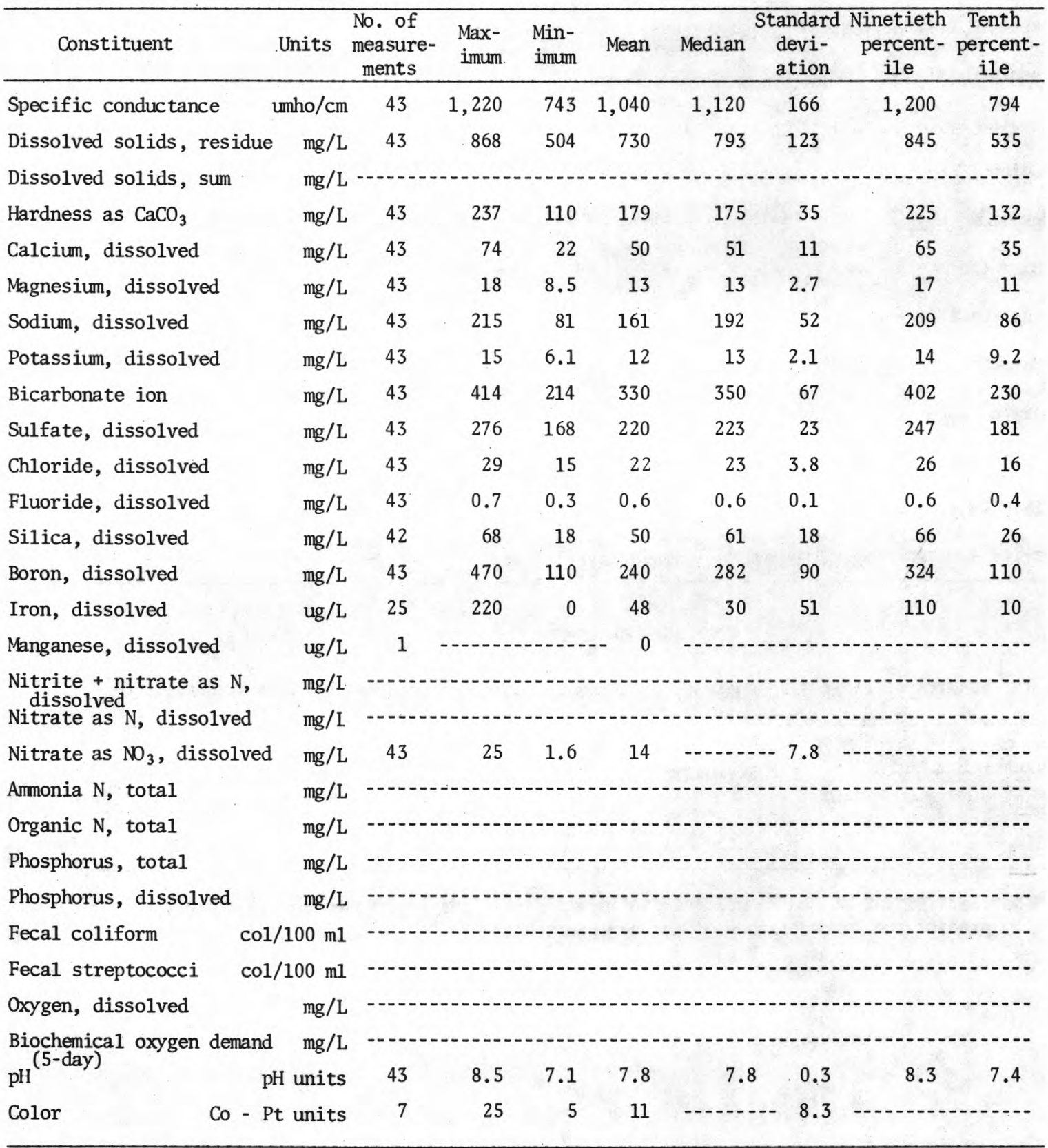


06678700 Brown Canyon Drain near Mitche11, Nebr.--Continued

Results of regression analyses relating concentrations of selected chemical constituents to specific conductance $(\mathrm{SpC})$

\begin{tabular}{|c|c|c|c|c|c|}
\hline $\begin{array}{l}\text { Dissolved } \\
\text { constituents } \\
(\mathrm{mg} / \mathrm{L})\end{array}$ & $\begin{array}{l}\text { No. of } \\
\text { measure- } \\
\text { ments }\end{array}$ & Regression equation & $\begin{array}{l}\text { Corre- } \\
\text { lation } \\
\text { coeffi- } \\
\text { cient }\end{array}$ & $\begin{array}{l}\text { Percent } \\
\text { explained } \\
\text { variance }\end{array}$ & $\begin{array}{c}\text { Standard } \\
\text { error of } \\
\text { estimate } \\
(\mathrm{mg} / \mathrm{L})\end{array}$ \\
\hline Dissolved solids, residue (RDS) & 43 & $\mathrm{RDS}=0.741(\mathrm{SpC})-41.460$ & 1.0 & 99 & 12 \\
\hline Dissolved solids, sum (SDS) & $\cdots$ & $-\cdots-1$ & & & \\
\hline Hardness as $\mathrm{CaCO}_{3}(\mathrm{TH})$ & 43 & $\mathrm{TH}=-0.158(\mathrm{SpC})+343.146$ & -.75 & 57 & 23 \\
\hline Calcium (Ca) & 43 & $\mathrm{Ca}=-0.042(\mathrm{SpC})+94.296$ & -.63 & 40 & 8.8 \\
\hline Magnesium (Mg) & 43 & $\mathrm{Mg}=-0.014(\mathrm{SpC})+27.157$ & -.84 & 70 & 1.5 \\
\hline Sodium (Na) & 43 & $\mathrm{Na}=0.308(\mathrm{SpC})-159.374$ & .98 & 97 & 9.3 \\
\hline Potassium (K) & 43 & $\mathrm{~K}=0.008(\mathrm{SpC})+3.377$ & .66 & 43 & 1.6 \\
\hline Bicarbonate $\left(\mathrm{HCO}_{3}\right)$ & 43 & $\mathrm{HCO}_{3}=0.383(\mathrm{SpC})-69.235$ & .95 & 90 & 22 \\
\hline Sulfate $\left(\mathrm{SO}_{4}\right)$ & 43 & $\mathrm{SO}_{4}=0.119(\mathrm{SpC})+95.664$ & .86 & 74 & 12 \\
\hline Chloride (C1) & 43 & $\mathrm{Cl}=0.020(\mathrm{SpC})+0.544$ & .88 & 78 & 1.8 \\
\hline Silica (Si) & 42 & $\mathrm{Si}=0.102(\mathrm{SpC})-55.630$ & .95 & 91 & 5.4 \\
\hline
\end{tabular}

Results of regression analyses relating specific conductance to water discharge (Q)

\begin{tabular}{ccccccc}
\hline $\begin{array}{c}\text { No. of } \\
\text { measure- } \\
\text { ments }\end{array}$ & $\begin{array}{c}\text { Water discharge } \\
\text { Maximum Minimum }\end{array}$ & Regression equation & $\begin{array}{c}\text { Correlation } \\
\text { coefficient }\end{array}$ & $\begin{array}{c}\text { Percent } \\
\text { explained } \\
\text { variance }\end{array}$ & $\begin{array}{c}\text { Standard error } \\
\text { of estimate }\end{array}$ \\
\hline 43 & 21 & 0.90 & $\mathrm{SpC}=1234 \mathrm{Q}^{-0.16573}$ & -0.94 & 89 & 0.024 \\
\hline
\end{tabular}


Location.--At gaging station at county road bridge, $0.2 \mathrm{mi}$ upstream from mouth and $2.5 \mathrm{miles}$ northwest of Mitchell, Scotts Bluff County.

Period of record.-- September 1961 to September 1965.

Statistical data for selected chemical constituents

\begin{tabular}{|c|c|c|c|c|c|c|c|c|c|}
\hline Constituent & Units & $\begin{array}{l}\text { No. of } \\
\text { measure- } \\
\text { ments }\end{array}$ & $\begin{array}{l}\text { Max- } \\
\text { imum }\end{array}$ & $\begin{array}{l}\text { Min- } \\
\text { imum }\end{array}$ & Mean & Median & $\begin{array}{c}\text { Standard } \\
\text { devi- } \\
\text { ation }\end{array}$ & $\begin{array}{c}\text { Ninetieth } \\
\text { percent- } \\
\text { ile }\end{array}$ & $\begin{array}{l}\text { Tenth } \\
\text { percent- } \\
\text { ile }\end{array}$ \\
\hline Specific conductance & umho/cm & 43 & 906 & 703 & 831 & 857 & 59 & 887 & 735 \\
\hline Dissolved solids, residue & $\mathrm{mg} / \mathrm{L}$ & 43 & 642 & 479 & 587 & 605 & 46 & 630 & 513 \\
\hline Dissolved solids, sum & $\mathrm{mg} / \mathrm{L}$ & - & -1 & 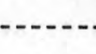 & ---- & 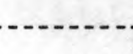 & 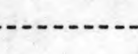 & $(-2+-2-1$ & 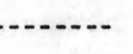 \\
\hline Hardness as $\mathrm{CaCO}_{3}$ & $\mathrm{mg} / \mathrm{L}$ & 43 & 358 & 255 & 314 & 323 & 31 & 347 & 265 \\
\hline Calcium, dissolved & $\mathrm{mg} / \mathrm{L}$ & 43 & 108 & 70 & 90 & 93 & 12 & 101 & 75 \\
\hline Magnesium, dissolved & $\mathrm{mg} / \mathrm{L}$ & 43 & 38 & 17. & 21 & 22 & 3.2 & 24 & 19 \\
\hline Sodium, dissolved & $\mathrm{mg} / \mathrm{L}$ & 43 & 63 & 48 & 56 & 57 & 3.0 & 60 & 52 \\
\hline Potasșium, dissolved & $\mathrm{mg} / \mathrm{L}$ & 43 & 17 & 7.6 & 13 & 14 & 2.5 & 16 & 9.1 \\
\hline Bicarbonate ion & $\mathrm{mg} / \mathrm{L}$ & 43 & 323 & 208 & 278 & 300 & 39 & 318 & 218 \\
\hline Sulfate, dissolved & $\mathrm{mg} / \mathrm{L}$ & 43 & 215 & 156 & 184 & 184 & 11 & 196 & 165 \\
\hline Chloride, dissolved & $\mathrm{mg} / \mathrm{L}$ & 43 & 20 & 12 & 14 & 15 & 1.6 & 17 & 12 \\
\hline Fluoride, dissolved & $\mathrm{mg} / \mathrm{L}$ & 43 & 0.6 & 0.1 & 0.4 & 0.4 & 0.1 & 0.5 & 0.4 \\
\hline Silica, dissolved & $\mathrm{mg} / \mathrm{L}$ & 43 & 54 & 20 & 42 & 49 & 11 & 53 & $\cdot 24$ \\
\hline Boron, dissolved & $\mathrm{mg} / \mathrm{L}$ & 43 & 160 & 70 & 111 & 112 & 22 & 144 & 89 \\
\hline Iron, dissolved & $\mathrm{ug} / \mathrm{L}$ & 25 & 140 & 0 & 46 & 30 & 39 & 104 & 13 \\
\hline Manganese, dissolved & $\mathrm{ug} / \mathrm{L}$ & 1 & $\ldots$ & 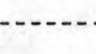 & 20 & & & & --- \\
\hline $\begin{array}{l}\text { Nitrite + nitrate as } \mathrm{N} \text {, } \\
\text { dissolved } \\
\text { Nitrate as } \mathrm{N} \text {, dissolved }\end{array}$ & $\begin{array}{l}\mathrm{mg} / \mathrm{L} \\
\mathrm{mg} / \mathrm{L}\end{array}$ & - & $-\pi$ & & $-\ldots$ & & & & -- \\
\hline Nitrate as $\mathrm{NO}_{3}$, dissolved & $\mathrm{mg} / \mathrm{L}$ & 43 & 11 & 0.20 & 7.5 & $\cdots$ & 3.0 & 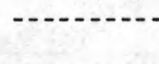 & - \\
\hline Ammonia $\mathrm{N}$, total & $\mathrm{mg} / \mathrm{L}$ & $-\cdots$ & & & & & & & 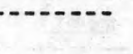 \\
\hline Organic $N$, total & $\mathrm{mg} / \mathrm{L}$ & - & 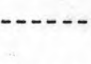 & & & & & & \\
\hline Phosphorus, total & $\mathrm{mg} / \mathrm{L}$ & & & & & & & & \\
\hline Phosphorus, dissolved & $\mathrm{mg} / \mathrm{L}$ & & & & & & & & \\
\hline Fecal coliform & $/ 100 \mathrm{ml}$ & $\cdots$ & 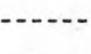 & & & & & & \\
\hline Fecal streptococci & $\mathrm{L} / 100 \mathrm{ml}-$ & & 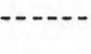 & & & & & & \\
\hline Oxygen, dissolved & $\mathrm{mg} / \mathrm{L}-$ & --- & & & & & & & \\
\hline $\begin{array}{l}\text { Biochemical oxygen demand } \\
\text { pH } 5 \text {-day) }\end{array}$ & mg/L - & 43 & 8.2 & 6.9 & 7.7 & 7.7 & 0.3 & 8.1 & 7.4 \\
\hline $\mathrm{Co}-\mathrm{Pt}$ & t units & 7 & 20 & 4 & 8 & 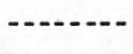 & 5.8 & $\cdots$ & (n.... \\
\hline
\end{tabular}


Results of regression analyses relating concentrations of selected chemical constituents to specific conductance $(\mathrm{SpC})$

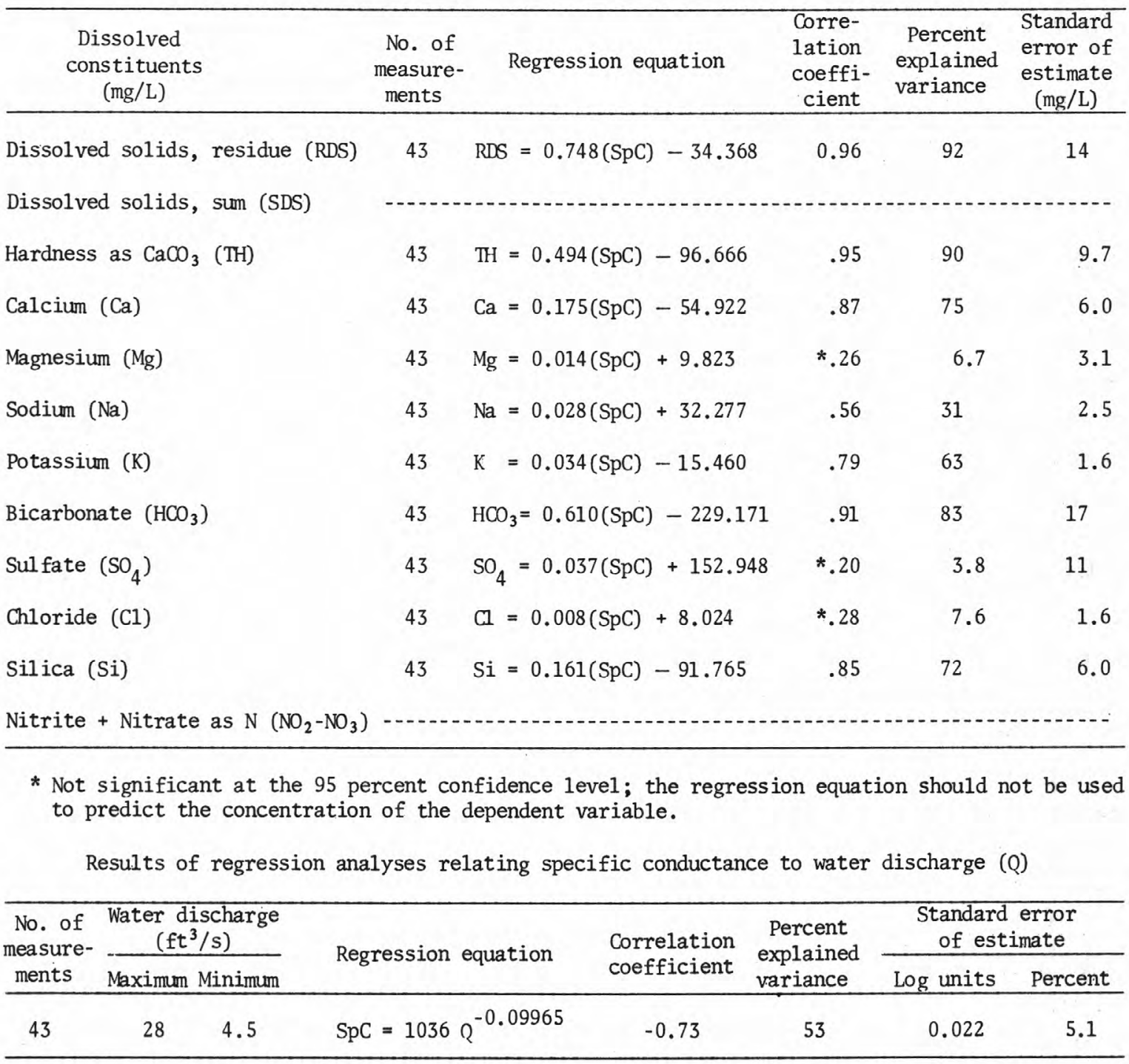




\section{NORTH PLATTE RIVER BASIN}

06680700 Winter Creek at Tri-State Canal near Scottsbluff, Nebr.

Location.--At gaging station, $15 \mathrm{ft}$ upstream from culvert under Tri-State canal and 5 mi northeast of Scottsbluff, Scotts Bluff County.

Period of record. - July 1961 to September 1965.

Statistical data for selected chemical constituents

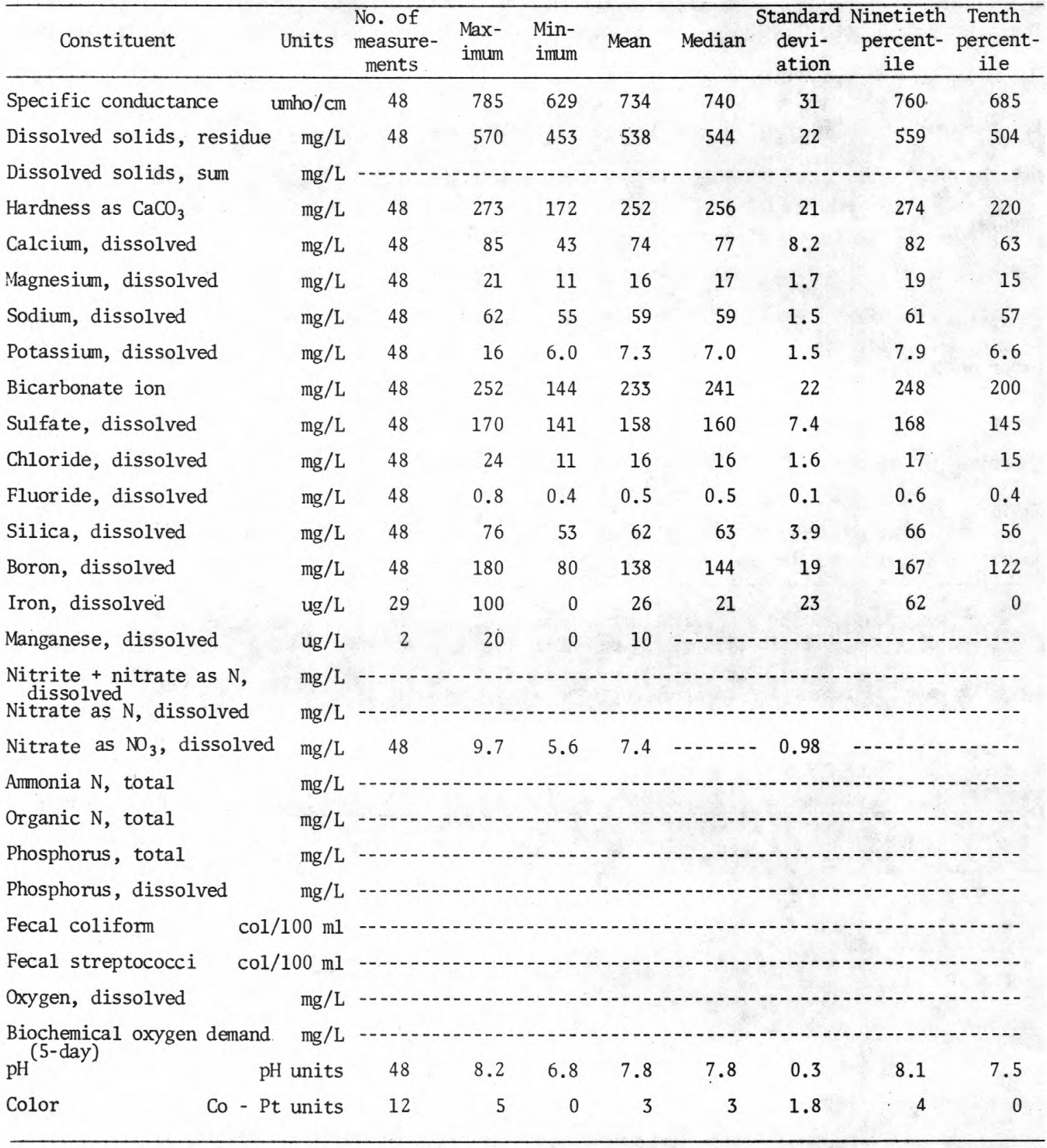


06680700 Winter Creek at Tri-State Canal near Scottsbluff, Nebr.--Continued

Results of regression analyses relating concentrations of selected chemical constituents to specific conductance $(\mathrm{SpC})$

\begin{tabular}{|c|c|c|c|c|c|}
\hline $\begin{array}{c}\text { Dissolved } \\
\text { constituents } \\
(\mathrm{mg} / \mathrm{L})\end{array}$ & $\begin{array}{l}\text { No. of } \\
\text { measure- } \\
\text { ments }\end{array}$ & Regression equation & $\begin{array}{l}\text { Corre- } \\
\text { lation } \\
\text { coeffi- } \\
\text { cient }\end{array}$ & $\begin{array}{l}\text { Percent } \\
\text { explained } \\
\text { variance }\end{array}$ & $\begin{array}{c}\text { Standard } \\
\text { error of } \\
\text { estimate } \\
(\mathrm{mg} / \mathrm{L})\end{array}$ \\
\hline Dissolved solids, residue (RDS) & 48 & $\mathrm{RDS}=0.642(\mathrm{SpC})+66.953$ & 0.90 & 82 & 9.5 \\
\hline Dissolved solids, sum (SDS) & --- & & & & \\
\hline Hardness as $\mathrm{CaCO}_{3}$ (TH) & 48 & $\mathrm{TH}=0.618(\mathrm{SpC})-201.923$ & .91 & 83 & 8.7 \\
\hline Calcium (Ca) & 48 & $\mathrm{Ca}=0.236(\mathrm{SpC})-98.991$ & .89 & 80 & 3.7 \\
\hline Magnesium (Mg) & 48 & $\mathrm{Mg}=0.005(\mathrm{SpC})+12.292$ & $* .10$ & 0.95 & 1.7 \\
\hline Sodium $(\mathrm{Na})$ & 48 & $\mathrm{Na}-0.004(\mathrm{SpC})+56.072$ & $* .07$ & 0.55 & 1.5 \\
\hline Potassium (K) & 48 & $\mathrm{~K}=0.011(\mathrm{SpC})-0.854$ & $* .22$ & 5.1 & 1.5 \\
\hline Bicarbonate $\left(\mathrm{HCO}_{3}\right)$ & 48 & $\mathrm{HCO}_{3}=0.652(\mathrm{SpC})-246.146$ & .91 & 83 & 9.2 \\
\hline Sulfate $\left(\mathrm{SO}_{4}\right)$ & 48 & $\mathrm{SO}_{4}=0.080(\mathrm{SpC})+99.203$ & .33 & 11 & 7.0 \\
\hline Chloride (Cl) & 48 & $\mathrm{Cl}=0.020(\mathrm{spC})+0.567$ & .40 & 16 & 1.5 \\
\hline Silica (Si) & 48 & $\mathrm{Si}=0.005(\mathrm{SpC})+58.531$ & *.04 & 0.17 & 3.9 \\
\hline
\end{tabular}

*Not significant at the 95 percent confidence level; the regression equation should not be used to predict the concentration of the dependent variable.

Results of regression analyses relating specific conductance to water discharge (Q)

\begin{tabular}{|c|c|c|c|c|c|c|c|}
\hline \multirow{2}{*}{$\begin{array}{l}\text { No. of } \\
\text { measure- } \\
\text { ments }\end{array}$} & \multicolumn{2}{|c|}{$\begin{array}{c}\text { Water discharge } \\
\left(\mathrm{ft}^{3} / \mathrm{s}\right) \\
\end{array}$} & \multirow{2}{*}{ Regression equation } & \multirow{2}{*}{$\begin{array}{l}\text { Correlation } \\
\text { coefficient }\end{array}$} & \multirow{2}{*}{$\begin{array}{l}\text { Percent } \\
\text { explained } \\
\text { variance }\end{array}$} & \multicolumn{2}{|c|}{$\begin{array}{c}\text { Standard error } \\
\text { of estimate }\end{array}$} \\
\hline & Maximum & Minimum & & & & Log units & Percent \\
\hline 48 & 52 & 14.5 & $\mathrm{SpC}=635 \mathrm{Q}^{0.04291}$ & 0.35 & 12 & 0.018 & 4.1 \\
\hline
\end{tabular}


NORTH PLATTE RIVER BASIN

06680800 Hale Drain near Scottsbluff, Nebr.

Location.--At Tri-State canal, $135 \mathrm{ft}$ downstream from culvert and 3 mi northeast of Scottsbluff, Scotts Bluff County.

Period of record.---September 1961 to September 1965.

Statistical data for selected chemical constituents

\begin{tabular}{|c|c|c|c|c|c|c|c|c|c|}
\hline Constituent & Units & $\begin{array}{l}\text { No. of } \\
\text { measure- } \\
\text { ments }\end{array}$ & $\begin{array}{l}\text { Max- } \\
\text { imum }\end{array}$ & $\begin{array}{l}\text { Min- } \\
\text { imum }\end{array}$ & Mean & Median & $\begin{array}{l}\text { Standard } \\
\text { devi- } \\
\text { ation }\end{array}$ & $\begin{array}{l}\text { Ninetieth } \\
\text { percent- } \\
\text { ile }\end{array}$ & $\begin{array}{l}\text { Tenth } \\
\text { percent- } \\
\text { ile }\end{array}$ \\
\hline Specific conductance & umho/cm & 43 & 934 & 660 & 794 & 802 & 49 & 842 & 733 \\
\hline Dissolved solids, residue & $\mathrm{mg} / \mathrm{L}$ & 43 & 612 & 476 & 566 & 570 & 31 & 602 & 523 \\
\hline Dissolved solids, sum & $\mathrm{mg} / \mathrm{L}$ & ---- & --1 & ---- & --- & -1 & (n........ & $-n$ & $-\cdots$ \\
\hline Hardness as $\mathrm{CaCO}_{3}$ & $\mathrm{mg} / \mathrm{L}$ & 43 & 252 & 142 & 222 & 226 & 24 & 246 & 185 \\
\hline Calcium, dissolved & $\mathrm{mg} / \mathrm{L}$ & 43 & 75 & 33 & 62 & 66 & 9.1 & 72 & 50 \\
\hline Magnesium, dissolved & $\mathrm{mg} / \mathrm{L}$ & 43 & 22 & 13 & 16 & 16 & 1.8 & 19 & 14 \\
\hline Sodium, dissolved & $\mathrm{mg} / \mathrm{L}$ & 43 & 94 & 62 & 84 & 87 & 7.2 & 91 & 74 \\
\hline Potassium, dissolved & $\mathrm{mg} / \mathrm{L}$ & 43 & 12 & 6.9 & 8.8 & 8.7 & 1.0 & 10 & 7.8 \\
\hline Bicarbonate ion & $\mathrm{mg} / \mathrm{L}$ & 43 & 288 & 172 & 255 & 262 & 27 & 284 & 220 \\
\hline Sulfate, dissolved & $\mathrm{mg} / \mathrm{L}$ & 43 & 184 & 143 & 167 & 164 & 10 & 180 & 156 \\
\hline Chloride, dissolved & $\mathrm{mg} / \mathrm{L}$ & 43 & 19 & 13 & 17 & 18 & 1.3 & 19 & 16 \\
\hline Fluoride, dissolved & $\mathrm{mg} / \mathrm{L}$ & 43 & 0.6 & 0.1 & 0.4 & 0.4 & 0.1 & 0.5 & 0.3 \\
\hline Silica, dissolved & $\mathrm{mg} / \mathrm{L}$ & 43 & 74 & 32 & 58 & 62 & 9.2 & 66 & 44 \\
\hline Boron, dissolved & $\mathrm{mg} / \mathrm{L}$ & 43 & 320 & 100 & 175 & 183 & 34 & 200 & $140^{t+1}$ \\
\hline Iron, dissolved & $\mathrm{ug} / \mathrm{L}$ & 25 & 160 & 0 & 42 & 28 & 41 & 88 & 12 \\
\hline Manganese, dissolved & ug/L & 1 & 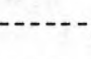 & $\ldots$ & 20 & & & & \\
\hline $\begin{array}{l}\text { Nitrite + nitrate as } \mathrm{N} \text {, } \\
\text { dissolved } \\
\text { Nitrate as N, dissolved }\end{array}$ & $\begin{array}{l}\mathrm{mg} / \mathrm{L} \\
\mathrm{mg} / \mathrm{L}\end{array}$ & & $-\infty$ & - & $-\ldots$ & & & & \\
\hline Nitrate as $\mathrm{NO}_{3}$, dissolved & $\mathrm{mg} / \mathrm{L}$ & 43 & 19 & 4.1 & 7.9 & & 2.3 & & \\
\hline Ammonia $\mathrm{N}$, total & $\mathrm{mg} / \mathrm{L}$ & & & & & & & & \\
\hline Organic $N$, total & $\mathrm{mg} / \mathrm{L}$ & & & & & & & & \\
\hline Phosphorus, total & $\mathrm{mg} / \mathrm{L}$ & & & & & & & & \\
\hline Phosphorus, dissolved & $\mathrm{mg} / \mathrm{L}$ & & & & & & & & \\
\hline Fecal coliform col & $1 / 100 \mathrm{ml}$ & & & & & & & & \\
\hline Fecal streptococci col & $\mathrm{l} / 100 \mathrm{ml}$ & & & & & & & & \\
\hline Oxygen, dissolved & $\mathrm{mg} / \mathrm{L}$ & & & & & & & & \\
\hline $\begin{array}{l}\text { Biochemical oxygen demand } \\
\mathrm{pH}^{(5-\text { day })}\end{array}$ & mits & 43 & 8.3 & 7.3 & 7.8 & 7.8 & 0.3 & 8.1 & 7.4 \\
\hline Co - P & t units & 7 & 10 & 4 & 7 & 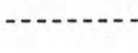 & 2.6 & & $-\cdots$ \\
\hline
\end{tabular}


Results of regression analyses relating concentrations of selected chemical constituents to specific conductance $(\mathrm{SpC})$

\begin{tabular}{|c|c|c|c|c|c|}
\hline $\begin{array}{l}\text { Dissolved } \\
\text { constituents } \\
(\mathrm{mg} / \mathrm{L})\end{array}$ & $\begin{array}{c}\text { No. of } \\
\text { measure- } \\
\text { ments }\end{array}$ & Regression equation & $\begin{array}{l}\text { Corre- } \\
\text { lation } \\
\text { coeffi- } \\
\text { cient }\end{array}$ & $\begin{array}{l}\text { Percent } \\
\text { explained } \\
\text { variance }\end{array}$ & $\begin{array}{c}\text { Standard } \\
\text { error of } \\
\text { estimate } \\
(\mathrm{mg} / \mathrm{L})\end{array}$ \\
\hline Dissolved solids, residue (RDS) & 43 & $\mathrm{RDS}=0.584(\mathrm{SpC})+101.700$ & 0.93 & 86 & 12 \\
\hline Dissolved solids, sum (SDS) & $-\ldots$ & & & & \\
\hline Hardness as $\mathrm{CaCO}_{3}(\mathrm{TH})$ & 43 & $\mathrm{TH}=0.351(\mathrm{SpC})-56.239$ & .70 & 50 & 18 \\
\hline Calcium (Ca) & 43 & $\mathrm{Ca}=0.130(\mathrm{SpC})-40.355$ & .69 & 48 & 6.6 \\
\hline Magnesium (Mg) & 43 & $\mathrm{Mg}=0.009(\mathrm{SpC})+8.818$ & $* .24$ & 6.0 & 1.8 \\
\hline Sodium (Na) & 43 & $\mathrm{Na}=0.083(\mathrm{SpC})+18.417$ & .56 & 32 & 6.0 \\
\hline Potassium (K) & 43 & $K=-0.0002(\mathrm{SpC})+8.984$ & $*_{-} .01$ & .02 & 1.0 \\
\hline Bicarbonate $\left(\mathrm{HCO}_{3}\right)$ & 43 & $\mathrm{HCO}_{3}=0.495(\mathrm{SpC})-137.492$ & .91 & 82 & 11 \\
\hline Sulfate $\left(\mathrm{SO}_{4}\right)$ & 43 & $\mathrm{SO}_{4}=0.067(\mathrm{SpC})+113.896$ & .33 & 11 & $9: 5$ \\
\hline Chloride (C1) & 43 & $\mathrm{Cl}=0.010(\mathrm{SpC})+8.746$ & .40 & 16 & 1.2 \\
\hline Silica (Si) & 43 & $\mathrm{Si}=0.064(\mathrm{SpC})+7.077$ & .34 & 12 & 8.8 \\
\hline
\end{tabular}

Results of regression analyses relating specific conductance to water discharge $(Q)$

\begin{tabular}{cccccccc}
\hline $\begin{array}{c}\text { No. of } \\
\text { measure- } \\
\text { ments }\end{array}$ & $\begin{array}{c}\text { Mater discharge } \\
\text { Maximum Minimum }\end{array}$ & Regression equation & $\begin{array}{c}\text { Correlation } \\
\text { coefficient }\end{array}$ & $\begin{array}{c}\text { Percent } \\
\text { explained } \\
\text { variance }\end{array}$ & \multicolumn{2}{c}{$\begin{array}{c}\text { Standard error } \\
\text { of estimate }\end{array}$} \\
\hline 43 & $20.3 \quad 1.4$ & $\mathrm{SpC}=7520^{0.03626}$ & 0.44 & 20 & 0.024 & 5.5 \\
\hline
\end{tabular}


06681300 Gering Drain at Mitche11-Gering Canal near Gering, Nebr.

Location. --At Mitchell-Gering canal, $2.8 \mathrm{mi}$ southwest of Gering, Scotts Bluff County.

Period of record. -- July 1961 to September 1965.

Statistical data for selected chemical constituents

\begin{tabular}{|c|c|c|c|c|c|c|c|c|c|}
\hline Constituent & Units & $\begin{array}{l}\text { No. of } \\
\text { measure- } \\
\text { ments }\end{array}$ & $\begin{array}{l}\text { Max- } \\
\text { imum }\end{array}$ & $\begin{array}{l}\text { Min- } \\
\text { imum }\end{array}$ & Mean & Median & $\begin{array}{l}\text { Standard } \\
\text { devi- } \\
\text { ation }\end{array}$ & $\begin{array}{l}\text { Ninetieth } \\
\text { percent- } \\
\text { ile }\end{array}$ & $\begin{array}{l}\text { Tenth } \\
\text { percent- } \\
\text { ile }\end{array}$ \\
\hline Specific conductance & umho/cm & 47 & 1,180 & 455 & 1,040 & 1,110 & 149 & 1,180 & 860 \\
\hline Dissolved solids, residue & $\mathrm{mg} / \mathrm{L}$ & 47 & 823 & 314 & 725 & 780 & 108 & 823 & 580 \\
\hline Dissolved solids, sum & $\mathrm{mg} / \mathrm{L}$ & --- & --1 & --- &.-- & ---1 & --- & $\cdots$ & $-\ldots$ \\
\hline Hardness as $\mathrm{CaCO}_{3}$ & $\mathrm{mg} / \mathrm{L}$ & 47 & 242 & 70 & 166 & 162 & 40 & 215 & 100 \\
\hline Calcium, dissolved & $\mathrm{mg} / \mathrm{L}$ & 47 & 69 & 11 & 47 & 49 & 13 & 62 & 25 \\
\hline Magnesium, dissolved & $\mathrm{mg} / \mathrm{L}$ & 47 & 19 & 6.9 & 12 & 11 & 3.4 & 17 & 8.5 \\
\hline Sodium, dissolved & $\mathrm{mg} / \mathrm{L}$ & 47 & 231 & 34 & 169 & 190 & 47 & 215 & 97 \\
\hline Potassium, dissolved & $\mathrm{mg} / \mathrm{L}$ & 47 & 17 & 7.3 & 11 & 11 & 1.5 & 13 & 9.5 \\
\hline Bicarbonate ion & $\mathrm{mg} / \mathrm{L}$ & 47 & 432 & 226 & 364 & 400 & 65 & 429 & 265 \\
\hline Sulfate, dissolved & $\mathrm{mg} / \mathrm{L}$ & 47 & 224 & 37 & 194 & 198 & 26 & 209 & 180 \\
\hline Chloride, dissolved & $\mathrm{mg} / \mathrm{L}$ & 43 & 32 & 5.4 & 24 & 26 & 4.6 & 29 & 19 \\
\hline Fluoride, dissolved & $\mathrm{mg} / \mathrm{L}$ & 45 & 0.8 & 0.3 & 0.6 & 0.6 & 0.1 & 0.7 & 0.5 \\
\hline Silica, dissolved & $\mathrm{mg} / \mathrm{L}$ & 45 & 70 & 25 & 52 & 60 & 13 & 64 & 34 \\
\hline Boron, dissolved & $\mathrm{mg} / \mathrm{L}$ & 45 & 500 & 20 & 343 & 405 & 113 & 429 & 175 \\
\hline Iron, dissolved & $\mathrm{ug} / \mathrm{L}$ & 27 & 100 & 10 & 41 & 31 & 30 & 92 & 10 \\
\hline Manganese, dissolved & $\mathrm{ug} / \mathrm{L}$ & - & 列 & -1 & 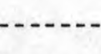 & & -1 & & $\ldots+1$ \\
\hline $\begin{array}{l}\text { Nitrite + nitrate as } \mathrm{N} \text {, } \\
\text { dissolved } \\
\text { Nitrate as } \mathrm{N} \text {, dissolved }\end{array}$ & $\begin{array}{l}\mathrm{mg} / \mathrm{L} \\
\mathrm{mg} / \mathrm{L}\end{array}$ & - & & & & & & & \\
\hline Nitrate as $\mathrm{NO}_{3}$, dissolved & $\mathrm{mg} / \mathrm{L}$ & 45 & 18 & 1.2 & 12 & & 4.8 & & -- \\
\hline Ammonia N, total & $\mathrm{mg} / \mathrm{L}$ & 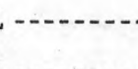 & - & & $\cdots$ & & - & & \\
\hline $\begin{array}{l}\text { Organic N, total } \\
\text { Phosphorus, total }\end{array}$ & $\begin{array}{l}\mathrm{mg} / \mathrm{L} \\
\mathrm{mg} / \mathrm{L}\end{array}$ & 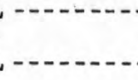 & $-\ldots$ & 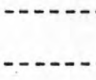 & 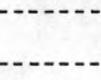 & & & & $\ldots$ \\
\hline Phosphorus, dissolved & $\mathrm{mg} / \mathrm{L}$ & & - & & & & & & \\
\hline Fecal coliform & $/ 100 \mathrm{ml}$ & - & $-2--2-1$ & & & & & & -- \\
\hline Fecal streptococci & $/ 100 \mathrm{~m} 1$ & & & & & & & & \\
\hline Oxygen, dissolved & $\mathrm{mg} / \mathrm{L}$ & & ----- & & & & & & -- \\
\hline $\begin{array}{l}\text { Biochemical oxygen demand } \\
\mathrm{pH}^{(5-\text { day })}\end{array}$ & $\begin{array}{l}\mathrm{mg} / \mathrm{L} \\
\mathrm{H} \text { units }\end{array}$ & 47 & 8.5 & 7.1 & 7.8 & 7.9 & 0.3 & 8.2 & 7.5 \\
\hline Co - Pt & t units & 10 & 35 & 3 & 10 & 8 & 9.3 & 16 & 5 \\
\hline
\end{tabular}


06681300 Gering Drain at Mitchell-Gering Canal near Gering, Nebr.--Continued

Results of regression analyses relating concentrations of selected chemical constituents to specific conductance $(\mathrm{SpC})$

\begin{tabular}{|c|c|c|c|c|c|}
\hline $\begin{array}{l}\text { Dissolved } \\
\text { constituents } \\
(\mathrm{mg} / \mathrm{L})\end{array}$ & $\begin{array}{l}\text { No. of } \\
\text { measure- } \\
\text { ments }\end{array}$ & Regression equation & $\begin{array}{l}\text { Corre- } \\
\text { lation } \\
\text { coeffi- } \\
\text { cient }\end{array}$ & $\begin{array}{l}\text { Percent } \\
\text { explained } \\
\text { variance }\end{array}$ & $\begin{array}{c}\text { Standard } \\
\text { error of } \\
\text { estimate } \\
(\mathrm{mg} / \mathrm{L})\end{array}$ \\
\hline Dissolved solids, residue (RDS) & 47 & $\mathrm{RDS}=0.722(\mathrm{SpC})--26.197$ & 0.99 & 99 & 12 \\
\hline Dissolved solids, sum (SDS) & & & & & \\
\hline Hardness as $\mathrm{CaCO}_{3}(\mathrm{TH})$ & 47 & $\mathrm{TH}=-0.129(\mathrm{SpC})+300.665$ & -.48 & 23 & 36 \\
\hline Calcium (Ca) & 47 & $\mathrm{Ca}=-0.026(\mathrm{SpC})+73.625$ & $*_{-} .28$ & 8.0 & 13 \\
\hline Magnesium (Mg) & 47 & $\mathrm{Mg}=-0.016(\mathrm{SpC})+28.070$ & -.69 & 47 & 2.5 \\
\hline Sodium $(\mathrm{Na})$ & 47 & $\mathrm{Na}-0.301(\mathrm{SpC})-144.068$ & .95 & 90 & 15 \\
\hline Potassium (K) & 47 & $K=0.003(\mathrm{SpC})+8.100$ & *.27 & 7.4 & 1.5 \\
\hline Bicarbonate $\left(\mathrm{HCO}_{3}\right)$ & 47 & $\mathrm{HCO}_{3}=0.403(\mathrm{SpC})-55.917$ & .92 & 84 & 26 \\
\hline Sulfate $\left(\mathrm{SO}_{4}\right)$ & 47 & $\mathrm{SO}_{4}=0.121(\mathrm{SpC})+67.822$ & .69 & 48 & 19 \\
\hline Chloride $(\mathrm{Cl})$ & 47 & $\mathrm{Cl}=0.029(\mathrm{SpC})-6.308$ & .93 & 86 & 1.7 \\
\hline Silica (Si) & 45 & $\mathrm{Si}=0.067(\mathrm{SpC})-17.679$ & .78 & 61 & 8.3 \\
\hline
\end{tabular}

* Not significant at the 95 percent confidence level; the regression equation should not be used to predict the concentration of the dependent variable.

Results of regression analyses relating specific conductance to water discharge (Q)

\begin{tabular}{|c|c|c|c|c|c|c|c|}
\hline \multirow{2}{*}{$\begin{array}{l}\text { No. of } \\
\text { measure- } \\
\text { ments }\end{array}$} & \multicolumn{2}{|c|}{$\begin{array}{c}\text { Water discharge } \\
\left(\mathrm{ft}^{3} / \mathrm{s}\right)\end{array}$} & \multirow{2}{*}{ Regression equation } & \multirow{2}{*}{$\begin{array}{l}\text { Correlation } \\
\text { coefficient }\end{array}$} & \multirow{2}{*}{$\begin{array}{l}\text { Percent } \\
\text { explained } \\
\text { variance }\end{array}$} & \multicolumn{2}{|c|}{$\begin{array}{c}\text { Standard error } \\
\text { of estimate }\end{array}$} \\
\hline & Maximum & Minimum & & & & Log units & Percent \\
\hline 47 & 170 & 5.2 & $\mathrm{SpC}=2032 Q^{-0.23724}$ & -0.88 & 78 & 0.035 & 8.1 \\
\hline
\end{tabular}


NORTH PLATTE RIVER BASIN

06682200 Alliance Drain near Minatare, Nebr.

Location.--At gaging station, $50 \mathrm{ft}$ north of county road, $0.1 \mathrm{mi}$ downstream from Tri-State canal and 4.5 mi north of Minatare, Scơtts Bluff County.

Period of record. --July 1961 to September 1965.

Statistical data for selected chemical constituents

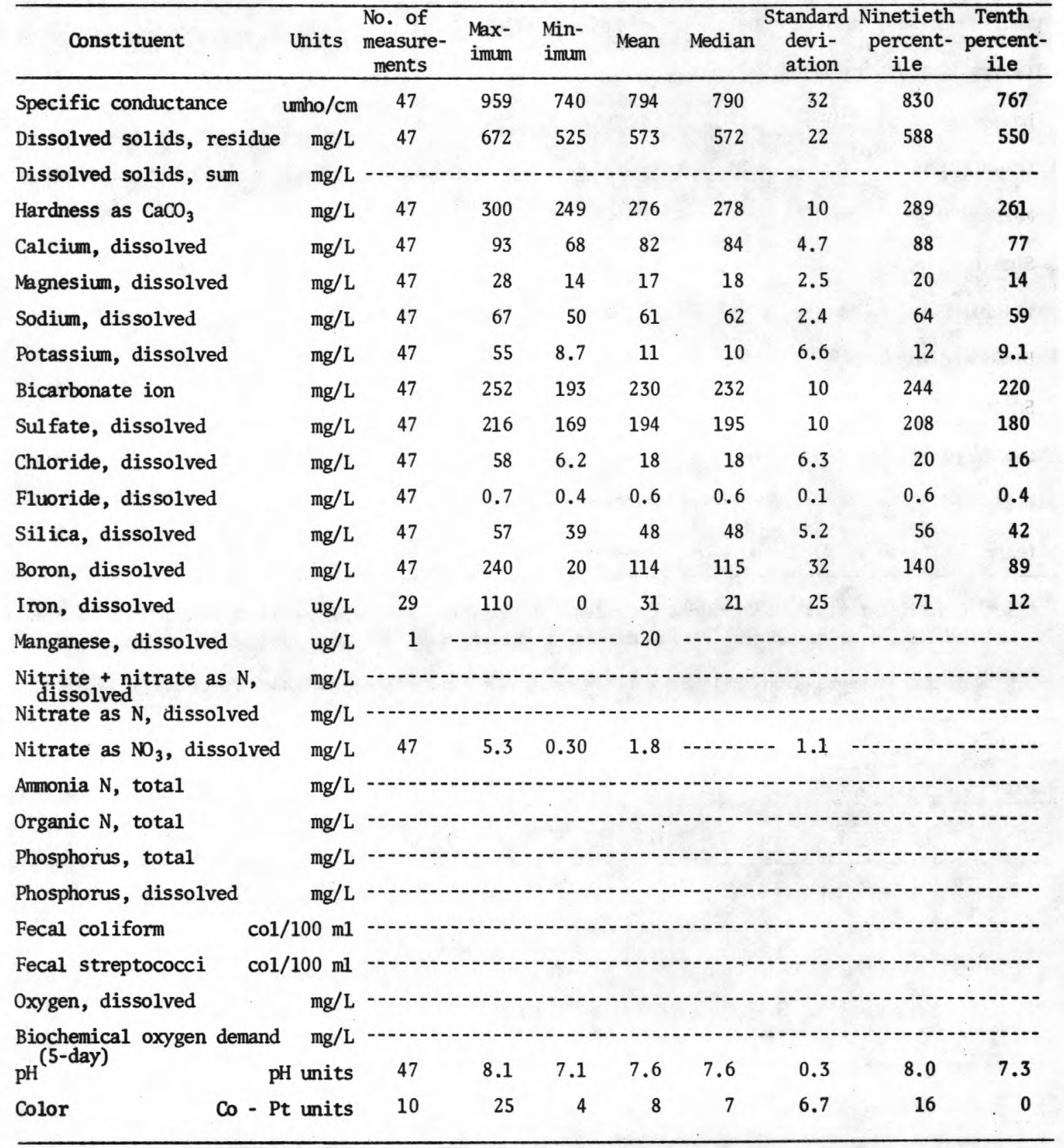


NORTH PLATTE RIVER BASIN

06682200 Alliance Drain near Minatare, Nebr.--Continued

Results of regression analyses relating concentrations of selected chemical constituents to specific conductance $(\mathrm{SpC})$

\begin{tabular}{|c|c|c|c|c|c|}
\hline $\begin{array}{l}\text { Dissolved } \\
\text { constituents } \\
(\mathrm{mg} / \mathrm{L})\end{array}$ & $\begin{array}{l}\text { No. of } \\
\text { measure- } \\
\text { ments }\end{array}$ & Regression equation & $\begin{array}{l}\text { Corre- } \\
\text { lation } \\
\text { coeffi- } \\
\text { cient }\end{array}$ & $\begin{array}{l}\text { Percent } \\
\text { explained } \\
\text { variance }\end{array}$ & $\begin{array}{c}\text { Standard } \\
\text { error of } \\
\text { estimate } \\
(\mathrm{mg} / \mathrm{L})\end{array}$ \\
\hline Dissolved solids, residue (RDS) & 47 & $\mathrm{RDS}=0.616(\mathrm{SpC})+84.392$ & 0.90 & 82 & 9.4 \\
\hline Dissolved solids, sum (SDS) & --- & 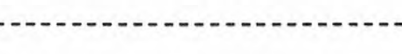 & & & $\cdots$ \\
\hline Hardness as $\mathrm{CaCO}_{3}(\mathrm{TH})$ & 47 & $\mathrm{TH}=0.181(\mathrm{SpC})+132.855$ & .56 & 32 & 8.5 \\
\hline Calcium (Ca) & 47 & $\mathrm{Ca}=0.055(\mathrm{SpC})+38.673$ & .37 & 14 & 4.4 \\
\hline Magnesium (Mg) & 47 & $\mathrm{Mg}=0.012(\mathrm{SpC})+7.594$ & $* .15$ & 2.4 & 2.5 \\
\hline Sodium ( $\mathrm{Na})$ & 47 & $\mathrm{Na}=0.019(\mathrm{Sp})+45.709$ & $* .25$ & 6.4 & 2.4 \\
\hline Potassium (K) & 47 & $\mathrm{~K}=0.170(\mathrm{SpC})-123.623$ & .82 & 66 & 3.9 \\
\hline Bicarbonate $\left(\mathrm{HCO}_{3}\right)$ & 47 & $\mathrm{HCO}_{3}=0.105(\mathrm{SpC})+147.208$ & .33 & 11 & 9.5 \\
\hline Sulfate $\left(\mathrm{SO}_{4}\right)$ & 47 & $\mathrm{SO}_{4}=0.109(\mathrm{SpC})+108.031$ & .34 & 12 & 9.6 \\
\hline Chloride (C1) & 47 & $\mathrm{Cl}=0.158(\mathrm{SpC})-107.036$ & .80 & 64 & 3.8 \\
\hline Silica (Si) & 47 & $S i=-0.020(\mathrm{SpC})+64.384$ & $*-.12$ & 1.5 & 5.2 \\
\hline
\end{tabular}

* Not significant at the 95 percent confidence level; the regression equation should not be used to predict the concentration of the dependent variable.

Results of regression analyses relating specific conductance to water discharge (Q)

\begin{tabular}{lllcccc}
\hline $\begin{array}{c}\text { No. of } \\
\text { measure- } \\
\text { ments }\end{array}$ & $\begin{array}{c}\text { Water discharge } \\
\left(\mathrm{ft}^{3} / \mathrm{s}\right)\end{array}$ & Maximum Minimum & Regression equation & $\begin{array}{c}\text { Correlation } \\
\text { coefficient }\end{array}$ & $\begin{array}{c}\text { Percent } \\
\text { explained } \\
\text { variance }\end{array}$ & $\begin{array}{c}\text { Standard error } \\
\text { of estimate }\end{array}$ \\
\hline 47 & 38 & 1.7 & $\mathrm{SpC}=774 \mathrm{Q}^{0.01023}$ & $* 0.19$ & 3.7 & 0.016 \\
\hline
\end{tabular}

* Not significant at the 95 percent confidence level; the regression equation should not be used to predict the concentration of the dependent variable. 
Location.--At gaging station at county highway bridge, $0.5 \mathrm{mi}$ downstream from Tri-State canal and $6.5 \mathrm{mi}$ northeast of Minatare, Scotts Bluff County.

Period of record.--July 1961 to September 1965.

Statistical data for selected chemical constituents

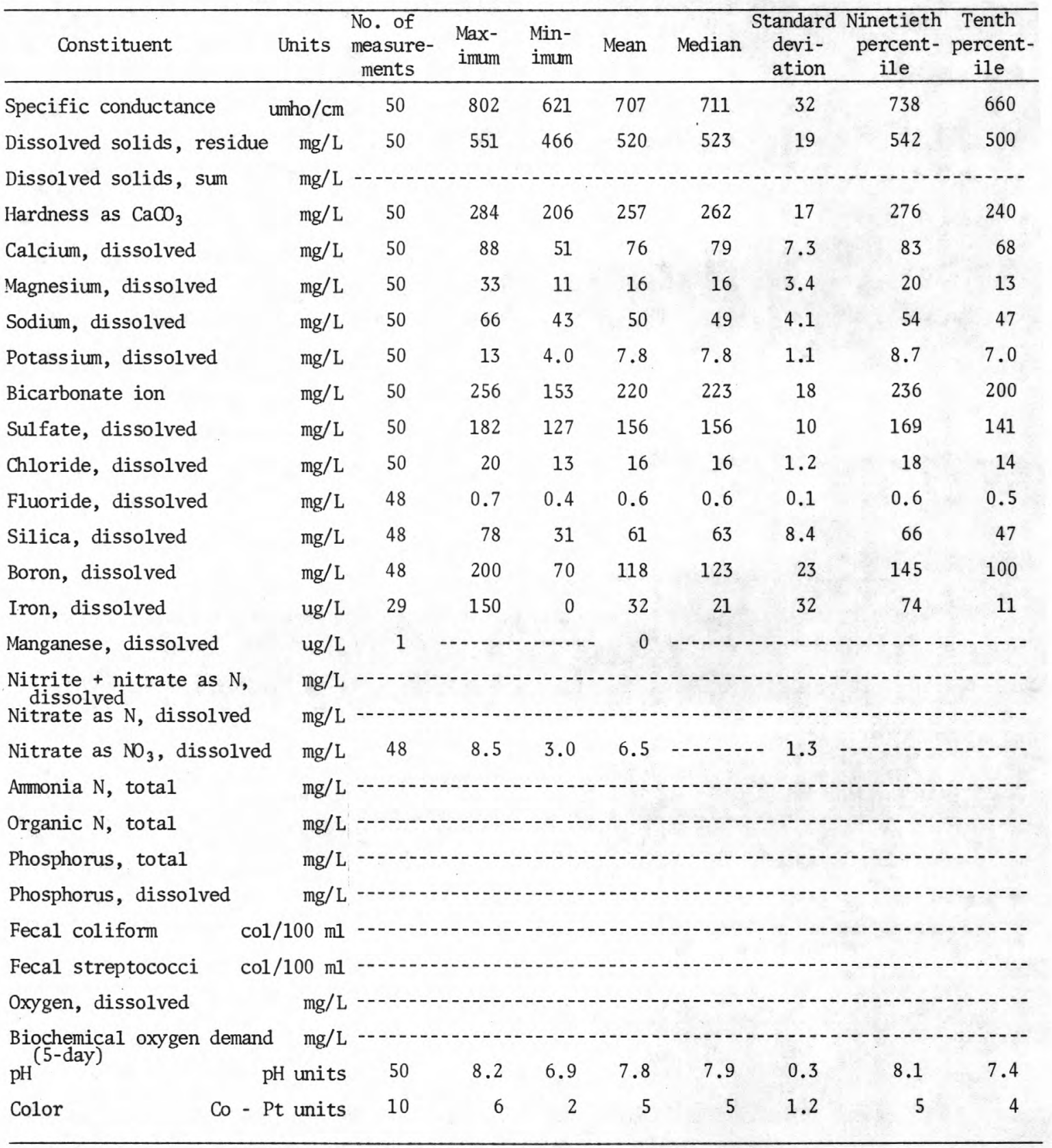




\section{NORTH PLATTE RIVER BASIN}

06682300 Ninemile Drain near Minatare, Nebr.--Continued

Results of regression analyses relating concentrations of selected chemical constituents to specific conductance $(\mathrm{SpC})$

\begin{tabular}{|c|c|c|c|c|c|}
\hline $\begin{array}{c}\text { Dissolved } \\
\text { constituents } \\
(\mathrm{mg} / \mathrm{L})\end{array}$ & $\begin{array}{l}\text { No. of } \\
\text { measure- } \\
\text { ments }\end{array}$ & Regression equation & $\begin{array}{l}\text { Corre- } \\
\text { lation } \\
\text { coeffi- } \\
\text { cient }\end{array}$ & $\begin{array}{l}\text { Percent } \\
\text { explained } \\
\text { variance }\end{array}$ & $\begin{array}{c}\text { Standard } \\
\text { error of } \\
\text { estimate } \\
(\mathrm{mg} / \mathrm{L})\end{array}$ \\
\hline Dissolved solids, residue (RDS) & 50 & $\mathrm{RDS}=0.512(\mathrm{SpC})+157.814$ & 0.85 & 73 & 10 \\
\hline Dissolved solids, sum (SDS) & & & & & \\
\hline Hardness as $\mathrm{CaCO}_{3}(\mathrm{TH})$ & 50 & $\mathrm{TH}=0.432(\mathrm{SpC})-47.890$ & .84 & 70 & 9.3 \\
\hline Calcium (Ca) & 50 & $\mathrm{Ca}=0.130(\mathrm{SpC})-15.676$ & .58 & 34 & 6.0 \\
\hline Magnesium (Mg) & 50 & $\mathrm{Mg}=0.024(\mathrm{SpC})-0.798$ & *.23 & 5.3 & 3.3 \\
\hline Sodium $(\mathrm{Na})$ & 50 & $\mathrm{Na}=0.088(\mathrm{SpC})-12.433$ & .70 & 48 & 3.0 \\
\hline Potassium $(K)$ & 50 & $\mathrm{~K}=0.008(\mathrm{SpC})+2.384$ & $* .23$ & 5.3 & 1.0 \\
\hline Bicarbonate $\left(\mathrm{HCO}_{3}\right)$ & 50 & $\mathrm{HCO}_{3}=0.512(\mathrm{SpC})-142.725$ & .90 & 81 & 8.2 \\
\hline Sulfate $\left(\mathrm{SO}_{4}\right)$ & 50 & $\mathrm{SO}_{4}=0.190(\mathrm{SpC})+21.444$ & .62 & 38 & 7.9 \\
\hline Chloride (C1) & 50 & $\mathrm{Cl}=0.014(\mathrm{SpC})+5.979$ & .37 & 14 & 1.1 \\
\hline Silica (Si) & 48 & $\mathrm{Si}=-0.063(\mathrm{SpC})+105.364$ & $*_{-} .25$ & 6.2 & 8.2 \\
\hline
\end{tabular}

* Not significant at the 95 percent confidence level; the regression equation should not be used to predict the concentration of the dependent variable.

Results of regression analyses relating specific conductance to water discharge (Q)

\begin{tabular}{ccccccc}
\hline $\begin{array}{c}\text { No. of } \\
\text { measure- } \\
\text { ments }\end{array}$ & $\begin{array}{c}\text { Water discharge } \\
\left(\mathrm{ft}^{3} / \mathrm{s}\right)\end{array}$ & Maximum Minimum & Regression equation & $\begin{array}{c}\text { Correlation } \\
\text { coefficient }\end{array}$ & $\begin{array}{c}\text { Percent } \\
\text { explained } \\
\text { variance }\end{array}$ & $\begin{array}{c}\text { Standard error } \\
\text { of estimate }\end{array}$ \\
\hline 50 & $140 \quad 11.5$ & $\mathrm{SpC}=620 \mathrm{Q}^{0.03992}$ & 0.57 & 33 & 0.016 & 3.7 \\
\hline
\end{tabular}




\section{NORTH PLATTE RIVER BASIN}

06682505 North Platte River at McGrew, Nebr.

Location.--Lat $41^{\circ} 45^{\prime} 42^{\prime \prime}$, long $103^{\circ} 25^{\prime} 02^{\prime \prime}$, in SW $W^{\frac{1}{4}}$ sec. 25 , T. 21 N., R. 53 W., Scotts Bluff County, Hydrologic Unit 10180009 , at bridge on county road $1.2 \mathrm{mi}$ north of State Highway $92,0.3 \mathrm{mi}$ downstream from Ninemile Creek and $0.9 \mathrm{mi}$ north of McGrew.

Period of record. --June 1973 to current year.

Statistical data for selected chemical constituents

\begin{tabular}{|c|c|c|c|c|c|c|c|c|c|}
\hline Constituent & Units & $\begin{array}{l}\text { No. of } \\
\text { measure- } \\
\text { ments }\end{array}$ & $\begin{array}{l}\text { Max- } \\
\text { imum }\end{array}$ & $\begin{array}{l}\text { Min- } \\
\text { imum }\end{array}$ & Mean & Median & $\begin{array}{c}\text { Standard } \\
\text { devi- } \\
\text { ation }\end{array}$ & $\begin{array}{l}\text { Ninetieth } \\
\text { percent- } \\
\text { ile }\end{array}$ & $\begin{array}{c}\text { Tenth } \\
\text { percent- } \\
\text { ile }\end{array}$ \\
\hline Specific conductance & umho/ $\mathrm{cm}$ & 51 & 1,090 & 705 & 922 & 950 & 93 & 1,000 & 790 \\
\hline Dissolved solids, residue & $\mathrm{mg} / \mathrm{L}$ & 32 & 715 & 482 & 624 & 640 & 56 & 677 & 545 \\
\hline Dissolved solids, sum & $\mathrm{mg} / \mathrm{L}$ & 18 & 660 & 458 & 615 & 637 & 52 & 655 & 528 \\
\hline Hardness as $\mathrm{CaCO}_{3}$ & $\mathrm{mg} / \mathrm{L}$ & 18 & 310 & 230 & 286 & 294 & 19 & 306 & 260 \\
\hline Calcium, dissolved & $\mathrm{mg} / \mathrm{L}$ & 18 & 91 & 64 & 80 & 82 & 6.6 & 88 & 70 \\
\hline Magnesium, dissolved & $\mathrm{mg} / \mathrm{L}$ & 18 & 23 & 18 & 21 & 22 & 1.2 & 23 & 19 \\
\hline Sodium, dissolved & $\mathrm{mg} / \mathrm{L}$ & 18 & 100 & 57 & 92 & 96 & 11 & 100 & 70 \\
\hline Potassium, dissolved & $\mathrm{mg} / \mathrm{L}$ & 18 & 12 & 5.6 & 9.6 & 10 & 1.5 & 12 & 7.6 \\
\hline Bicarbonate ion & $\mathrm{mg} / \mathrm{L}$ & 18 & 325 & 221 & 295 & 310 & 32 & 325 & 232 \\
\hline Sulfate, dissolved & $\mathrm{mg} / \mathrm{L}$ & 18 & 250 & 160 & 208 & 212 & 19 & 230 & 190 \\
\hline Chloride, dissolved & $\mathrm{mg} / \mathrm{L}$ & 50 & 28 & 14 & 21 & 22 & 3.0 & 25 & 17 \\
\hline Fluoride, dissolved & $\mathrm{mg} / \mathrm{L}$ & 18 & 0.7 & 0.4 & 0.5 & 0.5 & 0.1 & 0.6 & 0.4 \\
\hline Silica, dissolved & $\mathrm{mg} / \mathrm{L}$ & 18 & 42 & 15 & 33 & 37 & 7.9 & 41 & 22 \\
\hline Boron, dissolved & $\mathrm{mg} / \mathrm{L}$ & 18 & 210 & 110 & 156 & 160 & 22 & 183 & 130 \\
\hline Iron, dissolved & $\mathrm{ug} / \mathrm{L}$ & 18 & 70 & 0 & 27 & 21 & 19 & 60 & 0 \\
\hline Manganese, dissolved & $\mathrm{ug} / \mathrm{L}$ & 18 & 360 & 0 & 89 & 46 & 114 & 330 & 0 \\
\hline $\begin{array}{l}\text { Nitrite + nitrate as } \mathrm{N} \text {, } \\
\text { dissolved } \\
\text { Nitrate as }\end{array}$ & $\mathrm{mg} / \mathrm{L}$ & 24 & 3.6 & 0.64 & 1.9 & 1.9 & 0.86 & 2.9 & 0.76 \\
\hline Nitrate as $\mathrm{NO}_{3}$, dissolved & $\mathrm{mg} / \mathrm{L}$ & & & & & & & & \\
\hline Ammonia $\mathrm{N}$, total & $\mathrm{mg} / \mathrm{L}$ & 51 & 0.41 & 0.00 & 0.11 & 0.09 & 0.10 & 0.25 & 0.02 \\
\hline Organic $\mathrm{N}$, total & $\mathrm{mg} / \mathrm{L}$ & 51 & 3.0 & 0.09 & 0.90 & 0.86 & 0.46 & 1.2 & 0.50 \\
\hline Phosphorus, total & $\mathrm{mg} / \mathrm{L}$ & 51 & 4.4 & 0.08 & 0.39 & 0.16 & 0.75 & 0.43 & 0.12 \\
\hline Phosphorus, dissolved & $\mathrm{mg} / \mathrm{L}$ & 43 & 1.0 & 0.02 & 0.09 & 0.06 & 0.15 & 0.11 & 0.05 \\
\hline Fecal coliform & $\operatorname{col} / 100 \mathrm{ml}$ & 39 & 6,000 & 17 & - n & 363 & 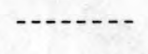 & 2,800 & 99 \\
\hline Fecal streptococci & $\mathrm{col} / 100 \mathrm{ml}$ & 48 & 6,300 & 16 & $-\cdots--$ & 560 & $\cdots \cdots$ & 2,300 & 120 \\
\hline Oxygen, dissolved & $\mathrm{mg} / \mathrm{L}$ & 52 & 12.8 & 5.8 & 9.5 & 9.8 & 1.5 & 11.6 & 7.4 \\
\hline \multirow{2}{*}{$\begin{array}{l}\text { Biochemical oxygen dem } \\
\mathrm{pH}^{(5-\text { day })}\end{array}$} & $\mathrm{mg} / \mathrm{L}$ & 52 & 11.0 & 0.7 & 3.0 & 2.5 & 1.8 & 5.1 & 1.4 \\
\hline & H units & 53 & - n & 6.9 & $-1-1$ & 7.6 & (n....... & 7.9 & 7.3 \\
\hline Co $-\mathrm{Pt}$ & t units & 18 & 25 & 1 & 10 & 7 & 7.7 & 21 & 2 \\
\hline
\end{tabular}


Results of regression analyses relating concentrations of selected chemical constituents to specific conductance $(\mathrm{SpC})$

\begin{tabular}{|c|c|c|c|c|c|}
\hline $\begin{array}{l}\text { Dissolved } \\
\text { constituents } \\
(\mathrm{mg} / \mathrm{L})\end{array}$ & $\begin{array}{l}\text { No. of } \\
\text { measure- } \\
\text { ments }\end{array}$ & Regression equation & $\begin{array}{l}\text { Corre- } \\
\text { lation } \\
\text { coeffi- } \\
\text { cient }\end{array}$ & $\begin{array}{l}\text { Percent } \\
\text { explained } \\
\text { variance }\end{array}$ & $\begin{array}{c}\text { Standard } \\
\text { error of } \\
\text { estimate } \\
(\mathrm{mg} / \mathrm{L})\end{array}$ \\
\hline Dissolved solids, residue (RDS) & 31 & $\mathrm{RDS}=0.478(\mathrm{SpC})+183.040$ & 0.84 & 72 & 30 \\
\hline Dissolved solids, sum (SDS) & 18 & $\mathrm{SDS}=0.558(\mathrm{SpC})+99.439$ & .91 & 83 & 22 \\
\hline Hardness as $\mathrm{CaCO}_{3}(\mathrm{TH})$ & 18 & $\mathrm{TH}=0.178(\mathrm{SpC})+121.235$ & .78 & 61 & 12 \\
\hline Calcium (Ca) & 18 & $\mathrm{Ca}=0.057(\mathrm{SpC})+27.108$ & .73 & 53 & 4.6 \\
\hline Magnesium (Mg) & 18 & $\mathrm{Mg}=0.006(\mathrm{SpC})+15.182$ & $* .42$ & 18 & 1.2 \\
\hline Sodium $(\mathrm{Na})$ & 18 & $\mathrm{Na}=0.120(\mathrm{SpC})-18.808$ & .90 & 81 & 5.0 \\
\hline Potassium (K) & 18 & $\mathrm{~K}=0.011(\mathrm{SpC})-0.651$ & .61 & 38 & 1.2 \\
\hline Bicarbonate $\left(\mathrm{HCO}_{3}\right)$ & 18 & $\mathrm{HCO}_{3}=0.293(\mathrm{SpC})+24.081$ & .76 & 58 & 22 \\
\hline Sulfate $\left(\mathrm{SO}_{4}\right)$ & 18 & $\mathrm{SO}_{4}=0.146(\mathrm{SpC})+72.755$ & .65 & 42 & 15 \\
\hline Chloride (C1) & 49 & $\mathrm{Cl}=0.026(\mathrm{spC})-3.261$ & .81 & 66 & 1.8 \\
\hline Silica (Si) & 18 & $\mathrm{Si}=0.052(\mathrm{SpC})-14.639$ & .55 & 30 & 6.8 \\
\hline Nitrite + Nitrate as $\mathrm{N}\left(\mathrm{NO}_{2}-\mathrm{NO}_{3}\right)$ & 24 & $\mathrm{HO}_{2}-\mathrm{NO}_{3}=0.008(\mathrm{SpC})-5.367$ & .88 & 77 & 0.42 \\
\hline
\end{tabular}

* Not significant at the 95 percent confidence level; the regression equation should not be used to predict the concentration of the dependent variable.

Results of regression analyses relating specific conductance to water discharge (Q)

\begin{tabular}{ccccccc}
\hline $\begin{array}{c}\text { No. of } \\
\text { measure- } \\
\text { ments }\end{array}$ & $\begin{array}{c}\text { Water discharge } \\
\text { Maximum Minimum }\end{array}$ & Regression equation & $\begin{array}{c}\text { Correlation } \\
\text { coefficient }\end{array}$ & $\begin{array}{c}\text { Percent } \\
\text { explained } \\
\text { variance }\end{array}$ & $\begin{array}{c}\text { Standard error } \\
\text { of estimate }\end{array}$ \\
\hline 51 & 5,530 & 331 & $S p C=2,206 \mathrm{Q}^{-0.12476}$ & -0.76 & 58 & 0.030 \\
\hline
\end{tabular}


Location.--Main channel gage: Lat $41^{\circ} 40^{\prime} 39^{\prime \prime}$, long $103^{\circ} 05^{\prime} 45^{\prime \prime}$, in $\mathrm{NW}^{\frac{1}{4}} \mathrm{SW}^{\frac{1}{4}} \mathrm{sec} .28, \mathrm{~T} .20$ N., R.50 W., Morrill County, Hydrologic Unit 10180009, on downstream side of pier near center of bridge on U.S. Highway 26, $0.5 \mathrm{mi}$ north of Bridgeport. Browns Creek channel gage: Lat $41^{\circ} 40^{\prime} 55^{\prime \prime}$, long $103^{\circ} 05^{\prime} 53^{\prime \prime}$, in $\mathrm{NW}^{\frac{1}{4}} \mathrm{NW} \frac{1}{4} \mathrm{sec}$. 28 , T.20 N., R. 50 W., Morrill County, on left bank 0.2 mi upstream from culvert on U.S. Highway 26 and $0.8 \mathrm{mi}$ north of Bridgeport.

Drainage area (revised) $.--25,300 \mathrm{mi}^{2}$, approximately, of which about $23,300 \mathrm{mi}^{2}$ contributes directly to surface runoff. Approximately $4,000 \mathrm{mi}^{2}$ in Great Divide basin is not included.

Period of record.--Water year 1971 to current year.

Statistical data for selected chemical constituents

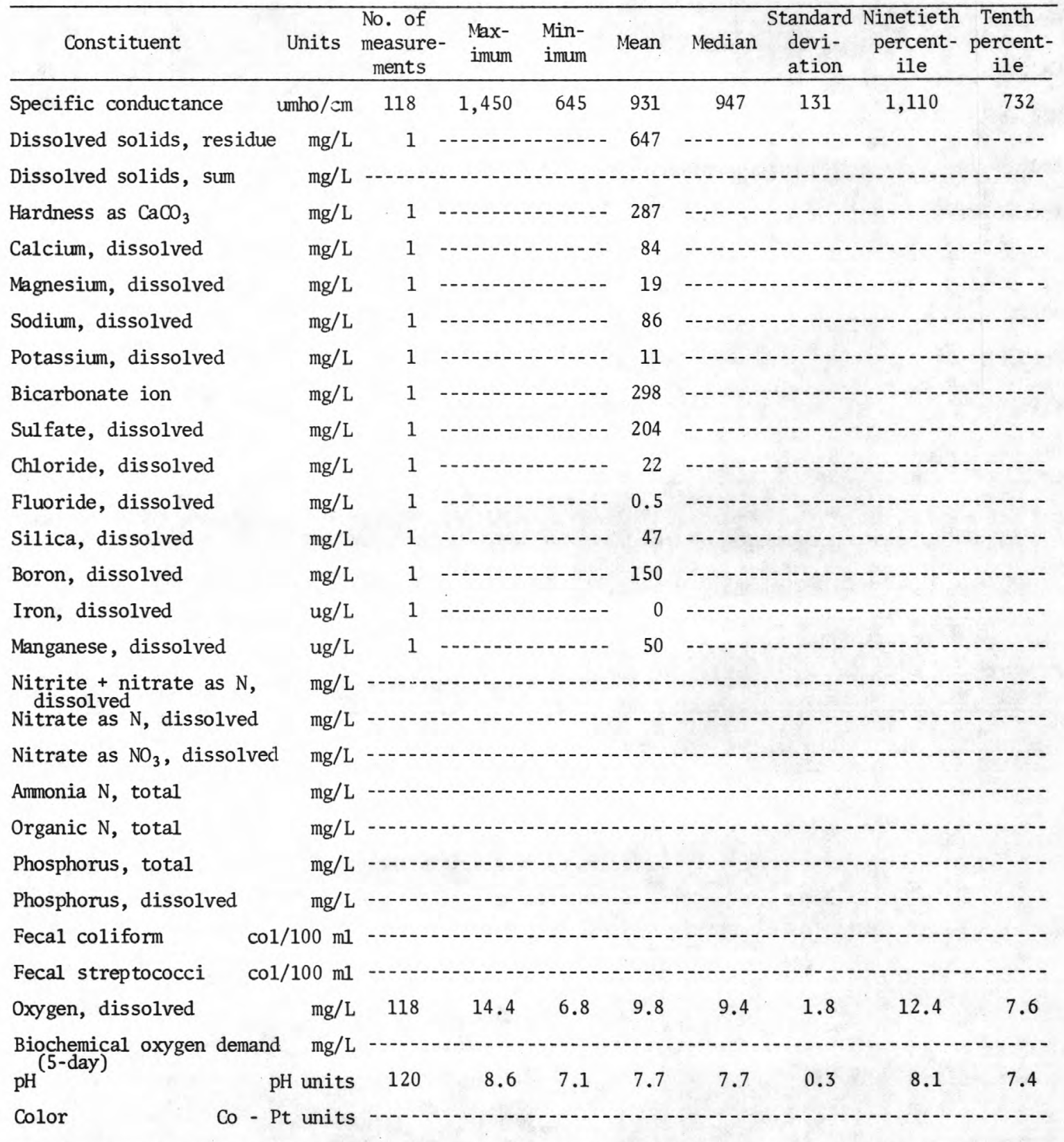


06684500 North Platte River at Bridgeport, Nebr.--Continued

Results of regression analyses relating concentrations of selected chemical constituents to specific conductance $(\mathrm{SpC})$

\begin{tabular}{|c|c|c|c|c|c|}
\hline $\begin{array}{l}\text { Dissolved } \\
\text { constituents } \\
(\mathrm{mg} / \mathrm{L})\end{array}$ & $\begin{array}{l}\text { No. of } \\
\text { measure- } \\
\text { ments }\end{array}$ & Regression equation & $\begin{array}{l}\text { Corre- } \\
\text { lation } \\
\text { coeffi- } \\
\text { cient }\end{array}$ & $\begin{array}{l}\text { Percent } \\
\text { explained } \\
\text { variance }\end{array}$ & $\begin{array}{c}\text { Standard } \\
\text { error of } \\
\text { estimate } \\
(\mathrm{mg} / \mathrm{L})\end{array}$ \\
\hline Dissolved solids, residue (RDS) & $-\cdots$ & 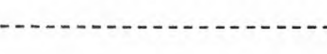 & $\cos$ & & -- \\
\hline Dissolved solids, sum (SDS) & $-\cdots-1$ & - & - & 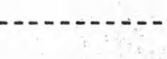 & 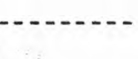 \\
\hline Hardness as $\mathrm{CaCO}_{3}(\mathrm{TH})$ & $\ldots \ldots$ & - & - & & $-B_{2}$ \\
\hline Calcium (Ca) & $-1-1$ & - & & $-\cdots-n$ & $\cdots$ \\
\hline Magnesium (Mg) & $-\cdots-1$ & 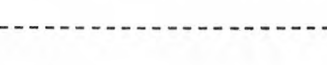 & & - & $--n-$ \\
\hline Sodium $(\mathrm{Na})$ & $-\cdots-1$ & - & & & -- \\
\hline Potassium (K) & $-\cdots-1$ & 烈 & $\ldots$ & 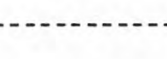 & 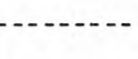 \\
\hline Bicarbonate $\left(\mathrm{HCO}_{3}\right)$ &.---- & - & & $--n-1$ & \\
\hline Sulfate $\left(\mathrm{SO}_{4}\right)$ & $---1--$ & 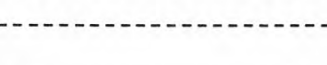 & $-0 .-$ & & - \\
\hline Chloride (Cl) & 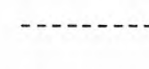 & 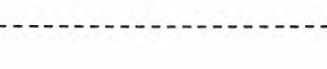 & 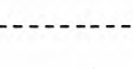 & $-1-2-1$ & \\
\hline Silica (Si) & $\cdots \cdots$ & $-\cdots$ & & & \\
\hline Nitrite + Nitrate as $\mathrm{N}\left(\mathrm{NO}_{2}-\mathrm{NO}_{3}\right)$ & $-\cdots--$ & & & & \\
\hline
\end{tabular}

Results of regression analyses relating specific conductance to water discharge (Q)

\begin{tabular}{ccccccc}
\hline $\begin{array}{c}\text { No. of } \\
\text { measure- } \\
\text { ments }\end{array}$ & $\begin{array}{c}\text { Water discharge } \\
\left(\mathrm{ft}^{3} / \mathrm{s}\right)\end{array}$ & Maximum Minimum & Regression equation & $\begin{array}{c}\text { Correlation } \\
\text { coefficient }\end{array}$ & $\begin{array}{c}\text { Percent } \\
\text { explained } \\
\text { variance }\end{array}$ & $\begin{array}{c}\text { Standard error } \\
\text { of estimate }\end{array}$ \\
\hline 118 & $10,500 \quad 169$ & $\mathrm{SpC}=1,472 \mathrm{Q}^{-0.06453}$ & -0.30 & 8.9 & 0.060 \\
\hline
\end{tabular}


NORTH PLATTE RIVER BASIN

06685000 Pumpkin Creek near Bridgeport, Nebr.

Location.--Lat $41^{\circ} 37^{\prime} 38^{\prime \prime}$, long $103^{\circ} 02^{\prime} 10^{\prime \prime}$, in SW/4 sec. 12, T.19 N., R.50 W., Morrill County, at gaging station at bridge on U.S. Highway 385 and State Highway 92, 0.5 mi upstream from mouth and $4 \mathrm{mi}$ southeast of Bridgeport.

Drainage area.--1,020 $\mathrm{mi}^{2}$, approximately.

Period of record. -- July 1970 to September 1973.

Statistical data for selected chemical constituents

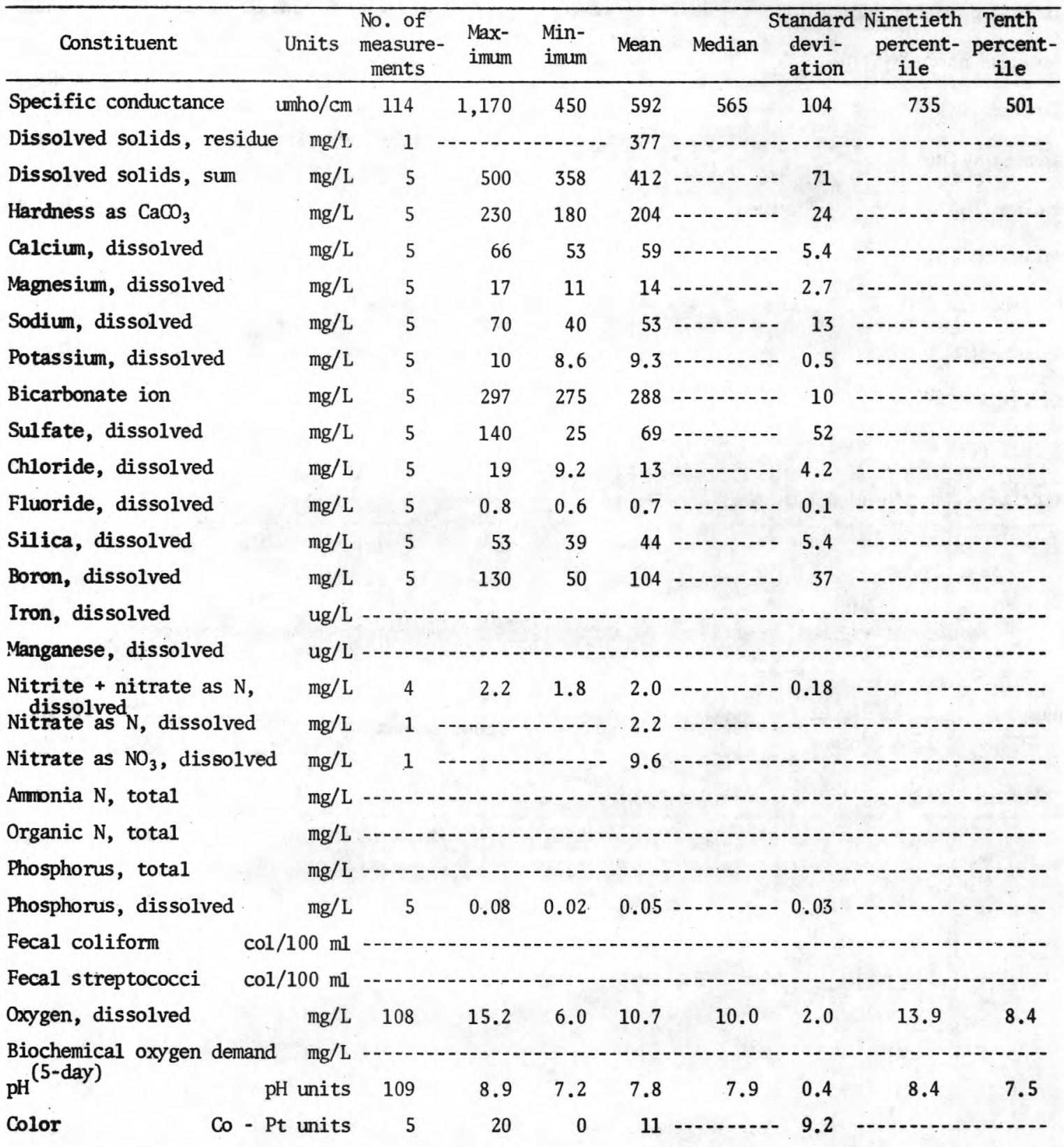


Results of regression analyses relating concentrations of selected chemical constituents to specific conductance $(\mathrm{SpC})$

\begin{tabular}{|c|c|c|c|c|c|}
\hline $\begin{array}{l}\text { Dissolved } \\
\text { constituents } \\
(\mathrm{mg} / \mathrm{L})\end{array}$ & $\begin{array}{l}\text { No. of } \\
\text { measure- } \\
\text { ments }\end{array}$ & Regression equation & $\begin{array}{l}\text { Corre- } \\
\text { lation } \\
\text { coeffi- } \\
\text { cient }\end{array}$ & $\begin{array}{l}\text { Percent } \\
\text { explained } \\
\text { variance }\end{array}$ & $\begin{array}{c}\text { Standard } \\
\text { error of } \\
\text { estimate } \\
(\mathrm{mg} / \mathrm{L})\end{array}$ \\
\hline Dissolved solids, residue (RDS) & $-\cdots$ & 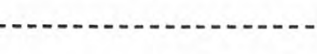 & - & $2-5-2$ & - \\
\hline Dissolved solids, sum (SDS) & $-\cdots-1$ & - - & 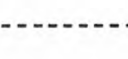 & 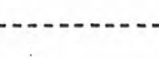 & -1 \\
\hline Hardness as $\mathrm{CaCO}_{3}(\mathrm{TH})$ & $\cdots-\cdots$ & 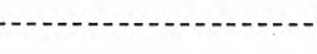 & thes & te- & $---2-1$ \\
\hline Calcium (Ca) & $-\ldots$ & - & $-\cdots$ & 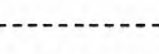 & 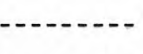 \\
\hline Magnesium (Mg) & $--1--1$ & 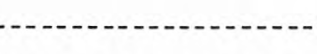 & & $-\cdots$ & 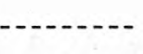 \\
\hline Sodium $(\mathrm{Na})$ & $\cdots-\cdots$ & 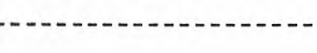 & $=--$ & $---n$ & $-\cdots$ \\
\hline Potassium (K) & 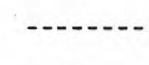 & - & $-\ldots$ & (n) & - \\
\hline Bicarbonate $\left(\mathrm{HCO}_{3}\right)$ & $-\cdots-1$ & 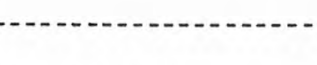 & $\ldots$ & $\cdots+\cdots$ & - n..... \\
\hline Sulfate $\left(\mathrm{SO}_{4}\right)$ & $-\cdots-1-1$ & 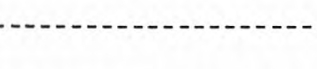 & . & 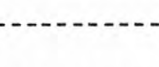 & 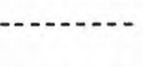 \\
\hline Chloride (Cl) & $-\cdots-1$ & - & $-\cdots-1$ & 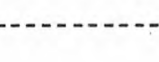 & 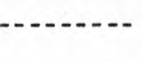 \\
\hline Silica (Si) & $-\cdots-1$ & (2) & 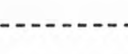 & - & \\
\hline Nitrite + Nitrate as $\mathrm{N}\left(\mathrm{NO}_{2}-\mathrm{NO}_{3}\right)$ & $-\cdots--$ & & & & \\
\hline
\end{tabular}

Results of regression analyses relating specific conductance to water discharge (Q)

\begin{tabular}{ccccccc}
\hline $\begin{array}{c}\text { No. of } \\
\text { measure- } \\
\text { ments }\end{array}$ & $\begin{array}{c}\text { Water discharge } \\
\left(\mathrm{ft}^{3} / \mathrm{s}\right)\end{array}$ & Maximum Minimum & Regression equation & $\begin{array}{c}\text { Correlation } \\
\text { coefficient }\end{array}$ & $\begin{array}{c}\text { Percent } \\
\text { explained } \\
\text { variance }\end{array}$ & $\begin{array}{c}\text { Standard error } \\
\text { of estimate }\end{array}$ \\
\hline 113 & $77 \quad 0.48$ & $\mathrm{SpC}=455 \mathrm{Q}^{0.09713}$ & 0.58 & 34 & 0.056 \\
\hline
\end{tabular}




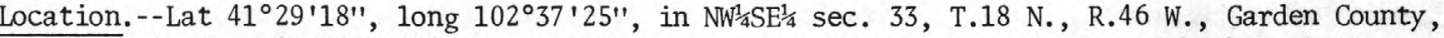
Hydrologic Unit 10180009, near right bank on downstream side of pier of highway bridge, $0.5 \mathrm{mi}$ south of Lisco.

Drainage area (revised) $--26,700 \mathrm{mi}^{2}$, approximately, of which about $24,700 \mathrm{mi}^{2}$ contributes directly to surface runoff. Approximately 4,000 $\mathrm{mi}^{2}$ in Great Divide basin is not included.

Period of record. --Water year 1970 to current year.

Statistical data for selected chemical constituents

\begin{tabular}{|c|c|c|c|c|c|c|c|c|c|}
\hline Constituent & Units & $\begin{array}{l}\text { No. of } \\
\text { measure- } \\
\text { ments }\end{array}$ & $\begin{array}{l}\text { Max- } \\
\text { imum }\end{array}$ & $\begin{array}{l}\text { Min- } \\
\text { imum }\end{array}$ & Mean & Median & $\begin{array}{c}\text { Standard } \\
\text { devi- } \\
\text { ation } \\
\end{array}$ & $\begin{array}{l}\text { Ninetieth } \\
\text { percent- } \\
\text { ile } \\
\end{array}$ & $\begin{array}{l}\text { Tenth } \\
\text { percent- } \\
\text { ile } \\
\end{array}$ \\
\hline Specific conductance & umho/cm & 263 & 1,230 & 640 & 887 & 898 & 90 & 994 & 758 \\
\hline Dissolved solids, residue & $\mathrm{mg} / \mathrm{L}$ & 25 & 670 & 482 & 601 & 610 & 50 & 663 & 516 \\
\hline Dissolved solids, sum & $\mathrm{mg} / \mathrm{L}$ & 65 & 658 & 458 & 584 & 590 & 48 & 638 & 520 \\
\hline Hardness as $\mathrm{CaCO}_{3}$ & $\mathrm{mg} / \mathrm{L}$ & 91 & 310 & 206 & 272 & 280 & 20 & 299 & 243 \\
\hline Calcium, dissolved & $\mathrm{mg} / \mathrm{L}$ & 90 & 90 & 53 & 77 & 78 & 7.2 & 85 & 65 \\
\hline Magnesium, dissolved & $\mathrm{mg} / \mathrm{L}$ & 90 & 23 & 14 & 20 & 20 & 1.3 & 22 & 18 \\
\hline Sodium, dissolved & $\mathrm{mg} / \mathrm{L}$ & 90 & 100 & 53 & 83 & 86 & 9.6 & 93 & 68 \\
\hline Potassium, dissolved & $\mathrm{mg} / \mathrm{L}$ & 90 & 20 & 6.0 & 9.9 & 10 & 1.8 & 12 & 7.6 \\
\hline Bicarbonate ion & $\mathrm{mg} / \mathrm{L}$ & 91 & 338 & 220 & 284 & 290 & 29 & 318 & 240 \\
\hline Sulfate, dissolved & $\mathrm{mg} / \mathrm{L}$ & 91 & 220 & 134 & 189 & 193 & 15 & 214 & 173 \\
\hline Chloride, dissolved & $\mathrm{mg} / \mathrm{L}$ & 91 & 29 & 12 & 20 & 21 & 2.6 & 23 & 17 \\
\hline Fluoride, dissolved & $\mathrm{mg} / \mathrm{L}$ & 38 & 0.7 & 0.3 & 0.5 & 0.5 & 0.1 & 0.6 & 0.5 \\
\hline Silica, dissolved & $\mathrm{mg} / \mathrm{L}$ & 67 & 42 & 13 & 35 & 37 & 7.1 & 42 & 24 \\
\hline Boron, dissolved & $\mathrm{mg} / \mathrm{L}$ & 37 & 190 & 90 & 138 & 140 & 26 & 175 & 101 \\
\hline Iron, dissolved & $\mathrm{ug} / \mathrm{L}$ & 27 & 140 & 0 & 37 & 28 & 39 & 82 & 0 \\
\hline Manganese, dissolved & $\mathrm{ug} / \mathrm{L}$ & 27 & 220 & 0 & 33 & 20 & 48 & 66 & 0 \\
\hline Nitrite + nitrate as $\mathrm{N}$, & $\mathrm{mg} / \mathrm{L}$ & 74 & 3.0 & 0.01 & 1.7 & 1.7 & 0.76 & 2.6 & 0.68 \\
\hline Nitrate as $N$, dissolved & $\mathrm{mg} / \mathrm{L}$ & 74 & 3.0 & 0.00 & 1.7 & 1.8 & 0.78 & 2.7 & 0.59 \\
\hline Nitrate as $\mathrm{NO}_{3}$, dissolved & $\mathrm{mg} / \mathrm{L}$ & & & & & & & & $\cdots$ \\
\hline Armonia $N$, total & $\mathrm{mg} / \mathrm{L}$ & 54 & 0.25 & 0.00 & 0.07 & 0.06 & 0.06 & 0.14 & 0.00 \\
\hline Organic $N$, total & $\mathrm{mg} / \mathrm{L}$ & 86 & 6.0 & 0.06 & 0.85 & 0.74 & 0.66 . & 1.3 & 0.38 \\
\hline Phosphorus, tota1 & $\mathrm{mg} / \mathrm{L}$ & 53 & 1.7 & 0.03 & 0.25 & 0.21 & 0.25 & 0.36 & 0.10 \\
\hline Phosphorus, dissolved & $\mathrm{mg} / \mathrm{L}$ & 83 & 0.14 & 0.00 & 0.06 & 0.06 & 0.03 & 0.10 & 0.02 \\
\hline Fecal coliform $\quad \mathrm{col} /$ & $\operatorname{col} / 100 \mathrm{ml}$ & 42 & 1,600 & 1 & - & $38-$ & & 350 & 2 \\
\hline Fecal streptococci $\mathrm{col} /$ & $\operatorname{col} / 100 \mathrm{ml}$ & 50 & 2,200 & 4 & - & $125-$ & & 723 & 41 \\
\hline Oxygen, dissolved & $\mathrm{mg} / \mathrm{L}$ & 222 & 15.2 & 6.8 & 9.9 & 9.6 & 1.8 & 12.5 & 7.7 \\
\hline \multirow{2}{*}{$\begin{array}{l}\text { Biochemical oxygen demand } \\
\mathrm{pH}^{(5-\text { day })}\end{array}$} & $\mathrm{mg} / \mathrm{L}$ & 90 & 10.0 & 0.5 & 2.5 & 2.3 & 1.6 & 4.3 & 1.1 \\
\hline & units & 263 & 9.0 & 7.1 & 7.8 & 7.8 & 0.4 & 8.3 & 7.4 \\
\hline $\mathrm{Co}-\mathrm{Pt}$ & t units & 85 & 120 & 0 & 14 & 8 & 16 & 29 & 3 \\
\hline
\end{tabular}


06686000 North Platte River at Lisco, Nebr.--Continued

Results of regression analyses relating concentrations of selected chemical constituents to specific conductance $(\mathrm{SpC})$

\begin{tabular}{|c|c|c|c|c|c|}
\hline $\begin{array}{c}\text { Dissolved } \\
\text { constituents } \\
(\mathrm{mg} / \mathrm{L})\end{array}$ & $\begin{array}{l}\text { No. of } \\
\text { measure- } \\
\text { ments }\end{array}$ & Regression equation & $\begin{array}{l}\text { Corre- } \\
\text { lation } \\
\text { coeffi- } \\
\text { cient }\end{array}$ & $\begin{array}{l}\text { Percent } \\
\text { explained } \\
\text { variance }\end{array}$ & $\begin{array}{c}\text { Standard } \\
\text { error of } \\
\text { estimate } \\
(\mathrm{mg} / \mathrm{L})\end{array}$ \\
\hline Dissolved solids, residue (RDS) & 24 & $\mathrm{RDS}=0.626(\mathrm{SpC})+64.033$ & 0.83 & 68 & 30 \\
\hline Dissolved solids, sum (SDS) & 66 & $\mathrm{SDS}=0.612(\mathrm{SpC})+53.755$ & .87 & 75 & 24 \\
\hline Hardness as $\mathrm{CaCO}_{3}(\mathrm{TH})$ & 91 & $\mathrm{TH}=0.194(\mathrm{SpC})+104.648$ & .69 & 47 & 15 \\
\hline Calcium (Ca) & 90 & $\mathrm{Ca}=0.071(\mathrm{SpC})+15.445$ & .72 & 51 & 5.1 \\
\hline Magnesium (Mg) & 90 & $\mathrm{Mg}=0.005(\mathrm{SpC})+15.118$ & .29 & 8.3 & 1.2 \\
\hline Sodium $(\mathrm{Na})$ & 90 & $\mathrm{Na}=0.109(\mathrm{SpC})-11.094$ & .82 & 68 & 5.4 \\
\hline Potassium (K) & 90 & $\mathrm{~K}=0.010(\mathrm{spC})+0.953$ & .42 & 17 & 1.6 \\
\hline Bicarbonate $\left(\mathrm{HCO}_{3}\right)$ & 91 & $\mathrm{HCO}_{3}=0.281(\mathrm{SpC})+40.225$ & .70 & 49 & 21 \\
\hline Sulfate $\left(\mathrm{SO}_{4}\right)$ & 91 & $\mathrm{SO}_{4}=0.149(\mathrm{SpC})+60.144$ & .70 & 49 & 11 \\
\hline Chloride (C1) & 91 & $\mathrm{Cl}=0.025(\mathrm{SpC})-1.528$ & .70 & 49 & 1.9 \\
\hline Silica (Si) & 67 & $\mathrm{Si}=0.072(\mathrm{SpC})-27.738$ & .70 & 49 & 5.1 \\
\hline Nitrite + Nitrate as $\mathrm{N}\left(\mathrm{NO}_{2}-\mathrm{NO}_{3}\right)$ & 74 & $\mathrm{NO}_{2}-\mathrm{NO}_{3}=0.007(\mathrm{SpC})-4.361$ & .65 & 42 & 0.58 \\
\hline
\end{tabular}

Results of regression analyses relating specific conductance to water discharge (Q)

\begin{tabular}{ccccccc}
\hline $\begin{array}{c}\text { No. of } \\
\text { measure- } \\
\text { ments }\end{array}$ & $\begin{array}{c}\text { Water discharge } \\
\left(\mathrm{ft}^{3} / \mathrm{s}\right)\end{array}$ & Maximum Minimum & Regression equation & $\begin{array}{c}\text { Correlation } \\
\text { coefficient }\end{array}$ & $\begin{array}{c}\text { Percent } \\
\text { explained } \\
\text { variance }\end{array}$ & $\begin{array}{c}\text { Standard error } \\
\text { of estimate }\end{array}$ \\
\hline 262 & $9,600 \quad 263$ & $\mathrm{SpC}=1,326 \mathrm{Q}^{-0.05577}$ & -0.29 & 8.7 & 0.047 & 11 \\
\hline
\end{tabular}


Location. --Lat $41^{\circ} 12^{\prime} 30^{\prime \prime}$, long $101^{\circ} 37^{\prime} 50^{\prime \prime}$, in SW sec. 1, T.14 N., R.38 W., Keith County, Hydrologic Unit 10180014 , on right bank $0.2 \mathrm{mi}$ downstream from diversion dam of Sutherland Reservoir supply canal and $2.5 \mathrm{mi}$ southwest of Keystone.

Drainage area (revised) $.--29,300 \mathrm{mi}^{2}$, approximately, of which about 25,800 $\mathrm{mi}^{2}$ contributes directly to surface runoff. Approximately 4,000 $\mathrm{mi}^{2}$ in Great Divide basin is not included.

Period of record.--Water year 1973 to current year.

Statistical data for selected chemical constituents

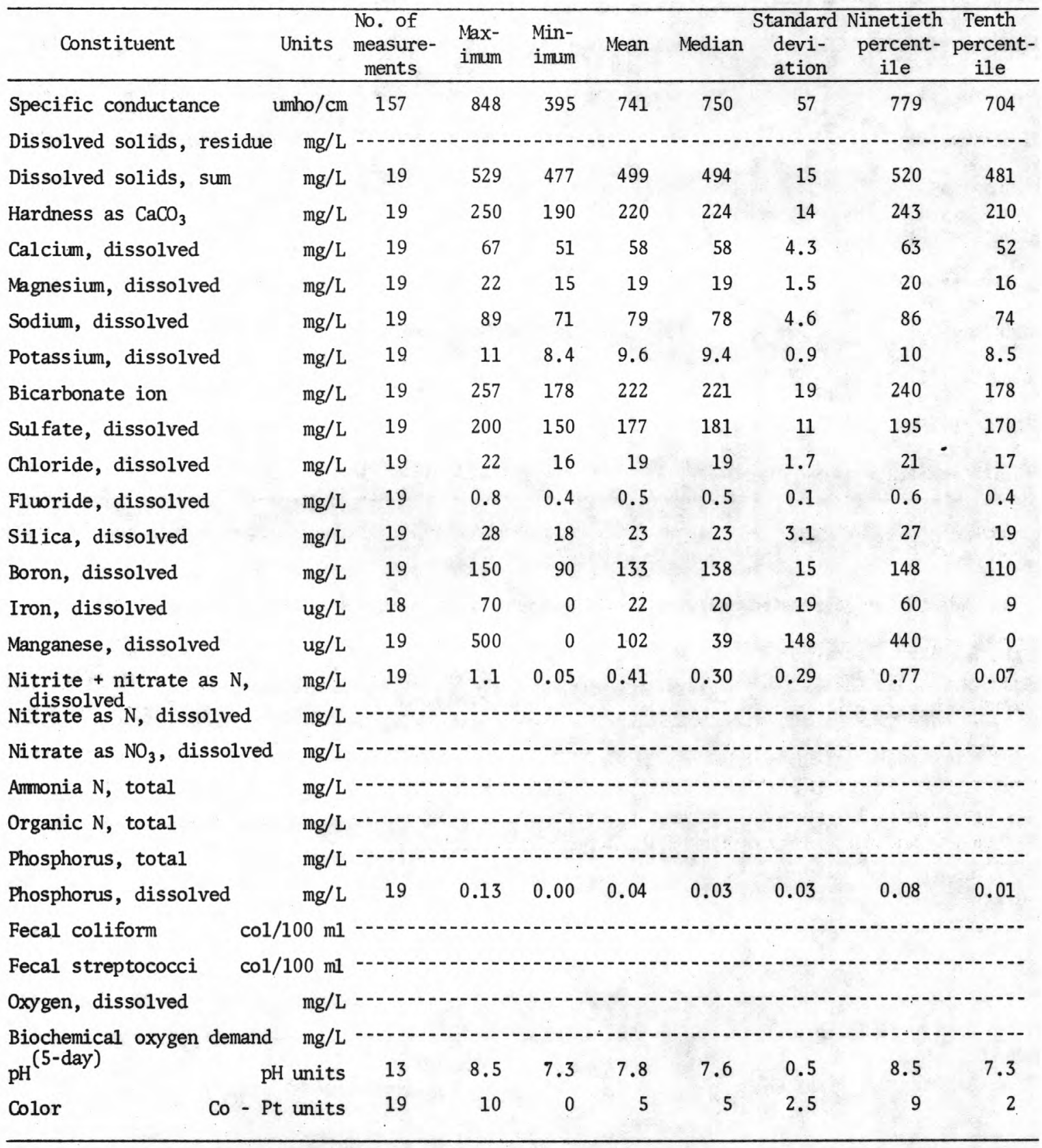


06690500 North Platte River near Keystone, Nebr.--Continued

Results of regression analyses relating concentrations of selected chemical constituents to specific conductance $(\mathrm{SpC})$

\begin{tabular}{|c|c|c|c|c|c|}
\hline $\begin{array}{l}\text { Dissolved } \\
\text { constituents } \\
(\mathrm{mg} / \mathrm{L})\end{array}$ & $\begin{array}{c}\text { No. of } \\
\text { measure- } \\
\text { ments }\end{array}$ & Regression equation & $\begin{array}{l}\text { Corre- } \\
\text { lation } \\
\text { coeffi- } \\
\text { cient }\end{array}$ & $\begin{array}{l}\text { Percent } \\
\text { explained } \\
\text { variance }\end{array}$ & $\begin{array}{c}\text { Standard } \\
\text { error of } \\
\text { estimate } \\
(\mathrm{mg} / \mathrm{L})\end{array}$ \\
\hline Dissolved solids, residue (RDS) & & & & & \\
\hline Dissolved solids, sum (SDS) & 19 & $\mathrm{SDS}=0.233(\mathrm{SpC})+318.663$ & 0.47 & 22 & 14 \\
\hline Hardness as $\mathrm{CaCO}_{3}(\mathrm{TH})$ & 19 & $\mathrm{TH}=0.009(\mathrm{SpC})+213.253$ & $* .02$ & 0.04 & 14 \\
\hline Calcium (Ca) & 19 & $\mathrm{Ca}=-0.013(\mathrm{SpC})+67.988$ & $*_{-} .10$ & .92 & 4.4 \\
\hline Magnesium (Mg) & 19 & $M g=0.004(S p C)+15.365$ & $* .08$ & .73 & 1.5 \\
\hline Sodium $(\mathrm{Na})$ & 19 & $\mathrm{Na}=0.066(\mathrm{SpC})+27.957$ & $* .43$ & 18 & 4.3 \\
\hline Potassium $(\mathrm{K})$ & 19 & $K=0.007(\mathrm{SpC})+4.014$ & $* .24$ & 5.8 & 0.9 \\
\hline Bicarbonate $\left(\mathrm{HCO}_{3}\right)$ & 19 & $\mathrm{HCO}_{3}=-0.174(\mathrm{SpC})+357.735$ & $*_{-} .28$ & 7.7 & 19 \\
\hline Sulfate $\left(\mathrm{SO}_{4}\right)$ & 19 & $\mathrm{SO}_{4}=0.193(\mathrm{SpC})+27.840$ & .53 & 28 & 9.5 \\
\hline Chloride (C1) & 19 & $\mathrm{Cl}=0.020(\mathrm{SpC})+3.009$ & *.35 & 12 & 1.7 \\
\hline Silica (Si) & 19 & $\mathrm{Si}=0.069(\mathrm{SpC})-30.985$ & .67 & 45 & 2.4 \\
\hline Nitrite + Nitrate as $\mathrm{N}\left(\mathrm{NO}_{2}-\mathrm{NO}_{3}\right)$ & 19 & $\mathrm{NO}_{2}-\mathrm{NO}_{3}=0.002(\mathrm{SpC})-1.374$ & *.24 & 5.9 & 0.29 \\
\hline
\end{tabular}

*Not significant at the 95 percent confidence level; the regression equation should not be used to predict the concentration of the dependent variable.

Results of regression analyses relating specific conductance to water discharge (Q)

\begin{tabular}{cccccccc}
\hline $\begin{array}{c}\text { No. of } \\
\text { measure- } \\
\text { ments }\end{array}$ & $\begin{array}{c}\text { Water discharge } \\
\text { Maximum Minimum }\end{array}$ & Regression equation & $\begin{array}{c}\text { Correlation } \\
\text { coefficient }\end{array}$ & $\begin{array}{c}\text { Percent } \\
\text { explained } \\
\text { variance }\end{array}$ & \multicolumn{2}{c}{$\begin{array}{c}\text { Standard error } \\
\text { of estimate }\end{array}$} \\
\hline 135 & $3,760 \quad 2.00$ & SpC $=756 Q^{-0.00440}$ & $*_{-0.11}$ & 1.1 & 0.045 & 10 \\
\hline
\end{tabular}

* Not significant at the 95 percent confidence level; the regression equation should not be used to predict the concentration of the dependent variable. 
The South Platte River basin in Nebraska lies mostly in western Nebraska, extending from the Wyoming and Colorado borders to the confluence of the North and South Platte Rivers in Lincoln County. Over half the basin in Nebraska is comprised of Lodgepole Creek drainage.

About 34,000 acres in the South Platte River and Lodgepole Creek valleys are irrigated by surface water diverted from both Lodgepole Creek and the South Platte River.

Descriptive statistics are available for four stations in the South P1atte River basin (fig. 6).

Specific conductance averaged $1,730 \mathrm{umho} / \mathrm{cm}$ at the South Platte River at Julesburg, Colo., between 1945 and 1969. At the South Platte River at Roscoe during the period 1975 to the present, the mean specific conductance is $1,890 u$ mho/ $\mathrm{cm}$. Sodium and calcium are the principal. cations and sulfate is the principal anion at both South Platte River stations. At Lodgepole Creek at Kimball, the mean specific conductance is 644 umho/cm. Calcium is the principal cation and bicarbonate is the principal anion.

Water in the South Platte River is the most highly mineralized water moving across any border of Nebraska. It is heavily used and reused for irrigation along its entire course across northeastern Colorado. Some dissolved chemical constituents greatly increase in concentration during the reuse. This is particularly true of sulfate, sodium, and chloride which are highly conservative constituents that tend to remain in solution once they are dissolved. On the other hand, calcium and bicarbonate are limited by solubility controls so that they do not increase in concentration very much.

Mean specific conductance is 9 percent higher at Roscoe than at Julesburg. Different periods of record may account for the increase. Use and recycling of river water for irrigation increased steadily in Colorado and Nebraska during 1949-69, the period of record for the South P1atte River at Julesburg. Increased water use since 1969 may have been accompanied by increased mineralization in the stream.and may account for the 9 percent increase between Julesburg and Roscoe.

Streamflow in Lodgepole Creek is mostly overland runoff. Low rainfall combined with diversion for irrigation causes much of Lodgepole Creek to be dry during summer months. Data for the station at Kimball probably are not completely representative of water quality in Lodgepole 

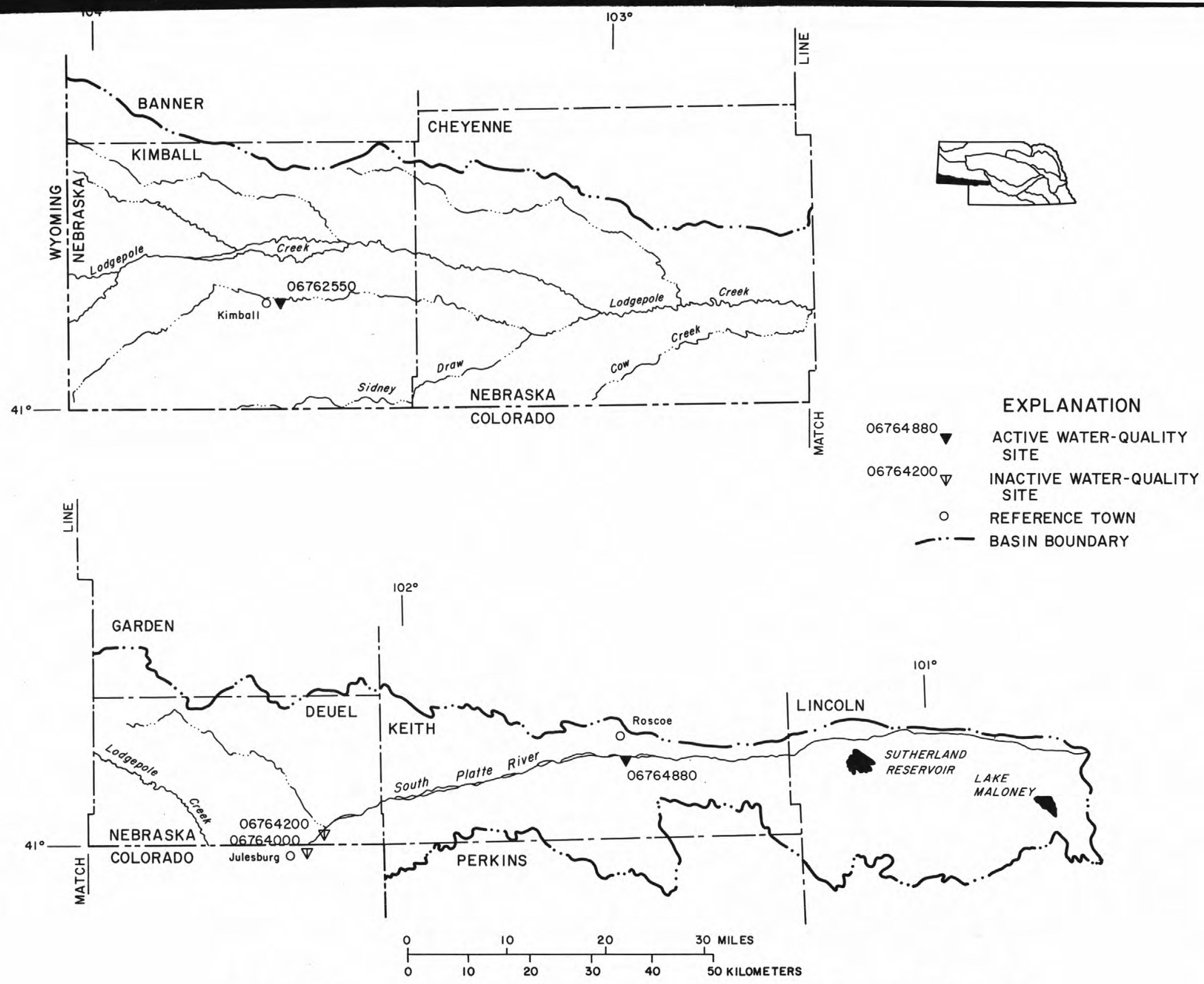

FIGURE 6.-Location of water-quality sampling sites, South Platte River basin 
Creek because of the proximity of the station to the sewage outfall from Kimball. At times during the summer, the only flow at the station may be effluent. Higher than normal concentrations of chloride, nitrite + nitrate, phosphorus and BOD are detected then. A1so higher concentrations of fecal coliform and fecal streptococcal bacteria are detected at these times.

Regression equations were developed between specific conductance and all 12 variables for the 2 Julesburg stations and the Lodgepole Creek station. Correlation coefficients are above 0.80 for most of the principal constituents. Data are sufficient only for regression equations between dissolved-solids residue and specific conductance and between chloride and specific conductance for the Roscoe station. 
SOUTH PLATTE RIVER BASIN

06762550 Lodgepole Creek at Kimball, Nebr.

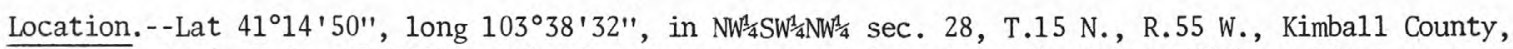
Hydrologic Unit 10190016, at bridge on county road $0.8 \mathrm{mi}$ north of U.S. Highway 30 at east edge of Kimball.

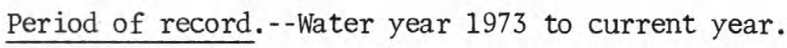

Statistical data for selected chemical constituents

\begin{tabular}{|c|c|c|c|c|c|c|c|c|c|}
\hline Constituent & Units & $\begin{array}{l}\text { No. of } \\
\text { measure- } \\
\text { ments }\end{array}$ & $\begin{array}{l}\text { Max- } \\
\text { imum }\end{array}$ & $\begin{array}{l}\text { Min- } \\
\text { imum }\end{array}$ & Mean & Median & $\begin{array}{c}\text { Standard } \\
\text { devi- } \\
\text { ation }\end{array}$ & $\begin{array}{l}\text { Ninetieth } \\
\text { percent- } \\
\text { ile } \\
\end{array}$ & $\begin{array}{l}\text { Tenth } \\
\text { percent- } \\
\text { ile }\end{array}$ \\
\hline Specific conductance & $\mathrm{umho} / \mathrm{cm}$ & 55 & 1,060 & 480 & 644 & 597 & 136 & 880 & 520 \\
\hline Dissolved solids, residue & $\mathrm{mg} / \mathrm{L}$ & 33 & 604 & 320 & 402 & 385 & 71 & 490 & 338 \\
\hline Dissolved solids, sum & $\mathrm{mg} / \mathrm{L}$ & 19 & 538 & 313 & 388 & $-\ldots \ldots$ & 64 & $\cdots \ldots$ & $-\cdots$ \\
\hline Hardness as $\mathrm{CaCO}_{3}$ & $\mathrm{mg} / \mathrm{L}$ & 18 & 310 & 190 & 220 & 220 & 26 & 240 & 200 \\
\hline Calcium, dissolved & $\mathrm{mg} / \mathrm{L}$ & 18 & 87 & 53 & 62 & 63 & 7.9 & 70 & 55 \\
\hline Magnesium, dissolved & $\mathrm{mg} / \mathrm{L}$ & 18 & 23 & 13 & 16 & 16 & 2.2 & 18 & 15 \\
\hline Sodium, dissolved & $\mathrm{mg} / \mathrm{L}$ & 18 & 72 & 26 & 38 & 37 & 10 & 49 & 29 \\
\hline Potassium, dissolved & $\mathrm{mg} / \mathrm{L}$ & 18 & 15 & 7.0 & 9.5 & 8.9 & 2.3 & 14 & 7.3 \\
\hline Bicarbonate ion & $\mathrm{mg} / \mathrm{L}$ & 18 & 299 & 234 & 264 & 270 & 22 & 290 & 238 \\
\hline Sulfate, dissolved & $\mathrm{mg} / \mathrm{L}$ & 18 & 140 & 30 & 46 & 41 & 25 & 69 & 31 \\
\hline Chloride, dissolved & $\mathrm{mg} / \mathrm{L}$ & 51 & 140 & 19 & 35 & 28 & 21 & 48 & 22 \\
\hline Fluoride, dissolved & $\mathrm{mg} / \mathrm{L}$ & 17 & 1.0 & 0.7 & 0.8 & 1.1 & 0.1 & 0.9 & 0.0 \\
\hline Silica, dissolved & $\mathrm{mg} / \mathrm{L}$ & 18 & 48 & 32 & 41 & 42 & 4.6 & 47 & 35 \\
\hline Boron, dissolved & $\mathrm{mg} / \mathrm{L}$ & 18 & 360 & 80 & 151 & 145 & 68 & 270 & 90 \\
\hline Iron, dissolved & $\mathrm{ug} / \mathrm{L}$ & 18 & 370 & 0 & 66 & 40 & 86 & 160 & 10 \\
\hline Manganese, dissolved & $\mathrm{ug} / \mathrm{L}$ & 18 & 60 & 0 & 22 & 20 & 17 & 50 & 0 \\
\hline $\begin{array}{l}\text { Nitrite }+ \text { nitrate as } N \text {, } \\
\text { dissolved } \\
\text { Nitrate as } N \text {, dissolved }\end{array}$ & $\begin{array}{l}\mathrm{mg} / \mathrm{L} \\
\mathrm{mg} / \mathrm{L}\end{array}$ & 27 & 4.7 & 1.1 & 2.2 & 2.2 & 0.82 & 2.9 & 1.3 \\
\hline Nitrate as $\mathrm{NO}_{3}$, dissolved & $\mathrm{mg} / \mathrm{L}$ & & & & & & & & \\
\hline Ammonia $\mathrm{N}$, total & $\mathrm{mg} / \mathrm{L}$ & 50 & 9.8 & 0.02 & 1.7 & 0.82 & 2.2 & 3.9 & 0.22 \\
\hline Organic $N$, total & $\mathrm{mg} / \mathrm{L}$ & 50 & 11 & 0.00 & 1.7 & 1.2 & 2.0 & 2.2 & 0.58 \\
\hline Phosphorus, total & $\mathrm{mg} / \mathrm{L}$ & 50 & 16 & 0.36 & 2.0 & 0.81 & 2.6 & 4.4 & 0.42 \\
\hline Phosphorus, dissolved & $\mathrm{mg} / \mathrm{L}$ & 44 & 6.6 & 0.24 & 1.2 & 0.64 & 1.5 & 3.7 & 0.33 \\
\hline Fecal coliform $\mathrm{col} /$ & $\mathrm{col} / 100 \mathrm{ml}$ & 43 & 180,000 & 170 & - & 8,400 & 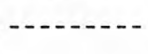 & $-58,000$ & 920 \\
\hline Fecal streptococci & $\operatorname{col} / 100 \mathrm{ml}$ & 47 & 140,000 & 120 & $-\ldots-\ldots$ & 4,200 & 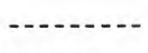 & $-15,000$ & 260 \\
\hline Oxygen, dissolved & $\mathrm{mg} / \mathrm{L}$ & 51 & 16.4 & 2.6 & 10.5 & 10.1 & 2.7 & 13.5 & 6.8 \\
\hline \multirow{2}{*}{$\begin{array}{l}\text { Biochemical oxygen demand } \\
\text { ( } 5 \text {-day) }\end{array}$} & $\mathrm{mg} / \mathrm{L}$ & 48 & 49 & 2.0 & 11 & 8.1 & 9.4 & 22 & 3.2 \\
\hline & H units & 53 & 8.5 & 7.0 & 7.7 & 7.8 & 0.3 & 8.2 & 7.3 \\
\hline $\mathrm{Co}-\mathrm{Pt}$ & $t$ units & 17 & 40 & 5 & 20 & 20 & 9.9 & 35 & 0 \\
\hline
\end{tabular}


Results of regression analyses relating concentrations of selected chemical constituents to specific conductance $(\mathrm{SpC})$

\begin{tabular}{|c|c|c|c|c|c|}
\hline $\begin{array}{l}\text { Dissolved } \\
\text { constituents } \\
(\mathrm{mg} / \mathrm{L})\end{array}$ & $\begin{array}{c}\text { No. of } \\
\text { measure- } \\
\text { ments }\end{array}$ & Regression equation & $\begin{array}{l}\text { Corre- } \\
\text { lation } \\
\text { coeffi- } \\
\text { cient }\end{array}$ & $\begin{array}{l}\text { Percent } \\
\text { explained } \\
\text { variance }\end{array}$ & $\begin{array}{c}\text { Standard } \\
\text { error of } \\
\text { estimate } \\
(\mathrm{mg} / \mathrm{L})\end{array}$ \\
\hline Dissolved solids, residue (RDS) & 34 & $\mathrm{RDS}=0.464(\mathrm{SpC})+107.629$ & 0.95 & 90 & 22 \\
\hline Dissolved solids, sum (SDS) & 19 & $\mathrm{SDS}=0.500(\mathrm{SpC})+75.966$ & .95 & 90 & 21 \\
\hline Hardness as $\mathrm{CaCO}_{3}(\mathrm{TH})$ & 19 & $\mathrm{TH}=0.107(\mathrm{SpC})+152.560$ & .49 & 24 & 23 \\
\hline Calcium (Ca) & 19 & $\mathrm{Ca}=0.030(\mathrm{SpC})+43.209$ & .46 & 21 & 7.2 \\
\hline Magnesium (Mg) & 19 & $\mathrm{Mg}=0.008(\mathrm{SpC})+10.915$ & .46 & 21 & 2.0 \\
\hline Sodium (Na) & 19 & $\mathrm{Na}=0.121(\mathrm{SpC})-34.131$ & .87 & 75 & 8.6 \\
\hline Potassium (K) & 19 & $\mathrm{~K}=0.22(\mathrm{SpC})-3.997$ & .90 & 82 & 1.3 \\
\hline Bicarbonate $\left(\mathrm{HCO}_{3}\right)$ & 19 & $\mathrm{HCO}_{3}=0.109(\mathrm{SpC})+197.104$ & .57 & 33 & 19 \\
\hline Sulfate $\left(\mathrm{SO}_{4}\right)$ & 19 & $\mathrm{SO}_{4}=0.154(\mathrm{SpC})-49.709$ & .76 & 58 & 16 \\
\hline Chloride (C1) & 53 & $\mathrm{Cl}=0.144(\mathrm{SpC})-55.919$ & .88 & 78 & 11 \\
\hline Silica (Si) & 19 & $\mathrm{Si}=0.017(\mathrm{SpC})+30.359$ & *.44 & 20 & 4.3 \\
\hline Nitrite + Nitrate as $\mathrm{N}\left(\mathrm{NO}_{2}-\mathrm{NO}_{3}\right)$ & 27 & $\mathrm{NO}_{2}-\mathrm{NO}_{3}=0.003(\mathrm{SpC})+0.317$ & *.25 & 6.1 & 0.81 \\
\hline
\end{tabular}

* Not significant at the 95 percent confidence level; the regression equation should not be used to predict the concentration of the dependent variable.

Results of regression analyses relating specific conductance to water discharge (Q)

\begin{tabular}{|c|c|c|c|c|c|c|c|}
\hline \multirow{2}{*}{$\begin{array}{c}\text { No. of } \\
\text { measure- } \\
\text { ments }\end{array}$} & \multicolumn{2}{|c|}{$\begin{array}{l}\text { Water discharge } \\
\left(\mathrm{ft}^{3} / \mathrm{s}\right)\end{array}$} & \multirow{2}{*}{ Regression equation } & \multirow{2}{*}{$\begin{array}{l}\text { Correlation } \\
\text { coefficient }\end{array}$} & \multirow{2}{*}{$\begin{array}{l}\text { Percent } \\
\text { explained } \\
\text { variance }\end{array}$} & \multicolumn{2}{|c|}{$\begin{array}{l}\text { Standard error } \\
\text { of estimate }\end{array}$} \\
\hline & Maximum & Minimum & & & & Log units & Percent \\
\hline 55 & 15 & 0.31 & $\mathrm{SpC}=730 \mathrm{Q}^{-0.11628}$ & -0.67 & 45 & 0.062 & 14 \\
\hline
\end{tabular}


Location. - -Lat $40^{\circ} 58^{\prime} 46^{\prime \prime}$, long $102^{\circ} 15^{\prime} 15^{\prime \prime}$, in $\mathrm{NW}^{\frac{1}{4}} \mathrm{NE}^{\frac{1}{4}}$ and $\mathrm{SE}^{\frac{1}{4}} \mathrm{NE}^{\frac{1}{4}}$ (two channels) sec. 33 , T. $12 \mathrm{~N} .$, R.44 W., Sedgwick County, at gaging station at bridge on U.S. Highway 385, 0.9 mi southeast of Julesburg, $3 \mathrm{mi}$ upstream from Colorado-Nebraska State 1ine, and $8 \mathrm{mi}$ downstream from Lodgepole Creek.

Drainage area.--23,138 sq $\mathrm{mi}$.

Period of record.--October 1945 to September 1969.

Statistical data for selected chemical constituents

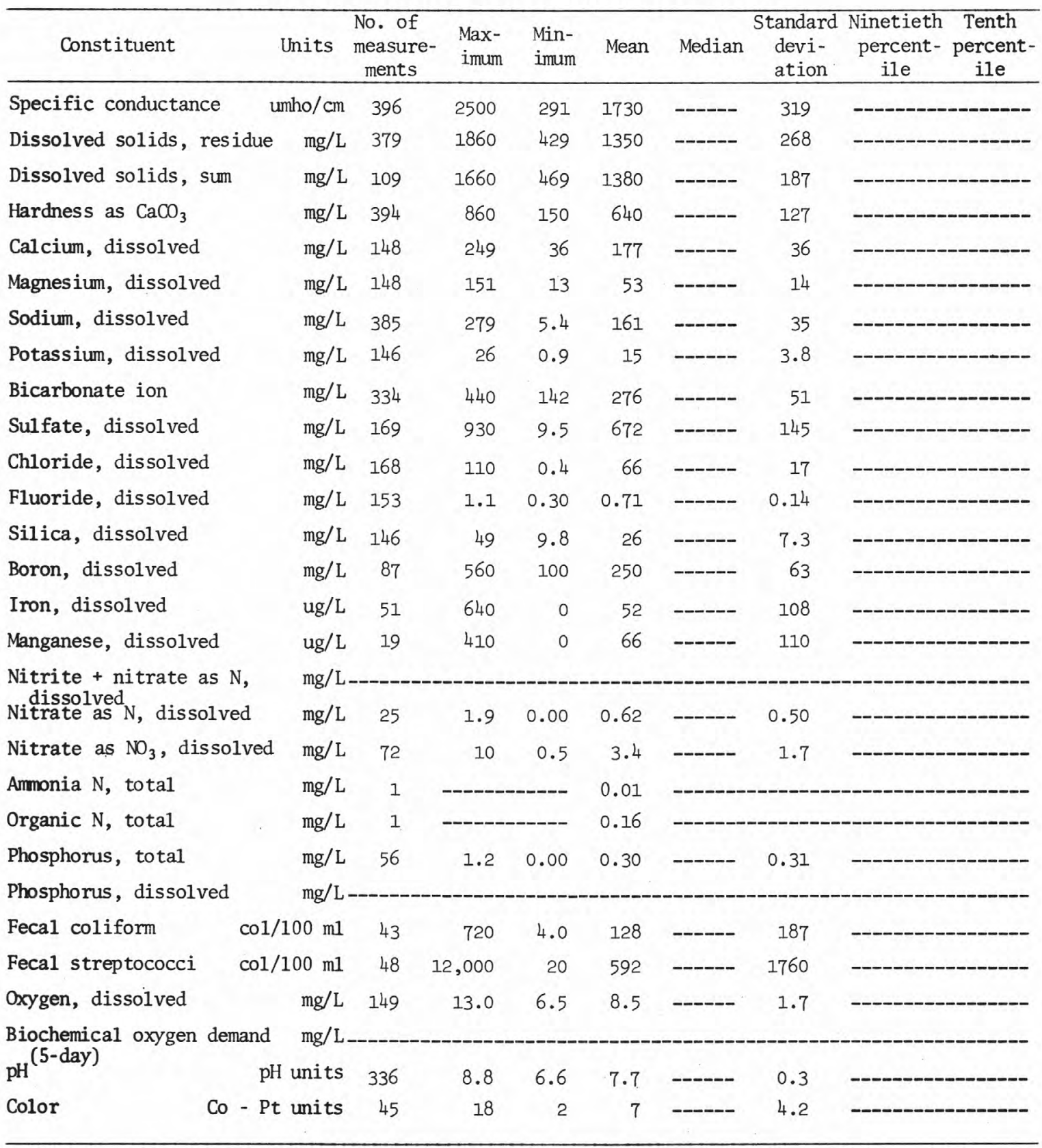


Results of regression analyses relating concentrations of selected chemical constituents to specific conductance $(\mathrm{SpC})$

\begin{tabular}{|c|c|c|c|c|c|}
\hline $\begin{array}{l}\text { Dissolved } \\
\text { constituents } \\
(\mathrm{mg} / \mathrm{L})\end{array}$ & $\begin{array}{l}\text { No. of } \\
\text { measure- } \\
\text { ments }\end{array}$ & Regression equation & $\begin{array}{l}\text { Corre- } \\
\text { lation } \\
\text { coeffi- } \\
\text { cient }\end{array}$ & $\begin{array}{l}\text { Percent } \\
\text { explained } \\
\text { variance }\end{array}$ & $\begin{array}{l}\text { Standard } \\
\text { error of } \\
\text { estimate } \\
(\mathrm{mg} / \mathrm{L})\end{array}$ \\
\hline Dissolved solids, residue (RDS) & 379 & $\mathrm{RDS}=0.837(\mathrm{SpC})-105.550$ & 0.98 & 95 & \\
\hline Dissolved solids, sum (SDS) & 110 & $\mathrm{SDS}=0.707(\mathrm{Spc})+76.068$ & .91 & 83 & \\
\hline Hardness as $\mathrm{CaCO}_{3}(\mathrm{TH})$ & 390 & $\mathrm{TH}=0.389(\mathrm{SpC})-34.578$ & .97 & 94 & \\
\hline Calcium (Ca) & 148 & $\mathrm{Ca}=0.097(\mathrm{SpC})+4.242$ & .88 & 78 & \\
\hline Magnesium (Mg) & 148 & $\mathrm{Mg}=0.032(\mathrm{SpC})-3.856$ & .75 & 56 & \\
\hline Sodium (Na) & 381 & $\mathrm{Na}=0.105(\mathrm{SpC})-20.875$ & .96 & 93 & \\
\hline Potassium (K) & 146 & $\mathrm{~K}=0.007(\mathrm{SpC})+3.529$ & .57 & 32 & \\
\hline Bicarbonate $\left(\mathrm{HCO}_{3}\right)$ & 334 & $\mathrm{HCO}_{3}=0.115(\mathrm{SpC})+76.412$ & .74 & 54 & \\
\hline Sulfate $\left(\mathrm{SO}_{4}\right)$ & 169 & $\mathrm{so}_{4}=0.425(\mathrm{spC})-77.642$ & .95 & 91 & \\
\hline Chloride (C1) & 168 & $\mathrm{Cl}=0.045(\mathrm{Spc})-12.774$ & .85 & 72 & \\
\hline Silica (Si) & 146 & $\mathrm{Si}=0.005(\mathrm{SpC})+16.478$ & .21 & 4.6 & \\
\hline Nitrite + Nitrate as $\mathrm{N}\left(\mathrm{NO}_{2}-\mathrm{NO}_{3}\right)$ & 24 & $\mathrm{NO}_{2}-\mathrm{NO}_{3}=0.00005(\mathrm{SpC})+0.528$ & $* .04$ & .16 & \\
\hline
\end{tabular}

*Not significant at the 95 percent confidence level; the regression equation should not be used to predict the concentration of the dependent variable.

Results of regression analyses relating specific conductance to water discharge (Q)

\begin{tabular}{|c|c|c|c|c|c|c|c|}
\hline \multirow{2}{*}{$\begin{array}{l}\text { No. of } \\
\text { measure- } \\
\text { ments }\end{array}$} & \multicolumn{2}{|c|}{$\begin{array}{l}\text { Water discharge } \\
\left(\mathrm{ft}^{3} / \mathrm{s}\right)\end{array}$} & \multirow{2}{*}{ Regression equation } & \multirow{2}{*}{$\begin{array}{l}\text { Correlation } \\
\text { coefficient }\end{array}$} & \multirow{2}{*}{$\begin{array}{c}\text { Percent } \\
\text { explained } \\
\text { variance }\end{array}$} & \multicolumn{2}{|c|}{$\begin{array}{c}\text { Standard error } \\
\text { of estimate }\end{array}$} \\
\hline & Maximum & Minimum & & & & Log units & Percent \\
\hline 395 & 18,500 & 9.7 & $\mathrm{SpC}=2316 \mathrm{Q}^{-.05775}$ & -0.36 & 13 & $--\cdots$ & $-\infty-\infty$ \\
\hline
\end{tabular}




\section{South Platte River near Julesburg, Colo.}

Location.--Lat $41^{\circ} 00^{\prime} 59^{\prime \prime}$, long $102^{\circ} 10^{\prime} 34^{\prime \prime}$, in $\mathrm{SE}^{\frac{1}{4}} \mathrm{NW} \mathrm{W}^{\frac{1}{4}} \mathrm{sec} .13$, T.12 N., R.43 W., Deuel County, Nebr. at diversion to Western Canal about $1.7 \mathrm{mi}$ downstream from Colorado-Nebraska State line, $4.7 \mathrm{mi}$ downstream from gaging station at Julesburg, and about $6 \mathrm{mi}$ northeast of Julesburg.

Drainage area.--23,200 $\mathrm{mi}^{2}$, approximately.

Period of record.--July 1969 to June 1973.

Statistical data for selected chemical constituents

\begin{tabular}{|c|c|c|c|c|c|c|c|c|c|}
\hline Constituent & Units & $\begin{array}{l}\text { No. of } \\
\text { measure- } \\
\text { ments }\end{array}$ & $\begin{array}{l}\text { Max- } \\
\text { imum }\end{array}$ & $\begin{array}{l}\text { Min- } \\
\text { imum }\end{array}$ & Mean & Median & $\begin{array}{c}\text { Standard } \\
\text { devi- } \\
\text { ation } \\
\end{array}$ & $\begin{array}{l}\text { Ninetieth } \\
\text { percent- } \\
\text { ile } \\
\end{array}$ & $\begin{array}{l}\text { Tenth } \\
\text { percent- } \\
\text { ile }\end{array}$ \\
\hline Specific conductance & umho/cm & 48 & 2160 & 897 & 1760 & 1830 & 274 & 2020 & 1300 \\
\hline Dissolved solids, residue & $\mathrm{mg} / \mathrm{L}$ & 35 & 1670 & 672 & 1380 & 1420 & 229 & 1600 & 1100 \\
\hline Dissolved solids, sum & $\mathrm{mg} / \mathrm{L}$ & 20 & 1550 & 1050 & 1360 & 1370 & 142 & 1560 & 1120 \\
\hline Hardness as $\mathrm{CaCO}_{3}$ & $\mathrm{mg} / \mathrm{L}$ & 40 & 770 & 340 & 652 & 680 & 102 & 740 & 510 \\
\hline Calcium, dissolved & $\mathrm{mg} / \mathrm{L}$ & 37 & 210 & 100 & 173 & 188 & 28 & 205 & 130 \\
\hline Magnesium, dissolved & $\mathrm{mg} / \mathrm{L}$ & 37 & 68 & 31 & 54 & 56 & 7.0 & 61 & 47 \\
\hline Sodium, dissolved & $\mathrm{mg} / \mathrm{L}$ & 21 & 208 & 140 & 176 & 180 & 20 & 200 & 152 \\
\hline Potassium, dissolved & $\mathrm{mg} / \mathrm{L}$ & 22 & 24 & 9.4 & 16 & 16 & 3.2 & 20 & 12 \\
\hline Bicarbonate ion & $\mathrm{mg} / \mathrm{L}$ & 32 & 358 & 181 & 279 & 278 & 48 & 327 & 200 \\
\hline Sulfate, dissolved & $\mathrm{mg} / \mathrm{L}$ & 40 & 810 & 283 & 657 & 678 & 118 & 782 & 530 \\
\hline Chloride, dissolved & $\mathrm{mg} / \mathrm{L}$ & 40 & 88 & 30 & 71 & 72 & 12 & 84 & 61 \\
\hline Fluoride, dissolved & $\mathrm{mg} / \mathrm{L}$ & 22 & 2.0 & 0.6 & 1.0 & 0.9 & 0.3 & 1.4 & 0.7 \\
\hline Silica, dissolved & $\mathrm{mg} / \mathrm{L}$ & 21 & 46 & 13 & 24 & 23 & 7.0 & 30 & 17 \\
\hline Boron, dissolved & $\mathrm{mg} / \mathrm{L}$ & 21 & 450 & 220 & 280 & 267 & 48 & 320 & 240 \\
\hline Iron, dissolved & $\mathrm{ug} / \mathrm{L}$ & 21 & 340 & 10 & 84 & 37 & 88 & 150 & 15 \\
\hline Manganese, dissolved & $\mathrm{ug} / \mathrm{L}$ & 18 & 600 & 10 & 98 & 60 & 133 & 180 & 20 \\
\hline $\begin{array}{l}\text { Nitrite + nitrate as } \mathrm{N} \text {, } \\
\text { dissolved }\end{array}$ & $\mathrm{mg} / \mathrm{L}$ & 25 & 4.7 & 0.01 & 1.8 & 1.6 & 1.2 & 3.4 & 0.60 \\
\hline Nitrate as $N$, dissolved & $\mathrm{mg} / \mathrm{L}$ & 23 & 5.1 & 0.06 & 2.1 & 2.1 & 1.3 & 3.8 & 0.61 \\
\hline $\begin{array}{l}\text { Nitrate as } \mathrm{NO}_{3} \text {, dissolved } \\
\text { Ammonia } \mathrm{N} \text {, total }\end{array}$ & $\begin{array}{l}\mathrm{mg} / \mathrm{L}- \\
\mathrm{mg} / \mathrm{L}\end{array}$ & & & & & & & & - \\
\hline Organic $N$, total & & & & & & & & & - \\
\hline Phosphorus, total & $\mathrm{mg} / \mathrm{L}$ & 36 & 5.0 & 0.05 & 0.58 & 0.36 & 0.82 & 1.0 & 0.12 \\
\hline Phosphorus, dissolved & $\mathrm{mg} / \mathrm{L}$ & 33 & 0.63 & 0.02 & 0.23 & 0.15 & 0.19 & 0.52 & 0.05 \\
\hline Fecal coliform & $1 / 100 \mathrm{ml}$ & & & & & & & & -- \\
\hline Fecal streptococci & $1 / 100 \mathrm{ml}$ & & & & & & & & --- \\
\hline Oxygen, dissolved & $\mathrm{mg} / \mathrm{L}-$ & 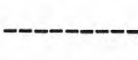 & & & & & & & -- \\
\hline \multirow{2}{*}{$\begin{array}{l}\text { Biochemical oxygen demand } \\
\text { pH }\end{array}$} & $\mathrm{mg} / \mathrm{L}$ & 1 & 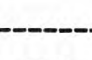 & --- & 2.3 & & & & -.-- \\
\hline & H units & 32 & 8.4 & 7.3 & 8.0 & 8.1 & 0.3 & 8.3 & 7.7 \\
\hline $\mathrm{Co}-\mathrm{Pt}$ & t units & 24 & 40 & 4 & 14 & 9 & 10 & 23 & 5.0 \\
\hline
\end{tabular}


06764200 South Platte River near Julesburg, Colo.--Continued

Results of regression analyses relating concentrations of selected chemical constituents to specific conductance $(\mathrm{SpC})$

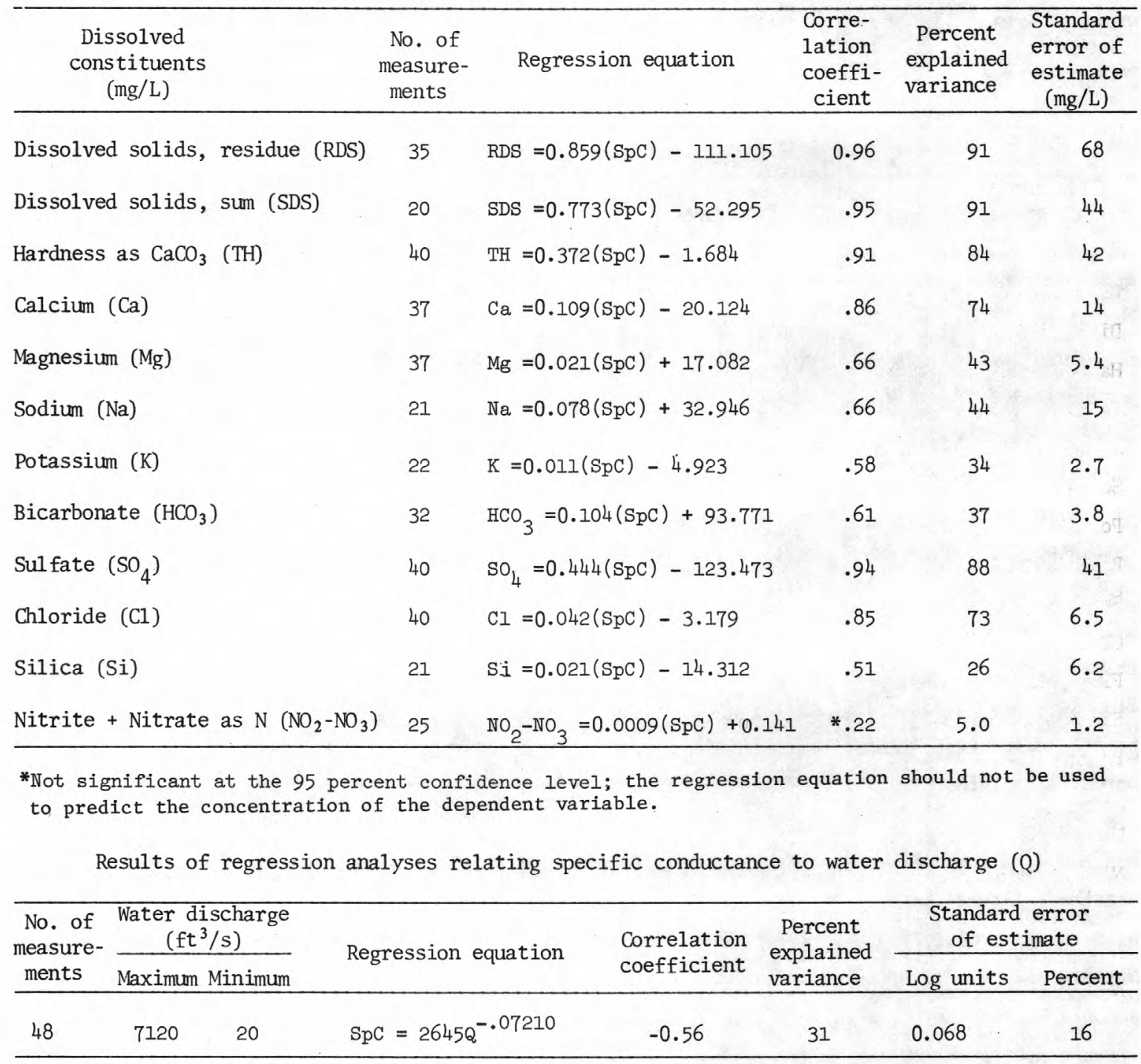




\section{SOUTH PLATTE RIVER BASIN}

06764880 South Platte River at Roscoe; Nebr.

Location.--Lat $41^{\circ} 07^{\prime} 33^{\prime \prime}$, long $101^{\circ} 34^{\prime} 35^{\prime \prime}$, in $\mathrm{NW}^{\frac{1}{4}} \mathrm{SW}^{\frac{1}{4}} \mathrm{sec} .4$, T.13 N., R.37 W., Keith County, Hydrologic Unit 10190018, at bridge on access road between U.S. Highway 30 and Interstate 80 , about $0.5 \mathrm{mi}$ southeast of Roscoe.

Period of record.--July 1975 to current year.

Statistical data for selected chemical constituents

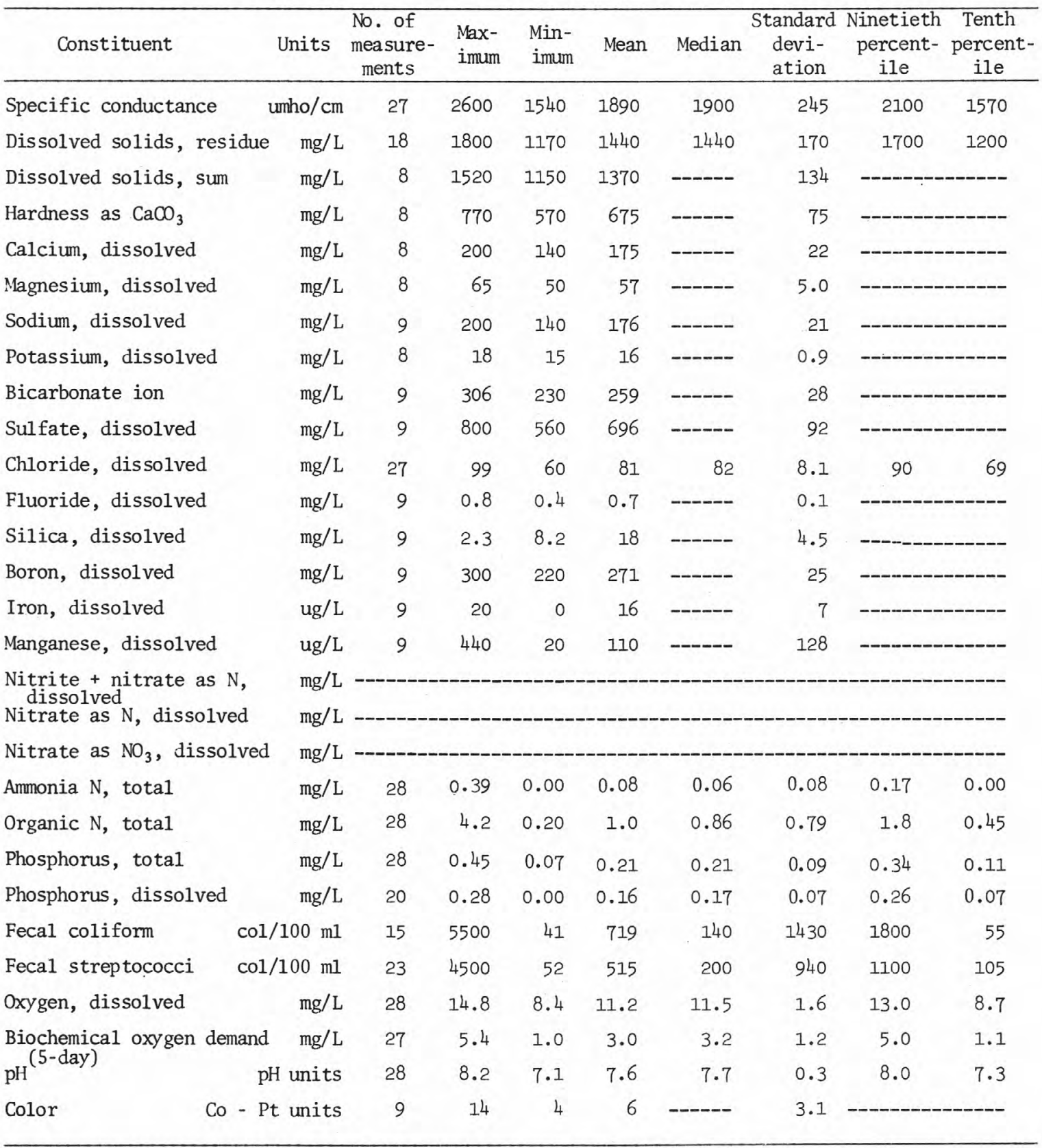


Results of regression analyses relating concentrations of selected chemical constituents to specific conductance $(\mathrm{SpC})$

\begin{tabular}{|c|c|c|c|c|c|}
\hline $\begin{array}{l}\text { Dissolved } \\
\text { constituents } \\
(\mathrm{mg} / \mathrm{L})\end{array}$ & $\begin{array}{l}\text { No. of } \\
\text { measure- } \\
\text { ments }\end{array}$ & Regression equation & $\begin{array}{l}\text { Corre- } \\
\text { lation } \\
\text { coeffi- } \\
\text { cient }\end{array}$ & $\begin{array}{l}\text { Percent } \\
\text { explained } \\
\text { variance }\end{array}$ & $\begin{array}{c}\text { Standard } \\
\text { error of } \\
\text { estimate } \\
(\mathrm{mg} / \mathrm{L})\end{array}$ \\
\hline Dissolved solids, residue (RDS) & 17 & $\mathrm{RDS}=0.486(\mathrm{SpC})+534.053$ & 0.76 & 58 & 114 \\
\hline \multicolumn{6}{|l|}{ Dissolved solids, sum (SDS) } \\
\hline \multicolumn{6}{|l|}{ Hardness as $\mathrm{CaCO}_{3}(\mathrm{TH})$} \\
\hline \multicolumn{6}{|l|}{ Calcium (Ca) } \\
\hline \multicolumn{6}{|l|}{ Magnesium (Mg) } \\
\hline \multicolumn{6}{|l|}{ Sodium $(\mathrm{Na})$} \\
\hline \multicolumn{6}{|l|}{ Potassium (K) } \\
\hline \multicolumn{6}{|l|}{ Bicarbonate $\left(\mathrm{HCO}_{3}\right)$} \\
\hline \multicolumn{6}{|l|}{ Sul fate $\left(\mathrm{SO}_{4}\right)$} \\
\hline Chloride (Cl) & 26 & $\mathrm{Cl}=0.019(\mathrm{SpC})+44.852$ & 0.57 & 33 & 6.8 \\
\hline Silica (Si) & $---\infty$ & & & & \\
\hline itrite + Nitrate as $\mathrm{N}\left(\mathrm{NO}_{2}-\mathrm{NO}_{3}\right.$ & & & & & \\
\hline
\end{tabular}

Results of regression analyses relating specific conductance to water discharge (Q)

\begin{tabular}{ccccccc}
\hline $\begin{array}{c}\text { No. of } \\
\text { measure- } \\
\text { ments }\end{array}$ & $\begin{array}{c}\text { Water discharge } \\
\left(\mathrm{ft}^{3} / \mathrm{s}\right)\end{array}$ & Maximum Minimum & Regression equation & $\begin{array}{c}\text { Correlation } \\
\text { coefficient }\end{array}$ & $\begin{array}{c}\text { Percent } \\
\text { explained } \\
\text { variance }\end{array}$ & $\begin{array}{c}\text { Standard error } \\
\text { of estimate }\end{array}$ \\
\hline 27 & $900 \quad 0.12$ & SpC $=1658 Q^{.02976}$ & 0.45 & 20 & 0.050 & 12 \\
\hline
\end{tabular}


The Middle Platte River basin is in central Nebraska. Including the Platte River and several tributaries, it extends from the confluence of the North and South Platte Rivers in Lincoln County to the confluence of the Platte and Loup Rivers in Platte County.

Of the approximately $2,765,000$ acres of land in the basin, about 1,$000 ; 000$ acres, or 36 percent, are irrigated farmland. This is the greatest amount of irrigated land in any of the 13 basins. About 230,000 acres are irrigated by diverted surface water, more than half of which are in the Tri-county area of Gosper, Phelps, and Kearney Counties (Nebraska Soil and water Conservation Commission, 1971). Water, during peak demand periods, is diverted from the Platte River into the Tricounty Supply Canal near North Platte.

Seventeen sampling sites in the basin have sufficient data for statistical analysis (fig. 7). Of these, nine are on the Platte River, six on tributaries, and two on canals or reservoirs. Of the sites on the Platte River, data for four represent the entire flow while data for five represent flow of individual channels. For the Platte River at Brady, data are available separately for channel 1, channel 4 , and for the combined flow of all channels. Likewise, for the Platte River near Overton, data are available for the north channel, the south channel, and combined flow. For the Platte River near Kearney, data are available for the north channel only.

Mean specific conductance of the Platte River increased from 727 umho/cm at Brady to 847 umho/cm at Grand Island and decreased to 828 umho/cm at Duncan. Generally, means of specific conductance for the tributaries are slightly higher. Tributaries like Spring Creek near Lexington carry irrigation return flow most of the year, which may account for their higher means. Principal cations at Platte River stations and at the Spring Creek, North Dry Creek, Tri-county Supply Canal, and Johnson Reservoir stations are calcium and sodium; principal anions are bicarbonate and sulfate.

The Wood River is the principal tributary entering the Platte River from the north in the Middle Platte River basin. Throughout the upper half of the Wood River basin, flow is maintained strictly by overland runoff. The mean specific conductance is $990 \mathrm{umho} / \mathrm{cm}$ at Wood River near Grand Island but is lower both upstream near Alda and downstream near Chapman. From Grand Island downstream, sodium and calcium are the principal cations; whereas upstream from Grand Island, calcium is the principal cation; bicarbonate is the principal anion throughout. 


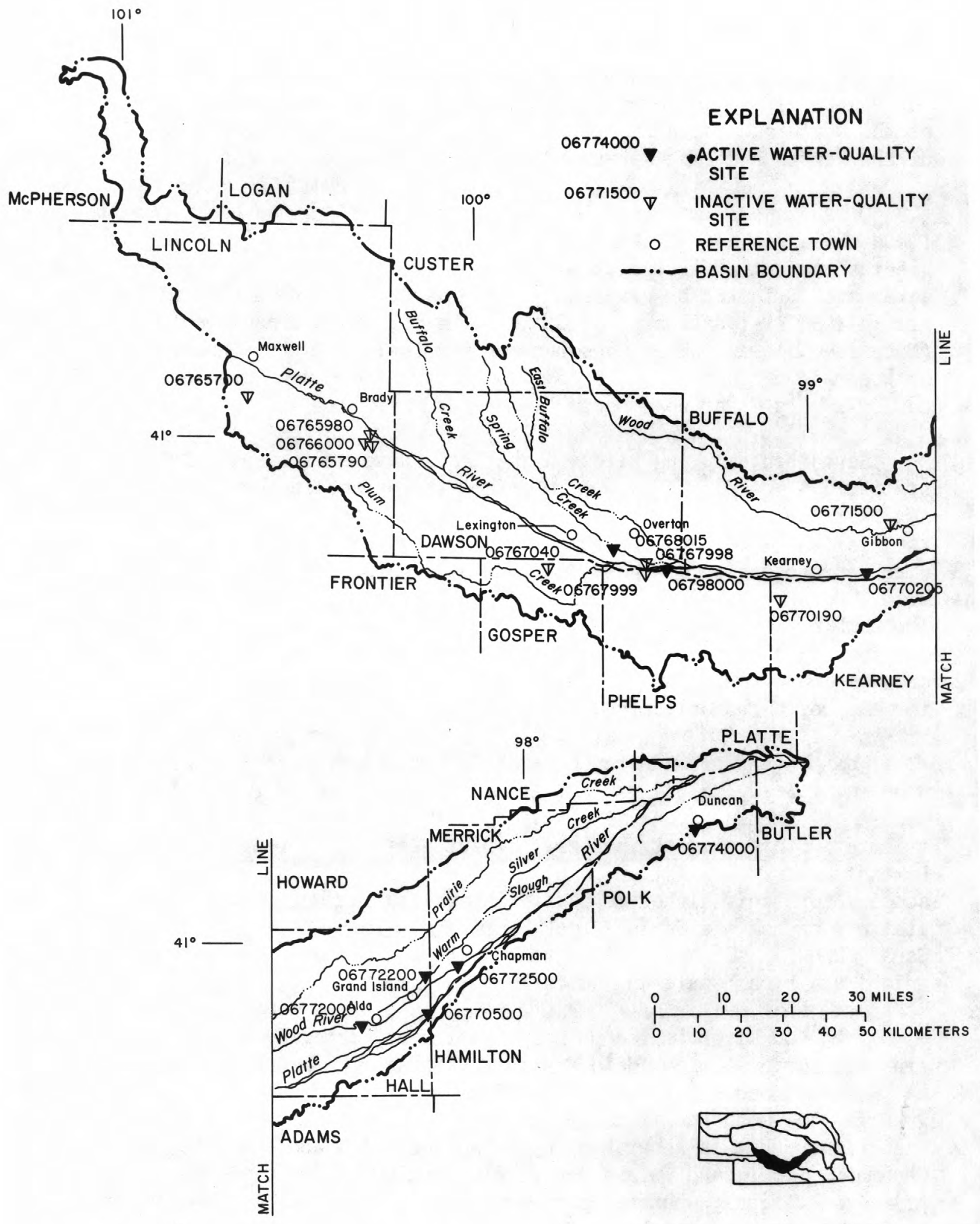

FIGURE 7.-Location of water-quality sampling sites, Middle Platte River basin 
The quality of water in the Wood River near Grand Island and near Chapman, in the North Channel of the Platte River near Kearney, and for part of its period of record in Spring Creek near Lexington is affected by sewage effluent. Mean concentrations of chloride, nitrite + nitrate, dissolved phosphorus, and BOD are noticeably higher at these stations than at stations not affected by effluent. The effect of the effluent can clearly be seen by comparing concentrations for Spring Creek at Lexington with those for a site not affected significantly by effluent. (See table 2.)

Table 2.--Comparison of concentrations of selected constituents to show the effect of sewage effluent on Spring Creek near Lexington

\begin{tabular}{lcc}
\hline \multicolumn{1}{c}{ Constituent } & $\begin{array}{c}\text { Platte River near } \\
\text { Overton (north channe1) }\end{array}$ & $\begin{array}{c}\text { Spring Creek near } \\
\text { Lexington }\end{array}$ \\
\hline Mean $\mathrm{NO}_{2}+\mathrm{NO}_{3}(\mathrm{mg} / \mathrm{L})$ & 0.59 & 2.3 \\
Mean chloride (mg/L) & 25 & 74 \\
Mean dissolved phosphorus (mg/L) & .08 & 1.6 \\
Mean biochemical oxygen demand (mg/L) & 2.7 & 12.3 \\
\hline
\end{tabular}

A similar comparison may be made to show the effects of sewage effluent on the Wood River near Grand Island. (See table 3.) The Wood River near Alda station is upstream from the Grand Island sewage-treatment plant, whereas the Wood River near Grand Island station is downstream.

Table 3.--Comparison of concentrations of selected constituents to show the effect of sewage effluent on Wood River near Grand Island

\begin{tabular}{lcc}
\hline \multicolumn{1}{c}{ Constituent } & $\begin{array}{c}\text { Wood River } \\
\text { near Alda }\end{array}$ & $\begin{array}{c}\text { Wood River near } \\
\text { Grand Island }\end{array}$ \\
\hline Mean chloride (mg/L) & 53 & 103 \\
Mean dissolved phosphorus (mg/L) & 1.2 & 3.0 \\
Median fecal coliform (col/100 ml) & 3,150 & 22,400 \\
\hline
\end{tabular}

Even with the larger amounts of nitrite + nitrate in water in the tributaries and with the massive irrigation development and its attendant use of fertilizers, there is no significant increase in nitrite + nitrate in Platte River water as it passes through the Middle P1atte River basin.

Results of regression analyses indicate that correlation coefficients generally exceed 0.8 for most constituents for Platte River stations in the basin. Exceptions are potassium, silica, nitrite + nitrate, and sometimes bicarbonate. There is no significant relationship between water discharge and specific conductance for most of these stations. 
This is because specific conductance, as for most of the constituents, varies over a much narrower range than does water discharge, or because the range of conductance may be controlled by factors other than water discharge.

Correlation coefficients for dissolved solids, sodium, and chloride generally exceed 0.9 at the downstream sites on Wood River, which means that changes in concentrations of these constituents are closely related to changes in specific conductance. 
06765700 Supply Canal (Tri-County Diversion) near Maxwe1l, Nebr.

Location.--Lat $41^{\circ} 03^{\prime} 50^{\prime \prime}$, long $100^{\circ} 38^{\prime} 50^{\prime \prime}$, in sec. 28 , T.13. N., R. 29 W., Lincoln County, at gaging station at Parshall flume near Maxwe11.

Period of record. --March 1951 to September 1972.

Statistical data for selected chemical constituents

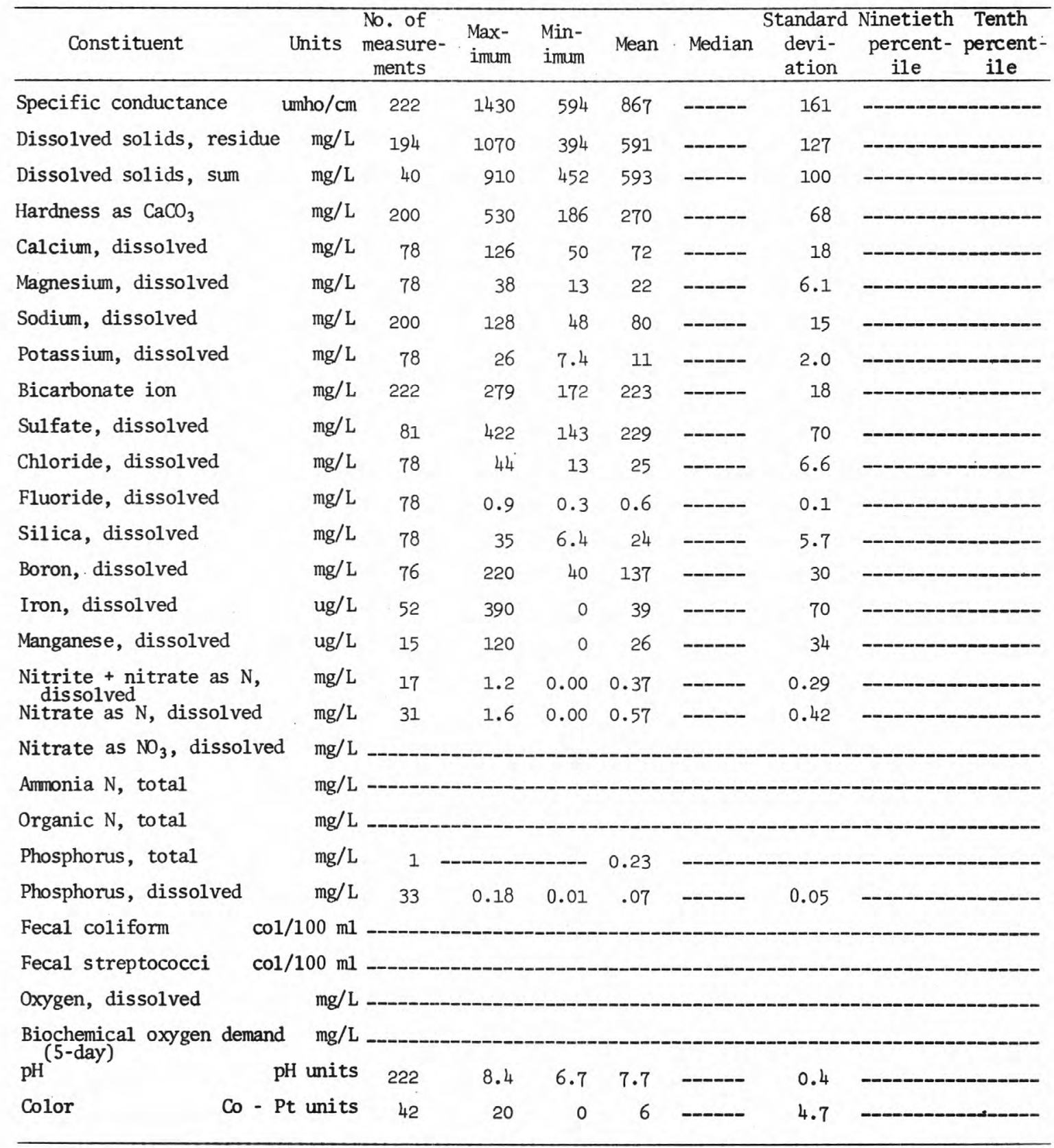


06765700 Supply Canal (Tri-County Diversion) near Maxwe11, Nebr.--Continued

Results of regression analyses relating concentrations of selected chemical constituents to specific conductance $(\mathrm{SpC})$

\begin{tabular}{|c|c|c|c|c|c|}
\hline $\begin{array}{l}\text { Dissolved } \\
\text { constituents } \\
(\mathrm{mg} / \mathrm{L})\end{array}$ & $\begin{array}{l}\text { No. of } \\
\text { measure- } \\
\text { ments }\end{array}$ & Regression equation & $\begin{array}{l}\text { Corre- } \\
\text { lation } \\
\text { coeffi- } \\
\text { cient }\end{array}$ & $\begin{array}{l}\text { Percent } \\
\text { explained } \\
\text { variance }\end{array}$ & $\begin{array}{c}\text { Standard } \\
\text { error of } \\
\text { estimate } \\
(\mathrm{mg} / \mathrm{L})\end{array}$ \\
\hline Dissolved solids, residue (RDS) & 193 & $\mathrm{RDS}=0.804(\mathrm{SpC})-98.691$ & 0.99 & 99 & -- \\
\hline Dissolved solids, sum (SDS) & 39 & $\mathrm{SDS}=0.796(\mathrm{SpC})-109.253$ & .98 & 97 & \\
\hline Hardness as $\mathrm{CaCO}_{3}(\mathrm{TH})$ & 199 & $\mathrm{TH}=0.442(\mathrm{SpC})-93.068$ & .97 & 94 & \\
\hline Calcium (Ca) & 77 & $\mathrm{Ca}=0.104(\mathrm{SpC})-17.723$ & .89 & 79 & \\
\hline Magnesium (Mg) & 77 & $\mathrm{Mg}=0.039(\mathrm{SpC})-11.768$ & .96 & 92 & \\
\hline Sodium $(\mathrm{Na})$ & 199 & $\mathrm{Na}=0.084(\mathrm{SpC})+7.479$ & .88 & 77 & \\
\hline Potassium $(\mathrm{K})$ & 77 & $K=0.004(\mathrm{SpC})+8.033$ & .26 & 6.9 & \\
\hline Bicarbonate $\left(\mathrm{HCO}_{3}\right)$ & 221 & $\mathrm{HCO}_{3}=0.056(\mathrm{spC})+175.065$ & .50 & 24 & -----1 \\
\hline Sulfate $\left(\mathrm{SO}_{4}\right)$ & 80 & $\mathrm{sO}_{4}=0.463(\mathrm{SpC})-171.775$ & .98 & 96 & \\
\hline Chloride (Cl) & 77 & $\mathrm{Cl}=0.040(\mathrm{SpC})-9.984$ & .92 & 84 & \\
\hline Silica (Si) & 77 & $\mathrm{Si}=-0.008(\mathrm{SpC})+31.313$ & $*_{-} .21$ & 4.3 & \\
\hline Nitrite + Nitrate as $\mathrm{N}\left(\mathrm{NO}_{2}-\mathrm{NO}_{3}\right)$ & 16 & $\mathrm{NO}_{2}-\mathrm{NO}_{3}=0.002(\mathrm{SpC})-1.201$ & .82 & 66 & \\
\hline
\end{tabular}

*Not significant at the 95 percent conficence level; the regression equation should not be used to predict the concentration of the dependent variable.

Results of regression analyses relating specific conductance to water discharge (Q)

\begin{tabular}{lcccccc}
\hline $\begin{array}{c}\text { No. of } \\
\text { measure- } \\
\text { ments }\end{array}$ & $\begin{array}{c}\text { Water discharge } \\
\left(\mathrm{ft}^{3} / \mathrm{s}\right)\end{array}$ & Maximum Minimum & Regression equation & $\begin{array}{c}\text { Correlation } \\
\text { coefficient }\end{array}$ & $\begin{array}{c}\text { Percent } \\
\text { explained } \\
\text { variance }\end{array}$ & $\begin{array}{c}\text { Standard error } \\
\text { of estimate }\end{array}$ \\
\hline 221 & 2240 & 606 & $S p C=308 Q^{.13987}$ & 0.22 & 4.7 & units \\
\hline
\end{tabular}




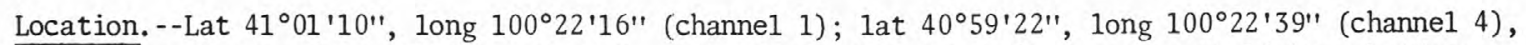
in secs. 11 and 23, T.12 N., R.27 W., Lincoln County, at gaging station at highway bridges $0.5 \mathrm{mi}$ and $2.5 \mathrm{mi}$, respectively, south of Brady, and $18 \mathrm{mi}$ downstream from confluence of North Platte and South Platte Rivers.

Drainage area.--60,200 $\mathrm{mi}^{2}$, approximately.

Period of record.--November 1950 to September 1972.

Statistical data for selected chemical constituents

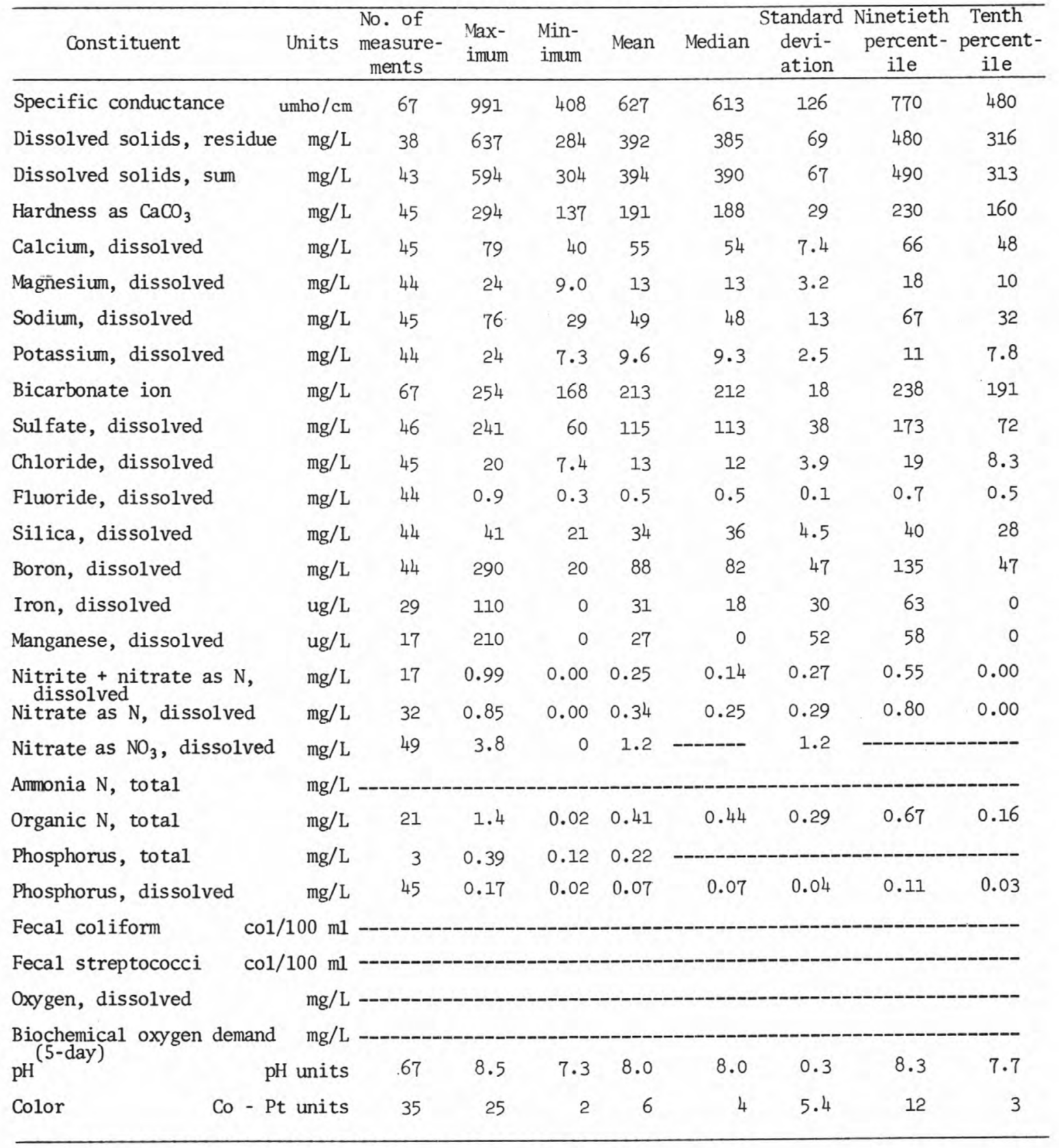


MIDDLE PLATTE RIVER BASIN

06765980 Platte River at Brady, Nebr. (Channel 1)--Continued

Results of regression analyses relating concentrations of selected chemical constituents to specific conductance (SpC)

\begin{tabular}{|c|c|c|c|c|c|}
\hline $\begin{array}{c}\text { Dissolved } \\
\text { constituents } \\
(\mathrm{mg} / \mathrm{L})\end{array}$ & $\begin{array}{l}\text { No. of } \\
\text { measure- } \\
\text { ments }\end{array}$ & Regression equation & $\begin{array}{l}\text { Corre- } \\
\text { lation } \\
\text { coeffi- } \\
\text { cient }\end{array}$ & $\begin{array}{l}\text { Percent } \\
\text { explained } \\
\text { variance }\end{array}$ & $\begin{array}{c}\text { Standard } \\
\text { error of } \\
\text { estimate } \\
(\mathrm{mg} / \mathrm{L})\end{array}$ \\
\hline Dissolved solids, residue (RDS) & 38 & $\mathrm{RDS}=0.670(\mathrm{SpC})+9.488$ & 0.96 & 92 & 20 \\
\hline Dissolved solids, sum (SDS) & 43 & $\mathrm{SDS}=0.657(\mathrm{spC})+4.714$ & .99 & 97 & 11 \\
\hline Hardness as $\mathrm{CaCO}_{3}(\mathrm{TH})$ & 45 & $\mathrm{TH}=0.263(\mathrm{SpC})+36.466$ & .92 & 85 & 11 \\
\hline Calcium (Ca) & 45 & $\mathrm{Ca}=0.059(\mathrm{SpC})+20.064$ & .83 & 70 & 4.1 \\
\hline Magnesium (Mg) & 44 & $\mathrm{Mg}=0.030(\mathrm{SpC})-4.632$ & .98 & 95 & .7 \\
\hline Sodium $(\mathrm{Na})$ & 45 & $\mathrm{Na}=0.125(\mathrm{SpC})-25.182$ & .96 & 92 & 3.9 \\
\hline Potassium (K) & 44 & $\mathrm{~K}=0.016(\mathrm{SpC})-.172$ & .68 & 46 & 1.9 \\
\hline Bicarbonate $\left(\mathrm{HCO}_{3}\right)$ & 67 & $\mathrm{HCO}_{3}=0.102(\mathrm{SpC})+148.652$ & .71 & 50 & 13 \\
\hline Sul fate $\left(\mathrm{SO}_{4}\right)$ & 46 & $\mathrm{sO}_{4}=0.349(\mathrm{spc})-91.666$ & .98 & 96 & 7.8 \\
\hline Chloride (Cl) & 45 & $\mathrm{Cl}=0.036(\mathrm{SpC})-8.425$ & .95 & 90 & 1.2 \\
\hline Silica (Si) & 44 & $\mathrm{Si}=-.026(\mathrm{Spc})+49.428$ & -.59 & 35 & 3.7 \\
\hline Nitrite + Nitrate as $\mathrm{N}\left(\mathrm{NO}_{2}-\mathrm{NO}_{3}\right)$ & 17 & $\mathrm{NO}_{2}-\mathrm{NO}_{3}=0.0008(\mathrm{SpC})-0.307$ & $* .24$ & 5.8 & .27 \\
\hline
\end{tabular}

*Not significant at the 95 percent confidence level; the regression equation should not be used to predict the concentration of the dependent variable.

Results of regression analyses relating specific conductance to water discharge (Q)

\begin{tabular}{llllccc}
\hline $\begin{array}{l}\text { No. of } \\
\text { measure- } \\
\text { ments }\end{array}$ & $\begin{array}{c}\text { Water discharge } \\
\left(\mathrm{ft}^{3} / \mathrm{s}\right)\end{array}$ & Maximum Minimum & Regression equation & $\begin{array}{c}\text { Correlation } \\
\text { coefficient }\end{array}$ & $\begin{array}{c}\text { Percent } \\
\text { explained } \\
\text { variance }\end{array}$ & \multicolumn{2}{c}{$\begin{array}{c}\text { Standard error } \\
\text { of estimate }\end{array}$} \\
\hline 67 & 9550 & 57 & SpC $=339 Q^{.11357}$ & 0.70 & 48 & 0.062 \\
\hline
\end{tabular}


Location.--Lat $41^{\circ} 01^{\prime} 10^{\prime \prime}$, long $100^{\circ} 22^{\prime} 16^{\prime \prime}$ (channel 1), lat $40^{\circ} 59^{\prime} 22^{\prime \prime}$, long $100^{\circ} 22^{\prime} 39^{\prime \prime}$ (channel 4), in sec. 11 and 23, T.12 N., R. 27 W., Lincoln County, at gaging station at highway bridges $0.5 \mathrm{mi}$ and $2.5 \mathrm{mi}$, respectively, south of Brady and $18 \mathrm{mi}$ downstream from confluence of North and South Platte Rivers.

Drainage area.--60,200 $\mathrm{mi}^{2}$, approximately.

Period of record.--November 1950 to September 1972.

Statistical data for selected chemical constituents

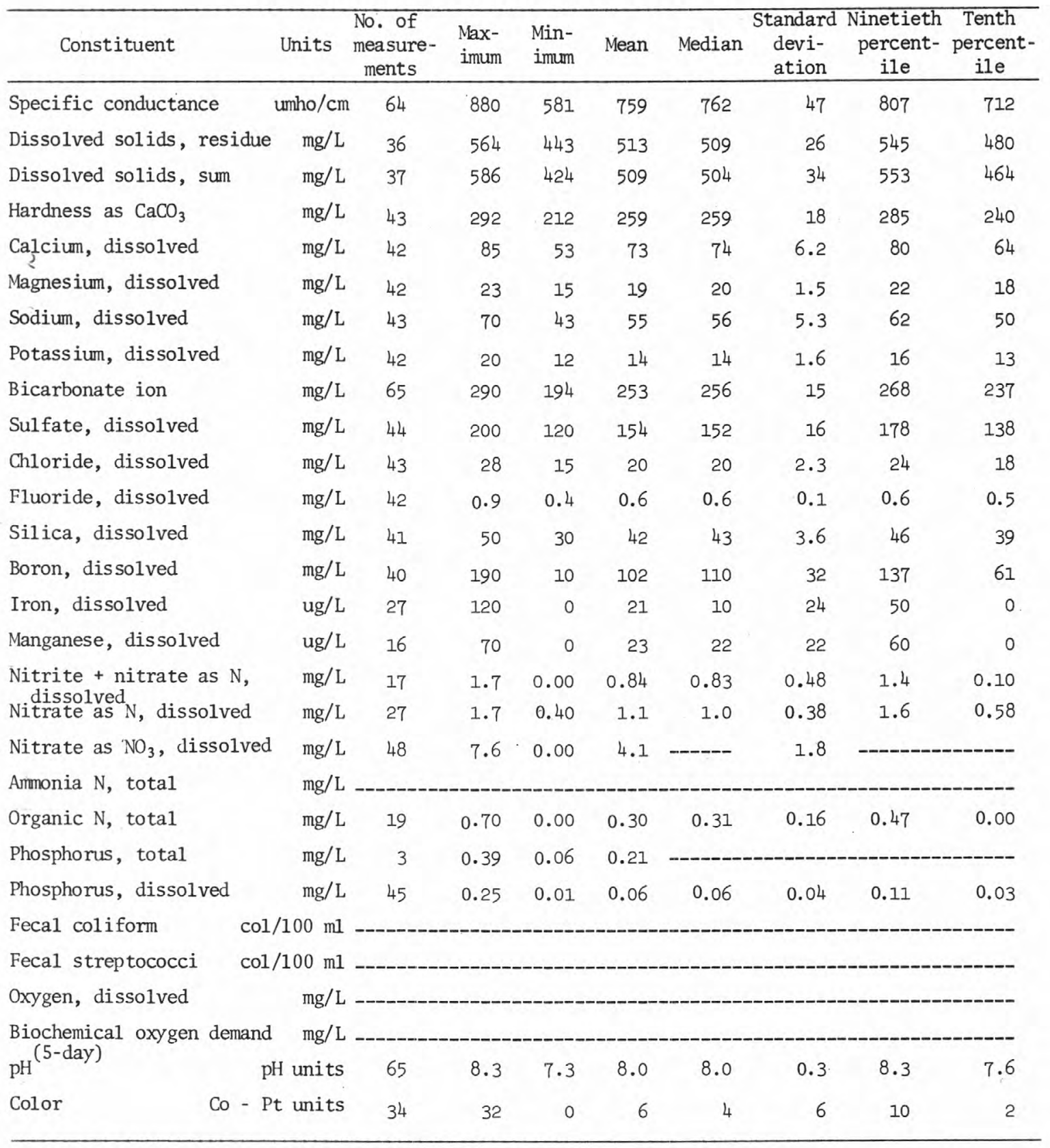


MIDDLE PLATTE RIVER BASIN

06765990 Platte River at Brady, Nebr. (Channel 4)--Continued

Results of regression analyses relating concentrations of selected chemical constituents to specific conductance $(\mathrm{SpC})$

\begin{tabular}{|c|c|c|c|c|c|}
\hline $\begin{array}{l}\text { Dissolved } \\
\text { constituents } \\
\left(\mathrm{mg} / \mathrm{L}_{1}\right)\end{array}$ & $\begin{array}{l}\text { No. of } \\
\text { measure- } \\
\text { ments }\end{array}$ & Regression equation & $\begin{array}{l}\text { Corre- } \\
\text { lation } \\
\text { coeffi- } \\
\text { cient }\end{array}$ & $\begin{array}{l}\text { Percent } \\
\text { explained } \\
\text { variance }\end{array}$ & $\begin{array}{c}\text { Standard } \\
\text { error of } \\
\text { estimate } \\
(\mathrm{mg} / \mathrm{L})\end{array}$ \\
\hline Dissolved solids, residue (RDS) & 35 & $\mathrm{RDS}=0.357(\mathrm{SpC})+250.770$ & 0.57 & 33 & 22 \\
\hline Dissolved solids, sum (SDS) & 36 & $\mathrm{SDS}=0.492(\mathrm{SpC})+140.602$ & .80 & 65 & 20 \\
\hline Hardness as $\mathrm{CaCO}_{3}(\mathrm{TH})$ & 42 & $\mathrm{TH}=0.239(\mathrm{SpC})+81.377$ & .73 & 54 & 12 \\
\hline Calcium (Ca) & 41 & $\mathrm{Ca}=0.064(\mathrm{SpC})+24.980$ & .62 & 38 & 4.4 \\
\hline Magnesium (Mg) & 41 & $\mathrm{Mg}=0.021(\mathrm{SpC})+3.289$ & .73 & 53 & 1.0 \\
\hline Sodium (Na) & 42 & $\mathrm{Na}=0.074(\mathrm{SpC})-.572$ & .74 & 54 & 3.6 \\
\hline Potassium (K) & 41 & $\mathrm{~K}=0.008(\mathrm{SpC})+7.623$ & $* .27$ & 7.2 & 1.6 \\
\hline Bicarbonate $\left(\mathrm{HCO}_{3}\right)$ & 64 & $\mathrm{HCO}_{3}=0.143(\mathrm{SpC})+145.355$ & .50 & 25 & 12 \\
\hline Sulfate $\left(\mathrm{SO}_{4}\right)$ & 43 & $\mathrm{SO}_{4}=0.220(\mathrm{SpC})-10.354$ & .73 & 53 & 11 \\
\hline Chloride $(\mathrm{Cl})$ & 42 & $\mathrm{Cl}=0.035(\mathrm{SpC})-5.932$ & .79 & 62 & 1.5 \\
\hline Silica (Si) & 40 & $\mathrm{Si}=0.016(\mathrm{SpC})+30.064$ & $* .23$ & 5.5 & 3.6 \\
\hline Nitrite + Nitrate as $\mathrm{N}\left(\mathrm{NO}_{2}-\mathrm{NO}_{3}\right)$ & 17 & $\mathrm{NO}_{2}-\mathrm{NO}_{3}=0.0008(\mathrm{SpC})+0.200$ & *.05 & .28 & .49 \\
\hline
\end{tabular}

*Not significant at the 95 percent confidence level; the regression equation should not be used to predict the concentration of the dependent variable.

Results of regression analyses relating specific conductance to water discharge (Q)

\begin{tabular}{lcccccccc}
\hline $\begin{array}{c}\text { No. of } \\
\text { measure- } \\
\text { ments }\end{array}$ & $\begin{array}{c}\text { Water discharge } \\
\left(\mathrm{ft}^{3} / \mathrm{s}\right)\end{array}$ & Maximum Minimum & Regression equation & $\begin{array}{c}\text { Correlation } \\
\text { coefficient }\end{array}$ & $\begin{array}{c}\text { Percent } \\
\text { explained } \\
\text { variance }\end{array}$ & \multicolumn{2}{c}{$\begin{array}{c}\text { Standard error } \\
\text { of estimate }\end{array}$} \\
\hline 64 & $120 \quad 24$ & $\mathrm{SpC}=664 \mathrm{Q}^{.03298}$ & $* 0.17$ & 3.0 & 0.027 & 6.2 \\
\hline
\end{tabular}




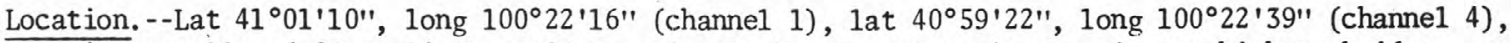
in sec. 11 and 23, T.12 N., R. 27 W., Lincoln County, at gaging station at highway bridges $0.5 \mathrm{mi}$ and $2.5 \mathrm{mi}$, respectively, south of Brady and $18 \mathrm{mi}$ downstream from confluence of North Platte and South Platte Rivers.

Drainage area. $--60,200 \mathrm{mi}^{2}$, approximately.

Period of record.--November 1950 to September 1972.

Statistical data for selected chemical constituents

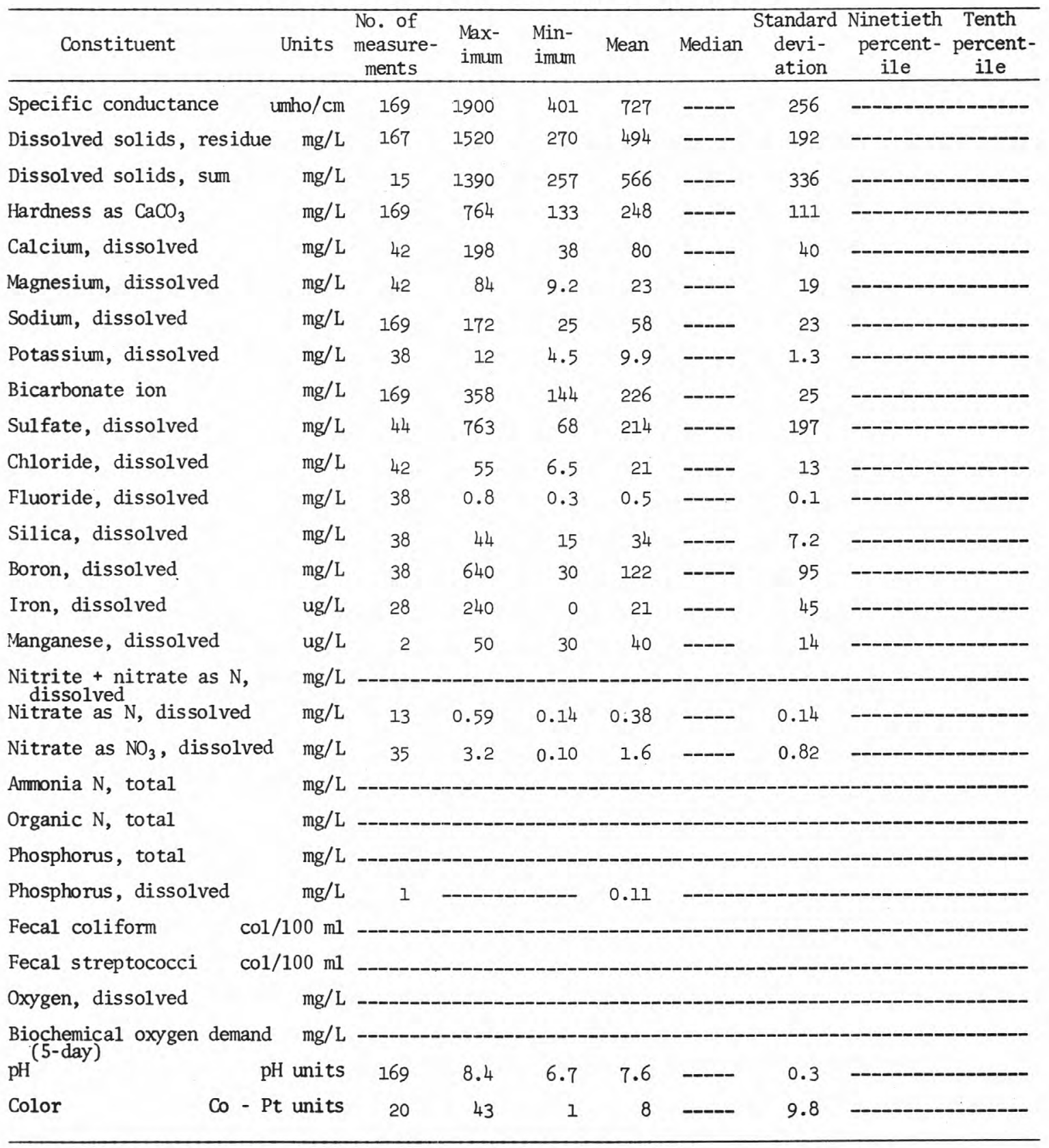




\section{MIDDLE PLATTE RIVER BASIN}

06766000 Platte River at Brady, Nebr.--Continued

Results of regression analyses relating concentrations of selected chemical constituents to specific conductance $(\mathrm{SpC})$

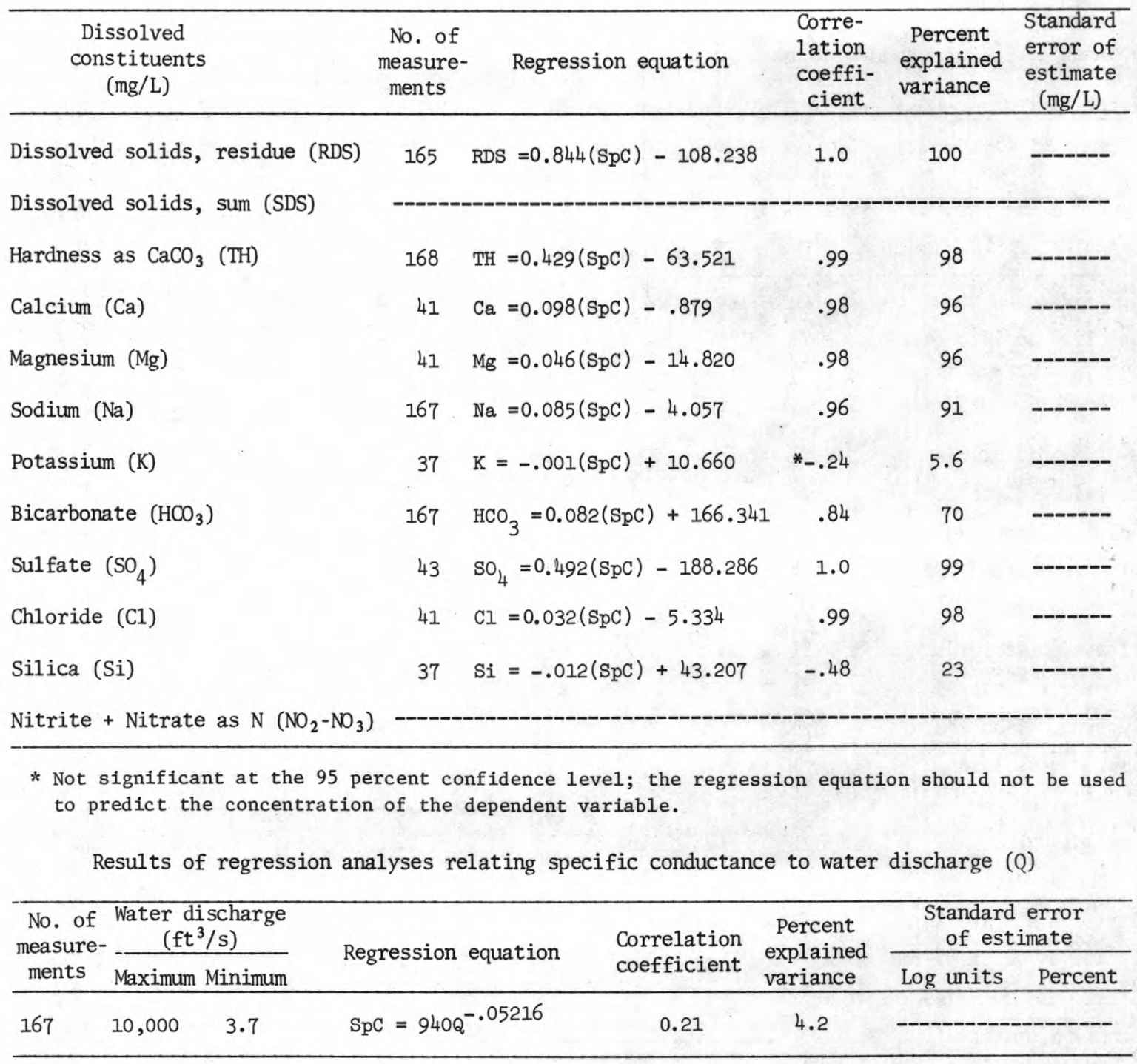


06767040 Johnson Reservoir below powerplant No. 2 near lexington, Nebr.

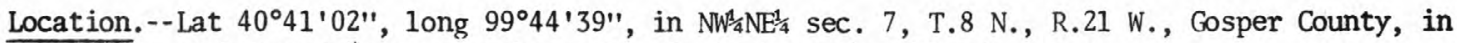
tail race below Johnson powerhouse No. 2, $6 \mathrm{mi}$ south of Lexington.

Period of record.--March 1957 to June 1971.

Statistical data for selected chemical constituents

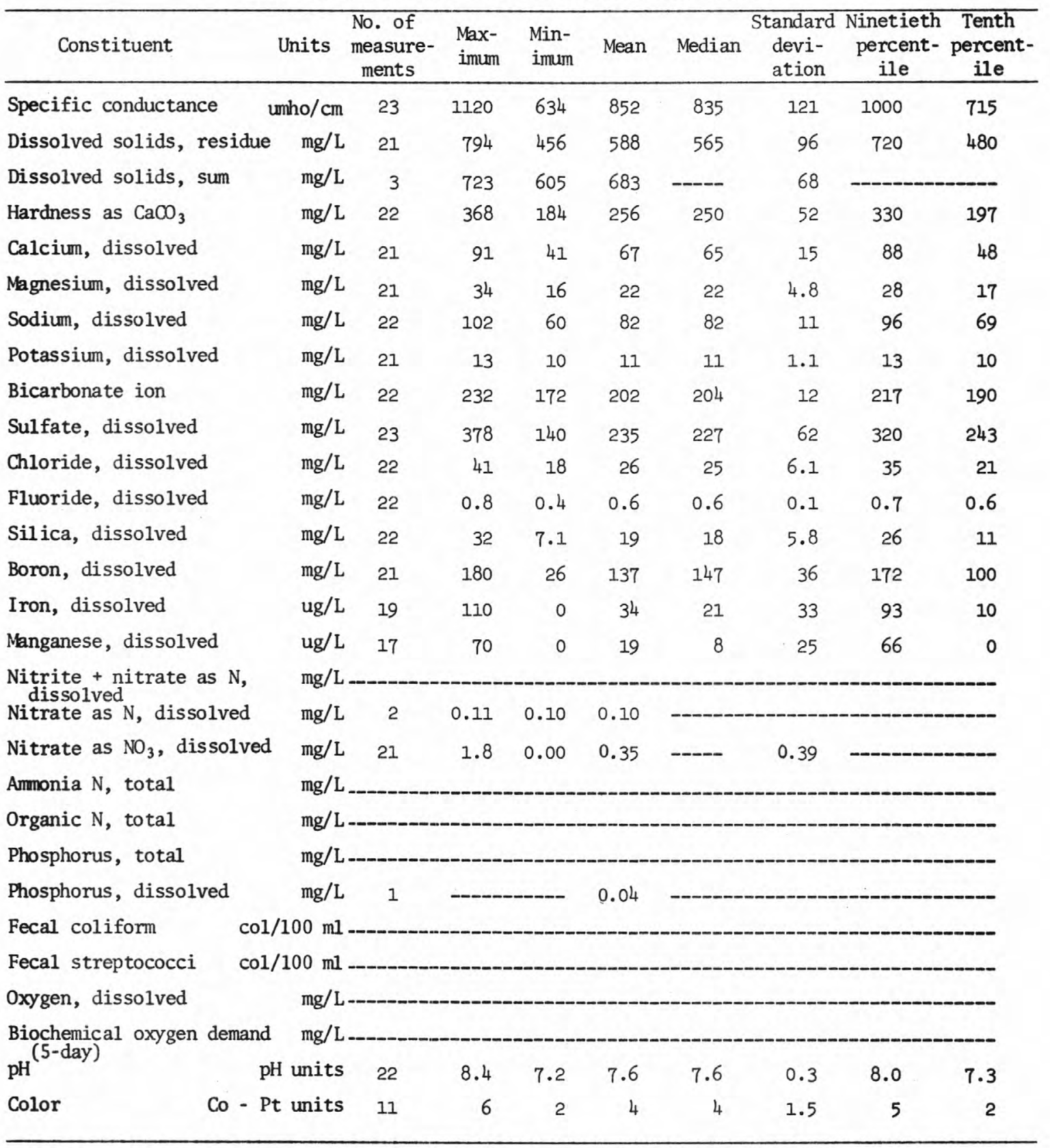


Results of regression analyses relating concentrations of selected chemical constituents to specific conductance (SpC)

\begin{tabular}{|c|c|c|c|c|c|}
\hline $\begin{array}{l}\text { Dissolved } \\
\text { constituents } \\
(\mathrm{mg} / \mathrm{L})\end{array}$ & $\begin{array}{l}\text { No. of } \\
\text { measure- } \\
\text { ments }\end{array}$ & Regression equation & $\begin{array}{l}\text { Corre- } \\
\text { lation } \\
\text { coeffi- } \\
\text { cient }\end{array}$ & $\begin{array}{l}\text { Percent } \\
\text { explained } \\
\text { variance }\end{array}$ & $\begin{array}{c}\text { Standard } \\
\text { error of } \\
\text { estimate } \\
(\mathrm{mg} / \mathrm{L})\end{array}$ \\
\hline Dissolved solids, residue (RDS) & 21 & $\mathrm{RDS}=0.822(\mathrm{SpC})-122.713$ & 1.0 & 99 & 9.5 \\
\hline Dissolved solids, sum (SDS) & ----- & & & & \\
\hline Hardness as $\mathrm{CaCO}_{3}(\mathrm{TH})$ & 22 & $\mathrm{TH}=0.405(\mathrm{SpC})-90.159$ & .96 & 92 & 15 \\
\hline Calcium (Ca) & 21 & $\mathrm{Ca}=0.116(\mathrm{SpC})-33.069$ & .90 & 80 & 6.8 \\
\hline Magnesium (Mg) & 21 & $\mathrm{Mg}=0.036(\mathrm{SpC})-8.330$ & .86 & 74 & 2.5 \\
\hline Sodium $(\mathrm{Na})$ & 22 & $\mathrm{Na}=0.079(\mathrm{SpC})+14.444$ & .91 & 82 & 4.6 \\
\hline Potassium (K) & 21 & $\mathrm{~K}=0.004(\mathrm{SpC})+7.804$ & $* .42$ & 18 & 1.0 \\
\hline Bicarbonate $\left(\mathrm{HCO}_{3}\right)$ & 22 & $\mathrm{HCO}_{3}=-.029(\mathrm{SpC})+227.339$ & $*-.30$ & 8.9 & 12 \\
\hline Sulfate $\left(\mathrm{SO}_{4}\right)$ & 23 & $\mathrm{SO}_{4}=0.507(\mathrm{SpC})-197.025$ & .98 & 97 & 11 \\
\hline Chloride (C1) & 22 & $\mathrm{Cl}=0.046(\mathrm{SpC})-13.884$ & .94 & 88 & 2.2 \\
\hline Silica (Si) & 22 & $\mathrm{Si}=-.018(\mathrm{SpC})+33.664$ & $*-.37$ & 14 & 5.5 \\
\hline
\end{tabular}

*Not significant at the 95 percent confidence level; the regression equation should not be used to predict the concentration of the dependent variable.

Results of regression analyses relating specific conductance to water discharge (Q)

\begin{tabular}{|c|c|c|c|c|c|c|}
\hline \multirow{2}{*}{$\begin{array}{l}\text { No. of } \\
\text { measure- } \\
\text { ments }\end{array}$} & \multirow{2}{*}{$\begin{array}{l}\text { Water discharge } \\
\frac{\left(\mathrm{ft}^{3} / \mathrm{s}\right)}{\text { Maximum Minimum }}\end{array}$} & \multirow{2}{*}{ Regression equation } & \multirow{2}{*}{$\begin{array}{l}\text { Correlation } \\
\text { coefficient }\end{array}$} & \multirow{2}{*}{$\begin{array}{l}\text { Percent } \\
\text { explained } \\
\text { variance }\end{array}$} & \multicolumn{2}{|c|}{$\begin{array}{c}\text { Standard error } \\
\text { of estimate }\end{array}$} \\
\hline & & & & & Log units & Percent \\
\hline
\end{tabular}




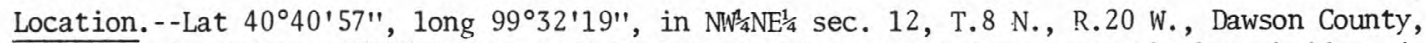
Hydrologic Unit 10200101, on left bank $600 \mathrm{ft}$ downstream from county highway bridge, $4 \mathrm{mi}$ south of Overton, and $4 \mathrm{mi}$ downstream from Plum Creek. Prior to June 2, 1976, (north channe1) gage at site $600 \mathrm{ft}$ upstream. South channel gage discontinued Jume 2, 1976.

$\underline{\text { Drainage area (revised) }} \cdot--57,700 \mathrm{mi}^{2}$, approximately.

Period of record.--October 1969 to September 1976.

Statistical data for selected chemical constituents

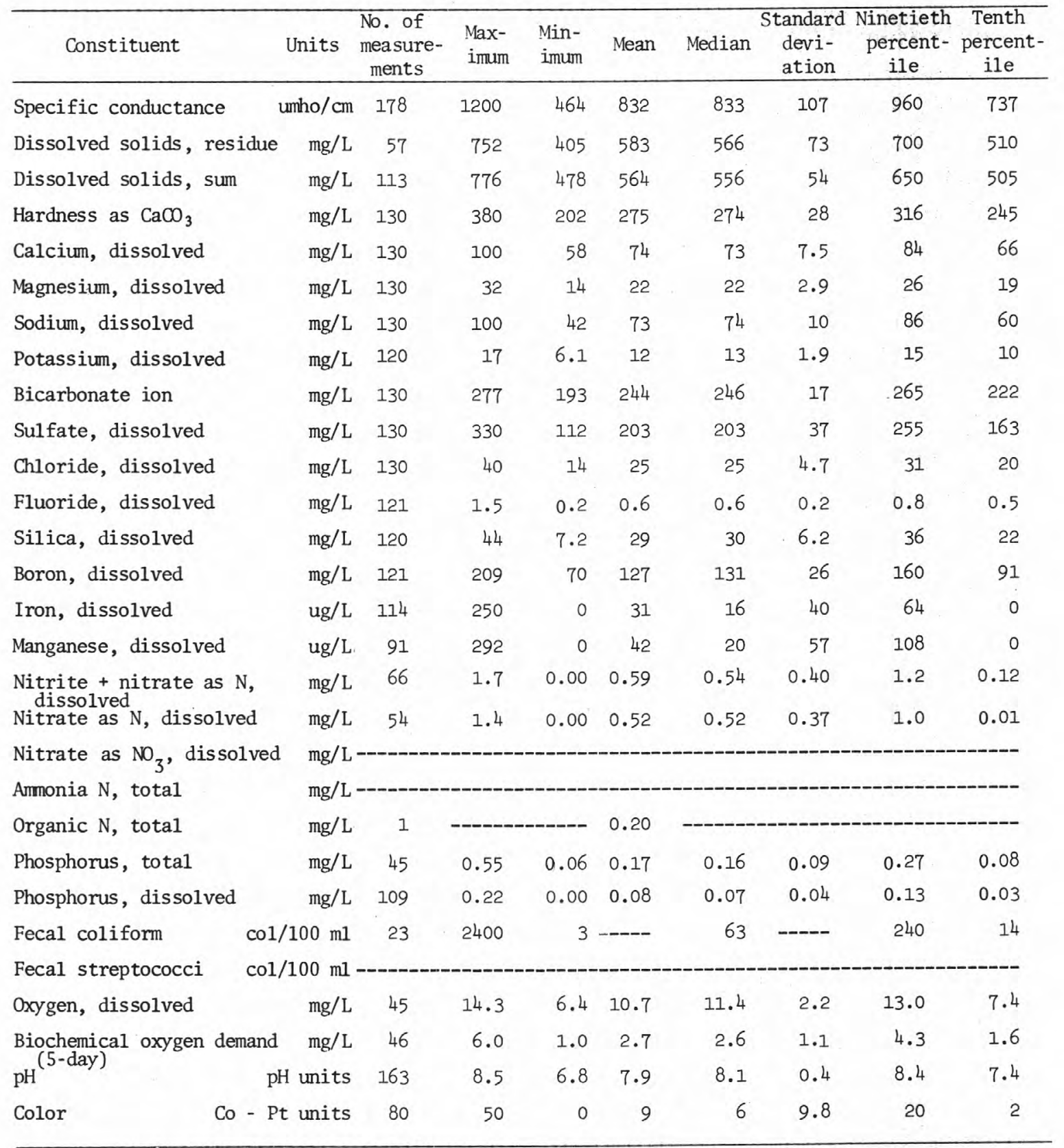


06767998 Platte River near Overton, Nebr. (North Channel)--Continued

Results of regression analyses relating concentrations of selected chemical constituents to specific conductance $(\mathrm{SpC})$

\begin{tabular}{|c|c|c|c|c|c|}
\hline $\begin{array}{l}\text { Dissolved } \\
\text { consti tuents } \\
(\mathrm{mg} / \mathrm{L})\end{array}$ & $\begin{array}{l}\text { No. of } \\
\text { measure- } \\
\text { ments }\end{array}$ & Regression equation & $\begin{array}{l}\text { Corre- } \\
\text { lation } \\
\text { coeffi- } \\
\text { cient }\end{array}$ & $\begin{array}{l}\text { Percent } \\
\text { explained } \\
\text { variance }\end{array}$ & $\begin{array}{c}\text { Standard } \\
\text { error of } \\
\text { estimate } \\
(\mathrm{mg} / \mathrm{L})\end{array}$ \\
\hline Dissolved solids, residue (RDS) & 57 & $\mathrm{RDS}=0.801(\mathrm{SpC})-80.424$ & 0.97 & 93 & 19 \\
\hline Dissolved solids, sum (SDS) & 114 & $\mathrm{SDS}=0.581(\mathrm{SpC})+71.104$ & .86 & 74 & 28 \\
\hline Hardness as $\mathrm{CaCO}_{3}(\mathrm{TH})$ & 130 & $\mathrm{TH}=0.280(\mathrm{SpC})+38.785$ & .82 & 66 & 16 \\
\hline Calcium (Ca) & 130 & $\mathrm{Ca}=0.064(\mathrm{SpC})+19.871$ & .70 & 49 & 5.4 \\
\hline Magnesium (Mg) & 130 & $\mathrm{Mg}=0.030(\mathrm{SpC})-3.034$ & .84 & 71 & 1.6 \\
\hline Sodium (Na) & 130 & $\mathrm{~N} a=0.095(\mathrm{SpC})-6.975$ & .78 & 60 & 6.3 \\
\hline Potassium (K) & 120 & $\mathrm{~K}=0.003(\mathrm{SpC})+9.924$ & *.12 & 1.4 & 1.9 \\
\hline Bicarbonate $\left(\mathrm{HCO}_{3}\right)$ & 130 & $\mathrm{HCO}_{3}=0.015(\mathrm{SpC})+231.446$ & $* .07$ & .49 & 17 \\
\hline Sulfate $\left(\mathrm{SO}_{4}\right)$ & 130 & $\mathrm{SO}_{4}=0.378(\mathrm{SpC})-115.842$ & .84 & 71 & 20 \\
\hline Chloride (C1) & 130 & $\mathrm{Cl}=0.042(\mathrm{SpC})-10.894$ & .74 & 55 & 3.1 \\
\hline Silica (Si) & 120 & $\mathrm{Si}=-.026(\mathrm{SpC})+50.381$ & -.34 & 12 & 5.9 \\
\hline Nitrite + Nitrate as $\mathrm{N}\left(\mathrm{NO}_{2}-\mathrm{NO}_{3}\right)$ & 66 & $\mathrm{NO}_{2}-\mathrm{NO}_{3}=0.0007(\mathrm{SpC})-0.012$ & $* .13$ & 1.8 & .40 \\
\hline
\end{tabular}

* Not significant at the 95 percent confidence level; the regression equation should not be used to predict the concentration of the dependent variable.

Results of regression analyses relating specific conductance to water discharge (Q)

\begin{tabular}{lllcccc}
\hline $\begin{array}{c}\text { No. of } \\
\text { measure- } \\
\text { ments }\end{array}$ & $\begin{array}{c}\text { Water discharge } \\
\left(\mathrm{ft}^{3} / \mathrm{s}\right)\end{array}$ & Maximum Minimum & Regression equation & $\begin{array}{c}\text { Correlation } \\
\text { coefficient }\end{array}$ & $\begin{array}{c}\text { Percent } \\
\text { explained } \\
\text { variance }\end{array}$ & $\begin{array}{c}\text { Standard error } \\
\text { of estimate }\end{array}$ \\
\hline 176 & $14,000 \quad 69$ & $\mathrm{SpC}=767 Q^{.01054}$ & 0.08 & 0.64 & 0.062 & 14 \\
\hline
\end{tabular}


06767999 Platte River near Overton, Nebr. (South Channel)

Location.--Lat $40^{\circ} 40^{\prime} 57^{\prime \prime}$, long $99^{\circ} 32^{\prime} 19^{\prime \prime}$, in NW' $\mathrm{NE}^{\frac{1}{4}} \mathrm{sec}$. 12, T.8 N., R.20 W., Dawson County, Hydrologic Unit 10200101, on left bank $600 \mathrm{ft}$ downstream from county highway bridge, $4 \mathrm{mi}$ south of Overton and $4 \mathrm{mi}$ downstream from Plum Creek. Prior to June 2, 1976, (north channel) gage at site $600 \mathrm{ft}$ upstream. South channel gage discontinued June 2, 1976.

Drainage area (revised.--57,700 $\mathrm{mi}^{2}$, approximately, of which about 52,900 $\mathrm{mi}^{2}$ contributes directly to surface runoff. Approximately 4,000 $\mathrm{mi}^{2}$ in Great Divide basin is not included.

Period of record.--October 1969 to September 1976.

Statistical data for selected chemical constituents

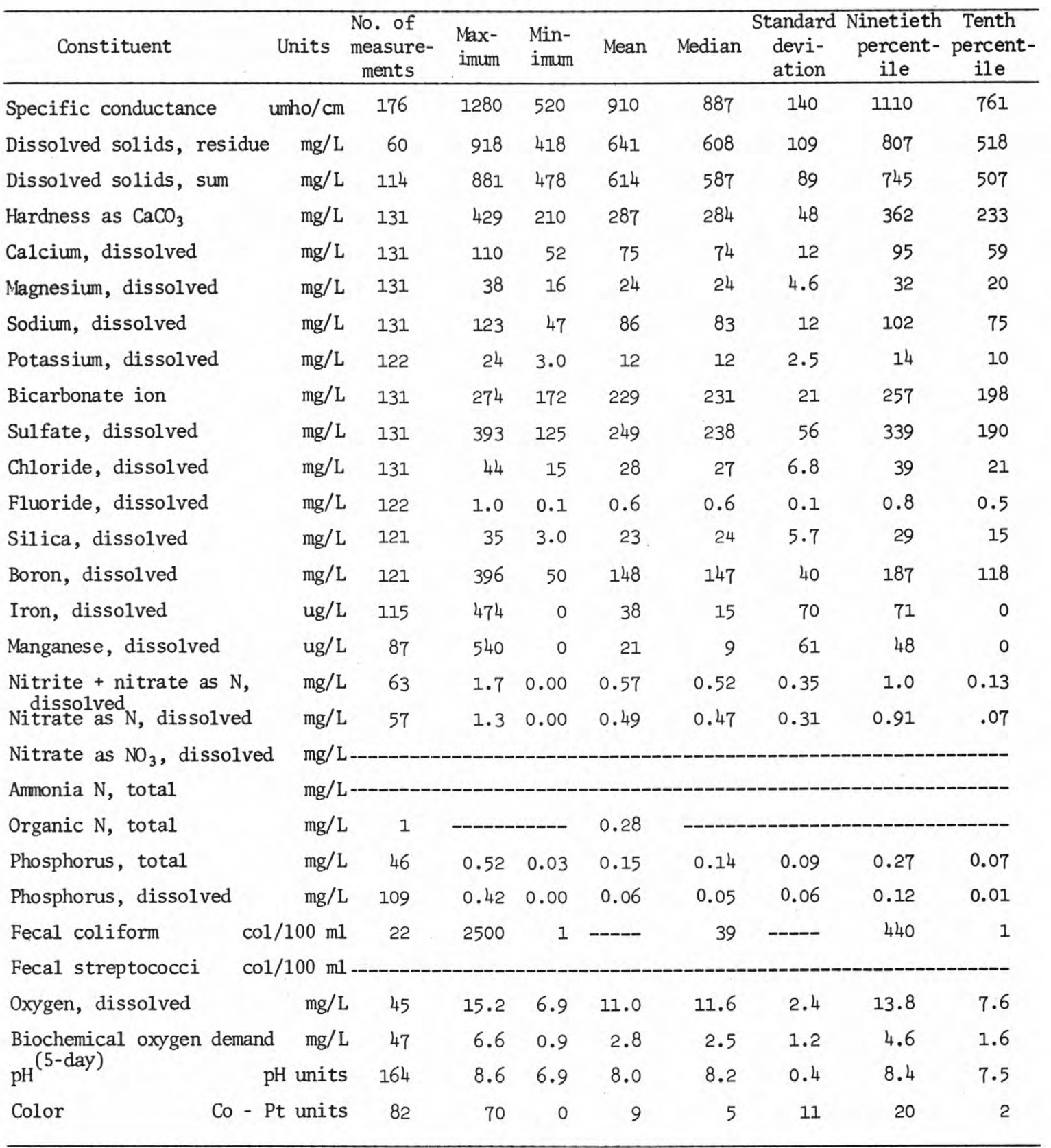


MIDDLE PLATTE RIVER BASIN

06767999 Platte River near Overton, Nebr. (South Channel)--Continued

Results of regression analyses relating concentrations of selected chemical constituents to specific conductance $(\mathrm{SpC})$

\begin{tabular}{|c|c|c|c|c|c|}
\hline $\begin{array}{l}\text { Dissolved } \\
\text { constituents } \\
(\mathrm{mg} / \mathrm{L})\end{array}$ & $\begin{array}{l}\text { No. of } \\
\text { measure- } \\
\text { ments }\end{array}$ & Regression equation & $\begin{array}{l}\text { Corre- } \\
\text { lation } \\
\text { coeffi- } \\
\text { cient }\end{array}$ & $\begin{array}{l}\text { Percent } \\
\text { explained } \\
\text { variance }\end{array}$ & $\begin{array}{c}\text { Standard } \\
\text { error of } \\
\text { estimate } \\
(\mathrm{mg} / \mathrm{L})\end{array}$ \\
\hline Dissolved solids, residue (RDS) & 60 & $\mathrm{RDS}=0.794(\mathrm{SpC})-77.859$ & 0.97 & 94 & 28 \\
\hline Dissolved solids, sum (SDS) & 114 & $\mathrm{SDS}=0.691(\mathrm{SpC})-20.546$ & .94 & 88 & 30 \\
\hline Hardness as $\mathrm{CaCO}_{3}(\mathrm{TH})$ & 131 & $\mathrm{TH}=0.361(\mathrm{SpC})-43.961$ & .92 & 86 & 18 \\
\hline Calcium (Ca) & 131 & $\mathrm{Ca}=0.088(\mathrm{spc})-6.271$ & .87 & 75 & 6.2 \\
\hline Magnesium (Mg) & 131 & $\mathrm{Mg}=0.034(\mathrm{spC})-6.982$ & .92 & 85 & 1.8 \\
\hline Sodium $(\mathrm{Na})$ & 131 & $\mathrm{Na}=0.089(\mathrm{SpC})+4.373$ & .87 & 76 & 6.1 \\
\hline Potassium (K) & 122 & $\mathrm{~K}=0.003(\mathrm{SpC})+9.140$ & $* .13$ & 1.8 & 2.4 \\
\hline Bicarbonate $\left(\mathrm{HCO}_{3}\right)$ & 131 & $\mathrm{HCO}_{3}=0.037(\mathrm{SpC})+194.880$ & .21 & 4.6 & 21 \\
\hline Sulfate $\left(\mathrm{SO}_{4}\right)$ & 131 & $\mathrm{sO}_{4}=0.432(\mathrm{SpC})-147.248$ & .94 & 88 & 20 \\
\hline Chloride (CI) & 131 & $\mathrm{Cl}=0.049(\mathrm{SpC})-16.401$ & .88 & 77 & 3.2 \\
\hline Silica (Si) & 121 & $\mathrm{Si}=-.007(\mathrm{SpC})+28.847$ & $*-.14$ & 2.0 & 5.7 \\
\hline Nitrite + Nitrate as $\mathrm{N}\left(\mathrm{NO}_{2}-\mathrm{NO}_{3}\right)$ & 63 & $\mathrm{NO}_{2}-\mathrm{NO}_{3}=0.002(\mathrm{SpC})-1.518$ & .68 & 47 & .26 \\
\hline
\end{tabular}

*Not significant at the 95 percent confidence level; the regression equation should not be used to predict the concentration of the dependent variable.

Results of regression analyses relating specific conductance to water discharge (Q)

\begin{tabular}{|c|c|c|c|c|c|c|}
\hline \multirow{2}{*}{$\begin{array}{l}\text { No. of } \\
\text { measure- } \\
\text { ments }\end{array}$} & \multirow{2}{*}{$\begin{array}{l}\text { Water discharge } \\
\frac{\left(\mathrm{ft}^{3} / \mathrm{s}\right)}{\text { Maximum Minimum }}\end{array}$} & \multirow{2}{*}{ Regression equation } & \multirow{2}{*}{$\begin{array}{l}\text { Correlation } \\
\text { coefficient }\end{array}$} & \multirow{2}{*}{$\begin{array}{l}\text { Percent } \\
\text { explained } \\
\text { variance } \\
\end{array}$} & \multicolumn{2}{|c|}{$\begin{array}{c}\text { Standard error } \\
\text { of estimate }\end{array}$} \\
\hline & & & & & Log units & Percent \\
\hline 174 & 5000 & $S p c=730 Q^{.03345}$ & 0.19 & 3.6 & 0.071 & 16 \\
\hline
\end{tabular}




\section{Platte River near Overton, Nebr.}

Location.--Lat $40^{\circ} 40^{\prime} 57^{\prime \prime}$, long $99^{\circ} 32^{\prime} 19^{\prime \prime}$, in $\mathrm{NW}^{3} \mathrm{NE}^{-1} \frac{1}{4} \mathrm{sec} .12$, T.8 N., R. 20 W., Dawson County, Hydrologic Unit 10200101, on left bank $600 \mathrm{ft}$ downstream from county highway bridge, $4 \mathrm{mi}$ south of Overton and $4 \mathrm{mi}$ downstream from Plum Creek. Prior to June 2, 1976, (north channe1) gage at site $600 \mathrm{ft}$ upstream. South channel gage discontinued June 2, 1976.

Drainage area (revised).--57,700 $\mathrm{mi}^{2}$, approximately, of which about 52,900 $\mathrm{mi}^{2}$ contributes directly to surface runoff. Approximately $4,000 \mathrm{mi}^{2}$ in Great Divide basin is not included.

Period of record.--Water years 1960 to 1968 , October 1976 to current year.

Statistical data for selected chemical constituents

\begin{tabular}{|c|c|c|c|c|c|c|c|c|c|}
\hline Constituent & Units & $\begin{array}{l}\text { No. of } \\
\text { measure- } \\
\text { ments }\end{array}$ & $\begin{array}{l}\text { Max- } \\
\text { imum }\end{array}$ & $\begin{array}{l}\text { Min- } \\
\text { imum }\end{array}$ & Mean & Median & $\begin{array}{c}\text { Standard } \\
\text { devi- } \\
\text { ation } \\
\end{array}$ & $\begin{array}{l}\text { Ninetieth } \\
\text { percent- } \\
\text { ile }\end{array}$ & $\begin{array}{l}\text { Tenth } \\
\text { percent- } \\
\text { ile } \\
\end{array}$ \\
\hline Specific conductance & umho/cm & 191 & 1190 & 421 & 824 & ---- & 116 & 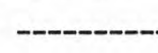 & --- \\
\hline Dissolved solids, residue & $\mathrm{mg} / \mathrm{L}$ & 177 & 866 & 270 & 558 & ----- & 89 & & --- \\
\hline Dissolved solids, sum & $\mathrm{mg} / \mathrm{L}$ & 15 & 610 & 487 & 555 & 548 & 36 & 600 & 498 \\
\hline Hardness as $\mathrm{CaCO}_{3}$ & $\mathrm{mg} / \mathrm{L}$ & 190 & 433 & 141 & 269 & ---- & 47 & & \\
\hline Calcium, dissolved & $\mathrm{mg} / \mathrm{L}$ & 77 & 107 & 47 & 72 & --- & 12 & & \\
\hline Magnesium, dissolved & $\mathrm{mg} / \mathrm{L}$ & 77 & 36 & 6.7 & 21 & --- & 4.4 & & \\
\hline Sodium, dissolved & $\mathrm{mg} / \mathrm{L}$ & 190 & 100 & 23 & 70 & ----- & 11 & & \\
\hline Potassium, dissolved & $\mathrm{mg} / \mathrm{L}$ & 77 & 24 & 10 & 12 & ----- & 1.8 & & \\
\hline Bicarbonate ion & $\mathrm{mg} / \mathrm{L}$ & 190 & 280 & 104 & 230 & ---- & 23 & & \\
\hline Sulfate, dissolved & $\mathrm{mg} / \mathrm{L}$ & 190 & 367 & 66 & 201 & ---- & 47 & & \\
\hline Chloride, dissolved & $\mathrm{mg} / \mathrm{L}$ & 77 & 37 & 8.6 & 23 & ----- & 4.4 & & \\
\hline Fluoride, dissolved & $\mathrm{mg} / \mathrm{L}$ & 76 & 0.8 & 0.3 & 0.5 & ---- & 0.1 & & \\
\hline Silica, dissolved & $\mathrm{mg} / \mathrm{L}$ & 77 & 36 & 11 & 27 & ---- & 5.6 & & \\
\hline Boron, dissolved & $\mathrm{mg} / \mathrm{L}$ & 77 & 440 & 50 & 131 & ---- & 48 & & \\
\hline Iron, dissolved & $\mathrm{ug} / \mathrm{L}$ & 62 & 130 & 0 & 23 & ----- & 29 & & \\
\hline Manganese, dissolved & $\mathrm{ug} / \mathrm{L}$ & 27 & 130 & 0 & 16 & 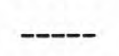 & 26 & & \\
\hline $\begin{array}{l}\text { Nitrite + nitrate as } \mathrm{N} \text {, } \\
\text { dissolved } \\
\text { Nitrate as } \mathrm{N} \text {, dissolved }\end{array}$ & $\begin{array}{l}\mathrm{mg} / \mathrm{L} \\
\mathrm{mg} / \mathrm{L}\end{array}$ & 13 & 2.0 & 0.51 & 1.0 & $\ldots$ & 0.41 & & \\
\hline Nitrate as $\mathrm{NO}_{3}$, dissolved & $\mathrm{mg} / \mathrm{L}-$ & & & & & & & & \\
\hline Ammonia $\mathrm{N}$, total & $\mathrm{mg} / \mathrm{L}-$ & & & & & & & & \\
\hline Organic $N$, total & $\mathrm{mg} / \mathrm{L}-$ & & & & & & & & \\
\hline Phosphorus, total & $\mathrm{mg} / \mathrm{L}-$ & & & & & & & & - \\
\hline Phosphorus, dissolved & $\mathrm{mg} / \mathrm{L}$ & 13 & 0.23 & 0.02 & 0.07 & 0.06 & 0.05 & 0.09 & 0.03 \\
\hline Fecal coliform & $/ 100 \mathrm{ml}-$ & & & & & & & & \\
\hline Fecal streptococci & $/ 100 \mathrm{ml}-$ & & & & & & & & \\
\hline Oxygen, dissolved & $\mathrm{mg} / \mathrm{L}-$ & & & & & & & & \\
\hline Biochemical oxygen demand $\mathrm{mg} / \mathrm{L}$ - & $\begin{array}{l}\mathrm{mg} / \mathrm{L}- \\
\mathrm{H} \text { units }\end{array}$ & 190 & 8.4 & 6.9 & 7.6 &.- & 0.3 & & \\
\hline Co $-\mathrm{Pt}$ & t unițs & 30 & 90 & 1 & 9 & ---- & 16 & & --- \\
\hline
\end{tabular}


Results of regression analyses relating concentrations of selected chemical constituents to specific conductance $(\mathrm{SpC})$

\begin{tabular}{|c|c|c|c|c|c|}
\hline $\begin{array}{l}\text { Dissolved } \\
\text { constituents } \\
(\mathrm{mg} / \mathrm{L})\end{array}$ & $\begin{array}{l}\text { No. of } \\
\text { measure- } \\
\text { ments }\end{array}$ & Regression equation & $\begin{array}{l}\text { Corre- } \\
\text { lation } \\
\text { coeffi- } \\
\text { cient }\end{array}$ & $\begin{array}{l}\text { Percent } \\
\text { explained } \\
\text { variance }\end{array}$ & $\begin{array}{c}\text { Standard } \\
\text { error of } \\
\text { estimate } \\
(\mathrm{mg} / \mathrm{L})\end{array}$ \\
\hline Dissolved solids, residue (RDS) & 177 & $\operatorname{RDS}=0.757(\mathrm{SpC})-61.463$ & 0.99 & 98 & 14 \\
\hline Dissolved solids, sum (SDS) & $-\cdots$ & & & & \\
\hline Hardness as $\mathrm{CaCO}_{3}(\mathrm{TH})$ & 187 & $\mathrm{TH}=0.378(\mathrm{SpC})-42 \cdot 325$ & .94 & 88 & 16 \\
\hline Calcium (Ca) & 74 & $\mathrm{Ca}=0.083(\mathrm{SpC})+4.051$ & .79 & 62 & 7.6 \\
\hline Magnesium (Mg) & 74 & $\mathrm{Mg}=0.032(\mathrm{SpC})-5.604$ & .84 & 71 & 2.5 \\
\hline Sodium (Na) & 187 & $\mathrm{Na}=0.080(\mathrm{SpC})+3.764$ & .84 & 71 & 6.0 \\
\hline Potassium (K) & 74 & $\mathrm{~K}=0.00006(\mathrm{SOC})+12.299$ & $* .004$ & .002 & 1.8 \\
\hline Bicarbonate $\left(\mathrm{HCO}_{3}\right)$ & 187 & $\mathrm{HCO}_{3}=0.119(\mathrm{SpC})+131.870$ & .60 & 36 & 18 \\
\hline Sulfate $\left(\mathrm{SO}_{4}\right)$ & 187 & $\mathrm{SO}_{4}=0.374(\mathrm{spC})-107.327$ & .92 & 85 & 18 \\
\hline Chloride (C1) & 74 & $\mathrm{Cl}=0.032(\mathrm{SpC})-3.699$ & .85 & 73 & 2.3 \\
\hline Silica (Si) & 74 & $\mathrm{Si}=0.014(\mathrm{SpC})+15.595$ & .30 & 9.0 & 5.2 \\
\hline
\end{tabular}

*Not significant at the 95 percent confidence level; the regression equation should not be used to predict the concentration of the dependent variable.

Results of regression analyses relating specific conductance to water discharge (Q)

\begin{tabular}{|c|c|c|c|c|c|c|c|}
\hline \multirow{2}{*}{$\begin{array}{l}\text { No. of } \\
\text { measure- } \\
\text { ments }\end{array}$} & \multicolumn{2}{|c|}{$\begin{array}{l}\text { Water discharge } \\
\left(\mathrm{ft}^{3} / \mathrm{s}\right) \\
\end{array}$} & \multirow{2}{*}{ Regression equation } & \multirow{2}{*}{$\begin{array}{l}\text { Correlation } \\
\text { coefficient }\end{array}$} & \multirow{2}{*}{$\begin{array}{l}\text { Percent } \\
\text { explained } \\
\text { variance }\end{array}$} & \multicolumn{2}{|c|}{$\begin{array}{c}\text { Standard error } \\
\text { of estimate }\end{array}$} \\
\hline & Maximum & Minimum & & & & Log units & Percent \\
\hline 189 & 13,300 & 129 & $\mathrm{SpC}=800 Q^{.00272}$ & $* 0.01$ & 0.02 & 0.065 & 15 \\
\hline
\end{tabular}


06768015 Spring Creek below Lexington, Nebr.

Location.--Lat $40^{\circ} 45^{\prime} 13^{\prime \prime}$, long $99^{\circ} 40^{\prime} 22^{\prime \prime}$, in $\mathrm{NW}^{\frac{1}{4}} \mathrm{SW}_{4}^{\frac{1}{4}} \mathrm{NW}^{\frac{1}{4}} \mathrm{sec}$. 13, T.9 N., R.21 W., Dawson County, Hydrologic Unit 10200101, at bridge on county road $0.5 \mathrm{mi}$ south of U.S. Highway $30,0.1 \mathrm{mi}$ downstream from Dawson County Drain No. 1 , and $3.2 \mathrm{mi}$ southeast of Lexington.

Period of record. - -Water year 1973 to current year.

Statistical data for selected chemical constituents

\begin{tabular}{|c|c|c|c|c|c|c|c|c|c|}
\hline Constituent & Units & $\begin{array}{l}\text { No. of } \\
\text { measure- } \\
\text { ments }\end{array}$ & $\begin{array}{l}\text { Max- } \\
\text { imum }\end{array}$ & $\begin{array}{l}\text { Min- } \\
\text { imum }\end{array}$ & Mean & Median & $\begin{array}{c}\text { Standard } \\
\text { devi- } \\
\text { ation }\end{array}$ & $\begin{array}{l}\text { Ninetieth } \\
\text { percent- } \\
\text { ile }\end{array}$ & $\begin{array}{l}\text { Tenth } \\
\text { percent- } \\
\text { ile }\end{array}$ \\
\hline Specific conductance & umho/cm & 54 & 2100 & 481 & 1260 & 1280 & 346 & 1720 & 840 \\
\hline Dissolved solids, residue & $\mathrm{mg} / \mathrm{L}$ & 33 & 1150 & 324 & 811 & 830 & 199 & 1010 & 580 \\
\hline Dissolved solids, sum & $\mathrm{mg} / \mathrm{L}$ & 17 & 1290 & 456 & 838 & 810 & 237 & 1150 & 536 \\
\hline Hardness as $\mathrm{CaCO}_{3}$ & $\mathrm{mg} / \mathrm{L}$ & 17 & 500 & 210 & 372 & 380 & 88 & 483 & 250 \\
\hline Calcium, dissolved & $\mathrm{mg} / \mathrm{L}$ & 17 & 130 & 60 & 103 & 100 & 24 & 127 & 69 \\
\hline Magnesium, dissolved & $\mathrm{mg} / \mathrm{L}$ & 17 & 42 & 14 & 28 & 29 & 7.2 & 38 & 20 \\
\hline Sodium, dissolved & $\mathrm{mg} / \mathrm{L}$ & 17 & 240 & 60 & 122 & 100 & 55 & 220 & 62 \\
\hline Potassium, dissolved & $\mathrm{mg} / \mathrm{L}$ & 17 & 39 & 12 & 27 & 28 & 6.9 & 35 & 18 \\
\hline Bicarbonate ion & $\mathrm{mg} / \mathrm{L}$ & 17 & 454 & 214 & 358 & 380 & 76 & 450 & 262 \\
\hline Sulfate, dissolved & $\mathrm{mg} / \mathrm{L}$ & 17 & 510 & 130 & 257 & 240 & 93 & 400 & 160 \\
\hline Chloride, dissolved & $\mathrm{mg} / \mathrm{L}$ & 50 & 290 & 16 & 74 & 57 & 57 & 170 & 26 \\
\hline Fluoride, dissolved & $\mathrm{mg} / \mathrm{L}$ & 17 & 1.0 & 0.3 & 0.6 & 0.6 & 0.2 & 0.8 & 0.4 \\
\hline Silica, dissolved & $\mathrm{mg} / \mathrm{L}$ & 17 & 68 & 22 & 39 & 39 & 10 & 51 & 29 \\
\hline Boron, dissolved & $\mathrm{mg} / \mathrm{L}$ & 17 & 360 & 130 & 210 & 185 & 69 & 330 & 160 \\
\hline Iron, dissolved & $\mathrm{ug} / \mathrm{L}$ & 17 & 250 & 20 & 82 & 50 & 61 & 160 & 30 \\
\hline Manganese, dissolved & $\mathrm{ug} / \mathrm{L}$ & 17 & 170 & 0 & 52 & 30 & 49 & 140 & 10 \\
\hline $\begin{array}{l}\text { Nitrite + nitrate as } \mathrm{N} \text {, } \\
\text { dissolved } \\
\text { Nitrate as } \mathrm{N} \text {, dissolved }\end{array}$ & $\begin{array}{l}\mathrm{mg} / \mathrm{L} \\
\mathrm{mg} / \mathrm{L}\end{array}$ & 24 & 5.5 & 0.12 & 2.3 & 2.2 & 1.4 & 3.9 & 0.6 \\
\hline Nitrate as $\mathrm{NO}_{3}$, dissolved & $\mathrm{mg} / \mathrm{L}$ & & & & & & & & \\
\hline Ammonia $\mathrm{N}$, total & $\mathrm{mg} / \mathrm{L}$ & 51 & 17 & .02 & 1.5 & .49 & 2.9 & 4.2 & .05 \\
\hline Organic $N$, total & $\mathrm{mg} / \mathrm{L}$ & 51 & 6.7 & 0.00 & 1.7 & 1.4 & 1.4 & 3.0 & 0.62 \\
\hline Phosphorus, total & $\mathrm{mg} / \mathrm{L}$ & 51 & 7.5 & 0.25 & 1.9 & 1.2 & 1.8 & 4.6 & 0.53 \\
\hline Phosphorus, dissolved & $\mathrm{mg} / \mathrm{L}$ & 41 & 6.7 & 0.05 & 1.6 & 0.94 & 1.8 & 4.6 & 0.24 \\
\hline Fecal coliform & $\operatorname{col} / 100 \mathrm{ml}$ & \multicolumn{2}{|c|}{32236000} & 260 & ---- & 5950 & -- -- & 65,500 & 833 \\
\hline Fecal streptococci & $\mathrm{col} / 100 \mathrm{ml}$ & 43 & 50000 & 50 & ---- & 2880 & $-\cdots$ & 23,000 & 437 \\
\hline Oxygen, dissolved & $\mathrm{mg} / \mathrm{L}$ & 49 & 17.0 & 0.8 & 9.0 & 8.4 & 3.3 & 13.3 & 4.6 \\
\hline \multirow{2}{*}{$\begin{array}{l}\text { Biochemical oxygen demand } \\
\mathrm{pH}^{(5-\text { day) }}\end{array}$} & $\mathrm{mg} / \mathrm{L}$ & 47 & 44.0 & 3.4 & $12 \cdot 3$ & 10 & 8.1 & 23.3 & 4.6 \\
\hline & units & 53 & 8.3 & $7 \cdot 3$ & 7.8 & 7.8 & 0.3 & 8.2 & 7.5 \\
\hline Co $-\mathrm{Pt}$ & $t$ units & 17 & 70 & 13 & 31 & .23 & 15 & 50 & 17 \\
\hline
\end{tabular}


Results of regression analyses relating concentrations of selected chemical constituents to specific conductance $(\mathrm{SpC})$

\begin{tabular}{|c|c|c|c|c|c|}
\hline $\begin{array}{l}\text { Dissolved } \\
\text { constituents } \\
(\mathrm{mg} / \mathrm{L})\end{array}$ & $\begin{array}{l}\text { No. of } \\
\text { measure- } \\
\text { ments }\end{array}$ & Regression equation & $\begin{array}{l}\text { Corre- } \\
\text { lation } \\
\text { coeffi- } \\
\text { cient }\end{array}$ & $\begin{array}{l}\text { Percent } \\
\text { explained } \\
\text { variance }\end{array}$ & $\begin{array}{c}\text { Standard } \\
\text { error of } \\
\text { estimate } \\
\text { (mg/L) }\end{array}$ \\
\hline Dissolved solids, residue (RDS) & 32 & $\mathrm{RDS}=0.525(\mathrm{SpC})+165.156$ & 0.89 & 79 & 92 \\
\hline Dissolved solids, sum (SDS) & 17 & $\mathrm{SDS}=0.601(\mathrm{SpC})+44.054$ & .93 & 87 & 90 \\
\hline Hardness as $\mathrm{CaCO}_{3}(\mathrm{TH})$ & 17 & $\mathrm{TH}=0.176(\mathrm{SpC})+140.257$ & .73 & 53 & 63 \\
\hline Calcium (Ca) & 17 & $\mathrm{Ca}=0.051(\mathrm{SpC})+35.746$ & .77 & 60 & 16 \\
\hline Magnesium (Mg) & 17 & $\mathrm{Mg}=0.012(\mathrm{SpC})+11.887$ & .62 & 39 & 5.8 \\
\hline Sodium (Na) & 17 & $\mathrm{Na}=0.129(\mathrm{SpC})-48.537$ & .86 & 75 & 28 \\
\hline Potassium (K) & 17 & $K=0.008(\mathrm{SpC})+16.260$ & $* .44$ & 20 & 6.4 \\
\hline Bicarbonate $\left(\mathrm{HCO}_{3}\right)$ & 17 & $\mathrm{HCO}_{3}=0.161(\mathrm{SpC})+144.546$ & .78 & 60 & 49 \\
\hline Sulfate $\left(\mathrm{SO}_{4}\right)$ & 17 & $\mathrm{SO}_{4}=0.149(\mathrm{SpC})+59.902$ & .59 & 34 & 78 \\
\hline Chloride $(\mathrm{Cl})$ & 49 & $\mathrm{Cl}=0.109(\mathrm{SpC})-62.874$ & .66 & 44 & 43 \\
\hline Silica (Si) & 17 & $\mathrm{Si}=0.021(\mathrm{SpC})+10.948$ & .78 & 61 & 6.5 \\
\hline Nitrite + Nitrate as $\mathrm{N}\left(\mathrm{NO}_{2}-\mathrm{NO}_{3}\right)$ & 24 & $\mathrm{NO}_{2}-\mathrm{NO}_{3}=0.003(\mathrm{SpC})-1.096$ & .65 & 42 & 1.1 \\
\hline
\end{tabular}

*Not significant at the 95 percent confidence level; the regression equation should not be used to predict the concentration of the dependent variable.

Results of regression analyses relating specific conductance to water discharge (Q)

\begin{tabular}{lccccccc}
\hline $\begin{array}{c}\text { No. of } \\
\text { measure } \\
\text { ments }\end{array}$ & $\begin{array}{c}\text { Water discharge } \\
\left(\mathrm{ft}^{3} / \mathrm{s}\right)\end{array}$ & Maximum Minimum & Regression equation & $\begin{array}{c}\text { Correlation } \\
\text { coefficient }\end{array}$ & $\begin{array}{c}\text { Percent } \\
\text { explained } \\
\text { variance }\end{array}$ & \multicolumn{2}{c}{$\begin{array}{c}\text { Standard error } \\
\text { of estimate }\end{array}$} \\
\hline 53 & $200 \quad 2$ & $\mathrm{SpC}=1868 \bar{Q}^{-0.17027}$ & 0.63 & 40 & 0.098 & 23 \\
\hline
\end{tabular}


Location.--Lat $40^{\circ} 36^{\prime} 00^{\prime \prime}$, long $99^{\circ} 08^{\circ} 30^{\prime \prime}$, in $\mathrm{SE}^{\frac{1}{4}} \mathrm{sec} .5$, T.7 N., R.16 W., Kearney County, at gaging station about $1,000 \mathrm{ft}$ north of east-west county road, $5.3 \mathrm{mi}$ upstream from mouth, $8 \mathrm{mi}$ southwest of Kearney, and $8.2 \mathrm{mi}$ north of Axtell.

Period of record.---February 1969 to September 1971.

Statistical data for selected chemical constituents

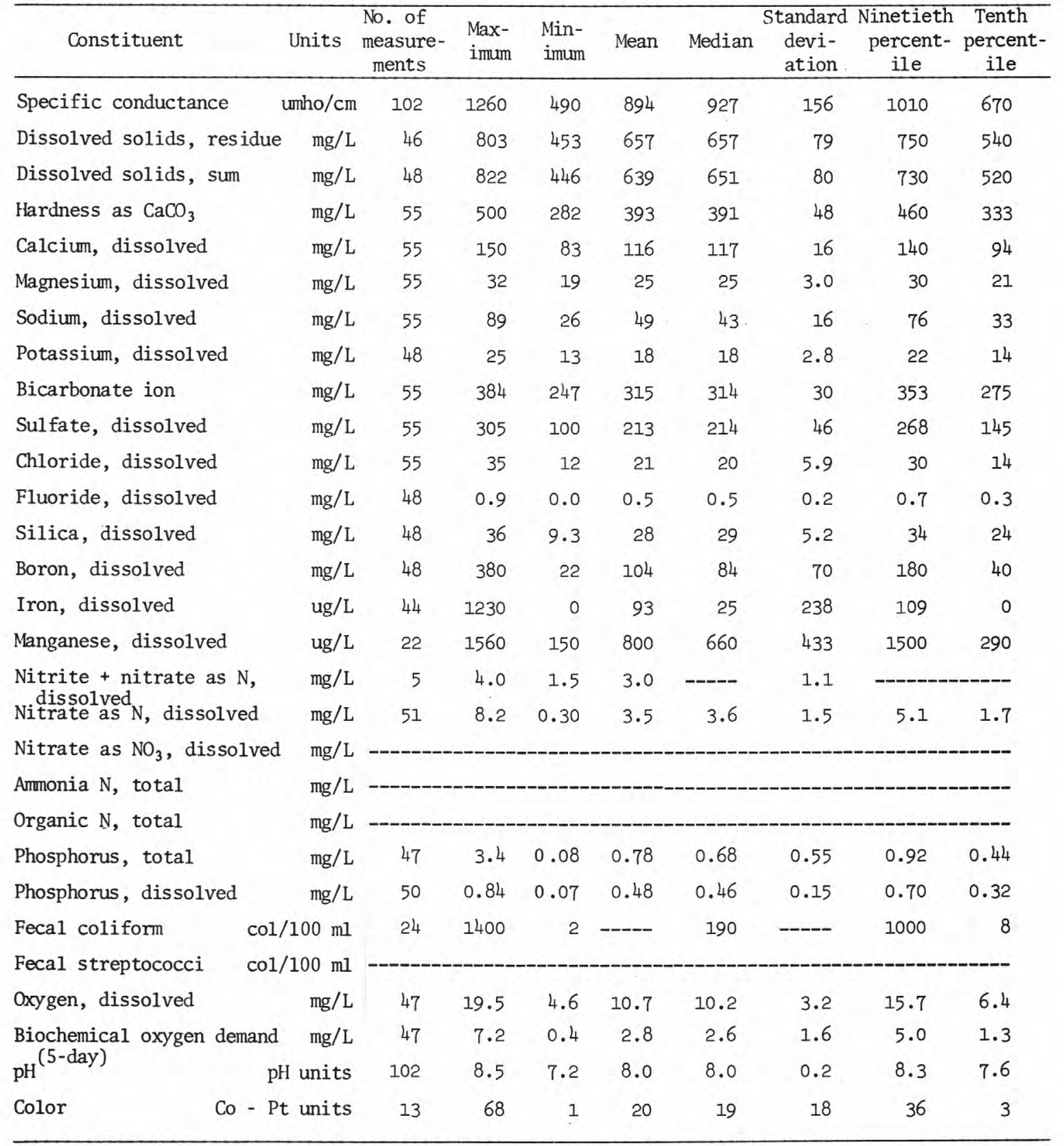


MIDDLE PLATTE RIVER BASIN

06770190 North Dry Creek near Kearney, Nebr.--Continued

Results of regression analyses relating concentrations of selected chemical constituents to specific conductance ( $\mathrm{SpC}$ )

\begin{tabular}{|c|c|c|c|c|c|}
\hline $\begin{array}{l}\text { Dissolved } \\
\text { constituents } \\
(\mathrm{mg} / \mathrm{L})\end{array}$ & $\begin{array}{l}\text { No. of } \\
\text { measure- } \\
\text { ments }\end{array}$ & Regression equation & $\begin{array}{l}\text { Corre- } \\
\text { lation } \\
\text { coeffi- } \\
\text { cient }\end{array}$ & $\begin{array}{l}\text { Percent } \\
\text { explained } \\
\text { variance }\end{array}$ & $\begin{array}{c}\text { Standard } \\
\text { error of } \\
\text { estimate } \\
(\mathrm{mg} / \mathrm{L})\end{array}$ \\
\hline Dissolved solids, residue (RDS) & 46 & $\operatorname{RDS}=0.696(\mathrm{SpC})+20.274$ & 0.93 & 86 & 30 \\
\hline Dissolved solids, sum (SDS) & 48 & $\mathrm{SDS}=0.676(\mathrm{SpC})+14.986$ & .94 & 89 & 27 \\
\hline Hardness as $\mathrm{CaCO}_{3}(\mathrm{TH})$ & 55 & $\mathrm{TH}=0.273(\mathrm{SpC})+141.160$ & .62 & 38 & 38 \\
\hline Calcium (Ca) & 55 & $\mathrm{Ca}=0.068(\mathrm{SpC})+53.221$ & .45 & 21 & 14 \\
\hline Magnesium (Mg) & 55 & $\mathrm{Mg}=0.024(\mathrm{SpC})+2.894$ & .84 & 71 & 1.7 \\
\hline Sodium (Na) & 55 & $\mathrm{Na}=0.108(\mathrm{SpC})-50.710$ & .72 & 52 & 11 \\
\hline Potassium (K) & 48 & $\mathrm{~K}=0.007(\mathrm{SpC})+10.844$ & .30 & 8.9 & 2.7 \\
\hline Bicarbonate $\left(\mathrm{HCO}_{3}\right)$ & 55 & $\mathrm{HCO}_{3}=0.050(\mathrm{SpC})+269.073$ & $* .18$ & 3.2 & 30 \\
\hline Sulfate $\left(\mathrm{SO}_{4}\right)$ & 55 & $\mathrm{SO}_{4}=0.395(\mathrm{SpC})-151.108$ & .92 & 84 & 19 \\
\hline Chloride (C1) & 55 & $\mathrm{Cl}=0.044(\mathrm{SpC})-19.552$ & .79 & 63 & 3.6 \\
\hline Silica (Si) & 48 & $\mathrm{Si}=-.007(\mathrm{SpC})+34.042$ & *-. 15 & 2.2 & 5.2 \\
\hline
\end{tabular}

*Not significant at the 95 percent confidence level; the regression equation should not be used to predict the concentration of the dependent variable.

Results of regression analyses relating specific conductance to water discharge (Q)

\begin{tabular}{llllccc}
\hline $\begin{array}{c}\text { No. of } \\
\text { measure- } \\
\text { ments }\end{array}$ & $\begin{array}{c}\text { Water discharge } \\
\text { Maximum Minimum }\end{array}$ & Regression equation & $\begin{array}{c}\text { Correlation } \\
\text { coefficient }\end{array}$ & $\begin{array}{c}\text { Percent } \\
\text { explained } \\
\text { variance }\end{array}$ & $\begin{array}{c}\text { Standard error } \\
\text { of estimate }\end{array}$ \\
\hline 102 & 25 & 5 & $\mathrm{SpC}=938 \mathrm{Q}^{-.02816}$ & $*-.05$ & 23 & 0.086 \\
\hline
\end{tabular}


Location. - -Lat $40^{\circ} 40^{\prime} 30^{\prime \prime}$, long $99^{\circ} 00^{\prime} 24^{\prime \prime}$, in $\mathrm{SW}^{\frac{1}{4}} \mathrm{NW}^{\frac{1}{4}} \mathrm{SW}^{\frac{1}{4}} \mathrm{sec}$. 10 , T.8 N., R, 15 W., Buffalo County, Hydrologic Unit 10200101, on county road, $0.2 \mathrm{mi}$ north of Interstate Highway I-80 (no access) and about $4.5 \mathrm{mi}$ southeast of Kearney.

Period of record.--Water year 1953 to current year.

Statistical data for selected chemical constituents

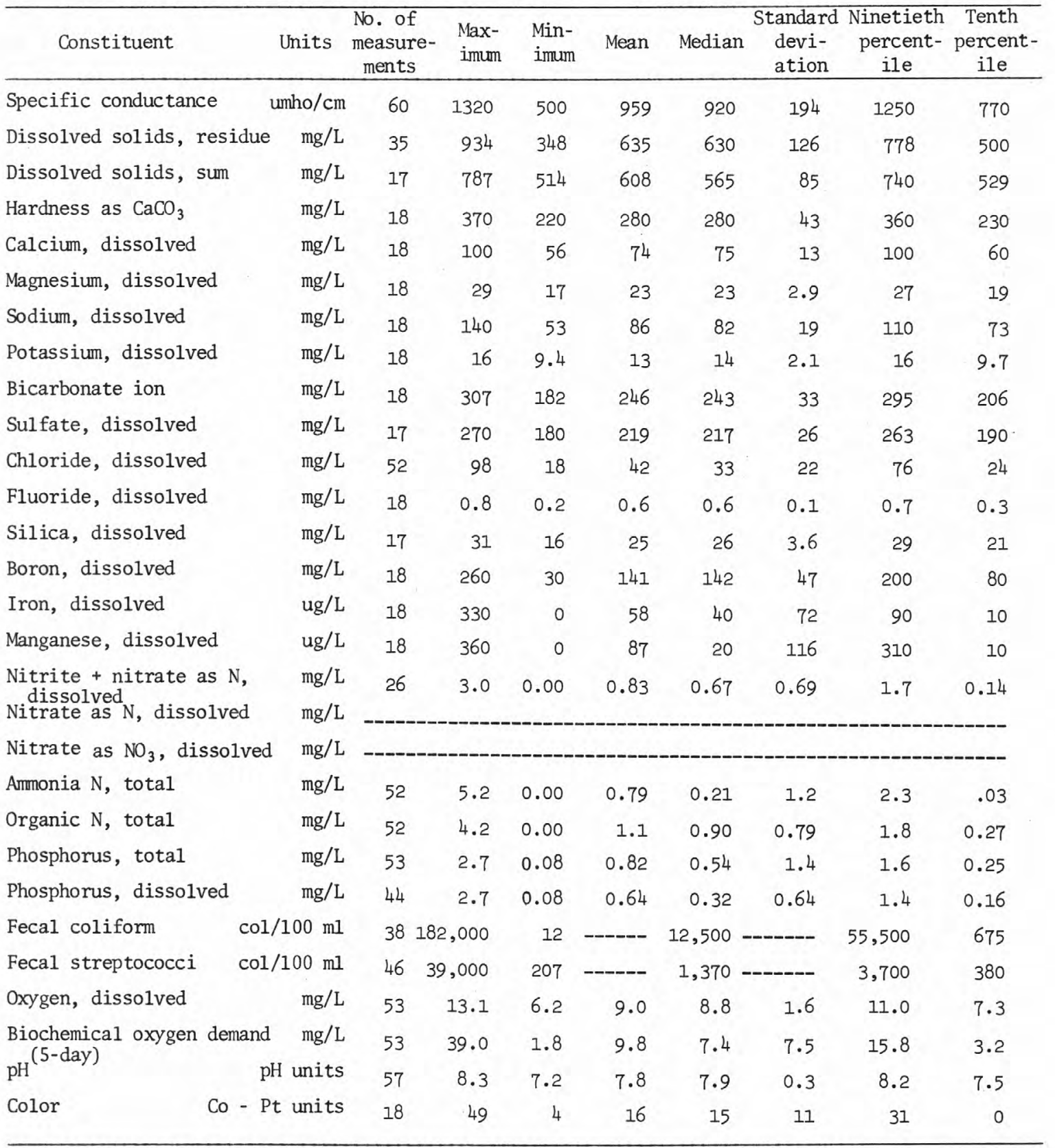


06770205 Platte River (North Channel) near Kearney, Nebr.--Continued

Results of regression analyses relating concentrations of selected chemical constituents to specific conductance $(\mathrm{SpC})$

\begin{tabular}{|c|c|c|c|c|c|}
\hline $\begin{array}{l}\text { Dissolved } \\
\text { constituents } \\
(\mathrm{mg} / \mathrm{L})\end{array}$ & $\begin{array}{l}\text { No. of } \\
\text { measure- } \\
\text { ments }\end{array}$ & Regression equation & $\begin{array}{l}\text { Corre- } \\
\text { lation } \\
\text { coeffi- } \\
\text { cient }\end{array}$ & $\begin{array}{l}\text { Percent } \\
\text { explained } \\
\text { variance }\end{array}$ & $\begin{array}{c}\text { Standard } \\
\text { error of } \\
\text { estimate } \\
(\mathrm{mg} / \mathrm{L})\end{array}$ \\
\hline Dissolved solids, residue (RDS) & 34 & $\mathrm{RDS}=0.543(\mathrm{SpC})+110.910$ & 0.91 & 82 & 54 \\
\hline Dissolved solids, sum (SDS) & 18 & $\mathrm{SDS}=0.482(\mathrm{SpC})+161.632$ & .86 & 74 & 44 \\
\hline Hardness as $\mathrm{CaCO}_{3}(\mathrm{TH})$ & 18 & $\mathrm{TH}=0.221(\mathrm{SpC})+76.038$ & .74 & 56 & 29 \\
\hline Calcium (Ca) & 18 & $\mathrm{Ca}=0.065(\mathrm{SpC})+14.150$ & .73 & 53 & 9.1 \\
\hline Magnesium $(\mathrm{Mg})$ & 18 & $\mathrm{Mg}=0.014(\mathrm{SpC})+9.348$ & .72 & 52 & 2.1 \\
\hline Sodium (Na) & 18 & $\mathrm{Na}=0.105(\mathrm{SpC})-11.312$ & .82 & 67 & 11 \\
\hline Potassium (K) & 18 & $\mathrm{~K}=0.006(\mathrm{SpC})+7.262$ & $* .44$ & 20 & 1.9 \\
\hline Bicarbonate $\left(\mathrm{HCO}_{3}\right)$ & 18 & $\mathrm{HCO}_{3}=0.153(\mathrm{SpC})+103.415$ & .67 & 45 & 25 \\
\hline Sulfate $\left(\mathrm{SO}_{4}\right)$ & 17 & $\mathrm{SO}_{4}=0.157(\mathrm{SpC})+72.947$ & .87 & 75 & 13 \\
\hline Chloride (C1) & 51 & $\mathrm{C} 1=0.090(\mathrm{SpC})-44.585$ & .80 & 64 & 13 \\
\hline Silica (Si) & 17 & $\mathrm{Si}=-.006(\mathrm{SpC})+31.161$ & $*-.25$ & 6.2 & 3.6 \\
\hline Nitrite + Nitrate as $\mathrm{N}\left(\mathrm{NO}_{2}-\mathrm{NO}_{3}\right)$ & 26 & $\mathrm{NO}_{2}-\mathrm{NO}_{3}=0.003(\mathrm{SpC})-1.948$ & .62 & 39 & .55 \\
\hline
\end{tabular}

*Not significant at the 95 percent confidence level; the regression equation should not be used to predict the concentration of the dependent variable.

Results of regression analyses relating specific conductance to water discharge (0)

\begin{tabular}{ccccccc}
\hline $\begin{array}{c}\text { No. of } \\
\text { measure- } \\
\text { ments }\end{array}$ & $\begin{array}{c}\text { Water discharge } \\
\left(\mathrm{ft}^{3} / \mathrm{s}\right)\end{array}$ & Maximum Minimum & Regression equation & $\begin{array}{c}\text { Correlation } \\
\text { coefficient }\end{array}$ & $\begin{array}{c}\text { Percent } \\
\text { explained } \\
\text { variance }\end{array}$ & $\begin{array}{c}\text { Standard error } \\
\text { of estimate }\end{array}$ \\
\hline 60 & 400 & 4.5 & $\mathrm{SpC}=164 \bar{Q}^{-0.13567}$ & -0.78 & 60 & 0.058 \\
\hline
\end{tabular}




\section{MIDDLE PLATTE RIVER BASIN}

06770500 Platte River near Grand Island, Nebr.

Location. --Lat $40^{\circ} 52^{\prime} 2^{\prime \prime}$, 1ong $98^{\circ} 16^{\prime} 54^{\prime \prime}$, in SW $W^{\frac{1}{4}} \mathrm{SW}^{\frac{1}{4}} \mathrm{sec}$. 31, T.11 N., R.8 W., Merrick County, Hydrologic Unit 10200101, on left bank $118 \mathrm{ft}$ downstream from bridge on U.S. Highway 34, $2 \mathrm{mi}$ upstream from Burlington Northern, Inc., bridge, and $5 \mathrm{mi}$ southeast of Grand Island.

Drainage area (revised).--58,800 $\mathrm{mi}^{2}$, approximately, of which about 54,000 $\mathrm{mi}^{2}$ contributes directly to surface runoff. Approximately 4,000 $\mathrm{mi}^{2}$ in Great Divide basin is not included.

Period of record.-- Water year 1972 to current year.

Statistical data for selected chemical constituents

\begin{tabular}{|c|c|c|c|c|c|c|c|c|c|}
\hline Constituent & Units & $\begin{array}{l}\text { No. of } \\
\text { measure- } \\
\text { ments }\end{array}$ & $\begin{array}{l}\text { Max- } \\
\text { imum }\end{array}$ & $\begin{array}{l}\text { Min- } \\
\text { imum }\end{array}$ & Mean & Median & $\begin{array}{c}\text { Standard } \\
\text { devi- } \\
\text { ation }\end{array}$ & $\begin{array}{c}\text { Ninetieth } \\
\text { percent- } \\
\text { ile }\end{array}$ & $\begin{array}{c}\text { Tenth } \\
\text { percent- } \\
\text { ile } \\
\end{array}$ \\
\hline Specific conductance & umho/cm & 64 & 1040 & 688 & 847 & 835 & 84 & 960 & 745 \\
\hline Dissolved solids, residue & $\mathrm{mg} / \mathrm{L}$ & 9 & 698 & 499 & 593 & $-\cdots$ & 71 & $-\cdots-$ & $-\cdots$ \\
\hline Dissolved solids, sum & $\mathrm{mg} / \mathrm{L}$ & 55 & 699 & 435 & 575 & 567 & 52 & 640 & 520 \\
\hline Hardness as $\mathrm{CaCO}_{3}$ & $\mathrm{mg} / \mathrm{L}$ & 56 & 350 & 210 & 272 & 273 & 31 & 320 & 235 \\
\hline Calcium, dissolved & $\mathrm{mg} / \mathrm{L}$ & 56 & 95 & 55 & 72 & 72 & 9.3 & 87 & 62 \\
\hline Magnesium, dissolved & $\mathrm{mg} / \mathrm{L}$ & 56 & 28 & 17 & 22 & 22 & 2.7 & 27 & 20 \\
\hline Sodium, dissolved & $\mathrm{mg} / \mathrm{L}$ & 56 & 95 & 53 & 80 & 80 & 8.2 & 89 & 70 \\
\hline Potassium, dissolved & $\mathrm{mg} / \mathrm{L}$ & 56 & 17 & 5.0 & 12 & 12 & 1.8 & 14 & 9.8 \\
\hline Bicarbonate ion & $\mathrm{mg} / \mathrm{L}$ & 56 & 280 & 182 & 227 & 228 & 18 & 249 & 200 \\
\hline Sulfate, dissolved & $\mathrm{mg} / \mathrm{L}$ & 56 & 300 & 160 & 222 & 223 & 31 & 275 & 190 \\
\hline Chloride, dissolved & $\mathrm{mg} / \mathrm{L}$ & 62 & 36 & 19 & 27 & 28 & 3.7 & 32 & 23 \\
\hline Fluoride, dissolved & $\mathrm{mg} / \mathrm{L}$ & 58 & 0.8 & 0.4 & 0.6 & 0.5 & 0.1 & 0.7 & 0.5 \\
\hline Silica, dissolved & $\mathrm{mg} / \mathrm{L}$ & 56 & 30 & 12 & 23 & 24 & 4.0 & 28 & 18 \\
\hline Boron, dissolved & $\mathrm{mg} / \mathrm{L}$ & 56 & 250 & 100 & 137 & 140 & 24 & 164 & 120 \\
\hline Iron, dissolved & $\mathrm{ug} / \mathrm{L}$ & 56 & 100 & 0 & 25 & 21 & 21 & 51 & 9 \\
\hline Manganese, dissolved & $\mathrm{ug} / \mathrm{L}$ & 56 & 230 & 0 & 11 & 0 & 31 & 22 & 0 \\
\hline $\begin{array}{l}\text { Nitrite }+ \text { nitrate as } N \text {, } \\
\text { dissolved } \\
\text { Nitrate as } N \text {, dissolved }\end{array}$ & $\begin{array}{l}\mathrm{mg} / \mathrm{L} \\
\mathrm{mg} / \mathrm{L}\end{array}$ & 34 & 1.3 & 0.00 & 0.43 & 0.38 & 0.37 & 0.85 & .02 \\
\hline Nitrate as $\mathrm{NO}_{3}$, dissolved & $\mathrm{mg} / \mathrm{L}$ & & & & & & & & \\
\hline Ammonia $\mathrm{N}$, total & $\mathrm{mg} / \mathrm{L}$ & 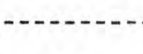 & - & -2 & 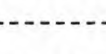 & 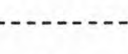 & & & -- \\
\hline Organic $N$, total & $\mathrm{mg} / \mathrm{L}$ & 52 & 3.0 & 0.25 & 0.91 & 0.79 & 0.52 & 1.4 & 0.40 \\
\hline Phosphorus, total & $\mathrm{mg} / \mathrm{L}$ & 52 & 3.5 & 0.06 & 0.23 & 0.16 & 0.47 & 0.26 & 0.10 \\
\hline Phosphorus, dissolved & $\mathrm{mg} / \mathrm{L}$ & 62 & 0.26 & 0.00 & 0.07 & 0.07 & 0.05 & 0.14 & 0.03 \\
\hline Fecal coliform & $\mathrm{col} / 100 \mathrm{ml}$ & 40 & 2060 & 3 & $\cdots-$ & 97 & $\cdots-$ & 870 & 11 \\
\hline Fecal streptococci & $\mathrm{col} / 100 \mathrm{ml}$ & 49 & 3200 & 10 & $-\cdots$ & 110 & $-\cdots$ & 997 & 25 \\
\hline Oxygen, dissolved & $\mathrm{mg} / \mathrm{L}$ & 63 & 14.2 & 5.0 & 10.3 & 10.0 & 1.8 & 12.7 & 8.1 \\
\hline $\begin{array}{l}\text { Biochemical oxygen demand } \\
\text { (5-day) }\end{array}$ & $\mathrm{mg} / \mathrm{L}$ & 48 & 31.0 & 1.4 & 7.8 & 6.2 & 5.8 & 15.0 & 2.0 \\
\hline $\mathrm{pH}^{(3-\text { ady) }}$ & $\mathrm{pH}$ units & 78 & 8.7 & 7.2 & 8.0 & 8.2 & 0.3 & 8.4 & 7.5 \\
\hline $\mathrm{Co}-\mathrm{Pt}$ & Co - Pt units & 56 & 50 & 1 & 11 & 8 & 8.8 & 20 & 3 \\
\hline
\end{tabular}


Results of regression analyses relating concentrations of selected chemical constituents to specific conductance $(\mathrm{SpC})$

\begin{tabular}{|c|c|c|c|c|c|}
\hline $\begin{array}{l}\text { Dissolved } \\
\text { constituents } \\
(\mathrm{mg} / \mathrm{L})\end{array}$ & $\begin{array}{l}\text { No. of } \\
\text { measure- } \\
\text { ments }\end{array}$ & Regression equation & $\begin{array}{l}\text { Corre- } \\
\text { lation } \\
\text { coeffi- } \\
\text { cient }\end{array}$ & $\begin{array}{l}\text { Percent } \\
\text { explained } \\
\text { variance }\end{array}$ & $\begin{array}{c}\text { Standard } \\
\text { error of } \\
\text { estimate } \\
(\mathrm{mg} / \mathrm{L})\end{array}$ \\
\hline Dissolved solids, residue (RDS) & & & & & \\
\hline Dissolved solids, sum (SDS) & 53 & $\mathrm{SDS}=0.547(\mathrm{SpC})+113.030$ & 0.86 & 74 & 27 \\
\hline Hardness as $\mathrm{CaCO}_{3}(\mathrm{TH})$ & 56 & $\mathrm{TH}=0.315(\mathrm{SpC})+6.879$ & .83 & 69 & 18 \\
\hline Calcium (Ca) & 56 & $\mathrm{Ca}=0.082(\mathrm{SpC})+2.923$ & .73 & 54 & 6.4 \\
\hline Magnesium (Mg) & 56 & $\mathrm{Mg}=0.026(\mathrm{SpC})+.504$ & .81 & 65 & 1.6 \\
\hline Sodium ( $\mathrm{Na})$ & 56 & $\mathrm{Na}=0.060(\mathrm{SpC})+28.840$ & .61 & 38 & 6.5 \\
\hline Potassium (K) & 56 & $K=-.002(\mathrm{SpC})+13.315$ & $*-.09$ & .80 & 1.8 \\
\hline Bicarbonate $\left(\mathrm{HCO}_{3}\right)$ & 56 & $\mathrm{HCO}_{3}=0.123(\mathrm{SpC})+123.730$ & .56 & 32 & 15 \\
\hline Sulfate $\left(\mathrm{SO}_{4}\right)$ & 56 & $\mathrm{SO}_{4}=0.293(\mathrm{SpC})-24.318$ & .79 & 62 & 19 \\
\hline Chloride (C1) & 62 & $\mathrm{C} 1=0.026(\mathrm{SpC})+5.451$ & .60 & 36 & 3.0 \\
\hline Silica (Si) & 56 & $\mathrm{Si}=0.002(\mathrm{SpC})+21.155$ & $* .04$ & .17 & 4.0 \\
\hline Nitrite + Nitrate as $\mathrm{N}\left(\mathrm{NO}_{2}-\mathrm{NO}_{3}\right)$ & 34 & $\mathrm{NO}_{2}-\mathrm{NO}_{3}=0.002(\mathrm{SpC})-1.650$ & .49 & 24 & .32 \\
\hline
\end{tabular}

*Not significant at the 95 percent confidence leve1; the regression equation should not be used to predict the concentration of the dependent variable.

Results of regression analyses relating specific conductance to water discharge (Q)

\begin{tabular}{llllcccc}
\hline \multirow{2}{*}{$\begin{array}{c}\text { No. of } \\
\text { measure- } \\
\text { ments }\end{array}$} & $\begin{array}{c}\text { Water discharge } \\
\left(\mathrm{ft}^{3} / \mathrm{s}\right)\end{array}$ & Maximum Minimum & Regression equation & $\begin{array}{c}\text { Correlation } \\
\text { coefficient }\end{array}$ & $\begin{array}{c}\text { Percent } \\
\text { explained } \\
\text { variance }\end{array}$ & \multicolumn{2}{c}{$\begin{array}{c}\text { Standard error } \\
\text { of estimate }\end{array}$} \\
\hline $64 \quad 14,000 \quad 26$ & $\mathrm{SpC}=698 Q^{.02662}$ & 0.33 & 11 & 0.040 & 9.2 \\
\hline
\end{tabular}




\section{MIDDLE PLATTE RIVER BASIN}

06771500 Wood River near Gibbon, Nebr.

Location.--Lat $40^{\circ} 46^{\prime} 17^{\prime \prime}$, long $98^{\circ} 47^{\prime} 51^{\prime \prime}$, in NW' $N^{1} W^{\frac{1}{4}} \mathrm{sec} .9$, T.9 N., R.13 W., Buffalo County, Hydrologic Unit 10200102, Buffalo County, on left bank $10 \mathrm{ft}$ downstream from bridge on county highway and $2.5 \mathrm{mi}$ northeast of Gibbon.

Drainage area. $--572 \mathrm{mi}^{2}$.

Period of record.--October 1974 to November 1976.

Statistical data for selected chemical constituents

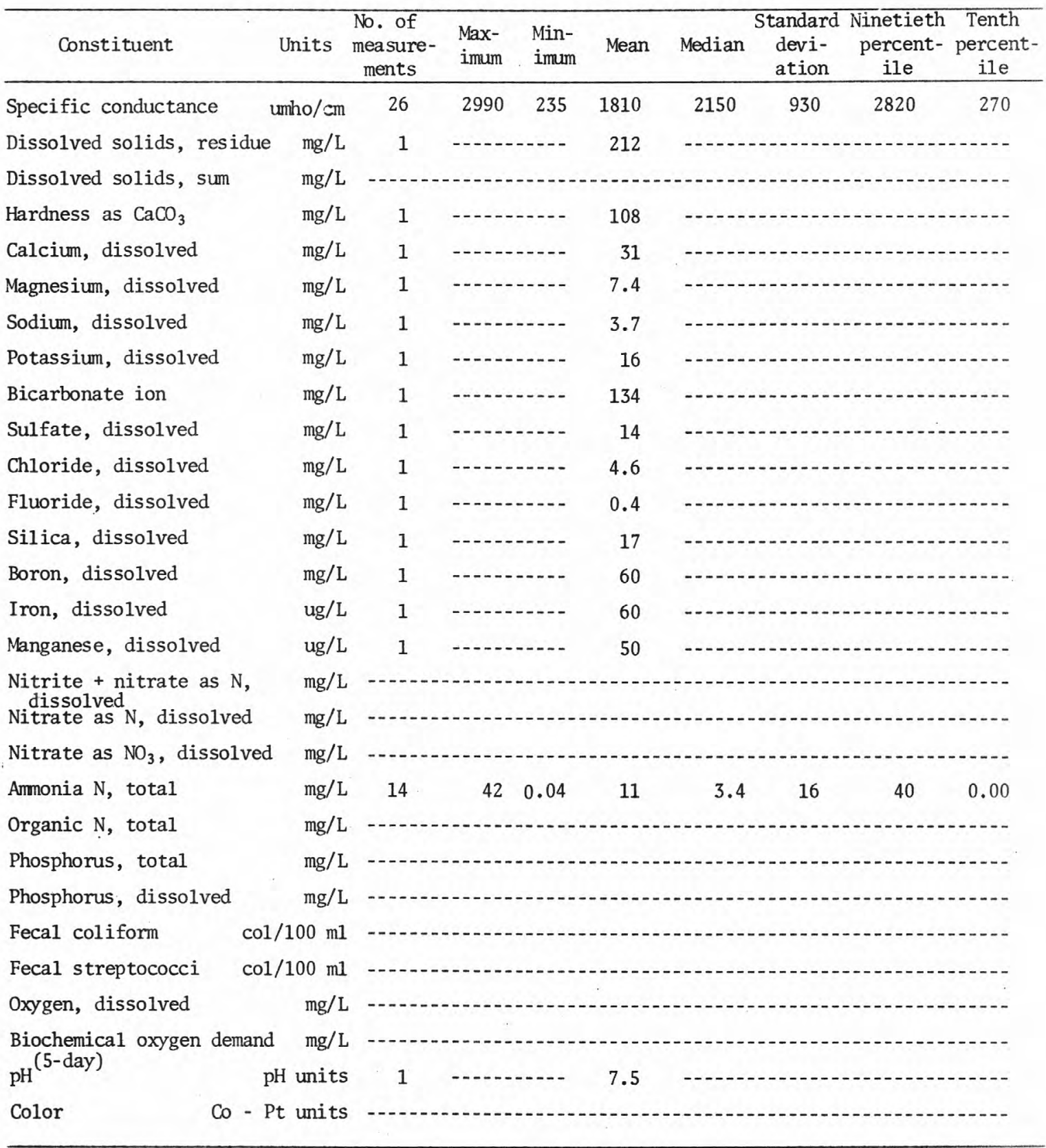


Location.--Lat $40^{\circ} 51^{\prime} 10^{\prime \prime}$, long $98^{\circ} 28^{\prime} 20^{\prime \prime}$, in $\mathrm{NE}^{\frac{1}{4}} \mathrm{SE}^{\frac{1}{4}} \mathrm{sec} .7$, T.10 N., R.10 W., Hall County, Hydrologic Unit 10200102, on right bank $1.2 \mathrm{mi}$ south of Alda, 2.2 mi upstream from old north channel of the Platte River, and $19 \mathrm{mi}$ upstream from present mouth.

Drainage area. $--628 \mathrm{mi}^{2}$.

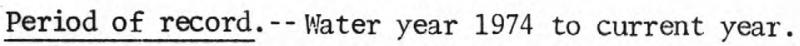

Statistical data for selected chemical constituents

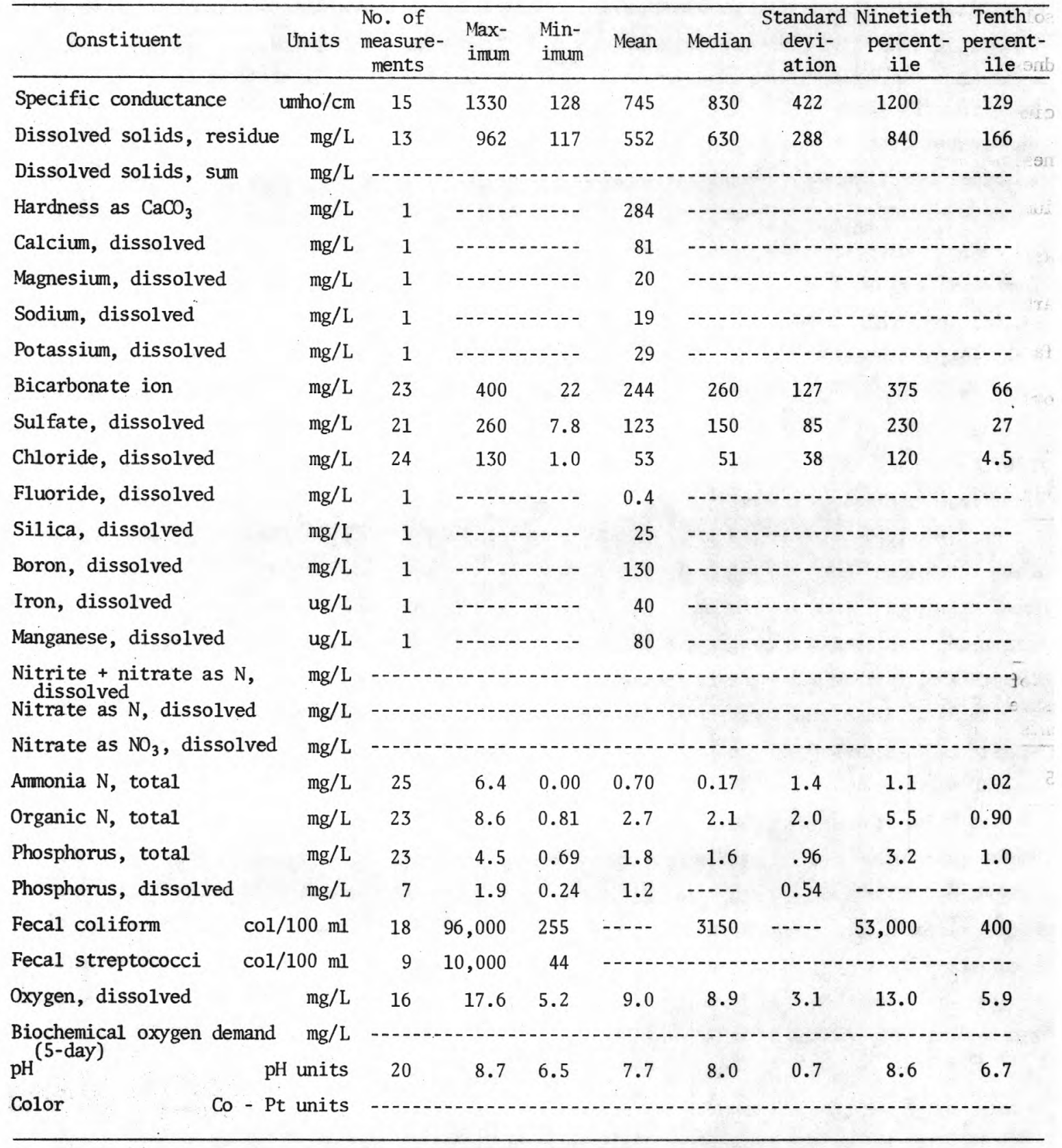




\section{MIDDLE PLATTE RIVER BASIN}

06772000 Wood River near Alda, Nebr.--Continued

Results of regression analyses relating concentrations of selected chemical constituents to specific conductance (SpC)

\begin{tabular}{|c|c|c|c|c|c|}
\hline $\begin{array}{l}\text { Dissolved } \\
\text { constituents } \\
(\mathrm{mg} / \mathrm{L})\end{array}$ & $\begin{array}{l}\text { No. of } \\
\text { measure- } \\
\text { ments }\end{array}$ & Regression equation & $\begin{array}{l}\text { Corre- } \\
\text { lation } \\
\text { coeffi- } \\
\text { cient }\end{array}$ & $\begin{array}{l}\text { Percent } \\
\text { explained } \\
\text { variance }\end{array}$ & $\begin{array}{c}\text { Standard } \\
\text { error of } \\
\text { estimate } \\
(\mathrm{mg} / \mathrm{L})\end{array}$ \\
\hline Dissolved solids, residue (RDS) & $-\ldots$ & - & -1 & & -- \\
\hline Dissolved solids, sum (SDS) & 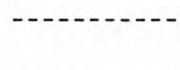 & 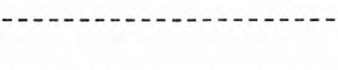 & $\ldots$ & & -- \\
\hline Hardness as $\mathrm{CaCO}_{3}(\mathrm{TH})$ & $-\ldots-n-1$ & 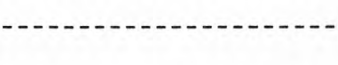 & 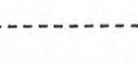 & & --- \\
\hline Calcium (Ca) & $-\cdots-1--1$ & 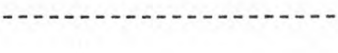 & & & -- \\
\hline Magnesium (Mg) & $---1--1--$ & 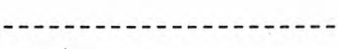 & $\ldots$ & $\ldots$ & -- \\
\hline Sodium (Na) & 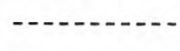 & - & --- & 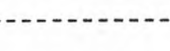 & -- \\
\hline Potassium $(\mathrm{K})$ & $\cdots-\cdots$ & - & & & \\
\hline Bicarbonate $\left(\mathrm{HCO}_{3}\right)$ & $15 \quad \mathrm{HCO}_{3}$ & $=0.212(\mathrm{SpC})+84.512$ & 0.67 & 45 & 103 \\
\hline Sulfate $\left(\mathrm{SO}_{4}\right)$ & $15 \quad \mathrm{SO}_{4}$ & $=0.182(\mathrm{SpC})-3.974$ & .84 & 71 & 51 \\
\hline Chloride (C1) & - n & 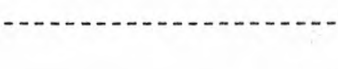 & & & \\
\hline Silica (Si) & - - n- & $-2-1-2-1-2-1-1$ & & & \\
\hline Nitrite + Nitrate as $\mathrm{N}\left(\mathrm{NO}_{2}-\mathrm{NO}_{3}\right)$ & - & & & & \\
\hline
\end{tabular}

Results of regression analyses relating specific conductance to water discharge (Q)

\begin{tabular}{ccccccc}
\hline $\begin{array}{c}\text { No. of } \\
\text { measure- } \\
\text { ments }\end{array}$ & $\begin{array}{c}\text { Water discharge } \\
\left(\mathrm{ft}^{3} / \mathrm{s}\right)\end{array}$ & Maximum Minimum & Regression equation & $\begin{array}{c}\text { Correlation } \\
\text { coefficient }\end{array}$ & $\begin{array}{c}\text { Percent } \\
\text { explained } \\
\text { variance }\end{array}$ & $\begin{array}{c}\text { Standard error } \\
\text { of estimate }\end{array}$ \\
\hline 15 & $472 \quad 1.3$ & $\mathrm{SpC}=1551 \mathrm{Q}^{-0.37092}$ & -0.84 & 70 & 0.210 & 50 \\
\hline
\end{tabular}


MIDDLE PLATTE RIVER BASIN

06772200 Wood River near Grand Island, Nebr.

Location.--Lat $40^{\circ} 56^{\circ} 05^{\prime \prime}$, long $98^{\circ} 16^{\prime} 56^{\prime \prime}$, in $\mathrm{SW}^{\frac{1}{4}} \mathrm{NW}^{\frac{1}{4}} \mathrm{SW}^{\frac{1}{4}} \mathrm{sec}$. 7, T.11 N., R.8 W., Merrick County, Hydrologic Unit 10200102, at bridge on county road $1.0 \mathrm{mi}$ south of U.S. Highway $30,3.0 \mathrm{mi}$ east of Grand Island.

Period of record.--Water year 1973 to current year.

Statistical data for selected chemical constituents

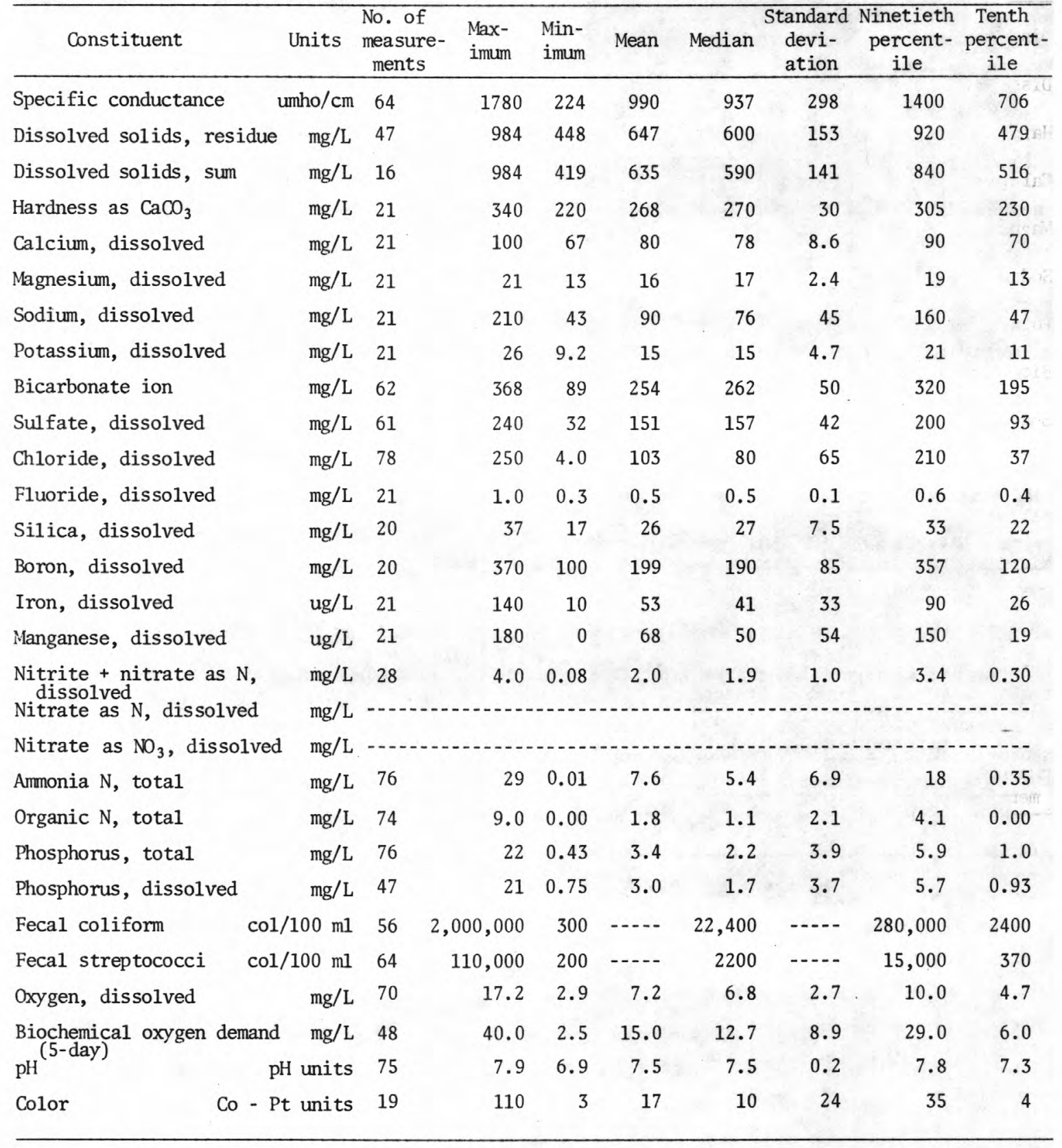


Results of regression analyses relating concentrations of selected chemical constituents to specific conductance $(\mathrm{SpC})$

\begin{tabular}{|c|c|c|c|c|c|}
\hline $\begin{array}{l}\text { Dissolved } \\
\text { constituents } \\
\text { (mg/L) }\end{array}$ & $\begin{array}{l}\text { No. of } \\
\text { measure- } \\
\text { ments }\end{array}$ & Regression equation & $\begin{array}{l}\text { Corre- } \\
\text { lation } \\
\text { coeffi- } \\
\text { cient }\end{array}$ & $\begin{array}{l}\text { Percent } \\
\text { explained } \\
\text { variance }\end{array}$ & $\begin{array}{c}\text { Standard } \\
\text { error of } \\
\text { estimate } \\
(\mathrm{mg} / \mathrm{L})\end{array}$ \\
\hline Dissolved solids, residue (RDS) & 48 & $\mathrm{RDS}=0.514(\mathrm{SpC})+124.162$ & 0.98 & 96 & 32 \\
\hline Dissolved solids, sum (SDS) & 17 & $\mathrm{SDS}=0.540(\mathrm{SpC})+83.038$ & .97 & 94 & 37 \\
\hline Hardness as $\mathrm{CaCO}_{3}(\mathrm{TH})$ & 21 & $\mathrm{TH}=0.020(\mathrm{SpC})+249.133$ & *.16 & 2.7 & 31 \\
\hline Calcium (Ca) & 21 & $\mathrm{Ca}=0.002(\mathrm{SpC})+77.817$ & *.07 & .48 & 8.8 \\
\hline Magnesium (Mg) & 21 & $\mathrm{Mg}=0.003(\mathrm{SpC})+13.748$ & *.29 & 8.2 & 2.4 \\
\hline Sodium $(\mathrm{Na})$ & 21 & $\mathrm{Na}=0.174(\mathrm{SpC})-77.292$ & .97 & 95 & 10 \\
\hline Potassium (K) & 21 & $\mathrm{~K}=0.011(\mathrm{SpC})+4.708$ & .60 & 36 & 3.8 \\
\hline Bicarbonate $\left(\mathrm{HCO}_{3}\right)$ & 54 & $\mathrm{NCO}_{3}=0.129(\mathrm{SpC})+131.891$ & .79 & 62 & 31 \\
\hline Sulfate $\left(\mathrm{SO}_{4}\right)$ & 50 & $\mathrm{SO}_{4}=0.088(\mathrm{SpC})+66.527$ & .63 & 40 & 33 \\
\hline Chloride (CI) & 64 & $\mathrm{C} 1=0.203(\mathrm{SpC})-100.321$ & .93 & 86 & 24 \\
\hline Silica (Si) & 21 & $\mathrm{Si}=0.005(\mathrm{SpC})+21.540$ & *.26 & 7.0 & 4.8 \\
\hline Nitrite + Nitrate as $\mathrm{N}\left(\mathrm{NO}_{2}-\mathrm{NO}_{3}\right)$ & 28 & $\mathrm{NO}_{2}-\mathrm{NO}_{3}=-.001(\mathrm{SpC})+3.277$ & $*-.30$ & 9.1 & 1.0 \\
\hline
\end{tabular}

*Not significant at the 95 percent confidence leve1; the regression equation should not be used to predict the concentration of the dependent variable.

Results of regression analyses relating specific conductance to water discharge (Q)

\begin{tabular}{cccccccc}
\hline $\begin{array}{c}\text { No.- of } \\
\text { measure- } \\
\text { ments }\end{array}$ & $\begin{array}{c}\text { Water discharge } \\
\text { Maximum Minimum }\end{array}$ & Regression equation & $\begin{array}{c}\text { Correlation } \\
\text { coefficient }\end{array}$ & $\begin{array}{c}\text { Percent } \\
\text { explained } \\
\text { variance }\end{array}$ & \multicolumn{2}{c}{$\begin{array}{c}\text { Standard error } \\
\text { of estimate }\end{array}$} \\
\hline 64 & 306 & 1.7 & $\mathrm{SpC}=1899 \mathrm{Q}^{-0.24546}$ & -0.72 & 52 & 0.099 & 23 \\
\hline
\end{tabular}


MIDDLE PLATTE RIVER BASIN

06772500 Wood River near Chapman, Nebr.

Location.--Lat $40^{\circ} 57^{\prime} 56^{\prime \prime}$, long $98^{\circ} 12^{\prime} 22^{\prime \prime}$, in $\mathrm{NE}^{\frac{1}{4}} \mathrm{SE}^{\frac{1}{4}} \mathrm{sec} .34, \mathrm{~T} .12$ N., R.8 W., Merrick County, Hydrologic Unit 10200102 , at county road bridge $2.5 \mathrm{mi}$ west and $4.0 \mathrm{mi}$ south of center of Chapman.

Drainage area.--700 $\mathrm{mi}^{2}$, approximately.

Period of record.--Water year 1968 to current year.

Statistical data for selected chemical constituents

\begin{tabular}{|c|c|c|c|c|c|c|c|c|c|}
\hline Constituent & Units & $\begin{array}{l}\text { No. of } \\
\text { measure- } \\
\text { ments }\end{array}$ & $\begin{array}{l}\text { Max- } \\
\text { imum }\end{array}$ & $\begin{array}{l}\text { Min- } \\
\text { imum }\end{array}$ & Mean & Median & $\begin{array}{c}\text { Standard } \\
\text { devi- } \\
\text { ation }\end{array}$ & $\begin{array}{l}\text { Ninetieth } \\
\text { percent- } \\
\text { ile }\end{array}$ & $\begin{array}{l}\text { Tenth } \\
\text { percent- } \\
\text { ile }\end{array}$ \\
\hline Specific conductance & umho/cm & 181 & 1570 & 158 & 806 & 788 & 248 & 1100 & 543 \\
\hline Dissolved solids, residue & $\mathrm{mg} / \mathrm{L}$ & 118 & 891 & 102 & 475 & 483 & 127 & 599 & 315 \\
\hline Dissolved solids, sum & $\mathrm{mg} / \mathrm{L}$ & 117 & 970 & 103 & 532 & 532 & 128 & 687 & 380 \\
\hline Hardness as $\mathrm{CaCO}_{3}$ & $\mathrm{mg} / \mathrm{L}$ & 154 & 330 & 56 & 238 & 251 & 46 & 281 & 186 \\
\hline Calcium, dissolved & $\mathrm{mg} / \mathrm{L}$ & 153 & 95 & 17 & 72 & 75 & 14 & 85 & 59 \\
\hline Magnesium, dissolved & $\mathrm{mg} / \mathrm{L}$ & 153 & 22 & 2.1 & 14 & 14 & 3.0 & 17 & 11 \\
\hline Sodium, dissolved & $\mathrm{mg} / \mathrm{L}$ & 154 & 190 & 3.0 & 66 & 58 & 37 & 118 & 30 \\
\hline Potassium, dissolved & $\mathrm{mg} / \mathrm{L}$ & 153 & 82 & 6.0 & 14 & 13 & 8.0 & 19 & 9.7 \\
\hline Bicarbonate ion & $\mathrm{mg} / \mathrm{L}$ & 153 & 410 & 67 & 223 & 215 & 53 & 292 & 180 \\
\hline Sulfate, dissolved & $\mathrm{mg} / \mathrm{L}$ & 153 & 260 & 12 & 125 & 127 & 46 & 178 & 63 \\
\hline Chloride, dissolved & $\mathrm{mg} / \mathrm{L}$ & 172 & 210 & 0.1 & 55 & 36 & 45 & 108 & 18 \\
\hline Fluoride, dissolved & $\mathrm{mg} / \mathrm{L}$ & 153 & 1.9 & 0.1 & 0.6 & 0.5 & 0.2 & 0.7 & 0.4 \\
\hline Silica, dissolved & $\mathrm{mg} / \mathrm{L}$ & 152 & 35 & 10 & 25 & 26 & 4.4 & 30 & 20 \\
\hline Boron, dissolved & $\mathrm{mg} / \mathrm{L}$ & 153 & 640 & 40 & 181 & 177 & 86 & 300 & 101 \\
\hline Iron, dissolved & $\mathrm{ug} / \mathrm{L}$ & 135 & 960 & 0 & 82 & 42 & 129 & 167 & 14 \\
\hline Manganese, dissolved & $\mathrm{ug} / \mathrm{L}$ & 96 & 500 & 0 & 120 & 77 & 123 & 290 & 10 \\
\hline $\begin{array}{l}\text { Nitrite }+ \text { nitrate as } \mathrm{N} \text {, } \\
\text { dissolved }\end{array}$ & $\mathrm{mg} / \mathrm{L}$ & 48 & 14 & 0.00 & 2.5 & 2.1 & 2.4 & 3.9 & 0.40 \\
\hline Nitrate as $N$, dissolved & $\mathrm{mg} / \mathrm{L}$ & 36 & 20 & 0.10 & 4.1 & 3.4 & 3.6 & 7.1 & 0.90 \\
\hline Nitrate as $\mathrm{NO}_{3}$, dissolved & $\mathrm{mg} / \mathrm{L}-$ & $\cdots$ & -- & 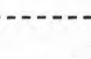 & $-\cdots$ & 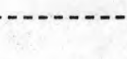 & --- & 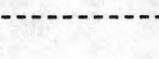 & -1 \\
\hline Ammonia $\mathrm{N}$, total & $\mathrm{mg} / \mathrm{L}$ & 54 & 22 & 0.00 & 5.2 & 2.4 & 6.4 & 16 & 0.04 \\
\hline Organic $N$, total & $\mathrm{mg} / \mathrm{L}$ & 54 & 21 & 0.00 & 2.2 & 1.0 & 3.7 & 5.0 & 0.00 \\
\hline Phosphorus, total & $\mathrm{mg} / \mathrm{L}$ & 64 & 14 & 0.33 & 3.0 & 2.2 & 2.5 & 6.4 & 1.0 \\
\hline Phosphorus, dissolved & $\mathrm{mg} / \mathrm{L}$ & 98 & 13 & 0.05 & 2.6 & 2.0 & 2.2 & 5.1 & 0.90 \\
\hline Fecal coliform & $\mathrm{col} / 100 \mathrm{ml}$ & 43 & 700,000 & 70 & $\cdots-$ & 12,800 & $\cdots--$ & 124,000 & 1120 \\
\hline Fecal streptococci & $\mathrm{col} / 100 \mathrm{ml}$ & 53 & 37,000 & 1 & $\cdots-$ & 1700 & $-\ldots$ & 16,000 & 200 \\
\hline Oxygen, dissolved & $\mathrm{mg} / \mathrm{L}$ & 128 & 11.6 & 1.8 & 7.4 & 7.6 & 1.6 & 9.5 & 5.2 \\
\hline $\begin{array}{l}\text { Biochemical oxygen demand } \\
\text { pH }(5 \text {-day) }\end{array}$ & $\mathrm{pH}$ units - & 72 & 47.0 & 0.30 & 13.1 & 10.6 & 9.4 & 25.2 & $\begin{array}{r}4.5 \\
---\end{array}$ \\
\hline Co $-\mathrm{Pt}$ & Co - Pt units & 94 & 280 & 2 & 19 & 10 & 36 & 29 & 4 \\
\hline
\end{tabular}


Results of regression analyses relating concentrations of selected chemical constituents to specific conductance $(\mathrm{SpC})$

\begin{tabular}{|c|c|c|c|c|c|}
\hline $\begin{array}{l}\text { Dissolved } \\
\text { constituents } \\
(\mathrm{mg} / \mathrm{L})\end{array}$ & $\begin{array}{l}\text { No. of } \\
\text { measure- } \\
\text { ments }\end{array}$ & Regression equation & $\begin{array}{l}\text { Corre- } \\
\text { lation } \\
\text { coeffi- } \\
\text { cient }\end{array}$ & $\begin{array}{l}\text { Percent } \\
\text { explained } \\
\text { variance }\end{array}$ & $\begin{array}{c}\text { Standard } \\
\text { error of } \\
\text { estimate } \\
(\mathrm{mg} / \mathrm{L})\end{array}$ \\
\hline Dissolved solids, residue (RDS) & 119 & $\mathrm{RDS}=0.579(\mathrm{SpC})+53.947$ & 0.96 & 93 & 33 \\
\hline Dissolved solids, sum (SDS) & 116 & $\mathrm{SDS}=0.556(\mathrm{SpC})+60.444$ & .98 & 95 & 28 \\
\hline Hardness as $\mathrm{CaCO}_{3}(\mathrm{TH})$ & 153 & $\mathrm{TH}=0.143(\mathrm{SpC})+125.798$ & .74 & 56 & 30 \\
\hline Calcium (Ca) & 152 & $\mathrm{Ca}=0.042(\mathrm{SpC})+39.302$ & .72 & 52 & 9.5 \\
\hline Magnesium (Mg) & 152 & $\mathrm{Mg}=0.009(\mathrm{SpC})+6.675$ & .74 & 55 & 2.1 \\
\hline Sodium $(\mathrm{Na})$ & 153 & $\mathrm{Na}=0.148(\mathrm{SpC})-50.844$ & .95 & 90 & 12 \\
\hline Potassium (K) & 152 & $\mathrm{~K}=0.007(\mathrm{SpC})+9.043$ & .20 & 4.0 & 7.9 \\
\hline Bicarbonate $\left(\mathrm{HCO}_{3}\right)$ & 153 & $\mathrm{HCO}_{3}=0.148(\mathrm{SpC})+105.976$ & .68 & 46 & 39 \\
\hline Sulfate $\left(\mathrm{SO}_{4}\right)$ & 153 & $\mathrm{SO}_{4}=0.140(\mathrm{SpC})+15.098$ & .73 & 53 & 31 \\
\hline Chloride (Cl) & 171 & $\mathrm{C} 1=0.159(\mathrm{SpC})-72.520$ & .88 & 77 & 22 \\
\hline Silica (Si) & 152 & $\mathrm{Si}=0.008(\mathrm{SpC})+19.372$ & .42 & 18 & 4.0 \\
\hline Nitrite + Nitrate as $\mathrm{N}\left(\mathrm{NO}_{2}-\mathrm{NO}_{3}\right)$ & 48 & $\mathrm{NO}_{2}-\mathrm{NO}_{3}=-.0009(\mathrm{SpC})+3.304$ & $*_{-} .08$ & .60 & 2.4 \\
\hline
\end{tabular}

*Not significant at the 95 percent confidence leve1; the regression equation should not be used to predict the concentration of the dependent variable.

Results of regression analyses relating specific conductance to water discharge (Q)

\begin{tabular}{llllcccc}
\hline \multirow{2}{*}{$\begin{array}{c}\text { No. of } \\
\text { measure- } \\
\text { ments }\end{array}$} & $\begin{array}{c}\text { Water discharge } \\
\left(\mathrm{ft}^{3} / \mathrm{s}\right)\end{array}$ & Maximum Minimum & Regression equation & $\begin{array}{c}\text { Correlation } \\
\text { coefficient }\end{array}$ & $\begin{array}{c}\text { Percent } \\
\text { explained } \\
\text { variance }\end{array}$ & \multicolumn{2}{c}{$\begin{array}{c}\text { Standard error } \\
\text { of estimate }\end{array}$} \\
\hline 181 & $1040 \quad .70$ & $\mathrm{SpC}=1488 \mathrm{Q}^{-0.23176}$ & -0.67 & 45 & 0.119 & 28 \\
\hline
\end{tabular}


Location.--Lat $41^{\circ} 22^{\prime} 04^{\prime \prime}$, long $97^{\circ} 29^{\prime} 40^{\prime \prime}$, in $\mathrm{SF}^{\frac{1}{4}} \mathrm{SW}^{\frac{3}{4}} \mathrm{sec}$. 12 , T.16 N., R.2 W., Platte County, Hydrologic Unit 10200103, on left bank $25 \mathrm{ft}$ downstream from highway bridge, $1.5 \mathrm{mi}$ south of Duncan, and $12 \mathrm{mi}$ upstream from Loup River.

Drainage area (revised).--60,900 $\mathrm{mi}^{2}$, approximately, of which about $56,100 \mathrm{mi}^{2}$ contributes directly to surface runoff. Approximately $4,000 \mathrm{mi}^{2}$ in Great Divide Basin is not iricluded.

Period of record.-- Water year 1965 to current year.

Statistical data for selected chemical constituents

\begin{tabular}{|c|c|c|c|c|c|c|c|c|c|}
\hline Constituent & Units & $\begin{array}{l}\text { No. of } \\
\text { measure- } \\
\text { ments }\end{array}$ & $\begin{array}{l}\text { Max- } \\
\text { imum }\end{array}$ & $\begin{array}{l}\text { Min- } \\
\text { imum }\end{array}$ & Mean & Median & $\begin{array}{l}\text { Standard } \\
\text { devi- } \\
\text { ation }\end{array}$ & $\begin{array}{l}\text { Ninetieth } \\
\text { percent- } \\
\text { ile }\end{array}$ & $\begin{array}{l}\text { Tenth } \\
\text { percent- } \\
\text { ile }\end{array}$ \\
\hline Specific conductance & umho/cm & 230 & 1560 & 236 & 828 & 836 & 146 & 1000 & 652 \\
\hline Dissolved solids, residue & $\mathrm{mg} / \mathrm{L}$ & 101 & 788 & 171 & 565 & 550 & 101 & 685 & 460 \\
\hline Dissolved solids, sum & $\mathrm{mg} / \mathrm{L}$ & 138 & 739 & 280 & 551 & 557 & 79 & 653 & 447 \\
\hline Hardness as $\mathrm{CaCO}_{3}$ & $\mathrm{mg} / \mathrm{L}$ & 182 & 392 & 80 & 263 & 264 & 43 & 317 & 222 \\
\hline Calcium, dissolved & $\mathrm{mg} / \mathrm{L}$ & 182 & 98 & 25 & 70 & 70 & 11 & 84 & 60 \\
\hline Magnesium, dissolved & $\mathrm{mg} / \mathrm{L}$ & 182 & 53 & 4.3 & 21 & 22 & 4.8 & 26 & 17 \\
\hline Sodium, dissolved & $\mathrm{mg} / \mathrm{L}$ & 182 & 103 & 13 & 73 & 77 & 14 & 91 & 55 \\
\hline Potassium, dissolved & $\mathrm{mg} / \mathrm{L}$ & 174 & 27 & 4.7 & 12 & 12 & 2.4 & 15 & 10 \\
\hline Bicarbonate ion & $\mathrm{mg} / \mathrm{L}$ & 181 & 284 & 104 & 223 & 224 & 27 & 254 & 192 \\
\hline Sulfate, dissolved & $\mathrm{mg} / \mathrm{L}$ & 182 & 311 & 25 & 208 & 208 & 46 & 270 & 156 \\
\hline Chloride, dissolved & $\mathrm{mg} / \mathrm{L}$ & 182 & 41 & 3.7 & 25 & 25 & 5.6 & 33 & 19 \\
\hline Fluoride, dissolved & $\mathrm{mg} / \mathrm{L}$ & 176 & 1.2 & 0.0 & 0.6 & 0.6 & 0.1 & 0.8 & 0.5 \\
\hline Silica, dissolved & $\mathrm{mg} / \mathrm{L}$ & 174 & 52 & 5.6 & 23 & 24 & 5.1 & 28 & 18 \\
\hline Boron, dissolved & $\mathrm{mg} / \mathrm{L}$ & 174 & 560 & 40 & 132 & 131 & 48 & 168 & 94 \\
\hline Iron, dissolved & $\mathrm{ug} / \mathrm{L}$ & 89 & 330 & 0 & 51 & 27 & 63 & 130 & 0 \\
\hline Manganese, dissolved & $\mathrm{ug} / \mathrm{L}$ & 63 & 457 & 0 & 44 & 27 & 71 & 87 & 0 \\
\hline Nitrite + nitrate as $\mathrm{N}$, & $\mathrm{mg} / \mathrm{L}$ & 75 & 1.7 & 0.00 & 0.48 & 0.44 & 0.38 & 1.0 & .02 \\
\hline Nitrate as $\mathrm{N}$, dissolved & $\mathrm{mg} / \mathrm{L}$ & 57 & 1.3 & 0.00 & 0.41 & 0.41 & 0.34 & 0.82 & 0.00 \\
\hline Nitrate as $\mathrm{NO}_{3}$, dissolved & $\mathrm{mg} / \mathrm{L}$ & & & & & & & & -- \\
\hline Ammonia $\mathrm{N}$, total & $\mathrm{mg} / \mathrm{L}$ & 1 & 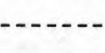 & -..- & 0.01 & & & & \\
\hline Organic $\mathrm{N}$, total & $\mathrm{mg} / \mathrm{L}$ & & & & & & & & -..- \\
\hline Phosphorus, total & $\mathrm{mg} / \mathrm{L}$ & 48 & 0.78 & 0.06 & 0.26 & 0.22 & 0.14 & 0.45 & 0.13 \\
\hline Phosphorus, dissolved & $\mathrm{mg} / \mathrm{L}$ & 124 & 0.46 & 0.00 & 0.12 & 0.10 & .08 & 0.21 & .04 \\
\hline Fecal coliform & $\mathrm{col} / 100 \mathrm{ml}$ & 24 & 5500 & 1 & $\cdots$ & 91 & $\cdots$ & 210 & 5 \\
\hline Fecal streptococci & $\mathrm{col} / 100 \mathrm{ml}$ & & & & & & & & -- \\
\hline Oxygen, dissolved & $\mathrm{mg} / \mathrm{L}$ & 47 & 14.2 & 5.2 & 10.6 & 10.1 & 2.4 & 13.5 & 7.3 \\
\hline \multirow{2}{*}{$\begin{array}{l}\text { Biochemical oxygen demand } \\
\mathrm{pH}^{(5-\text { day })}\end{array}$} & $\mathrm{mg} / \mathrm{L}$ & 47 & 7.8 & 0.9 & 3.2 & 2.9 & 1.9 & 6.1 & 1.2 \\
\hline & units & 223 & 8.7 & 6.8 & 7.9 & 8.0 & 0.4 & 8.4 & 7.5 \\
\hline $\mathrm{Co}-\mathrm{Pt}$ & units & 107 & 80 & 0 & 14 & 7 & 16 & 31 & 3 \\
\hline
\end{tabular}


Results of regression analyses relating concentrations of selected chemical constituents to specific conductance $(\mathrm{SpC})$

\begin{tabular}{|c|c|c|c|c|c|}
\hline $\begin{array}{l}\text { Dissolved } \\
\text { constituents } \\
(\mathrm{mg} / \mathrm{L})\end{array}$ & $\begin{array}{l}\text { No. of } \\
\text { measure- } \\
\text { ments }\end{array}$ & Regression equation & $\begin{array}{l}\text { Corre- } \\
\text { lation } \\
\text { coeffi- } \\
\text { cient }\end{array}$ & $\begin{array}{l}\text { Percent } \\
\text { explained } \\
\text { variance }\end{array}$ & $\begin{array}{c}\text { Standard } \\
\text { error of } \\
\text { estimate } \\
(\mathrm{mg} / \mathrm{L})\end{array}$ \\
\hline Dissolved solids, residue (RDS) & 101 & $\mathrm{RDS}=0.752(\mathrm{SpC})-51.607$ & 0.98 & 97 & 18 \\
\hline Dissolved solids, sum (SDS) & 136 & $\mathrm{SDS}=0.671(\mathrm{SpC})-5.446$ & .96 & 93 & 21 \\
\hline Hardness as $\mathrm{CaCO}_{3}(\mathrm{TH})$ & 182 & $\mathrm{TH}=0.315(\mathrm{SpC})+3.761$ & .93 & 87 & 16 \\
\hline Calcium (Ca) & 182 & $\mathrm{Ca}=0.073(\mathrm{SpC})+9.596$ & .84 & 71 & 6.0 \\
\hline Magnesium (Mg) & 182 & $\mathrm{Mg}=0.032(\mathrm{SpC})-5.144$ & .85 & 72 & 2.5 \\
\hline Sodium $(\mathrm{Na})$ & 182 & $\mathrm{Na}=0.103(\mathrm{SpC})-11.625$ & .92 & 84 & 5.7 \\
\hline Potassium (K) & 174 & $\mathrm{~K}=0.004(\mathrm{SpC})+8.753$ & .21 & 4.4 & 2.3 \\
\hline Bicarbonate $\left(\mathrm{HCO}_{3}\right)$ & 181 & $\mathrm{HCO}_{3}=0.155(\mathrm{SpC})+96.029$ & .72 & 52 & 19 \\
\hline Sulfate $\left(\mathrm{SO}_{4}\right)$ & 182 & $\mathrm{SO}_{4}=0.346(\mathrm{SpC})-76.432$ & .95 & 90 & 14 \\
\hline Chloride (C1) & 182 & $\mathrm{C} 1=0.038(\mathrm{SpC})-6.268$ & .87 & 76 & 2.8 \\
\hline Silica (Si) & 174 & $\mathrm{Si}=0.004(\mathrm{SpC})+20.342$ & $* .09$ & .80 & 5.1 \\
\hline Nitrite + Nitrate as $\mathrm{N}\left(\mathrm{NO}_{2}-\mathrm{NO}_{3}\right)$ & 75 & $\mathrm{NO}_{2}-\mathrm{NO}_{3}=-.0001(\mathrm{SpC})+0.589$ & $*-.04$ & .19 & .38 \\
\hline
\end{tabular}

*Not significant at the 95 percent confidence level; the regression equation should not.be used to predict the concentration of the dependent variable.

Results of regression analyses relating specific conductance to water discharge (Q)

\begin{tabular}{ccccccc}
\hline $\begin{array}{c}\text { No. of } \\
\text { measure- } \\
\text { ments }\end{array}$ & $\begin{array}{c}\text { Mater discharge } \\
\left(\mathrm{ft}^{3} / \mathrm{s}\right)\end{array}$ & Regression equation Minimum & $\begin{array}{c}\text { Correlation } \\
\text { coefficient }\end{array}$ & $\begin{array}{c}\text { Percent } \\
\text { explained } \\
\text { variance }\end{array}$ & $\begin{array}{c}\text { Standard error } \\
\text { of estimate }\end{array}$ \\
\hline 229 & $15,000 \quad 3.0$ & $\mathrm{SpC}=790 \mathrm{Q}^{.00418}$ & $* 0.03$ & 0.07 & 0.088 & 20 \\
\hline
\end{tabular}


The Loup River basin is the largest in surface area of all 13 basins in Nebraska. It is located exactly in the center of the State and streamflow in it originates within the borders of the State. The basin ends at the confluence of the Loup and Platte Rivers.

Over half of the Loup River basin is comprised of sandhills. Underlying the sandhills are saturated deposits of as much as $1,000 \mathrm{ft}$ in thickness. These deposits are the source of all streams originating in the basin. Only small amounts of overland runoff are carried by sandhills streams.

Downstream from the sandhil1s, Pleistocene sands and gravels are overlain by loess deposits and topography is hilly. Through the loesshills part of the basin, ground-water derived streamflow is supplemented by overland runoff.

Irrigation is not extensive in the Loup River basin at the present time because until the development of center-pivot irrigation systems, much of the land could not be irrigated by the systems then in existence because the land was too steeply sloping. Probably only about 450,000 acres or about 5 percent of the existing land area presently is irrigated. Of these acres, about 185,000 are irrigated by surface water diverted from the North Loup and Middle Loup Rivers.

Twelve sampling sites in the Loup River basin have sufficient data available for statistical analyses (fig. 8). Five of these sites are in the sandhills or near the edge of the sandhills. Seven are on the Loup River, including the Loup River Power Canal, or on the South, Middle, or North Loup Rivers. Cedar River and Beaver Creek are tributaries of the Loup River and drain approximately one-fifth of the basin. Water is diverted into the Loup River Power Canal near Genoa for electrical generation, and during most of the year nearly all the flow of the Loup River is diverted into this canal.

Mineralization of water in the Loup River basin, with the exception of Mud Creek, is low and varies over narrow ranges. Based on tenth and ninetieth percentile data, 80 percent of all specific conductance values can be expected to range between 160 and 350 umho/cm for stations in the Middle Loup drainage. Based on the same percentile data, specific conductances range typically between 130 and $260 \mathrm{umho} / \mathrm{cm}$ for stations in the North Loup drainage. Calcium is the principal cation and bicarbonate is the principal anion at all sampling sites in the basin. Silica is an important constituent throughout the basin. 


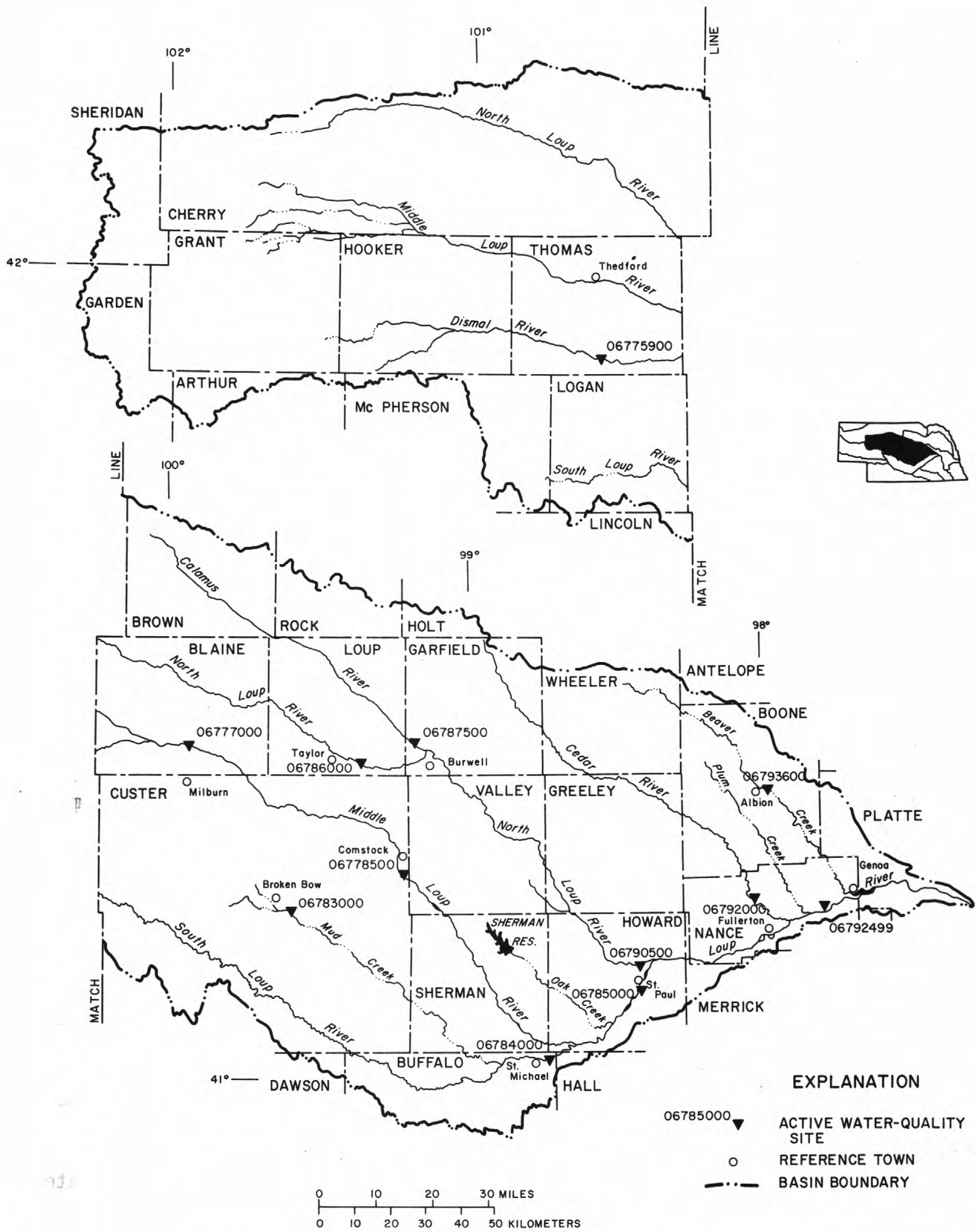

FIGURE 8.-Location of water-quality sampling sites, Loup River basin 
Mineralization increases in a downstream direction through the Middle Loup River system. After leaving the sandhills, concentrations of principal constituents increase about 35 percent from Comstock to St. Paul, accompanied by a 30 percent decrease in silica concentrations. This increase may be caused by several factors including the addition of more highly mineralized water from the South Loup River, discharge of more highly mineralized ground water from the loess hills, and possibly enrichment by irrigation return flow.

Water in the Loup River Power Canal (Sta. 06792499) is similar in mineralization to water in the Middle Loup at St. Paul (Sta. 06785000), even though water of lower mineralization from the North Loup River enters the Loup River between the two stations. The effect of this water of better quality may be offset by the seepage of more highly mineralized ground water into the stream between the two stations.

Median concentrations of fecal coliform and fecal streptococcal bacteria are low at all but one of the stations at which they were measured. Mud Creek near Broken Bow, located downstream from the city sewage outfall, the median fecal coliform bacteria concentration was 2,700 colonies per $100 \mathrm{ml}$ and the median fecal streptococcal bacteria concentration was 1,500 colonies per $100 \mathrm{ml}$. Also, mean concentrations of total and dissolved phosphorus are less than $1.0 \mathrm{mg} / \mathrm{L}$ for all stations except Mud Creek near Broken Bow.

Regression equations were developed between specific conductance and each of the 12 constituents for all stations except the South Loup River at St. Michael for which there were an insufficient number of measurements of each constituent. Correlation coefficients generally are above 0.7 for sandhills stations. Many of these coefficients are not significant at the 95 percent confidence leve1, especially for stations in the sandhills. In water from sandhills streams, natural variations in ion ratios between principal chemical constituents, such as sodium and potassium, tend to reduce correlation between individual constituents and conductance. Correlation coefficients are closer to 1.0 at downstream sites such as the Middle Loup River at St. Paul and the Loup River Power Canal because of the increased contribution of overland runoff to the streamflow. 
06775900 Dismal River near Thedford, Nebr.

Location.--Lat $41^{\circ} 46^{\prime} 45^{\prime \prime}$, long $100^{\circ} 31^{\prime} 30^{\prime \prime}$, in NE $\frac{1}{4} N^{\frac{1}{4}} \mathrm{sec} .23$, T. 21 N., R. 28 W., Thomas County, Hydrologic Unit 10210002, on right bank $25 \mathrm{ft}$ ups tream from bridge on State Highway 83, $2 \mathrm{mi}$ upstream from boundary of Nebraska National Forest (Bessey Division), and $14 \mathrm{mi}$ south of Thedford.

Drainage area.--960 $\mathrm{mi}^{2}$, approximately, of which about $30 \mathrm{mi}^{2}$ contributes directly to surface runoff.

Period of record. --Water year 1967 to current year.

Statistical data for selected chemical constituents

\begin{tabular}{|c|c|c|c|c|c|c|c|c|c|}
\hline Constituent & Units & $\begin{array}{l}\text { No. of } \\
\text { measure- } \\
\text { ments }\end{array}$ & $\begin{array}{l}\text { Max- } \\
\text { imum }\end{array}$ & $\begin{array}{l}\text { Min- } \\
\text { imum }\end{array}$ & Mean & Median & $\begin{array}{c}\text { Standard } \\
\text { devi- } \\
\text { ation }\end{array}$ & $\begin{array}{c}\text { Ninetieth } \\
\text { percent- } \\
\text { ile }\end{array}$ & $\begin{array}{l}\text { Tenth } \\
\text { percent- } \\
\text { ile }\end{array}$ \\
\hline Specific conductance & umho/cm & 138 & 218 & 132 & 176 & 177 & 12 & 189 & 160 \\
\hline Dissolved solids, residue & $\mathrm{mg} / \mathrm{L}$ & 72 & 190 & 142 & 157 & 155 & 8.1 & 168 & 145 \\
\hline Dissolved solids, sum & $\mathrm{mg} / \mathrm{L}$ & 75 & 172 & 138 & 155 & 154 & 5.7 & 164 & 229 \\
\hline Hardness as $\mathrm{CaCO}_{3}$ & $\mathrm{mg} / \mathrm{L}$ & 81 & 85 & 64 & 71 & 72 & 4.1 & 77 & 67 \\
\hline Calcium, dissolved & $\mathrm{mg} / \mathrm{L}$ & 82 & 26 & 20 & 23 & 23 & 1.4 & 25 & 22 \\
\hline Magnesium, dissolved & $\mathrm{mg} / \mathrm{L}$ & 81 & 4.8 & 1.8 & 3.5 & 3.5 & 0.4 & 3.9 & 3.3 \\
\hline Sodium, dissolved & $\mathrm{mg} / \mathrm{L}$ & 77 & 11 & 5.9 & 7.1 & 7.0 & 0.7 & 8.0 & 6.6 \\
\hline Potassium, dissolved & $\mathrm{mg} / \mathrm{L}$ & 78 & 9.2 & 3.2 & 5.3 & 5.3 & 0.7 & 5.9 & 4.8 \\
\hline Bicarbonate ion & $\mathrm{mg} / \mathrm{L}$ & 81 & 118 & 91 & 102 & 100 & 4.6 & 101 & 98 \\
\hline Sulfate, dissolved & $\mathrm{mg} / \mathrm{L}$ & 77 & 12 & 1.2 & 7.0 & 7.0 & 1.4 & 8.4 & 5.5 \\
\hline Ch1oride, dissolved & $\mathrm{mg} / \mathrm{L}$ & 82 & 4.4 & 0.4 & 1.2 & 1.2 & 0.6 & 1.8 & 0.7 \\
\hline Fluoride, dissolved & $\mathrm{mg} / \mathrm{L}$ & 83 & 0.5 & 0.2 & 0.3 & 0.3 & 0.06 & 0.4 & 0.3 \\
\hline Silica, dissolved & $\mathrm{mg} / \mathrm{L}$ & 77 & 62 & 44 & 56 & 57 & 3.0 & 59 & 52 \\
\hline Boron, dissolved & $\mathrm{mg} / \mathrm{L}$ & 79 & 60 & 0 & 20 & 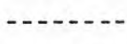 & 11 & -1 & --- \\
\hline Iron, dissolved & $\mathrm{ug} / \mathrm{L}$ & 68 & 490 & 0 & 60 & 28 & 94 & 122 & 4 \\
\hline Manganese, dissolved & $\mathrm{ug} / \mathrm{L}$ & 52 & 297 & 0 & 21 & 10 & 46 & 49 & 0 \\
\hline Nitrite + nitrate as $\mathrm{N}$, & $\mathrm{mg} / \mathrm{L}$ & 32 & 0.66 & 0.18 & 0.40 & 0.44 & 0.12 & 0.53 & 0.24 \\
\hline Nitrate as $\mathrm{N}$, dissolved & $\mathrm{mg} / \mathrm{L}$ & 26 & 0.70 & 0.00 & 0.40 & 0.48 & 0.21 & 0.61 & 0.00 \\
\hline Nitrate as $\mathrm{NO}_{3}$, dissolved & $\mathrm{mg} / \mathrm{L}$ & & & 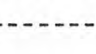 & - & -- & & & $-\cdots$ \\
\hline Ammonia $\mathrm{N}$, total & $\mathrm{mg} / \mathrm{L}$ & & & & & & & & -- \\
\hline Organic $N$, total & $\mathrm{mg} / \mathrm{L}$ & & & & & & & & -- \\
\hline Phosphorus, total & $\mathrm{mg} / \mathrm{L}$ & 20 & 0.41 & 0.16 & 0.23 & 0.23 & 0.05 & 0.28 & 0.19 \\
\hline Phosphorus, dissolved & $\mathrm{mg} / \mathrm{L}$ & 57 & 0.38 & 0.04 & 0.17 & 0.17 & 0.05 & 0.22 & 0.14 \\
\hline Fecal coliform & $/ 100 \mathrm{ml}$ & 18 & 500 & 23 & $-\cdots---$ & 99 & $\ldots \ldots$ & 207 & 33 \\
\hline Fecal streptococci & $/ 100 \mathrm{ml}$ & 22 & 840 & 4 & $-\cdots--$ & 120 & $\ldots \ldots$ & 325 & 12 \\
\hline Oxygen, dissolved & $\mathrm{mg} / \mathrm{L}$ & 80 & 12.3 & 6.2 & 9.4 & 9.3 & 1.4 & 11.0 & 7.7 \\
\hline \multirow{2}{*}{$\begin{array}{l}\text { Biochemical oxygen demand } \\
\mathrm{pH}^{(5-\text { day })}\end{array}$} & $\mathrm{mg} / \mathrm{L}$ & 74 & 8.4 & 0.3 & 1.4 & 1.0 & 1.4 & 2.5 & 0.6 \\
\hline & $H$ units & 138 & 8.7 & 6.5 & 7.8 & 7.9 & 0.4 & 8.3 & 7.4 \\
\hline Co $-\mathrm{Pt}$ & t units & 70 & 35 & 0 & 7 & 5 & 6.5 & 14 & 2 \\
\hline
\end{tabular}




\section{LOUP RIVER BASIN}

06775900 Dismal River near Thedford, Nebr.--Continued

Results of regression analyses relating concentrations of selected chemical constituents to specific conductance $(\mathrm{SpC})$

\begin{tabular}{|c|c|c|c|c|c|}
\hline $\begin{array}{l}\text { Dissolved } \\
\text { constituents } \\
(\mathrm{mg} / \mathrm{L})\end{array}$ & $\begin{array}{l}\text { No. of } \\
\text { measure- } \\
\text { ments }\end{array}$ & Regression equation & $\begin{array}{l}\text { Corre- } \\
\text { lation } \\
\text { coeffi- } \\
\text { cient }\end{array}$ & $\begin{array}{l}\text { Percent } \\
\text { explained } \\
\text { variance }\end{array}$ & $\begin{array}{c}\text { Standard } \\
\text { error of } \\
\text { estimate } \\
(\mathrm{mg} / \mathrm{L})\end{array}$ \\
\hline Dissolved solids, residue (RDS) & 72 & $\mathrm{RDS}=-0.043(\mathrm{SpC})+164.486$ & $*-0.04$ & 0.21 & 8.1 \\
\hline Dissolved solids, sum (SDS) & 74 & $\mathrm{SDS}=0.068(\mathrm{SpC})+142.582$ & $\star .10$ & 1.1 & 5.8 \\
\hline Hardness as $\mathrm{CaCO}_{3}(\mathrm{TH})$ & 81 & $\mathrm{TH}=0.074(\mathrm{SpC})+58.159$ & *.16 & 2.6 & 4.1 \\
\hline Calcium (Ca) & 82 & $\mathrm{Ca}=0.033(\mathrm{SpC})+16.911$ & *.21 & 4.6 & 1.4 \\
\hline Magnesium (Mg) & 81 & $\mathrm{Mg}=-0.004(\mathrm{SpC})+4.202$ & $*_{-} .09$ & 0.85 & 0.4 \\
\hline Sodium (Na) & 77 & $\mathrm{Na}=0.011(\mathrm{SpC})+5.056$ & *.14 & 2.0 & 0.7 \\
\hline Potassium (K) & 78 & $\mathrm{~K}=0.016(\mathrm{SpC})+2.402$ & *.20 & 4.0 & 0.7 \\
\hline Bicarbonate $\left(\mathrm{HCO}_{3}\right)$ & 81 & $\mathrm{HCO}_{3}=0.150(\mathrm{SpC})+74.501$ & .30 & 8.9 & 4.4 \\
\hline Sulfate $\left(\mathrm{SO}_{4}\right)$ & 77 & $\mathrm{SO}_{4}=-0.023(\mathrm{SpC})+11.161$ & $*-.15$ & 2.2 & 1.4 \\
\hline Chloride (C1) & 82 & $\mathrm{Cl}-0.002(\mathrm{SpC})+0.805$ & $* .04$ & 0.12 & 0.6 \\
\hline Silica (Si) & 77 & $\mathrm{Si}=-0.026(\mathrm{SpC})+57.266$ & $*_{-} .02$ & 0.07 & 3.0 \\
\hline Nitrite + Nitrate as $\mathrm{N}\left(\mathrm{NO}_{2}-\mathrm{NO}_{3}\right)$ & 32 & $\mathrm{NO}_{2}-\mathrm{NO}_{3}=-0.0009(\mathrm{SpC})+0.566$ & $*_{-} .08$. & 0.64 & 0.12 \\
\hline
\end{tabular}

* Not significant at the 95 percent confidence level; the regression equation should not be used to preduct the concentration of the dependent variable.

Results of regression analyses relating specific conductance to water discharge (Q)

\begin{tabular}{ccccccc}
\hline $\begin{array}{c}\text { No. of } \\
\text { measure- } \\
\text { ments }\end{array}$ & $\begin{array}{c}\text { Water discharge } \\
\text { Maximum Minimum }\end{array}$ & Regression equation & $\begin{array}{c}\text { Correlation } \\
\text { coefficient }\end{array}$ & $\begin{array}{c}\text { Percent } \\
\text { explained } \\
\text { variance }\end{array}$ & $\begin{array}{c}\text { Standard error } \\
\text { of estimate }\end{array}$ \\
\hline 125 & $233 \quad 176$ & $\mathrm{SpC}=250 \mathrm{Q}^{-0.06608}$ & $*-0.06$ & 0.32 & 0.030 \\
\hline
\end{tabular}

* Not significant at the 95 percent confidence level; the regression equation should not be used to predict the concentration of the dependent variable. 
06777000 Middle Loup River near Milburn, Nebr.

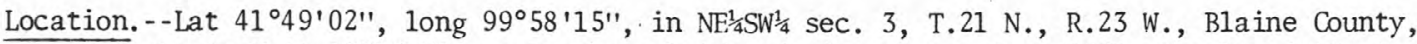
Hydrologic Unit 10210003, at Laughran bridge $9 \mathrm{mi}$ upstream from Rifle Creek and $15 \mathrm{mi}$ northwest of Milburn.

Drainage area. $--3,690 \mathrm{mi}^{2}$, approximately, of which $135 \mathrm{mi}^{2}$ contributes directly to surface runoff.

Period of record.--Water year 1970 to current year.

Statistical data for selected chemical constituents

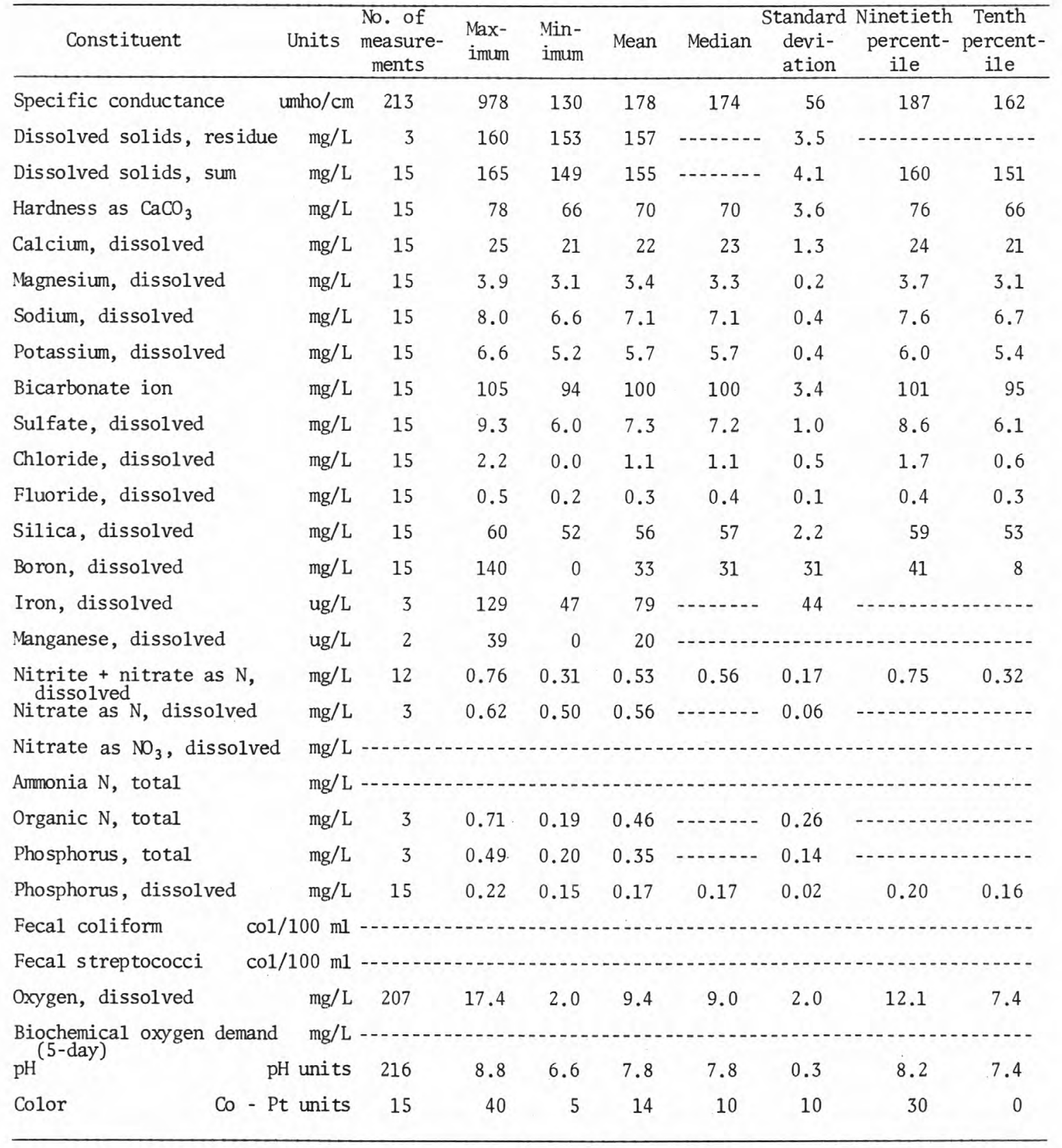




\section{LOUP RIVER BASIN}

06777000 Middle Loup River near Milburn, Nebr.--Continued

Results of regression analyses relating concentrations of selected chemical constituents to specific conductance $(\mathrm{SpC})$

\begin{tabular}{|c|c|c|c|c|c|}
\hline $\begin{array}{l}\text { Dissolved } \\
\text { constituents } \\
(\mathrm{mg} / \mathrm{L})\end{array}$ & $\begin{array}{l}\text { No. of } \\
\text { measure- } \\
\text { ments }\end{array}$ & Regression equation & $\begin{array}{l}\text { Corre- } \\
\text { lation } \\
\text { coeffi- } \\
\text { cient }\end{array}$ & $\begin{array}{l}\text { Percent } \\
\text { explained } \\
\text { variance }\end{array}$ & $\begin{array}{c}\text { Standard } \\
\text { error of } \\
\text { estimate } \\
(\mathrm{mg} / \mathrm{L})\end{array}$ \\
\hline Dissolved solids, residue (RDS) & \multicolumn{5}{|c|}{$\begin{array}{l}-1 \\
-1\end{array}$} \\
\hline Dissolved solids, sum (SDS) & 15 & $\mathrm{SDS}=0.311(\mathrm{SpC})+100.232$ & 0.67 & 45 & 3.1 \\
\hline Hardness as $\mathrm{CaCO}_{3}(\mathrm{TH})$ & 15 & $\mathrm{TH}=0.205(\mathrm{SpC})+33.538$ & $* .51$ & 26 & 3.2 \\
\hline Calcium (Ca) & 15 & $\mathrm{Ca}=0.070(\mathrm{SpC})+10.027$ & $* .48$ & 23 & 1.2 \\
\hline Magnesium (Mg) & 15 & $\mathrm{Mg}=0.006(\mathrm{SpC})+2.345$ & $* .22$ & 4.8 & 0.2 \\
\hline Sodium (Na) & 15 & $\mathrm{Na}=0.027(\mathrm{SpC})+2.339$ & .62 & 39 & 0.3 \\
\hline Potassium (K) & 15 & $\mathrm{~K}=0.024(\mathrm{SpC})+1.483$ & .59 & 34 & 0.3 \\
\hline Bicarbonate $\left(\mathrm{HCO}_{3}\right)$ & 15 & $\mathrm{HCO}_{3}=0.268(\mathrm{SpC})+51.872$ & .69 & 48 & 2.6 \\
\hline Sulfate $\left(\mathrm{SO}_{4}\right)$ & 15 & $\mathrm{SO}_{4}=0.004(\mathrm{SpC})+6.613$ & $* .04$ & 0.13 & 1.0 \\
\hline Chloride (C1) & 15 & $\mathrm{Cl}=-0.002(\mathrm{SpC})+1.433$ & $*_{-} .03$ & 0.10 & 0.5 \\
\hline Silica (Si) & 15 & $\mathrm{Si}=0.050(\mathrm{SpC})+47.566$ & $* .20$ & 3.9 & 2.3 \\
\hline
\end{tabular}

* Not significant at the 95 percent confidence level; the regression equation should not be used to predict the concentration of the dependent variable.

Results of regression analyses relating specific conductance to water discharge (Q)

\begin{tabular}{ccccccc}
\hline $\begin{array}{c}\text { No. of } \\
\text { measure- } \\
\text { ments }\end{array}$ & $\begin{array}{c}\text { Water discharge } \\
\left(\mathrm{ft}^{3} / \mathrm{s}\right)\end{array}$ & Maximum Minimum & Regression equation & $\begin{array}{c}\text { Correlation } \\
\text { coefficient }\end{array}$ & $\begin{array}{c}\text { Percent } \\
\text { explained } \\
\text { variance }\end{array}$ & $\begin{array}{c}\text { Standard error } \\
\text { of estimate }\end{array}$ \\
\hline 213 & $1,030 \quad 331$ & $\mathrm{SpC}=154 \mathrm{Q}^{0.01796}$ & $* 0.03$ & 0.08 & 0.032 & 7.4 \\
\hline
\end{tabular}

* Not significant at the 95 percent confidence level; the regression equation should not be used to predict the concentration of the dependent variable. 


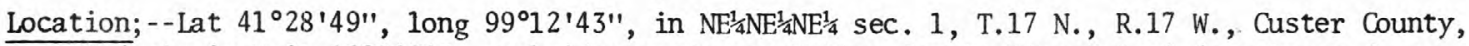
Hydrologic Unit 10210003, at bridge on Custer-Valley County line $0.3 \mathrm{mi}$ downstream from diversions for canals 3 and $4,1.3 \mathrm{mi}$ south of Burlington Northern, Inc., crossing, and $5.5 \mathrm{mi}$ southeast of Comstock.

Drainage area.--4,650 $\mathrm{mi}^{2}$, approximately, of which $430 \mathrm{mi}^{2}$ contributes directly to surface runoff.

Period of record. --Water year 1969 to current year.

Statistical data for selected chemical constituents

\begin{tabular}{|c|c|c|c|c|c|c|c|c|c|}
\hline Constituent & Units & $\begin{array}{l}\text { No. of } \\
\text { measure- } \\
\text { ments }\end{array}$ & $\begin{array}{l}\text { Max- } \\
\text { imum }\end{array}$ & $\begin{array}{l}\text { Min- } \\
\text { imum }\end{array}$ & Mean & Median & $\begin{array}{c}\text { Standard } \\
\text { devi- } \\
\text { ation } \\
\end{array}$ & $\begin{array}{l}\text { Ninetieth } \\
\text { percent- } \\
\text { ile }\end{array}$ & $\begin{array}{c}\text { Tenth } \\
\text { percent- } \\
\text { ile }\end{array}$ \\
\hline Specific conductance & umho/cm & 229 & 309 & 172 & 200 & 197 & $-\ldots$ & 223 & 180 \\
\hline Dissolved solids, residue & $\mathrm{mg} / \mathrm{L}$ & 3 & 169 & 161 & 166 & $\ldots \ldots$ & 4.4 & $\cdots$ & -- \\
\hline Dissolved solids, sum & $\mathrm{mg} / \mathrm{L}$ & 15 & 205 & 139 & 173 & 170 & 17 & 195 & 157 \\
\hline Hardness as $\mathrm{CaCO}_{3}$ & $\mathrm{mg} / \mathrm{L}$ & 15 & 110 & 71 & 89 & 90 & 12 & 100 & 72 \\
\hline Calcium, dissolved & $\mathrm{mg} / \mathrm{L}$ & 15 & 37 & 23 & 29 & 29 & 4.3 & 35 & 22 \\
\hline Magnesium, dissolved & $\mathrm{mg} / \mathrm{L}$ & 15 & 5.7 & 3.3 & 4.2 & 4.2 & 0.6 & 5.0 & 3.5 \\
\hline Sodium, dissolved & $\mathrm{mg} / \mathrm{L}$ & 15 & 9.6 & 6.0 & 7.6 & 7.6 & 0.8 & 8.6 & 6.6 \\
\hline Potassium, dissolved & $\mathrm{mg} / \mathrm{L}$ & 15 & 7.6 & 5.1 & 6.2 & 6.3 & 0.7 & 7.0 & 5.4 \\
\hline Bicarbonate ion & $\mathrm{mg} / \mathrm{L}$ & 15 & 156 & 95 & 121 & 112 & 16 & 140 & 107 \\
\hline Sulfate, dissolved & $\mathrm{mg} / \mathrm{L}$ & 15 & 11 & 6.0 & 7.9 & 7.6 & 1.5 & 10 & 6.1 \\
\hline Chloride, dissolved & $\mathrm{mg} / \mathrm{L}$ & 15 & 4.6 & 1.0 & 1.8 & 1.7 & 0.9 & 3.0 & 1.3 \\
\hline Fluoride, dissolved & $\mathrm{mg} / \mathrm{L}$ & 15 & 0.5 & 0.2 & 0.3 & 0.4 & 0.1 & 0.4 & 0.3 \\
\hline Silica, dissolved & $\mathrm{mg} / \mathrm{L}$ & 15 & 60 & 45 & 55 & 57 & 4.4 & 60 & 50 \\
\hline Boron, dissolved & $\mathrm{mg} / \mathrm{L}$ & 15 & 50 & 0 & 30 & 30 & 12 & 41 & 20 \\
\hline Iron, dissolved & $\mathrm{ug} / \mathrm{L}$ & 2 & 11 & 10 & 10 & --- & & & \\
\hline Manganese, dissolved & $\mathrm{ug} / \mathrm{L}$ & 2 & 202 & 0 & 101 & & & & \\
\hline $\begin{array}{l}\text { Nitrite + nitrate as } \mathrm{N}, \\
\text { dissolved }\end{array}$ & & 12 & 0.72 & 0.00 & 0.30 & 0.30 & 0.26 & 0.62 & 0.01 \\
\hline Nitrate as $\mathrm{N}$, dissolved & $\mathrm{mg} / \mathrm{L}$ & 3 & 0.56 & 0.38 & 0.48 & $\cdots+\cdots$ & 0.09 & & \\
\hline Nitrate as $\mathrm{NO}_{3}$, dissolved & $\mathrm{mg} / \mathrm{L}$ & & $\cdots$ & $\ldots-$ & & & & & \\
\hline Ammonia N, total & $\mathrm{mg} / \mathrm{L}$ & & & & & & & & \\
\hline Organic $N$, total & $\mathrm{mg} / \mathrm{L}$ & 3 & 0.52 & 0.35 & 0.43 & $-\cdots-1$ & 0.09 & & -- \\
\hline Phosphorus, total & $\mathrm{mg} / \mathrm{L}$ & 3 & 0.41 & 0.24 & 0.32 & $\cdots+\cdots$ & 0.09 & & \\
\hline Phosphorus, dissolved & $\mathrm{mg} / \mathrm{L}$ & 15 & 0.21 & 0.09 & 0.15 & 0.15 & 0.03 & 0.20 & 0.11 \\
\hline Fecal coliform col/ & $\operatorname{col} 1 / 100 \mathrm{ml}$ & & & & & & & & \\
\hline Fecal streptococci col/ & $\mathrm{col} / 100 \mathrm{ml}$ & & - & & & & & & \\
\hline Oxygen, dissolved & $\mathrm{mg} / \mathrm{L}$ & 224 & 13.6 & 7.1 & 9.7 & 9.4 & 1.6 & 12.0 & 7.8 \\
\hline $\begin{array}{l}\text { Biochemical oxygen demand } \\
\text { (5-day) }\end{array}$ & pH units & 232 & 8.9 & 6.6 & 7.9 & 7.9 & 0.4 & 8.4 & 7.4 \\
\hline Co - Pt & Co - Pt units & 15 & 50 & 4 & 20 & 20 & 12 & 32 & 5 \\
\hline
\end{tabular}


Results of regression analyses relating concentrations of selected chemical constituents to specific conductance (SpC)

\begin{tabular}{|c|c|c|c|c|c|}
\hline $\begin{array}{l}\text { Dissolved } \\
\text { constituents } \\
(\mathrm{mg} / \mathrm{L})\end{array}$ & $\begin{array}{l}\text { No. of } \\
\text { measure } \\
\text { ments }\end{array}$ & Regression equation & $\begin{array}{l}\text { Corre- } \\
\text { lation } \\
\text { coeffi- } \\
\text { cient }\end{array}$ & $\begin{array}{l}\text { Percent } \\
\text { explained } \\
\text { variance }\end{array}$ & $\begin{array}{c}\text { Standard } \\
\text { error of } \\
\text { estimate } \\
(\mathrm{mg} / \mathrm{L})\end{array}$ \\
\hline Dissolved solids, residue (RDS) & $-\cdots$ & & 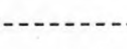 & & \\
\hline Dissolved solids, sum (SDS) & 15 & $\mathrm{SDS}=0.594(\mathrm{SpC})+48.519$ & 0.95 & 91 & 5.4 \\
\hline Hardness as $\mathrm{CaCO}_{3}$ (TH) & 15 & $\mathrm{TH}=0.378(\mathrm{SpC})+9.992$ & .89 & 80 & 5.4 \\
\hline Calcium (Ca) & 15 & $\mathrm{Ca}=0.137(\mathrm{spC})+0.104$ & .89 & 79 & 2.0 \\
\hline Magnesium (Mg) & 15 & $\mathrm{Mg}=0.011(\mathrm{SpC})+1.915$ & $* .50$ & 26 & 0.5 \\
\hline Sodium (Na) & 15 & $\mathrm{Na}-0.016(\mathrm{SpC})+4.124$ & .53 & 28 & 0.8 \\
\hline Potassium (K) & 15 & $K=0.020(\mathrm{spC})+2.026$ & .84 & 70 & 0.4 \\
\hline Bicarbonate $\left(\mathrm{HCO}_{3}\right)$ & 15 & $\mathrm{HCO}_{3} 0.576(\mathrm{SpC})+0.160$ & .98 & 95 & 3.7 \\
\hline Sulfate $\left(\mathrm{SO}_{4}\right)$ & 15 & $\mathrm{SO}_{4}=0.024(\mathrm{SpC})+2.990$ & $* .44$ & 20 & 1.4 \\
\hline Chloride (C1) & 15 & $\mathrm{Cl}=0.003(\mathrm{SpC})+1.225$ & $* .09$ & 0.76 & 1.0 \\
\hline Silica (Si) & 15 & $\mathrm{Si}=0.115(\mathrm{SpC})+30.561$ & .72 & 53 & 3.1 \\
\hline
\end{tabular}

* Not significant at the 95 percent confidence level; the regression equation should not be used to predict the concentration of the dependent variable.

Results of regression analyses relating specific conductance to water discharge (Q)

\begin{tabular}{ccccccc}
\hline $\begin{array}{c}\text { No. of } \\
\text { measure- } \\
\text { ments }\end{array}$ & $\begin{array}{c}\text { Water discharge } \\
\left(\mathrm{ft}^{3} / \mathrm{s}\right)\end{array}$ & Maximum Minimum & Regression equation & $\begin{array}{c}\text { Correlation } \\
\text { coefficient }\end{array}$ & $\begin{array}{c}\text { Percent } \\
\text { explained } \\
\text { variance }\end{array}$ & $\begin{array}{c}\text { Standard error } \\
\text { of estimate }\end{array}$ \\
\hline 227 & 1,980 & 6 & $\mathrm{SpC}=252 \mathrm{Q}^{-0.04054}$ & -0.50 & 25 & 0.039 \\
\hline
\end{tabular}


06783000 Mud Creek near Broken Bow, Nebr.

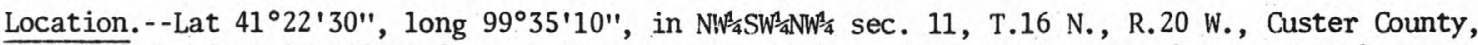
Hydrologic Unit 10210005, at bridge on State Highway 2, about 3 mi southeast of Broken Bow.

Period of record.--Water year 1973 to current year.

Statistical data for selected chemical constituents

\begin{tabular}{|c|c|c|c|c|c|c|c|c|c|}
\hline Constituent & Units & $\begin{array}{l}\text { No. of } \\
\text { measure- } \\
\text { ments }\end{array}$ & $\begin{array}{l}\text { Max- } \\
\text { imum }\end{array}$ & $\begin{array}{l}\text { Min- } \\
\text { imum }\end{array}$ & Mean & Median & $\begin{array}{c}\text { Standard } \\
\text { devi- } \\
\text { ation }\end{array}$ & $\begin{array}{c}\text { Ninetieth } \\
\text { percent- } \\
\text { ile } \\
\end{array}$ & $\begin{array}{l}\text { Tenth } \\
\text { percent- } \\
\text { ile }\end{array}$ \\
\hline Specific conductance & amho/cm & 52 & 886 & 205 & 668 & 682 & 143 & 837 & 500 \\
\hline Dissolved solids, residue & $\mathrm{mg} / \mathrm{L}$ & 35 & 539 & 127 & 422 & 443 & 88 & 512 & 320 \\
\hline Dissolved solids, sum & $\mathrm{mg} / \mathrm{L}$ & 17 & 507 & 197 & 420 & 420 & 80 & 500 & 332 \\
\hline Hardness as $\mathrm{CaCO}_{3}$ & $\mathrm{mg} / \mathrm{L}$ & 17 & 320 & 98 & 226 & 230 & 51 & 300 & 180 \\
\hline Calcium, dissolved & $\mathrm{mg} / \mathrm{L}$ & 17 & 99 & 32 & 71 & 71 & 16 & 95 & 54 \\
\hline Magnesium, dissolved & $\mathrm{mg} / \mathrm{L}$ & 17 & 17 & 4.4 & 12 & 12 & 2.9 & 15 & 9.3 \\
\hline Sodium, dissolved & $\mathrm{mg} / \mathrm{L}$ & 17 & 86 & 14 & 47 & 47 & 19 & 76 & 22 \\
\hline Potassium, dissolved & $\mathrm{mg} / \mathrm{L}$ & 17 & 31 & 11 & 17 & 17 & 4.4 & 20 & 12 \\
\hline Bicarbonate ion & $\mathrm{mg} / \mathrm{L}$ & 17 & 396 & 120 & 304 & 310 & 67 & 390 & 240 \\
\hline Sulfate, dissolved & $\mathrm{mg} / \mathrm{L}$ & 17 & 45 & 14 & 31 & 30 & 8.3 & 42 & 19 \\
\hline Chloride, dissolved & $\mathrm{mg} / \mathrm{L}$ & 51 & 120 & 9.8 & 48 & 47 & 21 & 75 & 26 \\
\hline Fluoride, dissolved & $\mathrm{mg} / \mathrm{L}$ & 17 & 0.6 & 0.1 & 0.3 & 0.3 & 0.1 & 0.5 & 0.2 \\
\hline Silica, dissolved & $\mathrm{mg} / \mathrm{L}$ & 17 & 58 & 8.9 & 45 & 48 & 11 & 52 & 38 \\
\hline Boron, dissolved & $\mathrm{mg} / \mathrm{L}$ & 17 & 360 & 60 & 188 & 175 & 77 & 280 & 110 \\
\hline Iron, dissolved & ug/L & 17 & 370 & 0 & 71 & 46 & 87 & 150 & 10 \\
\hline Manganese, dissolved & $\mathrm{ug} / \mathrm{L}$ & 17 & 460 & 20 & 178 & 125 & 132 & 430 & 50 \\
\hline $\begin{array}{l}\text { Nitrite }+ \text { nitrate as } N \text {, } \\
\text { dissolved } \\
\text { Nitrate as } N \text {, dissolved }\end{array}$ & $\begin{array}{l}\mathrm{mg} / \mathrm{L} \\
\mathrm{mg} / \mathrm{L}\end{array}$ & 25 & 1.6 & 0.1 & 0.6 & 0.4 & 0.44 & 1.4 & 0.2 \\
\hline Nitrate as $\mathrm{NO}_{3}$, dissolved & $\mathrm{mg} / \mathrm{L}$ & 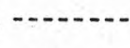 & & & & & & & --- \\
\hline Ammonia $\mathrm{N}$, total & $\mathrm{mg} / \mathrm{L}$ & 52 & 12 & 0.02 & 2.5 & 1.7 & 2.6 & 6.6 & 0.09 \\
\hline Organic N, total & $\mathrm{mg} / \mathrm{L}$ & 52 & 20 & 0.00 & 3.1 & 2.6 & 3.2 & 4.8 & 0.66 \\
\hline Phosphorus, total & $\mathrm{mg} / \mathrm{L}$ & 52 & 12 & 0.31 & 2.6 & 2.4 & 2.0 & 4.1 & 0.96 \\
\hline Phosphorus, dissolved & $\mathrm{mg} / \mathrm{L}$ & 45 & 7.5 & 0.27 & 2.1 & 2.0 & 1.3 & 3.4 & 0.81 \\
\hline Fecal coliform & $100 \mathrm{ml}$ & 41 & 200,000 & 33 & ----- & 2,700 & 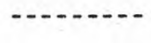 & 25,500 & 180 \\
\hline Fecal streptococci & $100 \mathrm{ml}$ & 51 & 120,000 & 16 & $\cdots--$ & 1,500 & & 8,300 & 360 \\
\hline Oxygen, dissolved & $\mathrm{mg} / \mathrm{L}$ & 54 & 12.2 & 2.5 & 7.0 & 6.9 & 1.9 & 9.7 & 4.4 \\
\hline \multirow{2}{*}{$\begin{array}{l}\text { Biochemical } \\
\text { (5-day) } \\
\mathrm{pH}^{(5)}\end{array}$} & $\mathrm{mg} / \mathrm{L}$ & 48 & 43.0 & 2.8 & 16.8 & 13.5 & 11.0 & 35.0 & 5.3 \\
\hline & \multirow[b]{2}{*}{ Co - Pt units } & 56 & 8.4 & 6.5 & 7.6 & 7.6 & 0.4 & 8.2 & 7.3 \\
\hline Co - Pt & & 17 & 140 & 10 & 42 & 30 & 32 & 90 & 18 \\
\hline
\end{tabular}


Results of regression analyses relating concentrations of selected chemical constituents to specific conductance $(\mathrm{SpC})$

\begin{tabular}{|c|c|c|c|c|c|}
\hline $\begin{array}{l}\text { Dissolved } \\
\text { constituents } \\
(\mathrm{mg} / \mathrm{L})\end{array}$ & $\begin{array}{l}\text { No. of } \\
\text { measure- } \\
\text { ments }\end{array}$ & Regression equation & $\begin{array}{l}\text { Corre- } \\
\text { lation } \\
\text { coeffi- } \\
\text { cient }\end{array}$ & $\begin{array}{l}\text { Percent } \\
\text { explained } \\
\text { variance }\end{array}$ & $\begin{array}{c}\text { Standard } \\
\text { error of } \\
\text { estimate } \\
(\mathrm{mg} / \mathrm{L})\end{array}$ \\
\hline Dissolved solids, residue (RDS) & 34 & $R D S=0.565(S p C)+44.915$ & 0.95 & 90 & 28 \\
\hline Dissolved solids, sum (SDS) & 17 & $S D S=0.553(S p C)+47.061$ & .98 & 96 & 15 \\
\hline Hardness as $\mathrm{CaCO}_{3}$ (TH) & 17 & $\mathrm{TH}=0.245(\mathrm{SpC})+61.321$ & .68 & 47 & 38 \\
\hline Calcium (Ca) & 17 & $\mathrm{Ca}=0.077(\mathrm{SpC})+18.763$ & .71 & 50 & 11 \\
\hline Magnesium (Mg) & 17 & $\mathrm{Mg}=0.014(\mathrm{SpC})+2.694$ & .66 & 44 & 2.2 \\
\hline Sodium (Na) & 17 & $\mathrm{Na}=0.103(\mathrm{SpC})-21.813$ & .78 & 62 & 12 \\
\hline Potassium (K) & 17 & $K=-0.008(\mathrm{SpC})+22.402$ & $*_{-} .27$ & 7.3 & 4.3 \\
\hline Bicarbonate $\left(\mathrm{HCO}_{3}\right)$ & 17 & $\mathrm{HCO}_{3}=0.412(\mathrm{SpC})+26.614$ & .88 & 77 & 33 \\
\hline Sulfate $\left(\mathrm{SO}_{4}\right)$ & 17 & $\mathrm{SO}_{4}=0.036(\mathrm{SpC})+6.366$ & .62 & 38 & 6.7 \\
\hline Chloride (C1) & 50 & $\mathrm{Cl}=0.096(\mathrm{SpC})-16.119$ & .66 & 43 & 16 \\
\hline Silica (Si) & 17 & $\mathrm{Si}=0.048(\mathrm{SpC})+12.529$ & .64 & 41 & 8.4 \\
\hline Nitrite + Nitrate as $\mathrm{N}\left(\mathrm{NO}_{2}-\mathrm{NO}_{3}\right)$ & 25 & $\mathrm{NO}_{2}-\mathrm{NO}_{3}=-0.0007(\mathrm{SpC})+1.054$ & $*-.23$ & 5.3 & 0.43 \\
\hline
\end{tabular}

* Not significant at the 95 percent confidence level; the regression equation should not be used to predict the concentration of the dependent variable.

Results of regression analyses relating specific conductance to water discharge (Q)

\begin{tabular}{cccccccc}
\hline $\begin{array}{c}\text { No. of } \\
\text { measure- }\end{array} \begin{array}{c}\text { Water discharge } \\
\text { ments }\end{array}$ & Maximum Minimum & Regression equation & $\begin{array}{c}\text { Correlation } \\
\text { coefficient }\end{array}$ & $\begin{array}{c}\text { Percent } \\
\text { explained } \\
\text { variance }\end{array}$ & $\begin{array}{c}\text { Standard error } \\
\text { of estimate }\end{array}$ \\
\hline 52 & 30 & 0.16 & $\mathrm{SpC}=703 \mathrm{Q}^{-0.14579}$ & -0.48 & 23 & 0.101 & 23 \\
\hline
\end{tabular}




\section{LOUP RIVER BASIN}

06784000 South Loup River at St. Michae1, Nebr.

Location.--Lat $41^{\circ} 01^{\prime} 53^{\prime \prime}$, long $98^{\circ} 44^{\prime} 25^{\prime \prime}$, in NE $\frac{1}{4} \mathrm{NE}^{\frac{1}{4}} \mathrm{sec} .11$, T.12 N., R.13 W., Buffalo County, Hydrologic Unit 10210004, $15 \mathrm{ft}$ upstream and $65 \mathrm{ft}$ right from right upstream comer of county highway bridge, $0.6 \mathrm{mi}$ northeast of St. Michael, and $3.4 \mathrm{mi}$ upstream from Sweet Creek.

Drainage area.--2,350 $\mathrm{mi}^{2}$, approximately, of which about $1,610 \mathrm{mi}^{2}$ contributes directly to surface runoff.

Period of record.-- Water years $1946-53,1974$ to current year.

Statistical data for selected chemical constituents

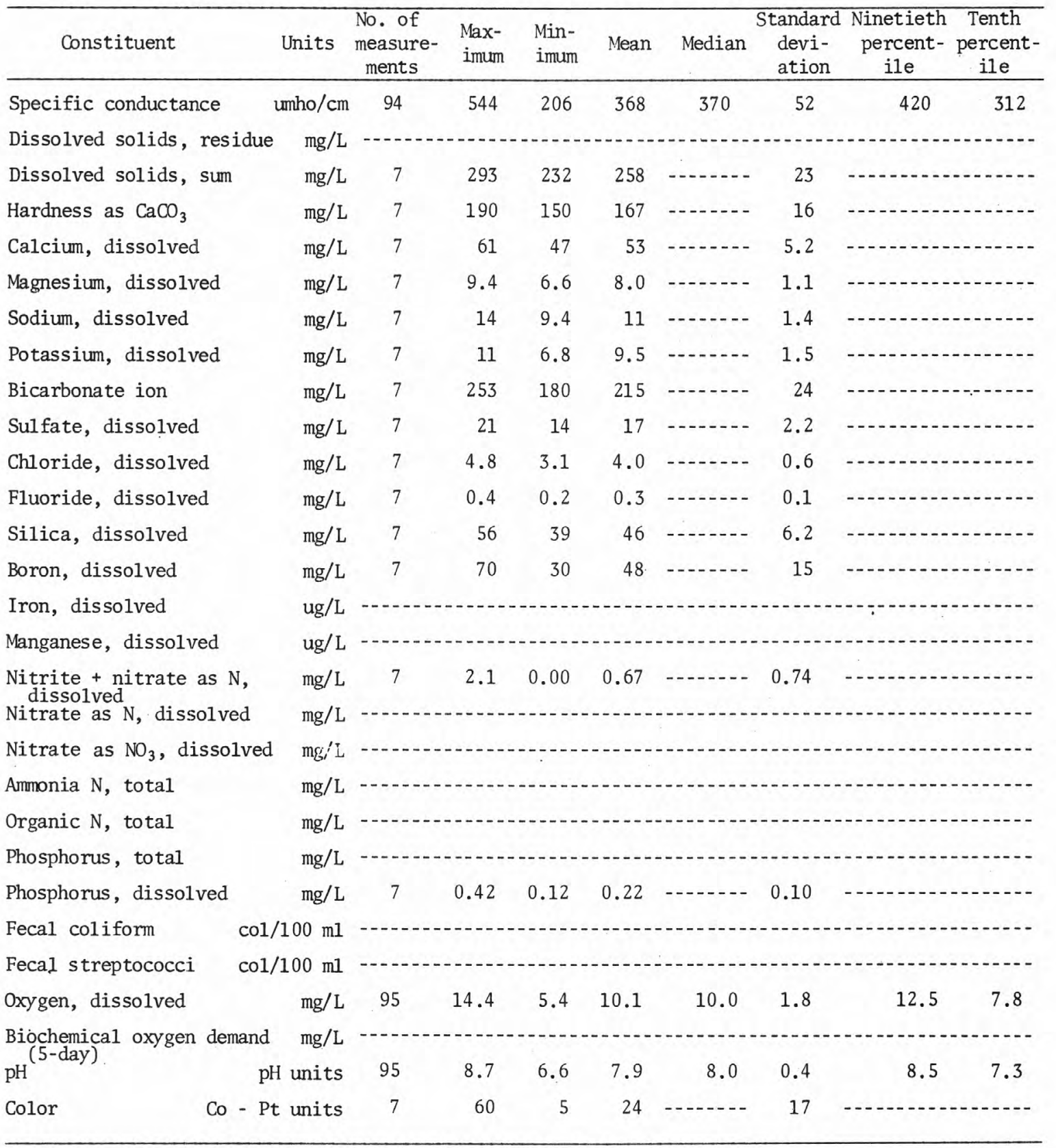


Results of regression analyses relating concentrations of selected chemical constituents to specific conductance (SpC)

\begin{tabular}{|c|c|c|c|c|c|}
\hline $\begin{array}{l}\text { Dissolved } \\
\text { constituents } \\
(\mathrm{mg} / \mathrm{L})\end{array}$ & $\begin{array}{l}\text { No. of } \\
\text { measure- } \\
\text { ments }\end{array}$ & Regression equation & $\begin{array}{l}\text { Corre- } \\
\text { lation } \\
\text { coeffi- } \\
\text { cient }\end{array}$ & $\begin{array}{l}\text { Percent } \\
\text { explained } \\
\text { variance }\end{array}$ & $\begin{array}{c}\text { Standard } \\
\text { error of } \\
\text { estimate } \\
(\mathrm{mg} / \mathrm{L})\end{array}$ \\
\hline Dissolved solids, residue (RDS) & 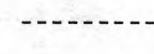 & & & & \\
\hline Dissolved solids, sum (SDS) & $-\cdots--$ & 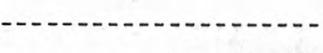 & & & \\
\hline Hardness as $\mathrm{CaCO}_{3}$ (TH) & ----- & & & & \\
\hline Calcium (Ca) & $-\cdots-1$ & - & & & \\
\hline Magnesium (Mg) & ---- & 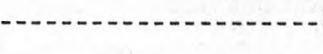 & & & \\
\hline Sodium (Na) & $-\cdots-1$ & 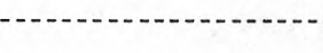 & & & \\
\hline Potassium (K) & ----1 & - & & & \\
\hline Bicarbonate $\left(\mathrm{HCO}_{3}\right)$ & ----- & (2) & & & \\
\hline Sulfate $\left(\mathrm{SO}_{4}\right)$ & $-\cdots-1$ & 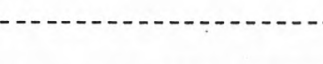 & $---n$ & . & $-2-1-2$ \\
\hline Chloride (C1) & $-\cdots-1$ & $-1-1-2-1-2-1-2-1$ & 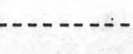 & $---n-\cdots$ & \\
\hline Silica (Si) & $-\cdots$ & ---- & & & \\
\hline Nitrite + Nitrate as $\mathrm{N}\left(\mathrm{NO}_{2}-\mathrm{NO}_{3}\right)$ & & & & & \\
\hline
\end{tabular}

Results of regression analyses relating specific conductance to water discharge (Q)

\begin{tabular}{llllccc}
\hline $\begin{array}{c}\text { No. of } \\
\text { measure- } \\
\text { ments }\end{array}$ & $\begin{array}{c}\text { Water discharge } \\
\left(\mathrm{ft}^{3} / \mathrm{s}\right)\end{array}$ & Maximum Minimum & Regression equation & $\begin{array}{c}\text { Correlation } \\
\text { coefficient }\end{array}$ & $\begin{array}{c}\text { Percent } \\
\text { explained } \\
\text { variance }\end{array}$ & $\begin{array}{c}\text { Standard error } \\
\text { of estimate }\end{array}$ \\
\hline 90 & $1,880 \quad 23$ & $\mathrm{SpC}=452 \mathrm{Q}^{-0.04485}$ & $*-0.18$ & 3.4 & 0.065 \\
\hline
\end{tabular}

* Not significant at the 95 percent confidence 1evel; the regression equation should not be used to predict the concentration of the dependent variable. 
06785000 Middle Loup River at St. Paul, Nebr.

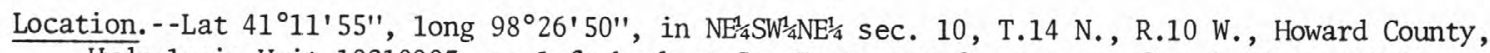
Hydrologic Unit 10210003, on left bank at St. Paul, $450 \mathrm{ft}$ upstream from bridge on U.S.

Highway 281 and $6 \mathrm{mi}$ upstream from confluence with North Loup River.

Drainage area. $--8,090 \mathrm{mi}^{2}$, approximately, of which about $3,130 \mathrm{mi}^{2}$ contributes directly to surface runoff.

Period of record. - -Water year 1969 to current year.

Statistical data for selected chemical constituents

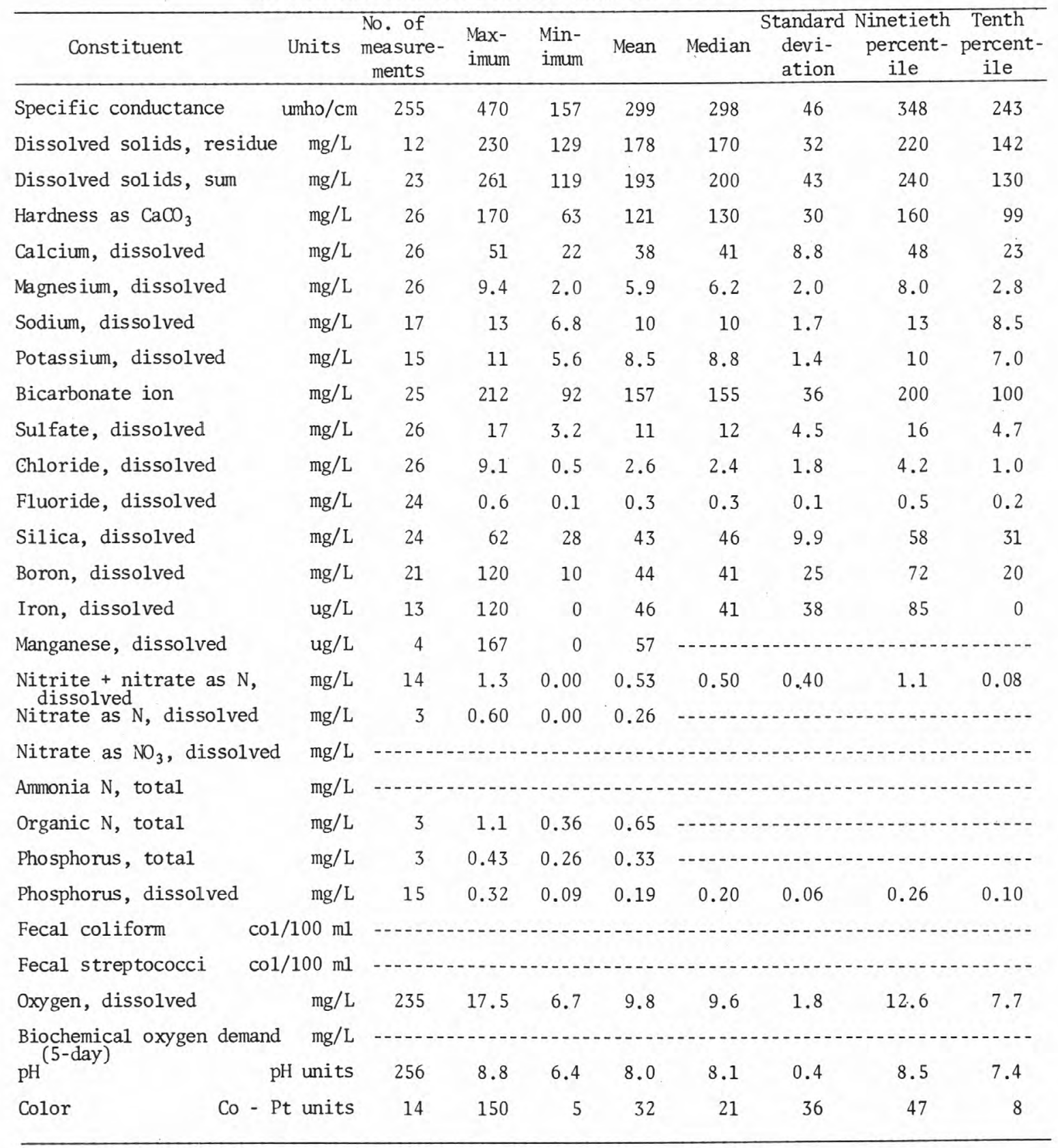


Results of regression analyses relating concentrations of selected chemical constituents to specific conductance $(\mathrm{SpC})$

\begin{tabular}{|c|c|c|c|c|c|}
\hline $\begin{array}{l}\text { Dissolved } \\
\text { constituents } \\
(\mathrm{mg} / \mathrm{L})\end{array}$ & $\begin{array}{l}\text { No. of } \\
\text { measure } \\
\text { ments }\end{array}$ & Regression equation & $\begin{array}{l}\text { Corre- } \\
\text { lation } \\
\text { coeffi- } \\
\text { cient }\end{array}$ & $\begin{array}{l}\text { Percent } \\
\text { explained } \\
\text { variance }\end{array}$ & $\begin{array}{l}\text { Standard } \\
\text { error of } \\
\text { estimate } \\
(\mathrm{mg} / \mathrm{L})\end{array}$ \\
\hline \multicolumn{6}{|c|}{ Dissolved solids, residue (RDS) } \\
\hline Dissolved solids, sum (SDS) & 23 & $\mathrm{SDS}=0.717(\mathrm{SpC})+2.654$ & 0.97 & 94 & 11 \\
\hline Hardness as $\mathrm{CaCO}_{3}(\mathrm{TH})$ & 26 & $\mathrm{TH}=0.430(\mathrm{SpC})+1.375$ & .94 & 88 & 10 \\
\hline Calcium (Ca) & 26 & $\mathrm{Ca}-0.124(\mathrm{SpC})+3.948$ & .93 & 87 & 3.2 \\
\hline Magnesium (Mg) & 26 & $\mathrm{Mg}=0.027(\mathrm{SpC})-1.601$ & .90 & 81 & 0,9 \\
\hline Sodium $(\mathrm{Na})$ & 17 & $\mathrm{Na}-0.030(\mathrm{SpC})+0.918$ & .89 & 80 & 0.8 \\
\hline Potassium (K) & 15 & $\mathrm{~K}=0.011(\mathrm{spC})+5.070$ & *.38 & 15 & 1.4 \\
\hline Bicarbonate $\left(\mathrm{HCO}_{3}\right)$ & 25 & $\mathrm{HCO}_{3}=0.556(\mathrm{SpC})+5.004$ & .96 & 92 & 10 \\
\hline Sulfate $\left(\mathrm{SO}_{4}\right)$ & 26 & $\mathrm{SO}_{4}=0.054(\mathrm{SpC})-4.039$ & .81 & 66 & 2.7 \\
\hline Chloride (C1) & 26 & $\mathrm{Cl}=0.019(\mathrm{SpC})-2.562$ & .69 & 48 & 1.3 \\
\hline Silica (Si) & 24 & $\mathrm{Si}-0.114(\mathrm{SpC})+12.394$ & .75 & 56 & 6.7 \\
\hline \multicolumn{6}{|c|}{ Nitrite + Nitrate as $\mathrm{N}\left(\mathrm{NO}_{2}-\mathrm{NO}_{3}\right)$} \\
\hline \multicolumn{6}{|c|}{$\begin{array}{l}\text { * Not significant at the } 95 \text { percent confidence level; the regression equation should not be used } \\
\text { to predict the concentration of the dependent variable. } \\
\text { Results of regression analyses relating specific conductance to water discharge (Q) }\end{array}$} \\
\hline \multirow{2}{*}{$\begin{array}{l}\begin{array}{l}\text { No. of Water discharge } \\
\text { measure- }\end{array} \\
\text { ments } \\
\text { maximum Minimum }\end{array}$} & \multirow{2}{*}{\multicolumn{2}{|c|}{ Regression equation }} & \multirow{2}{*}{$\begin{array}{l}\text { Percent } \\
\text { explained } \\
\text { variance } \\
\end{array}$} & \multicolumn{2}{|c|}{$\begin{array}{l}\text { Standard error } \\
\text { of estimate }\end{array}$} \\
\hline & & & & Log units & Percent \\
\hline $250 \quad 7,940 \quad 111$ & $\mathrm{SpC}=703 \mathrm{Q}$ & -0.13372 & 44 & 0.053 & 12 \\
\hline
\end{tabular}




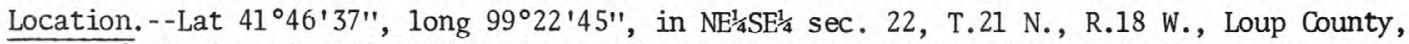
Hydrologic Unit 10210006 on left bank $64 \mathrm{ft}$ downstream from bridge on U.S. Highway 183 and $0.4 \mathrm{mi}$ north of Taylor.

Drainage area.--2,280 $\mathrm{mi}^{2}$, approximately, of which about $180 \mathrm{mi}^{2}$ contributes directly to surface runoff.

Period of record.--Water year 1974 to current year.

Statistical data for selected chemical constituents

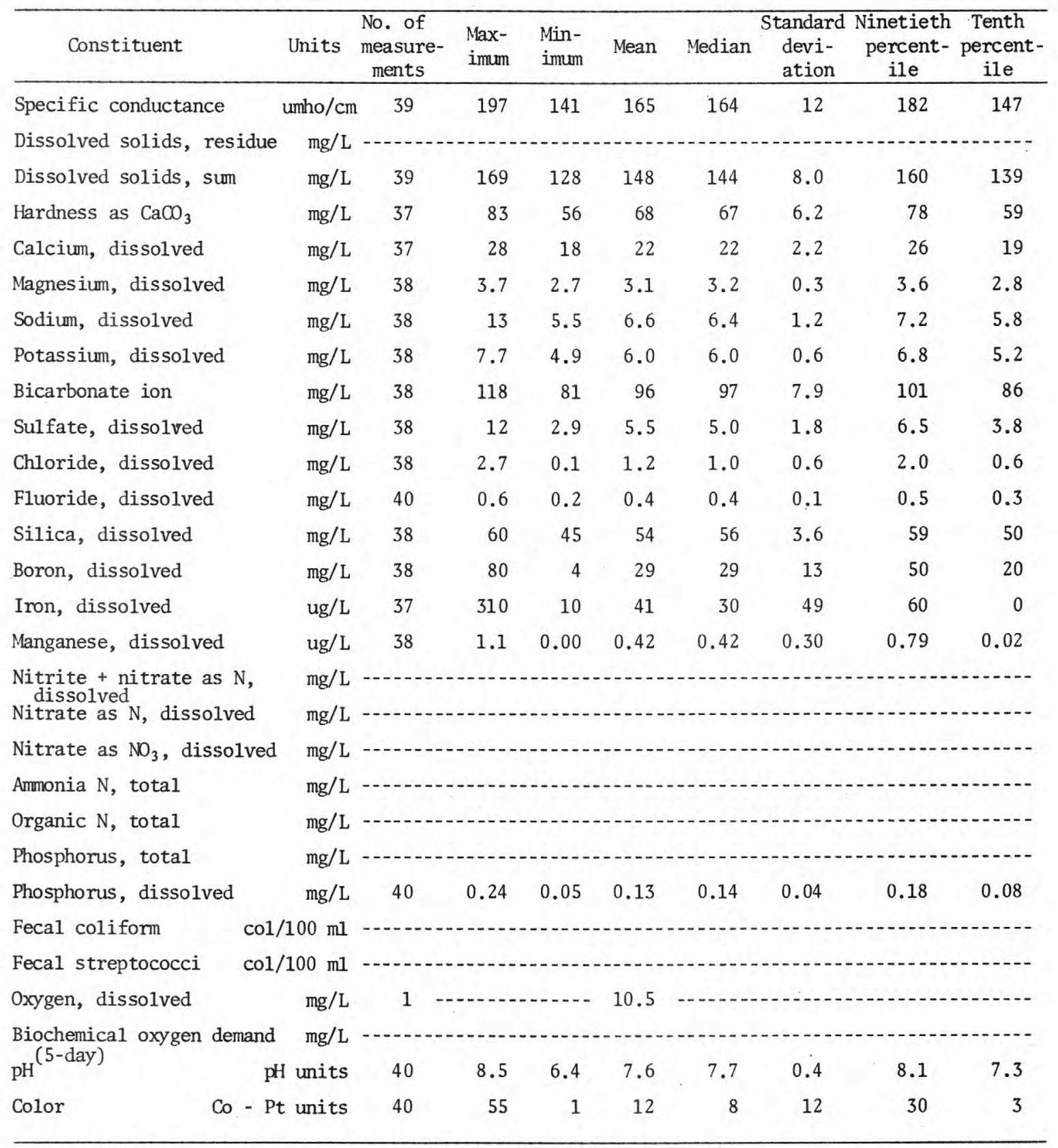


06786000 North Loup River at Taylor, Nebr.--Continued

Results of regression analyses relating concentrations of selected chemical constituents to specific conductance $(\mathrm{SpC})$

\begin{tabular}{|c|c|c|c|c|c|}
\hline $\begin{array}{c}\text { Dissolved } \\
\text { constituents } \\
(\mathrm{mg} / \mathrm{L})\end{array}$ & $\begin{array}{l}\text { No. of } \\
\text { measure- } \\
\text { ments }\end{array}$ & Regression equation & $\begin{array}{l}\text { Corre- } \\
\text { lation } \\
\text { coeffi- } \\
\text { cient }\end{array}$ & $\begin{array}{l}\text { Percent } \\
\text { explained } \\
\text { variance }\end{array}$ & $\begin{array}{c}\text { Standard } \\
\text { error of } \\
\text { estimate } \\
(\mathrm{mg} / \mathrm{L})\end{array}$ \\
\hline Dissolved solids, residue (RDS) & $-\ldots-1$ & 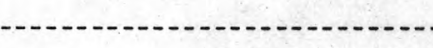 & -- & & \\
\hline Dissolved solids, sum (SDS) & 36 & $\mathrm{SDS}=0.438(\mathrm{SpC})+76.102$ & 0.63 & 40 & 6.6 \\
\hline Hardness as $\mathrm{CaCO}_{3}(\mathrm{TH})$ & 36 & $\mathrm{TH}=0.364(\mathrm{SpC})+6.987$ & .71 & 50 & 4.5 \\
\hline Calcium (Ca) & 36 & $\mathrm{Ca}=0.124(\mathrm{SpC})+1.220$ & .70 & 48 & 1.6 \\
\hline Magnesium (Mg) & 37 & $\mathrm{Mg}=0.013(\mathrm{SpC})+0.956$ & .53 & 28 & 0.3 \\
\hline Sodium $(\mathrm{Na})$ & 37 & $\mathrm{Na}=-0.002(\mathrm{SpC})+6.923$ & $*_{-} .02$ & 0.03 & 1.2 \\
\hline Potassium (K) & 37 & $K=0.023(\mathrm{SpC})+2.165$ & .46 & 22 & 0.5 \\
\hline Bicarbonate $\left(\mathrm{HCO}_{3}\right)$ & 37 & $\mathrm{HCO}_{3}=0.540(\mathrm{SpC})+6.635$ & .81 & 66 & 4.7 \\
\hline Sulfate $\left(\mathrm{SO}_{4}\right)$ & 37 & $\mathrm{SO}_{4}=0.017(\mathrm{SpC})+2.757$ & $* .11$ & 1.2 & 1.8 \\
\hline Chloride (Cl) & 37 & $\mathrm{Cl}=-0.001(\mathrm{SpC})+1.378$ & $*_{-} .03$ & .07 & 0.6 \\
\hline Silica (Si) & 37 & $\mathrm{Si}=0.122(\mathrm{SpC})+34.244$ & .41 & 17 & 3.3 \\
\hline Nitrite + Nitrate as $\mathrm{N}\left(\mathrm{NO}_{2}-\mathrm{NO}_{3}\right)$ & 37 & $\mathrm{NO}_{2}-\mathrm{NO}_{3}=-0.017(\mathrm{SpC})+3.228$ & -.66 & 44 & 0.23 \\
\hline
\end{tabular}

* Not significant at the 95 percent confidence level; the regression equation should not be used to predict the concentration of the dependent variable.

Results of regression analyses relating specific conductance to water discharge (Q)

\begin{tabular}{|c|c|c|c|c|c|c|c|}
\hline \multirow{2}{*}{$\begin{array}{l}\text { No. of } \\
\text { measure } \\
\text { ments }\end{array}$} & \multicolumn{2}{|c|}{$\begin{array}{l}\text { Water discharge } \\
\left(\mathrm{ft}^{3} / \mathrm{s}\right) \\
\end{array}$} & \multirow{2}{*}{ Regression equation } & \multirow{2}{*}{$\begin{array}{l}\text { Correlation } \\
\text { coefficient }\end{array}$} & \multirow{2}{*}{$\begin{array}{l}\text { Percent } \\
\text { explained } \\
\text { variance }\end{array}$} & \multicolumn{2}{|c|}{$\begin{array}{c}\text { Standard error } \\
\text { of estimate }\end{array}$} \\
\hline & Maximum & Minimum & & & & Log units & Percent \\
\hline 39 & 785 & 98 & $\mathrm{SpC}=298 \mathrm{Q}^{-0.09939}$ & -0.66 & 44 & 0.024 & 5.5 \\
\hline
\end{tabular}

* Not significant at the 95 percent confidence level; the regression equation should not be used to predict the concentration of the dependent variable. 


\section{LOUP RIVER BASIN}

06787500 Calamus River near Burwell, Nebr.

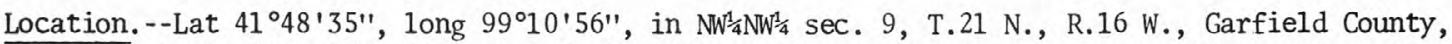
Hydrologic Unit 10210008, on left bank $130 \mathrm{ft}$ downstream from highway bridge, $1.5 \mathrm{mi}$ upstream from mouth, and $3 \mathrm{mi}$ northwest of Burwell.

Drainage area.--1,060 $\mathrm{mi}^{2}$, approximately, of which about $110 \mathrm{mi}^{2}$ contributes directly to surface runoff.

Period of record.--Water year 1972 to current year.

Statistical data for selected chemical constituents

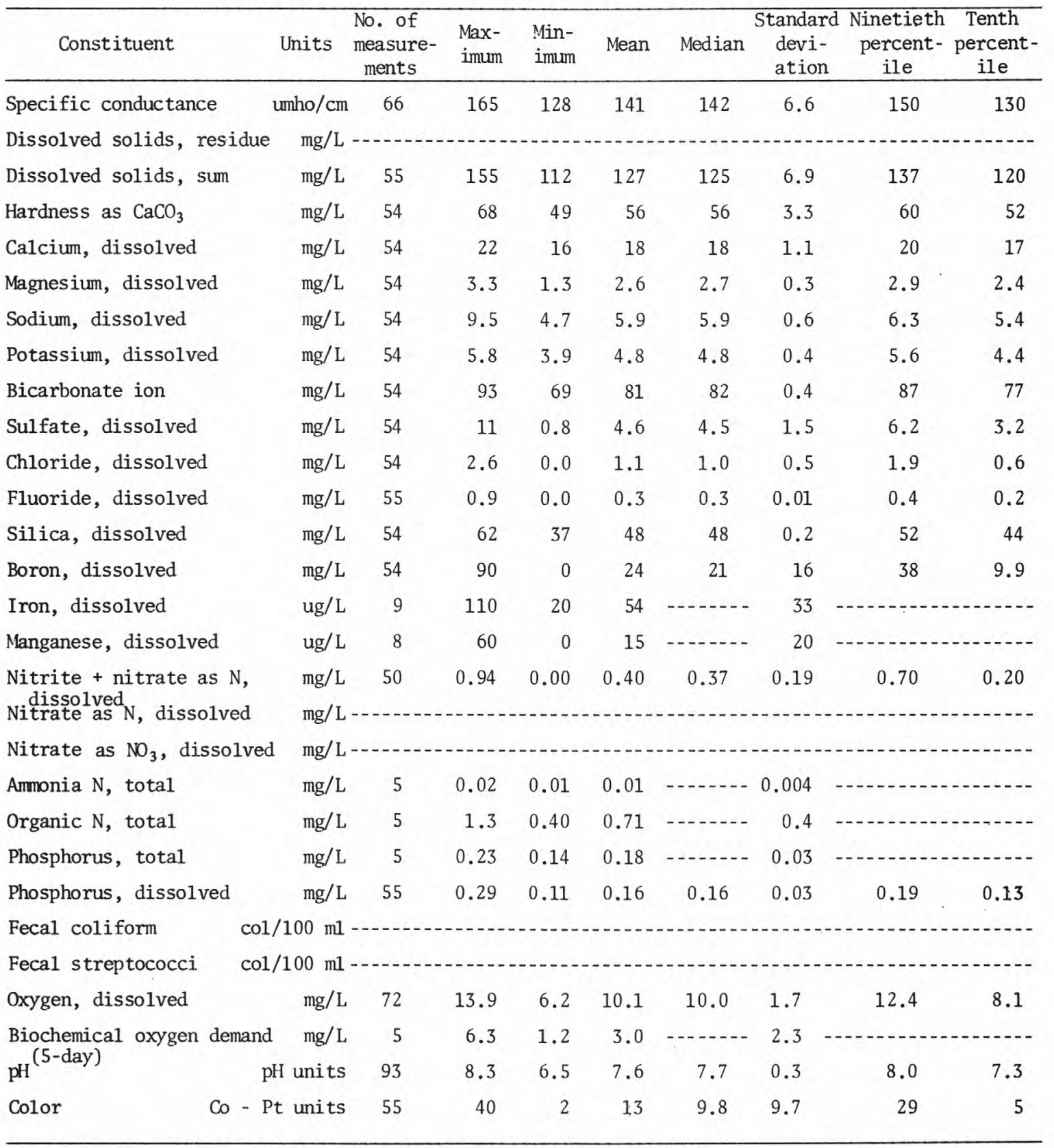


06787500 Calamus River near Burwell, Nebr.--Continued

Results of regression analyses relating concentrations of selected chemical constituents to specific conductance $(\mathrm{SpC})$

\begin{tabular}{|c|c|c|c|c|c|}
\hline $\begin{array}{c}\text { Dissolved } \\
\text { constituents } \\
(\mathrm{mg} / \mathrm{L})\end{array}$ & $\begin{array}{l}\text { No. of } \\
\text { measure- } \\
\text { ments }\end{array}$ & Regression equation & $\begin{array}{l}\text { Corre- } \\
\text { lation } \\
\text { coeffi- } \\
\text { cient }\end{array}$ & $\begin{array}{l}\text { Percent } \\
\text { explained } \\
\text { variance }\end{array}$ & $\begin{array}{l}\text { Standard } \\
\text { error of } \\
\text { estimate } \\
(\mathrm{mg} / \mathrm{L})\end{array}$ \\
\hline Dissolved solids, residue (RDS) & $\cdots+-$ & & & & - \\
\hline Dissolved solids, sum (SDS) & 54 & $\mathrm{SDS}=0.568(\mathrm{SpC})+46.981$ & 0.47 & 22 & 6.2 \\
\hline Hardness as $\mathrm{CaCO}_{3}(\mathrm{TH})$ & 54 & $\mathrm{TH}=0.298(\mathrm{SpC})+13.462$ & .51 & 26 & 2.9 \\
\hline Calcium ( $\mathrm{Ca}$ ) & 54 & $\mathrm{Ca}=0.103(\mathrm{SpC})+3.366$ & .50 & 25 & 1.0 \\
\hline Magnesium $(\mathrm{Mg})$ & 54 & $\mathrm{Mg}=0.012(\mathrm{SpC})+0.874$ & $* .25$ & 6.4 & 0.3 \\
\hline Sodium (Na) & 54 & $\mathrm{Na}=0.047(\mathrm{SpC})-0.785$ & .42 & 17 & 0.6 \\
\hline Potassium (K) & 54 & $\mathrm{~K}=0.024(\mathrm{SpC})-1.499$ & .32 & 10 & 0.4 \\
\hline Bicarbonate $\left(\mathrm{HCO}_{3}\right)$ & 54 & $\mathrm{HCO}_{3} 0.395(\mathrm{SpC})+25.560$ & .54 & 29 & 3.6 \\
\hline Sulfate $\left(\mathrm{SO}_{4}\right)$ & 54 & $\mathrm{SO}_{4}=0.063(\mathrm{SpC})-4.300$ & $* .24$ & 5.7 & 1.5 \\
\hline Chloride (C1) & 54 & $\mathrm{Cl}-0.018(\mathrm{SpC})-1.402$ & *.19 & 3.6 & 0.5 \\
\hline Silica (Si) & 54 & $\mathrm{Si}=0.062(\mathrm{SpC})+39.221$ & $* .08$ & 0.74 & 4.1 \\
\hline Nitrite + Nitrate as $\mathrm{N}\left(\mathrm{NO}_{2}-\mathrm{NO}_{3}\right)$ & 50 & $\mathrm{NO}_{2}-\mathrm{NO}_{3}=-0.002(\mathrm{SpC})+0.622$ & $2 *-.04$ & 0.21 & 0.19 \\
\hline
\end{tabular}

* Not significant at the 95 percent confidence level; the regression equation should not be used to predict the concentration of the dependent variable.

Results of regression analyses relating specific conductance to water discharge (Q)

\begin{tabular}{|c|c|c|c|c|c|c|c|}
\hline \multirow{2}{*}{$\begin{array}{l}\text { No. of } \\
\text { measure- } \\
\text { ments }\end{array}$} & \multicolumn{2}{|c|}{$\begin{array}{c}\text { Water discharge } \\
\left(\mathrm{ft}^{3} / \mathrm{s}\right) \\
\end{array}$} & \multirow{2}{*}{ Regression equation } & \multirow{2}{*}{$\begin{array}{l}\text { Correlation } \\
\text { coefficient }\end{array}$} & \multirow{2}{*}{$\begin{array}{c}\text { Percent } \\
\text { explained } \\
\text { variance } \\
\end{array}$} & \multicolumn{2}{|c|}{$\begin{array}{c}\text { Standard error } \\
\text { of estimate }\end{array}$} \\
\hline & Maximum & Minimum & & & & Log units & Percent \\
\hline 66 & 451 & 182 & $\mathrm{SpC}=193 \mathrm{Q}^{-0.05523}$ & $*-0.19$ & 3.6 & 0.020 & 4.6 \\
\hline
\end{tabular}

* Not significant at the 95 percent confidence level; the regression equation should not be used to predict the concentration of the dependent variable. 
06790500 North Loup River near St. Paul, Nebr.

Location. --Lat $41^{\circ} 15^{\prime} 35^{\prime \prime}$, 1ong $98^{\circ} 26^{\prime} 50^{\prime \prime}$, in $\mathrm{NW}^{\frac{1}{4}} \mathrm{NW}^{\frac{1}{4}} \mathrm{NE}^{\frac{1}{4}} \mathrm{sec}$. 22, T.15 N., R.10 W., Howard County, Hydrologic Unit 10210007, on right bank $310 \mathrm{ft}$ downstream from bridge on U.S. Highway 281, $3 \mathrm{mi}$ north of St. Paul, and $4 \mathrm{mi}$ upstream from confluence with Middle Loup River.

Drainage area. $--4,290 \mathrm{mi}^{2}$, approximately, of which about $1,240 \mathrm{mi}^{2}$ contributes directly to surface runoff.

Period of record. --Water years 1946-53, 1974 to current year.

Statistical data for selected chemical constituents

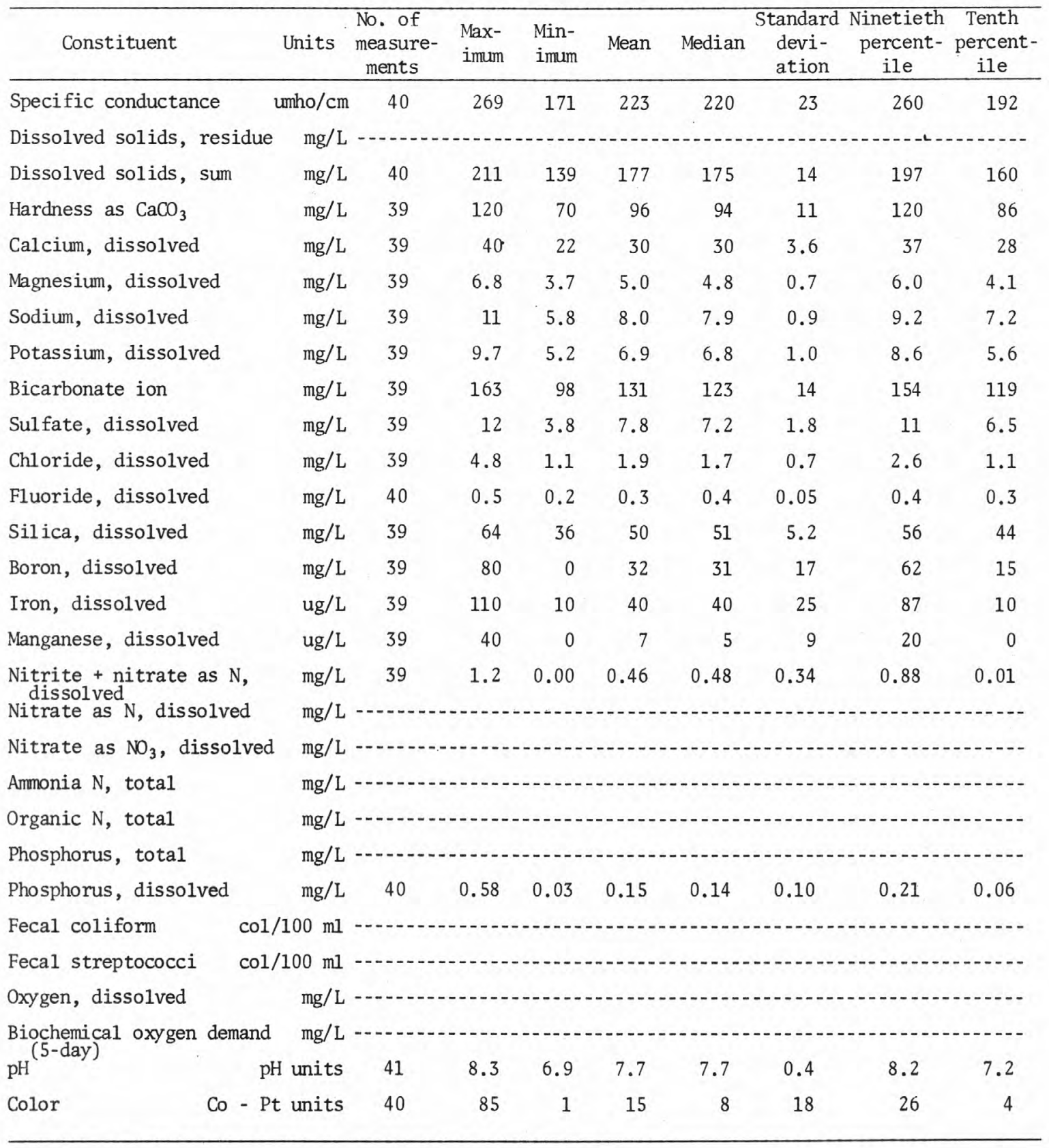


06790500 North Loup River near St. Paul, Nebr.--Continued

Results of regression analyses relating concentrations of selected chemical constituents to specific conductance $(\mathrm{SpC})$

\begin{tabular}{|c|c|c|c|c|c|}
\hline $\begin{array}{c}\text { Dissolved } \\
\text { constituents } \\
(\mathrm{mg} / \mathrm{L})\end{array}$ & $\begin{array}{l}\text { No. of } \\
\text { measure } \\
\text { ments }\end{array}$ & Regression equation & $\begin{array}{l}\text { Corre- } \\
\text { lation } \\
\text { coeffi- } \\
\text { cient }\end{array}$ & $\begin{array}{l}\text { Percent } \\
\text { explained } \\
\text { variance }\end{array}$ & $\begin{array}{c}\text { Standard } \\
\text { error of } \\
\text { estimate } \\
(\mathrm{mg} / \mathrm{L})\end{array}$ \\
\hline Dissolved solids, residue (RDS) & & & & & \\
\hline Dissolved solids, sum (SDS) & 42 & $\mathrm{SDS}=0.519(\mathrm{SpC})+61.268$ & 0.88 & 78 & 6.5 \\
\hline Hardness as $\mathrm{CaCO}_{3}(\mathrm{TH})$ & 42 & $\mathrm{TH}=0.405(\mathrm{SpC})+5.912$ & .87 & 76 & 5.5 \\
\hline Calcium (Ca) & 42 & $\mathrm{Ca}=0.127(\mathrm{SpC})+1.963$ & .85 & 72 & 1.9 \\
\hline Magnesium (Mg) & 42 & $\mathrm{Mg}=0.022(\mathrm{SpC})+0.118$ & .72 & 52 & 0.5 \\
\hline Sodium (Na) & 42 & $\mathrm{Na}=0.030(\mathrm{SpC})+1.347$ & .81 & 66 & 0.5 \\
\hline Potassium (K) & 42 & $\mathrm{~K}=0.031(\mathrm{SpC})+0.074$ & .68 & 46 & 0.8 \\
\hline Bicarbonate $\left(\mathrm{HCO}_{3}\right)$ & 42 & $\mathrm{HCO}_{3}=0.532(\mathrm{SpC})+11.893$ & .91 & 83 & 5.8 \\
\hline Sulfate $\left(\mathrm{SO}_{4}\right)$ & 42 & $\mathrm{SO}_{4}=0.044(\mathrm{SpC})-2.048$ & .59 & 35 & 1.4 \\
\hline Chloride (C1) & 42 & $\mathrm{Cl}=0.014(\mathrm{SpC})-1.127$ & .45 & 20 & 0.6 \\
\hline Silica (Si) & 42 & $\mathrm{Si}=0.020(\mathrm{SpC})+45.913$ & *.09 & 0.82 & 5.2 \\
\hline Nitrite + Nitrate as $\mathrm{N}\left(\mathrm{NO}_{2}-\mathrm{NO}_{3}\right)$ & 42 & $\mathrm{NO}_{2}-\mathrm{NO}_{3}=-0.007(\mathrm{SpC})+2.001$ & -.46 & 21 & 0.31 \\
\hline
\end{tabular}

* Not significant at the 95 percent confidence level; the regression equation should not be used to predict the concentration of the dependent variable.

Results of regression analyses relating specific conductance to water discharge $(Q)$

\begin{tabular}{|c|c|c|c|c|c|c|c|}
\hline \multirow{2}{*}{$\begin{array}{l}\text { No. of } \\
\text { measure- } \\
\text { ments }\end{array}$} & \multicolumn{2}{|c|}{$\begin{array}{l}\text { Water discharge } \\
\left(\mathrm{ft}^{3} / \mathrm{s}\right) \\
\end{array}$} & \multirow{2}{*}{ Regression equation } & \multirow{2}{*}{$\begin{array}{l}\text { Correlation } \\
\text { coefficient }\end{array}$} & \multirow{2}{*}{$\begin{array}{l}\text { Percent } \\
\text { explained } \\
\text { variance }\end{array}$} & \multicolumn{2}{|c|}{$\begin{array}{c}\text { Standard error } \\
\text { of estimate }\end{array}$} \\
\hline & Maximum & Minimum & & & & Log units & Percent \\
\hline 41 & 1,820 & 274 & $\mathrm{SpC}=391 \mathrm{Q}^{-0.08551}$ & -0.36 & 13 & 0.041 & 9.4 \\
\hline
\end{tabular}


Location.--Lat $41^{\circ} 23^{\prime} 45^{\prime \prime}$, long $98^{\circ} 00^{\prime} 15^{\prime \prime}$, in $\mathrm{NE}^{\frac{1}{4}} \mathrm{NE}^{\frac{1}{4}} \mathrm{sec} .4$, T.16 N., R.6 W., Nance County, Hydrologic Unit 10210010, near left bank on downstream side of pier of highway bridge, $3 \mathrm{mi}$ northwest of Fullerton and $7.2 \mathrm{mi}$ (revised) upstream from mouth.

Drainage area.--1,220 $\mathrm{mi}^{2}$, approximately, of which about $480 \mathrm{mi}^{2}$ contributes directly to surface runoff.

Period of record.--Water years 1953-59, 1974 to current year.

Statistical data for selected chemical constituents

\begin{tabular}{|c|c|c|c|c|c|c|c|c|c|}
\hline Constituent & Units & $\begin{array}{l}\text { No. of } \\
\text { measure- } \\
\text { ments }\end{array}$ & $\begin{array}{l}\text { Max- } \\
\text { imum }\end{array}$ & $\begin{array}{l}\text { Min- } \\
\text { imum }\end{array}$ & Mean & Median & $\begin{array}{c}\text { Standard } \\
\text { devi- } \\
\text { ation }\end{array}$ & $\begin{array}{l}\text { Ninetieth } \\
\text { percent- } \\
\text { ile }\end{array}$ & $\begin{array}{l}\text { Tenth } \\
\text { percent- } \\
\text { ile } \\
\end{array}$ \\
\hline Specific conductance & umho/cm & 45 & 344 & 194 & 276 & 276 & 31 & 312 & 240 \\
\hline Dissolved solids, residue & $\mathrm{mg} / \mathrm{L}$ & 6 & 301 & 136 & 199 & $-\cdots--$ & 56 & $-\cdots$ & $\cdots$ \\
\hline Dissolved solids, sum & $\mathrm{mg} / \mathrm{L}$ & 39 & 249 & 128 & 192 & 191 & 20 & 216 & 170 \\
\hline Hardness as $\mathrm{CaCO}_{3}$ & $\mathrm{mg} / \mathrm{L}$ & 41 & 170 & 81 & 124 & 127 & 15 & 145 & 111 \\
\hline Calcium, dissolved & $\mathrm{mg} / \mathrm{L}$ & 38 & 50 & 26 & 39 & 39 & 4.7 & 45 & 34 \\
\hline Magnesium, dissolved & $\mathrm{mg} / \mathrm{L}$ & 38 & 11 & 3.9 & 6.5 & 6.6 & 1.1 & 7.4 & 5.3 \\
\hline Sodium, dissolved & $\mathrm{mg} / \mathrm{L}$ & 41 & 26 & 3.8 & 8.5 & 8.1 & 3.0 & 9.4 & 6.9 \\
\hline Potassium, dissolved & $\mathrm{mg} / \mathrm{L}$ & 38 & 10 & 5.2 & 7.2 & 7.2 & 0.9 & 8.1 & 6.4 \\
\hline Bicarbonate ion & $\mathrm{mg} / \mathrm{L}$ & 42 & 213 & 110 & 164 & 164 & 17 & 185 & 143 \\
\hline Sulfate, dissolved. & $\mathrm{mg} / \mathrm{L}$ & 38 & 13 & 4.0 & 8.4 & 8.7 & 1.8 & 10 & 6.4 \\
\hline Chloride, dissolved & $\mathrm{mg} / \mathrm{L}$ & 39 & 25 & 0.1 & 2.4 & 1.7 & 3.8 & 3.0 & 1.2 \\
\hline Fluoride, dissolved & $\mathrm{mg} / \mathrm{L}$ & 41 & 0.4 & 0.2 & 0.3 & 0.3 & 0.06 & 0.3 & 0.2 \\
\hline Silica, dissolved & $\mathrm{mg} / \mathrm{L}$ & 38 & 53 & 17 & 38 & 38 & 6.4 & 46 & 32 \\
\hline Boron, dissolved & $\mathrm{mg} / \mathrm{L}$ & 39 & 70 & 2 & 35 & 30 & 13 & 52 & 25 \\
\hline Iron, dissolved & $\mathrm{ug} / \mathrm{L}$ & 39 & 1,100 & 0 & 70 & 40 & 172 & 87 & 0 \\
\hline Manganese, dissolved & $\mathrm{ug} / \mathrm{L}$ & 37 & 40 & 0 & 12 & 10 & 11 & 30 & 0 \\
\hline $\begin{array}{l}\text { Nitrite + nitrate as } \mathrm{N}, \\
\text { dissolved } \\
\text { Nitrate as } N \text {, dissolved }\end{array}$ & $\begin{array}{l}\mathrm{mg} / \mathrm{L} \\
\mathrm{mg} / \mathrm{L}\end{array}$ & 37 & 0.69 & 0.00 & 0.22 & 0.17 & 0.21 & 0.53 & 0.01 \\
\hline Nitrate as $\mathrm{NO}_{3}$, dissolved & $\mathrm{mg} / \mathrm{L}$ & $\cdots$ & & & & & & & -- \\
\hline Ammonia N, total & $\mathrm{mg} / \mathrm{L}$ & $-\cdots$ & & & 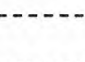 & & & & \\
\hline Organic N, total & $\mathrm{mg} / \mathrm{L}$ & $\cdots$ & & & & & & & -- \\
\hline Phosphorus, total & $\mathrm{mg} / \mathrm{L}$ & 10 & & & 02 & בית & & & $0_{12}$ \\
\hline Phosphorus, dissolved & $\mathrm{mg} / \mathrm{L}$ & 40 & 0.53 & 0.12 & 0.21 & 0.21 & 0.07 & 0.29 & 0.15 \\
\hline $\begin{array}{ll}\text { Fecal coliform } & \mathrm{col} / \\
\text { Fecal streptococci } & \mathrm{col} /\end{array}$ & $\mathrm{col} / 100 \mathrm{ml}$ & --- & - & 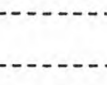 & --- & & & & -- \\
\hline Oxygen, dissolved & $\mathrm{mg} / \mathrm{L}$ & 2 & 9.3 & 8.3 & 8.8 & & & & \\
\hline $\begin{array}{l}\text { Biochemical oxygen demand } \\
\mathrm{pH}^{(5-\text { day })}\end{array}$ & $\mathrm{pH}$ units & 44 & 8.9 & 6.5 & 7.6 & 7.6 & 0.5 & 8.3 & 7.0 \\
\hline Co $-\mathrm{Pt}$ & Co - Pt units & 42 & 140 & 2 & 18 & 12 & 23 & 39 & 5 \\
\hline
\end{tabular}


Results of regression analyses relating concentrations of selected chemical constituents to specific conductance $(\mathrm{SpC})$

\begin{tabular}{|c|c|c|c|c|c|}
\hline $\begin{array}{l}\text { Dissolved } \\
\text { constituents } \\
\text { (mg/L) }\end{array}$ & $\begin{array}{l}\text { No. of } \\
\text { measure- } \\
\text { ments }\end{array}$ & Regression equation & $\begin{array}{c}\text { Corre- } \\
\text { lation } \\
\text { coeffi- } \\
\text { cient }\end{array}$ & $\begin{array}{l}\text { Percent } \\
\text { explained } \\
\text { variance }\end{array}$ & $\begin{array}{c}\text { Standard } \\
\text { error of } \\
\text { estimate } \\
(\mathrm{mg} / \mathrm{L})\end{array}$ \\
\hline \multicolumn{6}{|l|}{ Dissolved solids, residue (RDS) } \\
\hline Dissolved solids, sum (SDS) & 36 & $\mathrm{SDS}=0.567(\mathrm{SpC})+34.792$ & 0.76 & 58 & 12 \\
\hline Hardness as $\mathrm{CaCO}_{3}$ (TH) & 41 & $\mathrm{TH}=0.470(\mathrm{SpC})-6.938$ & .86 & 74 & 7.9 \\
\hline Calcium (Ca) & 38 & $\mathrm{Ca}=0.140(\mathrm{SpC})-0.365$ & .83 & 70 & 2.6 \\
\hline Magnesium (Mg) & 38 & $\mathrm{Mg}=0.032(\mathrm{SpC})-2.537$ & .81 & 66 & .6 \\
\hline Sodium (Na) & 41 & $\mathrm{Na}=0.020(\mathrm{SpC})+2.790$ & *.19 & 3.6 & $3.0^{2}$ \\
\hline Potassium (K) & 38 & $\mathrm{~K}=-0.004(\mathrm{SpC})+8.347$ & $*-.13$ & 1.6 & .95 \\
\hline Bicarbonate $\left(\mathrm{HCO}_{3}\right)$ & 42 & $\mathrm{HCO}_{3}=0.572(\mathrm{SpC})+5.433$ & .94 & 87 & 6.3 \\
\hline Sulfate $\left(\mathrm{SO}_{4}\right)$ & 38 & $\mathrm{SO}_{4}=0.013(\mathrm{SpC})+4.704$ & *.20 & 4.1 & 1.8 \\
\hline Chloride (C1) & 39 & $\mathrm{Cl}=0.003(\mathrm{SpC})+1.517$ & $* .02$ & .06 & 3.8 \\
\hline Silica (Si) & 38 & $\mathrm{Si}=0.136(\mathrm{SpC})-0.408$ & .59 & 35 & 5.2 \\
\hline Nitrite + Nitrate as $\mathrm{N}\left(\mathrm{NO}_{2}-\mathrm{NO}_{3}\right)$ & 37 & $\mathrm{NO}_{2}-\mathrm{NO}_{3}=0.006(\mathrm{SpC})+0.064$ & $* .07$ & .47 & .21 \\
\hline
\end{tabular}

* Not significant at the 95 percent confidence level; the regression equation should not be used to predict the concentration of the dependent variable.

Results of regression analyses relating specific conductance to water discharge (Q)

\begin{tabular}{lccccccc}
\hline $\begin{array}{c}\text { No. of } \\
\text { measure- } \\
\text { ments }\end{array}$ & $\begin{array}{c}\text { Water discharge } \\
\left(\mathrm{ft}^{3} / \mathrm{s}\right)\end{array}$ & Maximum Minimum & Regression equation & $\begin{array}{c}\text { Correlation } \\
\text { coefficient }\end{array}$ & $\begin{array}{c}\text { Percent } \\
\text { explained } \\
\text { variance }\end{array}$ & \multicolumn{2}{c}{$\begin{array}{c}\text { Standard error } \\
\text { of estimate }\end{array}$} \\
\hline 44 & $3,300 \quad 47$ & $\mathrm{SpC}=466 \mathrm{Q}^{-0.10168}$ & -0.54 & 29 & 0.044 & 10 \\
\hline
\end{tabular}


Location. --Lat $41^{\circ} 23^{\prime} 31^{\prime \prime}$, long $97^{\circ} 49^{\prime} 20^{\prime \prime}$, in NE $\frac{1}{4} W^{\frac{1}{4}}$ sec. 6, T.16 N., R.4 W., Nance County, Hydrologic Unit 10210009, at diversion structure, 2 mi upstream from gaging station, and $5.5 \mathrm{mi}$ southwest of Genoa.

Period of record.--Water year 1973 to current year.

Statistical data for selected chemical constituents

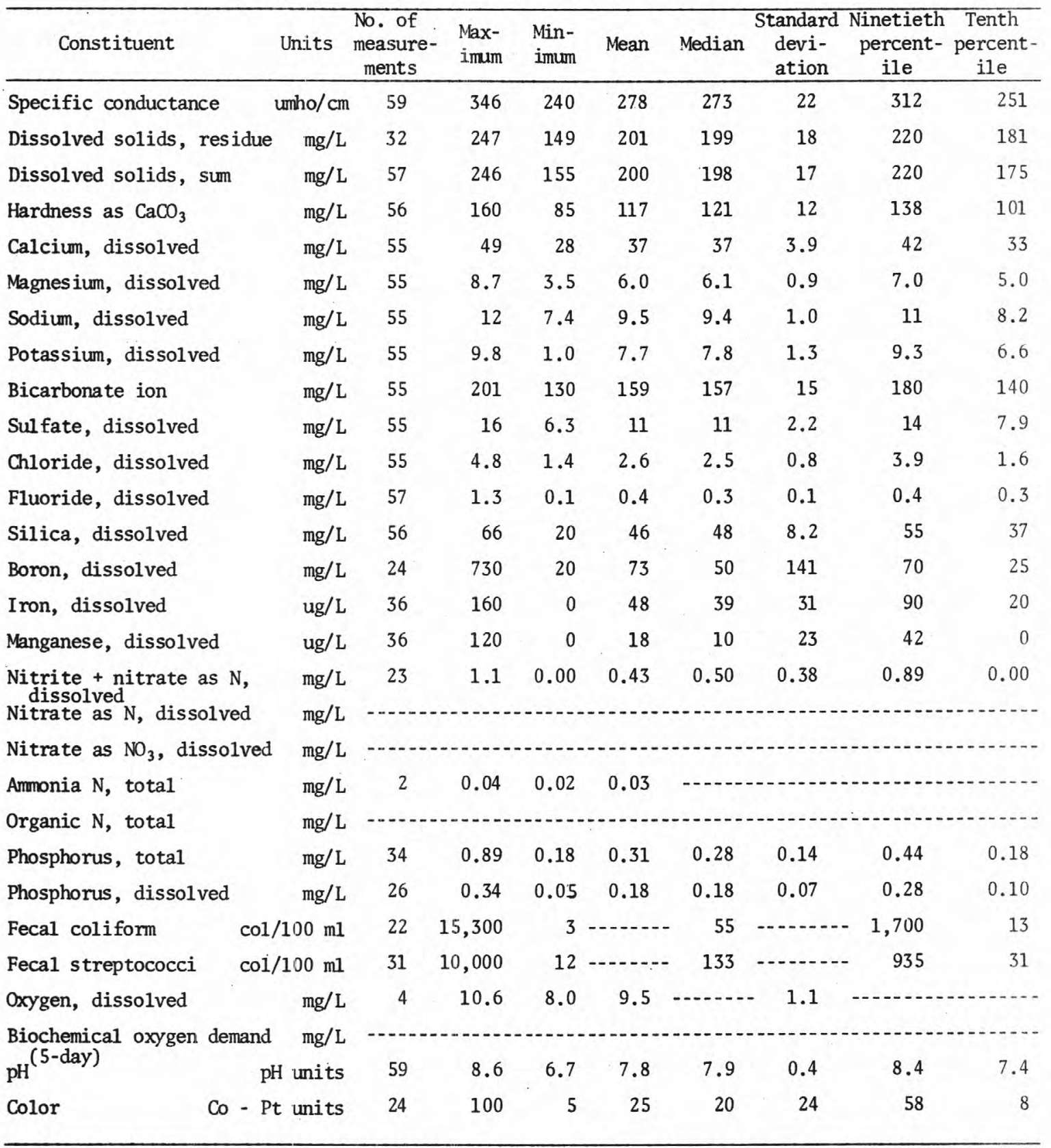


06792499 Loup River Power Canal at Diversion near Genoa, Nebr.--Continued

Results of regression analyses relating concentrations of selected chemical constituents to specific conductance (SpC)

\begin{tabular}{|c|c|c|c|c|c|}
\hline $\begin{array}{c}\text { Dissolved } \\
\text { constituents } \\
(\mathrm{mg} / \mathrm{L})\end{array}$ & $\begin{array}{l}\text { No. of } \\
\text { measure- } \\
\text { ments }\end{array}$ & Regression equation & $\begin{array}{l}\text { Corre- } \\
\text { lation } \\
\text { coeffi- } \\
\text { cient }\end{array}$ & $\begin{array}{l}\text { Percent } \\
\text { explained } \\
\text { variance }\end{array}$ & $\begin{array}{c}\text { Standard } \\
\text { error of } \\
\text { estimate } \\
(\mathrm{mg} / \mathrm{L})\end{array}$ \\
\hline Dissolved solids, residue (RDS) & 32 & $\mathrm{RDS}=0.647(\mathrm{SpC})+23.048$ & 0.80 & 64 & 11 \\
\hline Dissolved solids, sum (SDS) & 56 & $\mathrm{SDS}=0.553(\mathrm{SpC})+45.652$ & .71 & 51 & 12 \\
\hline Hardness as $\mathrm{CaCO}_{3}(\mathrm{TH})$ & 56 & $\mathrm{TH}=0.482(\mathrm{SpC})-16.972$ & .84 & 71 & 6.8 \\
\hline Calcium (Ca) & 55 & $\mathrm{Ca}=0.150(\mathrm{SpC})-4.750$ & .86 & 73 & 2.0 \\
\hline Magnesium (Mg) & 55 & $\mathrm{Mg}=0.022(\mathrm{SpC})-0.266$ & .57 & 33 & 0.7 \\
\hline Sodium (Na) & 55 & $\mathrm{Na}=0.027(\mathrm{SpC})+1.920$ & .58 & 33 & 0.8 \\
\hline Potassium (K) & 55 & $K=0.008(\mathrm{SpC})+5.310$ & $* .14$ & 2.0 & 1.3 \\
\hline Bicarbonate $\left(\mathrm{HCO}_{3}\right)$ & 55 & $\mathrm{HO}_{3}=0.575(\mathrm{SpC})-1.406$ & .84 & 71 & 8.2 \\
\hline Sulfate $\left(\mathrm{SO}_{4}\right)$ & 55 & $\mathrm{SO}_{4}=0.047(\mathrm{SpC})-2.168$ & .47 & 22 & 2.0 \\
\hline Chloride (C1) & 55 & $\mathrm{C} 1-0.012(\mathrm{SpC})-0.771$ & .33 & 11 & 0.8 \\
\hline Silica (Si) & 56 & $\mathrm{Si}=0.0005(\mathrm{SpC})+45.940$ & $* 0.00$ & 0.00 & 8.3 \\
\hline Nitrite + Nitrate as $\mathrm{N}\left(\mathrm{NO}_{2}-\mathrm{NO}_{3}\right)$ & 23 & $\mathrm{NO}_{2}-\mathrm{NO}_{3}=-0.002(\mathrm{SpC})+0.985$ & $*_{-} .11$ & 1.3 & 0.38 \\
\hline
\end{tabular}

* Not significant at the 95 percent confidence level; the regression equation should not be used tc predict the concentration of the dependent variable.

Results of regression analyses relating specific conductance to water discharge (Q)

\begin{tabular}{ccccccc}
\hline $\begin{array}{c}\text { No. of } \\
\text { measure- } \\
\text { ments }\end{array}$ & $\begin{array}{c}\text { Water discharge } \\
\left(\mathrm{ft}^{3} / \mathrm{s}\right)\end{array}$ & Maximum Minimum & Regression equation & $\begin{array}{c}\text { Correlation } \\
\text { coefficient }\end{array}$ & $\begin{array}{c}\text { Percent } \\
\text { explained } \\
\text { variance }\end{array}$ & $\begin{array}{c}\text { Standard error } \\
\text { of estimate }\end{array}$ \\
\hline 59 & $3,700 \quad 69$ & $\mathrm{SpC}=340 \mathrm{Q}^{-0.02830}$ & -0.27 & 7.1 & 0.033 & 7.6 \\
\hline
\end{tabular}




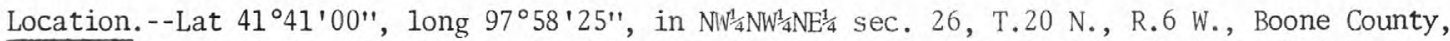
Hydrologic Unit 10210009, at bridge on county road $0.8 \mathrm{mi}$ east and $0.6 \mathrm{mi}$ southeast of junction of highways 14, 39, and 91 at east edge of Albion.

Period of record. --Water year 1973 to current year.

Statistical data for selected chemical constituents

\begin{tabular}{|c|c|c|c|c|c|c|c|c|c|}
\hline Constituent & Units & $\begin{array}{l}\text { No. of } \\
\text { measure- } \\
\text { ments }\end{array}$ & $\begin{array}{l}\text { Max- } \\
\text { imum }\end{array}$ & $\begin{array}{l}\text { Min- } \\
\text { imum }\end{array}$ & Mean & Median & $\begin{array}{c}\text { Standard } \\
\text { devi- } \\
\text { ation }\end{array}$ & $\begin{array}{l}\text { Ninetieth } \\
\text { percent- } \\
\text { ile }\end{array}$ & $\begin{array}{l}\text { Tenth } \\
\text { percent- } \\
\text { ile }\end{array}$ \\
\hline Specific conductance & umho/cm & 54 & 388 & 215 & 252 & 249 & 27 & 280 & 225 \\
\hline Dissolved solids, residue & $\mathrm{mg} / \mathrm{L}$ & 33 & 275 & 115 & 182 & 179 & 25 & 210 & 160 \\
\hline Dissolved solids, sum & $\mathrm{mg} / \mathrm{L}$ & 19 & 196 & 155 & 179 & 180 & 12 & 195 & 156 \\
\hline Hardness as $\mathrm{CaCO}_{3}$ & $\mathrm{mg} / \mathrm{L}$ & 18 & 130 & 94 & 113 & 118 & 8.6 & 127 & 98 \\
\hline Calcium, dissolved & $\mathrm{mg} / \mathrm{L}$ & 18 & 40 & 30 & 36 & 36 & 3.2 & 40 & 32 \\
\hline Magnesium, dissolved & $\mathrm{mg} / \mathrm{L}$ & 18 & 6.5 & 4.3 & 5.6 & 5.8 & 0.6 & 6.4 & 4.5 \\
\hline Sodium, dissolved & $\mathrm{mg} / \mathrm{L}$ & 18 & 10 & 6.9 & 8.3 & 8.6 & 1.0 & 9.3 & 7.1 \\
\hline Potassium, dissolved & $\mathrm{mg} / \mathrm{L}$ & 17 & 11 & 6.3 & 7.5 & 7.1 & 1.2 & 9.7 & 6.4 \\
\hline Bicarbonate ion & $\mathrm{mg} / \mathrm{L}$ & 18 & 174 & 123 & 149 & 150 & 14 & 170 & 129 \\
\hline Sulfate, dissolved & $\mathrm{mg} / \mathrm{L}$ & 18 & 29 & 4.3 & 8.6 & 6.9 & 5.7 & 14 & 4.7 \\
\hline Chloride, dissolved & $\mathrm{mg} / \mathrm{L}$ & 51 & 20 & 1.5 & 2.9 & 2.6 & 2.6 & 3.6 & 1.8 \\
\hline Fluoride, dissolved & $\mathrm{mg} / \mathrm{L}$ & 19 & 0.4 & 0.1 & 0.3 & 0.3 & 0.06 & 0.3 & 0.2 \\
\hline Silica, dissolved & $\mathrm{mg} / \mathrm{L}$ & 18 & 49 & 30 & 37 & 36 & 5.4 & 47 & 32 \\
\hline Boron, dissolved & $\mathrm{mg} / \mathrm{L}$ & 18 & 80 & 6 & 34 & 30 & 18 & 52 & 10 \\
\hline Iron, dissolved & $\mathrm{ug} / \mathrm{L}$ & 18 & 1,000 & 20 & 129 & 67 & 225 & 230 & 20 \\
\hline Manganese, dissolved & $\mathrm{ug} / \mathrm{L}$ & 18 & 120 & 0 & 28 & 19 & 33 & 100 & 10 \\
\hline $\begin{array}{l}\text { Nitrite + nitrate as } \mathrm{N}, \\
\text { dissolved } \\
\text { Nitrate as } \mathrm{N} \text {, dissolved }\end{array}$ & $\begin{array}{l}\mathrm{mg} / \mathrm{L} \\
\mathrm{mg} / \mathrm{L}\end{array}$ & 27 & 1.2 & 0.07 & 0.37 & 0.36 & 0.23 & 0.58 & 0.10 \\
\hline Nitrate as $\mathrm{NO}_{3}$, dissolved & $\mathrm{mg} / \mathrm{L}$ & & 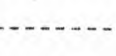 & & 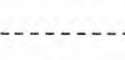 & 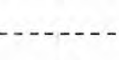 & & & 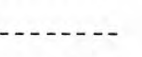 \\
\hline Anmonia $\mathrm{N}$, total & $\mathrm{mg} / \mathrm{L}$ & 54 & 1.1 & 0.00 & 0.22 & 0.19 & 0.19 & 0.40 & 0.00 \\
\hline Organic $N$, total & $\mathrm{mg} / \mathrm{L}$ & 54 & 3.8 & 0.15 & 0.86 & 0.77 & 0.56 & 1.3 & 0.31 \\
\hline Phosphorus, total & $\mathrm{mg} / \mathrm{L}$ & 54 & 1.6 & 0.28 & 0.55 & 0.52 & 0.22 & 0.80 & 0.36 \\
\hline Phosphorus, dissolved & $\mathrm{mg} / \mathrm{L}$ & 46 & 1.4 & 0.03 & 0.41 & 0.35 & 0.20 & 0.57 & 0.28 \\
\hline Fecal coliform & $\mathrm{col} / 100 \mathrm{ml}$ & 42 & 71,300 & 3 & (n...... & 530 & . & 4,800 & 11 \\
\hline Fecal streptococci & $\mathrm{col} / 100 \mathrm{ml}$ & 52 & 42,000 & 23 & (n...... & 430 & $\cdots$ & 4,000 & 72 \\
\hline Oxygen, dissolved & $\mathrm{mg} / \mathrm{L}$ & 55 & 14.0 & 7.1 & 9.8 & 9.6 & 1.6 & 12.0 & 7.7 \\
\hline \multirow{2}{*}{$\begin{array}{l}\text { Biochemical oxygen demand } \\
(5-\text { day })\end{array}$} & $\mathrm{mg} / \mathrm{L}$ & 52 & 9.4 & 1.1 & 3.7 & 2.8 & 2.1 & 7.0 & 1.7 \\
\hline & $H$ units & 59 & 8.5 & 6.8 & 7.6 & 7.7 & 0.3 & 8.0 & 7.2 \\
\hline $\mathrm{Co}-\mathrm{Pt}$ & Co - Pt units & 19 & 70 & 8 & 24 & 20 & 16 & 50 & 10 \\
\hline
\end{tabular}


Results of regression analyses relating concentrations of selected chemical constituents to specific conductance $(\mathrm{SpC})$

\begin{tabular}{|c|c|c|c|c|c|}
\hline $\begin{array}{l}\text { Dissolved } \\
\text { constituents } \\
(\mathrm{mg} / \mathrm{L})\end{array}$ & $\begin{array}{l}\text { No. of } \\
\text { measure- } \\
\text { ments }\end{array}$ & Regression equation & $\begin{array}{l}\text { Corre- } \\
\text { lation } \\
\text { coeffi- } \\
\text { cient }\end{array}$ & $\begin{array}{l}\text { Percent } \\
\text { explained } \\
\text { variance }\end{array}$ & $\begin{array}{c}\text { Standard } \\
\text { error of } \\
\text { estimate } \\
(\mathrm{mg} / \mathrm{L})\end{array}$ \\
\hline Dissolved solids, residue (RDS) & 33 & $\mathrm{RDS}=0.696(\mathrm{SpC})+7.473$ & 0.83 & 70 & 14 \\
\hline Dissolved solids, sum (SDS) & 18 & $\mathrm{SDS}=0.486(\mathrm{SpC})+55.201$ & .77 & 59 & 8.0 \\
\hline Hardness as $\mathrm{CaCO}_{3}(\mathrm{TH})$ & 18 & $T H=0.333(S p C)+27.709$ & .74 & 55 & 5.9 \\
\hline Calcium (Ca) & 18 & $\mathrm{Ca}=0.102(\mathrm{SpC})+9.480$ & .62 & 38 & 2.6 \\
\hline Magnesium (Mg) & 18 & $M g=0.028(S p C)-1.641$ & .83 & 69 & .4 \\
\hline Sodium $(\mathrm{Na})$ & 18 & $\mathrm{Na}=0.026(\mathrm{SpC})+1.672$ & .48 & 23 & .9 \\
\hline Potassium (K) & 17 & $\mathrm{~K}=0.014(\mathrm{SpC})+3.813$ & $* .23$ & 5.4 & $\begin{array}{r}1.2 \\
\text { if }\end{array}$ \\
\hline Bicarbonate $\left(\mathrm{HCO}_{3}\right)$ & 18 & $\mathrm{HCO}_{3}=0.640(\mathrm{SpC})-14.684$ & .86 & 74 & 7.5 \\
\hline Sulfate $\left(\mathrm{SO}_{4}\right)$ & 18 & $\mathrm{SO}_{4}=0.001(\mathrm{SpC})+8.298$ & $\star .003$ & 0.00 & 5.9 \\
\hline Chloride (C1) & 51 & $\mathrm{C} 1=0.073(\mathrm{SpC})-15.448$ & .76 & 58 & 1.7 \\
\hline Silica (Si) & 18 & $\mathrm{Si}=-0.011(\mathrm{SpC})+39.628$ & $*-.04$ & 0.14 & 5.6 \\
\hline Nitrite + Nitrate as $\mathrm{N}\left(\mathrm{NO}_{2}-\mathrm{NO}_{3}\right)$ & 27 & $\mathrm{NO}_{2}-\mathrm{NO}_{3}=0.005(\mathrm{SpC})-0.889$ & .64 & 42 & 0.18 \\
\hline
\end{tabular}

* Not significant at the 95 percent confidence level; the regression equation should not be used to predict the concentration of the dependent variable.

Results of regression analyses relating specific conductance to water discharge (Q)

\begin{tabular}{ccccccc}
\hline $\begin{array}{c}\text { No. of } \\
\text { measure- } \\
\text { ments }\end{array}$ & $\begin{array}{c}\text { Water discharge } \\
\left(\mathrm{ft}^{3} / \mathrm{s}\right)\end{array}$ & Maximum Minimum & Regression equation & $\begin{array}{c}\text { Correlation } \\
\text { coefficient }\end{array}$ & $\begin{array}{c}\text { Percent } \\
\text { explained } \\
\text { variance }\end{array}$ & $\begin{array}{c}\text { Standard error } \\
\text { of estimate }\end{array}$ \\
\hline 52 & $155 \quad 7.50$ & $\mathrm{SpC}=338 \mathrm{Q}^{-0.07601}$ & -0.40 & 16 & 0.040 \\
\hline
\end{tabular}

* Not significant at the 95 percent confidence level; the regression equation should not be used to predict the concentration of the dependent variable. 
The Flkhorn River basin is located in northeastern Nebraska. All streamflow in the basin originates within Nebraska. The basin ends at the confluence of the Elkhorn River and the Platte River west of Omaha. The western part of the basin, comprising about one-third of the total area, is sandhills. The rest of the basin generally lies within the glaciated part of Nebraska, which is characterized by deposits of glacial drift overlain by loess. In places, the drift is more than 200 $\mathrm{ft}$ thick. Topography of the area is rolling.

Irrigation is not extensive in the basin although development of pump irrigation is accelerating in the sandhills. By 1971, about 150,000 acres out of a total of about 4.5 million acres in the basin were under irrigation (Nebraska Soil and Water Conservation Commission, 1971). Only small amounts of land are irrigated by surface water and this by direct pumpage from the streams rather than by diversion.

Descriptive statistics were determined on data from seven sites in the Elkhorn River basin (fig. 9). Of these, two are on Logan Creek, the principal tributary of the Elkhorn River in the eastern part of the basin, and five are on the Elkhorn River and its branches. Mean specific conductance for the E1khorn River at Ewing is 220 umho/cm and for the South Fork E1khorn River at Ewing is 194 umho/cm. Mlost of the flow at Ewing is derived from ground water. From Fwing to Norfolk, mean specific conductance increases by 46 percent to $322 \mathrm{umho} / \mathrm{cm}$. The increase is due to higher concentrations of calcium and bicarbonate carried by streams draining the area to the south.

Mean specific conductance for 154 measurements on the Elkhorn River at Water 100 , the downstream most station in the basin, is $496 \mathrm{umho} / \mathrm{cm}$. This is an increase of 54 percent from Norfolk and is caused by the addition of more highly mineralized water from Logan Creek and other tributaries within the reach between Norfolk and Waterloo.

Mean specific conductance for 127 measurements of Logan Creek at Pender is 716 umho/cm. Logan Creek drains an area of loess and glacial drift. Ground water derived from this area is considerably more highly mineralized than that from the sandhills area. Glacial drift deposits and the loess and soil overlying them are rich in soluble chemical constituents including calcium and carbonate. This material is dissolved by percolating water which eventually discharges into the streams and causes the streams to be more highly mineralized. 


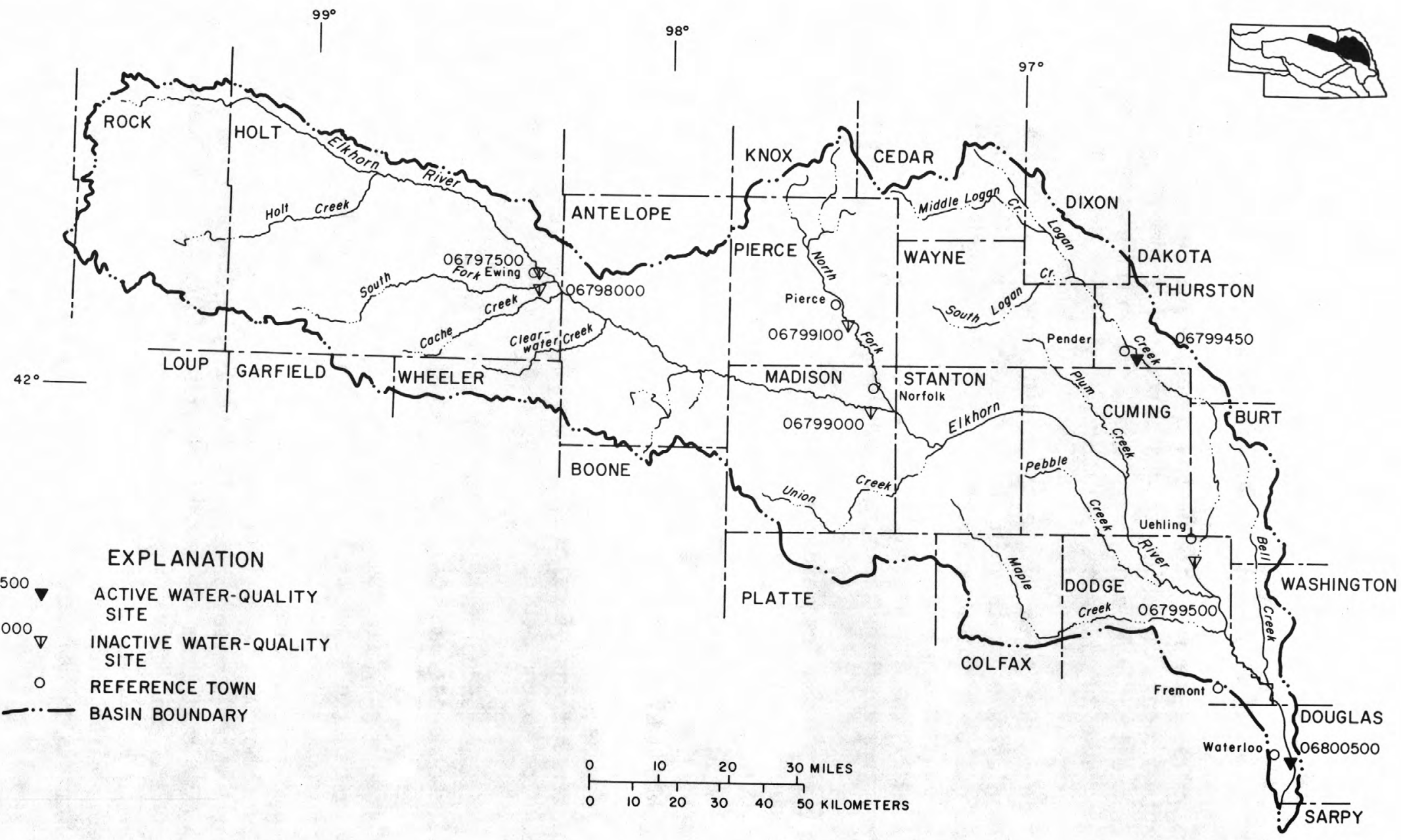

FIGURE 9.-Location of water-quality sampling sites, Elkhorn River basin 
Calcium is the principal cation and bicarbonate is the principal anion at all stations.

Fecal coliform and fecal streptococcal bacteria concentrations were measured only on Logan Creek at Pender and on Elkhorn River at Waterloo. Medians for the Pender station were 280 colonies/100 ml for fecal coliform bacteria and 332 colonies/100 $\mathrm{m} 1$ for fecal streptococci. Medians for the Water10o station were 9,600 and 3,130 colonies $/ 100 \mathrm{ml}$ for fecal coliform and fecal streptococcal bacteria, respectively. This station is only a few miles downstream from the outfall of the Fremont sewage-treatment plant. Mean BOD was $8.8 \mathrm{mg} / \mathrm{L}$ at the Waterloo station and the mean chloride was $13 \mathrm{mg} / \mathrm{L}$, the highest for any of the sites in the basin.

Regression equations were developed between specific conductance and each of the constituents having significant numbers of measurements. Correlation coefficients exceed 0.8 for nearly every regression between conductance and dissolved solids, hardness, calcium, magnesium, sodium, and bicarbonate for all stations. 
Location (revised).--Lat $42^{\circ} 16^{\prime} 03^{\prime \prime}$, long $98^{\circ} 20^{\prime} 11^{\prime \prime}$, at gaging station at bridge on State Highway $420,0.8 \mathrm{mi}$ north of Ewing, Holt County, and $1.5 \mathrm{mi}$ upstream from South Fork Elkhorn River.

Drainage area.--1,400 $\mathrm{mi}^{2}$, approximately, of which about $740 \mathrm{mi}^{2}$ contributes directly to surface runoff.

Period of record.--September 1960 to September 1966.

Statistical data for selected chemical constituents

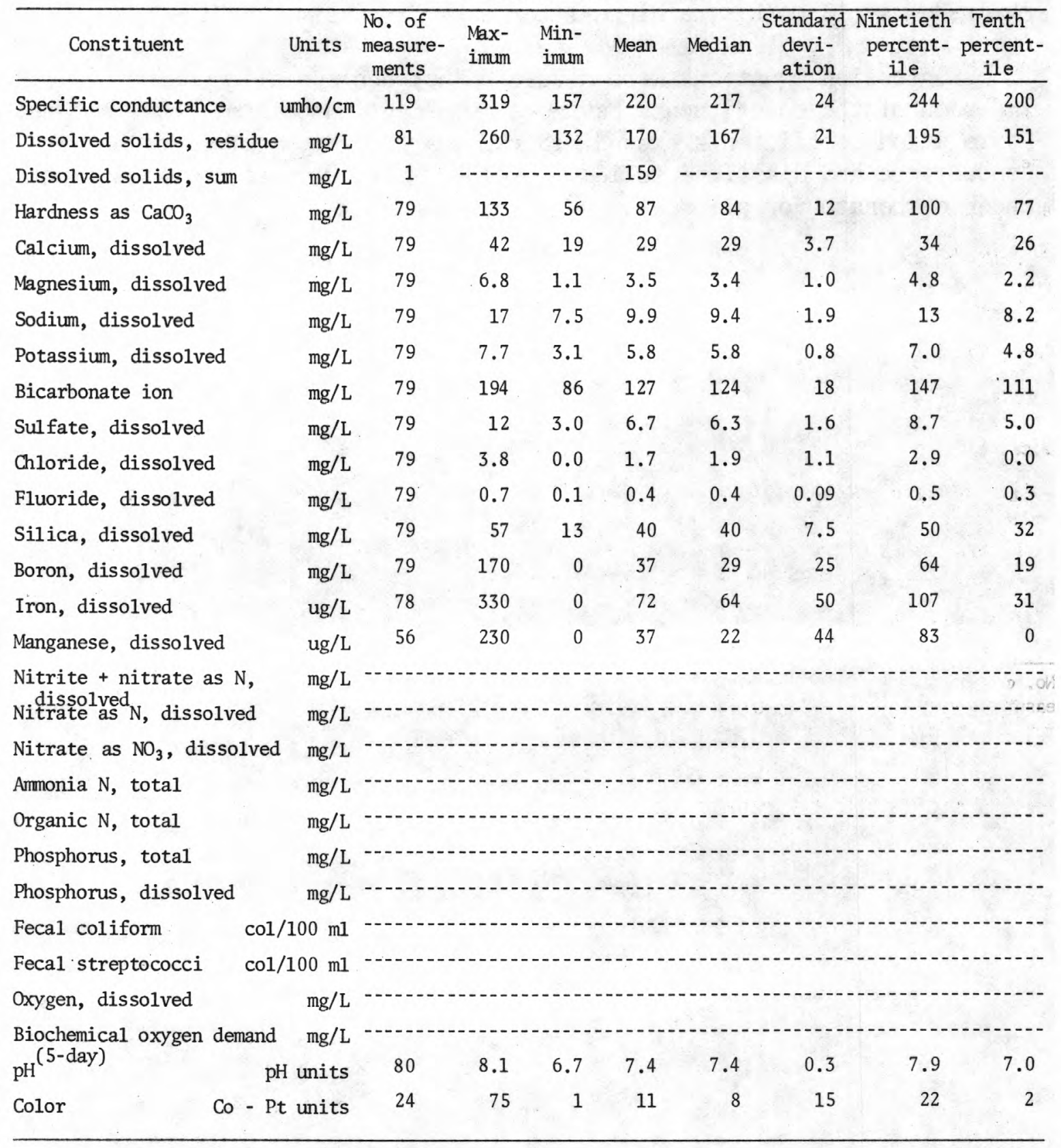


06797500 Elkhorn River at Ewing, Nebr.--Continued

Results of regression analyses relating concentrations of selected chemical constituents to specific conductance $(\mathrm{SpC})$

\begin{tabular}{|c|c|c|c|c|c|}
\hline $\begin{array}{c}\text { Dissolved } \\
\text { constituents } \\
(\mathrm{mg} / \mathrm{L})\end{array}$ & $\begin{array}{l}\text { No. of } \\
\text { measure- } \\
\text { ments }\end{array}$ & Regression equation & $\begin{array}{l}\text { Corre- } \\
\text { lation } \\
\text { coeffi- } \\
\text { cient }\end{array}$ & $\begin{array}{l}\text { Percent } \\
\text { explained } \\
\text { variance }\end{array}$ & $\begin{array}{c}\text { Standard } \\
\text { error of } \\
\text { estimate } \\
(\mathrm{mg} / \mathrm{L})\end{array}$ \\
\hline Dissolved solids, residue (RDS) & 81 & $\mathrm{RDS}=0.769(\mathrm{SpC})+0.156$ & 0.95 & 90 & 7.0 \\
\hline Dissolved solids, sum (SDS) & $-\ldots$ & $-1-2-1-1$ & & & 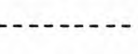 \\
\hline Hardness as $\mathrm{CaCO}_{3}(\mathrm{TH})$ & 79 & $\mathrm{TH}=0.430(\mathrm{SpC})-8.236$ & .96 & 93 & 3.2 \\
\hline Calcium (Ca) & 79 & $\mathrm{Ca}=0.129(\mathrm{SpC})+$ & .93 & 87 & 1.3 \\
\hline Magnesium (Mg) & 79 & $\mathrm{Mg}=0.026(\mathrm{SpC})-2.239$ & .66 & 43 & 0.8 \\
\hline Sodium (Na) & 79 & $\mathrm{Na}=0.061(\mathrm{SpC})-3.573$ & .86 & 74 & 1.0 \\
\hline Potassium (K) & 79 & $\mathrm{~K}=0.014(\mathrm{SpC})+2.774$ & .45 & 21 & 0.7 \\
\hline Bicarbonate $\left(\mathrm{HCO}_{3}\right)$ & 79 & $\mathrm{HCO}_{3}=0.643(\mathrm{SpC})-15.630$ & .97 & 95 & 4.1 \\
\hline Sulfate $\left(\mathrm{SO}_{4}\right)$ & 79 & $\mathrm{SO}_{4}=0.024(\mathrm{SpC})+1.428$ & .40 & 16 & 1.4 \\
\hline Chloride (C1) & 79 & $\mathrm{Cl}=-0.006(\mathrm{SpC})+2.987$ & $*-.14$ & 1.9 & 1.1 \\
\hline Silica (Si) & 79 & $\mathrm{Si}=0.013(\mathrm{SpC})+36.957$ & *.05 & 0.22 & 7.5 \\
\hline
\end{tabular}

* Not significant at the 95 percent confidence level; the regression equation should not be used to predict the concentration of the dependent variable.

Results of regression analyses relating specific conductance to water discharge $(Q)$

\begin{tabular}{ccccccc}
\hline $\begin{array}{c}\text { No. of } \\
\text { measure- } \begin{array}{c}\text { Water discharge } \\
\text { ments }\end{array}\end{array}$ & Maximum Minimum & Regression equation & $\begin{array}{c}\text { Correlation } \\
\text { coefficient }\end{array}$ & $\begin{array}{c}\text { Percent } \\
\text { explained } \\
\text { variance }\end{array}$ & $\begin{array}{c}\text { Standard error } \\
\text { of estimate }\end{array}$ \\
\hline 105 & 3,130 & 4.8 & $\mathrm{SpC}=191 \mathrm{Q}^{0.02983}$ & 0.27 & 7.5 & 0.044 \\
\hline
\end{tabular}


06798000 South Fork Elkhorn River at Ewing, Nebr.

Location.--Lat $42^{\circ} 15^{\prime} 04^{\prime \prime}$, 1ong $98^{\circ} 20^{\prime} 18^{\prime \prime}$, at gaging station at bridge on U.S. Highway 275 at southeast limits of Ewing, Holt County, and $0.8 \mathrm{mi}$ above mouth.

Drainage area.--320 $\mathrm{mi}^{2}$, approximately, of which about $190 \mathrm{mi}^{2}$ contributes directly to surface runoff.

Period of record.--September 1960 to September 1966.

Statistical data for selected chemical constituents

\begin{tabular}{|c|c|c|c|c|c|c|c|c|c|}
\hline Constituent & Units & $\begin{array}{l}\text { No. of } \\
\text { measure- } \\
\text { ments }\end{array}$ & $\begin{array}{l}\text { Max- } \\
\text { imum }\end{array}$ & $\begin{array}{l}\text { Min- } \\
\text { imum }\end{array}$ & Mean & Median & $\begin{array}{c}\text { Standard } \\
\text { devi- } \\
\text { ation }\end{array}$ & $\begin{array}{c}\text { Ninetieth } \\
\text { percent- } \\
\text { ile }\end{array}$ & $\begin{array}{c}\text { Tenth } \\
\text { percent- } \\
\text { ile }\end{array}$ \\
\hline Specific conductance & umho/cm & 84 & 285 & 166 & 194 & 188 & 22 & 218 & 171 \\
\hline Dissolved solids, residue & $\mathrm{mg} / \mathrm{L}$ & 81 & 243 & 127 & 156 & 153 & 19 & 176 & 140 \\
\hline Dissolved solids, sum & $\mathrm{mg} / \mathrm{L}$ & 1 & 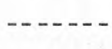 & $-\cdots$ & 134 & & & & --- \\
\hline Hardness as $\mathrm{CaCO}_{3}$ & $\mathrm{mg} / \mathrm{L}$ & 79 & 116 & 66 & 79 & 76 & 9.0 & 89 & 70 \\
\hline Calcium, dissolved & $\mathrm{mg} / \mathrm{L}$ & 79 & 39 & 22 & 26 & 26 & 3.1 & 30 & 24 \\
\hline Magnesium, dissolved & $\mathrm{mg} / \mathrm{L}$ & 79 & 5.9 & 0.4 & 3.0 & 3.0 & 0.8 & 4.2 & 2.1 \\
\hline Sodium, dissolved & $\mathrm{mg} / \mathrm{L}$ & 79 & 15 & 6.1 & 7.6 & 7.0 & 1.5 & 9.6 & 6.5 \\
\hline Potassium, dissolved & $\mathrm{mg} / \mathrm{L}$ & 79 & 7.6 & 3.7 & 4.6 & 4.5 & 0.8 & 6.0 & 3.9 \\
\hline Bicarbonate ion & $\mathrm{mg} / \mathrm{L}$ & 79 & 167 & 96 & 113 & 110 & 13 & 127 & 100 \\
\hline Sulfate, dissolved & $\mathrm{mg} / \mathrm{L}$ & 79 & 11 & 1.3 & 5.1 & 4.9 & 1.7 & 7.4 & 3.2 \\
\hline Chloride, dissolved & $\mathrm{mg} / \mathrm{L}$ & 79 & 3.4 & 0.0 & 0.8 & 0.8 & 0.8 & 2.0 & 0.0 \\
\hline Fluoride, dissolved & $\mathrm{mg} / \mathrm{L}$ & 79 & 0.5 & 0.1 & 0.3 & 0.3 & 0.08 & 0.4 & 0.1 \\
\hline Silica, dissolved & $\mathrm{mg} / \mathrm{L}$ & 79 & 62 & 21 & 43 & 44 & 5.2 & 48 & 37 \\
\hline Boron, dissolved & $\mathrm{mg} / \mathrm{L}$ & 79 & 100 & 0 & 25 & 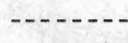 & 19 & $-\cdots$ & $-\cdots$ \\
\hline Iron, dissolved & $\mathrm{ug} / \mathrm{L}$ & 78 & 280 & 0 & 64 & 54 & 48 & 90 & 28 \\
\hline Manganese, dissolved & $\mathrm{ug} / \mathrm{L}$ & 55 & 110 & 0 & 22 & 10 & 29 & 67 & 0 \\
\hline $\begin{array}{l}\text { Nitrite + nitrate as } N \text {, } \\
\text { dissolved } \\
\text { Nitrate as } N \text {, dissolved }\end{array}$ & $\begin{array}{l}\mathrm{mg} / \mathrm{L} \\
\mathrm{mg} / \mathrm{L}\end{array}$ & & & & & & & - & \\
\hline Nitrate as $\mathrm{NO}_{3}$, dissolved & $\mathrm{mg} / \mathrm{L}$ & & & & & & & & \\
\hline Ammonia $\mathrm{N}$, total & $\mathrm{mg} / \mathrm{L}$ & & & & & & & & \\
\hline Organic $N$, total & $\mathrm{mg} / \mathrm{L}$ & & & & & & & & \\
\hline Phosphorus, total & $\mathrm{mg} / \mathrm{L}$ & & & & & & & & \\
\hline Phosphorus, dissolved & $\mathrm{mg} / \mathrm{L}$ & - & & & & & & & \\
\hline Fecal coliform & $/ 100 \mathrm{ml}$ & - & & & & & & & \\
\hline Fecal streptococci & $/ 100 \mathrm{ml}$ & & & & & & & & \\
\hline Oxygen, dissolved & $\mathrm{mg} / \mathrm{L}$ & & & & & & & & \\
\hline $\begin{array}{l}\text { Biochemical oxygen demand } \\
\mathrm{pH}^{(5-\text { day })}\end{array}$ & mg $/ \mathrm{L}$ & 80 & 8.4 & 6.7 & 7.4 & 7.4 & 0.3 & 8.0 & 7.1 \\
\hline Co - P & t units & 24 & 35 & 1 & 8 & 8 & 7.2 & 13 & 2 \\
\hline
\end{tabular}


06798000 South Fork E1khorn River at Ewing, Nebr.--Continued

Results of regression analyses relating concentrations of selected chemical constituents to specific conductance $(\mathrm{SpC})$

\begin{tabular}{|c|c|c|c|c|c|}
\hline $\begin{array}{c}\text { Dissolved } \\
\text { constituents } \\
\text { (mg/L) }\end{array}$ & $\begin{array}{l}\text { No. of } \\
\text { measure- } \\
\text { ments }\end{array}$ & Regression equation & $\begin{array}{l}\text { Corre- } \\
\text { lation } \\
\text { coeffi- } \\
\text { cient }\end{array}$ & $\begin{array}{l}\text { Percent } \\
\text { explained } \\
\text { variance }\end{array}$ & $\begin{array}{c}\text { Standard } \\
\text { error of } \\
\text { estimate } \\
(\mathrm{mg} / \mathrm{L})\end{array}$ \\
\hline Dissolved solids, residue (RDS) & 81 & $\mathrm{RDS}=0.816(\mathrm{SpC})-2.531$ & 0.94 & 89 & 6.3 \\
\hline Dissolved solids, sum (SDS) & $-\ldots$ & -- & - & & \\
\hline Hardness as $\mathrm{CaCO}_{3}(\mathrm{TH})$ & 79 & $\mathrm{TH}=0.395(\mathrm{SpC})+1.956$ & .97 & 95 & 2.0 \\
\hline Calcium (Ca) & 79 & $\mathrm{Ca}=0.128(\mathrm{SpC})+1.667$ & .93 & 87 & 1.1 \\
\hline Magnesium (Mg) & 79 & $\mathrm{Mg}=0.018(\mathrm{SpC})-0.483$ & .47 & 22 & 0.8 \\
\hline Sodium $(\mathrm{Na})$ & 79 & $\mathrm{Na}=0.064(\mathrm{SpC})-4.978$ & .93 & 86 & 0.6 \\
\hline Potassium (K) & 79 & $\mathrm{~K}=0.022(\mathrm{SpC})+0.351$ & .60 & 36 & 0.7 \\
\hline Bicarbonate $\left(\mathrm{HCO}_{3}\right)$ & 79 & $\mathrm{HCO}_{3}=0.572(\mathrm{SpC})+1.538$ & .96 & 93 & 3.5 \\
\hline Sulfate $\left(\mathrm{SO}_{4}\right)$ & 79 & $\mathrm{SO}_{4}=0.040(\mathrm{SpC})-2.654$ & .53 & 28 & 1.4 \\
\hline Chloride (C1) & 79 & $\mathrm{Cl}=0.006(\mathrm{SpC})-0.405$ & $* .17$ & 3.0 & 0.8 \\
\hline Silica (Si) & 79 & $\mathrm{Si}=-0.012(\mathrm{SpC})+44.952$ & $*-.05$ & 0.25 & 5.2 \\
\hline
\end{tabular}

*Not significant at the 95 percent confidence level; the regression equation should not be used to predict the concentration of the dependent variable.

Results of regression analyses relating specific conductance to water discharge (Q)

\begin{tabular}{|c|c|c|c|c|c|c|}
\hline \multirow{2}{*}{$\begin{array}{l}\text { No. of } \\
\text { measure- } \\
\text { ments }\end{array}$} & \multirow{2}{*}{$\begin{array}{l}\text { Water discharge } \\
\frac{\left(\mathrm{ft}^{3} / \mathrm{s}\right)}{\text { Maximum Minimum }}\end{array}$} & \multirow{2}{*}{ Regression equation } & \multirow{2}{*}{$\begin{array}{l}\text { Correlation } \\
\text { coefficient }\end{array}$} & \multirow{2}{*}{$\begin{array}{l}\text { Percent } \\
\text { explained } \\
\text { variance }\end{array}$} & \multicolumn{2}{|c|}{$\begin{array}{c}\text { Standard error } \\
\text { of estimate }\end{array}$} \\
\hline & & & & & Log units & Percent \\
\hline 84 & 490 & $\mathrm{SpC}=148 \mathrm{Q}^{0.06984}$ & 0.36 & 13 & 0.041 & 9.4 \\
\hline
\end{tabular}


Location.--Lat $42^{\circ} 00^{\prime} 20^{\prime \prime}$, long $97^{\circ} 28^{\prime} 40^{\prime \prime}$, in SW/4 sec. 31, T.24 N., R.1 W., Madison County, at gaging station at county road bridge, $3.5 \mathrm{mi}$ west-southwest of Norfolk, and $7 \mathrm{mi}$ upstream from North Fork Elkhom River.

Drainage area. $--2,790 \mathrm{mi}^{2}$, approximately, of which about $1,790 \mathrm{mi}^{2}$ contributes directly to surface runoff.

Period of record. -- September 1960 to September 1970.

Statistical data for selected chemical constituents

\begin{tabular}{|c|c|c|c|c|c|c|c|c|c|}
\hline Constituent & Units & $\begin{array}{l}\text { No. of } \\
\text { measure- } \\
\text { ments }\end{array}$ & $\begin{array}{l}\text { Max- } \\
\text { imum }\end{array}$ & $\begin{array}{l}\text { Min- } \\
\text { imum }\end{array}$ & Mean & Median & $\begin{array}{l}\text { Standard } \\
\text { devi- } \\
\text { ation } \\
\end{array}$ & $\begin{array}{l}\text { Ninetieth } \\
\text { percent- } \\
\text { ile }\end{array}$ & $\begin{array}{c}\text { Tenth } \\
\text { percent- } \\
\text { ile } \\
\end{array}$ \\
\hline Specific conductance & umho/cm & 157 & 682 & 182 & 322 & 322 & 47 & 347 & 278 \\
\hline Dissolved solids, residue & $\mathrm{mg} / \mathrm{L}$ & 129 & 343 & 141 & 220 & 221 & 28 & 247 & 187 \\
\hline Dissolved solids, sum & $\mathrm{mg} / \mathrm{L}$ & 38 & 287 & 140 & 214 & 218 & 28 & 243 & 170 \\
\hline Hardness as $\mathrm{CaCO}_{3}$ & $\mathrm{mg} / \mathrm{L}$ & 128 & 201 & 92 & 140 & 142 & 18 & 159 & 117 \\
\hline Calcium, dissolved & $\mathrm{mg} / \mathrm{L}$ & 128 & 66 & 29 & 45 & 46 & 5.6 & 51 & 37 \\
\hline Magnesium, dissolved & $\mathrm{mg} / \mathrm{L}$ & 128 & 9.8 & 3.0 & 6.8 & 7.0 & 1.2 & 8.1 & 5.2 \\
\hline Sodium, dissolved & $\mathrm{mg} / \mathrm{L}$ & 128 & 16 & $2.2^{\circ}$ & 9.3 & 9.3 & 2.0 & 12 & 7.3 \\
\hline Potassium, dissolved & $\mathrm{mg} / \mathrm{L}$ & 127 & 11 & 5.5 & 7.2 & 7.0 & 1.0 & 8.5 & 6.2 \\
\hline Bicarbonate ion & $\mathrm{mg} / \mathrm{L}$ & 128 & 266 & 114 & 190 & 193 & 24 & 212 & 158 \\
\hline Sulfate, dissolved & $\mathrm{mg} / \mathrm{L}$ & 128 & 17 & 3.8 & 8.6 & 8.4 & 1.8 & 11 & 6.9 \\
\hline Chloride, dissolved & $\mathrm{mg} / \mathrm{L}$ & 128 & 3.8 & 0.0 & 1.8 & $-\cdots-\cdots$ & 0.9 & 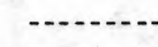 & $\cdots$ \\
\hline Fluoride, dissolved & $\mathrm{mg} / \mathrm{L}$ & 128 & 0.6 & 0.1 & 0.4 & 0.3 & 0.8 & 0.4 & 0.1 \\
\hline Silica, dissolved & $\mathrm{mg} / \mathrm{L}$ & 128 & 56 & 1.1 & 38 & 39 & 8.2 & 45 & 27 \\
\hline Boron, dissolved & $\mathrm{mg} / \mathrm{L}$ & 128 & 130 & 0 & 38 & 32 & 21 & 58 & 20 \\
\hline Iron, dissolved & $\mathrm{ug} / \mathrm{L}$ & 114 & 870 & 0 & 65 & 46 & 93 & 125 & 19 \\
\hline Manganese, dissolved & $\mathrm{ug} / \mathrm{L}$ & 74 & 160 & 0 & 39 & 28 & 40 & 104 & 0 \\
\hline $\begin{array}{l}\text { Nitrite + nitrate as } \mathrm{N}, \\
\text { dissolved } \\
\text { Nitrate as } \mathrm{N} \text {, dissolved }\end{array}$ & $\begin{array}{l}\mathrm{mg} / \mathrm{L} \\
\mathrm{mg} / \mathrm{L}\end{array}$ & 24 & 2.0 & 0.00 & 0.34 & 0.30 & 0.46 & 0.99 & 0.00 \\
\hline $\begin{array}{l}\text { Nitrate as } \mathrm{NO}_{3} \text {, dissolved } \\
\text { Ammonia } \mathrm{N} \text {, total }\end{array}$ & $\begin{array}{l}\mathrm{mg} / \mathrm{L} \\
\mathrm{mg} / \mathrm{L}\end{array}$ & 129 & 8.9 & 0.13 & 1.4 & 1.5 & 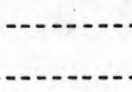 & & $\cdots$ \\
\hline $\begin{array}{l}\text { Organic N, total } \\
\text { Phosphorus, total }\end{array}$ & $\begin{array}{l}\mathrm{mg} / \mathrm{L} \\
\mathrm{mg} / \mathrm{L}\end{array}$ & $\cdots$ & & & & & & & \\
\hline Phosphorus, dissolved & $\mathrm{mg} / \mathrm{L}$ & 13 & 0.48 & 0.11 & 0.21 & 0.21 & 0.09 & 0.23 & 0.13 \\
\hline $\begin{array}{l}\text { Fecal coliform } \\
\text { Fecal streptococci }\end{array}$ & $\mathrm{col} / 100 \mathrm{ml}$ & $\cdots$ & & & & & & & $\cdots$ \\
\hline Oxygen, dissolved & $\mathrm{mg} / \mathrm{L}$ & $\cdots$ & & & & & & & - \\
\hline Biochemical oxygen demand & $\mathrm{mg} / \mathrm{L}$ & & & & & & -..... & & ....... \\
\hline $\mathrm{pH}^{(3-\text { day })}$ & H units & 129 & 8.6 & 6.7 & 7.7 & 7.7 & 0.3 & 8.1 & 7.3 \\
\hline Co - Pt & t units & 47 & 45 & 1 & 9 & 5 & 8.9 & 24 & 2 \\
\hline
\end{tabular}


Results of regression analyses relating concentrations of selected chemical constituents to specific conductance $(\mathrm{SpC})$

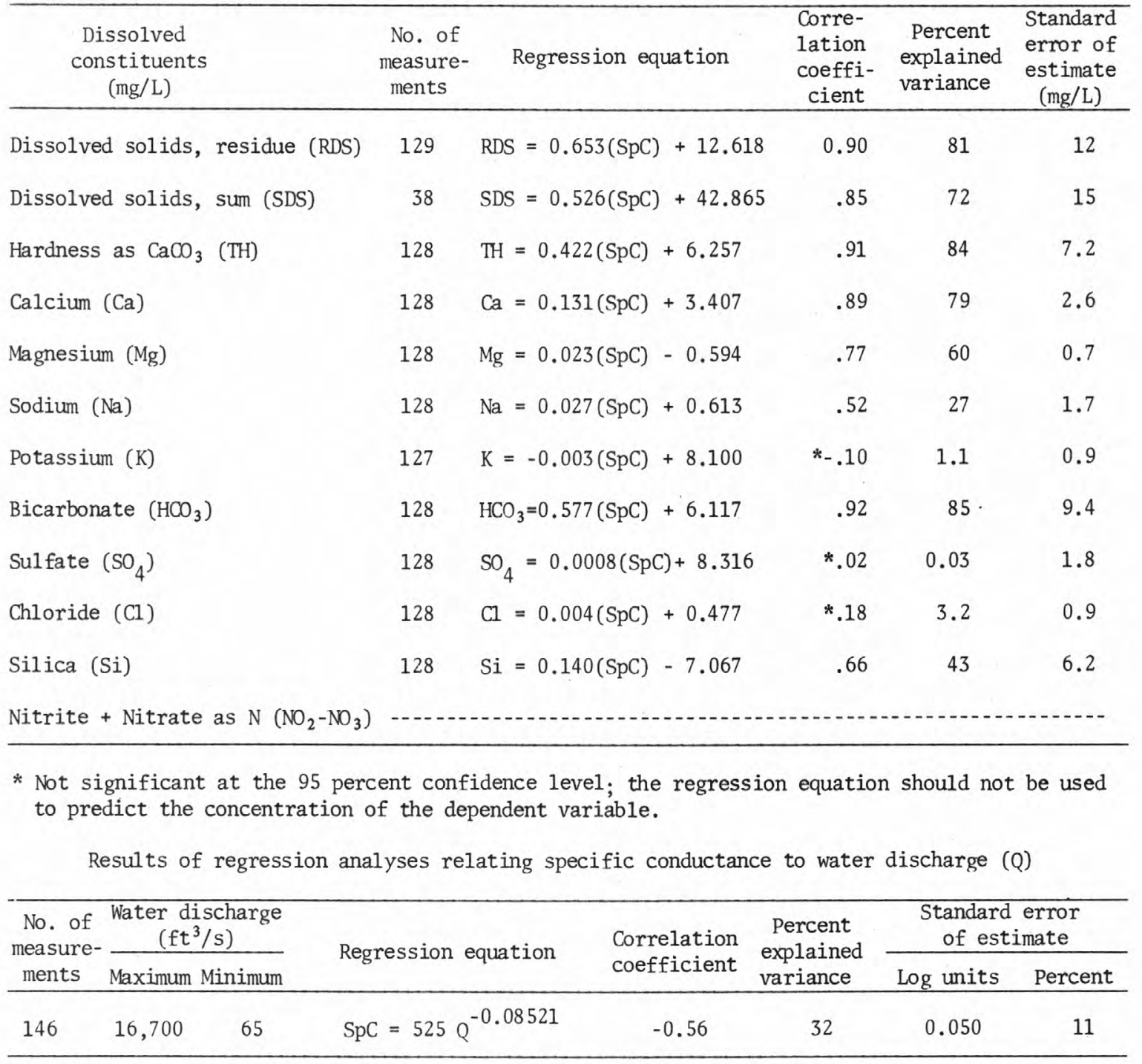


ELKHORN RIVER BASIN

06799100 North Fork Elkhorn River near Pierce, Nebr.

Location.--At gaging station, on left downstream wingwall of county road bridge, $2.5 \mathrm{mi}$ southeast of Pierce, Pierce County.

Drainage area.--520 $\mathrm{mi}^{2}$, approximately.

Period of record.--September 1960 to September 1964.

Statistical data for selected chemical constituents

\begin{tabular}{|c|c|c|c|c|c|c|c|c|c|}
\hline Constituent & Units & $\begin{array}{l}\text { No. of } \\
\text { measure- } \\
\text { ments }\end{array}$ & $\begin{array}{l}\text { Max- } \\
\text { imum }\end{array}$ & $\begin{array}{l}\text { Min- } \\
\text { imum }\end{array}$ & Mean & Median & $\begin{array}{c}\text { Standard } \\
\text { devi- } \\
\text { ation }\end{array}$ & $\begin{array}{l}\text { Ninetieth } \\
\text { percent- } \\
\text { ile }\end{array}$ & $\begin{array}{l}\text { Tenth } \\
\text { percent- } \\
\text { ile }\end{array}$ \\
\hline Specific conductance & umho/ $\mathrm{cm}$ & 56 & 567 & 175 & 445 & 439 & 56 & 510 & 412 \\
\hline Dissolved solids, residue & $\mathrm{mg} / \mathrm{L}$ & 53 & 367 & 149 & 289 & 283 & 36 & 333 & 262 \\
\hline Dissolved solids, sum & $\mathrm{mg} / \mathrm{L}$ & 1 & $-\cdots-$ & $-\ldots-1$ & 268 & $\ldots$ & & & --- \\
\hline Hardness as $\mathrm{CaCO}_{3}$ & $\mathrm{mg} / \mathrm{L}$ & 51 & 256 & 72 & 207 & 207 & 28 & 242 & 180 \\
\hline Calcium, dissolved & $\mathrm{mg} / \mathrm{L}$ & 51 & 77 & 23 & 63 & 63 & 8.5 & 73 & 56 \\
\hline Magnesium, dissolved & $\mathrm{mg} / \mathrm{L}$ & 51 & 18 & 3.6 & 12 & 12 & 2.5 & 16 & 9.7 \\
\hline Sodium, dissolved & $\mathrm{mg} / \mathrm{L}$ & 51 & 23 & 3.1 & 13 & 13 & 3.3 & 18 & 11 \\
\hline Potassium, dissolved & $\mathrm{mg} / \mathrm{L}$ & 51 & 11 & 3.0 & 6.1 & 5.6 & 1.6 & 7.9 & 4.4 . \\
\hline Bicarbonate ion & $\mathrm{mg} / \mathrm{L}$ & 51 & 308 & 92 & 252 & 256 & 32 & 285 & 214 \\
\hline Sulfate, dissolved & $\mathrm{mg} / \mathrm{L}$ & 51 & 56 & 7.5 & 24 & 20 & 9.6 & 37 & 16 \\
\hline Chloride, dissolved & $\mathrm{mg} / \mathrm{L}$ & 51 & 5.3 & 0.0 & 2.8 & 2.6 & 0.9 & 3.8 & 2.1 \\
\hline Fluoride, dissolved & $\mathrm{mg} / \mathrm{L}$ & 51 & 0.5 & 0.2 & 0.4 & 0.3 & 0.08 & 0.4 & 0.3 \\
\hline Silica, dissolved & $\mathrm{mg} / \mathrm{L}$ & 51 & 44 & 11 & 32 & 33 & 5.6 & 37 & 25 \\
\hline Boron, dissolved & $\mathrm{mg} / \mathrm{L}$ & 51 & 110 & 10 & 40 & 38 & 19 & 59 & 26 \\
\hline Iron, dissolved & $\mathrm{ug} / \mathrm{L}$ & 50 & 110 & 10 & 36 & 30 & 25 & 76 & 21 \\
\hline Manganese, dissolved & $\mathrm{ug} / \mathrm{L}$ & 29 & 470 & 0 & 156 & 118 & 145 & 360 & 0 \\
\hline $\begin{array}{l}\text { Nitrite + nitrate as } \mathrm{N} \text {, } \\
\text { dissolved } \\
\text { Nitrate as } N \text {, dissolved }\end{array}$ & $\begin{array}{l}\mathrm{mg} / \mathrm{L} \\
\mathrm{mg} / \mathrm{L}\end{array}$ & - & 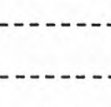 & & -- & & & & $-\cdots$ \\
\hline Nitrate as $\mathrm{NO}_{3}$, dissolved & $\mathrm{mg} / \mathrm{L}$ & 56 & 8.6 & 0.10 & 4.4 & 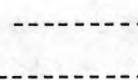 & 1.8 & & - \\
\hline Ammonia N, total & $\mathrm{mg} / \mathrm{L}$ & & & & & & & & \\
\hline Organic N, total & $\mathrm{mg} / \mathrm{L}$ & & & & & & & & \\
\hline $\begin{array}{l}\text { Phosphorus, total } \\
\text { Phosphorus, dissolved }\end{array}$ & $\mathrm{mg} / \mathrm{L}$ & & $\ldots$ & $-\ldots$ & 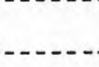 & - & & & -- \\
\hline Fecal coliform & $\operatorname{col} 1 / 100 \mathrm{ml}$ & & 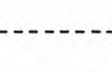 & 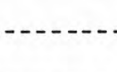 & 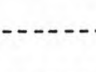 & & & & -- \\
\hline Fecal streptococci $\mathrm{col} /$ & $\operatorname{col} / 100 \mathrm{ml}$ & & & & & & & & $\cdots$ \\
\hline $\begin{array}{l}\text { Oxygen, dissolved } \\
\text { Biochemical oxygen demand }\end{array}$ & $\mathrm{mg} / \mathrm{L}$ & & 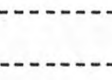 & --1 & -- & - & & & - \\
\hline $\mathrm{pH}^{(5-\text { day })}$ & $\mathrm{pH}$ units & 52 & 8.3 & 7.0 & 7.6 & 7.6 & 0.3 & 8.0 & 7.2 \\
\hline Co - Pt & Co - Pt units & 19 & 44 & 1 & 9 & 5 & 10 & 20 & 0 \\
\hline
\end{tabular}


06799100 North Fork Elkhorn River near Pierce, Nebr.--Continued

Results of regression analyses relating concentrations of selected chemical constituents to specific conductance $(\mathrm{SpC})$

\begin{tabular}{|c|c|c|c|c|c|}
\hline $\begin{array}{l}\text { Dissolved } \\
\text { constituents } \\
(\mathrm{mg} / \mathrm{L})\end{array}$ & $\begin{array}{l}\text { No. of } \\
\text { measure- } \\
\text { ments }\end{array}$ & Regression equation & $\begin{array}{l}\text { Corre- } \\
\text { lation } \\
\text { coeffi- } \\
\text { cient }\end{array}$ & $\begin{array}{l}\text { Percent } \\
\text { explained } \\
\text { variance }\end{array}$ & $\begin{array}{l}\text { Standard } \\
\text { error of } \\
\text { estimate } \\
(\mathrm{mg} / \mathrm{L})\end{array}$ \\
\hline Dissolved solids, residue (RDS) & 53 & $\mathrm{RDS}=0.590(\mathrm{SpC})+26.218$ & 0.96 & 92 & 10 \\
\hline Dissolved solids, sum (SDS) & $\cdots$ & & & & \\
\hline Hardness as $\mathrm{CaCO}_{3}(\mathrm{TH})$ & 51 & $\mathrm{TH}=0.464(\mathrm{SpC})+0.199$ & .97 & 94 & 6.7 \\
\hline Calcium (Ca) & 51 & $\mathrm{Ca}=0.134(\mathrm{SpC})+3.532$ & .92 & 86 & 3.3 \\
\hline Magnesium (Mg) & 51 & $\mathrm{Mg}=0.031(\mathrm{SpC})-1.833$ & .72 & 52 & 1.8 \\
\hline Sodium (Na) & 51 & $\mathrm{Na}=0.048(\mathrm{SpC})-8.096$ & .86 & 74 & 1.7 \\
\hline Potassium (K) & 51 & $K=-0.004(\mathrm{SpC})+7.951$ & $*-.16$ & 2.5 & 1.6 \\
\hline Bicarbonate $\left(\mathrm{HCO}_{3}\right)$ & 51 & $\mathrm{HCO}_{3}=0.509(\mathrm{SpC})+25.576$ & .93 & 86 & 12 \\
\hline Sulfate $\left(\mathrm{SO}_{4}\right)$ & 51 & $\mathrm{SO}_{4}=0.112(\mathrm{SpC})-26.041$ & .69 & 48 & 7.0 \\
\hline Chloride (C1) & 51 & $\mathrm{Cl}=0.005(\mathrm{SpC})+0.442$ & .36 & 13 & 0.8 \\
\hline Silica (Si) & 50 & $\mathrm{Si}=0.042(\mathrm{SpC})+13.448$ & .43 & 19 & 5.1 \\
\hline
\end{tabular}

* Not significant at the 95 percent confidence level; the regression equation should not be used to predict the concentration of the dependent variable.

Results of regression analyses relating specific conductance to water discharge (Q)

\begin{tabular}{ccccccc}
\hline $\begin{array}{c}\text { No. of } \\
\text { measure- } \\
\text { ments }\end{array}$ & $\begin{array}{c}\text { Mater discharge } \\
\left(\mathrm{ft}^{3} / \mathrm{s}\right)\end{array}$ & Maximum Minimum & Regression equation & $\begin{array}{c}\text { Correlation } \\
\text { coefficient }\end{array}$ & $\begin{array}{c}\text { Percent } \\
\text { explained } \\
\text { variance }\end{array}$ & $\begin{array}{c}\text { Standard error } \\
\text { of estimate }\end{array}$ \\
\hline 56 & $1,170 \quad 21$ & $\mathrm{SpC}=660 \mathrm{Q}^{-0.10048}$ & -0.45 & 20 & 0.061 \\
\hline
\end{tabular}


Location.--Lat $42^{\circ} 06^{\prime} 40^{\prime \prime}$, long $96^{\circ} 42^{\prime} 00^{\prime \prime}$, in NW/4 sec. 26, T. 25 N., R. 6 E., Thurston County, Hydrologic Unit 10220004, on right bank $200 \mathrm{ft}$ downstream from bridge on Nebraska State Highway 94 at Pender and $0.7 \mathrm{mi}$ downstream from Rattlesnake Creek.

Drainage area. $--731 \mathrm{mi}^{2}$, approximately.

Period of record.--Water years 1964-63, 1973 to current year.

Statistical data for selected chemical constituents

\begin{tabular}{|c|c|c|c|c|c|c|c|c|c|}
\hline Constituent & Units & $\begin{array}{l}\text { No. of } \\
\text { measure- } \\
\text { ments }\end{array}$ & $\begin{array}{l}\text { Max- } \\
\text { imum }\end{array}$ & $\begin{array}{l}\text { Min- } \\
\text { imum }\end{array}$ & Mean & Median & $\begin{array}{c}\text { Standard N } \\
\text { devi- } \\
\text { ation }\end{array}$ & $\begin{array}{l}\text { Ninetieth } \\
\text { percent- } \\
\text { ile }\end{array}$ & $\begin{array}{l}\text { Tenth } \\
\text { percent- } \\
\text { ile } \\
\end{array}$ \\
\hline Specific conductance & umho/cm & 127 & 870 & 287 & 716 & 730 & 102 & 803 & 600 \\
\hline Dissolved solids, residue & $\mathrm{mg} / \mathrm{L}$ & 111 & 595 & 204 & 474 & 483 & 60 & 525 & 408 \\
\hline Dissolved solids, sum & $\mathrm{mg} / \mathrm{L}$ & 29 & 535 & 173 & 433 & 467 & 97. & 502 & 190 \\
\hline Hardness as $\mathrm{CaCO}_{3}$ & $\mathrm{mg} / \mathrm{L}$ & 84 & 400 & 120 & 328 & 346 & 64 & 388 & 250 \\
\hline Calcium, dissolved & $\mathrm{mg} / \mathrm{L}$ & 85 & 120 & 34 & 92 & 96 & 19 & 113 & 66 \\
\hline Magnesium, dissolved & $\mathrm{mg} / \mathrm{L}$ & 85 & 40 & 7.6 & 24 & 25 & 6.0 & 29 & 17 \\
\hline Sodium, dissolved & $\mathrm{mg} / \mathrm{L}$ & 84 & 33 & 2.2 & 22 & 24 & 5.8 & 29 & 17 \\
\hline Potassium, dissolved & $\mathrm{mg} / \mathrm{L}$ & 84 & 13 & 4.8 & 7.6 & 6.9 & 2.0 & 12 & 5.6 \\
\hline Bicarbonate ion & $\mathrm{mg} / \mathrm{L}$ & 84 & 400 & 124 & 324 & 335 & 60 & 380 & 250 \\
\hline Sulfate, dissolved & $\mathrm{mg} / \mathrm{L}$ & 84 & 140 & 22 & 101 & 103 & 23 & 122 & 78 \\
\hline Chloride, dissolved & $\mathrm{mg} / \mathrm{L}$ & 126 & 23 & 1.4 & 7.2 & 6.0 & 3.4 & 12 & 4.2 \\
\hline Fluoride, dissolved & $\mathrm{mg} / \mathrm{L}$ & 85 & 0.8 & 0.1 & 0.3 & 0.3 & 0.1 & 0.5 & 0.2 \\
\hline Silica, dissolved & $\mathrm{mg} / \mathrm{L}$ & 84 & 28 & 3.2 & 20 & 21 & 4.8 & 25 & 13 \\
\hline Boron, dissolved & $\mathrm{mg} / \mathrm{L}$ & 85 & 170 & 10 & 76 & 78 & 24 & 102 & 51 \\
\hline Iron, dissolved & $\mathrm{ug} / \mathrm{L}$ & 68 & 310 & 0 & 32 & 21 & 50 & 59 & 0 \\
\hline Manganese, dissolved & $\mathrm{ug} / \mathrm{L}$ & 62 & 760 & 0 & 173 & 116 & 154 & 370 & 40 \\
\hline $\begin{array}{l}\text { Nitrite + nitrate as } \mathrm{N} \text {, } \\
\text { dissolved }\end{array}$ & $\mathrm{mg} / \mathrm{L}$ & 28 & 2.9 & 0.18 & 1.4 & 1.4 & 0.65 & 2.4 & 0.62 \\
\hline Nitrate as N, dissolved & $\mathrm{mg} / \mathrm{L}$ & 1 & $\cdots-1$ & 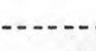 & -0.60 & $-\ldots$ & 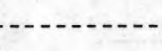 & & --- \\
\hline Nitrate as $\mathrm{NO}_{3}$, dissolved & $\mathrm{mg} / \mathrm{L}$ & & - & & 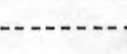 & & & & --- \\
\hline Ammonia $\mathrm{N}$, total & $\mathrm{mg} / \mathrm{L}$ & 55 & 3.5 & 0.00 & 0.32 & 0.14 & 0.53 & 0.63 & 0.01 \\
\hline Organic $N$, total & $\mathrm{mg} / \mathrm{L}$ & 53 & 36 & 0.04 & 2.1 & 0.56 & 5.8 & 1.9 & 0.25 \\
\hline Phosphorus, total & $\mathrm{mg} / \mathrm{L}$ & 55 & 8.3 & 0.11 & 0.65 & 0.36 & 1.2 & 0.85 & 0.24 \\
\hline Phosphorus, dissolved & $\mathrm{mg} / \mathrm{L}$ & 48 & 0.54 & 0.08 & 0.23 & 0.24 & 0.10 & 0.33 & 0.12 \\
\hline Fecal coliform & $\operatorname{col} / 100 \mathrm{ml}$ & \multicolumn{2}{|c|}{39290,000} & 16 & $-\cdots-1$ & 280 & - n & 3,200 & 100 \\
\hline Fecal streptococci & $\mathrm{col} / 100 \mathrm{ml}$ & \multicolumn{2}{|c|}{47150,000} & 4 & - - - - & 332 & - & 9,600 & 72 \\
\hline Oxygen, dissolved & $\mathrm{mg} / \mathrm{L}$ & 52 & 13.6 & 5.2 & 9.5 & 9.8 & 2.1 & 12.0 & 6.5 \\
\hline \multirow{2}{*}{$\begin{array}{l}\text { Biochemical oxygen dem } \\
\mathrm{pH}^{(5-\text { day })}\end{array}$} & $\mathrm{mg} / \mathrm{L}$ & 53 & 8.8 & 1.1 & 4.3 & 3.7 & 2.1 & 7.9 & 2.0 \\
\hline & & 129 & 8.6 & 6.5 & 7.8 & 7.8 & 0.3 & $8: 2$ & 7.5 \\
\hline $\mathrm{Co}-\mathrm{Pt}$ & $\begin{array}{r}\text { pH units } \\
\text { Co - Pt units }\end{array}$ & 46 & 380 & 1 & 20 & 5 & 57 & 46 & 3 \\
\hline
\end{tabular}


Results of regression analyses relating concentrations of selected chemical constituents to specific conductance $(\mathrm{SpC})$

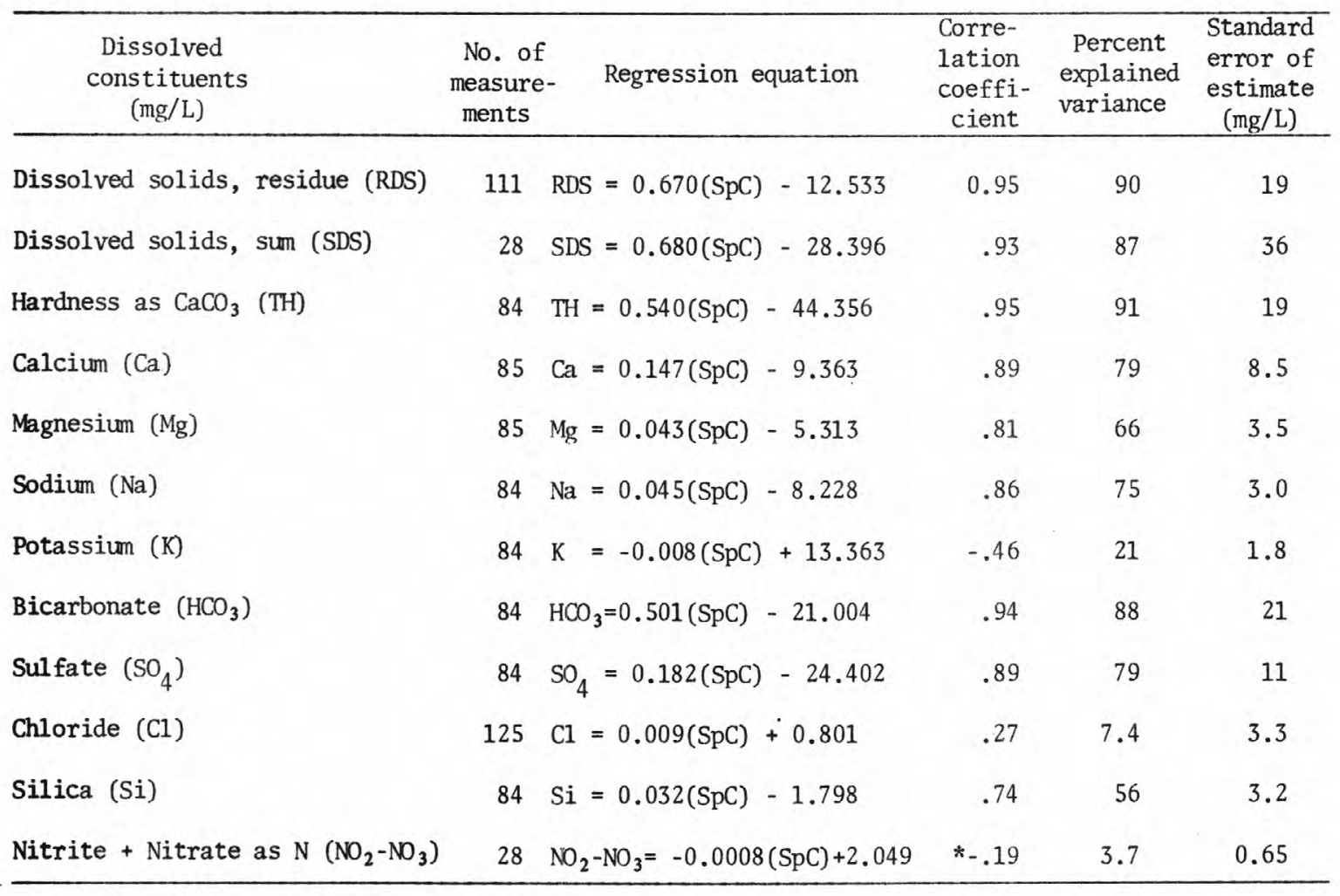

* Not significant at the 95 percent confidence level; the regression equation should not be used to predict the concentration of the dependent variable.

Results of regression analyses relating specific conductance to water discharge (Q)

\begin{tabular}{cccccc}
\hline $\begin{array}{l}\text { No. of Water discharge } \\
\text { measure- } \\
\text { ments }\end{array}$ & $\begin{array}{c}\left(\mathrm{ft}^{3} / \mathrm{s}\right) \\
\text { Maximum Minimum }\end{array}$ & Regression equation & $\begin{array}{c}\text { Correlation } \\
\text { coefficient }\end{array}$ & $\begin{array}{c}\text { Percent } \\
\text { explained } \\
\text { variance }\end{array}$ & $\begin{array}{c}\text { Standard error } \\
\text { of estimate }\end{array}$ \\
\hline 126 & $21,400 \quad 11.50$ & $S p C=1,2010^{-0.12082}$ & -0.64 & 42 & 0.063 \\
\hline
\end{tabular}


Location. --Lat $41^{\circ} 42^{\prime} 50^{\prime \prime}$, long $96^{\circ} 31^{\prime} 15^{\prime \prime}$, in SE $\frac{1}{4} \mathrm{SF}^{\frac{1}{4}} \mathrm{sec} .9$, T.20 N., R. 8 E., Dodge County, at gaging station at bridge on county road $2 \mathrm{mi}$ southwest of Uehling and $8 \mathrm{mi}$ upstream from mouth.

Drainage area. $--1,030 \mathrm{mi}^{2}$, approximately.

Period of record.--October 1968 to September 1971 .

Statistical data for selected chemical constituents

\begin{tabular}{|c|c|c|c|c|c|c|c|c|c|}
\hline Constituent & Units & $\begin{array}{l}\text { No. of } \\
\text { measure- } \\
\text { ments }\end{array}$ & $\begin{array}{l}\text { Max- } \\
\text { imum }\end{array}$ & $\begin{array}{l}\text { Min- } \\
\text { imum }\end{array}$ & Mean & Median & $\begin{array}{c}\text { Standard } \\
\text { devi- } \\
\text { ation }\end{array}$ & $\begin{array}{c}\text { Ninetieth } \\
\text { percent- } \\
\text { ile }\end{array}$ & $\begin{array}{l}\text { Tenth } \\
\text { percent- } \\
\text { ile }\end{array}$ \\
\hline Specific conductance & unho/cm & 71 & 888 & 276 & 676 & 698 & 113 & 780 & 530 \\
\hline Dissolved solids, residue & $\mathrm{mg} / \mathrm{L}$ & 40 & 580 & 178 & 444 & 455 & 87 & 540 & 330 \\
\hline Dissolved solids, sum & $\mathrm{mg} / \mathrm{L}$ & 42 & 570 & 178 & 427 & 447 & 84 & 503 & 330 \\
\hline Hardness as $\mathrm{CaCO}_{3}$ & $\mathrm{mg} / \mathrm{L}$ & 42 & 430 & 120 & 309 & 323 & 66 & 368 & 210 \\
\hline Calcium, dissolved & $\mathrm{mg} / \mathrm{L}$ & 43 & 120 & 36 & 85 & 88 & 19 & 102 & 62 \\
\hline Magnesium, dissolved & $\mathrm{mg} / \mathrm{L}$ & 42 & 31 & 7.4 & 24 & 26 & 5.5 & 28 & 16 \\
\hline Sodium, dissolved & $\mathrm{mg} / \mathrm{L}$ & 42 & 50 & 3.6 & 26 & 27 & 7.9 & 32 & 16 \\
\hline Potassium, dissolved & $\mathrm{mg} / \mathrm{L}$ & 42 & 17 & 4.8 & 7.8 & 7.1 & 2.5 & 10 & 6.0 \\
\hline Bicarbonate ion & $\mathrm{mg} / \mathrm{L}$ & 43 & 449 & 117 & 327 & 340 & 70 & 388 & 220 \\
\hline Sulfate, dissolved & $\mathrm{mg} / \mathrm{L}$ & 42 & 157 & 22 & 92 & 97 & 24 & 110 & 59 \\
\hline Chloride, dissolved & $\mathrm{mg} / \mathrm{L}$ & 42 & 20 & 2.0 & 7.3 & 6.4 & 2.9 & 11 & 4.8 \\
\hline Fluoride, dissolved & $\mathrm{mg} / \mathrm{L}$ & 42 & 0.8 & 0.1 & 0.4 & 0.4 & 0.1 & 0.6 & 0.2 \\
\hline Silica, dissolved & $\mathrm{mg} / \mathrm{L}$ & 42 & 28 & 5.2 & 18 & 19 & 5.1 & 25 & 13 \\
\hline Boron, dissolved & $\mathrm{mg} / \mathrm{L}$ & 42 & 135 & 20 & 81 & 84 & 28 & 118 & 40 \\
\hline Iron, dissolved & $\mathrm{ug} / \mathrm{L}$ & 41 & 1,000 & 0 & 90 & 47 & 165 & 170 & 0 \\
\hline Manganese, dissolved & $\mathrm{ug} / \mathrm{L}$ & 15 & 590 & 0 & 89 & 21 & 187 & 500 & 0 \\
\hline Nitrite + nitrate as $\mathrm{N}$, & $\mathrm{mg} / \mathrm{L}$ & 4 & 1.8 & 0.00 & 0.47 & ----- & 0.89 & $\cdots-$ & $-\cdots$ \\
\hline Nitrate as $N$, dissolved & $\mathrm{mg} / \mathrm{L}$ & 36 & 5.7 & 0.00 & 1.2 & 0.77 & 1.4 & 2.6 & 0.00 \\
\hline Nitrate as $\mathrm{NO}_{3}$, dissolved & $\mathrm{mg} / \mathrm{L}$ & 41 & 25 & 0.00 & 4.6 & $-\cdots-1$ & 5.9 & 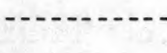 & -- \\
\hline Ammonia $\mathrm{N}$, total & $\mathrm{mg} / \mathrm{L}$ & $-\cdots$ & 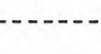 & 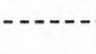 & 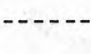 & & - & & -- \\
\hline Organic $N$, total & $\mathrm{mg} / \mathrm{L}$ & & & & & & & & \\
\hline Phosphorus, total & $\mathrm{mg} / \mathrm{L}$ & & & & & & & & \\
\hline Phosphorus, dissolved & $\mathrm{mg} / \mathrm{L}$ & 27 & 0.66 & 0.06 & 0.22 & 0.15 & 0.16 & 0.41 & 0.09 \\
\hline Fecal coliform & $/ 100 \mathrm{ml}$ & & & & & & & & ---- \\
\hline Fecal streptococci & $/ 100 \mathrm{ml}$ & & & & & & & & -- \\
\hline Oxygen, dissolved & $\mathrm{mg} / \mathrm{L}$ & & & & & & & & \\
\hline $\begin{array}{l}\text { Biochemical oxygen demand } \\
\mathrm{pH}^{(5-\text { day })}\end{array}$ & $\begin{array}{r}\mathrm{mg} / \mathrm{L} \\
\mathrm{H} \text { units }\end{array}$ & 43 & 8.3 & 7.3 & 7.9 & 8.0 & 0.2 & 8.2 & 7.8 \\
\hline $\mathrm{Co}-\mathrm{Pt}$ & t units & 30 & 35 & 1 & 7 & 5 & 7.3 & 20 & 1 \\
\hline
\end{tabular}


06799500 Logan Creek near Uehling, Nebr.--Continued

Results of regression analyses relating concentrations of selected chemical constituents to specific conductance $(\mathrm{SpC})$

\begin{tabular}{|c|c|c|c|c|c|}
\hline $\begin{array}{l}\text { Dissolved } \\
\text { constituents } \\
(\mathrm{mg} / \mathrm{L})\end{array}$ & $\begin{array}{l}\text { No. of } \\
\text { measure- } \\
\text { ments }\end{array}$ & Regression equation & $\begin{array}{l}\text { Corre- } \\
\text { lation } \\
\text { coeffi- } \\
\text { cient }\end{array}$ & $\begin{array}{l}\text { Percent } \\
\text { explained } \\
\text { variance }\end{array}$ & $\begin{array}{c}\text { Standard } \\
\text { error of } \\
\text { estimate } \\
(\mathrm{mg} / \mathrm{L})\end{array}$ \\
\hline Dissolved solids, residue (RDS) & 40 & $\mathrm{RDS}=0.698(\mathrm{SpC})-25.146$ & 0.96 & 92 & 25 \\
\hline Dissolved solids, sum (SDS) & 42 & $\mathrm{SDS}=0.697(\mathrm{SpC})-38.070$ & .98 & 96 & 16 \\
\hline Hardness as $\mathrm{CaCO}_{3}(\mathrm{TH})$ & 42 & $\mathrm{TH}=0.530(\mathrm{SpC})-44.181$ & .96 & 92 & 19 \\
\hline Calcium (Ca) & 43 & $\mathrm{Ca}=0.144(\mathrm{SpC})-11.227$ & .90 & 80 & 8.4 \\
\hline Magnesium (Mg) & 42 & $\mathrm{Mg}=0.041(\mathrm{SpC})-3.713$ & .89 & 80 & 2.5 \\
\hline Sodium $(\mathrm{Na})$ & 42 & $\mathrm{Na}=0.052(\mathrm{SpC})-8.828$ & .78 & 60 & 5.0 \\
\hline Potassium $(K)$ & 42 & $K=-0.006(\mathrm{SpC})+11.998$ & $*-.30$ & 9.0 & 2.4 \\
\hline Bicarbonate $\left(\mathrm{HCO}_{3}\right)$ & 43 & $\mathrm{HCO}_{3}=0.559(\mathrm{SpC})-45.892$ & .94 & 89 & 24 \\
\hline Sulfate $\left(\mathrm{SO}_{4}\right)$ & 42 & $\mathrm{SO}_{4}=0.171(\mathrm{SpC})-21.708$ & .85 & 72 & 13 \\
\hline Chloride (CI) & 42 & $\mathrm{Cl}=0.011(\mathrm{SpC})-0.289$ & .47 & 22 & 2.6 \\
\hline Silica (Si) & 42 & $\mathrm{Si}=0.026(\mathrm{SpC})+0.947$ & .60 & 36 & 4.2 \\
\hline
\end{tabular}

* Not significant at the 95 percent confidence level; the regression equation should not be used to predict the concentration of the dependent variable.

Results of regression analyses relating specific conductance to water discharge (Q)

\begin{tabular}{ccccccc}
\hline $\begin{array}{l}\text { No. of Water discharge } \\
\text { measure- } \\
\text { ments }\end{array}$ & Maximum Minimum & Regression equation & $\begin{array}{c}\text { Correlation } \\
\text { coefficient }\end{array}$ & $\begin{array}{c}\text { Percent } \\
\text { explained } \\
\text { variance }\end{array}$ & $\begin{array}{c}\text { Standard error } \\
\text { of estimate }\end{array}$ \\
\hline 62 & 4,480 & 6 & $\mathrm{SpC}=1,144 \mathrm{Q}^{-0.12354}$ & -0.56 & 32 & 0.077 \\
\hline
\end{tabular}




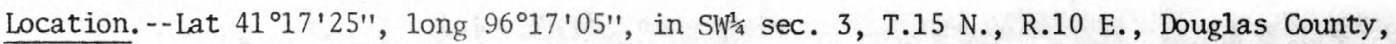
Hydrologic Unit 10220003, on right bank $100 \mathrm{ft}$. upstream from bridge at north edge of Waterloo and $3.5 \mathrm{mi}$ downstream from Rawhide Creek.

Drainage area.--6,900 $\mathrm{mi}^{2}$, approximately, of which about $5,870 \mathrm{mi}^{2}$ contributes directly to surface runoff.

Period of record.--Water year 1966 to current year.

Statistical data for selected chemical constituents

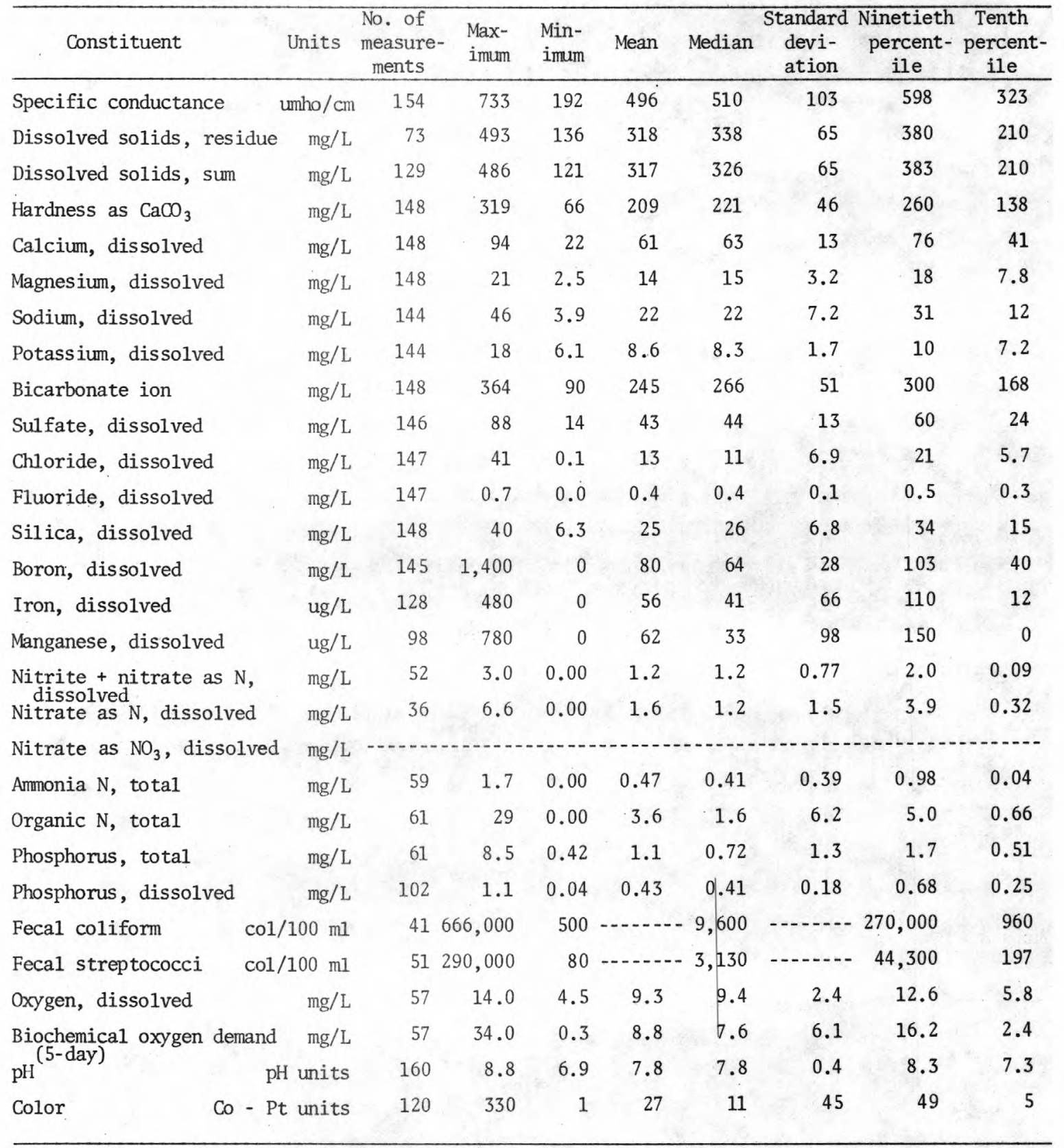


Results of regression analyses relating concentrations of selected chemical constituents to specific conductance $(\mathrm{SpC})$

\begin{tabular}{|c|c|c|c|c|c|}
\hline $\begin{array}{c}\text { Dissolved } \\
\text { constituents } \\
\text { (mg/L) }\end{array}$ & $\begin{array}{l}\text { No. of } \\
\text { measure- } \\
\text { ments }\end{array}$ & Regression equation & $\begin{array}{l}\text { Corre- } \\
\text { lation } \\
\text { coeffi- } \\
\text { cient }\end{array}$ & $\begin{array}{l}\text { Percent } \\
\text { explained } \\
\text { variance }\end{array}$ & $\begin{array}{c}\text { Standard } \\
\text { error of } \\
\text { estimate } \\
(\mathrm{mg} / \mathrm{L})\end{array}$ \\
\hline Dissolved solids, residue (RDS) & 73 & $\mathrm{RDS}=0.629(\mathrm{SpC})+11.816$ & 0.98 & 96 & 13 \\
\hline Dissolved solids, sum (SDS) & 125 & $\mathrm{SDS}=0.631(\mathrm{SpC})-1.227$ & .98 & 96 & 12 \\
\hline Hardness as $\mathrm{CaCO}_{3}(\mathrm{TH})$ & 146 & $\mathrm{TH}=0.428(\mathrm{SpC})-3.516$ & .95 & 91 & 14 \\
\hline Calcium (Ca) & 146 & $\mathrm{Ca}=0.122(\mathrm{SpC})+0.701$ & .93 & 86 & 5.0 \\
\hline Magnesium (Mg) & 146 & $\mathrm{Mg}=0.030(\mathrm{SpC})-1.361$ & .95 & 90 & 1.0 \\
\hline Sodium $(\mathrm{Na})$ & 142 & $\mathrm{Na}=0.064(\mathrm{SpC})-10.096$ & .87 & 76 & 3.5 \\
\hline Potassium (K) & 142 & $K=-0.005(\mathrm{SpC})+11.140$ & -.29 & 8.6 & 1.6 \\
\hline Bicarbonate $\left(\mathrm{HCO}_{3}\right)$ & 146 & $\mathrm{HCO}_{3}=0.464(\mathrm{SpC})+15.200$ & .93 & 86 & 19 \\
\hline Sulfate $\left(\mathrm{SO}_{4}\right)$ & 144 & $\mathrm{SO}_{4}=0.111(\mathrm{SpC})-11.850$ & .88 & 78 & 6.0 \\
\hline Chloride $(\mathrm{Cl})$ & 145 & $\mathrm{Cl}=0.046(\mathrm{SpC})-10.042$ & .68 & 46 & 5.1 \\
\hline Silica (Si) & 146 & $\mathrm{Si}=0.051(\mathrm{SpC})-0.262$ & .75 & 56 & 4.5 \\
\hline Nitrite + Nitrate as $\mathrm{N}\left(\mathrm{NO}_{2}-\mathrm{NO}_{3}\right)$ & 50 & $\mathrm{NO}_{2}-\mathrm{NO}_{3}=-0.001(\mathrm{SpC})+1.872$ & $*_{-} .19$ & 3.5 & 0.77 \\
\hline
\end{tabular}

* Not significant at the 95 percent confidence level; the regression equation should not be used to predict the concentration of the dependent variable.

Results of regression analyses relating specific conductance to water discharge (Q)

\begin{tabular}{|c|c|c|c|c|c|c|c|}
\hline \multirow{2}{*}{$\begin{array}{l}\text { No. of } \\
\text { measure- } \\
\text { ments }\end{array}$} & \multirow{2}{*}{\multicolumn{2}{|c|}{$\begin{array}{l}\text { Water discharge } \\
\frac{\left(\mathrm{ft}^{3} / \mathrm{s}\right)}{\text { Maximum Minimum }}\end{array}$}} & \multirow{2}{*}{ Regression equation } & \multirow{2}{*}{$\begin{array}{l}\text { Correlation } \\
\text { coefficient }\end{array}$} & \multirow{2}{*}{$\begin{array}{l}\text { Percent } \\
\text { explained } \\
\text { variance }\end{array}$} & \multicolumn{2}{|c|}{$\begin{array}{c}\text { Standard error } \\
\text { of estimate }\end{array}$} \\
\hline & & & & & & Log units & Percent \\
\hline 153 & 38,700 & 97 & $\mathrm{SpC}=1,765 \mathrm{Q}^{-0.19256}$ & -0.81 & 66 & 0.064 & 15 \\
\hline
\end{tabular}


The Lower Platte River basin includes all Platte River drainage from the confluence of the Loup and Platte Rivers to the mouth of the Platte, with the exception of the Elkhorn River basin. Included is Salt Creek which drains about half the Lower Platte River basin.

Much of the area drained by the Lower Platte River basin has been glaciated. Most of the glacial drift is overlain by loess; however, in some of the rest of the non-valley areas, soils have developed directly on the drift.

Total acres irrigated in the basin are slightly over 100,000 and most irrigation in the basin is by pumpage of ground water. Irrigation with surface water is limited to a few individuals who pump directly from the streams. With few exceptions, irrigated acreage is found only in the Platte River valley and in Todd Valley, a loess-covered ancestral channel of the Platte River in Saunders County.

Descriptive statistics are available for 16 stations in the Lower Platte River basin (fig. 10). Twelve of these are in the Salt Creek basin which, because of its proximity to the city of Lincoln and its unusual hydrologic conditions, has been studied extensively. 'With the exception of that from tributaries Stevens Creek, Wahoo Creek, and Silver Creek, streamflow in the Salt Creek basin is highly mineralized. The mean specific conductance of Salt Creek above Beal Slough was 1,190 umho/cm, of Salt Creek at Lincoln it was $4,850 \mathrm{umho} / \mathrm{cm}$, and of Salt Creek above Ashland it was $5,030 \mathrm{umho} / \mathrm{cm}$.

Seepage from saline deposits in the Dakota Formation of Cretaceous age is the cause of the highly mineralized water in Salt Creek. These deposits occur to the north and west of Lincoln at shallow depth. Water seeps directly into the streams from these deposits or moves into overlying Pleistocene deposits and from them seeps into the streams. Water in the lower reaches of Oak Creek and Little Salt Creek, which drain the area to the northwest of Lincoln and which enters Salt Creek in the Lincoln area is the most highly mineralized of any stream reaches in Nebraska.

As expected, sodium is the principal cation and chloride is the principal anion in water from all these reaches as well as in all reaches of Salt Creek downstream from Lincoln. Saline deposits in the Dakota formation occur at greater depth south than north and west of Lincoln, and less of the highly saline seepage from them reaches the stream. At Roca and above Beal Slough, sodium is the principal cation but bicarbonate 


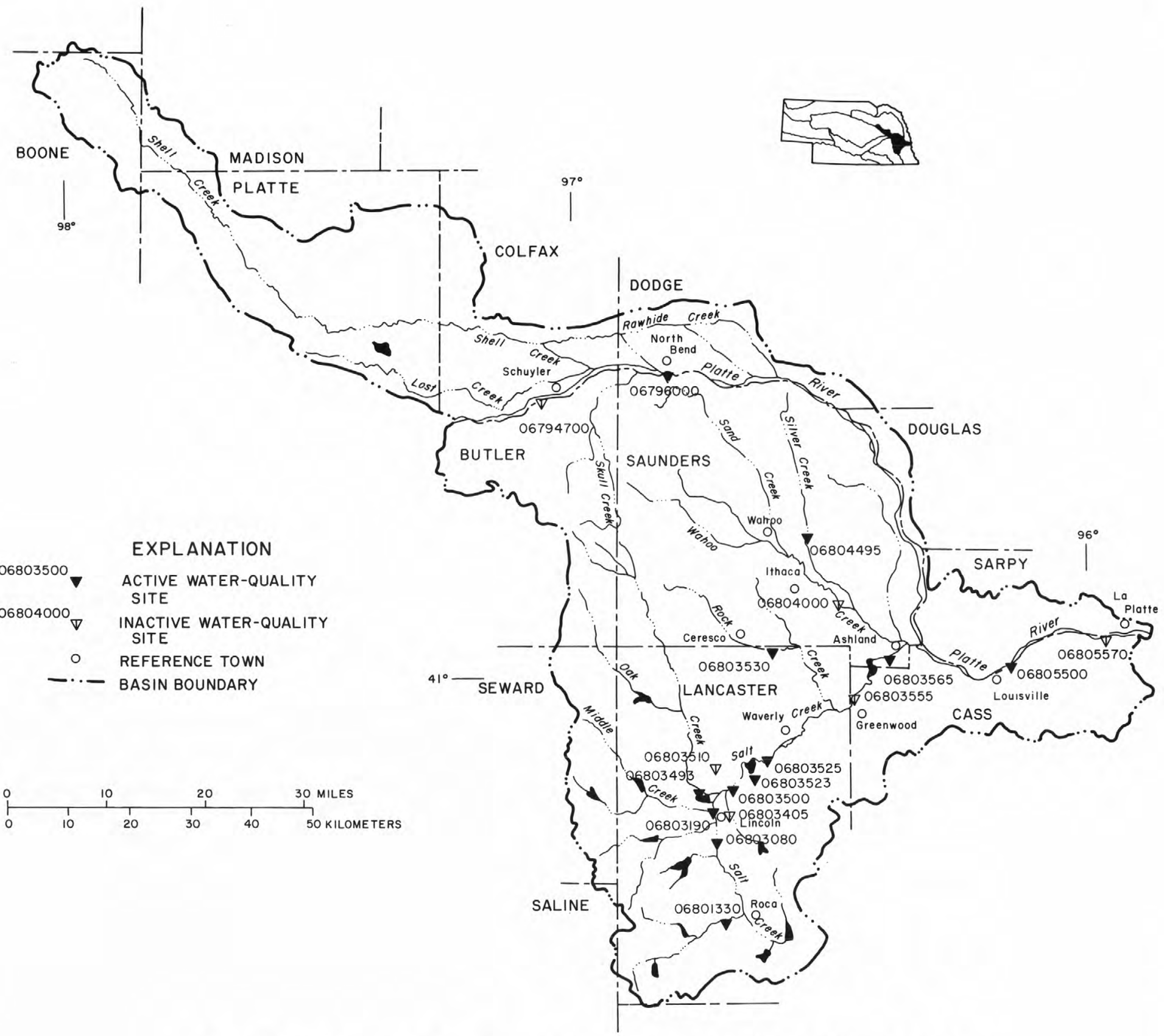

FIGURE 10.-Location of water-quality sampling sites, Lower Platte River basin 
and chloride are the principal anions. This is also true for Rock Creek near Ceresco. Calcium is the principal cation and bicarbonate the principal anion in Stevens, Wahoo, and Silver Creeks.

The effect of effluent from the Lincoln sewage-treatment plant on Salt Creek prior to 1977 can be seen in table 4. Medians for fecal coliform and fecal streptococcal bacteria increased dramatically at the first station (Salt Creek at Lincoln) downstream from the sewage-treatment plant. Thereafter, the medians decreased downstream. In 1977 treatment facilities were improved. Concentrations of the magnitudes shown in table 4 no longer apply.

Table 4.--Median fecal coliform and fecal streptococcal bacteria concentrations for stations on Salt Creek

\begin{tabular}{lrr}
\hline \multicolumn{1}{c}{ Station } & $\begin{array}{c}\text { Fecal coliform } \\
(\mathrm{col} / 100 \mathrm{ml})\end{array}$ & $\begin{array}{c}\text { Fecal streptococcal } \\
\text { bacteria } \\
(\text { col/100 m1) }\end{array}$ \\
\hline At Roca & 317 & 1,070 \\
Above. Beal Slough & 474 & 560 \\
At 14th Street, Lincoln & 568 & 910 \\
At Lincoln & 175,000 & 42,000 \\
Below Stevens Creek & 105,000 & 23,500 \\
At Greenwood & 7,000 & 1,000 \\
Above Ashland & 5,200 & 948 \\
\hline
\end{tabular}

Increases in means of dissolved and total phosphorus and of BOD follow the same pattern as the medians for the bacteria.

Salt Creek enters the Platte River about $10 \mathrm{mi}$ above Louisville. Whereas, the discharge contributed by Salt Creek averages only about 3.5 percent of the total flow of the Platte River at Louisville, during low flow in the Platte River the highly mineralized water from Salt Creek causes the quality of water in the Platte River to deteriorate considerably.

Some descriptive statistics are available for four stations on the Platte River near Schuyler, at North Bend, at Louisville, and at LaPlatte. Mean specific conductance for the Platte River at North Bend is 461 umho/cm; whereas, for the Platte River at Louisville it is 713 umho/cm, an increase of approximately 55 percent.

The influence of Salt Creek on the quality of water in the Platte River at Louisville also is reflected in mean chloride concentrations. 
At North Bend, it was $11 \mathrm{mg} / \mathrm{L}$ and at Louisville, it was $79 \mathrm{mg} / \mathrm{L}$. At North Bend, the principal cation is calcium and the principal anion is bicarbonate. At Louisville, bicarbonate also is the principal anion, but sodium and calcium are the principal cations.

Correlation coefficients between specific conductance and principal constituents are greater than 0.8 for the Platte River both near Schuyler and at North Bend. At Louisville, correlation coefficients between conductance and chloride and sodium are 0.92 and 0.98 , respectively; but those between conductance and other principal constituents are less than 0.7 .

A high degree of negative correlation between specific conductance and water discharge is observed for all main stem Salt Creek stations and most Salt Creek tributaries. This occurs because as overland runoff increases water discharge in the streams, it dilutes the highly mineralized base flow accordingly. 
Location.--Lat $41^{\circ} 24^{\prime} 50^{\prime \prime}$, long $97^{\circ} 03^{\prime} 35^{\prime \prime}$, in NE $\frac{1}{4} \mathrm{SE}^{\frac{1}{4}} \mathrm{sec}$. 27 , T.17 N., R.3 E., Colfax County, at bridge on State Highway 15, $2.6 \mathrm{mi}$ south of intersection of.U.S. Highway 30 and State Highway 15 at Schuyler, and $6.0 \mathrm{mi}$ upstream from She11 Creek.

Period of record.--October 1966 to September 1968.

Statistical data for selected chemical constituents

\begin{tabular}{|c|c|c|c|c|c|c|c|c|c|}
\hline Constituent & Unitș & $\begin{array}{l}\text { No. of } \\
\text { measure- } \\
\text { ments }\end{array}$ & $\begin{array}{l}\text { Max- } \\
\text { imum }\end{array}$ & $\begin{array}{l}\text { Min- } \\
\text { imum }\end{array}$ & Mean & Median & $\begin{array}{c}\text { Standard } \\
\text { devi- } \\
\text { ation }\end{array}$ & $\begin{array}{c}\text { Ninetieth } \\
\text { percent- } \\
\text { ile }\end{array}$ & $\begin{array}{l}\text { Tenth } \\
\text { percent- } \\
\text { ile }\end{array}$ \\
\hline Specific conductance & umho/cm & 27 & 709 & 250 & 383 & 367 & 107 & 520 & 272 \\
\hline Dissolved solids, residue & $\mathrm{mg} / \mathrm{L}$ & 27 & 484 & 184 & 267 & 252 & 70 & 350 & 282 \\
\hline Dissolved solids, sum & $\mathrm{mg} / \mathrm{L}$ & 13 & 329 & 180 & 243 & 240 & 46 & 290 & 183 \\
\hline Hardness as $\mathrm{CaCO}_{3}$ & $\mathrm{mg} / \mathrm{L}$ & 27 & 244 & 96 & 141 & 134 & 32 & 180 & 100 \\
\hline Calcium, dissolved & $\mathrm{mg} / \mathrm{L}$ & 27 & 68 & 30 & 42 & 40 & 8.6 & 51 & 32 \\
\hline Magnesium, dissolved & $\mathrm{mg} / \mathrm{L}$ & 27 & 18 & 5.1 & 8.8 & 8.0 & 3.1 & 14 & 5.4 \\
\hline Sodium, dissolved & $\mathrm{mg} / \mathrm{L}$ & 27 & 61 & 8.1 & 22 & 19 & 12 & 35 & 13 \\
\hline Potassium, dissolved & $\mathrm{mg} / \mathrm{L}$ & 27 & 11 & 6.0 & 8.3 & 8.2 & 1.2 & 9.8 & 6.4 \\
\hline Bicarbonate ion & $\mathrm{mg} / \mathrm{L}$ & 27 & 215 & 131 & 168 & 168 & 22 & 195 & 176 \\
\hline Sulfate, dissolved & $\mathrm{mg} / \mathrm{L}$ & 27 & 166 & 12 & 49 & 40 & 36 & 98 & 15 \\
\hline Chloride, dissolved & $\mathrm{mg} / \mathrm{L}$ & 27 & 18 & 1.6 & 6.4 & 5.4 & 4.1 & 12 & 3.0 \\
\hline Fluoride, dissolved & $\mathrm{mg} / \mathrm{L}$ & 27 & 0.5 & 0.1 & 0.4 & 0.4 & 0.09 & 0.4 & 0.3 \\
\hline Silica, dissolved & $\mathrm{mg} / \mathrm{L}$ & 27 & 56 & 21 & 40 & 42 & 7.0 & 48 & 32 \\
\hline Boron, dissolved & $\mathrm{mg} / \mathrm{L}$ & 27 & 100 & 0 & 46 & 41 & 24 & 100 & 30 \\
\hline Iron, dissolved & $\mathrm{ug} / \mathrm{L}$ & 14 & 90 & 20 & 32 & 30 & 19 & 50 & 23 \\
\hline Manganese, dissolved & $\mathrm{ug} / \mathrm{L}$ & 14 & 90 & 0 & 23 & 0 & 34 & 80 & 0 \\
\hline $\begin{array}{l}\text { Nitrite }+ \text { nitrate as } N \text {, } \\
\text { dissolved } \\
\text { Nitrate as } N \text {, dissolved }\end{array}$ & $\begin{array}{l}\mathrm{mg} / \mathrm{L} \\
\mathrm{mg} / \mathrm{L}\end{array}$ & & & & - & & & & -- \\
\hline Nitrate as $\mathrm{NO}_{3}$, dissolved & $\mathrm{mg} / \mathrm{L}$ & 27 & 3.0 & 0.00 & 0.70 & 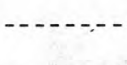 & 0.74 & & \\
\hline Ammonia $\mathrm{N}$, total & $\mathrm{mg} / \mathrm{L}$ & --- & (2) & & $x^{2}=$ & & & & \\
\hline Organic N, total & $\mathrm{mg} / \mathrm{L}$ & & & & & & & & \\
\hline $\begin{array}{l}\text { Phosphorus, total } \\
\text { Phosphorus, dissolved }\end{array}$ & $\begin{array}{l}\mathrm{mg} / \mathrm{L} \\
\mathrm{mg} / \mathrm{L}\end{array}$ & $\ldots$ & & $\cdots$ & & & & & \\
\hline Fecal coliform & $\operatorname{col} 1 / 100 \mathrm{ml}$ & & & & & & & & \\
\hline Fecal streptococci & $\mathrm{col} / 100 \mathrm{ml}$ & & & & & & & & \\
\hline \multicolumn{2}{|l|}{ Oxygen, dissolved } & & & & & & & & \\
\hline $\begin{array}{l}\text { Biochemical oxygen demand } \\
\text { pH } 5 \text {-day) }\end{array}$ & $\begin{array}{r}\mathrm{mg} / \mathrm{L} \\
\text { units }\end{array}$ & 27 & 8.2 & 7.2 & 7.7 & 7.7 & 0.2 & 8.1 & 7.4 \\
\hline $\mathrm{Co}-\mathrm{Pt}$ & $t$ units & 13 & 15 & 2 & 6 & 5 & 4 & 11 & 2 \\
\hline
\end{tabular}


Results of regression analyses relating concentrations of selected chemical constituents to specific conductance (SpC)

\begin{tabular}{|c|c|c|c|c|c|}
\hline $\begin{array}{c}\text { Dissolved } \\
\text { constituents } \\
(\mathrm{mg} / \mathrm{L})\end{array}$ & $\begin{array}{l}\text { No. of } \\
\text { measure- } \\
\text { ments }\end{array}$ & Regression equation & $\begin{array}{l}\text { Corre- } \\
\text { lation } \\
\text { coeffi- } \\
\text { cient }\end{array}$ & $\begin{array}{l}\text { Percent } \\
\text { explained } \\
\text { variance }\end{array}$ & $\begin{array}{c}\text { Standard } \\
\text { error of } \\
\text { estimate } \\
(\mathrm{mg} / \mathrm{L})\end{array}$ \\
\hline Dissolved solids, residue (RDS) & 27 & $\mathrm{RDS}=0.646(\mathrm{SpC})+19.564$ & 0.99 & 98 & 9.6 \\
\hline Dissolved solids, sum (SDS) & ---- & & & & - \\
\hline Hardness as $\mathrm{CaCO}_{3}(\mathrm{TH})$ & 27 & $\mathrm{TH}=0.296(\mathrm{SpC})+27.425$ & .98 & 96 & 6.2 \\
\hline Calcium (Ca) & 27 & $\mathrm{Ca}=0.076(\mathrm{spC})+12.902$ & .95 & 90 & 2.8 \\
\hline Magnesium (Mg) & 27 & $\mathrm{Mg}=0.026(\mathrm{spC})-1.123$ & .89 & 80 & 1.4 \\
\hline Sodium $(\mathrm{Na})$ & 27 & $\mathrm{Na}=0.110(\mathrm{SpC})-20.273$ & .98 & 96 & 2.4 \\
\hline Potassium (K) & 27 & $K=0.006(\mathrm{spC})+5.917$ & .54 & 29 & 1.0 \\
\hline Bicarbonate $\left(\mathrm{HCO}_{3}\right)$ & 27 & $\mathrm{HCO}_{3}=0.177(\mathrm{SpC})+99.996$ & .86 & 74 & 11 \\
\hline Sulfate $\left(\mathrm{SO}_{4}\right)$ & 27 & $\mathrm{SO}_{4}=0.328(\mathrm{SpC})-76.430$ & .98 & 96 & 6.8 \\
\hline Chloride (C1) & 27 & $\mathrm{Cl}=0.037(\mathrm{SpC})-7.958$ & .97 & 94 & 1.0 \\
\hline Silica (Si) & 27 & $\mathrm{Si}=-0.030(\mathrm{SpC})+51.649$ & -.45 & 21 & 6.3 \\
\hline
\end{tabular}

Results of regression analyses relating specific conductance to water discharge (Q)

\begin{tabular}{ccccccc}
\hline $\begin{array}{c}\text { No. of Water discharge } \\
\text { measure- } \\
\text { ments }\end{array}$ & Maximum Minimum & Regression equation & $\begin{array}{c}\text { Correlation } \\
\text { coefficient }\end{array}$ & $\begin{array}{c}\text { Percent } \\
\text { explained } \\
\text { variance }\end{array}$ & $\begin{array}{c}\text { Standard error } \\
\text { of estimate }\end{array}$ \\
\hline 27 & $14,600 \quad 770$ & $\mathrm{SpC}=467 \mathrm{Q}^{-0.02971}$ & $* 0.08$ & 0.61 & 0.113 & 26 \\
\hline
\end{tabular}

* Not significant at the 95 percent confidence level; the regression equation should not be used to predict the concentration of the dependent variable. 
Location. -- Lat $41^{\circ} 27^{\prime} 10^{\prime \prime}$, long $96^{\circ} 45^{\prime} 50^{\prime \prime}$, in $\mathrm{SE}^{\frac{1}{4}} \mathrm{sec}$ 7, T.17 N., R.6 E., Dodge County, Hydrologic Unit 10200201, on left bank $80 \mathrm{ft}$ upstream from bridge (new bridge in use during 1976) on State Highway 79, $1 \mathrm{mi}$ south of North Bend, and $5 \mathrm{mi}$ downstream from Shell Creek.

Drainage area (revised). $--77,100 \mathrm{mi}^{2}$, approximately, of which about $63,300 \mathrm{mi}^{2}$ contributes directly to surface runoff. Approximately $4,000 \mathrm{mi}^{2}$ in Great Divide Bas in is not included.

Period of record. --Water year 1973 to current year.

Statistical data for selected chemical constituents

\begin{tabular}{|c|c|c|c|c|c|c|c|c|c|}
\hline Constituent & Units & $\begin{array}{l}\text { No. of } \\
\text { measure- } \\
\text { ments }\end{array}$ & $\begin{array}{l}\text { Max- } \\
\text { imum }\end{array}$ & $\begin{array}{l}\text { Min- } \\
\text { imum }\end{array}$ & Mean & Median & $\begin{array}{c}\text { Standard } \\
\text { devi- } \\
\text { ation }\end{array}$ & $\begin{array}{l}\text { Ninetieth } \\
\text { percent- } \\
\text { ile }\end{array}$ & $\begin{array}{c}\text { Tenth } \\
\text { percent- } \\
\text { ile }\end{array}$ \\
\hline Specific conductance & umho/cm & 75 & 833 & 192 & 461 & 453 & 136 & 586 & 300 \\
\hline Dissolved solids, residue & $\mathrm{mg} / \mathrm{L}$ & 31 & 390 & 136 & 275 & 295 & 74 & 350 & 141 \\
\hline Dissolved solids, sum & $\mathrm{mg} / \mathrm{L}$ & 62 & 556 & 194 & 325 & 320 & 70 & 420 & 245 \\
\hline Hardness as $\mathrm{CaCO}_{3}$ & $\mathrm{mg} / \mathrm{L}$ & 70 & 280 & 80 & 167 & 169 & 40 & 222 & 123 \\
\hline Calcium, dissolved & $\mathrm{mg} / \mathrm{L}$ & 70 & 78 & 25 & 48 & 48 & 11 & 62 & 36 \\
\hline Magnesium, dissolved & $\mathrm{mg} / \mathrm{L}$ & 70 & 20 & 4.3 & 11 & 12 & 3.3 & 16 & 7.2 \\
\hline Sodium, dissolved & $\mathrm{mg} / \mathrm{L}$ & 70 & 66 & 3.1 & 30 & 31 & 13 & 44 & 12 \\
\hline Potassium, dissolved & $\mathrm{mg} / \mathrm{L}$ & 70 & 17 & 5.2 & 9.0 & 9.0 & 1.5 & 10 & 7.6 \\
\hline Bicarbonate ion & $\mathrm{mg} / \mathrm{L}$ & 69 & 264 & 92 & 181 & 184 & 33 & 218 & 140 \\
\hline Sulfate, dissolved & $\mathrm{mg} / \mathrm{L}$ & 70 & 220 & 8.5 & 73 & 71 & 39 & 120 & 19 \\
\hline Chloride, dissolved & $\mathrm{mg} / \mathrm{L}$ & 70 & 24 & 1.6 & 11 & 10 & 5.0 & 18 & $4: 0$ \\
\hline Fluoride, dissolved & $\mathrm{mg} / \mathrm{L}$ & 70 & 0.9 & 0.3 & 0.4 & 0.4 & 0.1 & 0.5 & 0.4 \\
\hline Silica, dissolved & $\mathrm{mg} / \mathrm{L}$ & 70 & 53 & 7.5 & 34 & 35 & 8.3 & 44 & 24 \\
\hline Boron, dissolved & $\mathrm{mg} / \mathrm{L}$ & 68 & 160 & 0 & 69 & 70 & 27 & 94 & 42 \\
\hline Iron, dissolved & $\mathrm{ug} / \mathrm{L}$ & 64 & 760 & 0 & 69 & 36 & 115 & 125 & 12 \\
\hline Manganese, dissolved & $\mathrm{ug} / \mathrm{L}$ & 64 & 120 & 0 & 16 & 10 & 22 & 48 & 0 \\
\hline $\begin{array}{l}\text { Nitrite + nitrate as } \mathrm{N}, \\
\text { dissolved } \\
\text { Nitrate as } \mathrm{N} \text {, dissolved }\end{array}$ & $\mathrm{mg} / \mathrm{L}$ & $\begin{array}{r}42 \\
1\end{array}$ & 1.2 & 0.00 & $\begin{array}{l}0.43 \\
0.80\end{array}$ & 0.40 & 0.36 & 0.90 & 0.00 \\
\hline Nitrate as $\mathrm{NO}_{3}$, dissolved & $\mathrm{mg} / \mathrm{L}$ & & & & & & & & 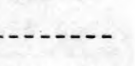 \\
\hline Ammonia $\mathrm{N}$, total & $\mathrm{mg} / \mathrm{L}$ & 52 & 0.39 & 0.00 & 0.08 & 0.06 & 0.08 & 0.17 & 0.00 \\
\hline Organic $N$, total & $\mathrm{mg} / \mathrm{L}$ & 53 & 4.7 & 0.32 & 1.1 & 1.0 & 0.74 & 2.0 & 0.45 \\
\hline Phosphorus, total & $\mathrm{mg} / \mathrm{L}$ & 56 & 0.73 & 0.14 & 0.32 & 0.28 & 0.13 & 0.49 & 0.19 \\
\hline Phosphorus, dissolved & $\mathrm{mg} / \mathrm{L}$ & 62 & 0.30 & 0.06 & 0.16 & 0.16 & 0.06 & 0.24 & 0.09 \\
\hline Fecal coliform & $\mathrm{col} / 100 \mathrm{ml}$ & 42 & 113,000 & 3 & 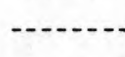 & 215 & 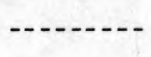 & 680 & 27 \\
\hline Fecal streptococci & $\mathrm{col} / 100 \mathrm{ml}$ & 50 & 13,600 & 20 & $\cdots-\cdots$ & 188 & 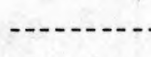 & 1,100 & 45 \\
\hline Oxygen, dissolved & $\mathrm{mg} / \mathrm{L}$ & 52 & 15.0 & 6.8 & 10.4 & 10.1 & 1.9 & 12.6 & 8.0 \\
\hline \multirow{2}{*}{$\begin{array}{l}\text { Biochemical oxygen demand } \\
\mathrm{pH}^{(5-\text { day })}\end{array}$} & $\mathrm{mg} / \mathrm{L}$ & 54 & 10.0 & 0.8 & 4.4 & 4.1 & 2.1 & 6.8 & 1.8 \\
\hline & $H$ units & 80 & 8.5 & 7.0 & 7.7 & 7.8 & 0.4 & 8.3 & 7.2 \\
\hline $\mathrm{Co}-\mathrm{Pt}$ & Co - Pt units & 63 & 90 & 3 & 23 & 20 & 18 & 49 & 8 \\
\hline
\end{tabular}


Results of regression analyses relating concentrations of selected chemical constituents to specific conductance $(\mathrm{SpC})$

\begin{tabular}{|c|c|c|c|c|c|}
\hline $\begin{array}{c}\text { Dissolved } \\
\text { constituents } \\
\text { (mg/L) }\end{array}$ & $\begin{array}{l}\text { No. of } \\
\text { measure- } \\
\text { ments }\end{array}$ & Regression equation & $\begin{array}{l}\text { Corre- } \\
\text { lation } \\
\text { coeffi- } \\
\text { cient }\end{array}$ & $\begin{array}{l}\text { Percent } \\
\text { explained } \\
\text { variance }\end{array}$ & $\begin{array}{c}\text { Standard } \\
\text { error of } \\
\text { estimate } \\
(\mathrm{mg} / \mathrm{L})\end{array}$ \\
\hline Dissolved solids, residue (RDS) & 33 & $\mathrm{RDS}=0.662(\mathrm{SpC})+8.194$ & 0.98 & 97 & 13 \\
\hline Dissolved solids, sum (SDS) & 63 & $\mathrm{SDS}=0.575(\mathrm{SpC})+43.080$ & .98 & 96 & 14 \\
\hline Hardness as $\mathrm{CaCO}_{3}(\mathrm{TH})$ & 71 & $\mathrm{TH}=0.283(\mathrm{SpC})+35.358$ & .97 & 93 & 10 \\
\hline Calcium (Ca) & 71 & $\mathrm{Ca}=0.074(\mathrm{SpC})+13.721$ & .95 & 90 & 3.4 \\
\hline Magnesium (Mg) & 71 & $\mathrm{Mg}=0.024(\mathrm{SpC})+0.209$ & .96 & 92 & 0.9 \\
\hline Sodium $(\mathrm{Na})$ & 71 & $\mathrm{Na}=0.093(\mathrm{SpC})-13.597$ & .97 & 94 & 3.2 \\
\hline Potassium (K) & 71 & $K=0.003(\mathrm{SpC})+7.607$ & 27 & 7.4 & 1.5 \\
\hline Bicarbonate $\left(\mathrm{HCO}_{3}\right)$ & 71 & $\mathrm{HCO}_{3}=0.203(\mathrm{SpC})+86.951$ & .84 & 70 & 18 \\
\hline Sulfate $\left(\mathrm{SO}_{4}\right)$ & 72 & $\mathrm{SO}_{4}=0.273(\mathrm{SpC})-54.453$ & .95 & 90 & 13 \\
\hline Chloride (Cl) & 73 & $\mathrm{Cl}=0.033(\mathrm{SpC})-4.304$ & .89 & 79 & 2.3 \\
\hline Silica (Si) & 72 & $\mathrm{Si}=0.008(\mathrm{SpC})+31.212$ & $* .13$ & 1.7 & 8.4 \\
\hline Nitrite + Nitrate as $\mathrm{N}\left(\mathrm{NO}_{2}-\mathrm{NO}_{3}\right)$ & 42 & $\mathrm{NO}_{2}-\mathrm{NO}_{3}=0.0005(\mathrm{SpC})+0.156$ & $* .18$ & 3.3 & 0.36 \\
\hline
\end{tabular}

* Not significant at the 95 percent confidence level; the regression equation should not be used to predict the concentration of the dependent variable.

Results of regression analyses relating specific conductance to water discharge (Q)

\begin{tabular}{|c|c|c|c|c|c|c|}
\hline \multirow{2}{*}{$\begin{array}{l}\text { No. of } \\
\text { measure- } \\
\text { ments } \\
\end{array}$} & \multirow{2}{*}{$\begin{array}{l}\text { Water discharge } \\
\frac{\left(\mathrm{ft}^{3} / \mathrm{s}\right)}{\text { Maximum Minimum }}\end{array}$} & \multirow{2}{*}{ Regression equation } & \multirow{2}{*}{$\begin{array}{l}\text { Correlation } \\
\text { coefficient }\end{array}$} & \multirow{2}{*}{$\begin{array}{l}\text { Percent } \\
\text { explained } \\
\text { variance } \\
\end{array}$} & \multicolumn{2}{|c|}{$\begin{array}{c}\text { Standard error } \\
\text { of estimate }\end{array}$} \\
\hline & & & & & Log units & Percent \\
\hline 76 & 73,600 & $\mathrm{SpC}=898 \mathrm{Q}^{-0.08550}$ & -0.30 & 9.4 & 0.129 & 30 \\
\hline
\end{tabular}




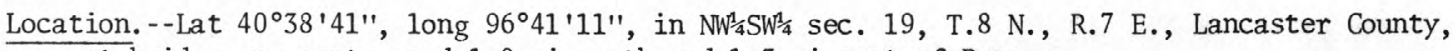
at bridge on county road $1.0 \mathrm{mi}$ south and $1.3 \mathrm{mi}$ east of Roca.

Period of record.--August 1971 to current year.

Statistical data for selected chemical constituents

\begin{tabular}{|c|c|c|c|c|c|c|c|c|c|}
\hline Constituent & Units & $\begin{array}{l}\text { No. of } \\
\text { measure- } \\
\text { ments }\end{array}$ & $\begin{array}{l}\text { Max- } \\
\text { imum }\end{array}$ & $\begin{array}{l}\text { Min- } \\
\text { imum }\end{array}$ & Mean & Median & $\begin{array}{c}\text { Standard } \\
\text { devi- } \\
\text { ation }\end{array}$ & $\begin{array}{c}\text { Ninetieth } \\
\text { percent- } \\
\text { ile }\end{array}$ & $\begin{array}{c}\text { Tenth } \\
\text { percent- } \\
\text { ile }\end{array}$ \\
\hline Specific conductance & umho/cm & 25 & 2,100 & 414 & 1,370 & 1,370 & 489 & 1,950 & 480 \\
\hline Dissolved solids, residue & $\mathrm{mg} / \mathrm{L}$ & 25 & 1,270 & 255 & 845 & 840 & 306 & 1,200 & 320 \\
\hline Dissolved solids, sum & $\mathrm{mg} / \mathrm{L}$ & 12 & 1,290 & 236 & 835 & 830 & 335 & 1,200 & 265 \\
\hline Hardness as $\mathrm{CaCO}_{3}$ & $\mathrm{mg} / \mathrm{L}$ & 16 & 410 & 150 & 317 & 335 & 84 & 393 & 160 \\
\hline Calcium, dissolved & $\mathrm{mg} / \mathrm{L}$ & 16 & 120 & 40 & 92 & 100 & 26 & 116 & 44 \\
\hline Magnesium, dissolved & $\mathrm{mg} / \mathrm{L}$ & 16 & 27 & 11 & 21 & 23 & 5.6 & 26 & 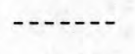 \\
\hline Sodium, dissolved & $\mathrm{mg} / \mathrm{L}$ & 16 & 310 & 25 & 173 & 170 & 90 & 300 & 27 \\
\hline Potassium, dissolved & $\mathrm{mg} / \mathrm{L}$ & 16 & 13 & 5.7 & 8.1 & 7.7 & 2.0 & 12 & 6.2 \\
\hline Bicarbonate ion & $\mathrm{mg} / \mathrm{L}$ & 16 & 311 & 139 & 252 & 270 & 57 & 303 & 156 \\
\hline Sulfate, dissolved & $\mathrm{mg} / \mathrm{L}$ & 16 & 230 & 45 & 137 & 140 & 55 & 200 & 49 \\
\hline Chloride, dissolved & $\mathrm{mg} / \mathrm{L}$ & 25 & 450 & 21 & 232 & 220 & 130 & 410 & 36 \\
\hline Fluoride, dissolved & $\mathrm{mg} / \mathrm{L}$ & 16 & 0.6 & 0.1 & 0.4 & 0.5 & 0.1 & 0.5 & 0.3 \\
\hline Silica, dissolved & $\mathrm{mg} / \mathrm{L}$ & 12 & 25 & 9.2 & 17 & 18 & 5.0 & 22 & 12 \\
\hline Boron, dissolved & $\mathrm{mg} / \mathrm{L}$ & 12 & 660 & 20 & 224 & 150 & 209 & 540 & 40 \\
\hline Iron, dissolved & $\mathrm{ug} / \mathrm{L}$ & 4 & 60 & 0 & 20 & 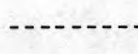 & 27 & $\ldots \ldots$ & $\cdots$ \\
\hline Manganese, dissolved & $\mathrm{ug} / \mathrm{L}$ & 4 & 770 & 40 & 375 & 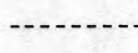 & 302 & & -- \\
\hline $\begin{array}{l}\text { Nitrite }+ \text { nitrate as } \mathrm{N}, \\
\text { dissolved } \\
\text { Nitrate as } N \text {, dissolved }\end{array}$ & $\begin{array}{l}\mathrm{mg} / \mathrm{L} \\
\mathrm{mg} / \mathrm{L}\end{array}$ & 16 & 4.3 & 0.00 & 1.1 & 0.76 & 1.1 & 2.0 & 0.06 \\
\hline Nitrate as $\mathrm{NO}_{3}$, dissolved & $\mathrm{mg} / \mathrm{L}$ & & & & & & & & -- \\
\hline Ammonia $\mathrm{N}$, total & $\mathrm{mg} / \mathrm{L}$ & 20 & 0.90 & 0.00 & 0.18 & 0.11 & 0.20 & 0.49 & 0.01 \\
\hline Organic $N$, total & $\mathrm{mg} / \mathrm{L}$ & 25 & 1.9 & 0.18 & 0.84 & 0.79 & 0.48 & 1.5 & 0.30 \\
\hline Phosphorus, total & $\mathrm{mg} / \mathrm{L}$ & 25 & 0.97 & 0.09 & 0.39 & 0.37 & 0.18 & 0.57 & 0.19 \\
\hline Phosphorus, dissolved & $\mathrm{mg} / \mathrm{L}$ & 22 & 0.45 & 0.14 & 0.25 & 0.24 & 0.08 & 0.38 & 0.17 \\
\hline Fecal coliform & $\mathrm{col} / 100 \mathrm{ml}$ & 21 & 38,000 & 16 & $\ldots$ & 317 & $\ldots$ & 965 & 120 \\
\hline Fecal streptococci & $\mathrm{col} / 100 \mathrm{ml}$ & 24 & 410,000 & 100 & 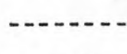 & 1,070 & $-\cdots$ & $--4,100$ & 270 \\
\hline Oxygen, dissolved & $\mathrm{mg} / \mathrm{L}$ & 25 & 15.3 & 5.8 & 9.6 & 9.5 & 2.8 & 13.0 & 6.4 \\
\hline $\begin{array}{l}\text { Biochemical oxygen demand } \\
\text { (5-day) }\end{array}$ & $\mathrm{mg} / \mathrm{L}$ & 24 & 9.0 & 1.0 & 3.3 & 2.7 & 2.0 & 5.1 & 1.4 \\
\hline $\mathrm{pH}$ & pH units & 32 & 8.2 & 6.5 & 7.6 & 7.7 & 0.4 & 8.0 & 7.3 \\
\hline Co - Pt & Co - Pt units & 16 & 200 & 5 & 37 & 11 & 64 & 51 & 0 \\
\hline
\end{tabular}


Results of regression analyses relating concentrations of selected chemical constituents to specific conductance $(\mathrm{SpC})$

\begin{tabular}{|c|c|c|c|c|c|}
\hline $\begin{array}{c}\text { Dissolved } \\
\text { constituents } \\
\text { (mg/L) }\end{array}$ & $\begin{array}{l}\text { No. of } \\
\text { measure- } \\
\text { ments }\end{array}$ & Regression equation & $\begin{array}{l}\text { Corre- } \\
\text { lation } \\
\text { coeffi- } \\
\text { cient }\end{array}$ & $\begin{array}{l}\text { Percent } \\
\text { explained } \\
\text { variance }\end{array}$ & $\begin{array}{c}\text { Standard } \\
\text { error of } \\
\text { estimate } \\
(\mathrm{mg} / \mathrm{L})\end{array}$ \\
\hline Dissolved solids, residue (RDS) & 25 & $\mathrm{RDS}=0.621(\mathrm{SpC})-4.586$ & 0.99 & 99 & 35 \\
\hline Dissolved solids, sum (SDS) & $-\cdots-$ & $---1--1--1$ & & & \\
\hline Hardness as $\mathrm{CaCO}_{3}(\mathrm{TH})$ & 16 & $\mathrm{TH}=0.146(\mathrm{SpC})+113.965$ & .94 & 89 & 29 \\
\hline Calcium (Ca) & 16 & $\mathrm{Ca}=0.044(\mathrm{SpC})+30.966$ & .94 & 88 & 9.2 \\
\hline Magnesium (Mg) & 16 & $\mathrm{Mg}=0.010(\mathrm{SpC})+7.373$ & .95 & 90 & 1.8 \\
\hline Sodium (Na) & 16 & $\mathrm{Na}=0.163(\mathrm{SpC})-54.745$ & .99 & 98 & 14 \\
\hline Potassium (K) & 16 & $K=0.0005(\mathrm{SpC})+7.496$ & $* .12$ & 1.6 & 2.1 \\
\hline Bicarbonate $\left(\mathrm{HCO}_{3}\right)$ & 16 & $\mathrm{HCO}_{3}=0.063(\mathrm{SpC})+163.313$ & .61 & 37 & 46 \\
\hline Sulfate $\left(\mathrm{SO}_{4}\right)$ & 16 & $\mathrm{SO}_{4}=0.097(\mathrm{SpC})+2.166$ & .96 & 92 & 16 \\
\hline Chloride (CI) & 25 & $\mathrm{Cl}=0.261(\mathrm{SpC})-124.435$ & .98 & 95 & 28 \\
\hline Silica (Si) & 12 & $\mathrm{Si}=0.006(\mathrm{SpC})+9.033$ & .66 & 44 & 3.9 \\
\hline Nitrite + Nitrate as $\mathrm{N}\left(\mathrm{NO}_{2}-\mathrm{NO}_{3}\right)$ & 16 & $\mathrm{NO}_{2}-\mathrm{NO}_{3}=0.00004(\mathrm{SpC})+1.005$ & $* .02$ & 0.04 & 1.1 \\
\hline
\end{tabular}

* Not significant at the 95 percent confidence level; the regression equation should not be used to predict the concentration of the dependent variable.

Results of regression analyses relating specific conductance to water discharge (Q)

\begin{tabular}{ccccccc}
\hline $\begin{array}{l}\text { No. of } \\
\text { measure- } \\
\text { ments }\end{array}$ & $\begin{array}{c}\text { Mater discharge } \\
\left(\mathrm{ft}^{3} / \mathrm{s}\right)\end{array}$ & Maximum Minimum & Regression equation & $\begin{array}{c}\text { Correlation } \\
\text { coefficient }\end{array}$ & $\begin{array}{c}\text { Percent } \\
\text { explained } \\
\text { variance }\end{array}$ & $\begin{array}{c}\text { Standard error } \\
\text { of estimate }\end{array}$ \\
\hline 25 & 225 & $\mathrm{SpC}=2,817 \mathrm{Q}^{-0.34398}$ & -0.83 & 69 & 0.114 \\
\hline
\end{tabular}


Location. --Lat $40^{\circ} 46^{\prime} 13^{\prime \prime}$, long $96^{\circ} 43^{\prime} 05^{\prime \prime}$, in $\mathrm{SW}^{\frac{1}{4}} \mathrm{SW}^{\frac{1}{4}} \mathrm{sec}$. 2, T.9 N., R.6 E., Lancaster County, Hydrologic Unit 10200203, at county road bridge $0.9 \mathrm{mi}$ west of U.S. Highway 77 and northeast corner of State Penitentiary at Lincoln.

Drainage area. $--221 \mathrm{mi}^{2}$.

Period of record. --Water year 1971 to current year.

Statistical data for selected chemical constituents

\begin{tabular}{|c|c|c|c|c|c|c|c|c|c|}
\hline Constituent & Units & $\begin{array}{l}\text { No. of } \\
\text { measure- } \\
\text { ments }\end{array}$ & $\begin{array}{l}\text { Max- } \\
\text { imum }\end{array}$ & $\begin{array}{l}\text { Min- } \\
\text { imum }\end{array}$ & Mean & Median & $\begin{array}{c}\text { Standard } \\
\text { devi- } \\
\text { ation }\end{array}$ & $\begin{array}{l}\text { Ninetieth } \\
\text { percent- } \\
\text { ile }\end{array}$ & $\begin{array}{l}\text { Tenth } \\
\text { percent- } \\
\text { ile }\end{array}$ \\
\hline Specific conductance & umho/cm & 89 & 2,500 & 347 & 1,190 & 1,190 & 491 & 1,780 & 477 \\
\hline Dissolved solids, residue & $\mathrm{mg} / \mathrm{L}$ & 77 & 1,420 & 216 & 736 & 760 & 281 & 1,100 & 320 \\
\hline Dissolved solids, sum & $\mathrm{mg} / \mathrm{L}$ & 32 & 1,380 & 207 & 686 & 703 & 286 & 1,000 & 300 \\
\hline Hardness as $\mathrm{CaCO}_{3}$ & $\mathrm{mg} / \mathrm{L}$ & 47 & 440 & 110 & 290 & 308 & 81 & 380 & 160 \\
\hline Calcium, dissolved & $\mathrm{mg} / \mathrm{L}$ & 47 & 130 & 25 & 83 & 91 & 24 & 113 & 46 \\
\hline Magnesium, dissolved & $\mathrm{mg} / \mathrm{L}$ & 47 & 29 & 8 & 20 & 21 & 5.2 & 26 & 12 \\
\hline Sodium, dissolved & $\mathrm{mg} / \mathrm{L}$ & 47 & 320 & 20 & 135 & 147 & 75 & 230 & 38 \\
\hline Potassium, dissolved & $\mathrm{mg} / \mathrm{L}$ & 47 & 14 & 5.2 & 8.0 & 7.8 & 1.7 & 10 & 6.0 \\
\hline Bicarbonate ion & $\mathrm{mg} / \mathrm{L}$ & 93 & 348 & 130 & 281 & 294 & 53 & 328 & 185 \\
\hline Sulfate, dissolved & $\mathrm{mg} / \mathrm{L}$ & 93 & 260 & 32 & 125 & 120 & 48 & 185 & 63 \\
\hline Chloride, dissolved & $\mathrm{mg} / \mathrm{L}$ & 106 & 480 & 14 & 160 & 150 & 109 & 308 & 32 \\
\hline Fluoride, dissolved & $\mathrm{mg} / \mathrm{L}$ & 44 & 0.9 & 0.0 & 0.4 & 0.4 & 0.1 & 0.5 & 0.3 \\
\hline Silica, dissolved & $\mathrm{mg} / \mathrm{L}$ & 33 & 29 & 4.8 & 16 & 16 & 5.5 & 22 & 8.2 \\
\hline Boron, dissolved & $\mathrm{mg} / \mathrm{L}$ & 21 & 730 & 10 & 166 & 110 & 172 & 350 & 55 \\
\hline Iron, dissolved & $\mathrm{ug} / \mathrm{L}$ & 28 & 160 & 0 & 31 & 20 & 33 & 66 & 10 \\
\hline Manganese, dissolved & $\mathrm{ug} / \mathrm{L}$ & 29 & 1,300 & 10 & 398 & 320 & 310 & 905 & 20 \\
\hline $\begin{array}{l}\text { Nitrite + nitrate as } \mathrm{N} \text {, } \\
\text { dissolved }\end{array}$ & $\mathrm{mg} / \mathrm{L}$ & 45 & 2.3 & 0.00 & 0.79 & 0.70 & 0.72 & 1.8 & 0.02 \\
\hline Nitrate as $\mathrm{N}$, dissolved & $\mathrm{mg} / \mathrm{L}$ & 7 & 2.1 & 0.10 & 0.72 & 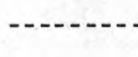 & 0.76 & & $\cdots$ \\
\hline Nitrate as $\mathrm{NO}_{3}$, dissolved & $\mathrm{mg} / \mathrm{L}$ & 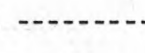 & -4 & & - & & & & 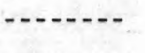 \\
\hline Ammonia $\mathrm{N}$, total & $\mathrm{mg} / \mathrm{L}$ & 69 & 1.1 & 0.00 & 0.17 & 0.11 & 0.18 & 0.41 & 0.02 \\
\hline Organic $N$, total & $\mathrm{mg} / \mathrm{L}$ & 103 & 7.0 & 0.00 & 0.88 & 0.58 & 0.93 & 1.9 & 0.21 \\
\hline Phosphorus, total & $\mathrm{mg} / \mathrm{L}$ & 103 & 2.4 & 0.09 & 0.46 & 0.37 & 0.35 & 0.66 & 0.23 \\
\hline Phosphorus, dissolved & $\mathrm{mg} / \mathrm{L}$ & 82 & 0.78 & 0.02 & 0.27 & 0.25 & 0.12 & 0.40 & 0.16 \\
\hline Fecal coliform & $\mathrm{col} / 100 \mathrm{ml}$ & 96 & 48,000 & 4 & 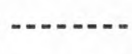 & 474 & 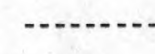 & 7,450 & 27 \\
\hline Fecal streptococci & $\mathrm{col} / 100 \mathrm{ml}$ & 81 & 240,000 & 8 & - n-n & 560 & 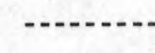 & 5,200 & 67 \\
\hline Oxygen, dissolved & $\mathrm{mg} / \mathrm{L}$ & 108 & 15.0 & 5.4 & 9.6 & 9.3 & 2.6 & 13.4 & 6.7 \\
\hline Biochemical oxygen demand & $\mathrm{mg} / \mathrm{L}$ & 92 & 16.6 & 1.2 & 4.0 & 3.5 & 2.4 & 6.5 & 1.8 \\
\hline $\mathrm{pH}^{(5-\text { day })} \quad \mathrm{pH}$ & H units & 139 & 9.2 & 6.6 & 7.6 & 7.7 & 0.4 & 8.0 & 7.2 \\
\hline Co $-\mathrm{Pt}$ & t units & 43 & 200 & 3 & 29 & 21 & 37 & 69 & 7 \\
\hline
\end{tabular}


06803080 Salt Creek above Beal Slough, at Lincoln, Nebr.--Continued

Results of regression analyses relating concentrations of selected chemical constituents to specific conductance $(\mathrm{SpC})$

\begin{tabular}{|c|c|c|c|c|c|}
\hline $\begin{array}{l}\text { Dissolved } \\
\text { constituents } \\
(\mathrm{mg} / \mathrm{L})\end{array}$ & $\begin{array}{l}\text { No. of } \\
\text { measure- } \\
\text { ments }\end{array}$ & Regression equation & $\begin{array}{l}\text { Corre- } \\
\text { lation } \\
\text { coeffi- } \\
\text { cient }\end{array}$ & $\begin{array}{l}\text { Percent } \\
\text { explained } \\
\text { variance }\end{array}$ & $\begin{array}{c}\text { Standard } \\
\text { error of } \\
\text { estimate } \\
(\mathrm{mg} / \mathrm{L})\end{array}$ \\
\hline Dissolved solids, residue (RDS) & 79 & $\mathrm{RDS}=0.551(\mathrm{SpC})+68.104$ & 0.97 & 95 & 66 \\
\hline Dissolved solids, sum (SDS) & 33 & $\mathrm{SDS}=0.558(\mathrm{SpC})+29.392$ & .96 & 92 & 82 \\
\hline Hardness as $\mathrm{CaCO}_{3}(\mathrm{TH})$ & 46 & $\mathrm{TH}=0.151(\mathrm{SpC})+106.896$ & .92 & 85 & 32 \\
\hline Calcium (Ca) & 46 & $\mathrm{Ca}=0.045(\mathrm{SpC})+29.411$ & .91 & 83 & 10 \\
\hline Magnesium (Mg) & 46 & $\mathrm{Mg}=0.010(\mathrm{SpC})+8.481$ & .91 & 83 & 2.2 \\
\hline Sodium (Na) & 46 & $\mathrm{Na}=0.146(\mathrm{SpC})-40.622$ & .96 & 93 & 20 \\
\hline Potassium (K) & 46 & $\mathrm{~K}=-0.001(\mathrm{SpC})+9.208$ & $*-.29$ & 8.3 & 1.6 \\
\hline Bicarbonate $\left(\mathrm{HCO}_{3}\right)$ & 71 & $\mathrm{HCO}_{3}=0.061(\mathrm{SpC})+196.876$ & .54 & 30 & 48 \\
\hline Sulfate $\left(\mathrm{SO}_{4}\right)$ & 73 & $\mathrm{SO}_{4}=0.098(\mathrm{SpC})+6.722$ & .93 & 86 & 20 \\
\hline Chloride (C1) & 87 & $\mathrm{Cl}=0.208(\mathrm{SpC})-76.424$ & .91 & 83 & 48 \\
\hline Silica (Si) & 33 & $\mathrm{Si}=0.004(\mathrm{SpC})+11.642$ & $* .33$ & 11 & 5.3 \\
\hline Nitrite + Nitrate as $\mathrm{N}\left(\mathrm{NO}_{2}-\mathrm{NO}_{3}\right)$ & 45 & $\mathrm{NO}_{2}-\mathrm{NO}_{3}=-0.001(\mathrm{SpC})+2.037$ & -.71 & 50 & 0.51 \\
\hline
\end{tabular}

* Not significant at the 95 percent confidence level; the regression equation should not be used to predict the concentration of the dependent variable.

Results of regression analyses relating specific conductance to water discharge $(Q)$

\begin{tabular}{ccccccc}
\hline $\begin{array}{c}\text { No. of } \\
\text { measure- } \\
\text { ments }\end{array}$ & $\begin{array}{c}\text { Water discharge } \\
\left(\mathrm{ft}^{3} / \mathrm{s}\right)\end{array}$ & Maximum Minimum & Regression equation & $\begin{array}{c}\text { Correlation } \\
\text { coefficient }\end{array}$ & $\begin{array}{c}\text { Percent } \\
\text { explained } \\
\text { variance }\end{array}$ & $\begin{array}{c}\text { Standard error } \\
\text { of estimate }\end{array}$ \\
\hline 89 & 491 & 3 & $\mathrm{SpC}=3,037 \mathrm{Q}^{-0.35480}$ & -0.85 & 72 & 0.109 \\
\hline
\end{tabular}


06803190 Salt Creek at 14 th Street at Lincoln, Nebr.

Location.--Lat $40^{\circ} 50^{\prime} 03^{\prime \prime}$, long 96 $42^{\prime} 03^{\prime \prime}$, in NE $\frac{1}{4} \mathrm{SE}^{\frac{1}{4}} \mathrm{sec}$. 14, T.10 N., R.6 E., Lancaster County, at bridge at 14 th Street, $2.1 \mathrm{mi}$ downstream from Middle Creek and $0.3 \mathrm{mi}$ upstream from confluence with Oak Creek.

Period of record.--March 1971 to current year.

Statistical data for selected chemical constituents

\begin{tabular}{|c|c|c|c|c|c|c|c|c|c|}
\hline Constituent & Units & $\begin{array}{l}\text { No. of } \\
\text { measure- } \\
\text { ments }\end{array}$ & $\begin{array}{l}\text { Max- } \\
\text { imum }\end{array}$ & $\begin{array}{l}\text { Min- } \\
\text { imum }\end{array}$ & Mean & Median & $\begin{array}{l}\text { Standard N } \\
\text { devi- } \\
\text { ation } \\
\end{array}$ & $\begin{array}{c}\text { Ninetieth } \\
\text { percent- } \\
\text { ile } \\
\end{array}$ & $\begin{array}{c}\text { Tenth } \\
\text { percent- } \\
\text { ile }\end{array}$ \\
\hline Specific conductance & $\mathrm{umh} \partial / \mathrm{cm}$ & 35 & 13,600 & 825 & 6,360 & 6,200 & 3,730 & 10,300 & 1,400 \\
\hline Dissolved solids, residue & $\mathrm{mg} / \mathrm{L}$ & 36 & 7,820 & 501 & 3,900 & 4,100 & 2,180 & 6,760 & 830 \\
\hline Dissolved solids, sum & $\mathrm{mg} / \mathrm{L}$ & 13 & 7,160 & 517 & 3,950 & 4,000 & 2,310 & 6,700 & 697 \\
\hline Hardness as $\mathrm{CaCO}_{3}$ & $\mathrm{mg} / \mathrm{L}$ & 26 & 560 & 140 & 347 & 380 & 113 & 470 & 255 \\
\hline Calcium, dissolved & $\mathrm{mg} / \mathrm{L}$ & 23 & 140 & 39 & 93 & 100 & 24 & 120 & 62 \\
\hline Magnesium, dissolved & $\mathrm{mg} / \mathrm{L}$ & 23 & 51 & 11 & 33 & 34 & 11 & 46 & 15 \\
\hline Sodium, dissolved & $\mathrm{mg} / \mathrm{L}$ & 24 & 2,800 & 100 & 1,430 & 1,620 & 797 & 2,430 & 290 \\
\hline Potassium, dissolved & $\mathrm{mg} / \mathrm{L}$ & 24 & 17 & 8.2 & 12 & 13 & 2.5 & 16 & 8.6 \\
\hline Bicarbonate ion & $\mathrm{mg} / \mathrm{L}$ & 26 & 396 & 161 & 310 & 340 & 75 & 390 & 200 \\
\hline Sulfate, dissolved & $\mathrm{mg} / \mathrm{L}$ & 26 & 620 & 65 & 292 & 315 & 153 & 465 & 112 \\
\hline Chloride, dissolved & $\mathrm{mg} / \mathrm{L}$ & 35 & 4,100 & 120 & 1,890 & 2,100 & 1,180 & 3,440 & 330 \\
\hline Fluoride, dissolved & $\mathrm{mg} / \mathrm{L}$ & 25 & 0.9 & 0.2 & 0.6 & 0.6 & 0.2 & 0.7 & 0.4 \\
\hline Silica, dissolved & $\mathrm{mg} / \mathrm{L}$ & 12 & 22 & 8.8 & 15 & 16 & 3.8 & 21 & 12 \\
\hline Boron, dissolved & $\mathrm{mg} / \mathrm{L}$ & 12 & 810 & 90 & 478 & 480 & 251 & 770 & 130 \\
\hline Iron, dissolved & $\mathrm{ug} / \mathrm{L}$ & 12 & 200 & 10 & 38 & 21 & 52 & 50 & 15 \\
\hline Manganese, dissolved & $\mathrm{ug} / \mathrm{L}$ & 12 & 810 & 80 & .384 & 440 & 237 & 640 & 110 \\
\hline $\begin{array}{l}\text { Nitrite }+ \text { nitrate as } \mathrm{N} \text {, } \\
\text { dissolved }\end{array}$ & $\mathrm{mg} / \mathrm{L}$ & 24 & 1.6 & 0.00 & 0.80 & 0.80 & 0.46 & 1.6 & 0.19 \\
\hline Nitrate as $N$, dissolved & $\mathrm{mg} / \mathrm{L}$ & 3 & 1.9 & 0.10 & 1.0 & $\cdots-\cdots$ & 0.90 & & $-\cdots$ \\
\hline Nitrate as $\mathrm{NO}_{3}$, dissolved & $\mathrm{mg} / \mathrm{L}$ & & 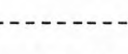 & 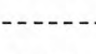 & & & & & \\
\hline Ammonia N, tota1 & $\mathrm{mg} / \mathrm{L}$ & 20 & 0.60 & 0.03 & 0.36 & 0.42 & 0.16 & 0.57 & 0.17 \\
\hline Organic $N$, total & $\mathrm{mg} / \mathrm{L}$ & 36 & 5.2 & 0.11 & 1.0 & 0.65 & 1.1 & 1.7 & 0.26 \\
\hline Phosphorus, tota1 & $\mathrm{mg} / \mathrm{L}$ & 36 & 1.6 & 0.20 & 0.47 & 0.40 & 0.31 & 0.66 & 0.20 \\
\hline Phosphorus, dissolved & $\mathrm{mg} / \mathrm{L}$ & 34 & 0.48 & 0.02 & 0.25 & 0.25 & 0.10 & 0.38 & 0.14 \\
\hline Fecal coliform & $/ 100 \mathrm{ml}$ & 30 & 120,000 & 10 & 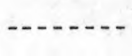 & 568 & $\ldots$ & 5,900 & 150 \\
\hline Fecal streptococci & $/ 100 \mathrm{ml}$ & 32 & 140,000 & 24 & - n & 910 & $\cdots$ & $-19,000$ & 150 \\
\hline Oxygen, dissolved & $\mathrm{mg} / \mathrm{L}$ & 37 & 18.4 & 5.0 & 10.8 & 10.2 & 2.7 & 14.3 & 7.2 \\
\hline \multirow{2}{*}{$\begin{array}{l}\text { Biochemica1 oxygen } \\
\mathrm{pH}^{(5-\text { day })}\end{array}$} & $\mathrm{mg} / \mathrm{L}$ & 36 & 9.6 & 1.2 & 3.6 & 3.1 & 2.0 & 6.1 & 1.5 \\
\hline & H units & 54 & 8.9 & 6.7 & 7.7 & 7.8 & 0.4 & 8.1 & 7.3 \\
\hline Co $-\mathrm{Pt}$ & $t$ units & 27 & 100 & 3 & 25 & 12 & 25 & 56 & 5 \\
\hline
\end{tabular}


06803190 Salt Creek at 14th Street at Lincoln, Nebr.--Continued

Results of regression analyses relating concentrations of selected chemical constituents to specific conductance $(\mathrm{SpC})$

\begin{tabular}{|c|c|c|c|c|c|}
\hline $\begin{array}{l}\text { Dissolved } \\
\text { constituents } \\
(\mathrm{mg} / \mathrm{L})\end{array}$ & $\begin{array}{l}\text { No. of } \\
\text { measure- } \\
\text { ments }\end{array}$ & Regression equation & $\begin{array}{l}\text { Corre- } \\
\text { lation } \\
\text { coeffi- } \\
\text { cient }\end{array}$ & $\begin{array}{l}\text { Percent } \\
\text { explained } \\
\text { variance }\end{array}$ & $\begin{array}{l}\text { Standard } \\
\text { error of } \\
\text { estimate } \\
(\mathrm{mg} / \mathrm{L})\end{array}$ \\
\hline Dissolved solids, residue (RDS) & 35 & $\mathrm{RDS}=0.583(\mathrm{SpC})+68.678$ & 0.99 & 98 & 287 \\
\hline Dissolved solids, sum (SDS) & & & & & \\
\hline Hardness as $\mathrm{CaCO}_{3}(\mathrm{TH})$. & 26 & $\mathrm{TH}=0.028(\mathrm{SpC})+162.801$ & .93 & 87 & 42 \\
\hline Calcium (Ca) & 23 & $\mathrm{Ca}=0.006(\mathrm{SpC})+49.893$ & .85 & 72 & 13 \\
\hline Magnesium (Mg) & 23 & $M g=0.003(\mathrm{SpC})+10.861$ & .96 & 92 & 3.2 \\
\hline Sodium (Na) & 24 & $\mathrm{Na}=0.217(\mathrm{SpC})-112.281$ & .99 & 99 & 80 \\
\hline Potassium (K) & 24 & $K=0.0004(\mathrm{SpC})+10.123$ & .52 & 27 & 2.2 \\
\hline Bicarbonate $\left(\mathrm{HCO}_{3}\right)$ & 26 & $\mathrm{HCO}_{3}=0.017(\mathrm{SpC})+198.589$ & .84 & 70 & 42 \\
\hline Sulfate $\left(\mathrm{SO}_{4}\right)$ & 26 & $\mathrm{SO}_{4}=0.039(\mathrm{SpC})+30.092$ & .99 & 98 & 20 \\
\hline Chloride (C1) & 35 & $\mathrm{Cl}=0.321(\mathrm{SpC})-208.640$ & 1.0 & 99 & 88 \\
\hline Silica (Si) & & 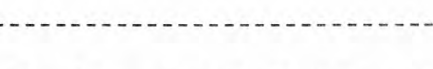 & & & \\
\hline Nitrite + Nitrate as $\mathrm{N}\left(\mathrm{NO}_{2}-\mathrm{NO}_{3}\right)$ & 23 & $\mathrm{NO}_{2}-\mathrm{NO}_{3}=-0.0001(\mathrm{SpC})+1.459$ & -.76 & 58 & 0.31 \\
\hline
\end{tabular}

Results of regression analyses relating specific conductance to water discharge (Q)

\begin{tabular}{ccccccc}
\hline $\begin{array}{c}\text { No. of } \\
\text { measure- } \\
\text { ments }\end{array}$ & $\begin{array}{c}\text { Water discharge } \\
\left(\mathrm{ft}^{3} / \mathrm{s}\right)\end{array}$ & Maximum Minimum & Regression equation & $\begin{array}{c}\text { Correlation } \\
\text { coefficient }\end{array}$ & $\begin{array}{c}\text { Percent } \\
\text { explained } \\
\text { variance }\end{array}$ & $\begin{array}{c}\text { Standard error } \\
\text { of estimate }\end{array}$ \\
\hline 35 & $593 \quad 8.1$ & $S p C=98,500 n^{-0.75825}$ & -0.96 & 92 & 0.096 \\
\hline
\end{tabular}


Location.--Lat $40^{\circ} 50^{\prime} 10^{\prime \prime}$, long $96^{\circ} 42^{\prime} 03^{\prime \prime}$, in $\mathrm{SE}^{\frac{1}{4}} \mathrm{NE}^{\frac{1}{4}} \mathrm{sec}$. 14, T.10 N., R. 6 E., Lancaster County, at bridge at 14th Street, $0.2 \mathrm{mi}$ upstream from confluence with Salt Creek.

Period of record. --March 1971 to current year.

Statistical data for selected chemical constituents

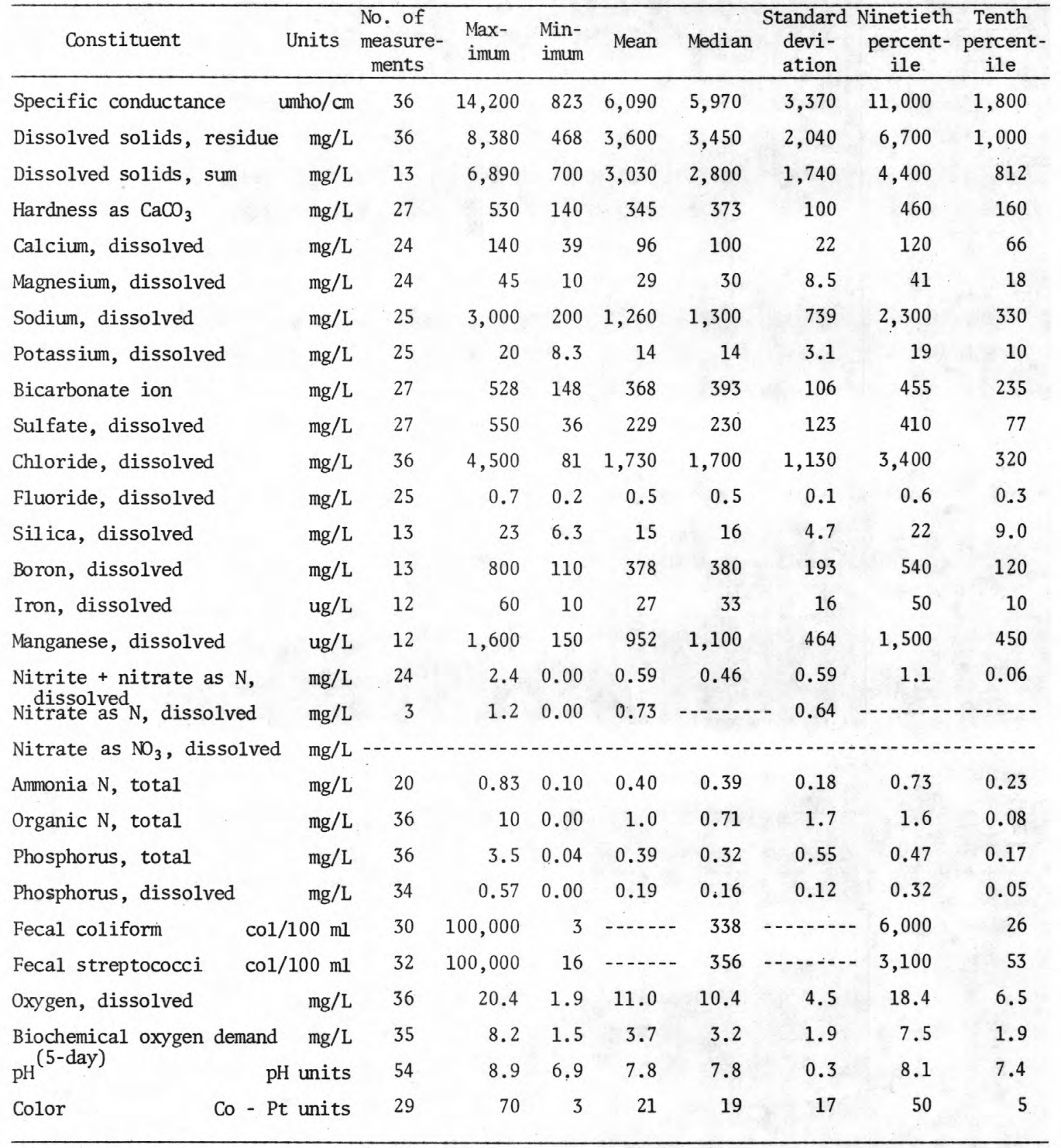


Results of regression analyses relating concentrations of selected chemical constituents to specific conductance $(\mathrm{SpC})$

\begin{tabular}{|c|c|c|c|c|c|}
\hline $\begin{array}{l}\text { Dissolved } \\
\text { constituents } \\
\text { (mg/L) }\end{array}$ & $\begin{array}{l}\text { No. of } \\
\text { measure- } \\
\text { ments }\end{array}$ & Regression equation & $\begin{array}{l}\text { Corre- } \\
\text { lation } \\
\text { coeffi- } \\
\text { cient }\end{array}$ & $\begin{array}{l}\text { Percent } \\
\text { explained } \\
\text { variance }\end{array}$ & $\begin{array}{l}\text { Standard } \\
\text { error of } \\
\text { estimate } \\
(\mathrm{mg} / \mathrm{L})\end{array}$ \\
\hline Dissolved solids, residue (RDS) & 36 & $\mathrm{RDS}=0.599(\mathrm{SpC})-54.144$ & 0.99 & 98 & 267 \\
\hline Dissolved solids, sum (SDS) & & $-\cdots$ & & & -1 \\
\hline Hardness as $\mathrm{CaCO}_{3}(\mathrm{TH})$ & 27 & $\mathrm{TH}=0.025(\mathrm{SpC})+189.699$ & .85 & 72 & 54 \\
\hline Calcium (Ca) & 24 & $\mathrm{Ca}=0.005(\mathrm{SpC})+61.377$ & .75 & 57 & 15 \\
\hline Magnesium (Mg) & 24 & $\mathrm{Mg}=0.002(\mathrm{SpC})+13.709$ & .90 & 81 & 3.8 \\
\hline Sodium $(\mathrm{Na})$ & 25 & $\mathrm{Na}=0.215(\mathrm{SpC})-129.099$ & .99 & 98 & 96 \\
\hline Potassium (K) & 25 & $\mathrm{~K}=0.0005(\mathrm{SpC})+10.253$ & .58 & 34 & 2.6 \\
\hline Bicarbonate $\left(\mathrm{HCO}_{3}\right)$ & 27 & $\mathrm{HCO}_{3}=0.024(\mathrm{SpC})+218.336$ & .78 & 61 & 68 \\
\hline Sulfate $\left(\mathrm{SO}_{4}\right)$ & 27 & $\mathrm{SO}_{4}=0.033(\mathrm{SpC})+24.995$ & .91 & 84 & 51 \\
\hline Chloride (Cl) & 36 & $\mathrm{Cl}=0.331(\mathrm{SpC})-282.043$ & .99 & 98 & 159 \\
\hline Silica (Si) & $-\cdots-1$ & - & & 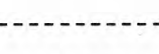 & \\
\hline Nitrite + Nitrate as $\mathrm{N}\left(\mathrm{NO}_{2}-\mathrm{NO}_{3}\right)$ & 24 & $\mathrm{NO}_{2}-\mathrm{NO}_{3}=-0.0001(\mathrm{SpC})+1.276$ & -.67 & 45 & 0.45 \\
\hline
\end{tabular}

Results of regression analyses relating specific conductance to water discharge (Q)

\begin{tabular}{|c|c|c|c|c|c|c|c|}
\hline \multirow{2}{*}{$\begin{array}{l}\text { No. of } \\
\text { measure- } \\
\text { ments }\end{array}$} & \multirow{2}{*}{\multicolumn{2}{|c|}{$\begin{array}{l}\text { Water discharge } \\
\frac{\left(\mathrm{ft}^{3} / \mathrm{s}\right)}{\text { Maximum Minimum }}\end{array}$}} & \multirow{2}{*}{ Regression equation } & \multirow{2}{*}{$\begin{array}{l}\text { Correlation } \\
\text { coefficient }\end{array}$} & \multirow{2}{*}{$\begin{array}{l}\text { Percent } \\
\text { explained } \\
\text { variance }\end{array}$} & \multicolumn{2}{|c|}{$\begin{array}{c}\text { Standard error } \\
\text { of estimate }\end{array}$} \\
\hline & & & & & & Log units & Percent \\
\hline 36 & 176 & 7 & $\mathrm{SpC}=42,100 \mathrm{Q}^{-0.68848}$ & -0.87 & 76 & 0.147 & 34 \\
\hline
\end{tabular}


06803405 Antelope Creek at Court Street at Lincoln, Nebr.

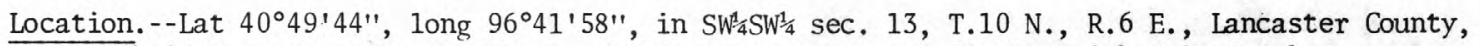
at bridge on Court Street about $0.1 \mathrm{mi}$ upstream from confluence with Salt Creek.

Period of record.--March 1971 to September 1977.

Statistical data for selected chemical constituents

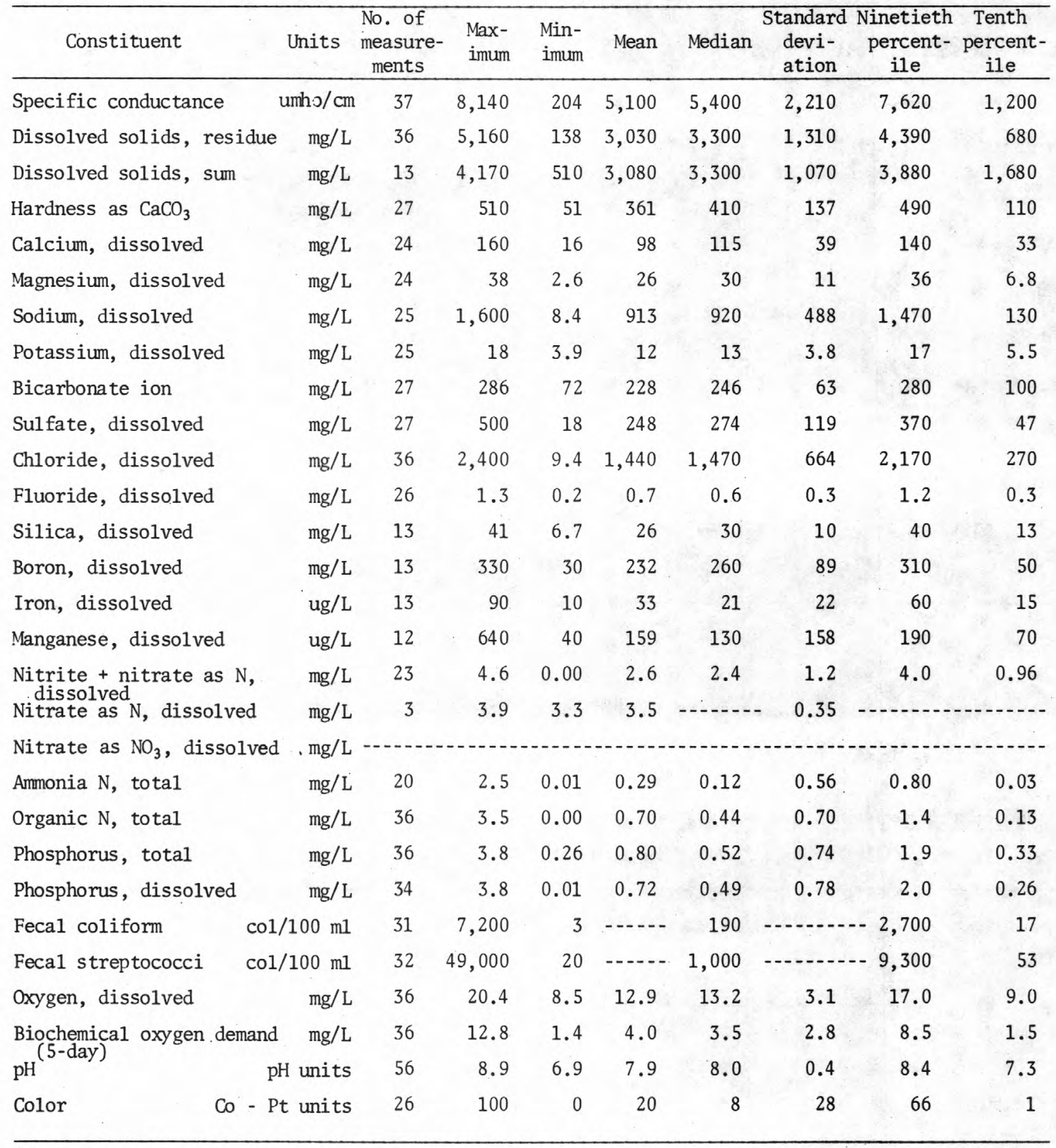


06803405 Antelope Creek at Court Street at Lincoln, Nebr.--Continued

Results of regression analyses relating concentrations of selected chemical constituents to specific conductance (SpC)

\begin{tabular}{|c|c|c|c|c|c|}
\hline $\begin{array}{c}\text { Dissolved } \\
\text { constituents } \\
(\mathrm{mg} / \mathrm{L})\end{array}$ & $\begin{array}{l}\text { No. of } \\
\text { measure- } \\
\text { ments }\end{array}$ & Regression equation & $\begin{array}{l}\text { Corre- } \\
\text { lation } \\
\text { coeffi- } \\
\text { cient }\end{array}$ & $\begin{array}{l}\text { Percent } \\
\text { explained } \\
\text { variance }\end{array}$ & $\begin{array}{l}\text { Standard } \\
\text { error of } \\
\text { estimate } \\
(\mathrm{mg} / \mathrm{L})\end{array}$ \\
\hline Dissolved solids, residue (RDS) & 36 & $\mathrm{RDS}=0.584(\mathrm{Sp})+32.780$ & 0.99 & 98 & 208 \\
\hline Dissolved solids, sum (SDS) & $-\ldots$ & (1, & 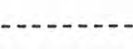 & $-\ldots$ & 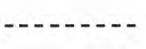 \\
\hline Hardness as $\mathrm{CaCO}_{3}(\mathrm{TH})$ & 28 & $\mathrm{TH}=0.050(\mathrm{SpC})+110.593$ & .89 & 80 & 63 \\
\hline Calcium (Ca) & 25 & $\mathrm{Ca}=0.013(\mathrm{SpC})+34.888$ & .85 & 72 & 21 \\
\hline Magnesium (Mg) & 25 & $\mathrm{Mg}=0.004(\mathrm{SpC})+5.653$ & .94 & 88 & 4.0 \\
\hline Sodium $(\mathrm{Na})$ & 26 & $\mathrm{Na}=0.195(\mathrm{SpC})-48.263$ & 1.0 & 99 & 42 \\
\hline Potassium (K) & 26 & $\mathrm{~K}=0.001(\mathrm{SpC})+6.543$ & .71 & 50 & 2.7 \\
\hline Bicarbonate $\left(\mathrm{HCO}_{3}\right)$ & 28 & $\mathrm{HCO}_{3}=0.023(\mathrm{SpC})+114.054$ & .88 & 78 & 30 \\
\hline Sulfate $\left(\mathrm{SO}_{4}\right)$ & 28 & $\mathrm{SO}_{4}=0.042(\mathrm{SpC})+35.450$ & .87 & 76 & 59 \\
\hline Chloride (Cl) & 36 & $\mathrm{Cl}=0.297(\mathrm{SpC})-84.830$ & .99 & 98 & 90 \\
\hline Silica (Si) & & (1) & & & \\
\hline Nitrite + Nitrate as $\mathrm{N}\left(\mathrm{NO}_{2}-\mathrm{NO}_{3}\right)$ & 22 & $\mathrm{NO}_{2}-\mathrm{NO}_{3}=0.0004(\mathrm{SpC})+0.682$ & .69 & 48 & 0.91 \\
\hline
\end{tabular}

Results of regression analyses relating specific conductance to water discharge $(Q)$

\begin{tabular}{ccccccc}
\hline $\begin{array}{c}\text { No. of } \\
\text { measure- } \\
\text { ments }\end{array}$ & $\begin{array}{c}\text { Water discharge } \\
\left(\mathrm{ft}^{3} / \mathrm{s}\right)\end{array}$ & Maximum Minimum & Regression equation & $\begin{array}{c}\text { Correlation } \\
\text { coefficient }\end{array}$ & $\begin{array}{c}\text { Percent } \\
\text { explained } \\
\text { variance }\end{array}$ & $\begin{array}{c}\text { Standard error } \\
\text { of estimate }\end{array}$ \\
\hline 37 & $67 \quad 1$ & SpC $=9,599 \mathrm{Q}^{-0.90032}$ & -0.95 & 91 & 0.114 \\
\hline
\end{tabular}


Location.--Lat $40^{\circ} 50^{\prime} 49^{\prime \prime}$, long $96^{\circ} 40^{\prime} 54^{\prime \prime}$, in NW $\mathrm{N}^{\frac{1}{4}} \mathrm{SW}^{\frac{1}{4}} \mathrm{sec}$. 7, T.10 N., R.7 E., Lancaster County, Hydrologic Unit 10200203, near center of channel on downstream side of pier of bridge on North 27 th Street at north edge of Lincoln, 1 mi downstream from Oak Creek.

Drainage area. $--684 \mathrm{mi}^{2}$.

Period of record.--Water years 1951-54, 1968 to current year.

Statistical data for selected chemical constituents

\begin{tabular}{|c|c|c|c|c|c|c|c|c|c|}
\hline Constituent & Units & $\begin{array}{l}\text { No. of } \\
\text { measure- } \\
\text { ments }\end{array}$ & $\begin{array}{l}\text { Max- } \\
\text { imum }\end{array}$ & $\begin{array}{l}\text { Min- } \\
\text { imum }\end{array}$ & Mean & Median & $\begin{array}{c}\text { Standard } \\
\text { devi- } \\
\text { ation } \\
\end{array}$ & $\begin{array}{l}\text { Ninetieth } \\
\text { percent- } \\
\text { ile }\end{array}$ & $\begin{array}{l}\text { Tenth } \\
\text { percent- } \\
\text { ile }\end{array}$ \\
\hline Specific conductance & umho/cm & 132 & 9,630 & 155 & 4,850 & 4,740 & 2,310 & 7,620 & 1,400 \\
\hline Dissolved solids, residue & $\mathrm{mg} / \mathrm{L}$ & 117 & 5,990 & 114 & 2,870 & 2,810 & 1,430 & 4,570 & 750 \\
\hline Dissolved solids, sum & $\mathrm{mg} / \mathrm{L}$ & 62 & 5,980 & 112 & 2,920 & 2,800 & 1,490 & 4,750 & 920 \\
\hline Hardness as $\mathrm{CaCO}_{3}$ & $\mathrm{mg} / \mathrm{L}$ & 75 & 483 & 37 & 311 & 338 & 103 & 416 & 125 \\
\hline Calcium, dissolved & $\mathrm{mg} / \mathrm{L}$ & 74 & 128 & 11 & 83 & 91 & 27 & 110 & 36 \\
\hline Magnesium, dissolved & $\mathrm{mg} / \mathrm{L}$ & 74 & 40 & 2.3 & 25 & 26 & 9.3 & 36 & 8.5 \\
\hline Sodium, dissolved & $\mathrm{mg} / \mathrm{L}$ & 71 & 2,130 & 36 & 993 & 947 & 531 & 1,760 & 260 \\
\hline Potassium, dissolved & $\mathrm{mg} / \mathrm{L}$ & 71 & 34 & 4.4 & 15 & 16 & 4.7 & 21 & 8.9 \\
\hline Bicarbonate ion & $\mathrm{mg} / \mathrm{L}$ & 74 & 474 & 47 & 316 & 348 & 94 & 406 & 160 \\
\hline Sulfate, dissolved & $\mathrm{mg} / \mathrm{L}$ & 72 & 450 & 17 & 236 & 246 & 106 & 358 & 55 \\
\hline Chloride, dissolved & $\mathrm{mg} / \mathrm{L}$ & 113 & 3,050 & 11 & 1,270 & 1,210 & 733 & 2,380 & 220 \\
\hline Fluoride, dissolved & $\mathrm{mg} / \mathrm{L}$ & 73 & 1.4 & 0.1 & 0.8 & 0.8 & 0.3 & 1.2 & 0.4 \\
\hline Silica, dissolved & $\mathrm{mg} / \mathrm{L}$ & 61 & 31 & 8.2 & 23 & 25 & 5.7 & 29 & 14 \\
\hline Boron, dissolved & $\mathrm{mg} / \mathrm{L}$ & 71 & 866 & 40 & 407 & 444 & 185 & 615 & 103 \\
\hline Iron, dissolved & $\mathrm{ug} / \mathrm{L}$ & 58 & 800 & 0 & 97 & 63 & 146 & 147 & 27 \\
\hline Manganese, dissolved & $\mathrm{ug} / \mathrm{L}$ & 43 & 1,430 & 74 & 486 & 370 & 313 & 853 & 150 \\
\hline Nitrite + nitrate as $\mathrm{N}$, & $\mathrm{mg} / \mathrm{L}$ & 52 & 8.3 & 0.00 & 1.6 & 1.2 & 1.8 & 2.0 & 0.53 \\
\hline Nitrate as $\mathrm{N}$, dissolved & $\mathrm{mg} / \mathrm{L}$ & 42 & 7.4 & 0.00 & 1.1 & 0.83 & 1.3 & 1.7 & 0.26 \\
\hline Nitrate as $\mathrm{NO}_{3}$, dissolved & $\mathrm{mg} / \mathrm{L}$ & & & & & & -5 & 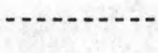 & ..... \\
\hline Ammonia $\mathrm{N}$, total & $\mathrm{mg} / \mathrm{L}$ & 60 & 15 & 0.01 & 5.9 & 5.4 & 3.7 & 11.0 & 1.2 \\
\hline Organic $N$, total & $\mathrm{mg} / \mathrm{L}$ & 111 & 17 & 0.00 & 2.6 & 1.9 & 2.9 & 6.2 & 0.22 \\
\hline Phosphorus, total & $\mathrm{mg} / \mathrm{L}$ & 96 & 11 & 0.30 & 3.9 & 3.4 & 2.2 & 6.8 & 1.2 \\
\hline Phosphorus, dissolved & $\mathrm{mg} / \mathrm{L}$ & 99 & 14 & 0.01 & 3.2 & 2.9 & 2.4 & 6.0 & 0.26 \\
\hline Fecal coliform & $\mathrm{col} / 100 \mathrm{ml}$ & 762,8 & 800,000 & 3 & \multicolumn{2}{|c|}{$\cdots-175,000$} & \multicolumn{2}{|c|}{$-1-895,000$} & 13 \\
\hline Fecal streptococci & $\mathrm{col} / 100 \mathrm{ml}$ & 72 & 770,000 & 1 & $\cdots+. .$. & 42,000 & \multicolumn{2}{|c|}{ - } & 11 \\
\hline Oxygen, dissolved & $\mathrm{mg} / \mathrm{L}$ & 93 & 17.0 & 0.60 & 8.5 & 8.6 & 2.6 & 11.8 & 4.9 \\
\hline \multirow{2}{*}{$\begin{array}{l}\text { Biochemical oxygen demand } \\
\text { (5H }\end{array}$} & $\mathrm{mg} / \mathrm{L}$ & 102 & $\cdots$ & 5.1 & 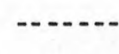 & 17.5 & $\cdots \cdots$ & 43.2 & 7.3 \\
\hline & $\mathrm{H}$ units & 153 & 8.3 & 6.7 & 7.5 & 7.5 & 0.3 & 7.9 & 7.1 \\
\hline Co $-\mathrm{Pt}$ & t units & 70 & 200 & 3 & 27 & 20 & 33 & 42 & 8 \\
\hline
\end{tabular}


Results of regression analyses relating concentrations of selected chemical constituents to specific conductance (SpC)

\begin{tabular}{|c|c|c|c|c|c|}
\hline $\begin{array}{l}\text { Dissolved } \\
\text { constituents } \\
(\mathrm{mg} / \mathrm{L})\end{array}$ & $\begin{array}{l}\text { No. of } \\
\text { measure- } \\
\text { ments }\end{array}$ & Regression equation & $\begin{array}{l}\text { Corre- } \\
\text { lation } \\
\text { coeffi- } \\
\text { cient }\end{array}$ & $\begin{array}{l}\text { Percent } \\
\text { explained } \\
\text { variance }\end{array}$ & $\begin{array}{c}\text { Standard } \\
\text { error of } \\
\text { estimate } \\
(\mathrm{mg} / \mathrm{L})\end{array}$ \\
\hline Dissolved solids, residue (RDS) & 119 & $\mathrm{RDS}=0.605(\mathrm{SpC})-70.027$ & 0.99 & 99 & 161 \\
\hline Dissolved solids, sum (SDS) & 62 & $\mathrm{SDS}=0.613(\mathrm{SpC})-128.190$ & .99 & 99 & 178 \\
\hline Hardness as $\mathrm{CaCO}_{3}(\mathrm{TH})$ & 76 & $\mathrm{TH}=0.037(\mathrm{SpC})+126.508$ & .90 & 80 & 46 \\
\hline Calcium (Ca) & 75 & $\mathrm{Ca}=0.009(\mathrm{SpC})+37.528$ & .86 & 74 & 14 \\
\hline Magnesium (Mg) & 75 & $M g=0.003(S p C)+7.733$ & .94 & 88 & 3.3 \\
\hline Sodium $(\mathrm{Na})$ & 72 & $\mathrm{Na}=0.218(\mathrm{SpC})-116.225$ & .99 & 98 & 75 \\
\hline Potassium (K) & 72 & $K=0.001(\mathrm{SpC})+9.283$ & .62 & 39 & 3.7 \\
\hline Bicarbonate $\left(\mathrm{HCO}_{3}\right)$ & 75 & $\mathrm{HCO}_{3}=0.030(\mathrm{SpC})+169.177$ & .78 & 60 & 60 \\
\hline Sulfate $\left(\mathrm{SO}_{4}\right)$ & 73 & $\mathrm{SO}_{4}=0.042(\mathrm{SpC})+24.677$ & .94 & 89 & 35 \\
\hline Chloride (C1) & 116 & $\mathrm{Cl}=0.317(\mathrm{SpC})-212.855$ & .97 & 95 & 167 \\
\hline Silica (Si) & 62 & $\mathrm{Si}=0.001(\mathrm{SpC})+16.114$ & .57 & 33 & 4.7 \\
\hline Nitrite + Nitrate as $\mathrm{N}\left(\mathrm{NO}_{2}-\mathrm{NO}_{3}\right)$ & 52 & $\mathrm{NO}_{2}-\mathrm{NO}_{3}=-0.00003(\mathrm{SpC})+1.698$ & $8 \stackrel{*}{-} 03$ & 0.09 & 1.8 \\
\hline
\end{tabular}

* Not significant at the 95 percent confidence level; the regression equation should not be used to predict the concentration of the dependent variable.

Results of regression analyses relating specific conductance to water discharge (Q)

\begin{tabular}{llllcccc}
\hline $\begin{array}{c}\text { No. of } \\
\text { measure- }\end{array}$ & $\begin{array}{c}\text { Water discharge } \\
\text { ments }\end{array}$ & Maximum Minimum & Regression equation & $\begin{array}{c}\text { Correlation } \\
\text { coefficient }\end{array}$ & $\begin{array}{c}\text { Percent } \\
\text { explained } \\
\text { variance }\end{array}$ & \multicolumn{2}{c}{$\begin{array}{c}\text { Standard error } \\
\text { of estimate }\end{array}$} \\
\hline 133 & $26,500 \quad 26$ & & SpC $=103,600 Q^{-0.66794}$ & -0.95 & 91 & 0.107 & 25 \\
\hline
\end{tabular}


06803510 Little Salt Creek near Lincoln, Nebr.

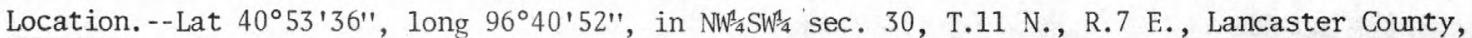
at gaging station at bridge on county road $1.6 \mathrm{mi}$ north of intersection of Interstate 80 and North 14 th Street, north of Lincoln.

Period of record. --April 1971 to September 1976.

Statistical data for selected chemical constituents

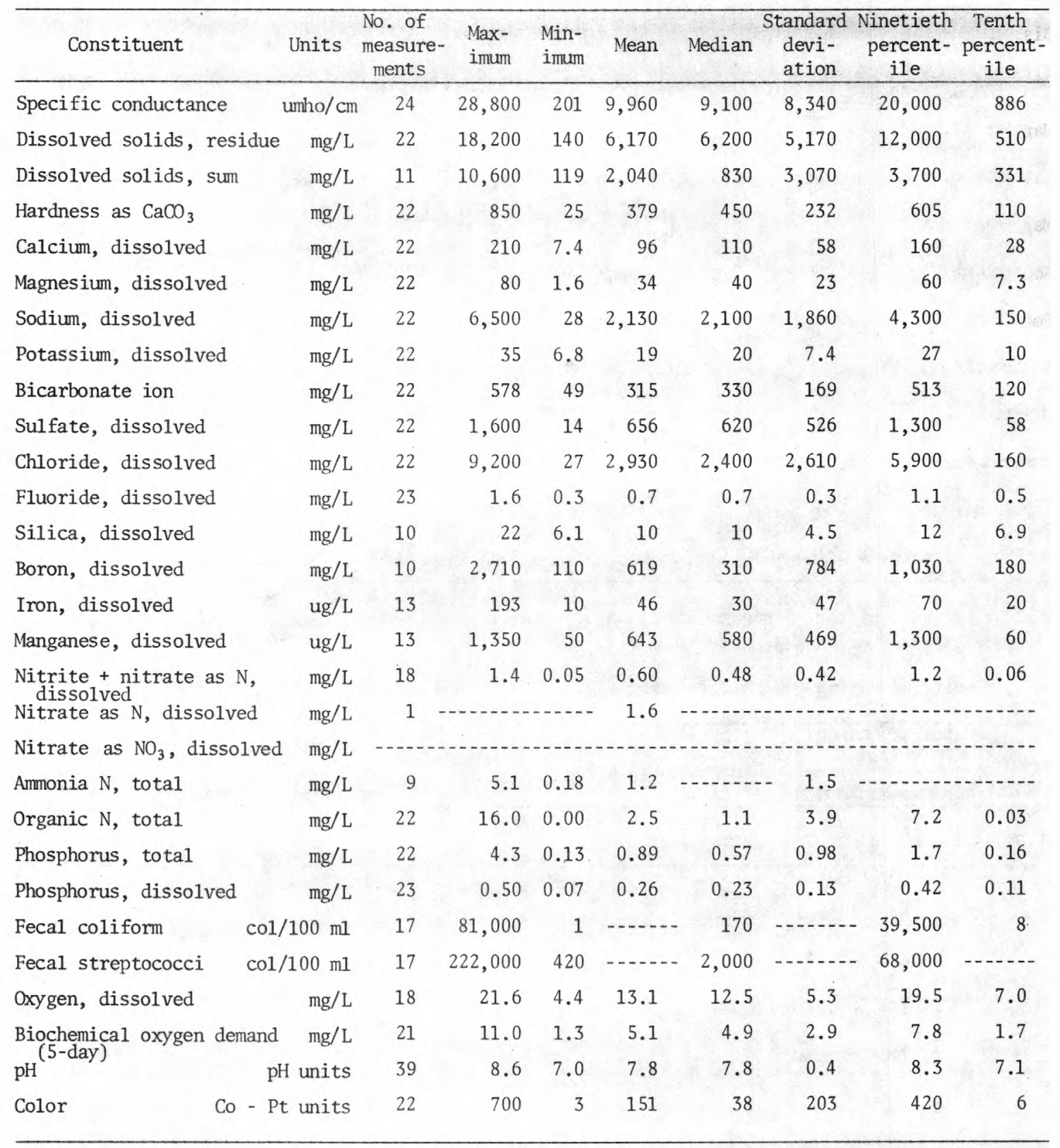


06803510 Little Salt Creek near Lincoln, Nebr.--Continued

Results of regression analyses relating concentrations of selected chemical constituents to specific conductance $(\mathrm{SpC})$

\begin{tabular}{|c|c|c|c|c|c|}
\hline $\begin{array}{l}\text { Dissolved } \\
\text { constituents } \\
(\mathrm{mg} / \mathrm{L})\end{array}$ & $\begin{array}{c}\text { No. of } \\
\text { measure- } \\
\text { ments }\end{array}$ & Regression equation & $\begin{array}{l}\text { Corre- } \\
\text { lation } \\
\text { coeffi- } \\
\text { cient } \\
\end{array}$ & $\begin{array}{l}\text { Percent } \\
\text { explained } \\
\text { variance }\end{array}$ & $\begin{array}{c}\text { Standard } \\
\text { error of } \\
\text { estimate } \\
(\mathrm{mg} / \mathrm{L})\end{array}$ \\
\hline Dissolved solids, residue (RDS) & 22 & $\mathrm{RDS}=0.612(\mathrm{SpC})-40.324$ & 0.99 & 99 & 550 \\
\hline \multicolumn{6}{|l|}{ Dissolved solids, sum (SDS) } \\
\hline Hardness as $\mathrm{CaCO}_{3}(\mathrm{TH})$ & 22 & $\mathrm{TH}=0.026(\mathrm{SpC})+119.906$ & .92 & 85 & 92 \\
\hline Calcium (Ca) & 22 & $\mathrm{Ca}=0.006(\mathrm{SpC})+36.097$ & .85 & 73 & 31 \\
\hline Magnesium (Mg) & 22 & $M g=0.003(\mathrm{SpC})+6.850$ & .98 & 96 & 4.8 \\
\hline Sodium (Na) & 22 & $\mathrm{Na}=0.221(\mathrm{SpC})-107.903$ & 1.0 & 99 & 143 \\
\hline Potassium (K) & 22 & $K=0.0007(\mathrm{SpC})+12.067$ & .82 & 67 & 4.4 \\
\hline Bicarbonate $\left(\mathrm{HCO}_{3}\right)$ & 22 & $\mathrm{HCO}_{3}=0.016(\mathrm{SpC})+152.407$ & .81 & 66 & 100 \\
\hline Sulfate $\left(\mathrm{SO}_{4}\right)$ & 22 & $\mathrm{SO}_{4}=0.062(\mathrm{SpC})+26.415$ & .99 & 98 & 73 \\
\hline Chloride $(\mathrm{Cl})$ & 22 & $\mathrm{Cl}-0.309(\mathrm{SpC})-205.824$ & 1.0 & 99 & 236 \\
\hline \multicolumn{6}{|l|}{ Silica (Si) } \\
\hline Nitrite + Nitrate as $\mathrm{N}\left(\mathrm{NO}_{2}-\mathrm{NO}_{3}\right)$ & 18 & $\mathrm{NO}_{2}-\mathrm{NO}_{3}=-0.00004(\mathrm{SpC})+0.972$ & -.74 & 55 & 0.29 \\
\hline
\end{tabular}

Results of regression analyses relating specific conductance to water discharge (Q)

\begin{tabular}{cccccccc}
\hline \multirow{2}{*}{$\begin{array}{c}\text { No. of } \\
\text { measure- } \\
\text { ments }\end{array}$} & $\begin{array}{c}\text { Water discharge } \\
\left(\mathrm{ft}^{3} / \mathrm{s}\right)\end{array}$ & Maximum Minimum & Regression equation & $\begin{array}{c}\text { Correlation } \\
\text { coefficient }\end{array}$ & $\begin{array}{c}\text { Percent } \\
\text { explained } \\
\text { variance }\end{array}$ & \multicolumn{2}{c}{$\begin{array}{c}\text { Standard error } \\
\text { of estimate }\end{array}$} \\
\hline 24 & $1,260 \quad 0.94$ & $S p C=31,530$ & Log units & Percent \\
\hline
\end{tabular}


06803523 Stevens Creek at Hwy. 6 near Lincoln, Nebr.

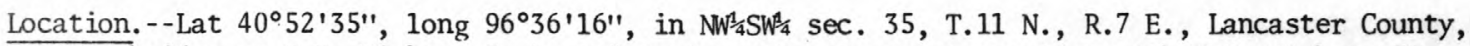
at bridge at U.S. Highway 6 about $2.6 \mathrm{mi}$ northeast of intersection with U.S. Highway 77, and about 1.2 mi upstream from confluence with Salt Creek.

Period of record.--March 1971 to current year.

Statistical data for selected chemical constituents

\begin{tabular}{|c|c|c|c|c|c|c|c|c|c|}
\hline Constituent & Units & $\begin{array}{l}\text { No. of } \\
\text { measure- } \\
\text { ments }\end{array}$ & $\begin{array}{l}\text { Max- } \\
\text { imum }\end{array}$ & $\begin{array}{l}\text { Min- } \\
\text { imum }\end{array}$ & Mean & Median & $\begin{array}{c}\text { Standard } \\
\text { devi- } \\
\text { ation }\end{array}$ & $\begin{array}{c}\text { Ninetieth } \\
\text { percent- } \\
\text { ile }\end{array}$ & $\begin{array}{l}\text { Tenth } \\
\text { percent- } \\
\text { ile }\end{array}$ \\
\hline Specific conductance & umho/cm & 36 & 892 & 89 & 672 & 678 & 131 & 780 & 540 \\
\hline Dissolved solids, residue & $\mathrm{mg} / \mathrm{L}$ & 36 & 616 & 80 & 438 & 443 & 85 & 510 & 360 \\
\hline Dissolved solids, sum & $\mathrm{mg} / \mathrm{L}$ & 21 & 563 & 61 & 416 & 430 & 104 & 490 & 320 \\
\hline Hardness as $\mathrm{CaCO}_{3}$ & $\mathrm{mg} / \mathrm{L}$ & 36 & 360 & 30 & 268 & 276 & 54 & 320 & 240 \\
\hline Calcium, dissolved & $\mathrm{mg} / \mathrm{L}$ & 33 & 100 & 8.3 & 75 & 77 & 16 & 90 & 62 \\
\hline Magnesium, dissolved & $\mathrm{mg} / \mathrm{L}$ & 33 & 28 & 2.2 & 20 & 20 & 4.3 & 24 & 17 \\
\hline Sodium, dissolved & $\mathrm{mg} / \mathrm{L}$ & 33 & 61 & 5.1 & 47 & 47 & 11 & 58 & 37 \\
\hline Potassium, dissolved & $\mathrm{mg} / \mathrm{L}$ & 33 & 11 & 4.2 & 6.7 & 6.5 & 1.6 & 8.6 & 4.9 \\
\hline Bicarbonate ion & $\mathrm{mg} / \mathrm{L}$ & 36 & 477 & 39 & 338 & 344 & 70 & 395 & 290 \\
\hline Sulfate, dissolved & $\mathrm{mg} / \mathrm{L}$ & 36 & 94 & 7.3 & 66 & 66 & 14 & 78 & 55 \\
\hline Chloride, dissolved & $\mathrm{mg} / \mathrm{L}$ & 36 & 43 & 1.1 & 20 & 19 & 8.9 & 35 & 12 \\
\hline Fluoride, dissolved & $\mathrm{mg} / \mathrm{L}$ & 33 & 0.8 & 0.0 & 0.4 & 0.4 & 0.2 & 0.5 & 0.2 \\
\hline Silica, dissolved & $\mathrm{mg} / \mathrm{L}$ & 21 & 29 & 5.9 & 19 & 20 & 6.4 & 26 & 13 \\
\hline Boron, dissolved & $\mathrm{mg} / \mathrm{L}$ & 21 & 320 & 40 & 134 & 113 & 75 & 250 & 70 \\
\hline Iron, dissolved & $\mathrm{ug} / \mathrm{L}$ & 12 & 50 & 10 & 19 & 20 & 12 & 30 & 12 \\
\hline Manganese, dissolved & $\mathrm{ug} / \mathrm{L}$ & 12 & 950 & 280 & 608 & 573 & 202 & 880 & 370 \\
\hline $\begin{array}{l}\text { Nitrite + nitrate as } \mathrm{N} \text {, } \\
\text { dissolved }\end{array}$ & $\mathrm{mg} / \mathrm{L}$ & 24 & 3.1 & 0.00 & 1.4 & 1.3 & 1.0 & 2.8 & 0.09 \\
\hline Nitrate as $\mathrm{N}$, dissolved & $\mathrm{mg} / \mathrm{L}$ & 3 & 1.8 & 0.00 & 1.1 & -1 & 0.99 & & -..... \\
\hline Nitrate as $\mathrm{NO}_{3}$, dissolved & $\mathrm{mg} / \mathrm{L}$ & & & & & & & & \\
\hline Anmonia $\mathrm{N}$, total & $\mathrm{mg} / \mathrm{L}$ & 20 & 1.3 & 0.10 & 0.39 & 0.28 & 0.32 & 0.99 & 0.14 \\
\hline Organic $N$, total & $\mathrm{mg} / \mathrm{L}$ & 36 & 4.0 & 0.17 & 0.83 & 0.63 & 0.73 & 1.4 & 0.27 \\
\hline Phosphorus, total & $\mathrm{mg} / \mathrm{L}$ & 36 & 0.85 & 0.21 & 0.44 & 0.41 & 0.14 & 0.67 & 0.29 \\
\hline Phosphorus, dissolved & $\mathrm{mg} / \mathrm{L}$ & 36 & 0.85 & 0.15 & 0.34 & 0.33 & 0.14 & 0.47 & 0.19 \\
\hline Fecal coliform & $\mathrm{col} / 100 \mathrm{ml}$ & 32 & 49,000 & 40 & 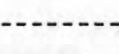 & 1,740 & $\cdots$ & 3,750 & 550 \\
\hline Fecal streptococci & $\mathrm{col} / 100 \mathrm{ml}$ & 32 & 124,000 & 180 & (n) & 1,040 & - & 5,900 & 390 \\
\hline Oxygen, dissolved & $\mathrm{mg} / \mathrm{L}$ & 36 & 14.5 & 3.8 & 9.6 & 8.9 & 2.6 & 14.0 & 7.2 \\
\hline \multirow{2}{*}{$\begin{array}{l}\text { Biochemical oxygen demand } \\
\text { (5-day) }\end{array}$} & $\mathrm{mg} / \mathrm{L}$ & 35 & 13.0 & 1.0 & 3.3 & 2.7 & 2.1 & 5.1 & 1.5 \\
\hline & $\mathrm{H}$ units & 56 & 8.3 & 5.8 & 7.7 & 7.8 & 0.4 & 8.2 & 7.3 \\
\hline $\mathrm{Co}-\mathrm{Pt}$ & Co - Pt units & 36 & 400 & 2 & 30 & 20 & 64 & 39 & 5 \\
\hline
\end{tabular}


Results of regression analyses relating concentrations of selected chemical constituents to specific conductance $(\mathrm{SpC})$

\begin{tabular}{|c|c|c|c|c|c|}
\hline $\begin{array}{l}\text { Dissolved } \\
\text { constituents } \\
(\mathrm{mg} / \mathrm{L})\end{array}$ & $\begin{array}{l}\text { No. of } \\
\text { measure- } \\
\text { ments }\end{array}$ & Regression equation & $\begin{array}{l}\text { Corre- } \\
\text { lation } \\
\text { coeffi- } \\
\text { cient }\end{array}$ & $\begin{array}{l}\text { Percent } \\
\text { explained } \\
\text { variance }\end{array}$ & $\begin{array}{c}\text { Standard } \\
\text { error of } \\
\text { estimate } \\
(\mathrm{mg} / \mathrm{L})\end{array}$ \\
\hline Dissolved solids, residue (RDS) & 36 & $\mathrm{RDS}=0.609(\mathrm{SpC})+28.205$ & 0.94 & 88 & 30 \\
\hline Dissolved solids, sum (SDS) & 21 & $S D S=0.628(S p C)+6.844$ & .98 & 96 & 21 \\
\hline Hardness as $\mathrm{CaCO}_{3}(\mathrm{TH})$ & 36 & $\mathrm{TH}=0.394(\mathrm{SpC})+2.396$ & .96 & 92 & 15 \\
\hline Calcium (Ca) & 33 & $\mathrm{Ca}=0.112(\mathrm{SpC})+0.242$ & .96 & 91 & 4.8 \\
\hline Magnesium (Mg) & 33 & $\mathrm{Mg}=0.030(\mathrm{SpC})-0.853$ & .96 & 92 & 1.2 \\
\hline Sodium (Na) & 34 & $\mathrm{Na}=0.069(\mathrm{SpC})+0.857$ & .86 & 74 & 5.8 \\
\hline Potassium $(\mathrm{K})$ & 33 & $K=-0.001(\mathrm{SpC})+7.384$ & $*_{-} .09$ & 0.80 & 1.6 \\
\hline Bicarbonate $\left(\mathrm{HCO}_{3}\right)$ & 36 & $\mathrm{HCO}_{3}=0.516(\mathrm{SpC})-8.102$ & .96 & 92 & 20 \\
\hline Sulfate $\left(\mathrm{SO}_{4}\right)$ & 36 & $\mathrm{SO}_{4}=0.091(\mathrm{SpC})+4.633$ & .82 & 67 & 8.5 \\
\hline Chloride $(\mathrm{Cl})$ & 36 & $\mathrm{Cl}=0.031(\mathrm{SpC})-0.589$ & .46 & 21 & 8.0 \\
\hline Silica (Si) & 21 & $\mathrm{Si}=0.028(\mathrm{SpC})+1.038$ & .70 & 49 & 4.6 \\
\hline Nitrite + Nitrate as $\mathrm{N}\left(\mathrm{NO}_{2}-\mathrm{NO}_{3}\right)$ & 24 & $\mathrm{NO}_{2}-\mathrm{NO}_{3}=0.002(\mathrm{SpC})+0.007$ & $* .27$ & 7.5 & 1.0 \\
\hline \multicolumn{6}{|c|}{$\begin{array}{l}\text { * Not significant at the } 95 \text { percent confidence level; the regression equation should not be used } \\
\text { to predict the concentration of the dependent variable. }\end{array}$} \\
\hline \multicolumn{6}{|c|}{ Results of regression analyses relating specific conductance to water discharge (Q) } \\
\hline \multirow{2}{*}{$\begin{array}{l}\begin{array}{l}\text { No. of Water discharge } \\
\text { measure- } \\
\text { ments }\end{array} \frac{\left(\mathrm{ft}^{3} / \mathrm{s}\right)}{\text { Maximum Minimum }} \\
\end{array}$} & \multirow{2}{*}{ egression equation } & \multirow{2}{*}{$\begin{array}{l}\text { Correlation } \\
\text { coefficient }\end{array}$} & $\begin{array}{l}\text { Percent } \\
\text { explained }\end{array}$ & \multicolumn{2}{|c|}{$\begin{array}{c}\text { Standard error } \\
\text { of estimate }\end{array}$} \\
\hline & & & ariance & Log units & Percent \\
\hline 2,390 & $C=759 Q$ & -0.14169 & 40 & 0.123 & 29 \\
\hline
\end{tabular}




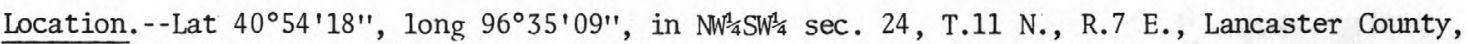
Hydrologic Unit 10200203, at bridge $0.5 \mathrm{mi}$ north of Interstate Highway 80 and 3 mi southwest of Waverly.

Drainage area. $--815 \mathrm{mi}^{2}$.

Period of record.--Water year 1971 to current year.

Statistical data for selected chemical constituents

\begin{tabular}{|c|c|c|c|c|c|c|c|c|c|}
\hline Constituent & Units & $\begin{array}{l}\text { No. of } \\
\text { measure- } \\
\text { ments }\end{array}$ & $\begin{array}{l}\text { Max- } \\
\text { imum }\end{array}$ & $\begin{array}{l}\text { Min- } \\
\text { imum }\end{array}$ & Mean & Median & $\begin{array}{l}\text { Standard } \\
\text { devi- } \\
\text { ation }\end{array}$ & $\begin{array}{c}\text { Ninetieth } \\
\text { percent- } \\
\text { ile }\end{array}$ & $\begin{array}{l}\text { Tenth } \\
\text { percent- } \\
\text { ile }\end{array}$ \\
\hline Specific conductance & umho/cm & 89 & 8,500 & 859 & 5,420 & 5,890 & 2,130 & 7,850 & 1,850 \\
\hline Dissolved solids, residue & $\mathrm{mg} / \mathrm{L}$ & 76 & 5,220 & 553 & 3,390 & 3,790 & 1,230 & 4,680 & 1,200 \\
\hline Dissolved solids, sum & $\mathrm{mg} / \mathrm{L}$ & 32 & 4,970 & 479 & 3,200 & 3,400 & 1,200 & 4,300 & 1,000 \\
\hline Hardness as $\mathrm{CaCO}_{3}$ & $\mathrm{mg} / \mathrm{L}$ & 46 & 410 & 160 & 334 & 348 & 57 & 390 & 250 \\
\hline Calcium, dissolved & $\mathrm{mg} / \mathrm{L}$ & 46 & 110 & 43 & 89 & 94 & 14 & 101 & 66 \\
\hline Magnesium, dissolved & $\mathrm{mg} / \mathrm{L}$ & 46 & 35 & 12 & 27 & 29 & 5.5 & 33 & 18 \\
\hline Sodium, dissolved & $\mathrm{mg} / \mathrm{L}$ & 47 & 1,700 & 100 & 1,130 & 1,180 & 417 & 1,630 & 440 \\
\hline Potassium, dissolved & $\mathrm{mg} / \mathrm{L}$ & 46 & 22 & 6.1 & 16 & 17 & 3.3 & 19 & 11 \\
\hline Bicarbonate ion & $\mathrm{mg} / \mathrm{L}$ & 95 & 446 & 168 & 355 & 378 & 60 & 406 & 270 \\
\hline Sulfate, dissolved & $\mathrm{mg} / \mathrm{L}$ & 95 & 470 & 63 & 294 & 325 & 94 & 396 & 167 \\
\hline Chloride, dissolved & $\mathrm{mg} / \mathrm{L}$ & 109 & 2,500 & 38 & 1,430 & 1,630 & 648 & 2,250 & 405 \\
\hline Fluoride, dissolved & $\mathrm{mg} / \mathrm{L}$ & 44 & 1.8 & 0.2 & 0.8 & 0.8 & 0.3 & 1.2 & 0.4 \\
\hline Silica, dissolved & $\mathrm{mg} / \mathrm{L}$ & 33 & 30 & 9.8 & 22 & 23 & 5.2 & 28 & 12 \\
\hline Boron, dissolved & $\mathrm{mg} / \mathrm{L}$ & 21 & 800 & 110 & 498 & 510 & 208 & 735 & 180 \\
\hline Iron, dissolved & $\mathrm{ug} / \mathrm{L}$ & 28 & 200 & 0 & 58 & 42 & 43 & 99 & 18 \\
\hline Manganese, dissolved & $\mathrm{ug} / \mathrm{L}$ & 28 & 1,100 & 16 & 372 & 310 & 271 & 735 & 90 \\
\hline $\begin{array}{l}\text { Nitrite + nitrate as } \mathrm{N} \text {, } \\
\text { dissolved }\end{array}$ & $\mathrm{mg} / \mathrm{L}$ & 45 & 4.2 & 0.00 & 0.95 & 0.83 & 0.71 & 1.7 & 0.21 \\
\hline Nitrate as N, dissolved & $\mathrm{mg} / \mathrm{L}$ & 8 & 4.1 & 0.30 & 1.3 & $\cdots \cdots-1$ & 1.2 & & --- \\
\hline Nitrate as $\mathrm{NO}_{3}$, dissolved & $\mathrm{mg} / \mathrm{L}$ & & & & 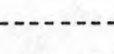 & & & & 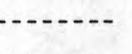 \\
\hline Ammonia $\mathrm{N}$, total & $\mathrm{mg} / \mathrm{L}$ & 76 & 11 & 0.01 & 4.0 & 4.0 & 2.7 & 7.7 & 0.78 \\
\hline Organic $N$, total & $\mathrm{mg} / \mathrm{L}$ & 106 & 10 & 0.00 & 1.7 & 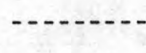 & 1.8 & & \\
\hline Phosphorus, total & $\mathrm{mg} / \mathrm{L}$ & 109 & 10 & 0.79 & 3.7 & 3.3 & 2.1 & 6.4 & 1.2 \\
\hline Phosphorus, dissolved & $\mathrm{mg} / \mathrm{L}$ & 84 & 10 & 0.15 & 3.3 & 3.1 & 2.0 & 5.9 & 0.54 \\
\hline Fecal coliform & $\mathrm{col} / 100 \mathrm{ml}$ & \multicolumn{2}{|c|}{$992,900,000$} & 10 & \multicolumn{2}{|c|}{$-105,000$} & \multicolumn{2}{|c|}{$-767,000$} & 201 \\
\hline Fecal streptococci & $\operatorname{col} / 100 \mathrm{ml}$ & 85 & 270,000 & 1 & $-\cdots-1$ & $-23,500$ & $\ldots$ & $-87,100$ & 162 \\
\hline Oxygen, dissolved & $\mathrm{mg} / \mathrm{L}$ & 114 & 12.5 & 0.0 & 7.0 & 7.1 & 2.5 & 10.1 & 3.6 \\
\hline $\begin{array}{l}\text { Biochemical oxygen demand } \\
\text { (5-day) }\end{array}$ & $\mathrm{mg} / \mathrm{L}$ & 98 & 43.0 & 0.8 & 11.6 & 10.1 & 7.0 & 20.0 & 4.8 \\
\hline $\mathrm{pH}$ & $\mathrm{pH}$ units & 145 & 8.5 & 6.5 & 7.5 & 7.6 & 0.3 & 7.9 & 7.2 \\
\hline Co - Pt & Co - Pt units & 44 & 100 & 7 & 27 & 20 & 18 & 47 & 12 \\
\hline
\end{tabular}


06803525 Salt Creek below Stevens Creek, near Waverly, Nebr.--Continued

Results of regression analyses relating concentrations of selected chemical constituents to specific conductance $(\mathrm{SpC})$

\begin{tabular}{|c|c|c|c|c|c|}
\hline $\begin{array}{l}\text { Dissolved } \\
\text { constituents } \\
\text { (mg/L) }\end{array}$ & $\begin{array}{l}\text { No. of } \\
\text { measure- } \\
\text { ments }\end{array}$ & Regression equation & $\begin{array}{l}\text { Corre- } \\
\text { lation } \\
\text { coeffi- } \\
\text { cient }\end{array}$ & $\begin{array}{l}\text { Percent } \\
\text { explained } \\
\text { variance }\end{array}$ & $\begin{array}{c}\text { Standard } \\
\text { error of } \\
\text { estimate } \\
(\mathrm{mg} / \mathrm{L})\end{array}$ \\
\hline Dissolved solids, residue (RDS) & 76 & $\mathrm{RDS}=0.584(\mathrm{SpC})+61.472$ & 0.98 & 97 & 224 \\
\hline Dissolved solids, sum (SDS) & 32 & $\mathrm{SDS}=0.576(\mathrm{SpC})+2.435$ & .99 & 99 & 128 \\
\hline Hardness as $\mathrm{CaCO}_{3}(\mathrm{TH})$ & 45 & $\mathrm{TH}=0.026(\mathrm{SpC})+185.047$ & .88 & 78 & 28 \\
\hline Calcium (Ca) & 45 & $\mathrm{Ca}=0.006(\mathrm{SpC})+53.227$ & .83 & 69 & 8.2 \\
\hline Magnesium (Mg) & 45 & $\mathrm{Mg}=0.002(\mathrm{spC})+12.499$ & .91 & 83 & 2.3 \\
\hline Sodium $(\mathrm{Na})$ & 45 & $\mathrm{Na}-0.209(\mathrm{SpC})-93.216$ & .99 & 98 & 68 \\
\hline Potassium $(K)$ & 45 & $\mathrm{~K}=0.001(\mathrm{spC})+8.854$ & .71 & 51 & 2.4 \\
\hline Bicarbonate $\left(\mathrm{HCO}_{3}\right)$ & 73 & $\mathrm{HCO}_{3}=0.025(\mathrm{SpC})+204.329$ & .81 & 65 & 38 \\
\hline Sulfate $\left(\mathrm{SO}_{4}\right)$ & 76 & $\mathrm{SO}_{4}=0.045(\mathrm{SpC})+41.671$ & .93 & 87 & 36 \\
\hline Chloride (CI) & 90 & $\mathrm{Cl}-0.301(\mathrm{SpC})-166.136$ & .97 & 94 & 168 \\
\hline Silica (Si) & 33 & $\mathrm{Si}=0.002(\mathrm{SpC})+9.718$ & .86 & 73 & 2.7 \\
\hline Nitrite + Nitrate as $\mathrm{N}\left(\mathrm{NO}_{2}-\mathrm{NO}_{3}\right)$ & 44 & $\mathrm{NO}_{2}-\mathrm{NO}_{3}=-0.0001(\mathrm{SpC})+1.656$ & -.43 & 19 & 0.65 \\
\hline
\end{tabular}

Results of regression analyses relating specific conductance to water discharge (Q)

\begin{tabular}{ccccccc}
\hline $\begin{array}{c}\text { No. of } \\
\text { measure- } \\
\text { ments }\end{array}$ & $\begin{array}{c}\text { Water discharge } \\
\text { Maximum Minimum }\end{array}$ & Regression equation & $\begin{array}{c}\text { Correlation } \\
\text { coefficient }\end{array}$ & $\begin{array}{c}\text { Percent } \\
\text { explained } \\
\text { variance }\end{array}$ & $\begin{array}{c}\text { Standard error } \\
\text { of estimate }\end{array}$ \\
\hline 89 & $1,300 \quad 48$ & $\mathrm{SpC}=127,300 \mathrm{Q}^{-0.68251}$ & -0.96 & 93 & 0.066 \\
\hline
\end{tabular}


Location.--Lat $41^{\circ} 00^{\prime} 56^{\prime \prime}$, long $96^{\circ} 32^{\prime} 39^{\prime \prime}$, in $\mathrm{NE}^{\frac{1}{4}} \mathrm{NE}^{\frac{1}{4}} \mathrm{sec}$. 17, T.12 N., R. 8 E., Lancaster County, Hydrologic Unit 10200203, on right bank $10 \mathrm{ft}$ downstream from bridge on east-west county road and $5.7 \mathrm{mi}$ southeast of Ceresco.

Drainage area. $--119 \mathrm{mi}^{2}$.

Period of record.--April 1970 to current year.

Statistical data for selected chemical constituents

\begin{tabular}{|c|c|c|c|c|c|c|c|c|c|}
\hline Constituent & Units & $\begin{array}{l}\text { No. of } \\
\text { measure- } \\
\text { ments }\end{array}$ & $\begin{array}{l}\text { Max- } \\
\text { imum }\end{array}$ & $\begin{array}{l}\text { Min- } \\
\text { imum }\end{array}$ & Mean & Median & $\begin{array}{c}\text { Standard } \\
\text { devi- } \\
\text { ation }\end{array}$ & $\begin{array}{c}\text { Ninetieth } \\
\text { percent- } \\
\text { ile }\end{array}$ & $\begin{array}{c}\text { Tenth } \\
\text { percent- } \\
\text { ile }\end{array}$ \\
\hline Specific conductance & umho/cm & 62 & 2,830 & 145 & 1,340 & 1,310 & 383 & 1,730 & 1,100 \\
\hline Dissolved solids, residue & $\mathrm{mg} / \mathrm{L}$ & 46 & 1,950 & 197 & 859 & 813 & 258 & 1,120 & 685 \\
\hline Dissolved solids, sum & $\mathrm{mg} / \mathrm{L}$ & 40 & 1,120 & 72 & 770 & 766 & 200 & 970 & 600 \\
\hline Hardness as $\mathrm{CaOO}_{3}$ & $\mathrm{mg} / \mathrm{L}$ & 51 & 390 & 68 & 266 & 272 & 58 & 309 & 212 \\
\hline Calcium, dissolved & $\mathrm{mg} / \mathrm{L}$ & 51 & 120 & 19 & 75 & 75 & 17 & 91 & 60 \\
\hline Magnesium, dissolved & $\mathrm{mg} / \mathrm{L}$ & 51 & 31 & 4.9 & 19 & 20 & 4.5 & 25 & 15 \\
\hline Sodium, dissolved & $\mathrm{mg} / \mathrm{L}$ & 51 & 470 & 35 & 193 & 184 & 63 & 260 & 152 \\
\hline Potassium, dissolved & $\mathrm{mg} / \mathrm{L}$ & 51 & 13 & 6.6 & 8.7 & 8.4 & 1.6 & 11 & 7.0 \\
\hline Bicarbonate ion & $\mathrm{mg} / \mathrm{L}$ & 63 & 399 & 60 & 312 & 317 & 63 & 364 & 220 \\
\hline Sulfate, dissolved & $\mathrm{mg} / \mathrm{L}$ & 50 & 290 & 31 & 128 & 125 & 41 & 183 & 97 \\
\hline Chloride, dissolved & $\mathrm{mg} / \mathrm{L}$ & 51 & 580 & 56 & 216 & 200 & 84 & 310 & 165 \\
\hline Fluoride, dissolved & $\mathrm{mg} / \mathrm{L}$ & 52 & 0.9 & 0.1 & 0.5 & 0.5 & 0.1 & 0.6 & 0.3 \\
\hline Silica, dissolved & $\mathrm{mg} / \mathrm{L}$ & 40 & 35 & 9.8 & 24 & 24 & 6.1 & 33 & 17 \\
\hline Boron, dissolved & $\mathrm{mg} / \mathrm{L}$ & 39 & 450 & 80 & 237 & 240 & 56 & 290 & 170 \\
\hline Iron, dissolved & $\mathrm{ug} / \mathrm{L}$ & 19 & 80 & 0 & 23 & 20 & 16 & 40 & 10 \\
\hline Manganese, dissolved & $\mathrm{ug} / \mathrm{L}$ & 19 & 1,900 & 60 & 653 & 650 & 534 & 1,600 & 120 \\
\hline $\begin{array}{l}\text { Nitrite + nitrate as } \mathrm{N}, \\
\text { dissolved }\end{array}$ & $\mathrm{mg} / \mathrm{L}$ & 37 & 4.1 & 0.00 & 1.4 & 1.3 & 1.2 & 3.1 & 0.06 \\
\hline Nitrate as $\mathrm{N}$, dissolved & $\mathrm{mg} / \mathrm{L}$ & 19 & 3.6 & 0.00 & 0.89 & 0.72 & 0.98 & 3.1 & 0.00 \\
\hline Nitrate as $\mathrm{NO}_{3}$, dissolved & $\mathrm{mg} / \mathrm{L}$ & & $\ldots$ & 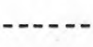 & 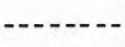 & & & & 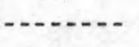 \\
\hline Ammonia $\mathrm{N}$, total & $\mathrm{mg} / \mathrm{L}$ & 31 & 3.8 & 0.04 & 0.38 & 0.22 & 0.66 & 0.55 & 0.07 \\
\hline Organic $\mathrm{N}$, total & $\mathrm{mg} / \mathrm{L}$ & 55 & 12.0 & 0.00 & 1.3 & 0.70 & 2.2 & 2.5 & 0.19 \\
\hline Phosphorus, total & $\mathrm{mg} / \mathrm{L}$ & 39 & 2.3 & 0.21 & 0.50 & 0.43 & 0.35 & 0.83 & 0.24 \\
\hline Phosphorus, dissolved & $\mathrm{mg} / \mathrm{L}$ & 64 & 1.9 & 0.01 & 0.33 & 0.30 & 0.23 & 0.48 & 0.19 \\
\hline Fecal coliform & $\mathrm{col} / 100 \mathrm{ml}$ & 28 & 19,000 & 25 & -....... & 527 & -....... & 3,300 & 45 \\
\hline Fecal streptococci & $\mathrm{col} / 100 \mathrm{ml}$ & 28 & 26,000 & 60 & -....- & 962 & - n. & 5,400 & 330 \\
\hline Oxygen, dissolved & $\mathrm{mg} / \mathrm{L}$ & 34 & 15.0 & 4.0 & 10.1 & 10.0 & 2.7 & 13.5 & 7.0 \\
\hline $\begin{array}{l}\text { Biochemical oxygen demand } \\
\text { (5-day) }\end{array}$ & $\mathrm{mg} / \mathrm{L}$ & 32 & 9.6 & 0.8 & 3.3 & 2.6 & 2.1 & 6.8 & 1.7 \\
\hline $\mathrm{pH}$ & units & 85 & 8.7 & 6.6 & 7.8 & 7.9 & 0.4 & 8.4 & 7.3 \\
\hline Co $-\mathrm{Pt}$ & units & 52 & 500 & 3 & 40 & 22 & 86 & 41 & 6 \\
\hline
\end{tabular}


06803530 Rock Creek near Ceresco, Nebr.--Continued

Results of regression analyses relating concentrations of selected chemical constituents to specific conductance $(\mathrm{SpC})$

\begin{tabular}{|c|c|c|c|c|c|}
\hline $\begin{array}{l}\text { Dissolved } \\
\text { constituents } \\
\text { (mg/L) }\end{array}$ & $\begin{array}{l}\text { No. of } \\
\text { measure- } \\
\text { ments }\end{array}$ & Regression equation & $\begin{array}{l}\text { Corre- } \\
\text { lation } \\
\text { coeffi- } \\
\text { cient }\end{array}$ & $\begin{array}{l}\text { Percent } \\
\text { explained } \\
\text { variance }\end{array}$ & $\begin{array}{c}\text { Standard } \\
\text { error of } \\
\text { estimate } \\
(\mathrm{mg} / \mathrm{L})\end{array}$ \\
\hline Dissolved solids, residue (RDS) & 44 & $\mathrm{RDS}=0.670(\mathrm{SpC})-83.347$ & 0.98 & 97 & 48 \\
\hline Dissolved solids, sum (SDS) & 37 & $S D S=0.565(S p C)+40.562$ & .98 & 97 & 30 \\
\hline Hardness as $\mathrm{CaCO}_{3}(\mathrm{TH})$ & 49 & $\mathrm{TH}=0.120(\mathrm{SpC})+100.929$ & .77 & 59 & 37 \\
\hline Calcium (Ca) & 49 & $\mathrm{Ca}=0.032(\mathrm{SpC})+30.694$ & .71 & 51 & 12 \\
\hline Magnesium (Mg) & 49 & $\mathrm{Mg}=0.010(\mathrm{SpC})+6.122$ & .79 & 63 & 2.8 \\
\hline Sodium (Na) & 49 & $\mathrm{Na}=0.164(\mathrm{SpC})-34.646$ & .98 & 95 & 14 \\
\hline Potassium (K) & 49 & $K=-0.0005(\mathrm{SpC})+9.447$ & $*_{-} .12$ & 1.4 & 1.7 \\
\hline Bicarbonate $\left(\mathrm{HCO}_{3}\right)$ & 61 & $\mathrm{HCO}_{3}=0.095(\mathrm{SpC})+182.640$ & .53 & 28 & 54 \\
\hline Sulfate $\left(\mathrm{SO}_{4}\right)$ & 48 & $\mathrm{SO}_{4}=0.106(\mathrm{SpC})-19.662$ & .97 & 93 & 11 \\
\hline Chloride (Cl) & 49 & $\mathrm{Cl}-0.216(\mathrm{SpC})-84.086$ & .96 & 93 & 22 \\
\hline Silica (Si) & 38 & $\mathrm{Si}=0.005(\mathrm{SpC})+16.995$ & $* .25$ & 6.1 & 6.0 \\
\hline Nitrite + Nitrate as $\mathrm{N}\left(\mathrm{NO}_{2}-\mathrm{NO}_{3}\right)$ & 37 & $\mathrm{NO}_{2}-\mathrm{NO}_{3}=-0.001(\mathrm{SpC})+2.768$ & $*_{-} .30$ & 9.3 & 1.2 \\
\hline
\end{tabular}

* Not significant at the 95 percent confidence level; the regression equation should not be used to predict the concentration of the dependent variable.

Results of regression analyses relating specific conductance to water discharge (Q)

\begin{tabular}{cccccccc}
\hline $\begin{array}{c}\text { No. of } \\
\text { measure- } \\
\text { ments }\end{array}$ & $\begin{array}{c}\text { Water discharge } \\
\text { Maximum Minimum }\end{array}$ & Regression equation & $\begin{array}{c}\text { Correlation } \\
\text { coefficient }\end{array}$ & $\begin{array}{c}\text { Percent } \\
\text { explained } \\
\text { variance }\end{array}$ & \multicolumn{2}{c}{$\begin{array}{c}\text { Standard error } \\
\text { of estimate }\end{array}$} \\
\hline 62 & 1,230 & 2.1 & $\mathrm{SpC}=2,292 \mathrm{Q}^{-0.24353}$ & -0.63 & 39 & 0.134 & 31 \\
\hline
\end{tabular}


Location.--Lat $40^{\circ} 57^{\prime} 56^{\prime \prime}$, long $96^{\circ} 27^{\prime} 01^{\prime \prime}$, at center of sec. 31, T.12 N., R.9 E., Cass County, at gaging station at county road bridge $0.5 \mathrm{mi}$ west of Greenwood.

Drainage area. $--1,051 \mathrm{mi}^{2}$.

Period of record.-- July 1971 to September 1972.

Statistical data for selected chemical constituents

\begin{tabular}{|c|c|c|c|c|c|c|c|c|c|}
\hline Constituent & Units & $\begin{array}{l}\text { No. of } \\
\text { measure- } \\
\text { ments }\end{array}$ & $\begin{array}{l}\text { Max- } \\
\text { imum }\end{array}$ & $\begin{array}{l}\text { Min- } \\
\text { imum }\end{array}$ & Mean & Median & $\begin{array}{l}\text { Standard } \\
\text { devi- } \\
\text { ation }\end{array}$ & $\begin{array}{l}\text { Ninetieth } \\
\text { percent- } \\
\text { ile }\end{array}$ & $\begin{array}{l}\text { Tenth } \\
\text { percent- } \\
\text { ile }\end{array}$ \\
\hline Specific conductance & umho/cm & 12 & 9,130 & 2,160 & 6,230 & 6,200 & 1,980 & 8,650 & 4,650 \\
\hline Dissolved solids, residue & $\mathrm{mg} / \mathrm{L}$ & 12 & 5,180 & 1,320 & 3,750 & 3,850 & 1,060 & 4,930 & 2,800 \\
\hline Dissolved solids, sum & $\mathrm{mg} / \mathrm{L}$ & & & & --- & 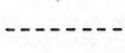 & $\sin ^{2}$ & -5 & $-1-1$ \\
\hline Hardness as $\mathrm{CaCO}_{3}$ & $\mathrm{mg} / \mathrm{L}$ & 12 & 440 & 260 & 360 & 360 & 52 & 420 & 310 \\
\hline Calcium, dissolved & $\mathrm{mg} / \mathrm{L}$ & 12 & 120 & 70 & 96 & 94 & 14 & 115 & 84 \\
\hline Magnesium, dissolved & $\mathrm{mg} / \mathrm{L}$ & 12 & 35 & 20 & 29 & 29 & 4.5 & 34 & 25 \\
\hline Sodium, dissolved & $\mathrm{mg} / \mathrm{L}$ & 12 & 1,800 & 340 & 1,190 & 1,250 & 420 & 1,730 & 830 \\
\hline Potassium, dissolved & $\mathrm{mg} / \mathrm{L}$ & 12 & 20 & 14 & 16 & 16 & 1.9 & 18 & 14 \\
\hline Bicarbonate ion & $\mathrm{mg} / \mathrm{L}$ & 12 & 426 & 286 & 360 & 363 & 41 & 410 & 290 \\
\hline Sulfate, dissolved & $\mathrm{mg} / \mathrm{L}$ & 12 & 520 & 140 & 312 & 330 & 107 & 420 & - n..... \\
\hline Chloride, dissolved & $\mathrm{mg} / \mathrm{L}$ & 12 & 2,700 & 470 & 1,710 & 1,800 & 624 & 2,500 & 1,200 \\
\hline Fluoride, dissolved & $\mathrm{mg} / \mathrm{L}$ & 12 & 1.1 & 0.4 & 0.8 & 0.8 & 0.2 & 1.0 & 0.6 \\
\hline Silica, dissolved & $\mathrm{mg} / \mathrm{L}$ & & & & & & & & \\
\hline Boron, dissolved & $\mathrm{mg} / \mathrm{L}$ & & & & & & & & \\
\hline Iron, dissolved & ug/L & 12 & 40 & 20 & 27 & 34 & 6 & 51 & 20 \\
\hline Manganese, dissolved & $\mathrm{ug} / \mathrm{L}$ & 12 & 1,100 & 60 & 441 & 380 & 350 & 657 & 90 \\
\hline $\begin{array}{l}\text { Nitrite }+ \text { nitrate as } \mathrm{N} \text {, } \\
\text { dissolved } \\
\text { Nitrate as } \mathrm{N} \text {, dissolved }\end{array}$ & $\begin{array}{l}\mathrm{mg} / \mathrm{L} \\
\mathrm{mg} / \mathrm{L}\end{array}$ & 12 & 7.1 & 0.57 & 1.8 & 1.2 & 1.9 & 4.3 & 0.60 \\
\hline $\begin{array}{l}\text { Nitrate as } \mathrm{NO}_{3} \text {, dissolved } \\
\text { Armonia } \mathrm{N} \text {, total }\end{array}$ & $\begin{array}{l}\mathrm{mg} / \mathrm{L} \\
\mathrm{mg} / \mathrm{L}\end{array}$ & & & & & & & & \\
\hline Organic $N$, total & $\mathrm{mg} / \mathrm{L}$ & 12 & 1.6 & 0.00 & 0.64 & 0.60 & 0.55 & 1.3 & 0.10 \\
\hline Phosphorus, total & $\mathrm{mg} / \mathrm{L}$ & 12 & 4.3 & 1.4 & 3.3 & 3.4 & 0.79 & 4.0 & 2.3 \\
\hline Phosphorus, dissolved & $\mathrm{mg} / \mathrm{L}$ & 12 & 4.0 & 1.0 & 3.0 & 3.2 & 0.82 & 3.9 & 2.0 \\
\hline Fecal coliform & $100 \mathrm{ml}$ & 11 & 60,000 & 330 & $-\cdots--$ & 7,000 & 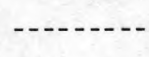 & 48,000 & 700 \\
\hline Fecal streptococci & $100 \mathrm{ml}$ & 11 & 21,000 & 170 & $-\cdots-1$ & 1,000 & 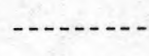 & $-17,500$ & 210 \\
\hline Oxygen, dissolved & $\mathrm{mg} / \mathrm{L}$ & 12 & 10.2 & 4.6 & 7.2 & 7.5 & 2.0 & 9.7 & 4.7 \\
\hline \multirow{2}{*}{$\begin{array}{l}\text { Biochemical oxygen demand } \\
\mathrm{pH}^{(5-\text { day })}\end{array}$} & $\mathrm{mg} / \mathrm{L}$ & 13 & 9.3 & 1.6 & 5.7 & 5.6 & 2.3 & 8.8 & 2.2 \\
\hline & I units & 23 & 7.9 & 6.7 & 7.5 & 7.6 & 0.3 & 7.9 & 7.1 \\
\hline Co - Pt & units & 12 & 60 & 10 & 22 & 30 & 13 & 30 & 0 \\
\hline
\end{tabular}


06803565 Salt Creek above Ashland, Nebr.

Location.--Lat $41^{\circ} 01^{\prime} 34^{\prime \prime}$, long $96^{\circ} 24^{\prime} 22^{\prime \prime}$, in $N^{p}-N^{\frac{1}{4}} \mathrm{sec}$. 10, T.12 N., R.9 E., Saunders County, Hydrologic Unit 10200203, at county road bridge $2 \mathrm{mi}$ southwest of Ashland.

Drainage area. $--1,118 \mathrm{mi}^{2}$.

Period of record.--Water year 1971 to current year.

Statistical data for selected chemical constituents

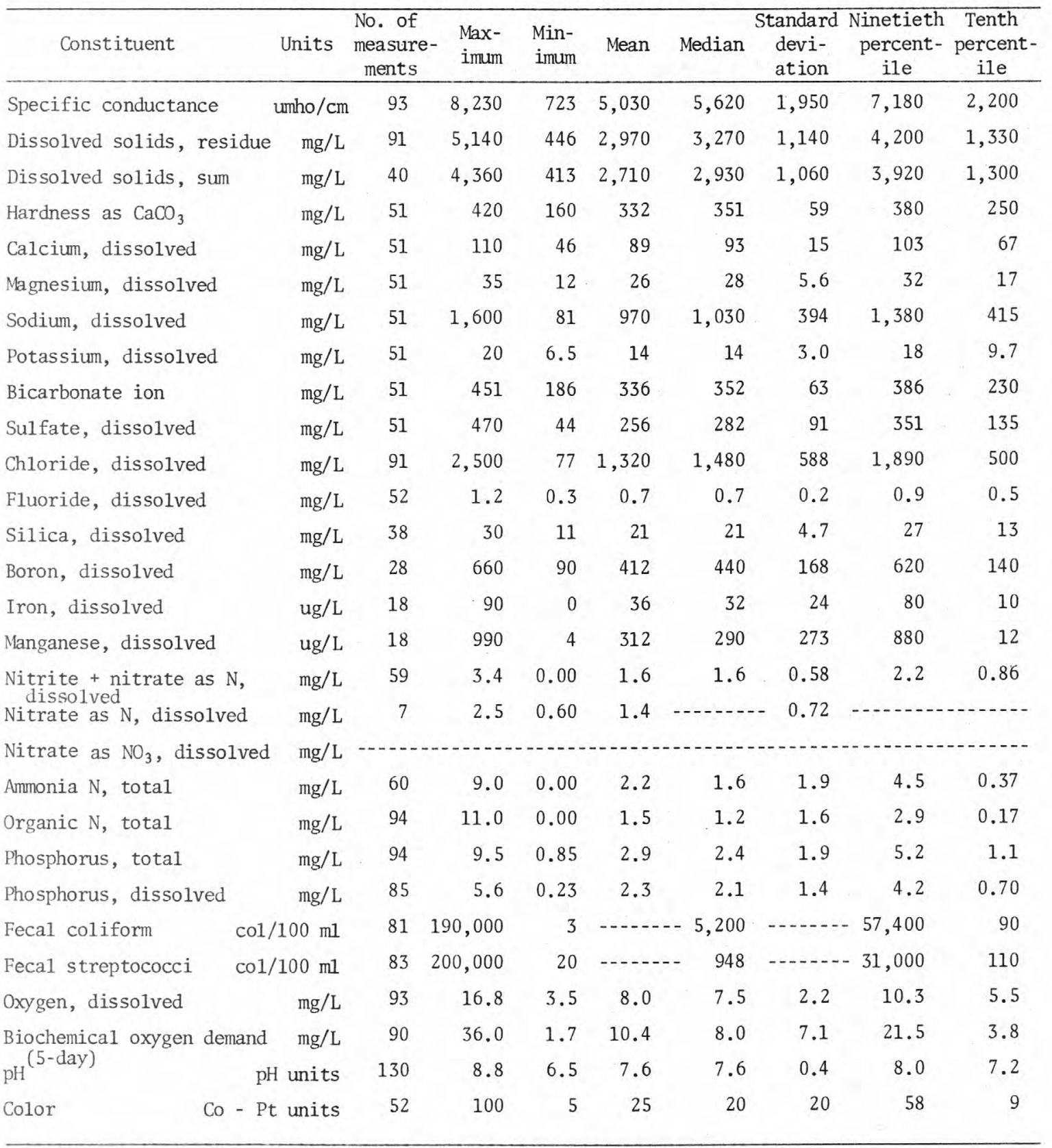


Results of regression analyses relating concentrations of selected chemical constituents to specific conductance $(\mathrm{SpC})$

\begin{tabular}{|c|c|c|c|c|c|}
\hline $\begin{array}{l}\text { Dissolved } \\
\text { constituents } \\
(\mathrm{mg} / \mathrm{L})\end{array}$ & $\begin{array}{l}\text { No. of } \\
\text { measure- } \\
\text { ments }\end{array}$ & Regression equation & $\begin{array}{l}\text { Corre- } \\
\text { lation } \\
\text { coeffi- } \\
\text { cient }\end{array}$ & $\begin{array}{l}\text { Percent } \\
\text { explained } \\
\text { variance }\end{array}$ & $\begin{array}{c}\text { Standard } \\
\text { error of } \\
\text { estimate } \\
(\mathrm{mg} / \mathrm{L})\end{array}$ \\
\hline Dissolved solids, residue (RDS) & 92 & $\mathrm{RDS}=0.580(\mathrm{SpC})+47.881$ & 0.98 & 97 & 208 \\
\hline Dissolved solids, sum (SDS) & 40 & $\mathrm{SDS}=0.566(\mathrm{SpC})+4.454$ & .99 & 99 & 111 \\
\hline Hardness as $\mathrm{CaCO}_{3}(\mathrm{TH})$ & 52 & $\mathrm{TH}=0.027(\mathrm{SpC})+192.517$ & .86 & 74 & 30 \\
\hline Calcium (Ca) & 52 & $\mathrm{Ca}=0.006(\mathrm{SpC})+55.662$ & .81 & 65 & 9.1 \\
\hline Magnesium (Mg) & 52 & $\mathrm{Mg}=0.003(\mathrm{SpC})+12.598$ & .90 & 80 & 2.5 \\
\hline Sodium $(\mathrm{Na})$ & 52 & $\mathrm{Na}-0.206(\mathrm{SpC})-89.356$ & .99 & 98 & 48 \\
\hline Potassium $(\mathrm{K})$ & 52 & $K=0.0009(\mathrm{SpC})+9.353$ & .56 & 32 & 2.5 \\
\hline Bicarbonate $\left(\mathrm{HCO}_{3}\right)$ & 52 & $\mathrm{HCO}_{3}=0.025(\mathrm{SpC})+203.822$ & .76 & 58 & 41 \\
\hline Sulfate $\left(\mathrm{SO}_{4}\right)$ & 52 & $\mathrm{SO}_{4}=0.039(\mathrm{SpC})+57.603$ & .81 & 65 & 54 \\
\hline Chloride (C1) & 92 & $\mathrm{C} 1-0.300(\mathrm{SpC})-164.521$ & .98 & 97 & 100 \\
\hline Silica (Si) & 39 & $S i=0.002(S p C)+13.167$ & .58 & 34 & 3.9 \\
\hline Nitrite + Nitrate as $\mathrm{N}\left(\mathrm{NO}_{2}-\mathrm{NO}_{3}\right)$ & 59 & $\mathrm{NO}_{2}-\mathrm{NO}_{3}=-0.00009(\mathrm{SpC})+2.025$ & -.31 & 9.8 & 0.55 \\
\hline
\end{tabular}

Results of regression analyses relating specific conductance to water discharge (Q)

\begin{tabular}{llllcccc}
\hline $\begin{array}{c}\text { No. of } \\
\text { measure- } \begin{array}{c}\text { Water discharge } \\
\text { ments }\end{array}\end{array} \frac{\left.\mathrm{ft}^{3} / \mathrm{s}\right)}{\text { Maximum Minimum }}$ & Regression equation & $\begin{array}{c}\text { Correlation } \\
\text { coefficient }\end{array}$ & $\begin{array}{c}\text { Percent } \\
\text { explained } \\
\text { variance }\end{array}$ & \multicolumn{2}{c}{$\begin{array}{c}\text { Standard error } \\
\text { of estimate }\end{array}$} \\
\hline 93 & $1,650 \quad 46$ & $\mathrm{SpC}=162,000 \mathrm{Q}^{-0.70218}$ & -0.92 & 86 & 0.090 & 21 \\
\hline
\end{tabular}




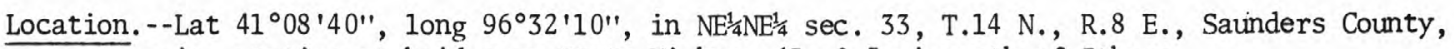
at gaging station at bridge on State Highway $63,0.5 \mathrm{mi}$ south of Ithaca.

Drainage area. $--272 \mathrm{mi}^{2}$, approximate1y.

Period of record.--October 1966 to September 1968 .

Statistical data for selected chemical constituents

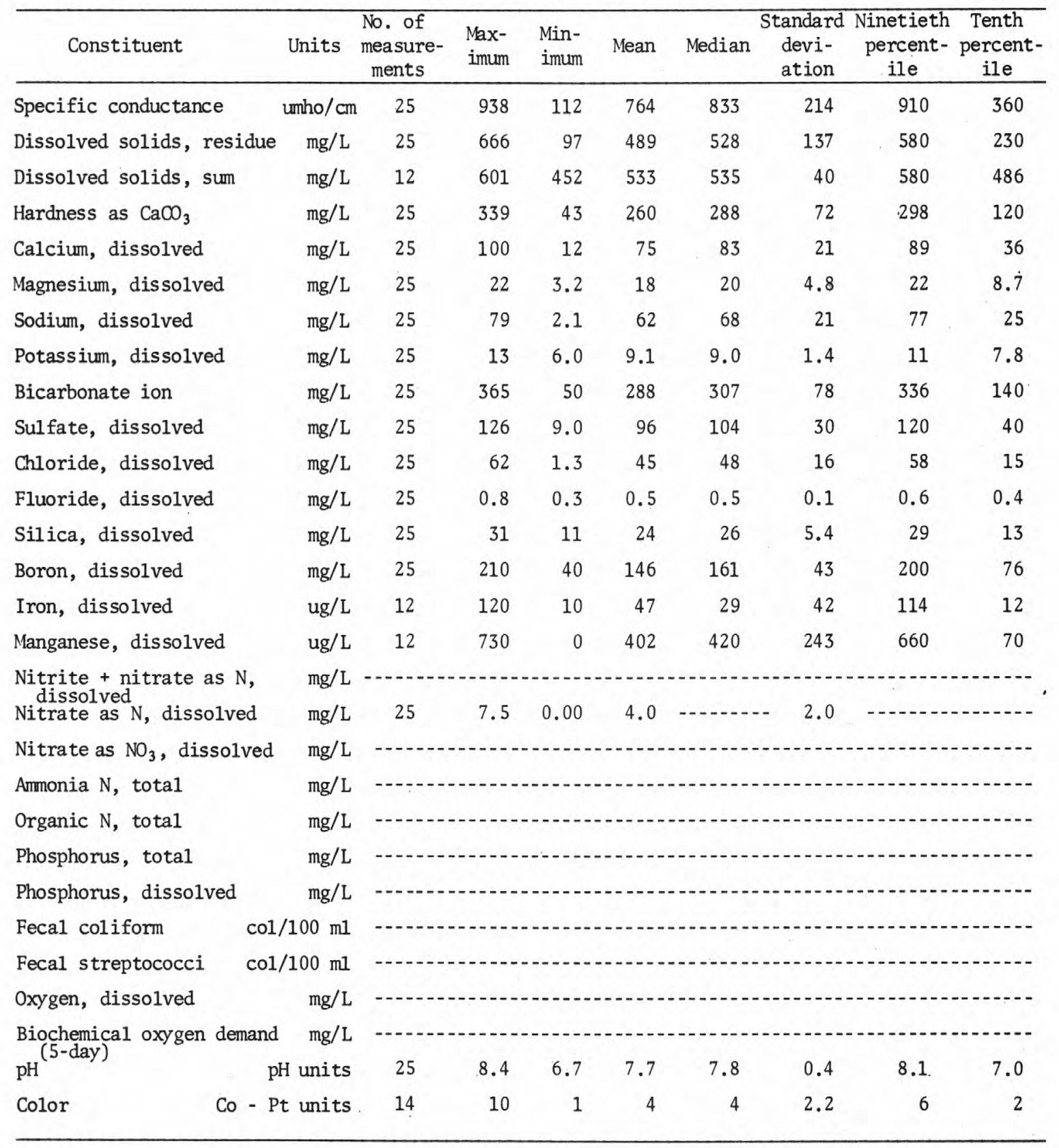


Results of regression analyses relating concentrations of selected chemical constituents to specific conductance $(\mathrm{SpC})$

\begin{tabular}{|c|c|c|c|c|c|}
\hline $\begin{array}{l}\text { Dissolved } \\
\text { constituents } \\
(\mathrm{mg} / \mathrm{L})\end{array}$ & $\begin{array}{l}\text { No. of } \\
\text { measure- } \\
\text { ments }\end{array}$ & Regression equation & $\begin{array}{l}\text { Corre- } \\
\text { lation } \\
\text { coeffi- } \\
\text { cient }\end{array}$ & $\begin{array}{l}\text { Percent } \\
\text { explained } \\
\text { variance }\end{array}$ & $\begin{array}{c}\text { Standard } \\
\text { error of } \\
\text { estimate } \\
(\mathrm{mg} / \mathrm{L})\end{array}$ \\
\hline Dissolved solids, residue (RDS) & 25 & $\mathrm{RDS}=0.637(\mathrm{SpC})+2.365$ & 0.99 & 98 & 18 \\
\hline Dissolved solids, sum (SDS) & & & & & \\
\hline Hardness as $\mathrm{CaCO}_{3}(\mathrm{TH})$ & 25 & $\mathrm{TH}=0.331(\mathrm{spC})+7.770$ & .98 & 97 & 12 \\
\hline Calcium (Ca) & 25 & $\mathrm{Ca}=0.097(\mathrm{spC})+0.971$ & .98 & 96 & 4.6 \\
\hline Magnesium (Mg) & 25 & $\mathrm{Mg}=0.022(\mathrm{SpC})+1.236$ & .96 & 93 & 1.3 \\
\hline Sodium ( $\mathrm{Na}$ ) & 25 & $\mathrm{Na}=0.095(\mathrm{SpC})-11.004$ & .98 & 96 & 4.0 \\
\hline Potassium (K) & 25 & $K=0.003(\mathrm{SpC})+6.837$ & .46 & 21 & 1.3 \\
\hline Bicarbonate $\left(\mathrm{HCO}_{3}\right)$ & 25 & $\mathrm{HCO}_{3}=0.351(\mathrm{SpC})+20.002$ & .97 & 94 & 20 \\
\hline Sulfate $\left(\mathrm{SO}_{4}\right)$ & 25 & $\mathrm{SO}_{4}=0.141(\mathrm{SpC})-11.400$ & .99 & 98 & 4.2 \\
\hline Chloride (C1) & 25 & $\mathrm{Cl}=0.073(\mathrm{SpC})-10.938$ & .97 & 94 & 4.1 \\
\hline Silica (Si) & 25 & $\mathrm{Si}=0.023(\mathrm{SpC})+6.361$ & .92 & 86 & 2.1 \\
\hline
\end{tabular}

Results of regression analyses relating specific conductance to water discharge $(Q)$

\begin{tabular}{ccccccc}
\hline $\begin{array}{c}\text { No. of } \\
\text { measure- } \\
\text { ments }\end{array}$ & $\begin{array}{c}\text { Water discharge } \\
\left(\mathrm{ft}^{3} / \mathrm{s}\right)\end{array}$ & Maximum Minimum & Regression equation & $\begin{array}{c}\text { Correlation } \\
\text { coefficient }\end{array}$ & $\begin{array}{c}\text { Percent } \\
\text { explained } \\
\text { variance }\end{array}$ & $\begin{array}{c}\text { Standard error } \\
\text { of estimate }\end{array}$ \\
\hline 25 & $11,400 \quad 19.4$ & $\mathrm{SpC}=2,584 \mathrm{Q}^{-0.32999}$ & -0.97 & 94 & 0.052 & 12 \\
\hline
\end{tabular}


LOWER PLATTE RIVER BASIN

06804495 Silver Creek near Wahoo, Nebr.

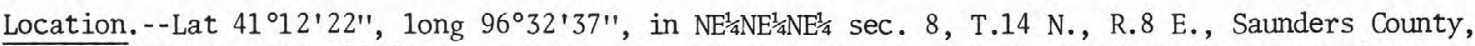
Hydrologic Unit 10200203, at bridge on county road $3.9 \mathrm{mi}$ east of intersection of First Street and U.S. Highway 77 in Wahoo.

Period of record.--Water year 1974 to current year.

Statistical data for selected chemical constituents

\begin{tabular}{|c|c|c|c|c|c|c|c|c|c|}
\hline Constituent & Units & $\begin{array}{l}\text { No. of } \\
\text { measure- } \\
\text { ments }\end{array}$ & $\begin{array}{l}\text { Max- } \\
\text { imum }\end{array}$ & $\begin{array}{l}\text { Min- } \\
\text { imum }\end{array}$ & Mean & Median & $\begin{array}{c}\text { Standard } \\
\text { devi- } \\
\text { ation }\end{array}$ & $\begin{array}{c}\text { Ninetieth } \\
\text { percent- } \\
\text { ile }\end{array}$ & $\begin{array}{l}\text { Tenth } \\
\text { percent- } \\
\text { ile }\end{array}$ \\
\hline Specific conductance & umho/cm & 48 & 713 & 75 & 539 & 546 & 99 & 620 & 432 \\
\hline Dissolved solids, residue & $\mathrm{mg} / \mathrm{L}$ & 31 & 408 & 220 & 337 & 338 & 39 & 390 & 300 \\
\hline Dissolved solids, sum & $\mathrm{mg} / \mathrm{L}$ & 16 & 439 & 36 & 328 & 343 & 87 & 390 & 271 \\
\hline Hardness as $\mathrm{CaCOO}_{3}$ & $\mathrm{mg} / \mathrm{L}$ & 16 & 250 & 17 & 210 & 228 & 53 & 240 & 190 \\
\hline Calcium, dissolved & $\mathrm{mg} / \mathrm{L}$ & 16 & 71 & 5.3 & 60 & 64 & 15 & 67 & 56 \\
\hline Magnesium, dissolved & $\mathrm{mg} / \mathrm{L}$ & 16 & 18 & 1.0 & 15 & 16 & 3.8 & 17 & 13 \\
\hline Sodium, dissolved & $\mathrm{mg} / \mathrm{L}$ & 16 & 57 & 2.4 & 28 & 28 & 12 & 43 & 20 \\
\hline Potassium, dissolved & $\mathrm{mg} / \mathrm{L}$ & 16 & 11 & 5.4 & 9.0 & 9.5 & 1.2 & 9.9 & 7.9 \\
\hline Bicarbonate ion & $\mathrm{mg} / \mathrm{L}$ & 16 & 296 & 18 & 260 & 280 & 66 & 293 & 245 \\
\hline Sulfate, dissolved & $\mathrm{mg} / \mathrm{L}$ & 16 & 43 & 7.7 & 34 & 38 & 9.6 & 42 & 21 \\
\hline Chloride, dissolved & $\mathrm{mg} / \mathrm{L}$ & 46 & 66 & 1.3 & 19 & 16 & 14 & 38 & 4.8 \\
\hline F1uoride, dissolved & $\mathrm{mg} / \mathrm{L}$ & 16 & 0.6 & 0.2 & 0.4 & 0.6 & 0.08 & 0.5 & 0.4 \\
\hline Silica, dissolved & $\mathrm{mg} / \mathrm{L}$ & 16 & 37 & 3.6 & 30 & 34 & 7.9 & 36 & 26 \\
\hline Boron, dissolved & $\mathrm{mg} / \mathrm{L}$ & 16 & 90 & 40 & 61 & 60 & 12 & 71 & 40 \\
\hline Iron, dissolved & $\mathrm{ug} / \mathrm{L}$ & 15 & 1,200 & 0 & 146 & 46 & 304 & 330 & 10 \\
\hline Manganese, dissolved & $\mathrm{ug} / \mathrm{L}$ & 15 & 430 & 10 & 237 & 220 & 133 & 423 & 50 \\
\hline $\begin{array}{l}\text { Nitrite + nitrate as } \mathrm{N} \text {, } \\
\text { dissolved }\end{array}$ & $\mathrm{mg} / \mathrm{L}$ & 21 & 4.9 & 1.1 & 1.7 & 1.5 & 0.81 & 2.1 & 1.2 \\
\hline Nitrate as N, dissolved & g/L & & & & 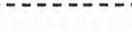 & & & & \\
\hline Nitrate as $\mathrm{NO}_{3}$, dissolved & $\mathrm{mg} / \mathrm{L}$ & & & & - & & & & -- \\
\hline Ammonia $\mathrm{N}$, total & $\mathrm{mg} / \mathrm{L}$ & 49 & 4.1 & 0.00 & 1.0 & 0.18 & 1.3 & 3.3 & 0.02 \\
\hline Organic $N$, total & $\mathrm{mg} / \mathrm{L}$ & 48 & 8.1 & 0.00 & 1.0 & 0.67 & 1.2 & 2.0 & 0.21 \\
\hline Phosphorus, total & $\mathrm{mg} / \mathrm{L}$ & 49 & 2.7 & 0.24 & 0.49 & 0.41 & 0.36 & 0.62 & 0.28 \\
\hline Phosphorus, dissolved. & $\mathrm{mg} / \mathrm{L}$ & 40 & 0.57 & 0.01 & 0.31 & 0.31 & 0.11 & 0.47 & 0.20 \\
\hline Fecal coliform & $\mathrm{col} / 100 \mathrm{ml}$ & 35 & 6,610 & 30 & $---n--$ & 380 & 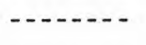 & 1,650 & 79 \\
\hline Fecal streptococci & $\mathrm{col} / 100 \mathrm{ml}$ & 45 & 7,670 & 68 & 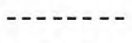 & 765 & $---\cdots$ & 2,200 & 230 \\
\hline Oxygen, dissolved & $\mathrm{mg} / \mathrm{L}$ & 46 & 22.5 & 3.7 & 10.4 & 10.1 & 3.0 & 12.8 & 7.2 \\
\hline \multirow{2}{*}{$\begin{array}{l}\text { Biochemical oxygen demand } \\
\text { (5-day) }\end{array}$} & $\mathrm{mg} / \mathrm{L}$ & 48 & 12.0 & 0.5 & 5.0 & 4.6 & 2.7 & 8.8 & 2.1 \\
\hline & $H$ units & 50 & 8.8 & 6.8 & 7.7 & 7.6 & 0.4 & 8.4 & 7.3 \\
\hline Co - Pt & $t$ units & 16 & 400 & 0 & 31 & 5 & 99 & 17 & 2 \\
\hline
\end{tabular}


Results of regression analyses relating concentrations of selected chemical constituents to specific conductance $(\mathrm{SpC})$

\begin{tabular}{|c|c|c|c|c|c|}
\hline $\begin{array}{l}\text { Dissolved } \\
\text { constituents } \\
\text { (mg/L) }\end{array}$ & $\begin{array}{c}\text { No. of } \\
\text { measure- } \\
\text { ments }\end{array}$ & Regression equation & $\begin{array}{l}\text { Corre- } \\
\text { lation } \\
\text { coeffi- } \\
\text { cient }\end{array}$ & $\begin{array}{l}\text { Percent } \\
\text { explained } \\
\text { variance }\end{array}$ & $\begin{array}{c}\text { Standard } \\
\text { error of } \\
\text { estimate } \\
(\mathrm{mg} / \mathrm{L})\end{array}$ \\
\hline Dissolved solids, residue (RDS) & 31 & $\mathrm{RDS}=0.401(\mathrm{SpC})+114.084$ & 0.80 & 64 & 24 \\
\hline Dissolved solids, sum (SDS) & 16 & $\mathrm{SDS}=0.633(\mathrm{SpC})-4.011$ & .97 & 94 & 22 \\
\hline Hardness as $\mathrm{CaCO}_{3}(\mathrm{TH})$ & 16 & $\mathrm{TH}=0.375(\mathrm{SpC})+13.212$ & .93 & 87 & 20 \\
\hline Calcium (Ca) & 16 & $\mathrm{Ca}=0.104(\mathrm{SpC})+5.387$ & .92 & 85 & 6.0 \\
\hline Magnesium (Mg) & 16 & $M g=0.027(\mathrm{spC})+0.604$ & .93 & 86 & 1.5 \\
\hline Sodium (Na) & 16 & $\mathrm{Na}-0.072(\mathrm{SpC})-9.578$ & .81 & 65 & 7.2 \\
\hline Potassium (K) & 16 & $K=0.008(\mathrm{SpC})+4.872$ & .87 & 76 & 0.6 \\
\hline Bicarbonate $\left(\mathrm{HCO}_{3}\right)$ & 16 & $\mathrm{HCO}_{3}=0.470(\mathrm{SpC})+13.490$ & .94 & 89 & 23 \\
\hline Sulfate $\left(\mathrm{SO}_{4}\right)$ & 16 & $\mathrm{SO}_{4}=0.058(\mathrm{SpC})+3.609$ & .81 & 65 & 5.8 \\
\hline Chloride $(\mathrm{Cl})$ & 47 & $\mathrm{Cl}=0.072(\mathrm{SpC})-20.049$ & .52 & 27 & 12 \\
\hline Silica (Si) & 16 & $\mathrm{Si}=0.052(\mathrm{SpC})+3.248$ & .87 & 76 & 4.0 \\
\hline Nitrite + Nitrate as $\mathrm{N}\left(\mathrm{NO}_{2}-\mathrm{NO}_{3}\right)$ & 21 & $\mathrm{NO}_{2}-\mathrm{NO}_{3}=-0.003(\mathrm{SpC})+3.558$ & $*_{-} .19$ & 3.5 & 0.81 \\
\hline
\end{tabular}

* Not significant at the 95 percent confidence level; the regression equation should not be used to predict the concentration of the dependent variable.

Results of regression analyses relating specific conductance to water discharge (Q)

\begin{tabular}{cccccccc}
\hline \multirow{2}{*}{$\begin{array}{c}\text { No. of } \\
\text { measure- }\end{array} \begin{array}{c}\text { Water discharge } \\
\text { ments }\end{array}$} & $\begin{array}{cccccc}\left.\mathrm{ft}^{3} / \mathrm{s}\right) \\
\text { Maximum Minimum }\end{array}$ & Regression equation & $\begin{array}{c}\text { Correlation } \\
\text { coefficient }\end{array}$ & $\begin{array}{c}\text { Percent } \\
\text { explained } \\
\text { variance }\end{array}$ & \multicolumn{2}{c}{$\begin{array}{c}\text { Standard error } \\
\text { of estimate }\end{array}$} \\
\hline 48 & 498 & 1.5 & $\mathrm{SpC}=741 \mathrm{Q}^{-0.20836}$ & -0.59 & 35 & 0.112 & 26 \\
\hline
\end{tabular}


Location.--Lat $41^{\circ} 00^{\prime} 55^{\prime \prime}$, long $96^{\circ} 09^{\prime} 28^{\prime \prime}$, in $\mathrm{NW}^{\frac{1}{4}} \mathrm{NW} \frac{1}{4} \mathrm{sec} .14, \mathrm{~T} .12 \mathrm{~N} ., \mathrm{R} .11$ E., Sarpy County, Hydrologic Unit 10200202, on left bank at upstream side of bridge on Nebraska Highway 50 , $1 \mathrm{mi}$ north of Louisville.

Drainage area (revised) $--85,800 \mathrm{mi}^{2}$, approximately, of which about $71,000 \mathrm{mi}^{2}$ contributes directly to surface runoff. Approximately $4,000 \mathrm{mi}^{2}$ in Great Divide Basin is not included.

Period of record. - -Water year 1972 to current year.

Statistical data for selected chemical constituents

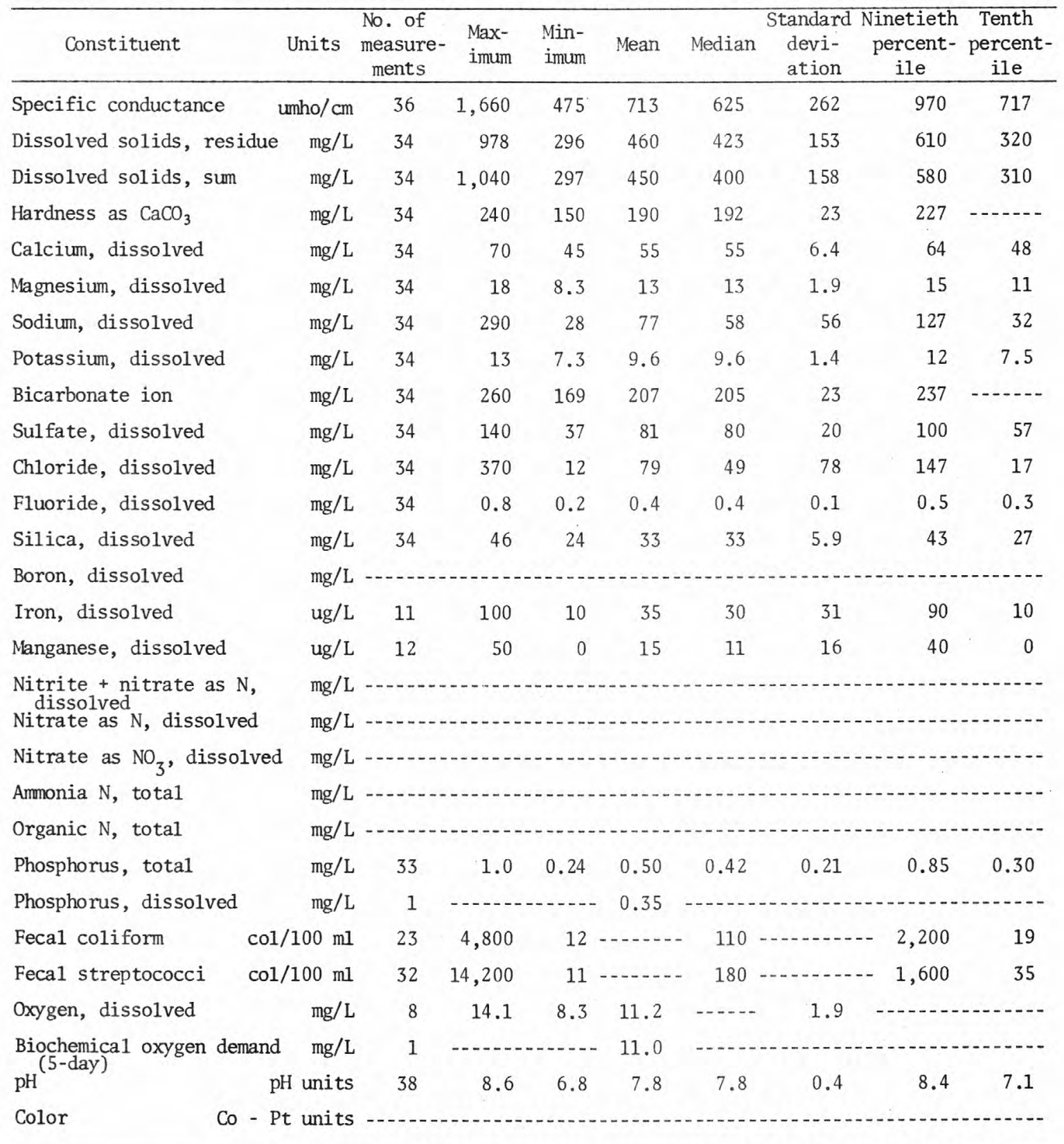


Results of regression analyses relating concentrations of selected chemical constituents to specific conductance $(\mathrm{SpC})$

\begin{tabular}{|c|c|c|c|c|c|}
\hline $\begin{array}{c}\text { Dissolved } \\
\text { constituents } \\
(\mathrm{mg} / \mathrm{L})\end{array}$ & $\begin{array}{l}\text { No. of } \\
\text { measure- } \\
\text { ments }\end{array}$ & Regression equation & $\begin{array}{c}\text { Corre- } \\
\text { lation } \\
\text { coeffi- } \\
\text { cient }\end{array}$ & $\begin{array}{c}\text { Percent } \\
\text { explained } \\
\text { variance }\end{array}$ & $\begin{array}{c}\text { Standard } \\
\text { error of } \\
\text { estimate } \\
(\mathrm{mg} / \mathrm{L})\end{array}$ \\
\hline Dissolved solids, residue (RDS) & 34 & $\mathrm{RDS}=0.558(\mathrm{SpC})+60.878$ & 0.98 & 96 & 29 \\
\hline Dissolved solids, sum (SDS) & 34 & $\mathrm{SDS}=0.579(\mathrm{SpC})+36.242$ & .99 & 98 & 23 \\
\hline Hardness as $\mathrm{CaCO}_{3}(\mathrm{TH})$ & 34 & $\mathrm{TH}=0.047(\mathrm{SpC})+156.638$ & .55 & 30 & 20 \\
\hline Calcium (Ca) & 34 & $\mathrm{Ca}=0.014(\mathrm{SpC})+45.426$ & .57 & 33 & 5.3 \\
\hline Magnesium (Mg) & 34 & $\mathrm{Mg}=0.004(\mathrm{SpC})+10.048$ & .52 & 27 & 1.6 \\
\hline Sodium $(\mathrm{Na})$ & 34 & $\mathrm{Na}=0.202(\mathrm{SpC})-68.001$ & .98 & 96 & 11 \\
\hline Potassium (K) & 34 & $\mathrm{~K}=0.003(\mathrm{SpC})+7.374$ & .57 & 33 & 1.2 \\
\hline Bicarbonate $\left(\mathrm{HCO}_{3}\right)$ & 34 & $\mathrm{HCO}_{3}=0.054(\mathrm{SpC})+168.241$ & .63 & 39 & 18 \\
\hline Sulfate $\left(\mathrm{SO}_{4}\right)$ & 34 & $\mathrm{SO}_{4}=0.049(\mathrm{SpC})+45.300$ & .67 & 45 & 15 \\
\hline Chloride (C1) & 34 & $\mathrm{Cl}=0.281(\mathrm{SpC})-121.556$ & .97 & 94 & 19 \\
\hline Silica (Si) & 34 & $\mathrm{Si}=-0.002(\mathrm{SpC})+34.563$ & $*_{-} .08$ & 0.66 & 5.9 \\
\hline
\end{tabular}

* Not significant at the 95 percent confidence level; the regression equation should not be used to predict the concentration of the dependent variable.

Results of regression analyses relating specific conductance to water discharge $(Q)$

\begin{tabular}{ccccccc}
\hline $\begin{array}{c}\text { No. of } \\
\text { measure- } \\
\text { ments }\end{array}$ & $\begin{array}{c}\text { Water discharge } \\
\left(\mathrm{ft}^{3} / \mathrm{s}\right)\end{array}$ & Maximum Minimum & Regression equation & $\begin{array}{c}\text { Correlation } \\
\text { coefficient }\end{array}$ & $\begin{array}{c}\text { Percent } \\
\text { explained } \\
\text { variance }\end{array}$ & $\begin{array}{c}\text { Standard error } \\
\text { of estimate }\end{array}$ \\
\hline 36 & $10,200 \quad 468$ & $\mathrm{SpC}=9,349 \mathrm{Q}^{-0.32216}$ & -0.75 & 57 & 0.087 & 20 \\
\hline
\end{tabular}




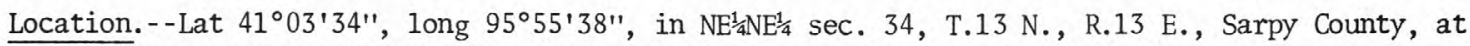
bridge on U.S. Highways 73 and $75,2.7 \mathrm{mi}$ upstream from mouth, and $1.0 \mathrm{mi}$ south of La Platte.

Period of record.--February 1974 to June 1975.

Statistical data for selected chemical constituents

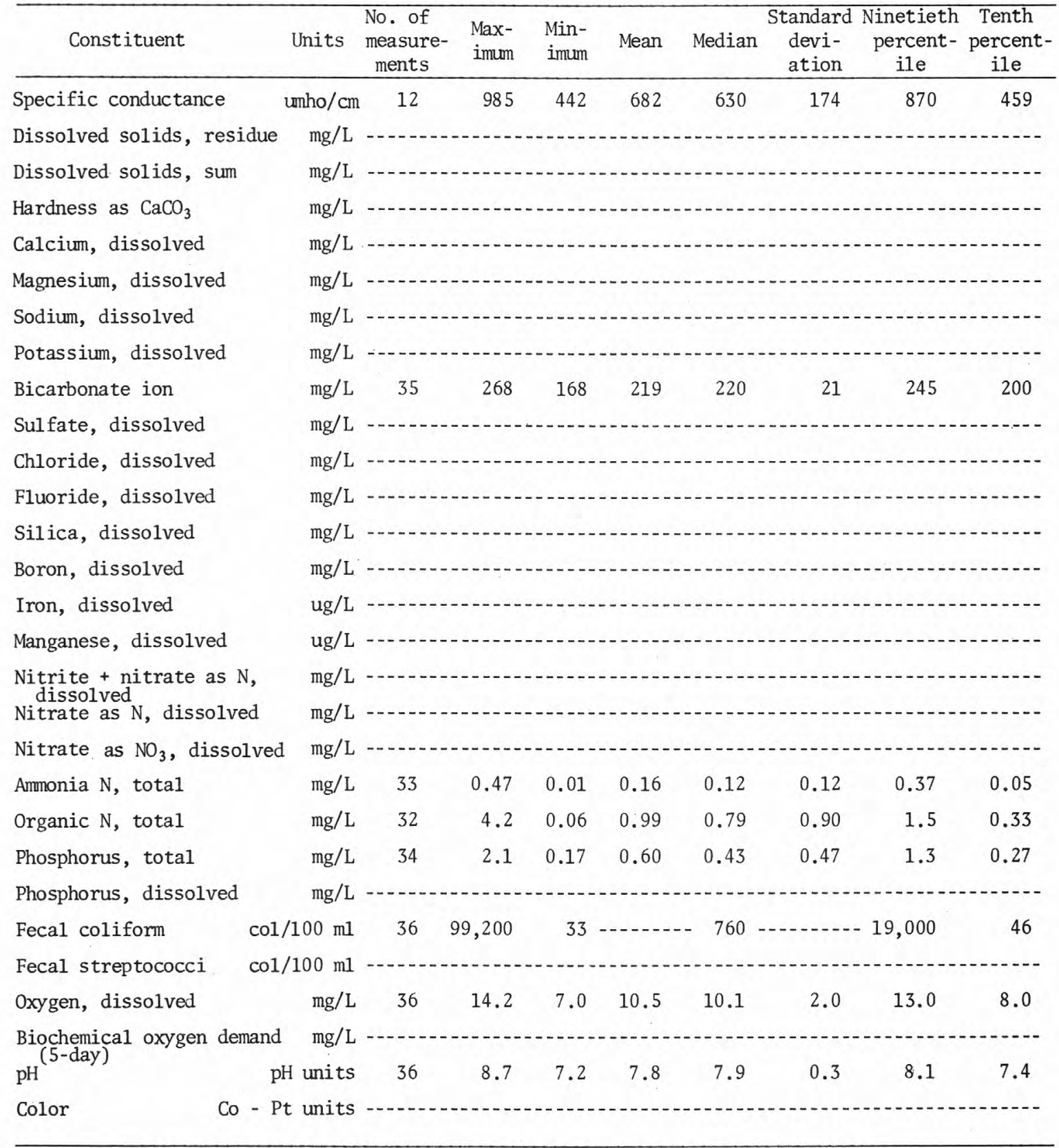


The Nemaha River basin consists mostly of three separate sma11 basins, the principal streams of which drain directly into the Missouri River. These include Weeping Water Creek in the north part of the basin, the Little Nemaha River in the middle part, and the Big Nemaha River in the south part. Part of the drainage basin of the Big Nemaha River is in northeast Kansas.

The entire Nemaha River basin lies within the glaciated part of the State. Part of the basin consists of loess-covered drift hills while the rest of the basin, excluding valleys, consists of glacial drift with soils developed directly upon it. Streamflow is characterized by high percentages of overland runoff.

Three sites, one on each of the principal streams, have sufficient water-quality data available for statistical analyses (fig. 11). These sites are all near the mouths of their respective streams and therefore the quality of water at each site is representative of outflow from its respective drainage. The mean specific conductance for 30 analyses for Weeping Water Creek near Union is $504 \mathrm{umho} / \mathrm{cm}$. Calcium is the principal cation and bicarbonate the principal anion. A mean nitrite + nitrate concentration of $4.8 \mathrm{mg} / \mathrm{L}$, a mean total phosphorus concentration of 1.7 $\mathrm{mg} / \mathrm{L}$, and a median fecal coliform bacteria concentration of $1,700 \mathrm{col} / 100$ $\mathrm{ml}$ may be due to sewage effluent and (or) feedlot runoff.

Fifty-six measurements of specific conductance at the Little Nemaha River at Auburn average 596 umho/cm. Like Weeping Water Creek, calcium and bicarbonate are the principal cation and anion, but the mean specific conductance is about 15 percent higher. Mean concentrations of nitrite + nitrate and total phosphorus are 2.2 and $0.65 \mathrm{mg} / \mathrm{L}$, respectively, and median concentrations for fecal coliform and fecal streptococcal bacteria are 2,970 and $1,380 \mathrm{col} / 100 \mathrm{ml}$, respectively. Sewage effluent from the city of Auburn enters the stream above the sampling site.

The average specific conductance of 56 measurements for the Big Nemaha River at Falls City is 657 umho/cm. For this station also, calcium and bicarbonate are the principal cation and anion. Total phosphorus and nitrite + nitrate means and fecal coliform and fecal streptococcal bacteria medians are similar to those for the Little Nemaha River at Auburn. Sewage effluent from Falls City enters the river above the sampling site.

Correlation coefficients are 0.8 or greater for all constituents except potassium, chloride, silica, and nitrite + nitrate for all three stations. 


\section{EXPLANATION}

06811500

ACTIVE WATER-QUALITY

$06805600 \quad$ SITE

INACTIVE WATER-QUALITY

SITE

- REFERENCE TOWN

- - BASIN BOUNDARY

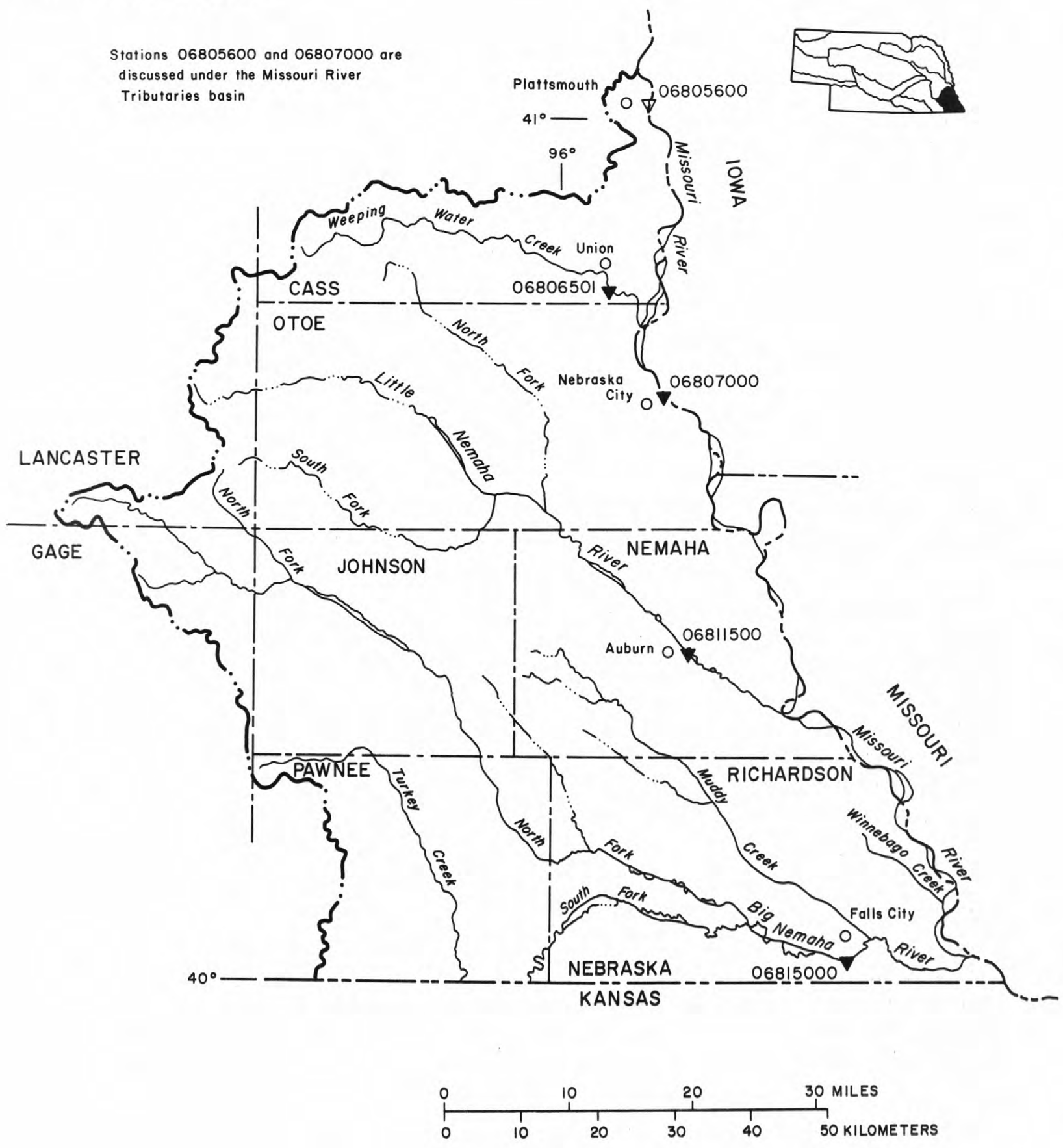

FIGURE 11.-Location of water-quality sampling sites, Nemaha River basin 


\section{NEMAHA RIVER BASIN}

06806501 Weeping Water Creek near Union, Nebr.

Location.--Lat $40^{\circ} 47^{\prime} 46^{\prime \prime}$, long $95^{\circ} 54^{\prime} 17^{\prime \prime}$, in $\mathrm{NE}^{\frac{1}{4}} \mathrm{NE}^{\frac{1}{4}} \mathrm{NW}^{\frac{1}{4}} \mathrm{sec}$. 36, T.10 N., R.13 E., Cass County, Hydrologic Unit 10240001 , at county road bridge $1.1 \mathrm{mi}$ downstream from gaging station, $1.6 \mathrm{mi}$ southeast of Union, and $3.9 \mathrm{mi}$ downstream from South Branch Weeping Water Creek.

Period of record.--Water year 1973 to current year.

Statistical data for selected chemical constituents

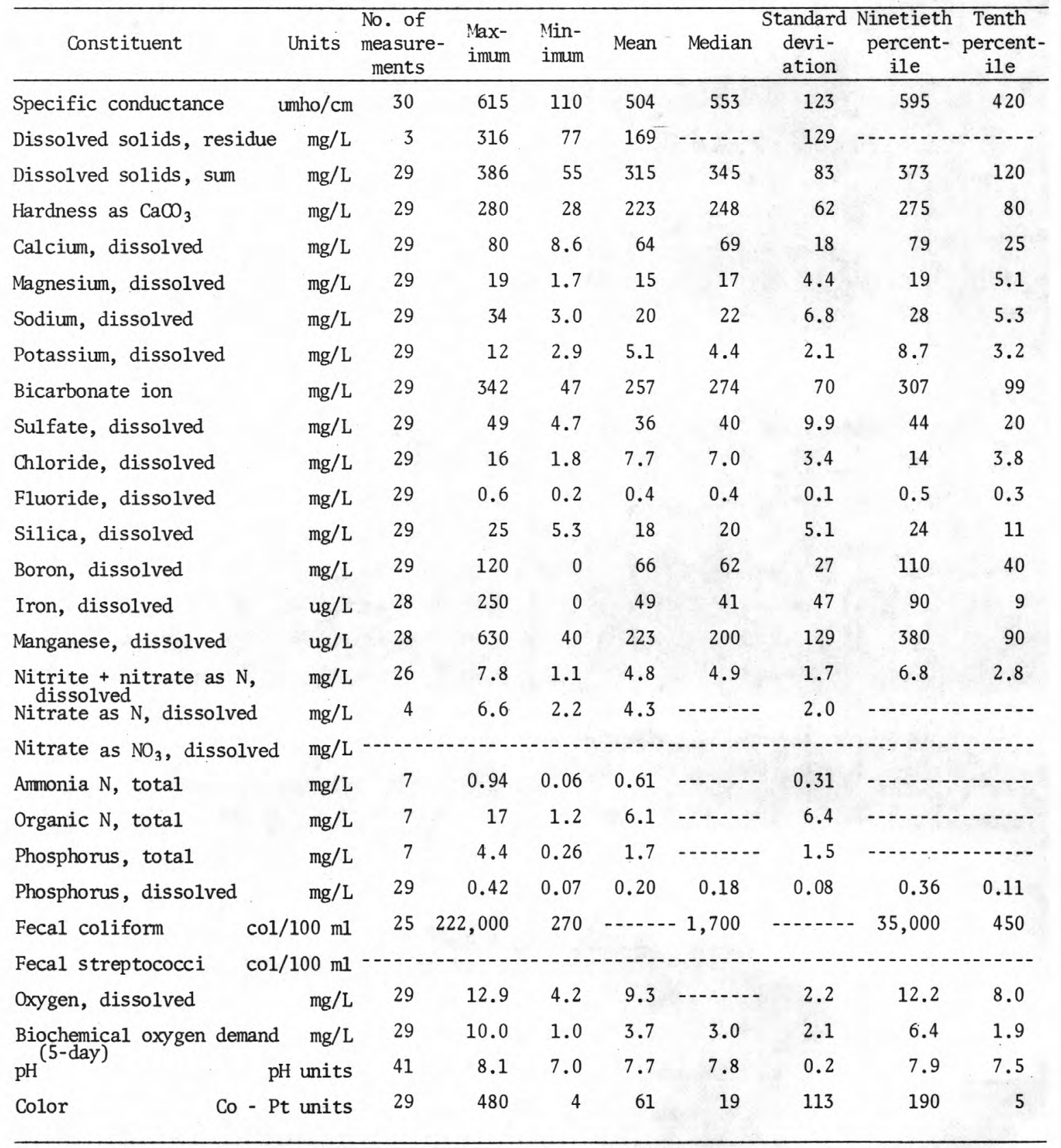


Results of regression analyses relating concentrations of selected chemical constituents to specific conductance $(\mathrm{SpC})$

\begin{tabular}{|c|c|c|c|c|c|}
\hline $\begin{array}{l}\text { Dissolved } \\
\text { constituents } \\
(\mathrm{mg} / \mathrm{L})\end{array}$ & $\begin{array}{l}\text { No. of } \\
\text { measure- } \\
\text { ments }\end{array}$ & Regression equation & $\begin{array}{l}\text { Corre- } \\
\text { lation } \\
\text { coeffi- } \\
\text { cient }\end{array}$ & $\begin{array}{l}\text { Percent } \\
\text { explained } \\
\text { variance }\end{array}$ & $\begin{array}{c}\text { Standard } \\
\text { error of } \\
\text { estimate } \\
(\mathrm{mg} / \mathrm{L})\end{array}$ \\
\hline Dissolved solids, residue (RDS) & & & & & \\
\hline Dissolved solids, sum (SDS) & 29 & $\mathrm{SDS}=0.657(\mathrm{SpC})-15.927$ & 0.99 & 99 & 9.1 \\
\hline Hardness as $\mathrm{CaCO}_{3}(\mathrm{TH})$ & 29 & $\mathrm{TH}=0.494(\mathrm{SpC})-26.085$ & .99 & 99 & 6.7 \\
\hline Calcium (Ca) & 29 & $\mathrm{Ca}=0.141(\mathrm{SpC})-7.058$ & .99 & 98 & 2.2 \\
\hline Magnesium (Mg) & 29 & $\mathrm{Mg}=0.035(\mathrm{SpC})-2.088$ & .99 & 98 & 0.7 \\
\hline Sodium $(\mathrm{Na})$ & 29 & $\mathrm{Na}=0.047(\mathrm{SpC})-3.321$ & .86 & 74 & 3.5 \\
\hline Potassium (K) & 29 & $K=-0.007(S p C)+8.693$ & -.43 & 18 & 1.9 \\
\hline Bicarbonate $\left(\mathrm{HCO}_{3}\right)$ & 29 & $\mathrm{HCO}_{3}=0.549(\mathrm{SpC})-19.512$ & .98 & 96 & 15 \\
\hline Sulfate $\left(\mathrm{SO}_{4}\right)$ & 29 & $\mathrm{SO}_{4}=0.072(\mathrm{SpC})+0.093$ & .91 & 83 & 4.1 \\
\hline Chloride (C1) & 29 & $\mathrm{Cl}=0.014(\mathrm{SpC})+0.542$ & .52 & 27 & 3.0 \\
\hline Silica (Si) & 29 & $\mathrm{Si}=0.036(\mathrm{SpC})+0.176$ & .88 & 77 & 2.5 \\
\hline Nitrite + Nitrate as $\mathrm{N}\left(\mathrm{NO}_{2}-\mathrm{NO}_{3}\right)$ & 26 & $\mathrm{NO}_{2}-\mathrm{NO}_{3}=0.010(\mathrm{SpC})-0.514$ & .48 & 23 & 1.5 \\
\hline
\end{tabular}

Results of regression analyses relating specific conductance to water discharge (Q)

\begin{tabular}{ccccccc}
\hline $\begin{array}{c}\text { No. of } \\
\text { measure- } \\
\text { ments }\end{array}$ & $\begin{array}{c}\text { Water discharge } \\
\text { Maximum Minimum }\end{array}$ & Regression equation & $\begin{array}{c}\text { Correlation } \\
\text { coefficient }\end{array}$ & $\begin{array}{c}\text { Percent } \\
\text { explained } \\
\text { variance }\end{array}$ & $\begin{array}{c}\text { Standard error } \\
\text { of estimate }\end{array}$ \\
\hline $\begin{array}{l}30 \\
2\end{array}$ & 4,290 & 7.1 & $\mathrm{SpC}=1,261 \mathrm{Q}^{-0.20944}$ & -0.68 & 47 & 0.125 \\
\hline
\end{tabular}


Location.--Lat $40^{\circ} 23^{\prime} 33^{\prime \prime}$, long $95^{\circ} 48^{\prime} 46^{\prime \prime}$, in NE $\frac{1}{4} \mathrm{NW} \frac{1}{4} \mathrm{sec} .23$, T. 5 N., R.14 E., Nemaha County, Hydrologic Unit 10240006, on left bank at downstream side of bridge on U.S. Highway 136, $1 \mathrm{mi}$ downstream from Longs Creek and Willow Creek and $1 \mathrm{mi}$ east of Auburn.

Drainage area. $--793 \mathrm{mi}^{2}$.

Period of record.-- Water year 1973 to current year.

Statistical data for selected chemical constituents

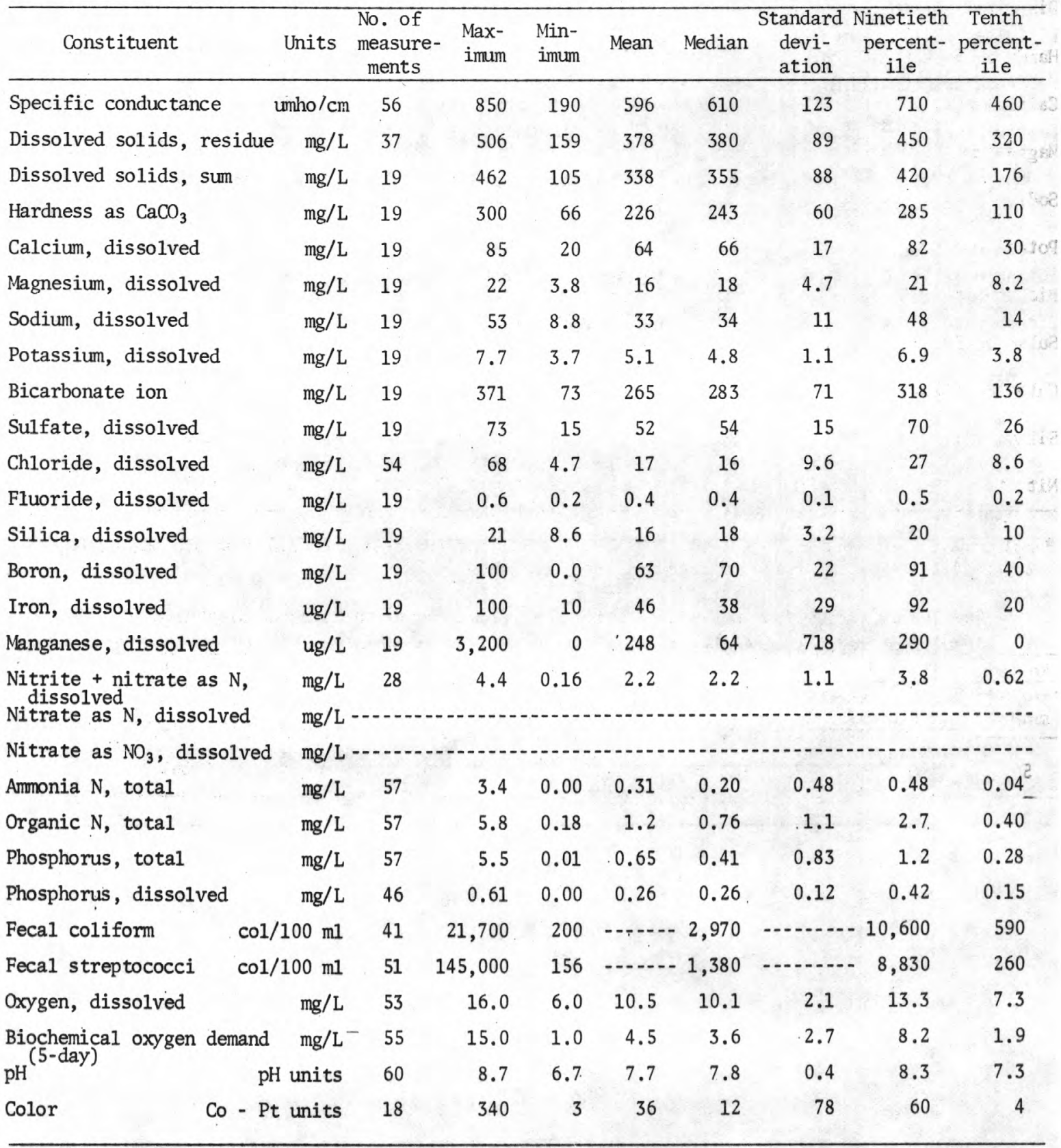




\section{NEMAHA RIVER BASIN}

06811500 Little Nemaha River at Auburn, Nebr.--Continued

Results of regression analyses relating concentrations of selected chemical constituents to specific conductance $(\mathrm{SpC})$

\begin{tabular}{|c|c|c|c|c|c|}
\hline $\begin{array}{l}\text { Dissolved } \\
\text { constituents } \\
(\mathrm{mg} / \mathrm{L})\end{array}$ & $\begin{array}{c}\text { No. of } \\
\text { measure- } \\
\text { ments }\end{array}$ & Regression equation & $\begin{array}{c}\text { Corre- } \\
\text { 1ation } \\
\text { coeffi- } \\
\text { cient }\end{array}$ & $\begin{array}{l}\text { Percent } \\
\text { explained } \\
\text { variance }\end{array}$ & $\begin{array}{c}\text { Standard } \\
\text { error of } \\
\text { estimate } \\
(\mathrm{mg} / \mathrm{L})\end{array}$ \\
\hline Dissolved solids, residue (RDS) & 37 & $\mathrm{RDS}=0.540(\mathrm{SpC})+48.620$ & 0.95 & 90 & 21 \\
\hline Dissolved solids, sum (SDS) & 19 & $\mathrm{SDS}=0.653(\mathrm{SpC})-24.810$ & .99 & 99 & 9.4 \\
\hline Hardness as $\mathrm{CaCO}_{3}$ (TH) & 19 & $\mathrm{TH}=0.446(\mathrm{SpC})-21.862$ & .99 & 98 & 9.2 \\
\hline Calcium (Ca) & 19 & $\mathrm{Ca}=0.127(\mathrm{SpC})-6.106$ & .98 & 96 & 3.4 \\
\hline Magnesium (Mg) & 19 & $\mathrm{Mg}=0.033(\mathrm{SpC})-2.620$ & .96 & 92 & 1.4 \\
\hline Sodium $(\mathrm{Na})$ & 19 & $\mathrm{Na}=0.074(\mathrm{SpC})-7.869$ & .88 & 78 & 5.3 \\
\hline Potassium (K) & 19 & $K=-0.005(S p C)+7.823$ & -.58 & 34 & 0.9 \\
\hline Bicarbonate $\left(\mathrm{HCO}_{3}\right)$ & 19 & $\mathrm{HCO}_{3}=0.527(\mathrm{SpC})-27.538$ & .99 & 98 & 11 \\
\hline Sul fate $\left(\mathrm{SO}_{4}\right)$ & 19 & $\mathrm{SO}_{4} 0.106(\mathrm{SpC})-7.082$ & .94 & 88 & 5.2 \\
\hline Chloride (Cl) & 54 & $\mathrm{Cl}=0.053(\mathrm{SpC})-14.218$ & .64 & 42 & 7.5 \\
\hline Silica (Si) & 19 & $S i=0.020(S p C)+5.633$ & .82 & 68 & 1.9 \\
\hline Nitrite + Nitrate as $\mathrm{N}\left(\mathrm{NO}_{2}-\mathrm{NO}_{3}\right)$ & 28 & $\mathrm{NO}_{2}-\mathrm{NO}_{3}=0.0005(\mathrm{SpC})+1.896$ & $* .05$ & 0.21 & 1.2 \\
\hline
\end{tabular}

* Not significant at the 95 percent confidence level; the regression equation should not be used to predict the concentration of the dependent variable.

Results of regression analyses relating specific conductance to water discharge (Q)

\begin{tabular}{cccccccc}
\hline $\begin{array}{c}\text { No. of } \\
\text { measure- } \\
\text { ments }\end{array}$ & $\begin{array}{c}\text { Water discharge } \\
\text { Maximum Minimum }\end{array}$ & Regression equation & $\begin{array}{c}\text { Correlation } \\
\text { coefficient }\end{array}$ & $\begin{array}{c}\text { Percent } \\
\text { explained } \\
\text { variance }\end{array}$ & \multicolumn{2}{c}{$\begin{array}{c}\text { Standard error } \\
\text { of estimate }\end{array}$} \\
\hline 56 & $1,030 \quad 1.2$ & $\mathrm{SpC}=1,105 \mathrm{Q}^{-0.13952}$ & -0.67 & 45 & 0.081 & 19 \\
\hline
\end{tabular}


Location. --Lat $40^{\circ} 02^{\prime} 00^{\prime \prime}$, 1ong $95^{\circ} 35^{\prime} 30^{\prime \prime}$, on line between secs. 22 and 23, T.1 N., R.16 E., Richardson County, Hydrologic Unit 10240008, near right bank on downstream side of pier of bridge on U.S. Highway 73, $1 \mathrm{mi}$ south of Falls City, and $13 \mathrm{mi}$ upstream from mouth.

Drainage area. $--1,340 \mathrm{mi}^{2}$.

Period of record.--Water years 1951,1973 to current year.

Statistical data for selected chemical constituents

\begin{tabular}{|c|c|c|c|c|c|c|c|c|c|}
\hline Constituent & Units & $\begin{array}{l}\text { No. of } \\
\text { measure- } \\
\text { ments }\end{array}$ & $\begin{array}{l}\text { Max- } \\
\text { imum }\end{array}$ & $\begin{array}{l}\text { Min- } \\
\text { imum }\end{array}$ & Mean & Median & $\begin{array}{c}\text { Standard } \\
\text { devi- } \\
\text { ation }\end{array}$ & $\begin{array}{c}\text { Ninetieth } \\
\text { percent- } \\
\text { ile }\end{array}$ & $\begin{array}{l}\text { Tenth } \\
\text { percent- } \\
\text { ile }\end{array}$ \\
\hline Specific conductance & umho $/ \mathrm{cm}$ & 56 & 1,020 & 260 & 657 & 677 & 147 & 795 & 450 \\
\hline Dissolved solids, residue & $\mathrm{mg} / \mathrm{L}$ & 35 & 638 & 251 & 437 & 418 & 81 & 570 & 350 \\
\hline Dissolved solids, sum & $\mathrm{mg} / \mathrm{L}$ & 20 & 568 & 142 & 356 & 370 & 107 & 530 & 210 \\
\hline Hardness as $\mathrm{CaCO}_{3}$ & $\mathrm{mg} / \mathrm{L}$ & 20 & 390 & 98 & 249 & 263 & 65 & 340 & 140 \\
\hline Calcium, dissolved & $\mathrm{mg} / \mathrm{L}$ & 20 & 110 & 30 & 70 & 74 & 18 & 97 & 40 \\
\hline Magnesium, dissolved & $\mathrm{mg} / \mathrm{L}$ & 20 & 28 & 5.6 & 18 & 20 & 5.3 & 25 & 9.6 \\
\hline Sodium, dissolved & $\mathrm{mg} / \mathrm{L}$ & 20 & 64 & 9.5 & 30 & 26 & 14 & 50 & 18 \\
\hline Potassium, dissolved & $\mathrm{mg} / \mathrm{L}$ & 20 & 7.0 & 3.5 & 5.1 & 4.8 & 1.0 & 6.6 & 4.1 \\
\hline Bicarbonate ion & $\mathrm{mg} / \mathrm{L}$ & 20 & 378 & 110 & 249 & 265 & 62 & 343 & 140 \\
\hline Sulfate, dissolved & $\mathrm{mg} / \mathrm{L}$ & 20 & 140 & 21 & 72 & 67 & 29 & 120 & 39 \\
\hline Chloride, dissolved & $\mathrm{mg} / \mathrm{L}$ & 55 & 61 & 6.2 & 25 & 23 & 12 & 38 & 12 \\
\hline Fluoride, dissolved & $\mathrm{mg} / \mathrm{L}$ & 20 & 0.5 & 0.2 & 0.3 & 0.3 & 0.07 & 0.4 & 0.3 \\
\hline Silica, dissolved & $\mathrm{mg} / \mathrm{L}$ & 20 & 18 & 7.3 & 11 & 9.8 & 3.1 & 17 & 7.3 \\
\hline Boron, dissolved & $\mathrm{mg} / \mathrm{L}$ & 20 & 120 & 20 & 74 & 72 & 29 & 116 & 50 \\
\hline Iron, dissolved & $\mathrm{ug} / \mathrm{L}$ & 20 & 200 & 10 & 55 & 40 & 51 & 170 & 23 \\
\hline Manganese, dissolved & $\mathrm{ug} / \mathrm{L}$ & 20 & 180 & 0 & 54 & 38 & 52 & 106 & 4 \\
\hline $\begin{array}{l}\text { Nitrite }+ \text { nitrate as } \mathrm{N}, \\
\text { dissolved } \\
\text { Nitrate as } N \text {, dissolved }\end{array}$ & $\begin{array}{l}\mathrm{mg} / \mathrm{L} \\
\mathrm{mg} / \mathrm{L}\end{array}$ & 28 & 3.8 & 0.12 & 1.8 & 2.0 & 1.1 & 3.2 & 0.30 \\
\hline Nitrate as $\mathrm{NO}_{3}$, dissolved & $\mathrm{mg} / \mathrm{L}$ & & - & & & & & & -- \\
\hline Ammonia $N$, total & $\mathrm{mg} / \mathrm{L}$ & 58 & 1.1 & 0.00 & 0.27 & 0.21 & 0.23 & 0.60 & 0.06 \\
\hline Organic $N$, total & $\mathrm{mg} / \mathrm{L}$ & 58 & 13 & 0.07 & 1.7 & 1.1 & 2.2 & 3.6 & 0.42 \\
\hline Phosphorus, total & $\mathrm{mg} / \mathrm{L}$ & 58 & 4.0 & 0.19 & 0.51 & 0.35 & 0.56 & 0.97 & 0.22 \\
\hline Phosphorus, dissolved & $\mathrm{mg} / \mathrm{L}$ & 48 & 0.50 & 0.04 & 0.19 & 0.19 & 0.08 & 0.29 & 0.09 \\
\hline Fecal coliform & $\mathrm{col} / 100 \mathrm{ml}$ & 40 & 53,000 & 400 & $-\cdots$ & 4,160 & 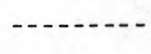 & $-24,000$ & 870 \\
\hline Fecal streptococci & $\operatorname{col} / 100 \mathrm{ml}$ & 51 & 500,000 & 40 & ----- & $-1,190$ & $-\ldots$ & $-20,000$ & 150 \\
\hline Oxygen, dissolved & $\mathrm{mg} / \mathrm{L}$ & 53 & 18.5 & 4.5 & 10.6 & 10.0 & 2.9 & 14.0 & 7.4 \\
\hline \multirow{2}{*}{$\begin{array}{l}\text { Biochemical oxygen demand } \\
\text { pH } 5 \text {-day) }\end{array}$} & $\mathrm{mg} / \mathrm{L}$ & 54 & 20.0 & 0.9 & 5.2 & 4.2 & 3.3 & 8.8 & 2.2 \\
\hline & I units & 60 & 8.6 & 6.9 & 7.8 & 7.8 & 0.4 & 8.4 & 7.4 \\
\hline Co $-\mathrm{Pt}$ & t units & 20 & 120 & 4 & 30 & 18 & 32 & 100 & 8 \\
\hline
\end{tabular}


06815000 Big Nemaha River at Falls City, Nebr.--Continued

Results of regression analyses relating concentrations of selected chemical constituents to specific conductance $(\mathrm{SpC})$

\begin{tabular}{|c|c|c|c|c|c|}
\hline $\begin{array}{c}\text { Dissolved } \\
\text { constituents } \\
(\mathrm{mg} / \mathrm{L})\end{array}$ & $\begin{array}{l}\text { No. of } \\
\text { measure- } \\
\text { ments }\end{array}$ & Regression equation & $\begin{array}{l}\text { Corre- } \\
\text { lation } \\
\text { coeffi- } \\
\text { cient }\end{array}$ & $\begin{array}{l}\text { Percent } \\
\text { explained } \\
\text { variance }\end{array}$ & $\begin{array}{c}\text { Standard } \\
\text { error of } \\
\text { estimate } \\
(\mathrm{mg} / \mathrm{L})\end{array}$ \\
\hline Dissolved solids, residue (RDS) & 35 & $\mathrm{RDS}=0.631(\mathrm{SpC})-3.472$ & 0.95 & 91 & 24 \\
\hline Dissolved solids, sum (SDS) & 19 & $\mathrm{SDS}=0.651(\mathrm{SpC})-31.842$ & .99 & 98 & 15 \\
\hline Hardness as $\mathrm{CaCO}_{3}(\mathrm{TH})$ & 19 & $\mathrm{TH}=0.414(\mathrm{SpC})+2.575$ & .94 & 88 & 23 \\
\hline Calcium (Ca) & 19 & $\mathrm{Ca}=0.110(\mathrm{SpC})+4.565$ & .91 & 83 & 7.6 \\
\hline Magnesium (Mg) & 19 & $\mathrm{Mg}=0.033(\mathrm{SpC})-1.771$ & .92 & 85 & 2.1 \\
\hline Sodium $(\mathrm{Na})$ & 19 & $\mathrm{Na}=0.084(\mathrm{SpC})-20.386$ & .88 & 78 & 6.6 \\
\hline Potassium (K) & 19 & $\mathrm{~K}=-0.003(\mathrm{SpC})+6.709$ & $*_{-} .38$ & 14 & 1.0 \\
\hline Bicarbonate $\left(\mathrm{HCO}_{3}\right)$ & 19 & $\mathrm{HCO}_{3}=0.368(\mathrm{SpC})+30.062$ & .87 & 75 & 32 \\
\hline Sulfate $\left(\mathrm{SO}_{4}\right)$ & 19 & $\mathrm{SO}_{4}=0.173(\mathrm{SpC})-31.320$ & .86 & 74 & 15 \\
\hline Chloride (C1) & 54 & $\mathrm{Cl}=0.058(\mathrm{SpC})-13.658$ & .68 & 46 & 8.6 \\
\hline Silica (Si) & 19 & $\mathrm{Si}=0.002(\mathrm{SpC})+9.529$ & *.11 & 1.2 & 3.2 \\
\hline Nitrite + Nitrate as $\mathrm{N}\left(\mathrm{NO}_{2}-\mathrm{NO}_{3}\right)$ & 28 & $\mathrm{NO}_{2}-\mathrm{NO}_{3}=-0.002(\mathrm{SpC})+2.870$ & $*_{-} .20$ & 4.0 & 1.1 \\
\hline
\end{tabular}

* Not significant at the 95 percent confidence level; the regression equation should not be used to predict the concentration of the dependent variable.

Results of regression analyses relating specific conductance to water discharge (Q)

\begin{tabular}{ccccccc}
\hline $\begin{array}{c}\text { No. of } \\
\text { measure- } \\
\text { ments }\end{array}$ & $\begin{array}{c}\text { Water discharge } \\
\left(\mathrm{ft}^{3} / \mathrm{s}\right)\end{array}$ & Maximum Minimum & Regression equation & $\begin{array}{c}\text { Correlation } \\
\text { coefficient }\end{array}$ & $\begin{array}{c}\text { Percent } \\
\text { explained } \\
\text { variance }\end{array}$ & $\begin{array}{c}\text { Standard error } \\
\text { of estimate }\end{array}$ \\
\hline 56 & 3,080 & 3.9 & $\mathrm{SpC}=1,219 \mathrm{Q}^{-0.12262}$ & -0.68 & 47 & 0.081 \\
\hline
\end{tabular}


The Republican River basin is in the southwest corner of the State and extends from the Colorado border east to Thayer County. The Republican River flows into Kansas a few miles west of Superior, Nebr.

The basin is characterized topographically as plains or dissected plains. However, in some of the western part of the basin sandhills exist. Otherwise, the basin is mantled mostly by loess overlying the Tertiary Ogallala Formation in its central part and by Pleistocene sands and gravels in its eastern part. Streams originating in the western part of the basin derive a considerably higher proportion of discharge from ground water than do streams originating elsewhere in the basin.

Water resources of the Republican River basin have been the most extensively developed of any basin in the State. Five major reservoirs for flood control and irrigation have been constructed in the basin. Nearly 100,000 acres of basin lands are irrigated by surface-water diversion.

Descriptive statistics were determined for water from six locations in the basin, all of which are on the mainstem of the Republican River (fig. 12). Mean specific conductance for the six stations varied from 500 to $569 u \mathrm{mho} / \mathrm{cm}$. The lowest mean of $500 \mathrm{umho} / \mathrm{cm}$ was for water from the Republican. River above Medicine Creek at Cambridge during the period 1950-56. With the exception of the Guide Rock station, the earliest sampling at the remaining stations was begun in 1968. The slight increase in mineralization since data collection was halted at Cambridge may result from the influence of return flow of diverted surface water used for irrigation. The influence of irrigation-return flow may be reduced somewhat by the influence of reservoirs acting as "sinks" for some constituents.

Calcium is the predominant cation and bicarbonate the predominant anion at each location. Mean concentrations of fluoride exceed $1.0 \mathrm{mg} / \mathrm{L}$ at the two sites furthest upstream, indicating the effect of seepage of ground water derived from the Ogallala Formation in the western part of the basin which is known to contain fluoride in these concentrations. Mean concentrations of nitrite + nitrate and total phosphorus are low throughout the basin. This may be an indicator of the effectiveness of the reservoirs as nutrient sinks. Median concentrations of fecal coliform and fecal streptococcal bacteria concentrations are all well below $1,000 \mathrm{col} / 100 \mathrm{ml}$, indicating that the stream contains very. little waste material from urban or agricultural area. 


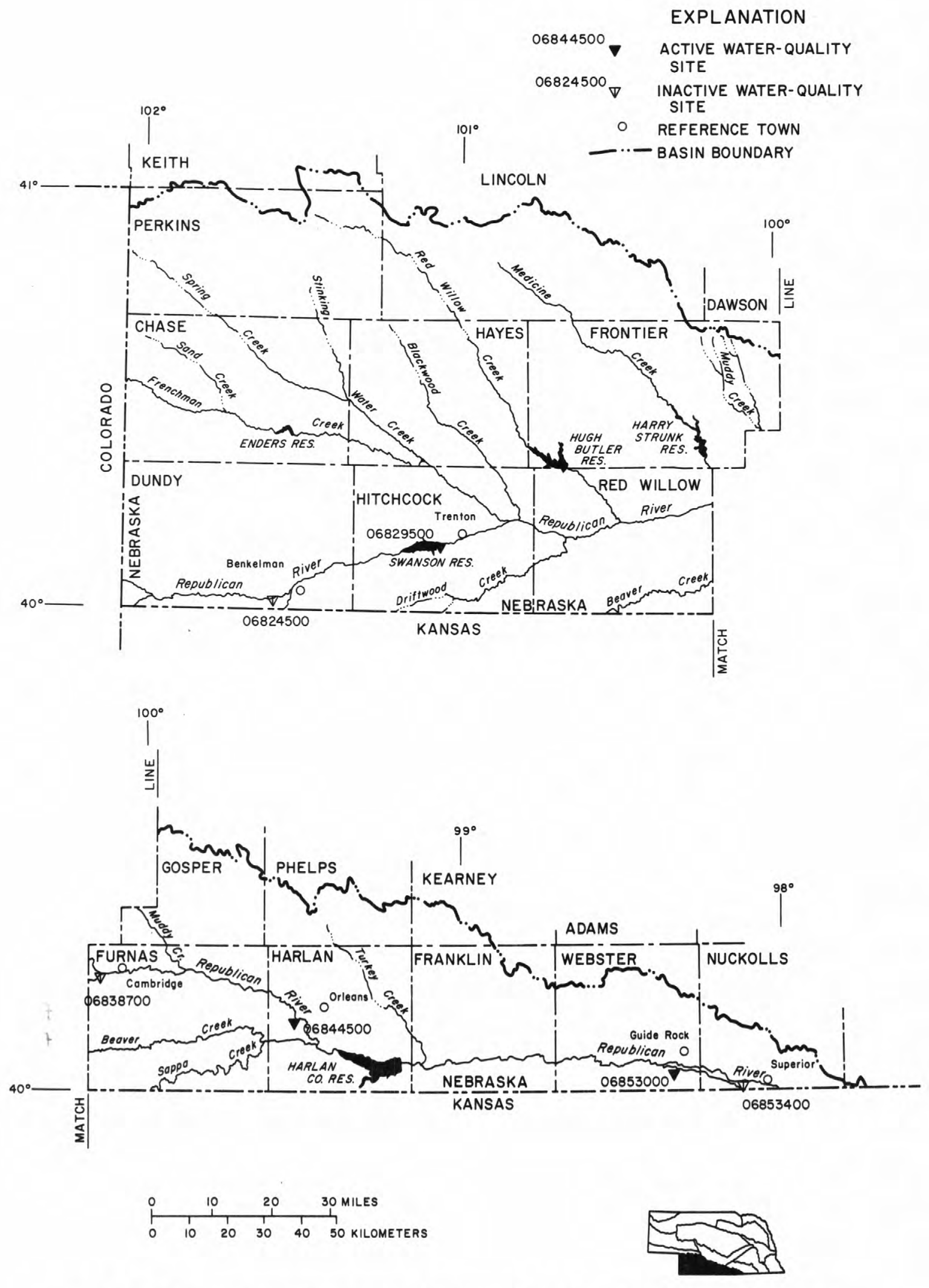

FIGURE 12.-Location of water-quality sampling sites, Republican River basin 
Regression equations between specific conductance and those chemical constituents for which sufficient data are available were determined for a11 stations. Correlation coefficients for regression equations relating dissolved solids to specific conductance were greater than 0.85 for all stations, and for regression equations relating principal constituents to specific conductance generally were greater than 0.7 for all stations, except for the Benkelman and Trenton stations. For the Cambridge station, correlation coefficients exceeded 0.8 except those for potassium and silica and several exceeded 0.9. Most measurements at Cambridge were made before the stream was regulated and the inclusion of high-flow data tended to result in better correlations than would be observed if more recent data were available. 


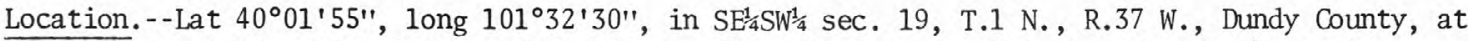
gaging station at bridge on U.S. Highway $34,0.6 \mathrm{mi}$ south of Burlington Northern, Inc., track, $1 \mathrm{mi}$ southwest of Benkelman, 2 mi upstream from South Fork Republican River, and $11 \mathrm{mi}$ downstream from Rock. Creek.

Drainage area.--4,830 $\mathrm{mi}^{2}$, approximately, of which about $1,230 \mathrm{mi}^{2}$ contributes directly to surface runoff.

Period of record. - - July 1969 to June 1972.

Statistical data for selected chemical constituents

\begin{tabular}{|c|c|c|c|c|c|c|c|c|c|}
\hline Constituent & Units & $\begin{array}{l}\text { No. of } \\
\text { measure- } \\
\text { ments }\end{array}$ & $\begin{array}{l}\text { Max- } \\
\text { imum }\end{array}$ & $\begin{array}{l}\text { Min- } \\
\text { imum }\end{array}$ & Mean & Median & $\begin{array}{c}\text { Standard } \\
\text { devi- } \\
\text { ation }\end{array}$ & $\begin{array}{c}\text { Ninetieth } \\
\text { percent- } \\
\text { ile }\end{array}$ & $\begin{array}{c}\text { Tenth } \\
\text { percent- } \\
\text { ile }\end{array}$ \\
\hline Specific conductance & umho/cm & 74 & 660 & 340 & 529 & 532 & 58 & 600 & 462 \\
\hline Dissolved solids, residue & $\mathrm{mg} / \mathrm{L}$. & 35 & 452 & 312 & 365 & 360 & 36 & 410 & 320 \\
\hline Dissolved solids, sum & $\mathrm{mg} / \mathrm{L}$ & 1 & 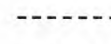 & ---- & 358 & & & & $\ldots-$ \\
\hline Hardness as $\mathrm{CaCO}_{3}$ & $\mathrm{mg} / \mathrm{L}$ & 35 & 250 & 160 & 208 & 213 & 21 & 235 & 180 \\
\hline Calcium, dissolved & $\mathrm{mg} / \mathrm{L}$ & 25 & 67 & 39 & 53 & 54 & 7.0 & 61 & 42 \\
\hline Magnesium, dissolved & $\mathrm{mg} / \mathrm{L}$ & 25 & 21 & 14 & 17 & 17 & 1.9 & 20 & 15 \\
\hline Sodium, dissolved & $\mathrm{mg} / \mathrm{L}$ & 18 & 36 & 23 & 28 & 29 & 3.4 & 34 & 24 \\
\hline Potassium, dissolved & $\mathrm{mg} / \mathrm{L}$ & 7 & 16 & 10 & 13 & ----- & 1.9 & & --- \\
\hline Bicarbonate ion & $\mathrm{mg} / \mathrm{L}$ & 16 & 278 & 196 & 242 & 240 & 20 & 260 & 216 \\
\hline Sulfate, dissolved & $\mathrm{mg} / \mathrm{L}$ & 37 & 117 & 19 & 72 & 71 & 21 & 100 & 50 \\
\hline Chloride, dissolved & $\mathrm{mg} / \mathrm{L}$ & 25 & 9.1 & 3.6 & 6.4 & 6.0 & 1.4 & 8.5 & 6.8 \\
\hline Fluoride, dissolved & $\mathrm{mg} / \mathrm{L}$ & 18 & 1.3 & 1.0 & 1.2 & 1.2 & 0.08 & 1.4 & 1.1 \\
\hline Silica, dissolved & $\mathrm{mg} / \mathrm{L}$ & 2 & 46 & 42 & 44 & ----- & & 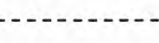 & --- \\
\hline Boron, dissolved & $\mathrm{mg} / \mathrm{L}$ & 18 & 140 & 0 & 90 & 94 & 29 & 120 & 60 \\
\hline Iron, dissolved & $\mathrm{ug} / \mathrm{L}$ & 6 & 790 & 17 & 386 & $-\cdots-1$ & 333 & 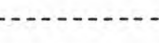 & --- \\
\hline Manganese, dissolved & $\mathrm{ug} / \mathrm{L}$ & 15 & 100 & 0 & 31 & 27 & 33 & 88 & 0 \\
\hline Nitrite + nitrate as $\mathrm{N}$, & $\mathrm{mg} / \mathrm{L}$ & 14 & 1.2 & 0.01 & 0.56 & 0.44 & 0.41 & 1.2 & 0.02 \\
\hline Nitrate as $N$, dissolved & $\mathrm{mg} / \mathrm{L}$ & 23 & 1.4 & 0.00 & 0.59 & 0.43 & 0.48 & 1.2 & 0.04 \\
\hline Nitrate as $\mathrm{NO}_{3}$, dissolved & $\mathrm{mg} / \mathrm{L}$ & 23 & 6.1 & 0.00 & 2.6 & ----- & 2.1 & - & --- \\
\hline Ammonia $\mathrm{N}$, total & $\mathrm{mg} / \mathrm{L}$ & 1 & ---- & -1 & 0.08 & ---- & & & \\
\hline Organic $N$, total & $\mathrm{mg} / \mathrm{L}$ & 1 & ---- & --- & 0.82 & $-\cdots$ & -- & & -- \\
\hline Phosphorus, total & $\mathrm{mg} / \mathrm{L}$ & 37 & 0.49 & 0.03 & 0.15 & 0.13 & 0.10 & 0.30 & 0.05 \\
\hline Phosphorus, dissolved & $\mathrm{mg} / \mathrm{L}$ & 13 & 0.19 & 0.00 & 0.07 & 0.04 & 0.06 & 0.15 & 0.01 \\
\hline Fecal coliform & $\mathrm{col} / 100 \mathrm{ml}$ & 23 & 1,500 & 0 & $\cdots-\cdots$ & 120 & $---\cdots--$ & 740 & 14 \\
\hline Fecal streptococci & $\mathrm{col} 1 / 100 \mathrm{ml}$ & & 西 & $-\cdots$ & & . & & . & $-1-$ \\
\hline Oxygen, dissolved & $\mathrm{mg} / \mathrm{L}$ & 37 & 12.7 & 6.2 & 9.9 & 10.0 & 1.9 & 12.3 & 7.6 \\
\hline Biochemical oxygen demand & $\mathrm{mg} / \mathrm{L}$ & 37 & 5.2 & 0.7 & 1.9 & 1.8 & 1.0 & 2.7 & 1.3 \\
\hline $\mathrm{pH}$ & $\mathrm{pH}$ units & 58 & 8.5 & 7.7 & 8.2 & 8.2 & 0.2 & 8.4 & 8.0 \\
\hline $\mathrm{Co}-\mathrm{Pt}$ & Co - Pt units & 7 & 26 & 3 & 10 & ------ & 9 & & \\
\hline
\end{tabular}


06824500 Republican River at Benkelman, Nebr.--Continued

Results of regression analyses relating concentrations of selected chemical constituents to specific conductance $(\mathrm{SpC})$

\begin{tabular}{|c|c|c|c|c|c|}
\hline $\begin{array}{l}\text { Dissolved } \\
\text { constituents } \\
(\mathrm{mg} / \mathrm{L})\end{array}$ & $\begin{array}{l}\text { No. of } \\
\text { measure- } \\
\text { ments }\end{array}$ & Regression equation & $\begin{array}{l}\text { Corre- } \\
\text { lation } \\
\text { coeffi- } \\
\text { cient }\end{array}$ & $\begin{array}{l}\text { Percent } \\
\text { explained } \\
\text { variance }\end{array}$ & $\begin{array}{c}\text { Standard } \\
\text { error of } \\
\text { estimate } \\
(\mathrm{mg} / \mathrm{L})\end{array}$ \\
\hline Dissolved solids, residue (RDS) & 35 & $\mathrm{RDS}=0.724(\mathrm{SpC})-17.646$ & 0.86 & 74 & 18 \\
\hline Dissolved solids, sum (SDS) & ---- & $-1-n-1$ & 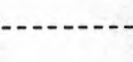 & & $-\ldots$ \\
\hline Hardness as $\mathrm{CaOO}_{3}(\mathrm{TH})$ & 35 & $\mathrm{TH}=0.328(\mathrm{SpC})+34.180$ & .66 & 43 & 16 \\
\hline Calcium (Ca) & 25 & $\mathrm{Ca}=0.95(\mathrm{SpC})-2.683$ & .60 & 36 & 5.7 \\
\hline Magnesium (Mg) & 25 & $\mathrm{Mg}=0.038(\mathrm{SpC})-2.716$ & .87 & 76 & 1.0 \\
\hline Sodium (Na) & 18 & $\mathrm{Na}=0.018(\mathrm{SpC})+19.019$ & $* .18$ & 3.3 & 3.5 \\
\hline \multicolumn{6}{|l|}{ Potassium (K) } \\
\hline Bicarbonate $\left(\mathrm{HCO}_{3}\right)$ & 16 & $\mathrm{HCO}_{3}=-0.173(\mathrm{SpC})+334.600$ & $*-.31$ & 9.8 & 19 \\
\hline Sulfate $\left(\mathrm{SO}_{4}\right)$ & 37 & $\mathrm{SO}_{4}=0.373(\mathrm{SpC})-125.910$ & .77 & 59 & 13 \\
\hline Chloride (C1) & 25 & $\mathrm{Cl}=0.017(\mathrm{SpC})-2.779$ & .53 & 28 & $1.2^{x}$ \\
\hline Silica (Si) & 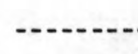 & 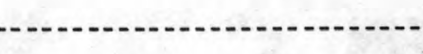 & 8 & - & 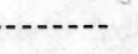 \\
\hline
\end{tabular}

* Not significant at the 95 percent confidence level; the regression equation should not be used to predict the concentration of the dependent variable.

Results of regression analyses relating specific conductance to water discharge (Q)

\begin{tabular}{ccccccc}
\hline $\begin{array}{c}\text { No. of } \\
\text { measure- } \\
\text { ments }\end{array}$ & $\begin{array}{c}\text { Water discharge } \\
\left(\mathrm{ft}^{3} / \mathrm{s}\right)\end{array}$ & Maximum Minimum & Regression equation & $\begin{array}{c}\text { Correlation } \\
\text { coefficient }\end{array}$ & $\begin{array}{c}\text { Percent } \\
\text { explained } \\
\text { variance }\end{array}$ & $\begin{array}{c}\text { Standard error } \\
\text { of estimate }\end{array}$ \\
\hline 74 & $220 \quad 0.78$ & $\mathrm{SpC}=518 \mathrm{Q}^{0.00368}$ & $* 0.04$ & 0.16 & 0.050 \\
\hline
\end{tabular}

* Not significant at the 95 percent confidence level; the regression equation should not be used to predict the concentration of the dependent variable. 
Location. --Lat $40^{\circ} 10^{\prime} 00^{\prime \prime}$, long $101^{\prime} 02^{\prime} 40^{\prime \prime}$, in $\mathrm{SE}^{\frac{1}{4}} \mathrm{sec}, 4$, T.2 N., R.33 W., Hitchcock County, at gaging station $300 \mathrm{ft}$ upstream from Elm Creek, 0.9 mi downstream from centerline of spillway of Trenton Dam, and $1.5 \mathrm{mi}$ southwest of Trenton.

Drainage area.--8,620 $\mathrm{mi}^{2}$, approximately, of which about $3,940 \mathrm{mi}^{2}$ contributes directly to surface runoff.

Period of record.-- Water years 1967 to current year.

Statistical data for selected chemical constituents

\begin{tabular}{|c|c|c|c|c|c|c|c|c|}
\hline Constituent & Units & $\begin{array}{l}\text { No. of } \\
\text { measure- } \\
\text { ments }\end{array}$ & $\begin{array}{l}\text { Max- } \\
\text { imum }\end{array}$ & $\begin{array}{l}\text { Min- } \\
\text { imum }\end{array}$ & Mean & Median & $\begin{array}{l}\text { Standard } \\
\text { devi- } \\
\text { ation }\end{array}$ & $\begin{array}{cc}\text { Ninetieth } & \text { Tenth } \\
\text { percent- percent- } \\
\text { ile } & \text { ile } \\
\end{array}$ \\
\hline Specific conductance & umho/cm & 240 & 970 & 260 & 561 & & 99 & \\
\hline Dissolved solids, residue & $\mathrm{mg} / \mathrm{L}$ & 141 & 504 & 232 & 378 & & 56 & \\
\hline Dissolved solids, sum & $\mathrm{mg} / \mathrm{L}$ & 149 & 499 & 215 & 372 & & 61 & \\
\hline Hardness as $\mathrm{CaCO}_{3}$ & $\mathrm{mg} / \mathrm{L}$ & 149 & 308 & 145 & 205 & & 31 & \\
\hline Calcium, dissolved & $\mathrm{mg} / \mathrm{L}$ & 150 & 94 & 30 & 56 & & 9.7 & \\
\hline Magnesium, dissolved & $\mathrm{mg} / \mathrm{L}$ & 149 & 27 & 9.0 & 16 & & 4.0 & \\
\hline Sodium, dissolved & $\mathrm{mg} / \mathrm{L}$ & 77 & 69 & 11 & 36 & & 11 & \\
\hline Potassium, dissolved & $\mathrm{mg} / \mathrm{L}$ & 74 & 23 & 3.6 & 11 & & 5.0 & \\
\hline Bicarbonate ion & $\mathrm{mg} / \mathrm{L}$ & 159 & 360 & 158 & 248 & & 35 & \\
\hline Sulfate, dissolved & $\mathrm{mg} / \mathrm{L}$ & 150 & 120 & 18 & 68 & & 22 & \\
\hline Chloride, dissolved & $\mathrm{mg} / \mathrm{L}$ & 154 & 19 & 1.0 & 7.8 & & 3.2 & \\
\hline Fluoride, dissolved & $\mathrm{mg} / \mathrm{L}$ & 140 & 1.8 & 0.4 & 1.1 & & 0.3 & \\
\hline Silica, dissolved & $\mathrm{mg} / \mathrm{L}$ & 148 & 96 & 4.1 & 49 & & 17 & \\
\hline Boron, dissolved & $\mathrm{mg} / \mathrm{L}$ & 50 & 830 & 90 & 214 & & 154 & \\
\hline Iron, dissolved & $\mathrm{ug} / \mathrm{L}$ & 134 & 600 & 0 & 103 & & 122 & \\
\hline Manganese, dissolved & $\mathrm{ug} / \mathrm{L}$ & 3 & 36 & 7 & 26 & & 16 & \\
\hline $\begin{array}{l}\text { Nitrite + nitrate as } \mathrm{N} \text {, } \\
\text { dissolved }\end{array}$ & $\mathrm{mg} / \mathrm{L}$ & 12 & 1.2 & 0.02 & 0.44 & & 0.38 & \\
\hline Nitrate as $N$, dissolved & $\mathrm{mg} / \mathrm{L}$ & 3 & 0.17 & 0.02 & 0.08 & & 0.08 & \\
\hline $\begin{array}{l}\text { Nitrate as } \mathrm{NO}_{3} \text {, dissolved } \\
\text { Anmonia } \mathrm{N} \text {, total }\end{array}$ & $\begin{array}{l}\mathrm{mg} / \mathrm{L} \\
\mathrm{mg} / \mathrm{L}\end{array}$ & 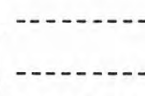 & & & & & & \\
\hline Organic $N$, total & $\mathrm{mg} / \mathrm{L}$ & 3 & 0.69 & 0.38 & 0.58 & & 0.18 & \\
\hline Phosphorus, total & $\mathrm{mg} / \mathrm{L}$ & 3 & 0.28 & 0.06 & 0.15 & & 0.12 & \\
\hline Phosphorus, dissolved & $\mathrm{mg} / \mathrm{L}$ & 15 & 0.28 & 0.00 & 0.04 & & 0.07 & \\
\hline $\begin{array}{ll}\text { Fecal coliform } & \text { col } \\
\text { Fecal streptococci } & \text { col }\end{array}$ & $\operatorname{col} 1 / 100 \mathrm{ml}$ & & & & & & & \\
\hline Oxygen, dissolved & $\mathrm{mg} / \mathrm{L}$ & 87 & 16.0 & 4.3 & 10.6 & & 2.8 & \\
\hline $\begin{array}{l}\text { Biochemical oxygen demand } \\
\text { pH }(5-\text { day })\end{array}$ & $\begin{array}{r}\mathrm{mg} / \mathrm{L} \\
\text { H units }\end{array}$ & 239 & 8.8 & 7.2 & 8.0 & & 0.4 & \\
\hline Co $-\mathrm{P}$ & t units & 20 & 32 & 0 & 8 & & 7.4 & \\
\hline
\end{tabular}


Results of regression analyses relating concentrations of selected chemical constituents to specific conductance $(\mathrm{SpC})$

\begin{tabular}{|c|c|c|c|c|c|}
\hline $\begin{array}{l}\text { Dissolved } \\
\text { constituents } \\
(\mathrm{mg} / \mathrm{L})\end{array}$ & $\begin{array}{l}\text { No. of } \\
\text { measure- } \\
\text { ments }\end{array}$ & Regression equation & $\begin{array}{l}\text { Corre- } \\
\text { lation } \\
\text { coeffi- } \\
\text { cient }\end{array}$ & $\begin{array}{l}\text { Percent } \\
\text { explained } \\
\text { variance }\end{array}$ & $\begin{array}{l}\text { Standard } \\
\text { error of } \\
\text { estimate } \\
(\mathrm{mg} / \mathrm{L})\end{array}$ \\
\hline Dissolved solids, residue (RDS) & 140 & $\mathrm{RDS}=0.675(\mathrm{SpC})+20.387$ & 0.88 & 78 & $-\cdot$ \\
\hline Dissolved solids, sum (SDS) & 148 & $\mathrm{SDS}=0.695(\mathrm{SpC})-0.680$ & .91 & 82 & -- \\
\hline Hardness as $\mathrm{CaCO}_{3}(\mathrm{TH})$ & 148 & $\mathrm{TH}=0.290(\mathrm{SpC})+49.614$ & .74 & 55 & -1 \\
\hline Calcium (Ca) & 149 & $\mathrm{Ca}=0.064(\mathrm{SpC})+22.076$ & .53 & 28 & -- \\
\hline Magnesium (Mg) & 148 & $\mathrm{Mg}=0.036(\mathrm{SpC})-3.422$ & .71 & 51 & -..- \\
\hline Sodium (Na) & 76 & $\mathrm{Na}=0.094(\mathrm{SpC})-16.703$ & .70 & 49 &.-- \\
\hline Potassium $(\mathrm{K})$ & 73 & $K=0.011(\mathrm{SpC})+5.270$ & *.17 & 3.0 & -- \\
\hline Bicarbonate $\left(\mathrm{HCO}_{3}\right)$ & 158 & $\mathrm{HCO}_{3}=0.270(\mathrm{SpC})+103.129$ & .61 & 37 & $-\cdots--$ \\
\hline Sulfate $\left(\mathrm{SO}_{4}\right)$ & 149 & $\mathrm{SO}_{4}=0.244(\mathrm{SpC})-63.092$ & .89 & 79 & - nut \\
\hline Chloride $(\mathrm{Cl})$ & 153 & $\mathrm{Cl}=0.025(\mathrm{SpC})-5.821$ & .62 & 39 & $-1 x^{2}$ \\
\hline Silica (Si) & 147 & $\mathrm{Si}=0.066(\mathrm{SpC})+13.513$ & .31 & 9.6 & (n........ \\
\hline
\end{tabular}

* Not significant at the 95 percent confidence level; the regression equation should not be used to predict the concentration of the dependent variable.

Results of regression analyses relating specific conductance to water discharge (Q)

\begin{tabular}{|c|c|c|c|c|c|c|c|}
\hline \multirow{2}{*}{$\begin{array}{l}\text { No. of } \\
\text { measure- } \\
\text { ments }\end{array}$} & \multicolumn{2}{|c|}{$\begin{array}{c}\text { Water discharge } \\
\left(\mathrm{ft}^{3} / \mathrm{s}\right) \\
\end{array}$} & \multirow{2}{*}{ Regression equation } & \multirow{2}{*}{$\begin{array}{l}\text { Correlation } \\
\text { coefficient }\end{array}$} & \multirow{2}{*}{$\begin{array}{l}\text { Percent } \\
\text { explained } \\
\text { variance }\end{array}$} & \multicolumn{2}{|c|}{$\begin{array}{c}\text { Standard error } \\
\text { of estimate }\end{array}$} \\
\hline & Maximum & Minimum & & & & Log units & Percent \\
\hline 239 & 3,150 & 0.68 & $\mathrm{SpC}=635 \mathrm{Q}^{-0.03532}$ & -0.47 & 22 & & \\
\hline
\end{tabular}


06838700 Republican River above Medicine Creek, at Cambridge, Nebr.

Location (revised).--At bridge on State Highway 47, 2 mi upstream from gaging station at Cambridge, Furnas County, $1.5 \mathrm{mi}$ upstream from Medicine Creek, and $3.3 \mathrm{mi}$ upstream from Cambridge diversion dam.

Drainage area.--13,200 $\mathrm{mi}^{2}$, approximately.

Period of record.-- December 1950 to September 1956.

Statistical data for selected chemical constituents.

\begin{tabular}{|c|c|c|c|c|c|c|c|c|}
\hline Constituent & Units & $\begin{array}{l}\text { No. of } \\
\text { measure- } \\
\text { ments }\end{array}$ & $\begin{array}{l}\text { Max- } \\
\text { imum }\end{array}$ & $\begin{array}{l}\text { Min- } \\
\text { imum }\end{array}$ & Mean & Median & $\begin{array}{l}\text { Standard } \\
\text { devi- } \\
\text { ation }\end{array}$ & $\begin{array}{cc}\text { Ninetieth } & \text { Tenth } \\
\text { percent- percent- } \\
\text { ile } & \text { ile } \\
\end{array}$ \\
\hline Specific conductance & umho/cm & 141 & 658 & 287 & 500 & & 86 & 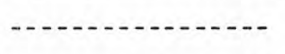 \\
\hline Dissolved solids, residue & $\mathrm{mg} / \mathrm{L}$ & 141 & 444 & 190 & 337 & $-\cdots-1$ & 60 & ------ \\
\hline Dissolved solids, sum & $\mathrm{mg} / \mathrm{L}$ & 65 & 440 & 182 & 333 & $-\cdots-1$ & 59 & ---+- \\
\hline Hardness as $\mathrm{CaCO}_{3}$ & $\mathrm{mg} / \mathrm{L}$ & 144 & 272 & 113 & 194 & $-\cdots-1$ & 35 & -- \\
\hline Calcium, dissolved & $\mathrm{mg} / \mathrm{L}$ & 84 & 82 & 34 & 54 & 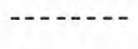 & 8.7 & $-\ldots$ \\
\hline Magnesium, dissolved & $\mathrm{mg} / \mathrm{L}$ & 84 & 22 & 6.8 & 15 & $\cdots-\cdots$ & 4.0 & 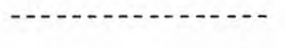 \\
\hline Sodium, dissolved & $\mathrm{mg} / \mathrm{L}$ & 144 & 45 & 7.0 & 27 & 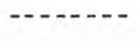 & 8.0 & 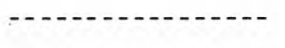 \\
\hline Potassium, dissolved & $\mathrm{mg} / \mathrm{L}$ & 65 & 18 & 8.4 & 14 & $\cdots+\cdots$ & 1.8 & $-\cdots--$ \\
\hline Bicarbonate ion & $\mathrm{mg} / \mathrm{L}$ & 141 & 355 & 158 & 255 & 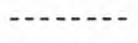 & 41 & --- \\
\hline Sulfate, dissolved & $\mathrm{mg} / \mathrm{L}$ & 143 & 78 & 3.0 & 42 & $\cdots-1$ & 16 & --- \\
\hline Chloride, dissolved & $\mathrm{mg} / \mathrm{L}$ & 84 & 13 & 1.0 & 8.2 & $-\cdots---$ & 2.8 & -- \\
\hline Fluoride, dissolved & $\mathrm{mg} / \mathrm{L}$ & 65 & 1.2 & 0.4 & 0.8 & $-\cdots--$ & 0.2 & --- \\
\hline Silica, dissolved & $\mathrm{mg} / \mathrm{L}$ & 65 & 62 & 24 & 43 & 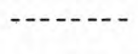 & 9.2 & \\
\hline Boron, dissolved & $\mathrm{mg} / \mathrm{L}$ & 81 & 220 & 0 & 99 & 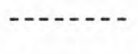 & 38 & \\
\hline Iron, dissolved & $\mathrm{ug} / \mathrm{L}$ & 62 & 140 & 0 & 32 & $-\cdots-$ & 34 & \\
\hline Manganese, dissolved & $\mathrm{ug} / \mathrm{L}$ & & - & - & 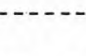 & & & \\
\hline $\begin{array}{l}\text { Nitrite + nitrate as } \mathrm{N} \text {, } \\
\text { dissolved } \\
\text { Nitrate as N, dissolved }\end{array}$ & $\begin{array}{l}\mathrm{mg} / \mathrm{L} \\
\mathrm{mg} / \mathrm{L}\end{array}$ & 84 & 1.7 & 0.09 & 0.79 & & 0.36 & \\
\hline Nitrate as $\mathrm{NO}_{3}$, dissolved & $\mathrm{mg} / \mathrm{L}$ & & & & & & & \\
\hline $\begin{array}{l}\text { Ammonia } N \text {, total } \\
\text { Organic } N \text {, total }\end{array}$ & $\mathrm{mg} / \mathrm{L}$ & & & & & & & \\
\hline $\begin{array}{l}\text { Urganic N, total } \\
\text { Phosphorus, total }\end{array}$ & $\mathrm{mg} / \mathrm{L}$ & & . & & & & & \\
\hline Phosphorus, dissolved & $\mathrm{mg} / \mathrm{L}$ & & & & & & & \\
\hline Fecal coliform & $/ 100 \mathrm{ml}$ & & & & & & & \\
\hline Fecal streptococci col/ & & & & & & & & \\
\hline Oxygen, dissolved & $\mathrm{mg} / \mathrm{L}$ & & & & & & & \\
\hline $\begin{array}{l}\text { Biochemical oxygen demand } \\
\text { (5-day) }\end{array}$ & H units & 141 & 8.5 & 7.1 & 7.8 & ----- & 0.3 & \\
\hline Co $-\mathrm{Pt}$ & t units & 38 & 20 & 3 & 9 & 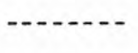 & 4.6 & \\
\hline
\end{tabular}


06838700 Republican River above Medicine Creek, at Cambridge, Nebr.--Continued

Results of regression analyses relating concentrations of selected chemical constituents to specific conductance $(\mathrm{SpC})$

\begin{tabular}{|c|c|c|c|c|c|}
\hline $\begin{array}{l}\text { Dissolved } \\
\text { constituents } \\
\text { (mg/L) }\end{array}$ & $\begin{array}{l}\text { No. of } \\
\text { measure- } \\
\text { ments }\end{array}$ & Regression equation & $\begin{array}{l}\text { Corre- } \\
\text { lation } \\
\text { coeffi- } \\
\text { cient }\end{array}$ & $\begin{array}{l}\text { Percent } \\
\text { explained } \\
\text { variance }\end{array}$ & $\begin{array}{c}\text { Standard } \\
\text { error of } \\
\text { estimate } \\
(\mathrm{mg} / \mathrm{L})\end{array}$ \\
\hline Dissolved solids, residue (RDS) & 140 & $\mathrm{RDS}=0.696(\mathrm{SpC})-11.052$ & 0.98 & 97 & --- \\
\hline Dissolved solids, sum (SDS) & 64 & $\mathrm{SDS}=0.692(\mathrm{SpC})-12.058$ & .99 & 98 & $\cdots$ \\
\hline Hardness as $\mathrm{CaCO}_{3}(\mathrm{TH})$ & 140 & $\mathrm{TH}=0.377(\mathrm{SpC})+4.246$ & .94 & 89. & \\
\hline Calcium (Ca) & 80 & $(\mathrm{a}=0.077(\mathrm{SpC})+14.358$ & .82 & 67 & \\
\hline Magnesium (Mg) & 80 & $\mathrm{Mg}=0.043(\mathrm{SpC})-6.634$ & .90 & 80 & \\
\hline Sodium (Na) & 140 & $\mathrm{Na}=0.082(\mathrm{SpC})-13.781$ & .90 & 81 & \\
\hline Potassium (K) & 61 & $K=0.006(\mathrm{SpC})+11.174$ & .27 & 7.5 & $\cdots$ \\
\hline Bicarbonate $\left(\mathrm{HCO}_{3}\right)$ & 140 & $\mathrm{HCO}_{3}=0.451(\mathrm{SpC})+29.852$ & .94 & 89 & \\
\hline Sulfate $\left(\mathrm{SO}_{4}\right)$ & 139 & $\mathrm{SO}_{4}=0.147(\mathrm{SpC})-32.309$ & .83 & 69 & 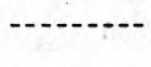 \\
\hline Chloride (C1) & 80 & $\mathrm{Cl}=0.029(\mathrm{SpC})-6.452$ & .88 & 78 & -........ \\
\hline Silica (Si) & 64 & $\mathrm{Si}=0.076(\mathrm{SpC})^{\circ}+4.585$ & .70 & 49 & \\
\hline
\end{tabular}

Results of regression analyses relating specific conductance to water discharge (Q)

\begin{tabular}{|c|c|c|c|c|c|c|c|}
\hline \multirow{2}{*}{$\begin{array}{c}\text { No. of } \\
\text { measure- } \\
\text { ments }\end{array}$} & \multicolumn{2}{|c|}{$\begin{array}{l}\text { Water discharge } \\
\left(\mathrm{ft}^{3} / \mathrm{s}\right)\end{array}$} & \multirow{2}{*}{ Regression equation } & \multirow{2}{*}{$\begin{array}{l}\text { Correlation } \\
\text { coefficient }\end{array}$} & \multirow{2}{*}{$\begin{array}{c}\text { Percent } \\
\text { explained } \\
\text { variance }\end{array}$} & \multicolumn{2}{|c|}{$\begin{array}{c}\text { Standard error } \\
\text { of estimate }\end{array}$} \\
\hline & Maximum & Minimum & & & & Log units & Percent \\
\hline 140 & 3,150 & 0.74 & $\mathrm{SpC}=594 \mathrm{Q}^{-0.03808}$ & -0.32 & 10 & & \\
\hline
\end{tabular}


Location.--Lat $40^{\circ} 07^{\prime} 53^{\prime \prime}$, long $99^{\circ} 30^{\prime} 08^{\prime \prime}$, in $\mathrm{NE}^{\frac{1}{4}} \mathrm{NE}^{\frac{1}{4}} \mathrm{sec}$. 19, T.2 N., R.19 W., Harlan County Hydrologic Unit 10250009, on right bank 18 ft downstream from bridge on State Highway 89, $200 \mathrm{ft}$ downstream from Burlington Northern, Inc., bridge, 2 mi west of Orleans, $2.8 \mathrm{mi}$ upstream from Sappa Creek, and 23 mi upstream from Harlan County Dam.

Drainage area.--15,640 $\mathrm{mi}^{2}$, approximately, of which about $8,910 \mathrm{mi}^{2}$ contributes directly to surface runoff.

Period of record. --Water year 1969 to current year.

Statistical data for selected chemical constituents

\begin{tabular}{|c|c|c|c|c|c|c|c|c|c|}
\hline Constituent & Units & $\begin{array}{l}\text { No. of } \\
\text { measure- } \\
\text { ments }\end{array}$ & $\begin{array}{l}\text { Max- } \\
\text { imum }\end{array}$ & $\begin{array}{l}\text { Min- } \\
\text { imum }\end{array}$ & Mean & Median & $\begin{array}{c}\text { Standard } \\
\text { devi- } \\
\text { ation }\end{array}$ & $\begin{array}{l}\text { Ninetieth } \\
\text { percent- } \\
\text { ile }\end{array}$ & $\begin{array}{l}\text { Tenth } \\
\text { percent- } \\
\text { ile }\end{array}$ \\
\hline Specific conductance $\quad u$ & umho/cm & 243 & 900 & 130 & 569 & 584 & 132 & 719 & 383 \\
\hline Dissolved solids, residue & $\mathrm{mg} / \mathrm{L}$ & 61 & 497 & 168 & 380 & 391 & 78 & 456 & 255 \\
\hline Dissolved solids, sum & $\mathrm{mg} / \mathrm{L}$ & 49 & 552 & 192 & 366 & 374 & 84 & 475 & 230 \\
\hline Hardness as $\mathrm{CaCO}_{3}$ & $\mathrm{mg} / \mathrm{L}$ & 72 & 340 & 120 & 212 & 218 & 42 & 259 & 148 \\
\hline Calcium, dissolved & $\mathrm{mg} / \mathrm{L}$ & 51 & 91 & 32 & 59 & 59 & 12 & 72 & 43 \\
\hline Magnesium, dissolved & $\mathrm{mg} / \mathrm{L}$ & 51 & 27 & 4.6 & 17 & 18 & 5.1 & 22 & 8.8 \\
\hline Sodium, dissolved & $\mathrm{mg} / \mathrm{L}$ & 26 & 52 & 13 & 37 & 36 & 8.8 & 50 & 28 \\
\hline Potassium, dissolved & $\mathrm{mg} / \mathrm{L}$ & 26 & 20 & 13 & 16 & 17 & 2.1 & 19 & 13 \\
\hline Bicarbonate ion & $\mathrm{mg} / \mathrm{L}$ & 72 & 404 & 176 & 274 & 277 & 46 & 330 & 207 \\
\hline Sulfate, dissolved & $\mathrm{mg} / \mathrm{L}$ & 61 & 94 & 10 & 52 & 52 & 18 & 76 & 25 \\
\hline Chloride, dissolved & $\mathrm{mg} / \mathrm{L}$ & 95 & 28 & 1.1 & 14 & 15 & 6.3 & 23 & 6.5 \\
\hline Fluoride, dissolved & $\mathrm{mg} / \mathrm{L}$ & 49 & 1.0 & 0.3 & 0.8 & 0.8 & 0.2 & 1.0 & 0.5 \\
\hline Silica, dissolved & $\mathrm{mg} / \mathrm{L}$ & 48 & 50 & 18 & 37 & 38 & 7.3 & 46 & 26 \\
\hline Boron, dissolved & $\mathrm{mg} / \mathrm{L}$ & 47 & 300 & 30 & 148 & 140 & 67 & 250 & 77 \\
\hline Iron, dissolved & $\mathrm{ug} / \mathrm{L}$ & 43 & 1,800 & 10 & 98 & 39 & 276 & 140 & 23 \\
\hline Manganese, dissolved & $\mathrm{ug} / \mathrm{L}$ & 21 & 80 & 0 & 23 & 12 & 24 & 59 & 0 \\
\hline $\begin{array}{l}\text { Nitrite + nitrate as } \mathrm{N}, \\
\text { dissolved }\end{array}$ & $\mathrm{mg} / \mathrm{L}$ & 30 & 2.3 & 0.00 & 1.1 & 1.1 & 0.79 & 2.2 & 0.01 \\
\hline $\begin{array}{l}\text { Nitrate as } \mathrm{N} \text {, dissolved } \\
\text { Nitrate as } \mathrm{NO}_{3} \text {, dissolved }\end{array}$ & $\begin{array}{l}\mathrm{mg} / \mathrm{L} \\
\mathrm{mg} / \mathrm{L}\end{array}$ & 3 & 1.1 & 0.01 & 0.38 & & & & \\
\hline Armonia $\mathrm{N}$, total & $\mathrm{mg} / \mathrm{L}$ & 56 & 1.8 & 0.00 & 0.16 & 0.11 & 0.25 & 0.29 & 0.02 \\
\hline Organic $N$, total & $\mathrm{mg} / \mathrm{L}$ & 59 & 8.9 & 0.00 & 1.3 & 0.97 & 1.6 & 2.0 & 0.33 \\
\hline Phosphorus, total & $\mathrm{mg} / \mathrm{L}$ & 58 & 2.7 & 0.19 & 0.45 & 0.32 & 0.46 & 0.60 & 0.22 \\
\hline Phosphorus, dissolved & $\mathrm{mg} / \mathrm{L}$ & 54 & 0.36 & 0.03 & 0.17 & 0.18 & 0.07 & 0.26 & 0.08 \\
\hline Fecal coliform & $\mathrm{col} / 100 \mathrm{ml}$ & 422 & 200,000 & 25 & -...- & 710 & \multicolumn{2}{|c|}{$\ldots-11,000$} & 84 \\
\hline Fecal streptococci & $\mathrm{col} / 100 \mathrm{ml}$ & 50 & 55,400 & 69 & -.....- & 510 & - n & 5,200 & 144 \\
\hline Oxygen, dissolved & $\mathrm{mg} / \mathrm{L}$ & 183 & 14.3 & 4.6 & 9.8 & 9.8 & 2.0 & 12.6 & 7.2 \\
\hline \multirow{2}{*}{$\begin{array}{l}\text { Biochemical oxygen demand } \\
(5-\text { day) } \\
\mathrm{pH}\end{array}$} & $\mathrm{mg} / \mathrm{L}$ & 54 & 25.0 & 0.3 & 6.6 & 5.0 & 5.8 & 15.0 & 1.7 \\
\hline & $H$ units & 240 & 8.8 & 7.1 & 8.0 & 8.1 & 0.3 & 8.4 & 7.6 \\
\hline $\mathrm{Co}-\mathrm{Pt}$ & $t$ units & 25 & 65 & 3 & 18 & 20 & 13 & 30 & 6 \\
\hline
\end{tabular}


Results of regression analyses relating concentrations of selected chemical constituents to specific conductance $(\mathrm{SpC})$

\begin{tabular}{|c|c|c|c|c|c|}
\hline $\begin{array}{l}\text { Dissolved } \\
\text { constituents } \\
(\mathrm{mg} / \mathrm{L})\end{array}$ & $\begin{array}{l}\text { No. of } \\
\text { measure- } \\
\text { ments. }\end{array}$ & Regression equation & $\begin{array}{l}\text { Corre- } \\
\text { lation } \\
\text { coeffi- } \\
\text { cient } \\
\end{array}$ & $\begin{array}{l}\text { Percent } \\
\text { explained } \\
\text { variance }\end{array}$ & $\begin{array}{c}\text { Standard } \\
\text { error of } \\
\text { estimate } \\
(\mathrm{mg} / \mathrm{L})\end{array}$ \\
\hline Dissolved solids, residue (RDS) & 61 & $\mathrm{RDS}=0.543(\mathrm{SpC})+70.170$ & 0.93 & 86 & 29 \\
\hline Dissolved solids, sum (SDS) & 48 & $\mathrm{SDS}=0.562(\mathrm{SpC})+52.322$ & .94 & 89 & 28 \\
\hline Hardness as $\mathrm{CaCO}_{3}$ (TH) & 72 & $\mathrm{TH}=0.327(\mathrm{SpC})+35.075$ & .93 & 97 & 15 \\
\hline Calcium (Ca) & 51 & $\mathrm{Ca}=0.073(\mathrm{SpC})+18.649$ & .86 & 73 & 6.0 \\
\hline Magnesium (Mg) & 51 & $\mathrm{Mg}=0.033(\mathrm{SpC})-1.623$ & .87 & 76 & 2.5 \\
\hline Sodium ( $\mathrm{Na}$ ) & 26 & $\mathrm{Na}=0.055(\mathrm{SpC})+2.991$ & .85 & 72 & 4.8 \\
\hline Potassium (K) & 26 & $\mathrm{~K}=0.007(\mathrm{SpC})+11.787$ & .46 & 21 & 1.9 \\
\hline Bicarbonate $\left(\mathrm{HCO}_{3}\right)$ & 72 & $\mathrm{HCO}_{3}=0.361(\mathrm{SpC})+77.512$ & .93 & 86 & 17 \\
\hline Sulfate $\left(\mathrm{SO}_{4}\right)$ & 61 & $\mathrm{SO}_{4}=0.127(\mathrm{SpC})-17.834$ & .87 & 75 & 9.2 \\
\hline Chloride (C1) & 95 & $\mathrm{Cl}=0.038(\mathrm{SpC})-7.950$ & .80 & 64 & 3.8 \\
\hline Silica (Si) & 48 & $\mathrm{Si}=0.027(\mathrm{SpC})+21.740$ & .51 & 26 & 6.3 \\
\hline Nitrite + Nitrate as $\mathrm{N}\left(\mathrm{NO}_{2}-\mathrm{NO}_{3}\right)$ & 30 & $\mathrm{NO}_{2}+\mathrm{NO}_{3}=0.006(\mathrm{SpC})-2.778$ & .44 & 20 & 0.72 \\
\hline
\end{tabular}

Results of regression analyses relating specific conductance to water discharge (Q)

\begin{tabular}{cccccccc}
\hline $\begin{array}{c}\text { No. of } \\
\text { measure } \\
\text { ments }\end{array}$ & $\begin{array}{c}\text { Water discharge } \\
\text { Maximum Minimum }\end{array}$ & Regression equation. & $\begin{array}{c}\text { Correlation } \\
\text { coefficient }\end{array}$ & $\begin{array}{c}\text { Percent } \\
\text { explained } \\
\text { variance }\end{array}$ & \multicolumn{2}{c}{$\begin{array}{c}\text { Standard error } \\
\text { of estimate }\end{array}$} \\
\hline 241 & 13,100 & 7.8 & $\mathrm{SpC}=791 \mathrm{Q}^{-0.07212}$ & -0.27 & 7.1 & 0.118 & 28 \\
\hline
\end{tabular}




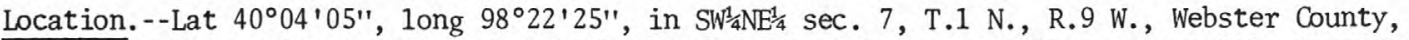
Hydrologic Unit 10250016, on left bank $300 \mathrm{ft}$ upstream from Willow Creek, $0.2 \mathrm{mi}$ downstream from Courtland diversion dam, and $2 \mathrm{mi}$. southwest of Guide Rock.

Drainage area.--22,040 $\mathrm{mi}^{2}$, approximately, of which about $14,550 \mathrm{mi}^{2}$ contributes directly to surface runoff.

Period of record.--Water year 1962 to current year.

Statistical data for selected chemical constituents

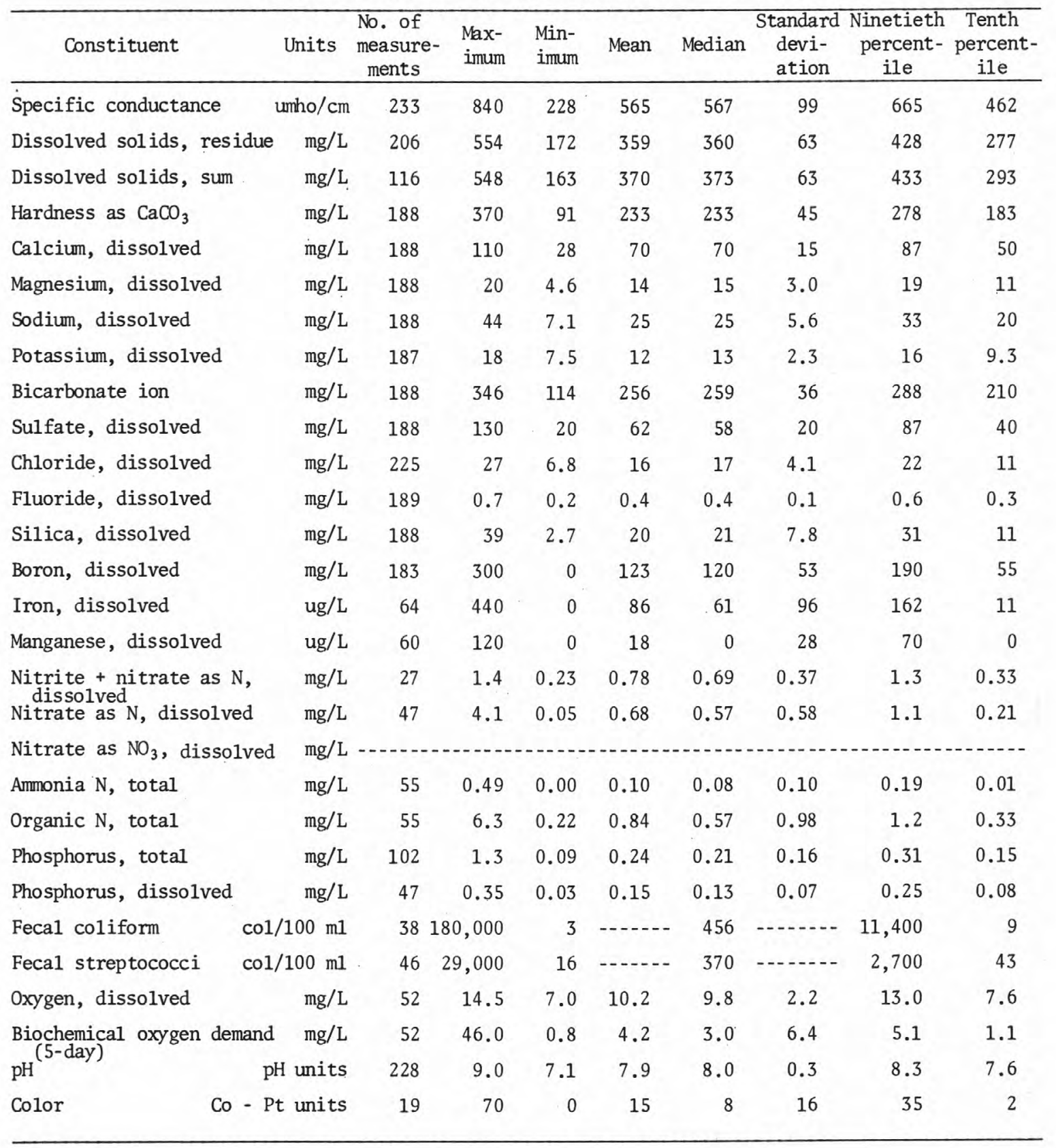


Results of regression analyses relating concentrations of selected chemical constituents to specific conductance $(\mathrm{SpC})$

\begin{tabular}{|c|c|c|c|c|c|}
\hline $\begin{array}{l}\text { Dissolved } \\
\text { constituents } \\
(\mathrm{mg} / \mathrm{L})\end{array}$ & $\begin{array}{l}\text { No. of } \\
\text { measure- } \\
\text { ments }\end{array}$ & Regression equation & $\begin{array}{l}\text { Corre- } \\
\text { lation } \\
\text { coeffi- } \\
\text { cient }\end{array}$ & $\begin{array}{l}\text { Percent } \\
\text { explained } \\
\text { variance }\end{array}$ & $\begin{array}{c}\text { Standard } \\
\text { error of } \\
\text { estimate } \\
(\mathrm{mg} / \mathrm{L})\end{array}$ \\
\hline Dissolved solids, residue (RDS) & 207 & $\mathrm{RDS}=0.610(\mathrm{SpC})+18.679$ & 0.92 & 85 & 24 \\
\hline Dissolved solids, sum (SDS) & 116 & $\mathrm{SDS}=0.593(\mathrm{SpC})+24.760$ & .92 & 85 & 25 \\
\hline Hardness as $\mathrm{CaCO}_{3}(\mathrm{TH})$ & 189 & $\mathrm{TH}=0.422(\mathrm{SpC})-0.044$ & .90 & 82 & 19 \\
\hline Calcium (Ca) & 189 & $\mathrm{Ca}=0.131(\mathrm{SpC})-2.751$ & .84 & 71 & 8.0 \\
\hline Magnesium (Mg) & 189 & $\mathrm{Mg}=0.022(\mathrm{SpC})+2.104$ & .72 & 51 & 2.1 \\
\hline Sodium (Na) & 189 & $\mathrm{Na}=0.043(\mathrm{SpC})+0.978$ & .74 & 54 & 3.8 \\
\hline Potassium (K) & 188 & $K=-0.005(\mathrm{SpC})+15.182$ & -.21 & 4.6 & 2.2 \\
\hline Bicarbonate $\left(\mathrm{HCO}_{3}\right)$ & 189 & $\mathrm{HCO}_{3}=0.321(\mathrm{SpC})+78.410$ & .85 & 72 & 19 \\
\hline Sulfate $\left(\mathrm{SO}_{4}\right)$ & 189 & $\mathrm{SO}_{4}=0.183(\mathrm{SpC})-39.697$ & .87 & 76 & 10 \\
\hline Chloride (C1) & 226 & $\mathrm{Cl}=0.031(\mathrm{SpC})-1.301$ & .74 & 56 & 2.8 \\
\hline Silica (Si) & 189 & $\mathrm{Si}=0.044(\mathrm{SpC})-3.784$ & .54 & 29 & 6.6 \\
\hline Nitrite + Nitrate as $\mathrm{N}\left(\mathrm{NO}_{2}-\mathrm{NO}_{3}\right)$ & 27 & $\mathrm{NO}_{2}-\mathrm{NO}_{3}=0.0001(\mathrm{SpC})+0.712$ & $* .03$ & 0.11 & 0.38 \\
\hline
\end{tabular}

* Not significant at the 95 percent confidence level; the regression equation should not be used to predict the concentration of the dependent variable.

Results of regression analyses relating specific conductance to water discharge (Q)

\begin{tabular}{cccccccc}
\hline $\begin{array}{c}\text { No. of } \\
\text { measure } \\
\text { ments }\end{array}$ & $\begin{array}{c}\text { Water discharge } \\
\text { Maximum Minimum }\end{array}$ & Regression equation & $\begin{array}{c}\text { Correlation } \\
\text { coefficient }\end{array}$ & $\begin{array}{c}\text { Percent } \\
\text { explained } \\
\text { variance }\end{array}$ & \multicolumn{2}{c}{$\begin{array}{c}\text { Standard error } \\
\text { of estimate }\end{array}$} \\
\hline 234 & $2,760 \quad 0.40$ & $\mathrm{SpC}=700 \mathrm{Q}^{-0.04666}$ & -0.31 & 9.8 & 0.082 & 19 \\
\hline
\end{tabular}




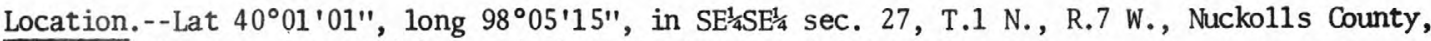
at bridge on State Highway 14 about 1 mi west of Superior.

Drainage area. $--22,300 \mathrm{mi}^{2}$.

Period of record. --July 1969 to September 1970.

Statistical data for selected chemical constituents

\begin{tabular}{|c|c|c|c|c|c|c|c|c|c|}
\hline Constituent & Units & $\begin{array}{l}\text { No. of } \\
\text { measure- } \\
\text { ments }\end{array}$ & $\begin{array}{l}\text { Max- } \\
\text { imum }\end{array}$ & $\begin{array}{l}\text { Min- } \\
\text { imum }\end{array}$ & Mean & Median & $\begin{array}{l}\text { Standard } \\
\text { devi- } \\
\text { ation }\end{array}$ & $\begin{array}{l}\text { Ninetieth } \\
\text { percent- } \\
\text { ile }\end{array}$ & $\begin{array}{l}\text { Tenth } \\
\text { percent- } \\
\text { ile }\end{array}$ \\
\hline Specific conductance & unho/cm & 45 & 659 & 220 & 511 & 527 & 113 & 620 & 280 \\
\hline Dissolved solids, residue & $\mathrm{mg} / \mathrm{L}$ & 20 & 458 & 190 & 344 & 347 & 71 & 420 & 260 \\
\hline Dissolved solids, sum & $\mathrm{mg} / \mathrm{L}$ & 3 & 379 & 150 & 275 & $-\ldots$ & 116 & 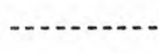 & $-\cdots$ \\
\hline Hardness as $\mathrm{CaCO}_{3}$ & $\mathrm{mg} / \mathrm{L}$ & 23 & 280 & 87 & 211 & 223 & 49 & 270 & 140 \\
\hline Calcium, dissolved & $\mathrm{mg} / \mathrm{L}$ & 10 & 86 & 25 & 66 & 76 & 19 & 85 & 46 \\
\hline Magnesium, dissolved & $\mathrm{mg} / \mathrm{L}$ & 10 & 18 & 5.9 & 14 & 16 & 3.4 & 17 & 11 \\
\hline Sodium, dissolved & $\mathrm{mg} / \mathrm{L}$ & 15 & 38 & 6.4 & 24 & 26 & 9.1 & 33 & 10 \\
\hline Potassium, dissolved & $\mathrm{mg} / \mathrm{L}$ & 8 & 17 & 8.2 & 13 & - n & 2.8 & $\cdots$ & --- \\
\hline Bicarbonate ion & $\mathrm{mg} / \mathrm{L}$ & 17 & 300 & 114 & 233 & 240 & 54 & 283 & 131 \\
\hline Sulfate, dissolved & $\mathrm{mg} / \mathrm{L}$ & 23 & 82 & 9.2 & 57 & 60 & 20 & 78 & 23 \\
\hline Chloride, dissolved & $\mathrm{mg} / \mathrm{L}$ & 10 & 19 & 5.1 & 15 & 18 & 5.0 & 19 & 7.0 \\
\hline Fluoride, dissolved & $\mathrm{mg} / \mathrm{L}$ & 15 & 0.7 & 0.3 & 0.6 & 0.6 & 0.1 & 0.7 & 0.4 \\
\hline Silica, dissolved & $\mathrm{mg} / \mathrm{L}$ & 4 & 33 & 9.4 & 16 & $-\cdots-1$ & 11 & & \\
\hline Boron, dissolved & $\mathrm{mg} / \mathrm{L}$ & 15 & 200 & 40 & 87 & 80 & 42 & 130 & 43 \\
\hline Iron, dissolved & $\mathrm{ug} / \mathrm{L}$ & 6 & 285 & 19 & 96 & - n & 97 & & \\
\hline Manganese, dissolved & $\mathrm{ug} / \mathrm{L}$ & 13 & 372 & 10 & 64 & 40 & 98 & 140 & 15 \\
\hline $\begin{array}{l}\text { Nitrite + nitrate as } \mathrm{N}, \\
\text { dissolved }\end{array}$ & $\mathrm{mg} / \mathrm{L}$ & 8 & 1.5 & 0.04 & 0.63 & 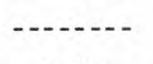 & 0.56 & & \\
\hline Nitrate as $N$, dissolved & $\mathrm{mg} / \mathrm{L}$ & 14 & 1.3 & 0.00 & 0.63 & 0.73 & 0.37 & 1.0 & 0.10 \\
\hline Nitrate as $\mathrm{NO}_{3}$, dissolved & $\mathrm{mg} / \mathrm{L}$ & 14 & 5.8 & 0.10 & 2.8 & 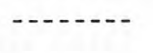 & 1.7 & & \\
\hline Ammonia $\mathrm{N}$, total & $\mathrm{mg} / \mathrm{L}$ & 2 & 0.21 & 0.20 & 0.20 & - n & 0.01 & & \\
\hline Organic N, total & $\mathrm{mg} / \mathrm{L}$ & 3 & 3.5 & 0.71 & 1.7 & $\cdots-\cdots$ & 1.5 & & \\
\hline Phosphorus, total & $\mathrm{mg} / \mathrm{L}$ & 23 & 2.2 & 0.17 & 0.49 & 0.33 & 0.48 & 1.2 & 0.15 \\
\hline Phosphorus, dissolved & $\mathrm{mg} / \mathrm{L}$ & 17 & 0.37 & 0.10 & 0.20 & 0.17 & 0.08 & 0.30 & 0.11 \\
\hline Fecal coliform & $\mathrm{col} / 100 \mathrm{ml}$ & 10 & 9,300 & 100 & $\cdots$ & 470 & - & 9,000 & 130 \\
\hline Fecal streptococci & $\mathrm{col} / 100 \mathrm{ml}$ & & & & & & & & $\cdots$ \\
\hline Oxygen, dissolved & $\mathrm{mg} / \mathrm{L}$ & 22 & 13.1 & 5.7 & 9.7 & 10.0 & 2.2 & 12.5 & 6.7 \\
\hline \multirow{2}{*}{$\begin{array}{l}\text { Biochemical oxygen dem } \\
\mathrm{pH}^{(5-\text { day })}\end{array}$} & $\mathrm{mg} / \mathrm{L}$ & 23 & 11.8 & 0.2 & 3.2 & 2.1 & 2.7 & 7.1 & 1.1 \\
\hline & $\mathrm{pH}$ units & 39 & 8.4 & 7.5 & 8.0 & 8.0 & 0.3 & 8.4 & 7.6 \\
\hline Co - Pt & Co - Pt units & 10 & 150 & 2 & 41 & 23 & 49 & 106 & 2 \\
\hline
\end{tabular}


Results of regression analyses relating concentrations of selected chemical constituents to specific conductance $(\mathrm{SpC})$

\begin{tabular}{|c|c|c|c|c|c|}
\hline $\begin{array}{l}\text { Dissolved } \\
\text { constituents } \\
(\mathrm{mg} / \mathrm{L})\end{array}$ & $\begin{array}{l}\text { No. of } \\
\text { measure- } \\
\text { ments }\end{array}$ & Regression equation & $\begin{array}{l}\text { Corre- } \\
\text { lation } \\
\text { coeffi- } \\
\text { cient }\end{array}$ & $\begin{array}{l}\text { Percent } \\
\text { explained } \\
\text { variance }\end{array}$ & $\begin{array}{c}\text { Standard } \\
\text { error of } \\
\text { estimate } \\
(\mathrm{mg} / \mathrm{L})\end{array}$ \\
\hline Dissolved solids, residue (RDS) & 20 & $\mathrm{RDS}=0.624(\mathrm{SpC})+22.193$ & 0.97 & 95 & 17 \\
\hline Dissolved solids, sum (SDS) & ---- & 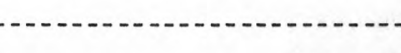 & . & & \\
\hline Hardness as $\mathrm{CaCO}_{3}(\mathrm{TH})$ & 23 & $\mathrm{TH}=0.377(\mathrm{SpC})+19.713$ & .92 & 84 & 20 \\
\hline Calcium (Ca) & ----- & 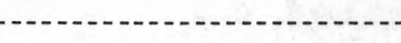 & & $-\cdots$ &.-- \\
\hline Magnesium (Mg) & ---- & 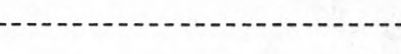 & & & \\
\hline Sodium (Na) & 15 & $\mathrm{Na}=0.051(\mathrm{SpC})-2.880$ & .79 & 62 & 5.8 \\
\hline \multicolumn{6}{|l|}{ Potassium (K) } \\
\hline Bicarbonate $\left(\mathrm{HCO}_{3}\right)$ & 17 & $\mathrm{HCO}_{3}=0.488(\mathrm{SpC})-6.349$ & .94 & 89 & 18 \\
\hline Sulfate $\left(\mathrm{SO}_{4}\right)$ & 23 & $\mathrm{SO}_{4}=0.152(\mathrm{SpC})-20.485$ & .92 & 84 & 7.9 \\
\hline Chloride (C1) & ----- & (a) & & 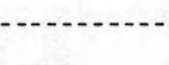 & \\
\hline Silica (Si) & $-\cdots---$ & & & & \\
\hline Nitrite + Nitrate as $\mathrm{N}\left(\mathrm{NO}_{2}-\mathrm{NO}_{3}\right)$ & & & & & \\
\hline
\end{tabular}

Results of regression analyses relating specific conductance to water discharge (Q)

\begin{tabular}{ccccccc}
\hline $\begin{array}{c}\text { No. of } \\
\text { measure- } \\
\text { ments }\end{array}$ & $\begin{array}{c}\text { Water discharge } \\
\left(\mathrm{ft}^{3} / \mathrm{s}\right)\end{array}$ & Maximum Minimum & Regression equation & $\begin{array}{c}\text { Correlation } \\
\text { coefficient }\end{array}$ & $\begin{array}{c}\text { Percent } \\
\text { explained } \\
\text { variance }\end{array}$ & $\begin{array}{c}\text { Standard error } \\
\text { of estimate }\end{array}$ \\
\hline 45 & 1,650 & 25 & $\mathrm{SpC}=1,169 \mathrm{Q}^{-0.15264}$ & -0.59 & 35 & 0.097 \\
\hline
\end{tabular}


The Big Blue River basin is in southeast Nebraska and the Big Blue River flows out of the State south of Barneston.

The upper part of the basin lies in an area of loess plains overlying Pleistocene sands and gravels deposited as a result of glaciation. This part of the basin is very level. The glaciated lower part of the basin is more rolling. It is characterized by loess deposits overlying glacial drift or soils developed on glacial drift.

Irrigation with ground water is highly developed in that part of the basin underlain by Pleistocene sand and grave1. In the glaciated area, it is developed only where buried Pleistocene channels exist. There are no major diversions of surface water for irrigation in the basin, but there are some private diversions for irrigation of individual property.

Descriptive statistics were determined for the quality of water at nine surface sampling sites (fig. 13). Of these, five are on the mainstem of the Big Blue River and two are on the West Fork Big Blue River. The other sites are on Lincoln Creek and Turkey Creek, major tributaries entering the Big Blue River from the west. Mean specific conductances in the Big Blue River basin range from 366 umho/cm at Surprise, in the upper end of the basin, to 622 below Beatrice. Several of the sites, including below Seward, near Crete, and below Beatrice on the Big Blue River, and below Hastings on the West Fork Big Blue River, are located immediately below the sewage outfall of their respective communities. Calcium is the principal cation and bicarbonate the principal anion for each station.

The station West Fork Big Blue River below Hastings is just below the Hastings sewage-plant outfa1l. Thuring dry weather, effluent constitutes most of the flow. Mean concentrations of $6.3 \mathrm{mg} / \mathrm{L}$ for nitrite + nitrate and of $5.5 \mathrm{mg} / \mathrm{L}$ for total phosphorus are the highest for those constituents of all stations included in this study. Mean concentrations of chloride and BOD also are high at this site as are the median concentrations of fecal coliform and fecal streptococcal bacteria.

The West Fork Big Blue River flows through a rural environment from Hastings to its mouth. A comparison of means or medians for several constituents (table 5) for West Fork Big Blue River at Hastings and near Dorchester, about $10 \mathrm{mi}$ upstream from the mouth, indicates the affects of dilution from ground-water seepage and overland runoff on the quality of streamflow. 


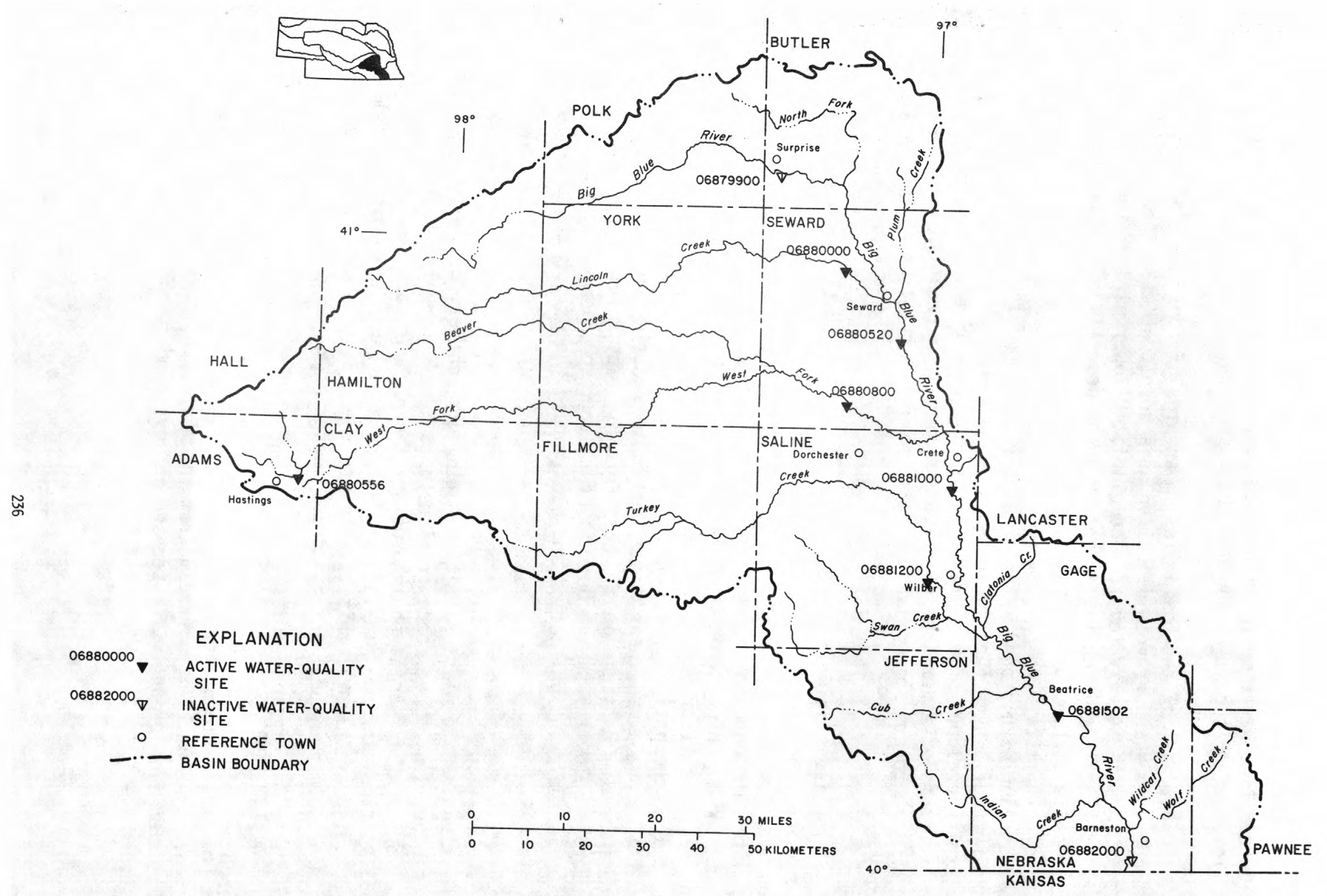

FIGURE 13.-Location of water-quality sampling sites, Big Blue River basin 
Table 5.--Comparison of mean and median concentrations of several constituents to show the effects of dilution from ground-water seepage and overland runoff on the quality of streamflow in the West Fork Big Blue River

Constituent
West Fork Big Blue River

Below Hastings Near Dorchester
Mean specific conductance, umho/cm

Mean chloride, $\mathrm{mg} / \mathrm{L}$

Mean nitrite + nitrate, $\mathrm{mg} / \mathrm{L}$

Mean total phosphorus, $\mathrm{mg} / \mathrm{L}$

Mean biochemical oxygen demand, $\mathrm{mg} / \mathrm{L}$

Median fecal coliform, col/100 ml

Median fecal streptococci, col/100 m1

$\begin{array}{rc}592 & 439 \\ 41 & 16 \\ 6.3 & 1.6 \\ 5.5 & .91 \\ 14.6 & 4.8 \\ 75,700 & 610 \\ 3,320 & 1,000\end{array}$

Fecal coliform and fecal streptococcal bacteria medians, BOD, and nitrite + nitrate, organic nitrogen, and total phosphorus means for the stations below Seward, near Crete, and below Beatrice all reflect the proximity to the sewage outfalls of their respective communities.

Turkey Creek near Wilber represents a special situation. Although calcium and bicarbonate are the predominate cation and anion most of the time, an examination of the data indicates that sodium and chloride usually are the predominant cation and anion under low-flow conditions. During dry weather, flow is maintained in Turkey Creek by a small amount of ground-water discharge. Migration of ground water through highly saline deposits of the Cretaceous Dakota Formation upward into Pleistocene deposits and then into the stream accounts for the change in predominate ions in Turkey Creek under low-flow conditions.

Regression data for all nine stations shows that correlation between specific conductance and principal cations, excluding potassium, and between specific conductance and bicarbonate is above 0.9 for all stations except West Fork Big Blue River below Hastings and Turkey Creek near Wilber. The large ratio of sewage-plant effluent to total streamflow affects ion ratios in such a way as to reduce correlation coefficients at the Hastings station. Seepage of saline water into Turkey Creek during periods of low discharge affects ion ratios with much the same result. 
BIG BLUE RIVER BASIN

06879900 Big Blue River at Surprise, Nebr.

Location.--Lat $41^{\circ} 06^{\prime} 05^{\prime \prime}$, long $97^{\circ} 18^{\prime} 35^{\prime \prime}$, in NW-4NW/4 sec. 15, T.13 N., R.1 E., Butler County, at gaging station $50 \mathrm{ft}$ downstream from bridge on county road at south edge of Surprise.

Drainage area. $--345 \mathrm{mi}^{2}$.

Period of record.---December 1950 to September 1956.

Statistical data for selected chemical constituents

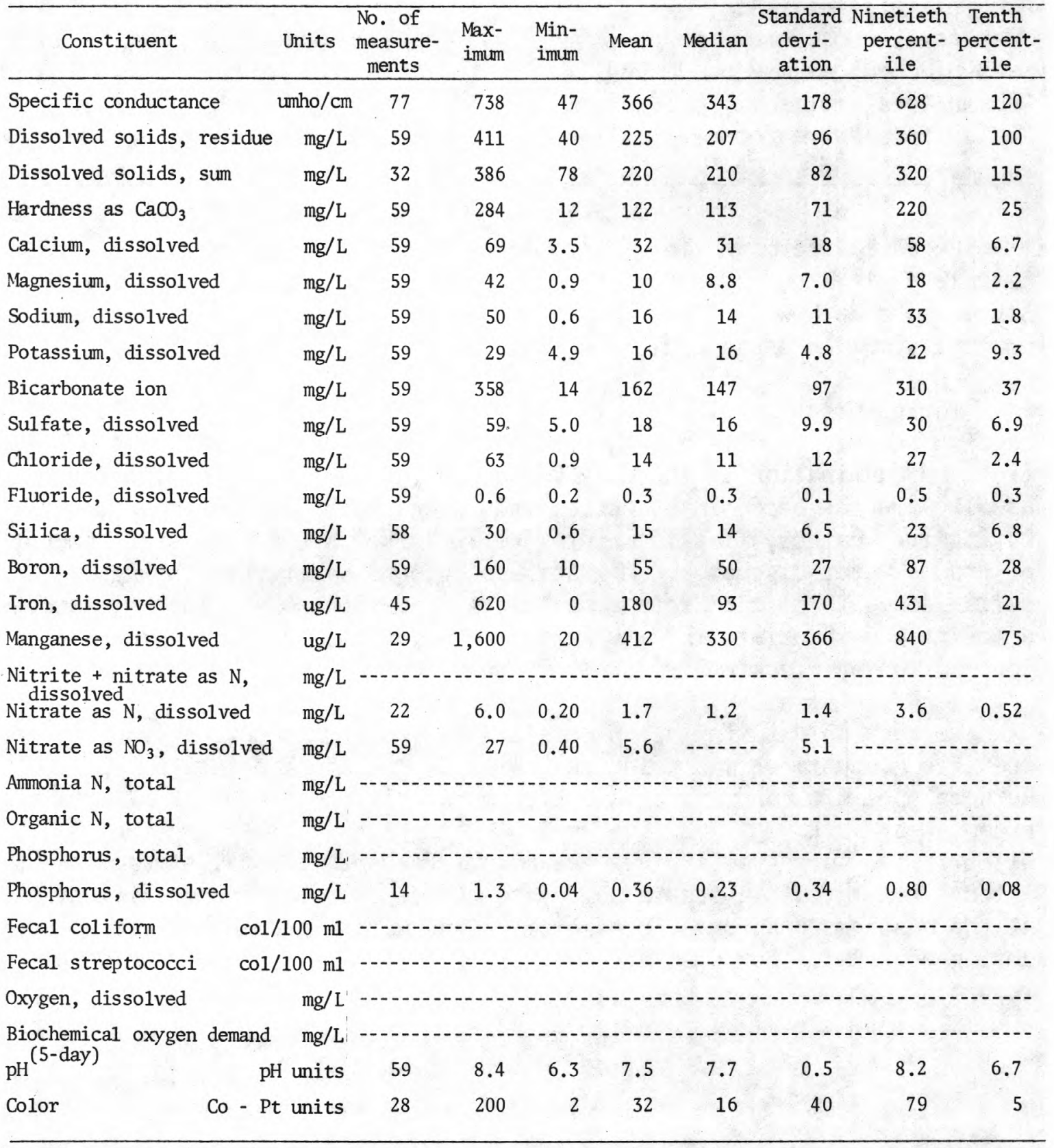


BIG BLUE RIVER BASIN

06879900 Big Blue River at Surprise, Nebr.--Continued

Results of regression analyses relating concentrations of selected chemical constituents to specific conductance $(\mathrm{SpC})$

\begin{tabular}{|c|c|c|c|c|c|}
\hline $\begin{array}{l}\text { Dissolved } \\
\text { constituents } \\
(\mathrm{mg} / \mathrm{L})\end{array}$ & $\begin{array}{l}\text { No. of } \\
\text { measure- } \\
\text { ments }\end{array}$ & Regression equation & $\begin{array}{l}\text { Corre- } \\
\text { lation } \\
\text { coeffi- } \\
\text { cient }\end{array}$ & $\begin{array}{l}\text { Percent } \\
\text { explained } \\
\text { variance }\end{array}$ & $\begin{array}{l}\text { Standard } \\
\text { error of } \\
\text { estimate } \\
(\mathrm{mg} / \mathrm{L})\end{array}$ \\
\hline Dissolved solids, residue (RDS) & 59 & $\mathrm{RDS}=0.531(\mathrm{SpC})+45.790$ & 0.97 & 94 & 23 \\
\hline Dissolved solids, sum (SDS) & 32 & $\mathrm{SDS}=0.551(\mathrm{SpC})+23.337$ & .99 & 99 & 9.4 \\
\hline Hardness as $\mathrm{CaCO}_{3}(\mathrm{TH})$ & 59 & $\mathrm{TH}=0.399(\mathrm{SpC})-13.089$ & .98 & 95 & 16 \\
\hline Calcium (Ca) & 59 & $\mathrm{Ca}=0.101(\mathrm{SpC})-1.846$ & .96 & 92 & 5.3 \\
\hline Magnesium (Mg) & 59 & $M g=0.036(S p C)-2.083$ & .90 & 81 & 3.1 \\
\hline Sodium (Na) & 59 & $\mathrm{Na}=0.061(\mathrm{SpC})-4.656$ & .94 & 88 & 4.1 \\
\hline Potassium (K) & 59 & $K=0.009(\mathrm{SpC})+13.154$ & .33 & 11 & 4.6 \\
\hline Bicarbonate $\left(\mathrm{HCO}_{3}\right)$ & 59 & $\mathrm{HCO}_{3}=0.543(\mathrm{SpC})-21.146$ & .98 & 95 & 21 \\
\hline Sulfate $\left(\mathrm{SO}_{4}\right)$ & 59 & $\mathrm{SO}_{4}=0.039(\mathrm{SpC})+4.714$ & .69 & 48 & 7.2 \\
\hline Chloride (CI) & 59 & $\mathrm{Cl}=0.044(\mathrm{SpC})-1.300$ & .68 & 46 & 8.5 \\
\hline Silica (Si) & 58 & $\mathrm{Si}=0.003(\mathrm{SpC})+13.549$ & $* .08$ & 0.71 & 6.6 \\
\hline
\end{tabular}

* Not significant at the 95 percent confidence 1evel; the regression equation should not be used to predict the concentration of the dependent variable.

Results of regression analyses relating specific conductance to water discharge (Q)

\begin{tabular}{ccccccc}
\hline $\begin{array}{c}\text { No. of } \\
\text { measure- } \\
\text { ments }\end{array}$ & $\begin{array}{c}\text { Water discharge } \\
\left(\mathrm{ft}^{3} / \mathrm{s}\right)\end{array}$ & Maximum Minimum & Regression equation & $\begin{array}{c}\text { Correlation } \\
\text { coefficient }\end{array}$ & $\begin{array}{c}\text { Percent } \\
\text { explained } \\
\text { variance }\end{array}$ & $\begin{array}{c}\text { Standard error } \\
\text { of estimate }\end{array}$ \\
\hline 75 & $3,390 \quad 0.02$ & $\mathrm{SpC}=354 \mathrm{Q}^{-0.18916}$ & -0.79 & 62 & 0.173 \\
\hline
\end{tabular}


Location.--Lat $40^{\circ} 54^{\prime} 57^{\prime \prime}$, long $97^{\circ} 08^{\prime} 43^{\prime \prime}$, in NW' $\mathrm{NE}^{\frac{1}{4}} \mathrm{sec}$. 24 , T.11 N., R.2 E., Seward County, Hydrologic Unit 10270201 , on left bank $20 \mathrm{ft}$ downstream from county road bridge, 2 mi west of Seward, and $2.5 \mathrm{mi}$ upstream from mouth.

Drainage area. $--446 \mathrm{mi}^{2}$.

Period of record.--Water years 1963-70, 1973 to current year.

Statistical data for selected chemical constituents

\begin{tabular}{|c|c|c|c|c|c|c|c|c|c|}
\hline Constituent & Units & $\begin{array}{l}\text { No. of } \\
\text { measure- } \\
\text { ments }\end{array}$ & $\begin{array}{l}\text { Max- } \\
\text { imum }\end{array}$ & $\begin{array}{l}\text { Min- } \\
\text { imum }\end{array}$ & Mean & Median & $\begin{array}{l}\text { Standard } \\
\text { devi- } \\
\text { ation }\end{array}$ & $\begin{array}{l}\text { Ninetieth } \\
\text { percent- } \\
\text { ile }\end{array}$ & $\begin{array}{l}\text { Tenth } \\
\text { percent- } \\
\text { ile }\end{array}$ \\
\hline Specific conductance & mho/ $/ \mathrm{cm}$ & 159 & 646 & 51 & 432 & 505 & 159 & 562 & 125 \\
\hline Dissolved solids, residue & $\mathrm{mg} / \mathrm{L}$ & 136 & 425 & 52 & 278 & 320 & 94 & 361 & 102 \\
\hline Dissolved solids, sum & $\mathrm{mg} / \mathrm{L}$ & 55 & 386 & 73 & 287 & 309 & 81 & 354 & 135 \\
\hline Hardness as $\mathrm{CaCO}_{3}$ & $\mathrm{mg} / \mathrm{L}$ & 119 & 270 & 13 & 168 & 202 & 77 & 247 & 27 \\
\hline Calcium, dissolved & $\mathrm{mg} / \mathrm{L}$ & 119 & 80 & 3.9 & 50 & 61 & 24 & 73 & 7.6 \\
\hline Magnesium, dissolved & $\mathrm{mg} / \mathrm{L}$ & 119 & 22 & 0.6 & 10 & 12 & 4.7 & 15 & 1.9 \\
\hline Sodium, dissolved & $\mathrm{mg} / \mathrm{L}$ & 119 & 33 & 0.6 & 20 & 24 & 9.2 & 29 & 2.5 \\
\hline Potassium, dissolved & $\mathrm{mg} / \mathrm{L}$ & 119 & 17 & 4.3 & 9.8 & 9.3 & 2.8 & 14 & 6.6 \\
\hline Bicarbonate ion & $\mathrm{mg} / \mathrm{L}$ & 119 & 366 & 0 & 224 & 268 & 106 & 328 & 32 \\
\hline Sulfate, dissolved & $\mathrm{mg} / \mathrm{L}$ & 119 & 76 & 4.8 & 25 & 27 & 11 & 36 & 8.3 \\
\hline Chloride, dissolved & $\mathrm{mg} / \mathrm{L}$ & 154 & 17 & 1.2 & 6.2 & 6.0 & 2.8 & 9.2 & 3.1 \\
\hline Fluoride, dissolved & $\mathrm{mg} / \mathrm{L}$ & 120 & 2.0 & 0.1 & 0.4 & 0.3 & 0.2 & 0.5 & 0.2 \\
\hline Silica, dissolved & $\mathrm{mg} / \mathrm{L}$ & 119 & 40 & 5.3 & 25 & 28 & 8.7 & 35 & 12 \\
\hline Boron, disșolved & $\mathrm{mg} / \mathrm{L}$ & 119 & 150 & 10 & 48 & 41 & 22 & 70 & 30 \\
\hline Iron, dissolved & $\mathrm{ug} / \mathrm{L}$ & 97 & 630 & 0 & 90 & 44 & 112 & 244 & 14 \\
\hline Manganese, dissolved & $\mathrm{ug} / \mathrm{L}$ & 75 & 1,000 & 0 & 263 & 205 & 198 & 487 & 62 \\
\hline Nitrite + nitrate as $\mathrm{N}$, & $\mathrm{mg} / \mathrm{L}$ & 28 & 2.9 & 0.03 & 1.3 & 1.3 & 0.61 & 2.2 & 0.60 \\
\hline Nitrate as $\mathrm{N}$, dissolved & $\mathrm{mg} / \mathrm{L}$ & 25 & 6.5 & 0.00 & 1.6 & 1.3 & 1.6 & 2.9 & 0.01 \\
\hline Nitrate as $\mathrm{NO}_{3}$, dissolved & $\mathrm{mg} / \mathrm{L}$ & & & & & & & & (n) \\
\hline Ammonia N, total & $\mathrm{mg} / \mathrm{L}$ & 55 & 1.6 & 0.00 & 0.21 & 0.10 & 0.34 & 0.73 & 0.02 \\
\hline Organic $\mathrm{N}$, total & $\mathrm{mg} / \mathrm{L}$ & 55 & 5.6 & 0.10 & 1.3 & 0.87 & 1.0 & 2.4 & 0.37 \\
\hline Phosphorus, total & $\mathrm{mg} / \mathrm{L}$ & 56 & 1.9 & 0.20 & 0.60 & 0.56 & 0.32 & 0.99 & 0.26 \\
\hline Phosphorus, dissolved & $\mathrm{mg} / \mathrm{L}$ & 63 & 0.93 & 0.11 & 0.38 & 0.37 & 0.15 & 0.54 & 0.20 \\
\hline Fecal coliform $\quad \mathrm{col} / 1$ & $\operatorname{col} / 100 \mathrm{ml}$ & 43 & 26,000 & 3 & -....- & 350 & - n & 2,500 & 20 \\
\hline Fecal streptococci & $\operatorname{col} / 100 \mathrm{ml}$ & 52 & 50,000 & 6 & $\cdots+-$ & 590 & (n) & 3,000 & 26 \\
\hline Oxygen, dissolved & $\mathrm{mg} / \mathrm{L}$ & 55 & 17.4 & 4.6 & 9.9 & 9.2 & 3.3 & 15.0 & 6.3 \\
\hline \multirow{2}{*}{$\begin{array}{l}\text { Biochemical oxygen demand } \\
\mathrm{pH}^{(5-\text {-day })}\end{array}$} & $\mathrm{mg} / \mathrm{L}$ & 53 & 26.0 & 1.0 & 4.8 & 3.6 & 4.1 & 8.9 & 1.8 \\
\hline & pH units & 161 & 8.6 & 3.9 & 7.6 & 7.8 & 0.6 & 8.2 & 7.0 \\
\hline Co - Pt & Co - Pt units & 65 & 900 & 1 & 45 & 10 & 120 & 130 & 2 \\
\hline
\end{tabular}


Results of regression analyses relating concentrations of selected chemical constituents to specific conductance $(\mathrm{SpC})$

\begin{tabular}{|c|c|c|c|c|c|}
\hline $\begin{array}{l}\text { Dissolved } \\
\text { constituents } \\
(\mathrm{mg} / \mathrm{L})\end{array}$ & $\begin{array}{l}\text { No. of } \\
\text { measure- } \\
\text { ments }\end{array}$ & Regression equation & $\begin{array}{l}\text { Corre- } \\
\text { lation } \\
\text { coeffi- } \\
\text { cient }\end{array}$ & $\begin{array}{l}\text { Percent } \\
\text { explained } \\
\text { variance }\end{array}$ & $\begin{array}{c}\text { Standard } \\
\text { error of } \\
\text { estimate } \\
(\mathrm{mg} / \mathrm{L})\end{array}$ \\
\hline Dissolved solids, residue (RDS) & 138 & $\mathrm{RDS}=0.557(\mathrm{SpC})+46.120$ & 0.97 & 94 & 23 \\
\hline Dissolved solids, sum (SDS) & 54 & $\mathrm{SDS}=0.589(\mathrm{SpC})+24.564$ & .98 & 96 & 16 \\
\hline Hardness as $\mathrm{CaCO}_{3}(\mathrm{TH})$ & 119 & $\mathrm{TH}=0.458(\mathrm{SpC})-19.598$ & .99 & 98 & 10 \\
\hline Calcium (Ca) & 119 & $\mathrm{Ca}=0.139(\mathrm{SpC})-6.928$ & .99 & 98 & 3.7 \\
\hline Magnesium (Mg) & 119 & $\mathrm{Mg}=0.027(\mathrm{SpC})-0.570$ & .96 & 93 & 1.2 \\
\hline Sodium (Na) & 119 & $\mathrm{Na}=0.053(\mathrm{SpC})-2.052$ & .97 & 93 & 2.4 \\
\hline Potassium (K) & 119 & $\mathrm{~K}=-0.008(\mathrm{SpC})+12.913$ & -.46 & 21 & 2.5 \\
\hline Bicarbonate $\left(\mathrm{HCO}_{3}\right)$ & 119 & $\mathrm{HCO}_{3}=0.622(\mathrm{SpC})-30.318$ & .99 & 97 & 17 \\
\hline Sulfate $\left(\mathrm{SO}_{4}\right)$ & 119 & $\mathrm{SO}_{4}=0.047(\mathrm{SpC})+5.797$ & .73 & 53 & 7.5 \\
\hline Chloride (Cl) & 156 & $\mathrm{C} 1=0.009(\mathrm{SpC})+2.243$ & .52 & 27 & 2.4 \\
\hline Silica (Si) & 119 & $\mathrm{Si}=0.040(\mathrm{SpC})+9.151$ & .76 & 58 & 5.7 \\
\hline Nitrite + Nitrate as $\mathrm{N}\left(\mathrm{NO}_{2}-\mathrm{NO}_{3}\right)$ & 28 & $\mathrm{NO}_{2}-\mathrm{NO}_{3}=-0.0007(\mathrm{SpC})+1.686$ & $*_{-} .13$ & 1.7 & 0.62 \\
\hline
\end{tabular}

* Not significant at the 95 percent confidence level; the regression equation should not be used to predict the concentration of the dependent variable.

Results of regression analyses relating specific conductance to water discharge (Q)

\begin{tabular}{ccccccc}
\hline $\begin{array}{c}\text { No. of } \\
\text { measure- } \\
\text { ments }\end{array}$ & $\begin{array}{c}\text { Water discharge } \\
\left(\mathrm{ft}^{3} / \mathrm{s}\right)\end{array}$ & Maximum Minimum & Regression equation & $\begin{array}{c}\text { Correlation } \\
\text { coefficient }\end{array}$ & $\begin{array}{c}\text { Percent } \\
\text { explained } \\
\text { variance }\end{array}$ & $\begin{array}{c}\text { Standard error } \\
\text { of estimate }\end{array}$ \\
\hline 159 & $4,510 \quad 1.3$ & $\mathrm{SpC}=1,136 \mathrm{Q}^{-0.36424}$ & -0.92 & 84 & 0.106 \\
\hline
\end{tabular}


Location.--Lat $40^{\circ} 52^{\prime} 15^{\prime \prime}$, long $97^{\circ} 04^{\prime} 28^{\prime \prime}$, in $\mathrm{NE}^{\frac{1}{4}} \mathrm{NE}^{\frac{1}{4}} \mathrm{NW} \mathrm{W}^{\frac{1}{4}}$ sec. 3, T.10 N., R.3 E., Seward County, Hydrologic Unit 10270202, at bridge on county road about 2.5 mi southeast of Seward.

Period of record.--Water year 1973 to current year.

Statistical data for selected chemical constituents

\begin{tabular}{|c|c|c|c|c|c|c|c|c|c|}
\hline Constituent & Units & $\begin{array}{l}\text { No. of } \\
\text { measure- } \\
\text { ments }\end{array}$ & $\begin{array}{l}\text { Max- } \\
\text { imum }\end{array}$ & $\begin{array}{l}\text { Min- } \\
\text { imum }\end{array}$ & Mean & Median & $\begin{array}{l}\text { Standard } \\
\text { devi- } \\
\text { ation }\end{array}$ & $\begin{array}{l}\text { Ninetieth } \\
\text { percent- } \\
\text { ile }\end{array}$ & $\begin{array}{l}\text { Tenth } \\
\text { percent- } \\
\text { ile }\end{array}$ \\
\hline Specific conductance & umho/cm & 56 & 881 & 213 & 605 & 624 & 164 & 776 & 280 \\
\hline Dissolved solids, residue & $\mathrm{mg} / \mathrm{L}$ & 35 & 533 & 130 & 391 & 408 & 105 & 500 & 200 \\
\hline Dissolved solids, sum & $\mathrm{mg} / \mathrm{L}$ & 19 & 562 & 145 & 393 & 397 & 106 & 510 & 160 \\
\hline Hardness as $\mathrm{CaCO}_{3}$ & $\mathrm{mg} / \mathrm{L}$ & 18 & 360 & 82 & 248 & 248 & 74 & 350 & 86 \\
\hline Calcium, dissolved & $\mathrm{mg} / \mathrm{L}$ & 18 & 100 & 23 & 71 & 74 & 21 & 97 & 24 \\
\hline Magnesium, dissolved & $\mathrm{mg} / \mathrm{L}$ & 18 & 27 & 5.3 & 18 & 18 & 5.5 & 26 & 6.9 \\
\hline Sodium, dissolved & $\mathrm{mg} / \mathrm{L}$ & 18 & 55 & 11 & 37 & 39 & 11 & 49 & 16 \\
\hline Potassium, dissolved & $\mathrm{mg} / \mathrm{L}$ & 18 & 19 & 8.6 & 11 & 12 & 2.4 & 14 & 8.9 \\
\hline Bicarbonate ion & $\mathrm{mg} / \mathrm{L}$ & 18 & 401 & 97 & 293 & 310 & 85 & 390 & 108 \\
\hline Sul fate, dissolved & $\mathrm{mg} / \mathrm{L}$ & 18 & 110 & 22 & 70 & 66 & 25 & 93 & 31 \\
\hline Chloride, dissolved & $\mathrm{mg} / \mathrm{L}$ & 53 & 86 & 3.6 & 16 & 14 & 12 & 24 & 7.6 \\
\hline Fluoride, dissolved & $\mathrm{mg} / \mathrm{L}$ & 19 & 0.5 & 0.2 & 0.3 & 0.3 & 0.1 & 0.4 & 0.3 \\
\hline Silica, dissolved & $\mathrm{mg} / \mathrm{L}$ & 18 & 30 & 8.7 & 21 & 23 & 6.1 & 29 & 9.8 \\
\hline Boron, dissolved & $\mathrm{mg} / \mathrm{L}$ & 18 & 120 & 40 & 79 & 78 & 23 & 113 & 50 \\
\hline Iron, dissolved & $\mathrm{ug} / \mathrm{L}$ & 18 & 140 & 10 & 44 & 30 & 36 & 110 & 10 \\
\hline Manganese, dissolved & $\mathrm{ug} / \mathrm{L}$ & 18 & 700 & 0 & 232 & 260 & 205 & 650 & 80 \\
\hline $\begin{array}{l}\text { Nitrite + nitrate as } \mathrm{N}, \\
\text { dissolved } \\
\text { Nitrate as } \mathrm{N} \text {, dissolved }\end{array}$ & $\begin{array}{l}\mathrm{mg} / \mathrm{L} \\
\mathrm{mg} / \mathrm{L}\end{array}$ & 28 & 3.1 & 0.55 & 1.6 & 1.7 & 0.57 & 2.3 & 0.73 \\
\hline Nitrate as $\mathrm{NO}_{3}$, dissolved & $\mathrm{mg} / \mathrm{L}$ & - & & & $\cdots$ & 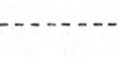 & $\cdots$ & $\cdots$ & -.... \\
\hline Ammonia $\mathrm{N}$, total & $\mathrm{mg} / \mathrm{L}$ & 56 & 2.1 & 0.00 & 0.51 & 0.38 & 0.48 & 1.1 & 0.02 \\
\hline Organic N, total & $\mathrm{mg} / \mathrm{L}$ & 56 & 9.0 & 0.28 & 1.6 & 1.4 & 1.3 & 2.7 & 0.70 \\
\hline Phosphorus, total & $\mathrm{mg} / \mathrm{L}$ & 56 & 2.6 & 0.37 & 0.92 & 0.83 & 0.43 & 1.4 & 0.55 \\
\hline Phosphorus, dissolved & $\mathrm{mg} / \mathrm{L}$ & 47 & 1.0 & 0.27 & 0.60 & 0.53 & 0.20 & 0.90 & 0.36 \\
\hline Fecal coliform & $\mathrm{col} / 100 \mathrm{ml}$ & 43 & 36,200 & 400 & $\cdots$ & 2,800 & $\cdots$ & 14,000 & 595 \\
\hline Fecal streptococci & $\mathrm{col} 1 / 100 \mathrm{ml}$ & 51 & 100,000 & 300 & 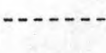 & 1,370 & ....- & 5,230 & 570 \\
\hline Oxygen, dissolved & $\mathrm{mg} / \mathrm{L}$ & 55 & 16.8 & 2.9 & 8.4 & 7.6 & 3.6 & 14.3 & 4.1 \\
\hline \multirow{2}{*}{$\begin{array}{l}\text { Biochemical oxygen demand } \\
\text { (5-day) }\end{array}$} & $\mathrm{mg} / \mathrm{L}$ & 51 & 44.0 & 1.8 & 13.2 & 12.0 & 9.6 & 21.3 & 4.8 \\
\hline & H units & 60 & 8.7 & 6.6 & 7.6 & 7.6 & 0.4 & 8.0 & 7.2 \\
\hline $\mathrm{Co}-\mathrm{Pt}$ & t units & 19 & 700 & 4 & 62 & 20 & 156 & 100 & 5 \\
\hline
\end{tabular}


Results of regression analyses relating concentrations of selected chemical constituents to specific conductance $(\mathrm{SpC})$

\begin{tabular}{|c|c|c|c|c|c|}
\hline $\begin{array}{c}\text { Dissolved } \\
\text { constituents } \\
(\mathrm{mg} / \mathrm{L})\end{array}$ & $\begin{array}{l}\text { No. of } \\
\text { measure- } \\
\text { ments }\end{array}$ & Regression equation & $\begin{array}{l}\text { Corre- } \\
\text { lation } \\
\text { coeffi- } \\
\text { cient }\end{array}$ & $\begin{array}{l}\text { Percent } \\
\text { explained } \\
\text { variance }\end{array}$ & $\begin{array}{c}\text { Standard } \\
\text { error of } \\
\text { estimate } \\
(\mathrm{mg} / \mathrm{L})\end{array}$ \\
\hline Dissolved solids, residue (RDS) & 35 & $\mathrm{RDS}=0.650(\mathrm{SpC})-0.502$ & 0.96 & 93 & 29 \\
\hline Dissolved solids, sum (SDS) & 18 & $\mathrm{SDS}=0.635(\mathrm{SpC})-8.043$ & .98 & 97 & 19 \\
\hline Hardness as $\mathrm{CaCO}_{3}(\mathrm{TH})$ & 18 & $\mathrm{TH}=0.435(\mathrm{SpC})-27.448$ & .99 & 98 & 10 \\
\hline Calcium (Ca) & 18 & $\mathrm{Ca}=0.124(\mathrm{SpC})-7.609$ & .99 & 98 & 2.9 \\
\hline Magnèsium (Mg) & 18 & $\mathrm{Mg}=0.031(\mathrm{SpC})-2.349$ & .97 & 94 & 1.4 \\
\hline Sodium $(\mathrm{Na})$ & 18 & $\mathrm{Na}=0.062(\mathrm{SpC})-1.555$ & .96 & 92 & 3.2 \\
\hline Potassium (K) & 18 & $K=-0.005(S p C)+14.337$ & $*_{-} .34$ & 11 & 2.4 \\
\hline Bicarbonate $\left(\mathrm{HCO}_{3}\right)$ & 18 & $\mathrm{HCO}_{3}=0.496(\mathrm{SpC})-20.610$ & .99 & 98 & 13 \\
\hline Sulfate $\left(\mathrm{SO}_{4}\right)$ & 18 & $\mathrm{SO}_{4}=0.128(\mathrm{SpC})-11.156$ & .88 & 77 & 12 \\
\hline Chloride $(\mathrm{Cl})$ & 53 & $\mathrm{Cl}=0.021(\mathrm{SpC})+2.732$ & .29 & 83 & 12 \\
\hline Silica (Si) & 18 & $\mathrm{Si}=0.022(\mathrm{SpC})+7.356$ & .62 & 38 & 4.9 \\
\hline Nitrite + Nitrate as $\mathrm{N}\left(\mathrm{NO}_{2}-\mathrm{NO}_{3}\right)$ & 28 & $\mathrm{NO}_{2}-\mathrm{NO}_{3}=0.0003(\mathrm{SpC})+1.437$ & *.08 & 0.60 & 0.58 \\
\hline
\end{tabular}

* Not significant at the 95 percent confidence level; the regression equation should not be used to predict the concentration of the dependent variable.

Results of regression analyses relating specific conductance to water discharge (0)

\begin{tabular}{ccccccc}
\hline $\begin{array}{c}\text { No. of } \\
\text { measure- } \\
\text { ments }\end{array}$ & $\begin{array}{c}\text { Water discharge } \\
\left(\mathrm{ft}^{3} / \mathrm{s}\right)\end{array}$ & Maximum Minimum & Regression equation & $\begin{array}{c}\text { Correlation } \\
\text { coefficient }\end{array}$ & $\begin{array}{c}\text { Percent } \\
\text { explained } \\
\text { variance }\end{array}$ & $\begin{array}{c}\text { Standard error } \\
\text { of estimate }\end{array}$ \\
\hline 56 & 650 & 5.9 & $\mathrm{SpC}=1,094 \mathrm{Q}^{-0.18452}$ & -0.59 & 35 & 0.122 \\
\hline
\end{tabular}


06880556 West Fork Big Blue River below Hastings, Nebr.

Location.--Lat $40^{\circ} 36^{\prime} 09^{\prime \prime}$, long $98^{\circ} 20^{\prime} 02^{\prime \prime}$, in NW $W^{\frac{1}{4}} \mathrm{NW}^{\frac{1}{4}} \mathrm{SW}^{\frac{1}{4}}$ sec. 3, T.7 N. R.9 W., Adams County, Hydrologic Unit 10270203, at bridge on county road $1.4 \mathrm{mi}$ north of U.S. Highway 6 and about $1.5 \mathrm{mi}$ northeast of Hastings.

Period of record.--Water year 1973 to current year.

Statistical data for selected chemical constituents

\begin{tabular}{|c|c|c|c|c|c|c|c|c|c|}
\hline Constituent & Units & $\begin{array}{l}\text { No. of } \\
\text { measure- } \\
\text { ments }\end{array}$ & $\begin{array}{l}\text { Max- } \\
\text { imum }\end{array}$ & $\begin{array}{l}\text { Min- } \\
\text { imum }\end{array}$ & Mean & Median & $\begin{array}{c}\text { Standard } \\
\text { devi- } \\
\text { ation }\end{array}$ & $\begin{array}{c}\text { Ninetieth } \\
\text { percent- } \\
\text { ile }\end{array}$ & $\begin{array}{l}\text { Tenth } \\
\text { percent- } \\
\text { ile }\end{array}$ \\
\hline Specific conductance & umho/cm & 60 & 980 & 105 & 592 & 613 & 188 & 810 & 333 \\
\hline Dissolved solids, residue & $\mathrm{mg} / \mathrm{L}$ & 35 . & 510 & 140 & 375 & 393 & 100 & 478 & 210 \\
\hline Dissolved solids, sum & $\mathrm{mg} / \mathrm{L}$ & 17 & 493 & 72 & 349 & 330 & 109 & 460 & 171 \\
\hline Hardness as $\mathrm{CaCO}_{3}$ & $\mathrm{mg} / \mathrm{L}$ & 17 & 190 & 33 & 156 & 172 & 38 & 186 & 95 \\
\hline Calcium, dissolved & $\mathrm{mg} / \mathrm{L}$ & 17 & 59 & 10 & 49 & 54 & 12 & 59 & 28 \\
\hline Magnesium, dissolved & $\mathrm{mg} / \mathrm{L}$ & 17 & 10 & 2.0 & 8.3 & 8.8 & 2.0 & 9.9 & 6.0 \\
\hline Sodium, dissolved & $\mathrm{mg} / \mathrm{L}$ & 17 & 88 & 7.9 & 52 & 44 & 25 & 85 & 16 \\
\hline Potassium, dissolved & $\mathrm{mg} / \mathrm{L}$ & 17 & 14 & 7.5 & 11 & 11 & 2.1 & 14 & 9.1 \\
\hline Bicarbonate ion & $\mathrm{mg} / \mathrm{L}$ & 17 & 252 & 50 & 183 & 190 & 47 & 230 & 106 \\
\hline Sulfate, dissolved & $\mathrm{mg} / \mathrm{L}$ & 17 & 82 & 7.3 & 52 & 52 & 17 & 79 & 31 \\
\hline Chloride, dissolved & $\mathrm{mg} / \mathrm{L}$ & 52 & 77 & 3.6 & 41 & 41 & 20 & 68 & 15 \\
\hline Fluoride, dissolved & $\mathrm{mg} / \mathrm{L}$ & 17 & 1.6 & 0.3 & 0.6 & 0.5 & 0.3 & 1.1 & 0.3 \\
\hline Silica, dissolved & $\mathrm{mg} / \mathrm{L}$ & 17 & 35 & 7.9 & 26 & 28 & 6.4 & 32 & 18 \\
\hline Boron, dissolved & $\mathrm{mg} / \mathrm{L}$ & 17 & 530 & 50 & 212 & 145 & 149 & 450 & 60 \\
\hline Iron, dissolved & $\mathrm{ug} / \mathrm{L}$ & 17 & 400 & 20 & 96 & 58 & 95 & 230 & 30 \\
\hline Manganese, dissolved & $\mathrm{ug} / \mathrm{L}$ & 17 & 190 & 10 & 48 & 32 & 43 & 90 & 20 \\
\hline Nitrite + nitrate as $\mathrm{N}$, & $\mathrm{mg} / \mathrm{L}$ & 26 & 15 & 0.04 & 6.3 & 6.0 & 4.1 & 12 & 1.5 \\
\hline $\begin{array}{l}\text { Nitrate as } \mathrm{N} \text {, dissolved } \\
\text { Nitrate as } \mathrm{NO}_{3} \text {, dissolved }\end{array}$ & $\begin{array}{l}\mathrm{mg} / \mathrm{L} \\
\mathrm{mg} / \mathrm{L}\end{array}$ & & & & & & & & --- \\
\hline Ammonia $\mathrm{N}$, total & $\mathrm{mg} / \mathrm{L}$ & 53 & 8.3 & 0.01 & 2.5 & 2.1 & 2.0 & 4.5 & 0.20 \\
\hline Organic $N$, total & $\mathrm{mg} / \mathrm{L}$ & 51 & 19 & 0.00 & 2.1 & 1.4 & 2.8 & 3.2 & 0.18 \\
\hline Phosphorus, total & $\mathrm{mg} / \mathrm{L}$ & 53 & 11 & 0.90 & 5.5 & 5.8 & 3.0 & 9.1 & 1.8 \\
\hline Phosphorus, dissolved & $\mathrm{mg} / \mathrm{L}$ & 44 & 9.8 & 0.25 & 4.9 & 5.5 & 3.0 & 8.5 & 1.4 \\
\hline Fecal coliform & $\operatorname{col} / 100 \mathrm{ml}$ & 39 & 860,000 & 3,300 & $-\cdots--$ & 75,700 & 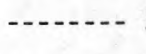 & 510,000 & 6,800 \\
\hline Fecal streptococci & $\operatorname{col} / 100 \mathrm{ml}$ & 47 & 60,000 & 400 & $-\ldots$ & 3,320 & 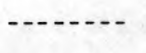 & 30,000 & 890 \\
\hline Oxygen, dissolved & $\mathrm{mg} / \mathrm{L}$ & 53 & 10.8 & 2.8 & 6.8 & 6.9 & 1.6 & 9.2 & 5.3 \\
\hline Biochemical oxygen demand & $\mathrm{mg} / \mathrm{L}$ & 54 & 48.0 & 3.6 & 14.6 & 12.4 & 9.0 & 27.5 & 7.3 \\
\hline $\mathrm{pH}$ & $\mathrm{pH}$ units & 57 & 7.9 & 7.1 & 7.4 & 7.4 & 0.2 & 7.7 & 7.2 \\
\hline Co $-P t$ & Co - Pt units & 17 & 300 & 7 & 45 & 24 & 71 & 130 & 10 \\
\hline
\end{tabular}


Results of regression analyses relating concentrations of selected chemical constituents to specific conductance $(\mathrm{SpC})$

\begin{tabular}{|c|c|c|c|c|c|}
\hline $\begin{array}{l}\text { Dissolved } \\
\text { constituents } \\
(\mathrm{mg} / \mathrm{L})\end{array}$ & $\begin{array}{l}\text { No. of } \\
\text { measure- } \\
\text { ments }\end{array}$ & Regression equation & $\begin{array}{l}\text { Corre- } \\
\text { lation } \\
\text { coeffi- } \\
\text { cient }\end{array}$ & $\begin{array}{l}\text { Percent } \\
\text { explained } \\
\text { variance }\end{array}$ & $\begin{array}{l}\text { Standard } \\
\text { error of } \\
\text { estimate } \\
(\mathrm{mg} / \mathrm{L})\end{array}$ \\
\hline Dissolved solids, residue (RDS) & 36 & $\mathrm{RDS}=0.479(\mathrm{SpC})+81.862$ & 0.90 & 81 & 44 \\
\hline Dissolved solids, sum (SDS) & 17 & $\mathrm{SDS}=0.536(\mathrm{SpC})+33.902$ & .97 & 94 & 28 \\
\hline Hardness as $\mathrm{CaCO}_{3}(\mathrm{TH})$ & 17 & $\mathrm{TH}=0.156(\mathrm{SpC})+64.921$ & .80 & 64 & 24 \\
\hline Calcium (Ca) & 17 & $\mathrm{Ca}=0.050(\mathrm{SpC})+20.233$ & .79 & 62 & 7.9 \\
\hline Magnesium (Mg) & 17 & $\mathrm{Mg}=0.008(\mathrm{SpC})+3.768$ & .76 & 58 & 1.3 \\
\hline Sodium $(\mathrm{Na})$ & 17 & $\mathrm{Na}=0.120(\mathrm{SpC})-17.888$ & .94 & 88 & 9.2 \\
\hline Potassium (K) & 17 & $K=0.009(\mathrm{SpC})+6.086$ & .83 & 68 & 1.2 \\
\hline Bicarbonate $\left(\mathrm{HCO}_{3}\right)$ & 17 & $\mathrm{HCO}_{3}=0.192(\mathrm{SpC})+70.456$ & .80 & 65 & 29 \\
\hline Sulfate $\left(\mathrm{SO}_{4}\right)$ & 17 & $\mathrm{SO}_{4}=0.075(\mathrm{SpC})+8.604$ & .85 & 72 & 9.5 \\
\hline Chloride (Cl) & 53 & $\mathrm{Cl}=0.092(\mathrm{SpC})-14.254$ & .86 & 74 & 10 \\
\hline Silica (Si) & 17 & $\mathrm{Si}=0.030(\mathrm{SpC})+9.060$ & .92 & 85 & 2.5 \\
\hline Nitrite + Nitrate as $\mathrm{N}\left(\mathrm{NO}_{2}-\mathrm{NO}_{3}\right)$ & 26 & $\mathrm{NO}_{2}-\mathrm{NO}_{3}=0.017(\mathrm{SpC})-3.052$ & .77 & 59 & 2.7 \\
\hline
\end{tabular}

Results of regression analyses relating specific conductance to water discharge $(Q)$

\begin{tabular}{ccccccc}
\hline $\begin{array}{c}\text { No. of } \\
\text { measure- } \\
\text { ments }\end{array}$ & $\begin{array}{c}\text { Water discharge } \\
\text { Maximum Minimum }\end{array}$ & Regression equation & $\begin{array}{c}\text { Correlation } \\
\text { coefficient }\end{array}$ & $\begin{array}{c}\text { Percent } \\
\text { explained } \\
\text { variance }\end{array}$ & $\begin{array}{c}\text { Standard error } \\
\text { of estimate }\end{array}$ \\
\hline 60 & 422 & 2.5 & $\mathrm{SpC}=1,336 \mathrm{Q}^{-0.33887}$ & -0.73 & 54 & 0.122 \\
\hline
\end{tabular}


BIG BLUE RIVER BASIN

06880800 West Fork Big Blue River near Dorchester, Nebr.

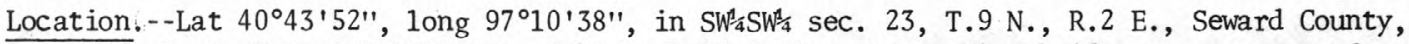
Hydrologic Unit 10270203 , on right bank $60 \mathrm{ft}$ downstream from bridge on county road, $6.2 \mathrm{mi}$ northwest of Dorchester, and $19 \mathrm{mi}$ upstream from mouth.

Drainage area.--1,206 $\mathrm{mi}^{2}$.

Period of record.--Water years $1963-70,1973$ to current year.

Statistical data for selected chemical constituents

\begin{tabular}{|c|c|c|c|c|c|c|c|c|c|}
\hline Constituent & Units & $\begin{array}{l}\text { No. of } \\
\text { measure- } \\
\text { ments }\end{array}$ & $\begin{array}{l}\text { Max- } \\
\text { imum }\end{array}$ & $\begin{array}{l}\text { Min- } \\
\text { imum }\end{array}$ & Mean & Median & $\begin{array}{c}\text { Standard } \\
\text { devi- } \\
\text { ation }\end{array}$ & $\begin{array}{l}\text { Ninetieth } \\
\text { percent- } \\
\text { ile }\end{array}$ & $\begin{array}{c}\text { Tenth } \\
\text { percent- } \\
\text { ile }\end{array}$ \\
\hline Specific conductance & umho/cm & 166 & 701 & 78 & 439 & 504 & 156 & 583 & 137 \\
\hline Dissolved solids, residue & $\mathrm{mg} / \mathrm{L}$ & 146 & 415 & 71 & 286 & 327 & 87 & 365 & 140 \\
\hline Dissolved solids, sum & $\mathrm{mg} / \mathrm{L}$ & 62 & 407 & 78 & 275 & 320 & 91 & 356 & 98 \\
\hline Hardness as $\mathrm{CaCO}_{3}$ & $\mathrm{mg} / \mathrm{L}$ & 128 & 260 & 21 & 156 & 198 & 67 & 199 & 35 \\
\hline Calcium, dissolved & $\mathrm{mg} / \mathrm{L}$ & 128 & 79 & 6.1 & 47 & 57 & 21 & 66 & 10 \\
\hline Magnesium, dissolved & $\mathrm{mg} / \mathrm{L}$ & 128 & 15 & 1.5 & 9.3 & 11 & 3.6 & 13 & 2.8 \\
\hline Sodium, dissolved & $\mathrm{mg} / \mathrm{L}$ & 128 & 50 & 2.0 & 24 & 27 & 11 & 36 & 5.3 \\
\hline Potassium, dissolved & $\mathrm{mg} / \mathrm{L}$ & 127 & 16 & 4.5 & 9.2 & 8.9 & 2.2 & 13 & 6.9 \\
\hline Bicarbonate ion & $\mathrm{mg} / \mathrm{L}$ & 129 & 299 & 26 & 192 & 231 & 80 & 264 & 48 \\
\hline Sulfate, dissolved & $\mathrm{mg} / \mathrm{L}$ & 127 & 63 & 7.0 & 35 & 40 & 14 & 50 & 10 \\
\hline Chloride, dissolved & $\mathrm{mg} / \mathrm{L}$ & 163 & 43 & 2.0 & 16 & 16 & 7.3 & 24 & 4.9 \\
\hline Fluoride, dissolved & $\mathrm{mg} / \mathrm{L}$ & 128 & 0.6 & 0.1 & 0.4 & 0.4 & 0.1 & 0.5 & 0.3 \\
\hline Silica, dissolved & $\mathrm{mg} / \mathrm{L}$ & 127 & 36 & 4.7 & 24 & 26 & 6.9 & 32 & 14 \\
\hline Boron, dissolved & $\mathrm{mg} / \mathrm{L}$ & 127 & 320 & 10 & 61 & 55 & 33 & 85 & 31 \\
\hline Iron, dissolved & $\mathrm{ug} / \mathrm{L}$ & 98 & 660 & 0 & 68 & 36 & 99 & 143 & 8 \\
\hline Manganese, dissolved & $\mathrm{ug} / \mathrm{L}$ & 77 & 1,000 & 0 & 150 & 129 & 140 & 273 & 23 \\
\hline Nitrite + nitrate as $\mathrm{N}$, & $\mathrm{mg} / \mathrm{L}$ & 28 & 2.9 & 0.28 & 1.6 & 1.8 & 0.65 & 2.3 & 0.44 \\
\hline Nitrate as N, dissolved & $\mathrm{mg} / \mathrm{L}$ & 24 & 3.7 & 0.00 & 1.6 & 1.4 & 1.1 & 3.2 & 0.07 \\
\hline Nitrate as $\mathrm{NO}_{3}$, dissolved & $\mathrm{mg} / \mathrm{L}$ & & & & 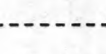 & & & & \\
\hline Ammonia $\mathrm{N}$, total & $\mathrm{mg} / \mathrm{L}$ & 57 & 1.6 & 0.00 & 0.31 & 0.20 & 0.34 & 0.64 & 0.04 \\
\hline Organic $N$, total & $\mathrm{mg} / \mathrm{L}$ & 57 & 7.3 & 0.09 & 1.4 & 1.2 & 1.2 & 2.2 & 0.33 \\
\hline Phosphorus, total & $\mathrm{mg} / \mathrm{L}$ & 57 & 3.8 & 0.52 & 0.91 & 0.83 & 0.45 & 1.2 & 0.62 \\
\hline Phosphorus, dissolved & $\mathrm{mg} / \mathrm{L}$ & 63 & 1.6 & 0.15 & 0.59 & 0.58 & 0.20 & 0.77 & 0.37 \\
\hline Fecal coliform & $\operatorname{col} / 100 \mathrm{ml}$ & \multicolumn{2}{|c|}{44125,000} & 5 & 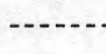 & 610 & - n & $-5,800$ & 45 \\
\hline Fecal streptococci & $\operatorname{col} / 100 \mathrm{ml}$ & \multicolumn{2}{|c|}{52143,000} & 25 & $-\cdots--$ & $-1,000$ & $-1-1$ & 9,400 & 86 \\
\hline Oxygen, dissolved & $\mathrm{mg} / \mathrm{L}$ & 51 & 15.0 & 5.5 & 9.4 & 8.8 & 2.2 & 12.3 & 6.9 \\
\hline \multirow{2}{*}{$\begin{array}{l}\text { Biochemical oxyger } \\
\mathrm{pH}^{(5-\text { day })}\end{array}$} & $\mathrm{mg} / \mathrm{L}$ & 53 & 16.0 & 0.8 & 4.8 & 4.1 & 3.0 & 8.4 & 1.9 \\
\hline & units & 170 & 8.5 & 6.5 & 7.6 & 7.6 & 0.4 & 8.1 & 7.0 \\
\hline Co $-P t$ & $t$ units & 70 & 450 & 1 & 33 & 10 & 68 & 71 & 3 \\
\hline
\end{tabular}


06880800 West Fork Big Blue River near Dorchester, Nebr.--Continued

Results of regression analyses relating concentrations of selected chemical constituents to specific conductance $(\mathrm{SpC})$

\begin{tabular}{|c|c|c|c|c|c|}
\hline $\begin{array}{l}\text { Dissolved } \\
\text { constituents } \\
\text { (mg/L) }\end{array}$ & $\begin{array}{l}\text { No. of } \\
\text { measure- } \\
\text { ments }\end{array}$ & Regression equation & $\begin{array}{l}\text { Corre- } \\
\text { lation } \\
\text { coeffi- } \\
\text { cient }\end{array}$ & $\begin{array}{l}\text { Percent } \\
\text { explained } \\
\text { variance }\end{array}$ & $\begin{array}{c}\text { Standard } \\
\text { error of } \\
\text { estimate } \\
(\mathrm{mg} / \mathrm{L})\end{array}$ \\
\hline Dissolved solids, residue (RDS) & 146 & $\mathrm{RDS}=0.544(\mathrm{SpC})+50.259$ & 0.98 & 96 & 17 \\
\hline Dissolved solids, sum (SDS) & 61 & $\mathrm{SDS}=0.599(\mathrm{spC})+14.733$ & .99 & 98 & 12 \\
\hline Hardness as $\mathrm{CaCO}_{3}(\mathrm{TH})$ & 128 & $\mathrm{TH}=0.410(\mathrm{SpC})-15.096$ & .99 & 97 & 11 \\
\hline Calciưm (Ca) & 128 & $\mathrm{Ca}=0.128(\mathrm{SpC})-6.124$ & .98 & 96 & 3.9 \\
\hline Magnesium (Mg) & 128 & $\mathrm{Mg}=0.022(\mathrm{SpC})+0.122$ & .97 & 94 & 0.9 \\
\hline Sodium (Na) & 128 & $\mathrm{Na}=0.065(\mathrm{SpC})-3.454$ & .96 & 92 & 3.1 \\
\hline Potassium (K) & 127 & $K=-0.007(S p C)+12.085$ & -.51 & 26 & 1.9 \\
\hline Bicarbonate $\left(\mathrm{HCO}_{3}\right)$ & 129 & $\mathrm{HCO}_{3}=0.497(\mathrm{SpC})-15.992$ & .99 & 97 & 13 \\
\hline Sulfate $\left(\mathrm{SO}_{4}\right)$ & 127 & $\mathrm{SO}_{4}=0.083(\mathrm{SpC})+0.299$ & .95 & 91 & 4.2 \\
\hline Chloride (C1) & 163 & $\mathrm{Cl}=0.038(\mathrm{SpC})-0.954$ & .80 & 64 & 4.4 \\
\hline Silica (Si) & 127 & $\mathrm{Si}=0.032(\mathrm{SpC})+10.800$ & .76 & 57 & 4.5 \\
\hline Nitrite + Nitrate as $\mathrm{N}\left(\mathrm{NO}_{2}-\mathrm{NO}_{3}\right)$ & 28 & $\mathrm{NO}_{2}-\mathrm{NO}_{3}=-0.001(\mathrm{SpC})+2.255$ & $*_{-} .29$ & 8.4 & 0.6 \\
\hline
\end{tabular}

* Not significant at the 95 percent confidence level; the regression equation should not be used to predict the concentration of the dependent variable.

Results of regression analyses relating specific conductance to water discharge (Q)

\begin{tabular}{|c|c|c|c|c|c|c|c|}
\hline \multirow{2}{*}{$\begin{array}{l}\text { No. of } \\
\text { measure- } \\
\text { ments }\end{array}$} & \multirow{2}{*}{\multicolumn{2}{|c|}{$\begin{array}{l}\begin{array}{l}\text { Water discharge } \\
\left(\mathrm{ft}^{3} / \mathrm{s}\right)\end{array} \\
\text { Maximum Minimum }\end{array}$}} & \multirow{2}{*}{ Regression equation } & \multirow{2}{*}{$\begin{array}{l}\text { Correlation } \\
\text { coefficient }\end{array}$} & \multirow{2}{*}{$\begin{array}{l}\text { Percent } \\
\text { explained } \\
\text { variance }\end{array}$} & \multicolumn{2}{|c|}{$\begin{array}{c}\text { Standard error } \\
\text { of estimate }\end{array}$} \\
\hline & & & & & & Log units & Percent \\
\hline 166 & 3,400 & 15 & $\mathrm{SpC}=3,570 \mathrm{Q}^{-0.46838}$ & -0.93 & 87 & 0.084 & 19 \\
\hline
\end{tabular}


Location. --Lat $40^{\circ} 35^{\prime} 47^{\prime \prime}$, long $96^{\circ} 57^{\prime} 36^{\prime \prime}$, in SW $^{\frac{1}{4}} \mathrm{SE}^{\frac{1}{4}} \mathrm{sec}$ 3, T.7 N., R.4 E., Saline County, Hydrologic Unit 10270202 on downstream side of right pier of highway bridge, $1.8 \mathrm{mi}$ south of Missouri Pacific Railroad station in Crete, 3.3 mi downstream from Walnut Creek, and $3.6 \mathrm{mi}$ upstream from Squaw Creek.

Drainage area. $--2,716 \mathrm{mi}^{2}$.

Period of record.--Water years 1961-63, 1968 to current year.

Statistical data for selected chemical constituents

\begin{tabular}{|c|c|c|c|c|c|c|c|c|c|}
\hline Constituent & Units & $\begin{array}{l}\text { No. of } \\
\text { measure- } \\
\text { ments }\end{array}$ & $\begin{array}{l}\text { Max- } \\
\text { imum }\end{array}$ & $\begin{array}{l}\text { Min- } \\
\text { imum }\end{array}$ & Mean & Median & $\begin{array}{c}\text { Standard } \\
\text { devi- } \\
\text { ation }\end{array}$ & $\begin{array}{l}\text { Ninetieth } \\
\text { percent- } \\
\text { ile }\end{array}$ & $\begin{array}{l}\text { Tenth } \\
\text { percent- } \\
\text { ile }\end{array}$ \\
\hline Specific conductance & mho/cm & 81 & 745 & 65 & 461 & 520 & 192 & 656 & 152 \\
\hline Dissolved solids, residue & $\mathrm{mg} / \mathrm{L}$ & 60 & 468 & 54 & 320 & 343 & 101 & 420 & 170 \\
\hline Dissolved solids, sum & $\mathrm{mg} / \mathrm{L}$ & 19 & 432 & 79 & 245 & 250 & 131 & 400 & 96 \\
\hline Hardness as $\mathrm{CaCO}_{3}$ & $\mathrm{mg} / \mathrm{L}$ & 39 & 280 & 17 & 139 & 140 & 84 & 252 & 33 \\
\hline Calcium, dissolved & $\mathrm{mg} / \mathrm{L}$ & 39 & 84 & 5.0 & 42 & 45 & 26 & 78 & 9.8 \\
\hline Magnesium, dissolved & $\mathrm{mg} / \mathrm{L}$ & 39 & 18 & 1.0 & 8.3 & 8.3 & 5.1 & 15 & 1.4 \\
\hline Sodium, dissolved & $\mathrm{mg} / \mathrm{L}$ & 39 & 45 & 2.2 & 20 & 17 & 12 & 36 & 4.0 \\
\hline Potassium, dissolved & $\mathrm{mg} / \mathrm{L}$ & 38 & 16 & 7.0 & 9.7 & 9.6 & 1.7 & 12 & 7.5 \\
\hline Bicarbonate ion & $\mathrm{mg} / \mathrm{L}$ & 39 & 326 & 28 & 165 & 170 & 99 & 295 & 43 \\
\hline Sulfate, dissolved & $\mathrm{mg} / \mathrm{L}$ & 39 & 74 & 1.0 & 34 & 34 & 19 & 60 & 11 \\
\hline Chloride, dissolved & $\mathrm{mg} / \mathrm{L}$ & 78 & 36 & 0.0 & 15 & 15 & 9.6 & 26 & 4.4 \\
\hline Fluoride, dissolved & $\mathrm{mg} / \mathrm{L}$ & 39 & 0.8 & 0.2 & 0.4 & 0.3 & 0.1 & 0.5 & 0.3 \\
\hline Silica, dissolved & $\mathrm{mg} / \mathrm{L}$ & 39 & 33 & 7.7 & 18 & 20 & 6.9 & 28 & 9.2 \\
\hline Boron, dissolved & $\mathrm{mg} / \mathrm{L}$ & 39 & 130 & 10 & 58 & 58 & 24 & 87 & 33 \\
\hline Iron, dissolved & $\mathrm{ug} / \mathrm{L}$ & 37 & 310 & 0 & 79 & 56 & 70 & 170 & 13 \\
\hline Manganese, dissolved & $\mathrm{ug} / \mathrm{L}$ & 33 & 460 & 0 & 102 & 30 & 128 & 250 & 0 \\
\hline $\begin{array}{l}\text { Nitrite + nitrate as } \mathrm{N}, \\
\text { dissolved } \\
\text { Nitrate as N, dissolved }\end{array}$ & $\begin{array}{l}\mathrm{mg} / \mathrm{L} \\
\mathrm{mg} / \mathrm{L}\end{array}$ & 33 & 3.4 & 0.00 & 1.6 & 1.7 & 0.76 & 2.5 & 0.55 \\
\hline Nitrate as $\mathrm{NO}_{3}$, dissolved & $\mathrm{mg} / \mathrm{L}$ & -- & & & & & & & \\
\hline Anmonia $\mathrm{N}$, total & $\mathrm{mg} / \mathrm{L}$ & 54 & 1.4 & 0.01 & 0.44 & 0.36 & 0.34 & 0.86 & 0.07 \\
\hline Organic $N$, total & $\mathrm{mg} / \mathrm{L}$ & 54 & 17 & 0.20 & 2.1 & 1.4 & 3.1 & 3.0 & 0.49 \\
\hline Phosphorus, total & $\mathrm{mg} / \mathrm{L}$ & 59 & 5.9 & 0.56 & 1.1 & 0.77 & 0.93 & 1.6 & 0.69 \\
\hline Phosphorus, dissolved & $\mathrm{mg} / \mathrm{L}$ & 50 & 0.91 & 0.21 & 0.50 & 0.52 & 0.13 & 0.64 & 0.34 \\
\hline Fecal coliform & $\mathrm{col} / 100 \mathrm{ml}$ & 46 & 345,000 & 10 & $\ldots$ & 6,800 & - & 81,000 & 120 \\
\hline Fecal streptococci & $\mathrm{col} / 100 \mathrm{ml}$ & 56 & 145,000 & 36 & -....... & 2,900 & - & $-43,000$ & 148 \\
\hline Oxygen, dissolved & $\mathrm{mg} / \mathrm{L}$ & 57 & 15.0 & 0.0 & 8.9 & 8.4 & 2.6 & 12.3 & 6.4 \\
\hline $\begin{array}{l}\text { Biochemical oxygen demand } \\
\text { (5-day) }\end{array}$ & $\mathrm{mg} / \mathrm{L}$ & 56 & 35.0 & 1.9 & 11.8 & 10.4 & 6.6 & 21.0 & 3.8 \\
\hline $\mathrm{pH}$ & $\mathrm{pH}$ units & 81 & 8.4 & 6.2 & 7.5 & 7.6 & 0.4 & 8.0 & 6.9 \\
\hline $\mathrm{Co}_{0}-\mathrm{Pt}$ & Co - Pt units & 25 & 500 & 0 & 152 & 38 & 188 & 450 & 5 \\
\hline
\end{tabular}


Results of regression analyses relating concentrations of selected chemical constituents to specific conductance (SpC)

\begin{tabular}{|c|c|c|c|c|c|}
\hline $\begin{array}{c}\text { Dissolved } \\
\text { constituents } \\
(\mathrm{mg} / \mathrm{L})\end{array}$ & $\begin{array}{l}\text { No. of } \\
\text { measure- } \\
\text { ments }\end{array}$ & Regression equation & $\begin{array}{l}\text { Corre- } \\
\text { lation } \\
\text { coeffi- } \\
\text { cient }\end{array}$ & $\begin{array}{l}\text { Percent } \\
\text { explained } \\
\text { variance }\end{array}$ & $\begin{array}{c}\text { Standard } \\
\text { error of } \\
\text { estimate } \\
(\mathrm{mg} / \mathrm{L})\end{array}$ \\
\hline Dissolved solids, residue (RDS) & 60 & $\mathrm{RDS}=0.535(\mathrm{SpC})+57.424$ & 0.92 & 85 & 39 \\
\hline Dissolved solids, sum (SDS) & 19 & $\mathrm{SDS}=0.588(\mathrm{SpC})+14.242$ & .99 & 99 & 15 \\
\hline Hardness as $\mathrm{CaCO}_{3}(\mathrm{TH})$ & 39 & $\mathrm{TH}=0.422(\mathrm{SpC})-16.319$ & .99 & 98 & 10 \\
\hline Calcium (Ca) & 39 & $\mathrm{Ca}=0.129(\mathrm{SpC})-5.411$ & .99 & 98 & 3.5 \\
\hline Magnesium (Mg) & 39 & $\mathrm{Mg}=0.025(\mathrm{SpC})-0.739$ & .96 & 92 & 1.4 \\
\hline Sodium (Na) & 39 & $\mathrm{Na}=0.061(\mathrm{SpC})-2.580$ & .98 & 95 & 2.7 \\
\hline Potassium (K) & 38 & $\mathrm{~K}=-0.002(\mathrm{SpC})+10.376$ & $*-.21$ & 4.6 & 1.7 \\
\hline Bicarbonate $\left(\mathrm{HCO}_{3}\right)$ & 39 & $\mathrm{HCO}_{3}=0.495(\mathrm{SpC})-17.069$ & .99 & 97 & 16 \\
\hline Sulfate $\left(\mathrm{SO}_{4}\right)$ & 39 & $\mathrm{SO}_{4}=0.093(\mathrm{SpC})-0.040$ & .96 & 92 & 5.6 \\
\hline Chloride (C1) & 78 & $\mathrm{CI}=0.034(\mathrm{SpC})-0.648$ & .81 & 65 & 4.7 \\
\hline Silica (Si) & 39 & $\mathrm{Si}=0.030(\mathrm{SpC})+7.432$ & .85 & 73 & 3.6 \\
\hline Nitrite + Nitrate as $\mathrm{N}\left(\mathrm{NO}_{2}-\mathrm{NO}_{3}\right)$ & 32 & $\mathrm{NO}_{2}-\mathrm{NO}_{3}=-0.001(\mathrm{SpC})+2.321$ & -.37 & 13 & 0.72 \\
\hline
\end{tabular}

* Not significant at the 95 percent confidence level; the regression equation should not be used to predict the concentration of the dependent variable.

Results of regression analyses relating specific conductance to water discharge (Q)

\begin{tabular}{lllccc}
\hline $\begin{array}{l}\text { No. of } \\
\text { measure- } \\
\text { ments }\end{array}$ & $\begin{array}{c}\text { Water discharge } \\
\text { Maximum Minimum }\end{array}$ & Regression equation & $\begin{array}{c}\text { Correlation } \\
\text { coefficient }\end{array}$ & $\begin{array}{c}\text { Percent } \\
\text { explained } \\
\text { variance }\end{array}$ & $\begin{array}{c}\text { Standard error } \\
\text { of estimate }\end{array}$ \\
\hline 81 & $19,900 \quad 11$ & $\mathrm{SpC}=2,975 \mathrm{Q}^{-0.36769}$ & -0.87 & 75 & 0.130 \\
\hline
\end{tabular}


BIG BLUE RIVER BASIN

06881200 Turkey Creek near Wilber, Nebr.

Location.--Lat $40^{\circ} 28^{\prime} 48^{\prime \prime}$, long $97^{\circ} 00^{\prime} 43^{\prime \prime}$, in $\mathrm{NE}^{\frac{1}{4}} \mathrm{NE}^{\frac{1}{4}} \mathrm{sec}$. 19, T.6 N., R.4 E., Saline County, Hydrologic Unit 10270204, on left bank near downstream side of bridge on State Highway 41, $2.8 \mathrm{mi}$ west of Wilber.

Drainage area. $--460 \mathrm{mi}^{2}$.

Period of record.--Water years 1966-70, 1973 to current year.

Statistical data for selected chemical constituents

\begin{tabular}{|c|c|c|c|c|c|c|c|c|c|}
\hline Constituent & Units & $\begin{array}{l}\text { No. of } \\
\text { measure- } \\
\text { ments }\end{array}$ & $\begin{array}{l}\text { Max- } \\
\text { imum }\end{array}$ & $\begin{array}{l}\text { Min- } \\
\text { imum }\end{array}$ & Mean & Median & $\begin{array}{c}\begin{array}{c}\text { Standard } \\
\text { devi- } \\
\text { ation }\end{array} \\
\end{array}$ & $\begin{array}{l}\text { Ninetieth } \\
\text { percent- } \\
\text { ile }\end{array}$ & $\begin{array}{l}\text { Tenth } \\
\text { percent- } \\
\text { ile }\end{array}$ \\
\hline Specific conductance & umho/ $\mathrm{cm}$ & 134 & 1,890 & 73 & 536 & 529 & 304 & 738 & 180 \\
\hline Dissolved solids, residue & $\mathrm{mg} / \mathrm{L}$ & 113 & 1,060 & 52 & 329 & 329 & 162 & 450 & 130 \\
\hline Dissolved solids, sum & $\mathrm{mg} / \mathrm{L}$ & 58 & 989 & 63 & 305 & 308 & 140 & 430 & 130 \\
\hline Hardness as $\mathrm{CaCO}_{3}$ & $\mathrm{mg} / \mathrm{I}$ & 93 & 280 & 21 & 165 & 187 & 68 & 229 & 47 \\
\hline Calcium, dissolved & $\mathrm{mg} / \mathrm{L}$ & 93 & 87 & 5.2 & 49 & 54 & 21 & 71 & 12 \\
\hline Magnesium, dissolved & $\mathrm{mg} / \mathrm{L}$ & 93 & 16 & 1.4 & 10 & 12 & 3.7 & 14 & 3.2 \\
\hline Sodium, dissolved & $\mathrm{mg} / \mathrm{L}$ & 94 & 300 & 2.1 & 36 & 32 & 36 & 46 & 6.1 \\
\hline Potassium, dissolved & $\mathrm{mg} / \mathrm{L}$ & 93 & 15 & 2.8 & 8.2 & 7.9 & 2.8 & 12 & 4.8 \\
\hline Bicarbonate ion & $\mathrm{mg} / \mathrm{L}$ & 94 & 326 & 24 & 196 & 218 & 81 & 273 & 48 \\
\hline Sulfate, dissolved & $\mathrm{mg} / \mathrm{L}$ & 94 & 70 & 6.8 & 41 & 44 & 15 & 56 & 15 \\
\hline Chloride, dissolved & $\mathrm{mg} / \mathrm{L}$ & 130 & 430 & 1.6 & 44 & 26 & 73 & 68 & 8.3 \\
\hline Fluoride, dissolved & $\mathrm{mg} / \mathrm{L}$ & 94 & 0.6 & 0.2 & 0.4 & 0.4 & 0.1 & 0.5 & 0.3 \\
\hline Silica, dissolved & $\mathrm{mg} / \mathrm{L}$ & 93 & 33 & 3.9 & 21 & 21 & 5.6 & 28 & 14 \\
\hline Boron, dissolved & $\mathrm{mg} / \mathrm{L}$ & 93 & 250 & 7 & 61 & 56 & 34 & 94 & 28 \\
\hline Iron, dissolved & $\mathrm{ug} / \mathrm{L}$ & 73 & 360 & 0 & 78 & 46 & 83 & 200 & 16 \\
\hline Manganese, dissolved & $\mathrm{ug} / \mathrm{L}$ & 57 & 440 & 0 & 114 & 78 & 98 & 235 & 16 \\
\hline $\begin{array}{l}\text { Nitrite + nitrate as } \mathrm{N} \text {, } \\
\text { dissolved }\end{array}$ & $\mathrm{mg} / \mathrm{L}$ & 28 & 2.1 & 0.06 & 0.80 & 0.70 & 0.52 & 1.7 & 0.12 \\
\hline Nitrate as $\mathrm{N}$, dissolved & $\mathrm{mg} / \mathrm{L}$ & 23 & 3.6 & 0.00 & 0.79 & 0.50 & 0.98 & 2.0 & 0.00 \\
\hline Nitrate as $\mathrm{NO}_{3}$, dissolved & $\mathrm{mg} / \mathrm{L}$ & & & & & 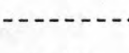 & & 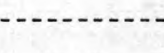 & 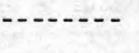 \\
\hline Anmonia $\mathrm{N}$, total & $\mathrm{mg} / \mathrm{L}$ & 56 & 2.5 & 0.00 & 0.33 & 0.16 & 0.47 & 0.78 & 0.03 \\
\hline Organic N, total & $\mathrm{mg} / \mathrm{L}$ & 56 & 8.0 & 0.15 & 1.3 & 1.1 & 1.2 & 2.3 & 0.40 \\
\hline Phosphorus, total & $\mathrm{mg} / \mathrm{L}$ & 56 & 1.9 & 0.21 & 0.61 & 0.57 & 0.30 & 0.94 & 0.30 \\
\hline Phosphorus, dissolved & $\mathrm{mg} / \mathrm{L}$ & 63 & 1.2 & 0.00 & 0.38 & 0.37 & 0.16 & 0.50 & 0.23 \\
\hline Fecal coliform & $\mathrm{col} / 100 \mathrm{ml}$ & 44 & 30,000 & 3 & - & 488 & $-\cdots+-$ & 3,400 & 12 \\
\hline Fecal streptococci & $\mathrm{col} / 100 \mathrm{ml}$ & 52 & 130,000 & 8 & -1--- & 1,140 & 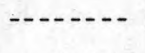 & 10,200 & 24 \\
\hline Oxygen, dissolved & $\mathrm{mg} / \mathrm{L}$ & 53 & 15.3 & 3.0 & 9.1 & 8.5 & 2.6 & 12.7 & 6.3 \\
\hline \multirow{2}{*}{$\begin{array}{l}\text { Biochemical oxygen demand } \\
\text { (5-day) }\end{array}$} & $\mathrm{mg} / \mathrm{L}$ & 54 & 44.0 & 0.3 & 5.1 & 4.0 & 5.8 & 7.9 & 2.0 \\
\hline & H units & 136 & 8.5 & 6.4 & 7.6 & 7.7 & 0.4 & 8.2 & 7.0 \\
\hline $\mathrm{Co}-\mathrm{Pt}$ & $t$ units & 48 & 500 & 1 & 49 & 20 & 99 & 82 & 4 \\
\hline
\end{tabular}


BIG BLUE RIVER BASIN

06881200 Turkey Creek near Wilber, Nebr.--Continued

Results of regression analyses relating concentrations of selected chemical constituents to specific conductance $(\mathrm{SpC})$

\begin{tabular}{|c|c|c|c|c|c|}
\hline $\begin{array}{l}\text { Dissolved } \\
\text { constituents } \\
(\mathrm{mg} / \mathrm{L})\end{array}$ & $\begin{array}{l}\text { No. of } \\
\text { measure- } \\
\text { ments }\end{array}$ & Regression equation & $\begin{array}{l}\text { Corre- } \\
\text { lation } \\
\text { coeffi- } \\
\text { cient }\end{array}$ & $\begin{array}{l}\text { Percent } \\
\text { explained } \\
\text { variance }\end{array}$ & $\begin{array}{c}\text { Standard } \\
\text { error of } \\
\text { estimate } \\
(\mathrm{mg} / \mathrm{L})\end{array}$ \\
\hline Dissolved solids, residue (RDS) & 111 & $\mathrm{RDS}=0.544(\mathrm{SpC})+42.624$ & 0.99 & 98 & 21 \\
\hline Dissolved solids, sum (SDS) & 56 & $\mathrm{SDS}=0.535(\mathrm{SpC})+37.458$ & .99 & 98 & 19 \\
\hline Hardness as $\mathrm{CaCO}_{3}(\mathrm{TH})$ & 93 & $\mathrm{TH}=0.198(\mathrm{SpC})+67.857$ & .71 & 50 & 48 \\
\hline Calcium (Ca) & 92 & $\mathrm{Ca}=0.059(\mathrm{SpC})+20.247$ & .68 & 46 & 16 \\
\hline Magnesium (Mg) & 92 & $\mathrm{Mg}=0.012(\mathrm{SpC})+4.198$ & .78 & 61 & 2.3 \\
\hline Sodium $(\mathrm{Na})$ & 93 & $\mathrm{Na}=0.136(\mathrm{SpC})-30.838$ & .91 & 83 & 15 \\
\hline Potassium $(\mathrm{K})$ & 92 & $K=-0.004(\mathrm{SpC})+9.965$ & -.30 & 9.4 & 2.7 \\
\hline Bicarbonate $\left(\mathrm{HCO}_{3}\right)$ & 94 & $\mathrm{HCO}_{3}=0.246(\mathrm{SpC})+75.734$ & .73 & 54 & 55 \\
\hline Sulfate $\left(\mathrm{SO}_{4}\right)$ & 93 & $\mathrm{SO}_{4}=0.050(\mathrm{SpC})+16.639$ & .81 & 66 & 8.8 \\
\hline Chloride $(\mathrm{Cl})$ & 128 & $\mathrm{Cl}=0.215(\mathrm{SpC})-72.270$ & .90 & 81 & 32 \\
\hline Silica (Si) & 92 & $\mathrm{Si}=0.013(\mathrm{SpC})+14.590$ & .55 & 30 & 4.7 \\
\hline Nitrite + Nitrate as $\mathrm{N}\left(\mathrm{NO}_{2}-\mathrm{NO}_{3}\right)$ & 28 & $\mathrm{NO}_{2}-\mathrm{NO}_{3}=-0.001(\mathrm{SpC})+1.491$ & -.56 & 31 & 0.44 \\
\hline
\end{tabular}

Results of regression analyses relating specific conductance to water discharge (Q)

\begin{tabular}{|c|c|c|c|c|c|c|c|}
\hline \multirow{2}{*}{$\begin{array}{l}\text { No. of } \\
\text { measure- } \\
\text { ments }\end{array}$} & \multicolumn{2}{|c|}{$\begin{array}{l}\text { Water discharge } \\
\left(\mathrm{ft}^{3} / \mathrm{s}\right)\end{array}$} & \multirow{2}{*}{ Regression equation } & \multirow{2}{*}{$\begin{array}{l}\text { Correlation } \\
\text { coefficient }\end{array}$} & \multirow{2}{*}{$\begin{array}{c}\text { Percent } \\
\text { explained } \\
\text { variance }\end{array}$} & \multicolumn{2}{|c|}{$\begin{array}{c}\text { Standard error } \\
\text { of estimate }\end{array}$} \\
\hline & Maximum & Minimum & & & & Log units & Percent \\
\hline 133 & 4,070 & 0.10 & $\mathrm{SpC}=1,080 \mathrm{Q}^{-0.30393}$ & -0.88 & 77 & 0.125 & 29 \\
\hline
\end{tabular}


06881502 Big Blue River below Beatrice, Nebr.

Location.--Lat $40^{\circ} 14^{\prime} 55^{\prime \prime}$, long 96 $42^{\prime} 46^{\prime \prime}$, in SE$\frac{1}{4} \mathrm{SE}^{\frac{1}{4}} \mathrm{Sec} .2$, T.3 N., R.6 E., Gage County, Hydrologic Unit 10270202, at pipeline bridge about $2 \mathrm{mi}$ downstream from bridge on U.S. Highway $77,1.3 \mathrm{mi}$ southeast of Beatrice.

Period of record. - -Water year 1973 to current year.

Statistical data for selected chemical constituents

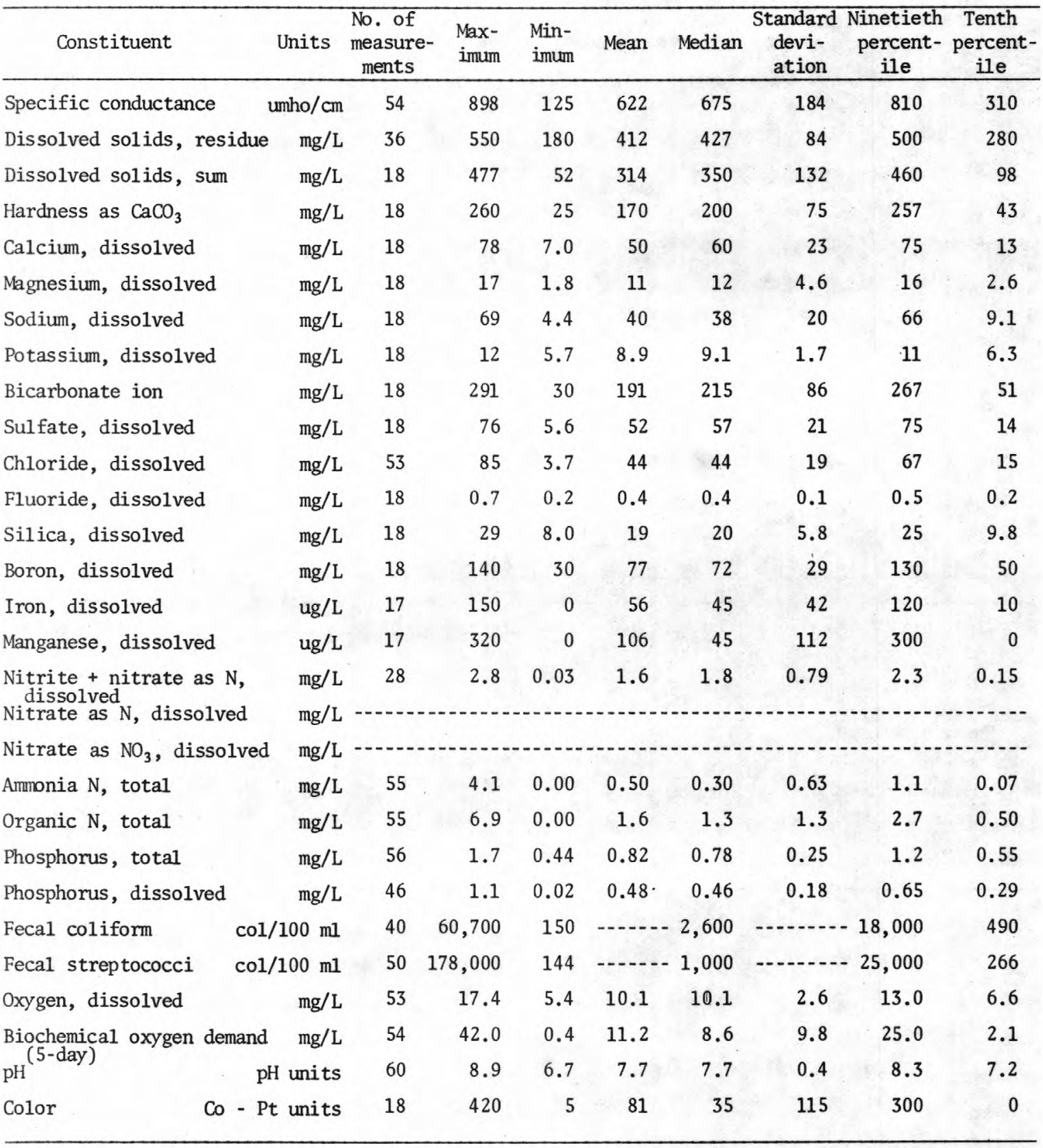


Results of regression analyses relating concentrations of selected chemical constituents to specific conductance (SpC)

\begin{tabular}{|c|c|c|c|c|c|}
\hline $\begin{array}{l}\text { Dissolved } \\
\text { constituents } \\
(\mathrm{mg} / \mathrm{L})\end{array}$ & $\begin{array}{l}\text { No. of } \\
\text { measure- } \\
\text { ments }\end{array}$ & Regression equation & $\begin{array}{l}\text { Corre- } \\
\text { lation } \\
\text { coeffi- } \\
\text { cient }\end{array}$ & $\begin{array}{l}\text { Percent } \\
\text { explained } \\
\text { variance }\end{array}$ & $\begin{array}{c}\text { Standard } \\
\text { error of } \\
\text { estimate } \\
(\mathrm{mg} / \mathrm{L})\end{array}$ \\
\hline Dissolved solids, residue (RDS) & 35 & $\mathrm{RDS}=0.545(\mathrm{SpC})+51.018$ & 0.99 & 98 & 13 \\
\hline Dissolved solids, sum (SDS) & 18 & $\mathrm{SDS}=0.611(\mathrm{SpC})-4.538$ & 1.0 & 99 & 11 \\
\hline Hardness as $\mathrm{CaCO}_{3}(\mathrm{TH})$ & 18 & $\mathrm{TH}=0.344(\mathrm{SpC})-9.769$ & .99 & 98 & 12 \\
\hline Calcium (Ca) & 18 & $\mathrm{Ca}=0.103(\mathrm{SpC})-3.557$ & .99 & 97 & 3.8 \\
\hline Magnesium (Mg) & 18 & $\mathrm{Mg}=0.020(\mathrm{SpC})-0.032$ & .97 & 94 & 1.2 \\
\hline Sodium $(\mathrm{Na})$ & 18 & $\mathrm{Na}=0.092(\mathrm{SpC})-8.123$ & .98 & 96 & 4.2 \\
\hline Potassium (K) & 18 & $\mathrm{~K}=0.001(\mathrm{SpC})+8.382$ & $* .13$ & 1.6 & 1.7 \\
\hline Bicarbonate $\left(\mathrm{HCO}_{3}\right)$ & 18 & $\mathrm{HCO}_{3}=0.390(\mathrm{SpC})-12.291$ & .98 & 96 & 16 \\
\hline Sulfate $\left(\mathrm{SO}_{4}\right)$ & 18 & $\mathrm{SO}_{4} 0.095(\mathrm{SpC})+1.830$ & .96 & 92 & 6.1 \\
\hline Chloride (C1) & 52 & $\mathrm{Cl}=0.090(\mathrm{SpC})-12.493$ & .84 & 71 & 10 \\
\hline Silica (Si) & 18 & $\mathrm{Si}=0.023(\mathrm{SpC})+6.553$ & .87 & 76 & 3.0 \\
\hline Nitrite + Nitrate as $\mathrm{N}\left(\mathrm{NO}_{2}-\mathrm{NO}_{3}\right)$ & 28 & $\mathrm{NO}_{2}-\mathrm{NO}_{3}=0.0001(\mathrm{SpC})+1.544$ & $* .02$ & 0.05 & 0.81 \\
\hline
\end{tabular}

* Not significant at the 95 percent confidence level; the regression equation should not be used to predict the concentration of the dependent variable.

Results of regression analyses relating specific conductance to water discharge (Q)

\begin{tabular}{ccccccc}
\hline $\begin{array}{c}\text { No. of } \\
\text { measure- } \\
\text { ments }\end{array}$ & $\begin{array}{c}\text { Water discharge } \\
\text { Maximum Minimum }\end{array}$ & Regression equation & $\begin{array}{c}\text { Correlation } \\
\text { coefficient }\end{array}$ & $\begin{array}{c}\text { Percent } \\
\text { explained } \\
\text { variance }\end{array}$ & $\begin{array}{c}\text { Standard error } \\
\text { of estimate }\end{array}$ \\
\hline 54 & 7,260 & 25.7 & $\mathrm{SpC}=3,214 \mathrm{Q}^{-0.31326}$ & -0.82 & 67 & 0.105 \\
\hline
\end{tabular}




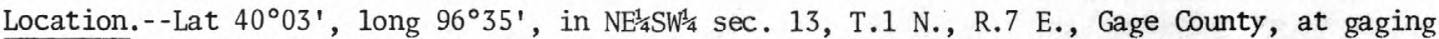
station in tailrace of powerplant, 0.8 mi northwest of Barneston, 2 mi upstream from Plum Creek, and $5 \mathrm{mi}$ upstream from Nebraska-Kansas State line.

Drainage area.- $-4,444 \mathrm{mi}^{2}$.

Period of record.--October 1966 to September 1968.

Statistical data for selected chemical constituents

\begin{tabular}{|c|c|c|c|c|c|c|c|c|c|}
\hline Constituent & Units & $\begin{array}{l}\text { No. of } \\
\text { measure- } \\
\text { ments }\end{array}$ & $\begin{array}{l}\text { Max- } \\
\text { imum }\end{array}$ & $\begin{array}{l}\text { Min- } \\
\text { imum }\end{array}$ & Mean & Median & $\begin{array}{c}\text { Standard } \\
\text { devi- } \\
\text { ation }\end{array}$ & $\begin{array}{l}\text { Ninetieth } \\
\text { percent- } \\
\text { ile }\end{array}$ & $\begin{array}{l}\text { Tenth } \\
\text { percent- } \\
\text { ile }\end{array}$ \\
\hline Specific conductance & umho/cm & 44 & 737 & 89 & 538 & 595 & 173 & 690 & 210 \\
\hline Dissolved solids, residue & $\mathrm{mg} / \mathrm{L}$ & 41 & 468 & 69 & 338 & 370 & 103 & 432 & 150 \\
\hline Dissolved solids, sum & $\mathrm{mg} / \mathrm{L}$ & 16 & 441 & 100 & 325 & 370 & 103 & 410 & 126 \\
\hline Hardness as $\mathrm{CaCO}_{3}$ & $\mathrm{mg} / \mathrm{L}$ & 41 & 254 & 28 & 182 & 196 & 61 & 238 & 74 \\
\hline Calcium, dissolved & $\mathrm{mg} / \mathrm{L}$ & 41 & 78 & 7.0 & 54 & 58 & 19 & 72 & 22 \\
\hline Magnesium, dissolved & $\mathrm{mg} / \mathrm{L}$ & 41 & 17 & 2.4 & 12 & 13 & 3.8 & 15 & 4.7 \\
\hline Sodium, dissolved & $\mathrm{mg} / \mathrm{L}$ & 41 & 57 & 1.6 & 40 & 46 & 16 & 56 & 13 \\
\hline Potassium, dissolved & $\mathrm{mg} / \mathrm{L}$ & 41 & 18 & 6.2 & 8.7 & 8.0 & 2.6 & 11 & 8.5 \\
\hline Bicarbonate ion & $\mathrm{mg} / \mathrm{L}$ & 41 & 292 & 32 & 212 & 240 & 73 & 277 & 76 \\
\hline Sulfate, dissolved & $\mathrm{mg} / \mathrm{L}$ & 41 & 68 & 5.2 & 48 & 53 & 16 & 64 & 21 \\
\hline Chloride, dissolved & $\mathrm{mg} / \mathrm{L}$ & 41 & 53 & 1.2 & 34 & 37 & 14 & 49 & 7.9 \\
\hline Fluoride, dissolved & $\mathrm{mg} / \mathrm{L}$ & 41 & 0.5 & 0.2 & 0.4 & 0.4 & 0.1 & 0.4 & 0.3 \\
\hline Silica, dissolved & $\mathrm{mg} / \mathrm{L}$ & 41 & 29 & 4.7 & 19 & 20 & 5.9 & 26 & 11 \\
\hline Boron, dissolved & $\mathrm{mg} / \mathrm{L}$ & 41 & 170 & 20 & 69 & 69 & 26 & 93 & 40 \\
\hline Iron, dissolved & $\mathrm{ug} / \mathrm{L}$ & 14 & 510 & 0 & 81 & 19 & 150 & 320 & 0 \\
\hline Manganese, dissolved & $\mathrm{ug} / \mathrm{L}$ & 14 & 490 & 0 & 122 & 110 & 128 & 197 & 0 \\
\hline $\begin{array}{l}\text { Nitrite + nitrate as } N \text {, } \\
\text { dissolved } \\
\text { Nitrate as } N \text {, dissolved }\end{array}$ & $\begin{array}{l}\mathrm{mg} / \mathrm{L} \\
\mathrm{mg} / \mathrm{L}\end{array}$ & 3 & 1.7 & 0.50 & 0.93 & ----1 & 0.66 & & -- \\
\hline Nitrate as $\mathrm{NO}_{3}$, dissolved & $\mathrm{mg} / \mathrm{L}$ & 41 & 26 & 0.10 & 8.3 & 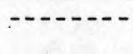 & 5.4 & & \\
\hline Ammonia $\mathrm{N}$, total & $\mathrm{mg} / \mathrm{L}$ & $-\cdots--$ & -1 & $-\ldots$ & $\ldots$ & & & & -- \\
\hline Organic $N$, total & $\mathrm{mg} / \mathrm{L}$ & & $\ldots$ & & $\ldots$ & & & & \\
\hline Phosphorus, total & $\mathrm{mg} / \mathrm{L}$ & & -- & -- & -- & 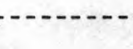 & & & -- \\
\hline Phosphorus, dissolved & $\mathrm{mg} / \mathrm{L}$ & 1 & 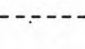 & 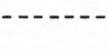 & 0.40 & & & & \\
\hline Fecal coliform & $1 / 100 \mathrm{~m} 1$ & & & & & & & & (n) \\
\hline $\begin{array}{l}\text { Fecal streptococci } \\
\text { Oxygen, dissolved }\end{array}$ & $\begin{array}{r}\mathrm{mg} / \mathrm{L}\end{array}$ & 4 & 4.0 & 2.6 & 3.2 & & 0.6 & & -1 \\
\hline $\begin{array}{l}\text { Biochemical oxygen demand } \\
\text { (5H }\end{array}$ & $\mathrm{mg} / \mathrm{L}$ & 44 & 8.4 & 6.1 & 7.6 & 7.7 & 0.4 & 8.1 & 7.0 \\
\hline $\mathrm{Co}-\mathrm{P}$ & t units & 26 & 70 & 1 & 12 & 7.5 & 15 & 37 & 4.5 \\
\hline
\end{tabular}


Results of regression analyses relating concentrations of selected chemical constituents to specific conductance (SpC)

\begin{tabular}{|c|c|c|c|c|c|}
\hline $\begin{array}{c}\text { Dissolved } \\
\text { constituents } \\
\text { (mg/L) }\end{array}$ & $\begin{array}{l}\text { No. of } \\
\text { measure- } \\
\text { ments }\end{array}$ & Regression equation & $\begin{array}{l}\text { Corre- } \\
\text { lation } \\
\text { coeffi- } \\
\text { cient } \\
\end{array}$ & $\begin{array}{l}\text { Percent } \\
\text { explained } \\
\text { variance }\end{array}$ & $\begin{array}{c}\text { Standard } \\
\text { error of } \\
\text { estimate } \\
(\mathrm{mg} / \mathrm{L})\end{array}$ \\
\hline Dissolved solids, residue (RDS) & 41 & $\mathrm{RDS}=0.580(\mathrm{SpC})+22.540$ & 1.0 & 99 & 10 \\
\hline Dissolved solids, sum (SDS) & 16 & $\mathrm{SDS}=0.587(\mathrm{SpC})+12.886$ & .99 & 99 & 11 \\
\hline Hardness as $\mathrm{CaCO}_{3}(\mathrm{TH})$ & 41 & $\mathrm{TH}=0.344(\mathrm{SpC})-4.757$ & .99 & 98 & 8.6 \\
\hline Calcium (Ca) & 41 & $\mathrm{Ca}=0.104(\mathrm{SpC})-2.727$ & .98 & 97 & 3.3 \\
\hline Magnesium (Mg) & 41 & $\mathrm{Mg}=0.020(\mathrm{SpC})+0.473$ & .96 & 92 & 1.1 \\
\hline Sodium $(\mathrm{Na})$ & 41 & $\mathrm{Na}=0.089(\mathrm{SpC})-7.708$ & .98 & 97 & 2.7 \\
\hline Potassium (K) & 41 & $K=-0.007(S p C)+12.471$ & -.48 & 23 & 2.3 \\
\hline Bicarbonate $\left(\mathrm{HCO}_{3}\right)$ & 41 & $\mathrm{HCO}_{3}=0.406(\mathrm{SpC})-8.050$ & .99 & 98 & 11 \\
\hline Sulfate $\left(\mathrm{SO}_{4}\right)$ & 41 & $\mathrm{SO}_{4} 0.089(\mathrm{SpC})+0.312$ & .98 & 96 & 3.4 \\
\hline Chloride (C1) & 41 & $\mathrm{Cl}=0.075(\mathrm{SpC})-7.244$ & .95 & 90 & 4.6 \\
\hline Silica (Si) & 41 & $\mathrm{Si}=0.015(\mathrm{SpC})+10.955$ & .46 & 21 & 5.3 \\
\hline
\end{tabular}

Results of regression analyses relating specific conductance to water discharge (Q)

\begin{tabular}{ccccccc}
\hline $\begin{array}{c}\text { No. of } \\
\text { measure- } \\
\text { ments }\end{array}$ & $\begin{array}{c}\text { Water discharge } \\
\text { Maximum Minimum }\end{array}$ & Regression equation & $\begin{array}{c}\text { Correlation } \\
\text { coefficient }\end{array}$ & $\begin{array}{c}\text { Percent } \\
\text { explained } \\
\text { variance }\end{array}$ & $\begin{array}{c}\text { Standard error } \\
\text { of estimate }\end{array}$ \\
\hline 44 & $26,000 \quad 31$ & $\mathrm{SpC}=1,971 \mathrm{Q}^{-0.27778}$ & -0.86 & 74 & 0.112 \\
\hline
\end{tabular}


The Little Blue River basin is in south-central Nebraska between the Big Blue and Republican River basins. The Little Blue River leaves Nebraska just south of Steele City in Jefferson County.

Most of the basin is composed of flatlands underlain by loesscovered Pleistocene sand and gravel deposits. Topography becomes somewhat rolling in the eastern glaciated part of the basin where loess mantles glacial drift. In places in the eastern part of the basin, bedrock of Cretaceous age outcrops along valley sides. Streamflow is maintained mostly by discharge of ground water supplemented by overland runoff in the upstream part of the basin. Overland runoff comprises most of the streamflow in the eastern part of the basin.

Descriptive statistics were determined for two locations on the Little Blue River. One is at Deweese in the upper part of the basin and the other is at Hollenberg, Kans., just downstream from the NebraskaKansas border (fig. 14). At Deweese, the mean specific conductance is $358 \mathrm{umho} / \mathrm{cm}$. At Hollenberg, the mean conductance is $465 \mathrm{umho} / \mathrm{cm}$, an increase of 30 percent over the mean for Deweese. The mean concentration of sodium increased 92 percent and the mean for chloride increased 350 percent from Deweese to Hollenberg. Seepage of small amounts of more highly mineralized water from bedrock in the lower part of the basin probably accounts for these increased concentrations. Calcium is the principal cation and bicarbonate the principal anion at both stations.

Concentrations of most constituents associated with water pollution are not high at the Hollenberg station. Medians for fecal coliform and fecal streptococcal bacteria are 600 and $290 \mathrm{col} / 100 \mathrm{ml}$, respectively. However, the mean BOD concentration of $10.8 \mathrm{mg} / \mathrm{L}$ indicates some effect of effluent from the Fairbury sewage plant approximately 15 mi upstream.

Results of regression analyses indicate that with the exception of potassium and nitrite + nitrate, correlation coefficients are all above 0.87 for all constituents at both stations. 


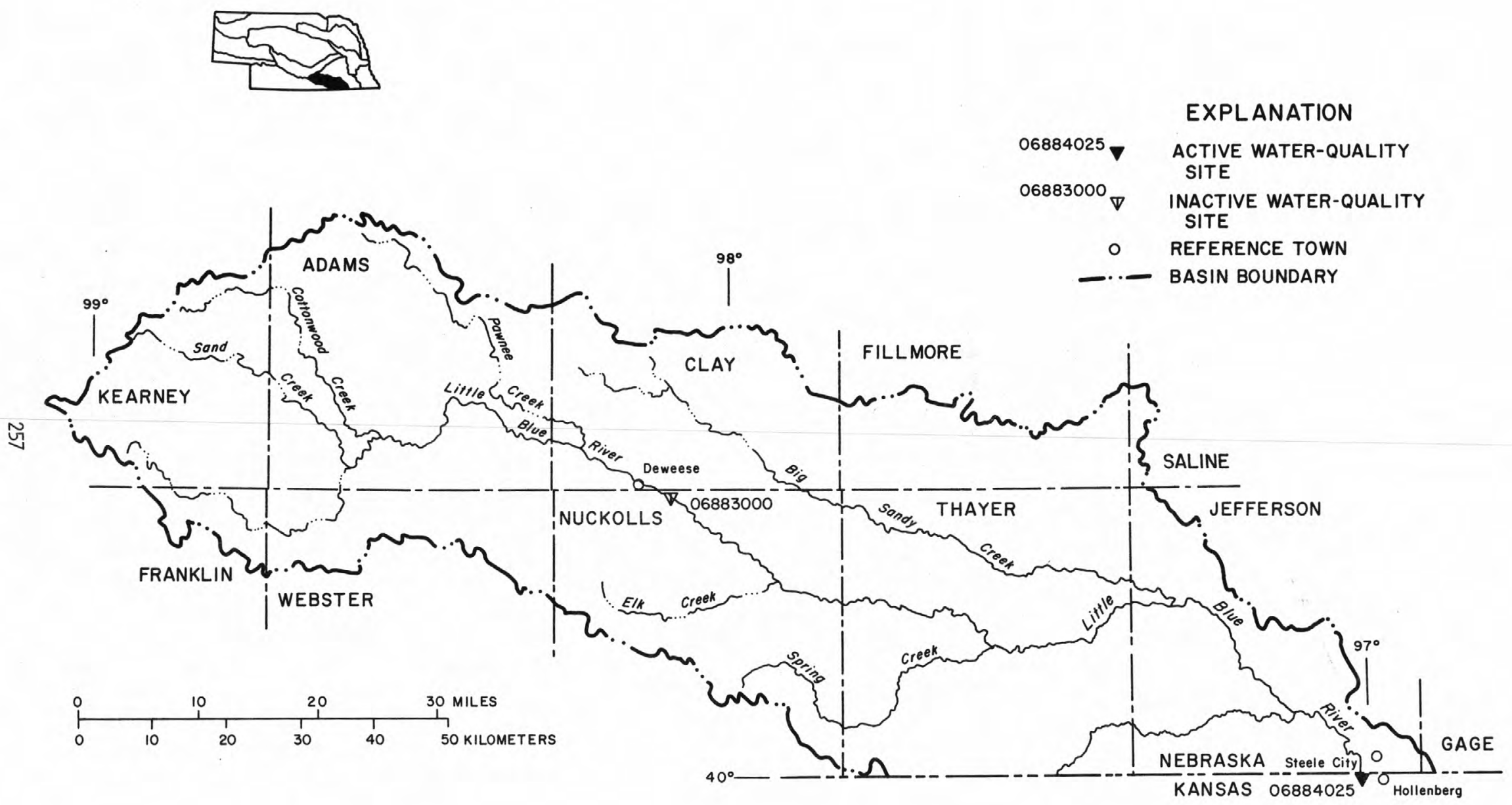

FIGURE 14.-Location of water-quality sampling sites, Little Blue River basin 


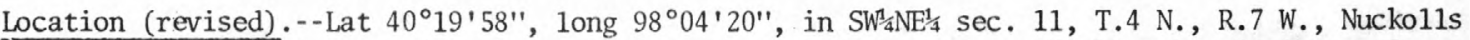
County, at gaging station about 1,500 ft above bridge on State Highway 14, $1 \mathrm{mi}$ upstream from Walnut Creek, $3.2 \mathrm{mi}$ southeast of Deweese, and $6 \mathrm{mi}$ northwest of Angus.

Drainage area. $--979 \mathrm{mi}^{2}$.

Period of record.--August 1956 to September 1958, October 1959 to June 1960, November 1960 to September 1968 .

Statistical data for selected chemical constituents

\begin{tabular}{|c|c|c|c|c|c|c|c|c|}
\hline Constituent & Units & $\begin{array}{l}\text { No. of } \\
\text { measure- } \\
\text { ments }\end{array}$ & $\begin{array}{l}\text { Max- } \\
\text { imum }\end{array}$ & $\begin{array}{l}\text { Min- } \\
\text { imum }\end{array}$ & Mean & Median & $\begin{array}{c}\text { Standard } \\
\text { devi- } \\
\text { ation }\end{array}$ & $\begin{array}{l}\text { Ninetieth Tenth } \\
\text { percent- percent- } \\
\text { ile } \quad \text { ile } \\
\end{array}$ \\
\hline Specific conductance & umho $/ \mathrm{cm}$ & 203 & 758 & 63 & 358 & & 124 & \\
\hline Dissolved solids, residue & $\mathrm{mg} / \mathrm{L}$ & 171 & 312 & 61 & 233 & & 69 & \\
\hline Dissolved solids, sum & $\mathrm{mg} / \mathrm{L}$ & 38 & 294 & 66 & 219 & & 77 & \\
\hline Hardness as $\mathrm{CaCO}_{3}$ & $\mathrm{mg} / \mathrm{L}$ & 175 & 197 & 21 & 148 & & 57 & \\
\hline Calcium, dissolved & $\mathrm{mg} / \mathrm{L}$ & 171 & 70 & 6.5 & 47 & & 19 & \\
\hline Magnesium, dissolved & $\mathrm{mg} / \mathrm{L}$ & 171 & 13 & 1.0 & 7.5 & & 2.7 & \\
\hline Sodium, dissolved & $\mathrm{mg} / \mathrm{L}$ & 175 & 19 & 1.1 & 12 & & 5.1 & \\
\hline Potassium, dissolved & $\mathrm{mg} / \mathrm{L}$ & 171 & 13 & 4.3 & 7.6 & & 1.6 & \\
\hline Bicarbonate ion & $\mathrm{mg} / \mathrm{L}$ & 175 & 235 & 27 & 176 & & 67 & \\
\hline Sulfate, dissolved & $\mathrm{mg} / \mathrm{L}$ & 170 & 36 & 3.3 & 27 & & 8.8 & \\
\hline Chloride, dissolved & $\mathrm{mg} / \mathrm{L}$ & 170 & 11 & 0.0 & 6.9 & & 2.9 & \\
\hline Fluoride, dissolved & $\mathrm{mg} / \mathrm{L}$ & 171 & 0.6 & 0.0 & 0.3 & & 0.1 & \\
\hline Silica, dissolved & $\mathrm{mg} / \mathrm{L}$ & 171 & 39 & 9.9 & 28 & & 6.1 & \\
\hline Boron, dissolved & $\mathrm{mg} / \mathrm{L}$ & 171 & 130 & 0 & 38 & & 19 & \\
\hline Iron, dissolved & $\mathrm{ug} / \mathrm{L}$ & 147 & 630 & 0 & 62 & & 99 & \\
\hline Manganese, dissolved & $\mathrm{ug} / \mathrm{L}$ & 70 & 520 & 0 & 63 & 41 & 82 & 160 \\
\hline $\begin{array}{l}\text { Nitrite + nitrate as } \mathrm{N} \text {, } \\
\text { dissolved } \\
\text { Nitrate as } \mathrm{N} \text {, dissolved }\end{array}$ & $\mathrm{mg} / \mathrm{L}$ & 22 & 1.3 & 0.0 & 0.39 & -- & 0.37 & \\
\hline Nitrate as $\mathrm{NO}_{3}$, dissolved & $\mathrm{mg} / \mathrm{L}$ & 171 & 6.3 & 0.0 & 1.3 & & 1.3 & \\
\hline Ammonia $\mathrm{N}$, total & $\mathrm{mg} / \mathrm{L}$ & & & & & & & \\
\hline Organic $\mathrm{N}$, total & $\mathrm{mg} / \mathrm{L}$ & & & & & & & \\
\hline Phosphorus, total & $\mathrm{mg} / \mathrm{L}$ & & & & & & & \\
\hline Phosphorus, dissolved & $\mathrm{mg} / \mathrm{L}$ & & & & & & & \\
\hline $\begin{array}{l}\text { Fecal collform } \\
\text { Fecal streptoco }\end{array}$ & $1 / 100 \mathrm{ml}$ & - & & & & & & - \\
\hline Oxygen, dissolved & $\mathrm{mg} / \mathrm{L}$ & $\cdots$ & & & & & & \\
\hline $\begin{array}{l}\text { Biochemical oxygen demand } \\
\mathrm{pH}^{(5-\text { day })}\end{array}$ & $\mathrm{mg} / \mathrm{L}$ & 175 & 8.3 & 6.3 & 7.5 & $-\cdots$ & 0.4 & \\
\hline Co - P & Pt units & 44 & 40 & 0 & 7 & $\ldots \ldots$ & 6.8 & 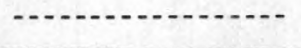 \\
\hline
\end{tabular}


Results of regression analyses relating concentrations of selected chemical constituents to specific conductance $(\mathrm{SpC})$

\begin{tabular}{|c|c|c|c|c|c|}
\hline $\begin{array}{l}\text { Dissolved } \\
\text { constituents } \\
(\mathrm{mg} / \mathrm{L})\end{array}$ & $\begin{array}{l}\text { No. of } \\
\text { measure- } \\
\text { ments }\end{array}$ & Regression equation & $\begin{array}{l}\text { Corre- } \\
\text { lation } \\
\text { coeffi- } \\
\text { cient }\end{array}$ & $\begin{array}{l}\text { Percent } \\
\text { explained } \\
\text { variance }\end{array}$ & $\begin{array}{c}\text { Standard } \\
\text { error of } \\
\text { estimate } \\
(\mathrm{mg} / \mathrm{L})\end{array}$ \\
\hline Dissolved solids, residue (RDS) & 170 & $R D S=0.562(S p C)+35.140$ & 0.99 & 98 & \\
\hline Dissolved solids, sum (SDS) & 37 & $\operatorname{SDS}=0.602(\mathrm{SpC})+14.579$ & 1.0 & 99 & $\ldots \ldots$ \\
\hline Hardness as $\mathrm{CaCO}_{3}(\mathrm{TH})$ & 174 & $\mathrm{TH}=0.462(\mathrm{SpC})-13.727$ & 1.0 & 99 & \\
\hline Calcium (Ca) & 170 & $\mathrm{Ca}=0.152(\mathrm{SpC})-6.188$ & .99 & 98 & \\
\hline Magnesium (Mg) & 170 & $M g=0.020(S p C)+0.341$ & .92 & 85 & -- \\
\hline Sodium (Na) & 174 & $\mathrm{Na}=0.041(\mathrm{SpC})-1.954$ & .98 & 97 & \\
\hline Potassium $(K)$ & 170 & $K=-0.007(S p C)+10.182$ & -.55 & 30 & \\
\hline Bicarbonate $\left(\mathrm{HCO}_{3}\right)$ & 174 & $\mathrm{HCO}_{3}=0.538(\mathrm{SpC})-11.970$ & 1.0 & 99 & \\
\hline Sulfate $\left(\mathrm{SO}_{4}\right)$ & 169 & $\mathrm{SO}_{4}=0.071(\mathrm{SpC})+1.648$ & .97 & 94 & \\
\hline Chloride $(\mathrm{Cl})$ & 170 & $\mathrm{Cl}=0.022(\mathrm{SpC})-0.808$ & .94 & 88 & \\
\hline Silica (Si) & 170 & $\mathrm{Si}=0.044(\mathrm{SpC})+12.168$ & .88 & 77 & -...... \\
\hline
\end{tabular}

Results of regression analyses relating specific conductance to water discharge (Q)

\begin{tabular}{lcccccc}
\hline $\begin{array}{l}\text { No. of } \\
\text { measure- } \\
\text { ments }\end{array}$ & $\begin{array}{c}\text { Water discharge } \\
\left(\mathrm{ft}^{3} / \mathrm{s}\right)\end{array}$ & Maximum Minimum & Regression equation & $\begin{array}{c}\text { Correlation } \\
\text { coefficient }\end{array}$ & $\begin{array}{c}\text { Percent } \\
\text { explained } \\
\text { variance }\end{array}$ & \multicolumn{2}{c}{$\begin{array}{c}\text { Standard error } \\
\text { of estimate }\end{array}$} \\
\hline 191 & 9,860 & 26 & $\mathrm{SpC}=2,179 \mathrm{Q}^{-0.40140}$ & -0.91 & 85 & Log units Percent \\
\hline
\end{tabular}


Location. --Lat $39^{\circ} 58^{\prime} 48^{\prime \prime}$, long $97^{\circ} 00^{\prime} 16^{\prime \prime}, \mathrm{NE}^{\frac{1}{4}} \mathrm{SW}^{\frac{1}{4}} \mathrm{sec} .8$, T.1 S., R.4 E., Washington County, Hydrologic Unit 10270207 on right bank, 2 ft downstream from bridge on county road, $0.6 \mathrm{mi}$ west of Hollenberg, Kans., and $1.75 \mathrm{mi}$ downstream from Nebraska-Kansas State line.

Drainage area. $--2,752 \mathrm{mi}^{2}$.

Period of record. --Water year 1972 to current year.

Statistical data for selected chemical constituents

\begin{tabular}{|c|c|c|c|c|c|c|c|c|c|}
\hline Constituent & Units & $\begin{array}{l}\text { No. of } \\
\text { measure- } \\
\text { ments }\end{array}$ & $\begin{array}{c}\text { Max- } \\
\text { imum }\end{array}$ & $\begin{array}{l}\text { Min- } \\
\text { imum }\end{array}$ & Mean & Median & $\begin{array}{c}\text { Standard } \\
\text { devi- } \\
\text { ation } \\
\end{array}$ & $\begin{array}{l}\text { Ninetieth } \\
\text { percent- } \\
\text { ile }\end{array}$ & $\begin{array}{l}\text { Tenth } \\
\text { percent- } \\
\text { ile }\end{array}$ \\
\hline Specific conductance & umho/cm & 68 & 693 & 110 & 465 & 521 & 180 & 658 & 145 \\
\hline Dissolved solids, residue & $\mathrm{mg} / \mathrm{L}$ & 42 & 439 & 78 & 320 & 352 & 87 & 402 & 190 \\
\hline Dissolved solids, sum & $\mathrm{mg} / \mathrm{L}$ & 21 & 410 & 93 & 247 & 275 & 116 & 370 & 100 \\
\hline Hardness as $\mathrm{CaCO}_{3}$ & $\mathrm{mg} / \mathrm{L}$ & 27 & 270 & 45 & 137 & 150 & 69 & 220 & 53 \\
\hline Calcium, dissolved. & $\mathrm{mg} / \mathrm{L}$ & 27 & 87 & 14 & 43 & 41 & 22 & 71 & 17 \\
\hline Magnesium, dissolved & $\mathrm{mg} / \mathrm{L}$ & 27 & 13 & 1.9 & 7.1 & 7.4 & 3.5 & 12 & 2.6 \\
\hline Sodium, dissolved & $\mathrm{mg} / \mathrm{L}$ & 27 & 49 & 3.8 & 23 & 26 & 15 & 40 & 7.3 \\
\hline Potassium, dissolved & $\mathrm{mg} / \mathrm{L}$ & 27 & 12 & 4.8 & 8.2 & 8.4 & 1.5 & 9.6 & 6.4 \\
\hline Bicarbonate ion & $\mathrm{mg} / \mathrm{L}$ & 21 & 274 & 50 & 165 & 190 & 83 & 250 & 80 \\
\hline Sulfate, dissolved & $\mathrm{mg} / \mathrm{L}$ & 27 & 72 & 9.7 & 31 & 30 & 17 & 48 & 11 \\
\hline Chloride, dissolved & $\mathrm{mg} / \mathrm{L}$ & 62 & 73 & 3.8 & 31 & 36 & 16 & 46 & 6.3 \\
\hline Fluoride, dissolved & $\mathrm{mg} / \mathrm{L}$ & 27 & 1.1 & 0.2 & 0.4 & 0.4 & 0.2 & 0.6 & 0.2 \\
\hline Silica, dissolved & $\mathrm{mg} / \mathrm{L}$ & 21 & 28 & 8.2 & 18 & 19 & 7.3 & 27 & 14 \\
\hline Boron, dissolved & $\mathrm{mg} / \mathrm{L}$ & 21 & 100 & 30 & 60 & 59 & 18 & 90 & 53 \\
\hline Iron, dissolved & $\mathrm{ug} / \mathrm{L}$ & 20 & 240 & 0 & 55 & 40 & 53 & 120 & 24 \\
\hline Manganese, dissolved & $\mathrm{ug} / \mathrm{L}$ & 20 & 130 & 0 & 36 & 22 & 37 & 72 & 0 \\
\hline $\begin{array}{l}\text { Nitrite + nitrate as } \mathrm{N}, \\
\text { dissolved }\end{array}$ & $\mathrm{mg} / \mathrm{L}$ & 38 & 6.9 & 0.02 & 1.4 & 1.3 & 1.1 & 2.4 & 0.27 \\
\hline Nitrate as N, dissolved & $\mathrm{mg} / \mathrm{L}$ & 6 & 6.8 & 0.02 & 2.0 & - & 2.4 & &.-- \\
\hline Nitrate as $\mathrm{NO}_{3}$, dissolved & $\mathrm{mg} / \mathrm{L}$ & & & & & & & & 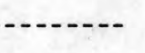 \\
\hline Anmonia $\mathrm{N}$, total & $\mathrm{mg} / \mathrm{L}$ & 61 & 4.7 & 0.00 & 0.38 & 0.19 & 0.68 & 0.66 & 0.05 \\
\hline Organic $N$, total & $\mathrm{mg} / \mathrm{L}$ & 57 & 9.6 & 0.04 & 1.7 & 0.89 & 2.0 & 4.0 & 0.36 \\
\hline Phosphorus, total & $\mathrm{mg} / \mathrm{L}$ & 58 & 5.5 & 0.17 & 0.68 & 0.47 & 0.85 & 1.2 & 0.29 \\
\hline Phosphorus, dissolved & $\mathrm{mg} / \mathrm{L}$ & 49 & 0.79 & 0.15 & 0.28 & 0.29 & 0.10 & 0.37 & 0.22 \\
\hline Fecal coliform & $\mathrm{col} 1 / 100 \mathrm{ml}$ & 48 & 83,000 & 0 & $-\ldots$ & 600 & - & 18,500 & 32 \\
\hline Fecal streptococci & $\operatorname{col} / 100 \mathrm{ml}$ & 59 & 280,000 & 36 & 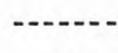 & 290 & - & 20,000 & 67 \\
\hline Oxygen, dissolved & $\mathrm{mg} / \mathrm{L}$ & 63 & 17.8 & 4.3 & 9.7 & 9.6 & 2.6 & 12.9 & 6.7 \\
\hline \multirow{2}{*}{$\begin{array}{l}\text { Biochemical oxygen demand } \\
\text { (5-day) }\end{array}$} & $\mathrm{mg} / \mathrm{L}$ & 61 & 42.0 & 0.6 & 10.8 & 6.6 & 10.7 & 26.0 & 2.1 \\
\hline & H units & 70 & 8.6 & 6.6 & 7.6 & 7.7 & 0.4 & 8.2 & 7.1 \\
\hline $\mathrm{Co}-\mathrm{Pt}$ & Co - Pt units & 20 & 900 & 4 & 144 & 28 & 229 & 520 & 6 \\
\hline
\end{tabular}


Results of regression analyses relating concentrations of selected chemical constituents to specific conductance $(\mathrm{SpC})$

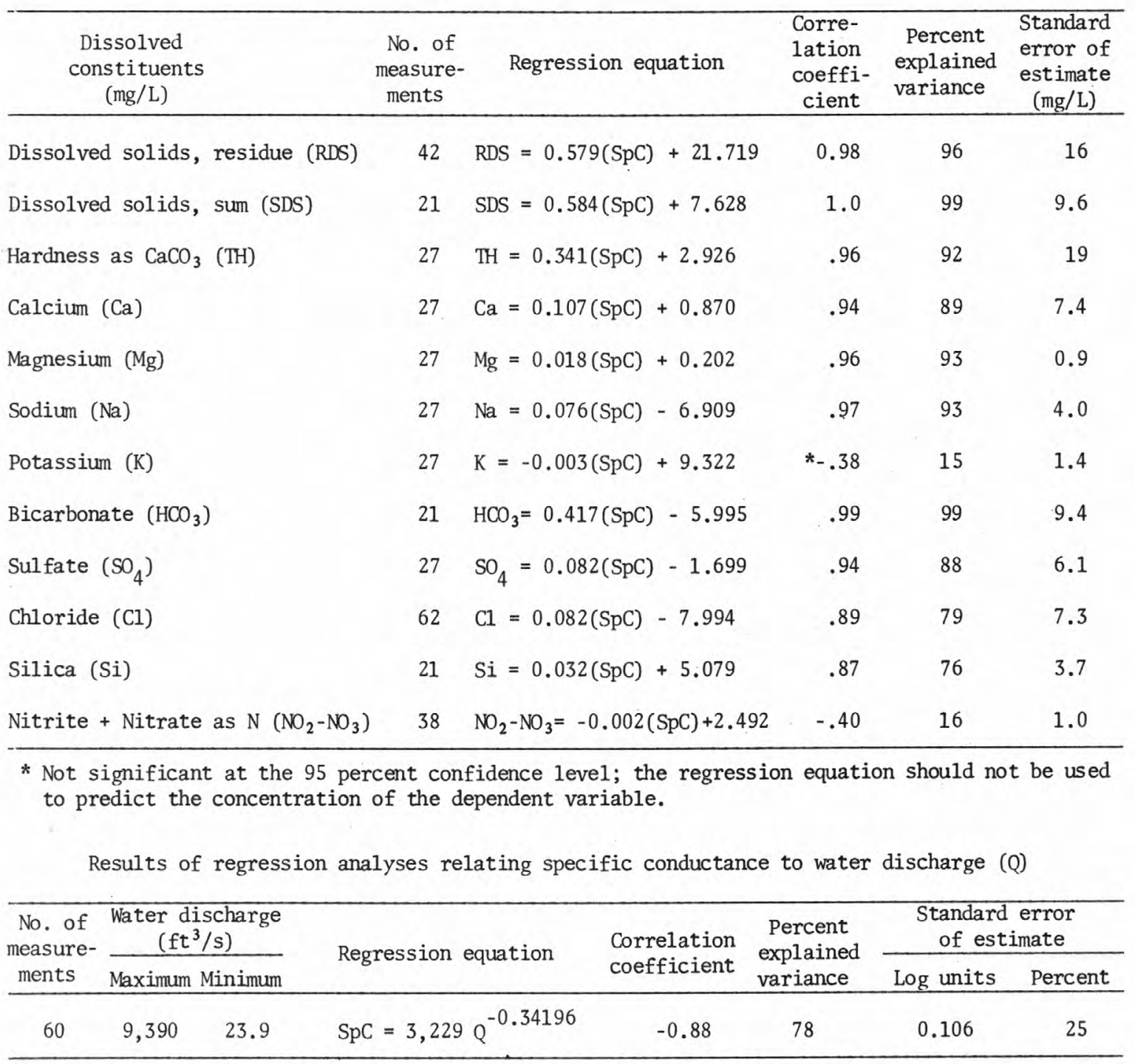


The quality of water at the 109 sampling sites for which statistical data have been presented depends on several factors. One of these is the quality of ground-water seepage, which itself depends on the availability of soluble constituents and the texture of the soils and subsoils in the recharge area of the aquifer from which the water is derived. Water from sites in the upper part of the Loup River basin and the middle part of the Niobrara River basin is derived almost completely from the discharge of ground water from areas where sma11 amounts of soluble constituents occur, and soils are sandy. As a result, the least mineralized streamflow in Nebraska occurs in these areas.

The quality of ground-water seepage also is important in other parts of the State. Even though overland runoff may account for nearly all the discharge in many streams in eastern Nebraska, if the stream flows the year around, base flow usually is maintained by ground-water seepage. In some of these streams, such as several in the Salt Creek basin, this seepage is derived from highly saline aquifers so that at low and medium flows these streams may be highly mineralized.

The second factor on which water quality at the sampling sites depends is the quality of overland runoff. Overland runoff accounts for most of the streamflow in eastern Nebraska. Following storm events, overland runoff usually contains smaller amounts of dissolved-chemical constituents. With the exception of sandhills streams, whose contributing drainage areas are but a small fraction of their total drainage areas, an inverse relationship between specific conductance and water discharge usually exists for all sampling sites; that is, specific conductance decreases as water discharge increases. This relationship may be poorly defined in locations where a high degree of regulation exists in the stream basin (such as the Republican River basin).

A third factor, quality of irrigation return flow, affects water quality in streams. For example, quality of water in the North Platte River basin is affected by return flow of surface water diverted upstream for irrigation use. Increases in dissolved solids, sodium, and sulfate concentrations are evident as a result of concentration by evapotranspiration, leaching of soils, or other processes.

Lastly, effluent from sewage-treatment plants or runoff from large feedlots may affect the quality of streamflow significantly, especially if the amount of effluent is large in proportion to the amount of streamflow. Usually, quality of water at sampling sites below effluent discharges 
is characterized by increases in BOD, chloride, and bacteria and nutrient constituents. Several stations in the Big Blue River basin and on the mainstem of the Missouri River located below effluent discharges are characterized by high values of these constituents.

\section{Application of Descriptive Statistics}

The tables of descriptive statistics for each station can be used to compare quality of water at stations within basins, to compare quality of water outflowing from various basins, and to compare the quality of water at any station or stations with criteria for water use.

\section{Intra-basin water quality}

Comparison of water quality based on descriptive statistics can show similarities and differences in quality between stations within a basin. Generally, in basins such as the Loup, Elkhorn, Little Blue, and Big Blue, for which the source of water is entirely within the State, water is more highly mineralized at downstream sampling sites than at upstream sites as a result of seepage of more highly mineralized ground water in the lower part of each basin. For some basins where water originates partly outside the State, water flowing into the State is more highly mineralized than is water which originates in Nebraska. For example, as a result of the inflow of ground water low in dissolved chemical constituents, the mean specific conductance for the Niobrara River is less near the mouth at Verdel than at the most upstream sampling site above Box Butte Reservoir. In still other basins, like the Middle Platte or the Republican, there is little, if any, overall difference in water quality between upstream and downstream sites.

In general, with two principal exceptions, more than one area of mantle rock and geomorphology characterize each basin in the State. For example, the upper part of the Elkhorn River basin is in the sandhills, but the lower part is in loess-covered glacial drift hills. The differences in mantle rock, geomorphology, and proportions of overland runoff to groundwater inflow cause the quality of streamflow to differ by area within a basin. This can be illustrated by comparing the quality of water in the Elkhorn River at Ewing with the quality of water in Logan Creek at Pender. Mean conductance is 220 umho/cm at Ewing where runoff is largely from the sandhills, and is $716 \mathrm{umho} / \mathrm{cm}$ at Pender where runoff is largely from the glacial drift area even though calcium and bicarbonate are the principal ions in both streams. 
The Missouri Tributaries basin and the Nemaha River basin are both located in loess-covered glacial drift hills. Insufficient data exist for these basins to compare data from unstream and downstream locations on the same stream. However, water quality is very similar both in type and degree of mineralization in the three major streams comprising the Nemaha River basin.

\section{Inter-basin water quality}

Comparison of water quality between basins is difficult because of water-quality differences within basins. However, comparison of outflow from the various basins is simple and highly instructive. Tables 6 and 7 contain data for the most downstream site in each of the basins or for the most representative station where downstream order may not be meaningful.

According to table 6 , calcium is the principal cation and bicarbonate the principal anion in outflow from seven and eight of the basins in the State, respectively. Sodium is the principal cation in the White River Hat Creek basin and sulfate is the principal anion in the White River Hat Creek basin and in the South Platte River basin. Sodium and calcium occur almost equally in water leaving the Missouri tributaries, North Platte, South P1atte, Middle Platte, and Lower Platte basins. Sulfate and bicarbonate occur almost equally in water leaving the Missouri tributaries, North Platte, and Middle P1atte basins. Based on specific conductance, water leaving the South Platte River basin is the most mineralized, and water leaving the Niobrara River basin is the least mineralized.

In table 7 are data for comparison of constituents often associated with water pollution for each basin where information is available. Those stations having the highest means and medians are below outfalls of sewage-treatment plants.

\section{Water-quality criteria}

Descriptive statistics for the individual stations can be used to estimate the suitability of water for many uses. Criteria have been established for most major uses of water including public drinking-water supply, irrigation, industry, and recreation and are discussed by the Environmental Protection Agency (1976). By comparing the descriptive statistics for a station in question with the criteria for a particular use, the suitability of water for that use can be determined. 
Table 6.--Comparison of mean concentrations of chemical constituents in outflow from each of 13 river basins in Nebraska

\begin{tabular}{|c|c|c|c|c|c|c|c|c|}
\hline Basin & Station & $\begin{array}{c}\text { Specific } \\
\text { conductance } \\
\text { umho/cm }\end{array}$ & $\begin{array}{c}\mathrm{Ca} \\
\mathrm{mg} / \mathrm{L}\end{array}$ & $\mathrm{Mg}$ & $\begin{array}{c}\mathrm{Na} \\
\mathrm{mg} / \mathrm{L}\end{array}$ & $\begin{array}{l}\mathrm{HCO}_{3} \\
\mathrm{mg} / \mathrm{L}\end{array}$ & $\begin{array}{l}\mathrm{SO}_{4} \\
\mathrm{mg} / \mathrm{L}\end{array}$ & $\begin{array}{c}\mathrm{C} 1 \\
\mathrm{mg} / \mathrm{L}\end{array}$ \\
\hline White - Hat & White R. near Whitney & 1,320 & 83 & 23 & 157 & 313 & 416 & 14 \\
\hline Niobrara & Niobrara R. near Verde1 & 266 & 36 & 4.8 & 9.9 & 142 & 16 & 1.9 \\
\hline $\begin{array}{l}\text { Missouri } \\
\text { Tributaries }\end{array}$ & Missouri R. at Nebraska City & 676 & 61 & 19 & 54 & 197 & 164 & 17 \\
\hline North Platte & North Platte R. near Keystone & 741 & 58 & 19 & 79 & 222 & 177 & 19 \\
\hline South Platte & South Platte R. at Roscoe & 1890 & 175 & 57 & 176 & 259 & 696 & 81 \\
\hline Middle Platte & Platte R. near Duncan & 828 & 70 & 21 & 73 & 223 & 208 & 25 \\
\hline Loup & $\begin{array}{l}\text { Loup River Power Canal at } \\
\text { diversion near Genoa }\end{array}$ & 278 & 37 & 6.1 & 9.4 & 157 & 11 & 2.5 \\
\hline Elkhorn & E1khorn R. at Water10o & 476 & 61 & 14 & 22 & 245 & 43 & 13 \\
\hline Lower Platte & Platte R. at Louisville & 713 & 55 & 13 & 77 & 207 & 81 & 79 \\
\hline Nemaha & Big Nemaha R. at Falls City & 657 & 70 & 18 & 30 & 249 & 72 & 25 \\
\hline Repub1ican & Republican R. at Superior & 511 & 66 & 14 & 24 & 233 & 57 & 15 \\
\hline Big Blue & Big Blue R. at Barneston & 538 & 54 & 12 & 40 & 212 & 48 & 34 \\
\hline Little Blue & $\begin{array}{l}\text { Little Blue R. at Hollenberg, } \\
\text { Kans. }\end{array}$ & 405 & 43 & 7.1 & 23 & 165 & 31 & 31 \\
\hline
\end{tabular}


Table 7.--Comparison of nitrite + nitrate, total phosphorus, BOD, fecal coliform and fecal streptococci in outflow from 13 river basins in Nebraska

\begin{tabular}{|c|c|c|c|c|c|c|}
\hline \multirow[t]{2}{*}{ Basin } & \multirow[t]{2}{*}{ Station } & \multicolumn{3}{|c|}{$\begin{array}{c}\text { Mean concentrations } \\
\mathrm{mg} / \mathrm{L}\end{array}$} & \multicolumn{2}{|c|}{$\begin{array}{c}\text { Median concentrations } \\
\text { colonies } / 100 \mathrm{~m} 1\end{array}$} \\
\hline & & $\mathrm{NO}_{2}+\mathrm{NO}_{3}$ & Total P & BOD & $\begin{array}{c}\text { Fecal } \\
\text { coliform }\end{array}$ & $\begin{array}{c}\text { Fecal } \\
\text { streptococci }\end{array}$ \\
\hline White - Hat & White R. near Whitney & 0.59 & 0.27 & 2.5 & 100 & --- \\
\hline Niobrara & Niobrara R. near Verdel & .40 & .27 & 3.2 & 32 & 118 \\
\hline $\begin{array}{l}\text { Missouri } \\
\text { Tributaries }\end{array}$ & Missouri R. at Nebraska City & .68 & .24 & $-\cdots$ & $-\cdots$ & $-\cdots$ \\
\hline North Platte & North Platte R. near Keystone & .41 & --- & $-\cdots$ & $\cdots$ & --- \\
\hline South Platte & South Platte R. at. Roscoe & --- & .21 & 3.0 & 140 & 200 \\
\hline Middle Platte & Platte R. near Duncan & .48 & .26 & 3.2 & 91 & --- \\
\hline Loup & $\begin{array}{l}\text { Loup R. Power Canal at } \\
\text { diversion near Genoa }\end{array}$ & .43 & .31 & --- & 55 & 133 \\
\hline Elkhorn & Elkhorn R. at Waterloo & 1.2 & 1.1 & 8.8 & 9600 & 3130 \\
\hline Lower Platte & Platte R. at Louisville & --- & .50 & $-\ldots$ & 110 & 180 \\
\hline Nemaha & Big Nemaha R. at Falls City & 1.8 & .51 & 5.2 & 4160 & 1190 \\
\hline Republican & Republican R. at Superior & .63 & .49 & 3.2 & 470 &.-- \\
\hline Big Blue & Big Blue R. at Barneston & $-\cdots$ & $-\cdots$ & --- & $-\cdots$ & $-\cdots$ \\
\hline Little Blue & $\begin{array}{l}\text { Little Blue R. at Hollenberg, } \\
\text { Kans. }\end{array}$ & 1.4 & .68 & 10.8 & 600 & 290 \\
\hline
\end{tabular}


Probably the most useful of the descriptive statistics for comparison to water quality criteria are those which measure frequency--the percentiles, including the median, and the range. The maximum and minimum which determine the range, represent only unusual conditions. For many uses, the 10 th and 90 th percentiles may be more representative of the actual range of occurrence of most constituents and therefore a better indication of what the quality of water at a station truly is like.

The process of using descriptive statistics to determine the suitability of water for a particular use is complicated in that, for most uses, a variety of constituents or properties and their relation to each other must be considered simultaneously. In determining suitability for irrigation, for example, total dissolved solids, boron concentrations, the ratio of sodium concentrations to those of calcium and magnesium, and the relation of bicarbonate concentrations to calcium and magnesium, must all be considered.

For an example of how the tables for each station can be used, table 8 compares irrigation criteria for dissolved solids and boron with frequency statistics for those constituents from representative outflow stations in each basin. Both total dissolved solids maximu and 90th percentile concentrations exceed the criteria for seven of the basins. However, minimum and 10th percentile concentrations exceed the criteria for only one of the basins. It should be pointed out that even though total dissolved solids concentrations exceed the criteria, use of the water for irrigation is not precluded because many other factors are involved (U.S. Salinity Laboratory, 1954).

Maximum concentrations for boron are less than the criteria for outflow from all 13 basins.

\section{Application of Regression Equations}

In genera1, but with numerous exceptions, for all sampling sites where sufficient data are available, dissolved-solids sum and residue, hardness, calcium, magnesium, and bicarbonate concentrations correlate well with specific conductance. Exceptions and reasons for exceptions have been noted in the sections concerning each basin. Sodium, chloride, and sulfate concentrations sometimes correlate well with specific conductance. Nitrite + nitrate, silica, and potassium concentrations correlate poorly with specific conductance in most cases. Specific conductance correlates well with water discharge for some sites. 
Table 8.--Examples showing comparison of frequency statistics with irrigation criteria for two constituents

\begin{tabular}{|c|c|c|c|c|c|c|c|c|c|c|c|c|c|}
\hline \multirow[b]{2}{*}{$\begin{array}{l}\text { River } \\
\text { basin }\end{array}$} & \multirow[b]{2}{*}{ Station } & \multicolumn{6}{|c|}{ Total dissolved solids (mg/L) } & \multicolumn{6}{|c|}{ Boron, dissolved (ug/L) } \\
\hline & & Criteria & $\begin{array}{l}\text { Max- } \\
\text { imum }\end{array}$ & $\begin{array}{l}90 \text { th } \\
\text { per- } \\
\text { centile }\end{array}$ & Median & $\begin{array}{c}10 \text { th } \\
\text { per- } \\
\text { centile }\end{array}$ & $\begin{array}{l}\text { Min- } \\
\text { imum }\end{array}$ & Criteria & $\begin{array}{l}\text { Max- } \\
\text { imum }\end{array}$ & $\begin{array}{l}90 \text { th } \\
\text { per- } \\
\text { centile }\end{array}$ & Median & $\begin{array}{l}10 \text { th } \\
\text { per- } \\
\text { centile }\end{array}$ & $\begin{array}{l}\text { Min- } \\
\text { imum }\end{array}$ \\
\hline White - Hat & White R. near Whitney & 500 & 2,290 & 1,700 & 813 & 470 & 298 & 750 & 740 & 420 & 207 & 87 & 60 \\
\hline Niobrara & Niobrara R. near Verdel & & 262 & 230 & 197 & 180 & 165 & & 60 & 50 & 30 & 20 & 0 \\
\hline $\begin{array}{l}\text { Missouri } \\
\text { tributaries }\end{array}$ & $\begin{array}{l}\text { Missouri R. at Nebraska } \\
\text { City }\end{array}$ & & 643 & --- & -- & -- & 217 & & 320 & -- & --- & -- & 40 \\
\hline North Platte & No.Platte R. near Keystone & & 529 & 520 & 494 & 481 & 477 & & 150 & 148 & 138 & 110 & 90 \\
\hline South Platte & So.Platte R. at Roscoe & & 1,800 & 1,700 & 1,440 & 1,200 & 1,170 & & 300 & -- & $-\cdot$ & -- & 220 \\
\hline Middle Platte & Platte R. near Duncan & & 788 & 685 & 550 & 460 & 171 & & 560 & 164 & 131 & 94 & 40 \\
\hline Loup & $\begin{array}{l}\text { Loup R. Power Canal at } \\
\text { diversion near Genoa }\end{array}$ & & 247 & 220 & 199 & 181 & 149 & & 730 & 70 & 50 & 25 & 20 \\
\hline E1khorn & E1khorn R. at Waterloo & & 493 & 380 & 338 & 210 & 136 & & 480 & 110 & 64 & 12 & 0 \\
\hline Lower Platte & Platte R. at Louisville & & 978 & 610 & 423 & 320 & 296 & & -- & -- & $-\cdot$ & $--\cdot$ & -- \\
\hline Nemaha & Big Nemaha R. at Falls City & & 638 & 570 & 418 & 350 & 251 & & 120 & 116 & 72 & 50 & 20 \\
\hline Republican & Republican R. at Superior & & 458 & 420 & 347 & 260 & 190 & & 200 & 130 & 80 & 43 & 40 \\
\hline Big Blue & Big Blue R. at Barneston & & 468 & 432 & 370 & 150 & 69 & & 170 & 93 & 69 & 40 & 20 \\
\hline Little Blue & $\begin{array}{l}\text { Little Blue R. at Hollen- } \\
\text { berg, Kans. }\end{array}$ & 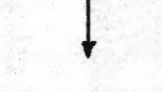 & 439 & 402 & 352 & 190 & 78 & $\downarrow$ & 100 & 90 & 59 & 53 & 30 \\
\hline
\end{tabular}


The regression equations in this report can be used in several ways. If specific conductance is known for a given time for a given sampling site, concentrations of other constituents can be estimated by substituting the known conductance into the appropriate equations. Assuming conditions are similar to those occurring during the period of record for each station, the reliability of the computed concentrations compared to the actual ones is related to the correlation coefficient, the standard error of estimate, and the level of significance. For sampling sites having water low in mineralization, differences between computed and actual concentrations may be within the range of the measurement error of the analytical method.

In table 9, three examples are given in which concentrations computed from the regression equations are compared to concentrations obtained by laboratory measurement. The three stations for which the examples are shown are distributed across the State in three different topographic and geologic settings. Streamflow in Little Nemaha River near Auburn is maintained mostly by overland runoff. That in the Calamus River near Burwell is maintained almost completely by discharge of ground water into the stream. That in the North Platte River near Lisco is maintained by controlled releases of water upstream and by irrigation return flow from both overland runoff and subsurface drainage.

Correlation coefficients for the first example, Little Nemaha River at Auburn, generally are high for the relationships between conductance and the selected constituents. The maximum difference between computed and actual concentrations is 6.2 percent for magnesium. This difference of $1 \mathrm{mg} / \mathrm{L}$ probably is not much greater than the measurement errors in the analytical method for magnesium.

Correlation coefficients for the second example, Calamus River near Burwe11, generally are poor, but differences between the laboratory measurement and the computed values, with the exception of sulfate, are very small. This indicates that when concentrations of constituents are consistently very low, the accuracy and precision of the analytical methods used to determine these concentrations may explain more of the variation in concentrations than does specific conductance.

For the third example, North Platte River at Lisco, the greatest percent difference between actual and computed concentrations for the constituents examined is 6.8 percent. Correlation coefficients are not as good as those for the Auburn station; however, because the water is more highly mineralized, the percent differences between actual and computed concentrations for some constituents are actually very low. 
Table 9.--Comparison between concentrations of chemical constituents determined by chemical analyses with those concentrations computed from regression equations

\begin{tabular}{ccccc}
\hline Constituent & $\begin{array}{c}\text { (A) } \\
\text { Actual } \\
\text { analysis }\end{array}$ & $\begin{array}{c}\text { Computed } \\
\text { value }\end{array}$ & $\begin{array}{l}\text { lation } \\
\text { coeffi } \\
\text { cient }\end{array}$ & $\frac{\mid A-B}{A} \times 100$ \\
\hline
\end{tabular}

Little Nemaha River at Auburn - March 1, 1977

$\begin{array}{lrrrr}\text { Specific conductance, umho/cm } & 598 & -1 . & -. .1 & \\ \text { Dissolved solids sum, mg/L } & 351 & 365 & 0.99 & 4.0 \\ \text { Calcium, mg/L } & 69 & 70 & .98 & 1.4 \\ \text { Magnesium, mg/L } & 16 & 17 & .96 & 6.2 \\ \text { Sodium, mg/L } & 34 & 36 & .88 & 5.9 \\ \text { Bicarbonate, mg/L } & 284 & 287 & .99 & 1.1 \\ \text { Sulfate, mg/L } & 55 & 56 & .94 & 1.8 \\ \text { Chloride, mg/L } & 17 & 18 & .64 & 5.9\end{array}$

Calamus River near Burwe11 - September 9, 1977

Specific conductance, umho/cm 135

Dissolved solids sum, mg/L $\quad 127$

Calcium, mg/L

Magnesium, $\mathrm{mg} / \mathrm{L}$

Sodium, mg/L

Bicarbonate, $\mathrm{mg} / \mathrm{L}$

Sulfate, $\mathrm{mg} / \mathrm{L}$

Chloride, mg/L
127
19

2.4

5.6

80

2.7

1.1
124

17

2.5

5.5

79

4.2

1.0
.47

.50

*. 25

.42

.54

*.24

*.19
2.4

10

4.2

1.8

1.2

56

9.1

North Platte River at Lisco - May 17, 1977

Specific conductance, umho/cm 922

Dissolved solids sum, mg/L $\quad 606$

Calcium, mg/L

Magnesium, mg/L

Sodium, $\mathrm{mg} / \mathrm{L}$

Bicarbonate, $\mathrm{mg} / \mathrm{L}$

Sulfate, $\mathrm{mg} / \mathrm{L}$

Chloride, $\mathrm{mg} / \mathrm{L}$

21

87

280

210

22

618
80
20
89
299
197
22

.87

.72

.29

.82

.70

.70

.70
2.0

0

4.8

2.3

6.8

6.2

0

* Not significant at 95 percent confidence leve1; the regression equation should not be used to predict the concentration of the dependent variable. 
Another way regression equations may be used is to reduce the frequency of measurement for some constituents for individual stations, provided that basin characteristics have not changed from the period of time that the data on which the regression equation is based was collected. Also, if daily specific conductance measurements are available for a station, mean daily constituent concentrations can be simulated. Furthermore, if the standard errors of estimate of the regression relationships are satisfactory and if both daily specific conductance and mean daily water discharge data are available, mean daily constituent loads can be simulated.

If regression data are verified periodically by laboratory measurements at regular intervals, changes in regression coefficients with time may be useful in detecting effects of changes in the environment caused by major human activities. Such changes in regression coefficients are more likely to be observed in small streams than in large streams.

Based on the available data for the 109 stations covered by this report, it appears that with only a few exceptions, use of regression equations generated for an individual station is appropriate only for that station. Small intra-basin differences in water quality preclude the use for one station of regression data generated for another. Therefore, any simulation of water-quality data using the regression equations generated for a particular station should be considered appropriate only for that location on the stream or for a short distance upstream or downstream from that location. 
Barr, A. J., Goodnight, J. H., Sall, J. P., and Helwig, J. T., 1976, A user's guide to SAS-76: Raleigh, N.C., SAS Institute, Inc., 331 p.

Benta11, R., et a1, 1971, Water supplies and the land--the Elkhorn River basin of Nebraska: Lincoln, University of Nebraska, Conservation and Survey Division, Resource Atlas no. 1, 51 p.

Benta11, R., and Shaffer, F. B., 1979, Availability and use of water in Nebraska, 1975: Lincoln, University of Nebraska, Conservation and Survey Division, Nebraska Water Survey Paper 48, 121 p.

Condra, G. E.,' and Reed, E. C., 1959, The geological section of Nebraska: Lincoln, University of Nebraska, Conservation and Survey Division, Nebraska Geological Survey Bulletin 14a, 82 p.

Engberg, R. A., and Spalding, R. F., 1978, Ground-water quality atlas of Nebraska: Lincoln, University of Nebraska, Conservation and Survey Division, Resource Atlas no. 3, 39 p.

Ham, J. D., 1970, Study and interpretation of the chemical characteristics of natural water; $2 \mathrm{~d}$ edition: U.S. Geological Survey Water-Supply Paper 1473, $363 \mathrm{p}$.

Irwin, G. A., and Lemons, M., 1975, A summary of selected chemical-quality conditions in 66 California streams, 1950-72: Meno Park, Calif., U.S. Geological Survey open-file report, 104 p.

Keech, C. F., and Bentall, Ray, 1971, Dunes on the Plains--the Sandhills region of Nebraska: Lincoln, University of Nebraska, Conservation and Survey Division, Resource Report no. 4, 18 p.

Lane, W. L., 1975, Extraction of information on inorganic water quality: Colorado State University, Hydrology Papers No. 73, 74 p.

Lawson, M. P., Dewey, K. F., and Neild, R. E., 1977, Climatic atlas of Nebraska: Lincoln, Nebr., University of Nebraska Press, 88 p.

McKee, J. E., and Wolf, H. W., eds., 1963, Water quality criteria, 2d ed: California State Water Quality Control Board Publication No. 3-A, 550 p.

Missouri River Basin Commission, 1975, Water quality technical paper, Level B study of Platte River basin, Nebraska: Missouri River Basin Commission, Technical Paper, $164 \mathrm{p}$. 
National Academy of Sciences - National Academy of Engineering, Environmental Studies Board, Committee on Water Quality Criteria, 1973, Water quality criteria, 1972: Washington, D.C., U.S. Environmental Protection Agency, EPA R.3-73-033, 594 p.

Nebraska Soil and Water Conservation Commission, 1971, Report on the framework study: Lincoln, Nebraska Soil and Water Conservation Commission, State Water Plan Publication no. 101, 268 p.

University of Nebraska, 1966, reprinted 1969, Groundwater atlas of Nebraska: Lincoln, University of Nebraska, Conservation and Survey Division, Miscellaneous Publication, $15 \mathrm{p}$.

U.S. Environmental Protection Agency, 1976, Quality criteria for water: Washington, D.C., U.S. Government Printing Office, EPA-440/9-76-023, $501 \mathrm{p}$.

U.S. Geological Survey, published annually 1964-74, Water resources data for Nebraska--Part 2, Water-quality records: Lincoln, Nebr., U.S. Geological Survey open-file reports.

U.S. Geological Survey, published annually, 1975-78, Water-resources data for Nebraska: Lincoln, Nebr., U.S. Geological Survey open-file reports.

U.S. Salinity Laboratory Staff, 1954, Diagnosis and improvement of saline and alkal ine soils: U.S. Department of Agriculture Handbook 60, 160 p. 



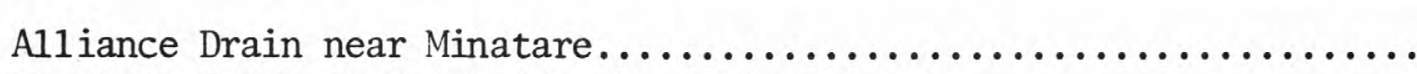

Antelope Creek at Court Street at Lincoln.......................

Beaver Creek near Albion....................................

Big Blue River at Barneston................................

at Surprise.

below Beatrice........................................

below Seward.....................................

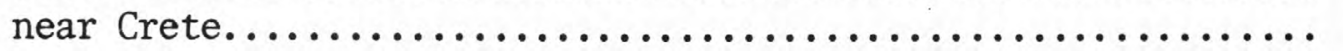

West Fork below Hastings.............................

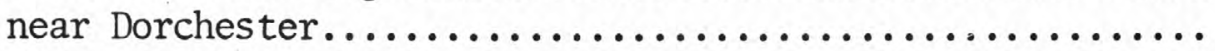

Big Nemaha River at Falls City.

Page

68,69

188,189

155,156

254,255

238,239

252,253

242,243

248,249

244,245

246,247

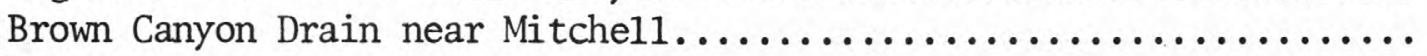

Calamus River near Burwel1...............................

Cedar River near Fullerton.

218,219

58,59

147,148

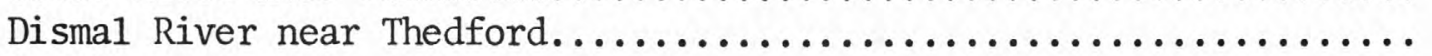

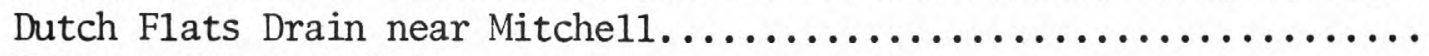

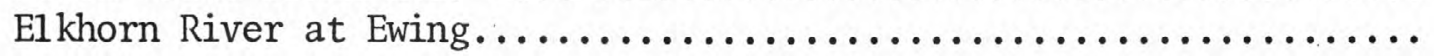

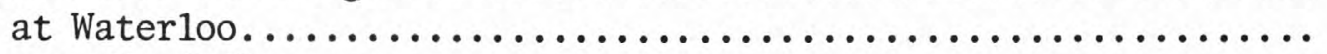

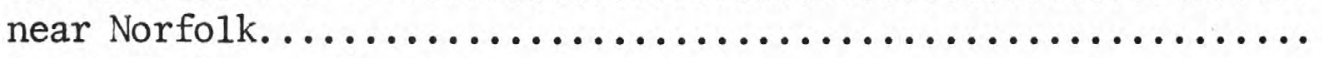

North Fork near Pierce............................

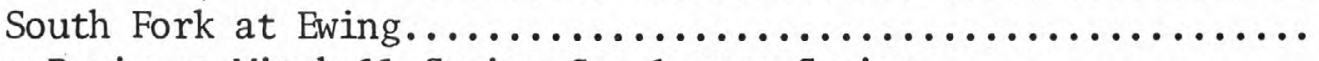

Gering Drain at Mitchell-Gering Canal near Gering................

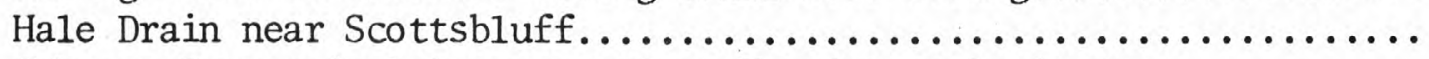

Johnson Reservoir below powerplant No. 2 near Lexington...........

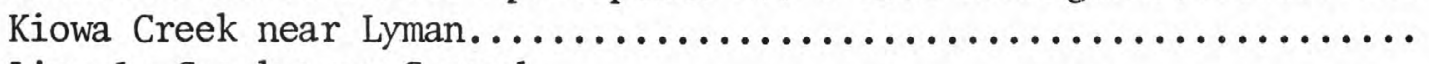

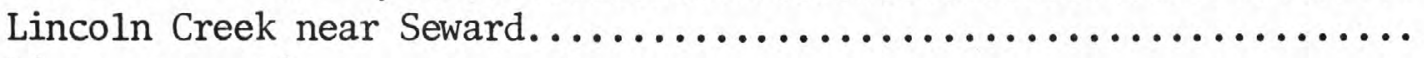

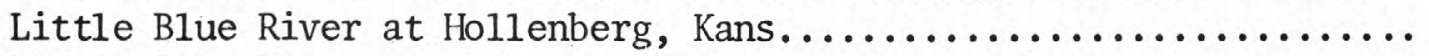

Little Blue River near Deweese.............................

Little Nemaha River at Auburn...............................

Little Salt Creek near Lincoln.............................

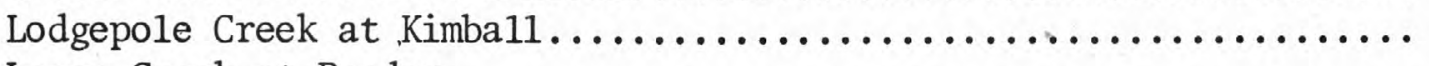

Logan Creek at Pender....................................

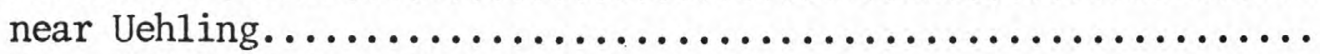

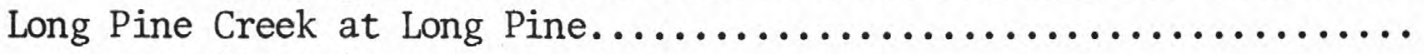

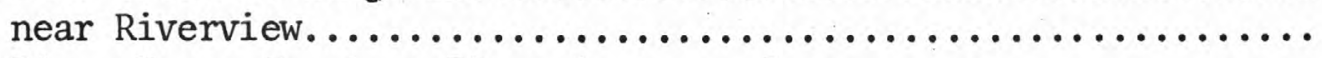

Loup River Power Canal at Diversion near Genoa.................. Middle Loup River at St. Paul.............................

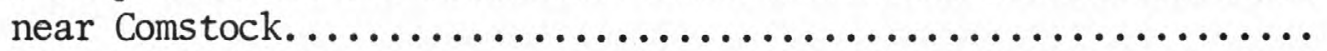

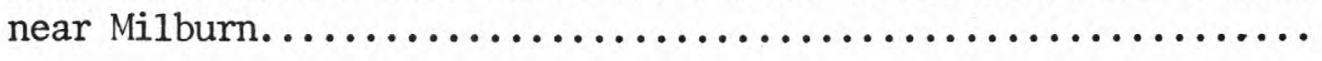
151,152 133,134 60,61 160,161 172,173 164,165 166,167 162,163

66,67

64,65

105, 106

54,55 240,241 260,261 258,259 216,217 194,195

85,86

168,169 170,171 29,30

31,32 153,154 143,144 137,138 135,136 


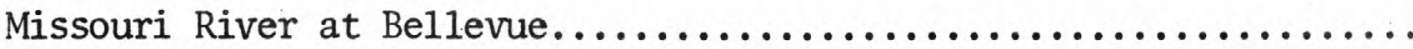

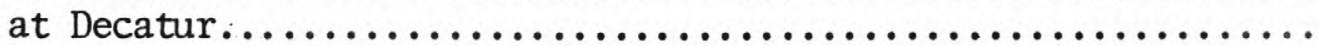

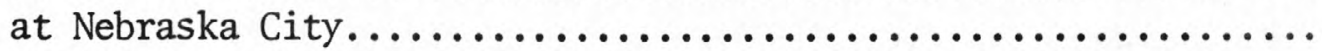

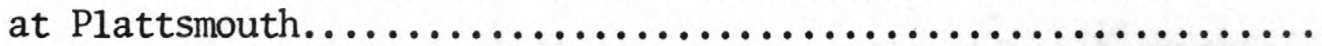

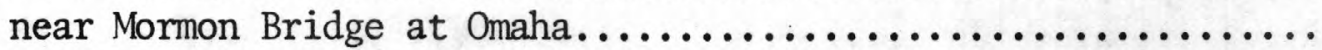

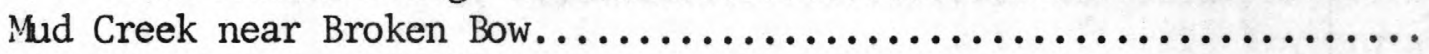

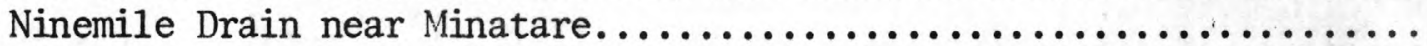

Niobrara River above Box Butte Reservoir.....................

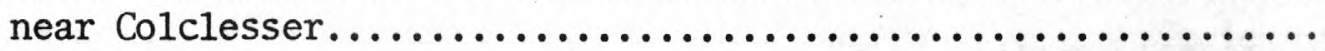

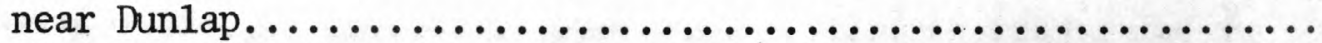

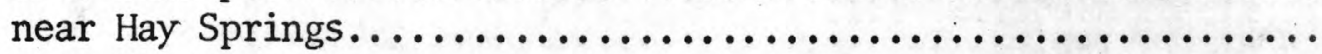

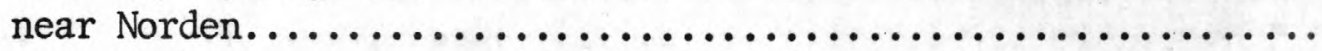

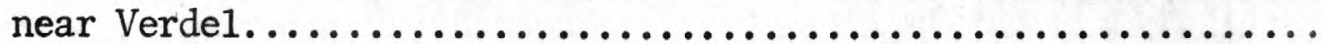

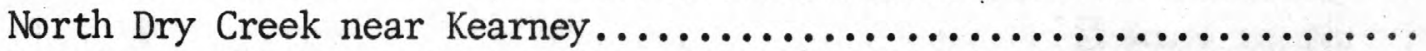

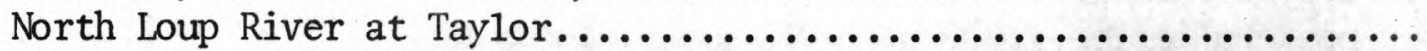

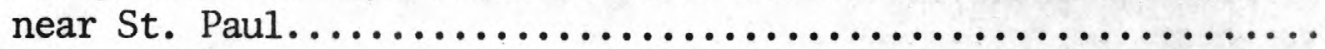

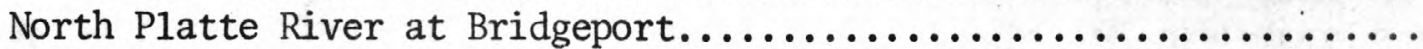

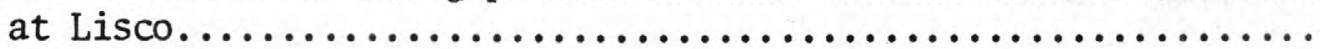

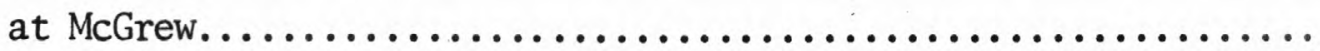

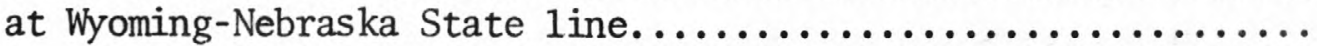

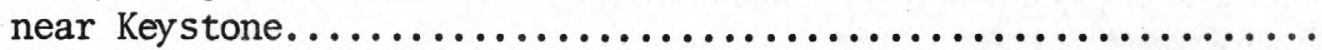

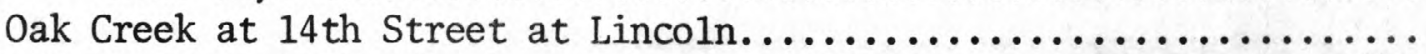
Platte River at Brady.

at Brady (channe1 1)

at Brady (channel 4 )

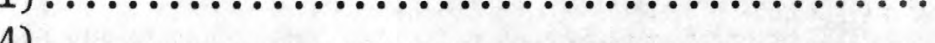

at LaPlatte.

at Louisville.

at North Bend.

near Schuyler.

near Duncan.

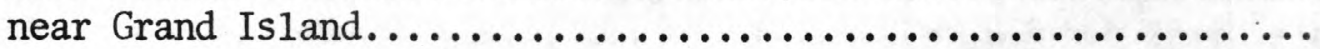

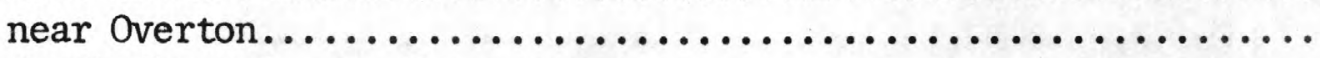

North Channel near Kearney.

near Overton.

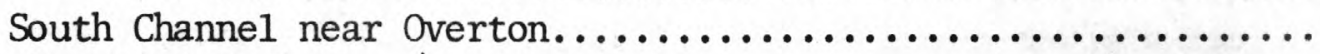

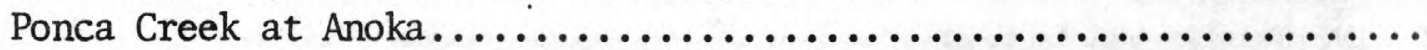

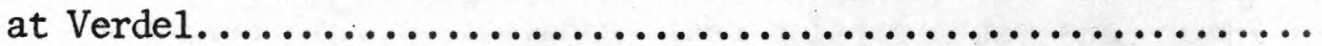

Pumpkin Creek near Bridgeport............................

Republican River above Medicine Creek at Cambridge..............

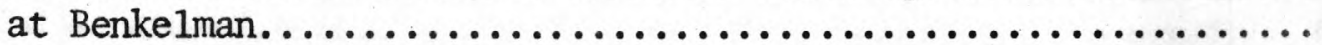

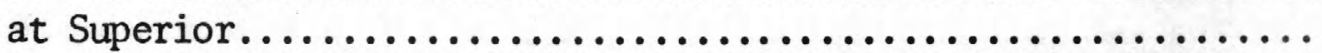

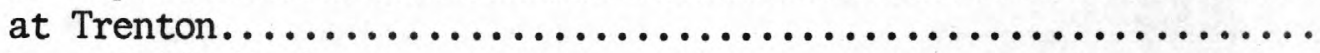

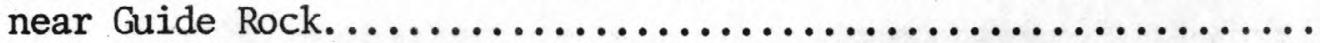

near Orleans. 
Pock Creek near Ceresco P. Page

Rock Creek near Ceresco................................. 200, 201

Salt Creek above Ashland............................... 203, 204

above Beal Slough at Lincoln........................ 184, 185

at 14 th Street at Lincoln............................ 186, 187

at Greenwood.......................................... 202

at Lincoln..................................... 192, 193

below Stevens Creek near Waverly...................... 198, 199

near Roca...................................... 182, 183

Sheep Creek near Morrill............................. 56, 57

Silver Creek near Wahoo.............................. 207, 208

South Loup River at St. Michae1........................ 141, 142

South Platte River at Julesburg, Colo..................... 87, 88

at Roscoe...................................... 91, 92

near Julesburg, Colo.............................. 89, 90

Spring Creek below Lexington........................... 113, 114

Stevens Creek at Highway 6 near Lincoln..................... 196, 197

Supply Canal (Tri-county diversion) near Maxwell............. 97, 98

Turkey Creek near Wilber.............................. 250, 251

Wahoo Creek at Ithaca................................. 205, 206

Weeping Water Creek near Union......................... 214, 215

White River near Whitney............................... 15, 16

Winter Creek at Tri-State Canal near Scottsbluff............... 62, 63

Wood River near Alda................................. 122, 123

near Chapman....................................... 126, 127

near Gibbon...................................... 121

near Grand Island................................... 124, 125 

